Cochrane Database of Systematic Reviews

\title{
Perioperative intravenous ketamine for acute postoperative pain in adults (Review)
}

Brinck ECV, Tiippana E, Heesen M, Bell RF, Straube S, Moore RA, Kontinen V

Brinck ECV, Tiippana E, Heesen M, Bell RF, Straube S, Moore RA, Kontinen V.

Perioperative intravenous ketamine for acute postoperative pain in adults.

Cochrane Database of Systematic Reviews 2018, Issue 12. Art. No.: CD012033. DOI: 10.1002/14651858.CD012033.pub4.

www.cochranelibrary.com 
TABLE OF CONTENTS

HEADER

ABSTRACT

PLAIN LANGUAGE SUMMARY

SUMMARY OF FINDINGS

BACKGROUND

OBJECTIVES

METHODS

RESULTS

Figure 1.

Figure 2.

Figure 3.

DISCUSSION

AUTHORS' CONCLUSIONS

ACKNOWLEDGEMENTS

REFERENCES

CHARACTERISTICS OF STUDIES

DATA AND ANALYSES

Analysis 1.1. Comparison 1: Perioperative ketamine versus control in a non-stratified study population, Outcome 1: Opioid consumption at 24 hours

Analysis 1.2. Comparison 1: Perioperative ketamine versus control in a non-stratified study population, Outcome 2: Opioid consumption at 48 hours

Analysis 1.3. Comparison 1: Perioperative ketamine versus control in a non-stratified study population, Outcome 3: Pain intensity at rest at 24 hours

Analysis 1.4. Comparison 1: Perioperative ketamine versus control in a non-stratified study population, Outcome 4: Pain intensity during movement at 24 hours

Analysis 1.5. Comparison 1: Perioperative ketamine versus control in a non-stratified study population, Outcome 5: Pain intensity at rest at 48 hours

Analysis 1.6. Comparison 1: Perioperative ketamine versus control in a non-stratified study population, Outcome 6: Pain intensity during movement at 48 hours

Analysis 1.7. Comparison 1: Perioperative ketamine versus control in a non-stratified study population, Outcome 7: Time to first request for analgesia/trigger of PCA

Analysis 1.8. Comparison 1: Perioperative ketamine versus control in a non-stratified study population, Outcome 8: CNS adverse events - all studies

Analysis 1.9. Comparison 1: Perioperative ketamine versus control in a non-stratified study population, Outcome 9: Hyperalgesia

Analysis 1.10. Comparison 1: Perioperative ketamine versus control in a non-stratified study population, Outcome 10: CNS adverse events - studies with events

Analysis 1.11. Comparison 1: Perioperative ketamine versus control in a non-stratified study population, Outcome 11: Postoperative nausea and vomiting - all studies

Analysis 2.1. Comparison 2: Pre-incisional and postoperative ketamine versus control in a non-stratified patient population, Outcome 1: Opioid consumption at 24 hours

Analysis 2.2. Comparison 2: Pre-incisional and postoperative ketamine versus control in a non-stratified patient population, Outcome 2: Opioid consumption at 48 hours

Analysis 2.3. Comparison 2: Pre-incisional and postoperative ketamine versus control in a non-stratified patient population, Outcome 3: Pain intensity at 24 hours

Analysis 2.4. Comparison 2: Pre-incisional and postoperative ketamine versus control in a non-stratified patient population, Outcome 4: Pain intensity at 48 hours

Analysis 2.5. Comparison 2: Pre-incisional and postoperative ketamine versus control in a non-stratified patient population, Outcome 5: Time to first request for analgesia/first trigger of PCA

Analysis 3.1. Comparison 3: Perioperative ketamine versus control co-administered with nitrous oxide in a non-stratified study population, Outcome 1: Opioid consumption at 24 hours

Analysis 3.2. Comparison 3: Perioperative ketamine versus control co-administered with nitrous oxide in a non-stratified study population, Outcome 2: Opioid consumption at 48 hours 
Analysis 3.3. Comparison 3: Perioperative ketamine versus control co-administered with nitrous oxide in a non-stratified study population, Outcome 3: Pain intensity at rest at 24 hours

Analysis 3.4. Comparison 3: Perioperative ketamine versus control co-administered with nitrous oxide in a non-stratified study population, Outcome 4: Pain intensity during movement at 24 hours

Analysis 3.5. Comparison 3: Perioperative ketamine versus control co-administered with nitrous oxide in a non-stratified study population, Outcome 5: Pain intensity at rest at 48 hours

Analysis 3.6. Comparison 3: Perioperative ketamine versus control co-administered with nitrous oxide in a non-stratified study population, Outcome 6: Pain intensity during movement at 48 hours

Analysis 4.1. Comparison 4: CNS adverse events in studies with benzodiazepine premedication, Outcome 1: CNS adverse events

Analysis 5.1. Comparison 5: Perioperative ketamine versus control: thoracotomy, Outcome 1: Opioid consumption at 24 hours Analysis 5.2. Comparison 5: Perioperative ketamine versus control: thoracotomy, Outcome 2: Opioid consumption at 48 hours Analysis 5.3. Comparison 5: Perioperative ketamine versus control: thoracotomy, Outcome 3: Pain intensity at rest at 24 hours Analysis 5.4. Comparison 5: Perioperative ketamine versus control: thoracotomy, Outcome 4: Pain intensity during movement at 24 hours

Analysis 5.5. Comparison 5: Perioperative ketamine versus control: thoracotomy, Outcome 5: Pain intensity at rest at 48 hours

Analysis 5.6. Comparison 5: Perioperative ketamine versus control: thoracotomy, Outcome 6: Pain intensity during movement at 48 hours

Analysis 6.1. Comparison 6: Perioperative ketamine versus control: major orthopaedic surgery, Outcome 1: Opioid consumption at 24 hours

Analysis 6.2. Comparison 6: Perioperative ketamine versus control: major orthopaedic surgery, Outcome 2: Opioid consumption at 48 hours

Analysis 6.3. Comparison 6: Perioperative ketamine versus control: major orthopaedic surgery, Outcome 3: Pain intensity at rest at 24 hours

Analysis 6.4. Comparison 6: Perioperative ketamine versus control: major orthopaedic surgery, Outcome 4: Pain intensity during movement at 24 hours

Analysis 6.5. Comparison 6: Perioperative ketamine versus control: major orthopaedic surgery, Outcome 5: Pain intensity at rest at 48 hours

Analysis 6.6. Comparison 6: Perioperative ketamine versus control: major orthopaedic surgery, Outcome 6: Pain intensity during movement at 48 hours

Analysis 7.1. Comparison 7: Perioperative ketamine versus control: major abdominal surgery, Outcome 1: Opioid consumption at 24 hours

Analysis 7.2. Comparison 7: Perioperative ketamine versus control: major abdominal surgery, Outcome 2: Opioid consumption at 48 hours

Analysis 7.3. Comparison 7: Perioperative ketamine versus control: major abdominal surgery, Outcome 3: Pain intensity at rest at 24 hours

Analysis 7.4. Comparison 7: Perioperative ketamine versus control: major abdominal surgery, Outcome 4: Pain intensity during movement at 24 hours

Analysis 7.5. Comparison 7: Perioperative ketamine versus control: major abdominal surgery, Outcome 5: Pain intensity at rest at 48 hours

Analysis 7.6. Comparison 7: Perioperative ketamine versus control: major abdominal surgery, Outcome 6: Pain intensity during movement at 48 hours

Analysis 8.1. Comparison 8: Perioperative ketamine versus control: total abdominal hysterectomy, Outcome 1: Opioid consumption at 24 hours

Analysis 8.2. Comparison 8: Perioperative ketamine versus control: total abdominal hysterectomy, Outcome 2: Opioid consumption at 48 hours

Analysis 8.3. Comparison 8: Perioperative ketamine versus control: total abdominal hysterectomy, Outcome 3: Pain intensity at rest at 24 hours

Analysis 9.1. Comparison 9: Perioperative ketamine versus control: laparoscopic procedures, Outcome 1: Opioid consumption at 24 hours

Analysis 9.2. Comparison 9: Perioperative ketamine versus control: laparoscopic procedures, Outcome 2: Opioid consumption at 48 hours

Analysis 9.3. Comparison 9: Perioperative ketamine versus control: laparoscopic procedures, Outcome 3: Pain intensity at rest at 24 hours

APPENDICES 
[Intervention Review]

\section{Perioperative intravenous ketamine for acute postoperative pain in adults}

Elina CV Brinck ${ }^{1}$, Elina Tiippana ${ }^{2}$, Michael Heesen ${ }^{3}$, Rae Frances Bell ${ }^{4}$, Sebastian Straube ${ }^{5}$, R Andrew Moore ${ }^{6}$, Vesa Kontinen ${ }^{7}$

1Department of Anesthesiology, Intensive Care and Pain Medicine, Division of Anesthesiology, Töölö Hospital, Helsinki University and Helsinki University Hospital, Helsinki, Finland. 2Department of Anesthesiology, Intensive Care and Pain Medicine, Division of Anesthesiology, Hyvinkää Hospital, Helsinki University and Helsinki University Hospital, Helsinki, Finland. ${ }^{3}$ Department of Anaesthesia and Intensive Care, Kantonsspital Baden, Baden, Switzerland. ${ }^{4}$ Regional Centre of Excellence in Palliative Care, Haukeland University Hospital, Bergen, Norway. ${ }^{5}$ Department of Medicine, Division of Preventive Medicine, University of Alberta, Edmonton, Canada. 6Plymouth, UK. ${ }^{7}$ Department of Anesthesiology, Intensive Care and Pain Medicine, Division of Anesthesiology, Jorvi Hospital, Helsinki University and Helsinki University Hospital, Helsinki, Finland

Contact address: Elina CV Brinck, elina.brinck@hus.fi.

Editorial group: Cochrane Pain, Palliative and Supportive Care Group.

Publication status and date: Stable (no update expected for reasons given in 'What's new'), published in Issue 1, 2021.

Citation: Brinck ECV, Tiippana E, Heesen M, Bell RF, Straube S, Moore RA, Kontinen V. Perioperative intravenous ketamine for acute postoperative pain in adults. Cochrane Database of Systematic Reviews 2018, Issue 12. Art. No.: CD012033. DOI: 10.1002/14651858.CD012033.pub4.

Copyright (c 2018 The Cochrane Collaboration. Published by John Wiley \& Sons, Ltd.

\section{A B S T R A C T}

\section{Background}

Inadequate pain management after surgery increases the risk of postoperative complications and may predispose for chronic postsurgical pain. Perioperative ketamine may enhance conventional analgesics in the acute postoperative setting.

\section{Objectives}

To evaluate the efficacy and safety of perioperative intravenous ketamine in adult patients when used for the treatment or prevention of acute pain following general anaesthesia.

\section{Search methods}

We searched CENTRAL, MEDLINE and Embase to July 2018 and three trials registers (metaRegister of controlled trials, ClinicalTrials.gov and the World Health Organization (WHO) International Clinical Trials Registry Platform (ICTRP)) together with reference checking, citation searching and contact with study authors to identify additional studies.

\section{Selection criteria}

We sought randomised, double-blind, controlled trials of adults undergoing surgery under general anaesthesia and being treated with perioperative intravenous ketamine. Studies compared ketamine with placebo, or compared ketamine plus a basic analgesic, such as morphine or non-steroidal anti-inflammatory drug (NSAID), with a basic analgesic alone.

\section{Data collection and analysis}

Two review authors searched for studies, extracted efficacy and adverse event data, examined issues of study quality and potential bias, and performed analyses. Primary outcomes were opioid consumption and pain intensity at rest and during movement at 24 and 48 hours postoperatively. Secondary outcomes were time to first analgesic request, assessment of postoperative hyperalgesia, central nervous system (CNS) adverse effects, and postoperative nausea and vomiting. We assessed the evidence using GRADE and created a 'Summary of findings' table. 


\section{Main results}

We included 130 studies with 8341 participants. Ketamine was given to 4588 participants and 3753 participants served as controls. Types of surgery included ear, nose or throat surgery, wisdom tooth extraction, thoracotomy, lumbar fusion surgery, microdiscectomy, hip joint replacement surgery, knee joint replacement surgery, anterior cruciate ligament repair, knee arthroscopy, mastectomy, haemorrhoidectomy, abdominal surgery, radical prostatectomy, thyroid surgery, elective caesarean section, and laparoscopic surgery. Racemic ketamine bolus doses were predominantly $0.25 \mathrm{mg}$ to $1 \mathrm{mg}$, and infusions 2 to $5 \mu \mathrm{g} / \mathrm{kg} / \mathrm{minute}$; 10 studies used only S-ketamine and one only R-ketamine. Risk of bias was generally low or uncertain, except for study size; most had fewer than 50 participants per treatment arm, resulting in high heterogeneity, as expected, for most analyses. We did not stratify the main analysis by type of surgery or any other factor, such as dose or timing of ketamine administration, and used a non-stratified analysis.

Perioperative intravenous ketamine reduced postoperative opioid consumption over 24 hours by $8 \mathrm{mg}$ morphine equivalents (95\% $\mathrm{Cl} 6$ to 9 ; $19 \%$ from $42 \mathrm{mg}$ consumed by participants given placebo, moderate-quality evidence; 65 studies, 4004 participants). Over 48 hours, opioid consumption was $13 \mathrm{mg}$ lower ( $95 \% \mathrm{Cl} 10$ to 15; 19\% from $67 \mathrm{mg}$ with placebo, moderate-quality evidence; 37 studies, 2449 participants).

Perioperative intravenous ketamine reduced pain at rest at 24 hours by $5 / 100 \mathrm{~mm}$ on a visual analogue scale $(95 \% \mathrm{Cl} 4$ to $7 ; 19 \%$ lower from 26/100 mm with placebo, high-quality evidence; 82 studies, 5004 participants), and at 48 hours by $5 / 100 \mathrm{~mm} \mathrm{(95 \%} \mathrm{Cl} 3$ to $7 ; 22 \%$ lower from $23 / 100 \mathrm{~mm}$, high-quality evidence; 49 studies, 2962 participants). Pain during movement was reduced at $24 \mathrm{hours}$ (6/100 mm, $14 \%$ lower from 42/100 mm, moderate-quality evidence; 29 studies, 1806 participants), and 48 hours (6/100 mm, 16\% lower from $37 \mathrm{~mm}$, lowquality evidence; 23 studies, 1353 participants).

Results for primary outcomes were consistent when analysed by pain at rest or on movement, operation type, and timing of administration, or sensitivity to study size and pain intensity. No analysis by dose was possible. There was no difference when nitrous oxide was used. We downgraded the quality of the evidence once if numbers of participants were large but small-study effects were present, or twice if numbers were small and small-study effects likely but testing not possible.

Ketamine increased the time for the first postoperative analgesic request by 54 minutes ( $95 \% \mathrm{Cl} 37$ to 71 minutes), from a mean of 39 minutes with placebo (moderate-quality evidence; 31 studies, 1678 participants). Ketamine reduced the area of postoperative hyperalgesia by $7 \mathrm{~cm}^{2}(95 \% \mathrm{Cl}-11.9$ to -2.2$)$, compared with placebo (very low-quality evidence; 7 studies 333 participants). We downgraded the quality of evidence because of small-study effects or because the number of participants was below 400 .

CNS adverse events occurred in 52 studies, while 53 studies reported of absence of CNS adverse events. Overall, 187/3614 (5\%) participants receiving ketamine and $122 / 2924(4 \%)$ receiving control treatment experienced an adverse event (RR $1.2,95 \% \mathrm{Cl} 0.95$ to 1.4 ; high-quality evidence; 105 studies, 6538 participants). Ketamine reduced postoperative nausea and vomiting from $27 \%$ with placebo to $23 \%$ with ketamine ( $R R 0.88,95 \% \mathrm{Cl} 0.81$ to 0.96 ; the number needed to treat to prevent one episode of postoperative nausea and vomiting with perioperative intravenous ketamine administration was 24 (95\% Cl 16 to 54; high-quality evidence; 95 studies, 5965 participants).

\section{Authors' conclusions}

Perioperative intravenous ketamine probably reduces postoperative analgesic consumption and pain intensity. Results were consistent in different operation types or timing of ketamine administration, with larger and smaller studies, and by higher and lower pain intensity. CNS adverse events were little different with ketamine or control. Perioperative intravenous ketamine probably reduces postoperative nausea and vomiting by a small extent, of arguable clinical relevance.

\section{PLAIN LANGUAGE SUMMARY}

\section{Ketamine venous injection for acute pain after operation in adults}

\section{Bottom line}

Ketamine injected into a vein at the time of operation reduces pain, nausea and vomiting, and use of opioid (morphine-like) painkillers after operation.

\section{Background}

Poor pain management after an operation increases the risk of complications, decreases quality of life, and increases the risk for chronic pain. Painkillers such as paracetamol and non-steroidal anti-inflammatory drugs (ibuprofen, diclofenac), alone may be insufficient. Opioids (strong painkillers), often cause side effects. Studies suggested that ketamine used by injection during an operation helps to relieve pain after the operation.

\section{Study characteristics}

In July 2018 we searched for randomised clinical trials where ketamine was injected before, during, or after operation in adults having an operation under general anaesthesia. Important outcomes were opioid use and pain at 24 and 48 hours after the operation, time to first request for a painkiller, and ketamine-related side effects. We found 130 eligible studies with 8341 participants. 


\section{Key findings}

Compared to people given control treatment, those given intravenous ketamine used less opioid painkiller (by about 1 part in 10), and had less pain (by about 2 parts in 10; moderate- or high-quality evidence). Ketamine may be more effective in operations that are likely to cause more intense pain. People given ketamine requested painkillers 54 minutes later than those who did not receive ketamine (moderatequality evidence). Ketamine reduced the risk of postoperative nausea and vomiting by a small amount (high-quality evidence). Ketamine produced no increased risk of central nervous system side effects (hallucination, nightmares or double vision) (high-quality evidence).

Future research should assess ketamine's effect after operations that are accompanied by intense pain such as thoracotomy, back surgery, or amputations. Additionally, assessing ketamine's effects among particular patient groups, for example, the elderly or individuals with a history of substance abuse would be of interest.

\section{Quality of the evidence}

We rated the quality of the evidence from studies using four levels: very low, low, moderate, or high. Very low-quality evidence means that we are very uncertain about the results. High-quality evidence means that we are very confident in the results.

We found the quality of evidence for most outcomes to be moderate. Many of the studies were small, which was the main reason for downgrading the evidence from high to moderate. We tested the results by operation type, timing of ketamine injection, and by looking at larger studies, and those with more pain were consistent, and provided confidence in the results. There was sufficient evidence to allow conclusions about ketamine's effect on pain, painkiller consumption and side effects after operation. 


\section{SUMMARY OF FINDINGS}

\section{Summary of findings 1. Perioperative intravenous ketamine compared to placebo for acute postoperative pain in adults}

Perioperative intravenous ketamine compared to placebo for acute postoperative pain: non-stratified analysis

Patient or population: adults undergoing any type of surgery

Settings: immediate postoperative period

Intervention: intravenous ketamine given before, during, or after surgery

Comparison: intravenous placebo

\begin{tabular}{|c|c|c|c|c|c|}
\hline \multirow[t]{2}{*}{ Outcomes } & \multirow[t]{2}{*}{ Details } & \multirow{2}{*}{$\begin{array}{l}\text { Number of partici- } \\
\text { pants } \\
\text { (studies) }\end{array}$} & \multicolumn{2}{|c|}{ Absolute values and effect of ketamine } & \multirow{2}{*}{$\begin{array}{l}\text { Quality of the evi } \\
\text { dence } \\
\text { (GRADE) }\end{array}$} \\
\hline & & & $\begin{array}{l}\text { Measured values with } \\
\text { placebo }\end{array}$ & $\begin{array}{l}\text { Difference with perioperative intravenous } \\
\text { ketamine } \\
(95 \% \mathrm{Cl})\end{array}$ & \\
\hline \multirow{2}{*}{$\begin{array}{l}\text { Opioid consump- } \\
\text { tion } \\
\text { (mg morphine } \\
\text { equivalents) }\end{array}$} & 24 hours & $\begin{array}{l}4004 \\
\text { (65 RCTs) }\end{array}$ & $\begin{array}{l}\text { Median } 31 \mathrm{mg} \\
\text { (mean } 42 \mathrm{mg} \text { ) }\end{array}$ & $\begin{array}{l}\text { MD } 7.6 \mathrm{mg} \text { lower } \\
\text { (8.9 lower to } 6.4 \text { lower) }\end{array}$ & Moderate ${ }^{1}$ \\
\hline & 48 hours & $\begin{array}{l}2449 \\
(37 \mathrm{RCTs})\end{array}$ & $\begin{array}{l}\text { Median } 59 \text { mg } \\
\text { (mean } 67 \mathrm{mg} \text { ) }\end{array}$ & $\begin{array}{l}\text { MD } 12.6 \mathrm{mg} \text { lower } \\
\text { (15 lower to } 10 \text { lower) }\end{array}$ & Moderate ${ }^{1}$ \\
\hline \multirow[t]{4}{*}{$\begin{array}{l}\text { Pain intensity } \\
(0-100 \mathrm{~mm} \text { VAS. } 7\end{array}$} & At rest 24 hours & $\begin{array}{l}5004 \\
\text { (82 RCTs) }\end{array}$ & $\begin{array}{l}\text { Median } 25 \mathrm{~mm} \\
\text { (mean } 26 \mathrm{~mm} \text { ) }\end{array}$ & $\begin{array}{l}\text { MD } 5 \mathrm{~mm} \text { (VAS) lower } \\
\text { (6.6 lower to } 3.6 \text { lower) }\end{array}$ & High$^{2}$ \\
\hline & On movement 24 hours & $\begin{array}{l}1806 \\
\text { (29 RCTs) }\end{array}$ & $\begin{array}{l}\text { Median } 43 \mathrm{~mm} \\
\text { (mean } 42 \mathrm{~mm} \text { ) }\end{array}$ & $\begin{array}{l}\text { MD } 6 \text { mm (VAS) lower } \\
\text { (11 lower to } 0.5 \text { lower) }\end{array}$ & Moderate ${ }^{1}$ \\
\hline & At rest 48 hours & $\begin{array}{l}2962 \\
\text { (49 RCTs) }\end{array}$ & $\begin{array}{l}\text { Median } 21 \mathrm{~mm} \\
\text { (mean } 23 \mathrm{~mm} \text { ) }\end{array}$ & $\begin{array}{l}\text { MD } 5 \mathrm{~mm} \text { (VAS) lower } \\
\text { (6.7 lower to } 3.4 \text { lower) }\end{array}$ & High $^{2}$ \\
\hline & On movement 48 hours & $\begin{array}{l}1353 \\
\text { (23 RCTs) }\end{array}$ & $\begin{array}{l}\text { Median } 37 \mathrm{~mm} \\
\text { (mean } 37 \mathrm{~mm} \text { ) }\end{array}$ & $\begin{array}{l}\text { MD } 6 \mathrm{~mm} \text { (VAS) lower } \\
\text { (10 lower to } 1.3 \text { lower) }\end{array}$ & Low $^{3}$ \\
\hline
\end{tabular}




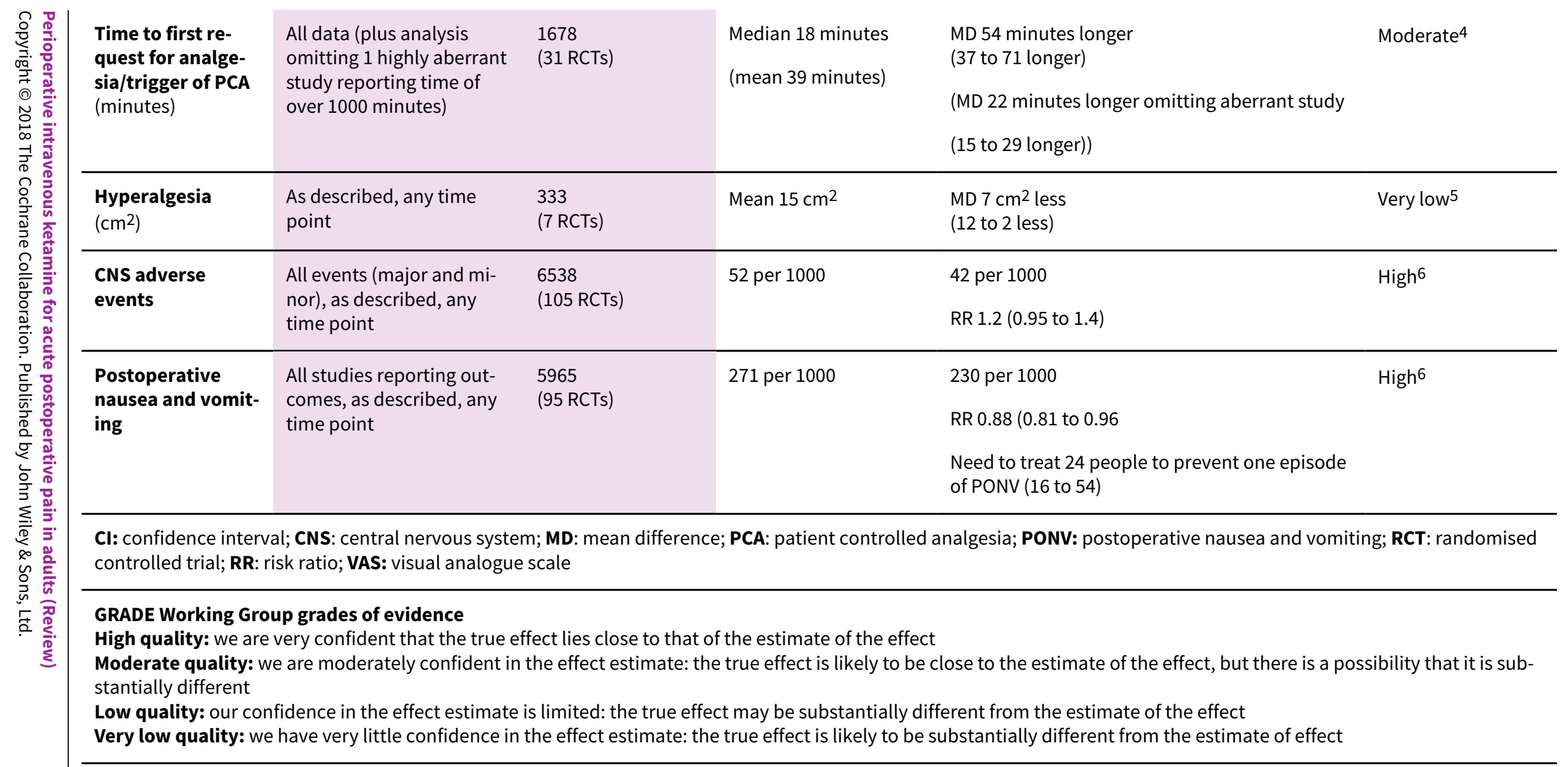

1 Downgraded once for small study effect.

2 Not downgraded for small study effect because no reduction in effect with larger studies.

3 Downgraded once for small study effect, and once because fewer than 1500 participants.

4 Downgraded once because all studies small, more than 1500 participants but not possible to test for small-study effects.

5 Downgraded three times because fewer than 400 participants.

6 Not downgraded: consistent across large body of data.

7 Lower VAS means less pain. 


\section{B A C K G R O U N D}

\section{Description of the condition}

Inadequate pain management after surgery increases the risk of postoperative complications and it is one of the major risk factors associated with chronic postsurgical pain (Prabhakar 2014; Kehlet 2006). Chronic postsurgical pain is defined as pain that persists for longer than three months (VanDenKerkhof 2013). It adversely affects quality of life and delays rehabilitation and return to usual activities.

Pre-emptive analgesia aims to reduce the risk of acute pain becoming chronic (Katz 2009), but conventional analgesics, such as paracetamol alone, may be insufficient in the acute postoperative period. Adverse events may also limit analgesic use, as is the case with non-steroidal anti-inflammatory drugs (NSAIDs). Neuraxial blocks are not applicable to all patients, as they may mask complications after certain types of surgery (such as spinal surgery), or anticoagulation may limit their use.

Opioids are the most effective drugs for the treatment of acute postoperative pain. These are also widely used for alleviating chronic pain, both malignant and non-malignant, although the use of opioids for chronic non-cancer has recently come to be viewed critically. Several adverse events may accompany the prolonged use of opioids, as well as the development of opioid tolerance and dependency (Macintyre 2010). In addition, the findings of several recent trials have associated opioid use with opioidinduced hyperalgesia, which is characterised by an activation of pronociception. This appears clinically as a paradoxical increase in pain as a result of opioid administration, assuming that there are no other underlying factors (disease progression or a surgical complication). Opioid-induced hyperalgesia results in decreased opioid analgesic efficacy and is distinguishable from opioid tolerance, a condition in which escalating opioid doses may restore analgesic effect.

The development of opioid-induced hyperalgesia is thought to result from neuroplastic changes in the peripheral and central nervous system (CNS), involving both cellular and neural mechanisms (Lee 2011). Firstly, the perturbated action of glutamatergic $\mathrm{N}$-methyl-D-aspartate (NMDA) receptors plays a central role in the development of opioid-induced hyperalgesia. Secondly, continuous opioid administration leads to increased levels of spinal dynorphins, which results in excessive synthesis and release of excitatory neuropeptides, shifting the balance between antinociceptive and pronociceptive systems towards the latter. Thirdly, the descending pathway processing spinal nociceptive impulses reacts to the prolonged opioid administration in a way that results in altered expression and release of different neuropeptides, thus favouring the pronociceptive system (Angst 2006; Mao 2002; Silverman 2009).

The clinical risk factors associated with opioid-induced hyperalgesia are opioid dose and duration of treatment. Genetic factors may also be relevant (Colvin 2010). Susceptibility to opioid-induced hyperalgesia may differ between individual opioid medications (Mao 2002), and can restrict opioid use in pain therapy. Methods to modulate opioid-induced hyperalgesia include the addition of adjuvant therapy that has NMDA-receptor antagonist activity, such as ketamine (Lee 2011; Low 2012).

\section{Description of the intervention}

Ketamine is a phencyclidine derivative, first synthesised in 1962. It is a racemic mixture of two optical isomers: $\mathrm{R}(-)$ and $\mathrm{S}(+)-$ enantiomers. Hepatic cytochrome P450 enzymes metabolise ketamine to norketamine, an active metabolite (Mion 2013; Sigtermans 2009). S-ketamine is approved for clinical use in countries such as Finland and Germany. Its pharmacodynamics are complex. In addition to the competitive antagonism of glutamatergic NMDA receptors, ketamine also inhibits HCN1 ion channels in the forebrain, contributing to its hypnotic action (Benarroch 2013; Chen 2009; Zhou 2013).

Previous reviews have suggested that ketamine is an effective adjuvant drug for the treatment of acute postoperative pain, but it is associated with significant adverse events (Bell 2006; Elia 2005; Laskowski 2011; Schmid 1999; Subramaniam 2004). Both its analgesic effects and adverse events are dose-related and the optimal dose or route of administration are still unknown. Ketamine can be administered either intravenously during general anaesthesia or intravenously via patient-controlled analgesia (PCA) after surgery.

Ketamine causes a dissociative anaesthesia in which the eyes remain open while laryngeal, corneal and pupillary reflexes are conserved. Sensory input reaches the cortical sensory areas but is not perceived, due to suppression of association areas (Aroni 2009). Ketamine does not suppress either respiratory or myocardial function, or haemodynamics, therefore it is a useful anaesthetic agent for critically ill patients, battlefield injuries, or for procedural sedation and analgesia (Eikermann 2012). Its adverse effects are dose-dependent and include hypersalivation, nausea and vomiting; its psychotomimetic effects include vivid dreams, blurred vision, hallucinations, nightmares and delirium. These effects are more common in adult patients and in women (Aroni 2009). Sketamine is purported to have fewer adverse effects and a shorter sedation time than racemic ketamine (Geisslinger 1993; Marland 2013). For analgesic purposes, subanaesthetic doses (a dose that is below that required to produce anaesthesia), of ketamine are used. This 'low-dose' is defined as a bolus dose of $1 \mathrm{mg} / \mathrm{kg}$ intravenous, and for continuous intravenous administration, a dose under $1.2 \mathrm{mg} / \mathrm{kg} /$ hour (Peltoniemi 2016). The analgesic potency of S-ketamine is approximately twice that of racemic ketamine (Arendt-Nielsen 1996).

Benzodiazepine premedication reduces the psychotomimetic adverse reactions of both enantiomers. Ketamine combined with the common anaesthetic agent nitrous oxide, an NMDA-receptor antagonist, has exhibited neurotoxic effects in animal studies (Begon 2001; Bulutcu 2002; Jevtović-Todorović 1998). Clinically, this neurotoxic effect might present as psychotomimetic reactions. In animal studies, neurotoxic effects have been prevented by the co-administration of $\gamma$-aminobutyric acid (GABA)-ergic agents, for example benzodiazepines (Beals 2003; Jevtovic-Todorovic 2000). It could also be hypothesised that concurrent administration of nitrous oxide with ketamine could abolish the analgesic effects of ketamine as nitrous oxide acts as a weak antagonist to NMDAreceptors.

\section{How the intervention might work}

Numerous clinical trials have examined the analgesic properties of ketamine. It has been useful in the treatment of neuropathic pain 
(Fisher 2000), and as an adjuvant to opioids in the treatment of refractory pain in people with cancer (Bredlaw 2013). Its analgesic effect is probably mediated via inhibition of NMDA receptors in nociceptive neurons and activation of descending inhibitory monoaminergic pain pathways (Hirota 2011). NMDA receptors play an active role in the processing of nociception in the dorsal horn ganglia of the spinal cord and also play a role in chronic pain states (Ruschweyeh 2011; Sandkühler 2012). Low doses of ketamine alleviate pain because they reduce NMDA receptormediated secondary hyperalgesia and the wind-up phenomenon, as well as opioid-induced hyperalgesia via an interaction with opioid receptors (Hirota 2011). Wind-up is a phenomenon whereby responses of dorsal horn neurons increase during repetitive, constant-intensity, C-fibre stimuli (i.e. increased duration and magnitude of the cell responses). Blockade of NMDA receptors has been shown in animal studies to prevent the development of increased pain sensitivity and opioid tolerance (Bell 2006; Mao 2002; Price 2000). Additionally, inhibition of microglial BK channels may contribute to the analgesic effects of ketamine (Hayashi 2011).

Ketamine has been used as an effective adjuvant for analgesia postoperatively, since it reduces pain and opioid requirements (Elia 2005; Subramaniam 2004), and is used as an adjuvant to opioids for cancer pain, though with inadequate evidence (Bell 2017). Bell and colleagues found that ketamine also reduces postoperative nausea and vomiting (Bell 2006). It is of particular benefit for painful procedures including thoracic, upper abdominal and major orthopaedic surgeries (Laskowski 2011). Ketamine may, in addition to its opioid-sparing effect, reduce the development of chronic postoperative pain via inhibition of NMDA receptors and reduction of wind-up and central sensitisation. The optimal dose and route of administration for this indication are as yet unclear.

\section{Why it is important to do this review}

Numerous clinical trials and previous reviews have suggested that ketamine is an effective adjuvant drug for acute postoperative pain treatment, but that it has significant adverse effects. Ketamine is widely used in the perioperative setting, with intravenous administration the most common route. Both analgesic and adverse effects are dose-dependent and the optimal dose is still unknown.

A previous Cochrane Review on this topic included trials using different routes of administration (Bell 2006). This review focused on the efficacy and tolerability of ketamine for acute postoperative pain. Earlier reviews on this topic have also included studies where ketamine has been administered intramuscularly, epidurally, subcutaneously and intravenously (Elia 2005; Schmid 1999; Subramaniam 2011). A large number of trials have since been published and it is important to review the current literature using updated Cochrane methodology. This current review is expected to provide important information regarding the optimal dosing of ketamine in the perioperative setting, and to establish a current evidence base for its efficacy and tolerability in the treatment of acute postoperative pain.

\section{O B JE C T IVES}

To evaluate the efficacy and safety of perioperative intravenous ketamine in adult patients when used for the treatment or prevention of acute pain following general anaesthesia.

\section{METHODS}

\section{Criteria for considering studies for this review}

\section{Types of studies}

We included randomised, prospective, double-blind studies in which:

- participants received ketamine alone or placebo alone as a study drug;

- ketamine was administered in addition to a basic analgesic such as opioid or NSAID in one study group, and compared with a group receiving the same basic analgesic (but without ketamine) in another group;

- pain intensity, use of opioids, or time to first opioid request were reported outcomes;

- the minimum size was 10 participants per arm who completed the study (Moore 1998; Moore 2008).

We required full journal publication, with the exception of online clinical trial results, summaries of otherwise unpublished clinical trials and abstracts with sufficient data for analysis. We did not include short abstracts (e.g. meeting reports).

\section{Types of participants}

We included adults aged 18 years and above undergoing a surgical procedure under general anaesthesia.

\section{Types of interventions}

We included people treated intravenously with ketamine (racemic ketamine or S-ketamine), during general anaesthesia as a bolus dose or as a continuous infusion or, if administered in the postoperative period, via a patient-controlled analgesia device (PCA) or as a continuous intravenous infusion.

\section{Types of outcome measures}

\section{Primary outcomes}

- Our primary outcome for studies using PCA or opioid as rescue medication was total consumption of opioids in milligrams of morphine equivalents for up to 48 hours after surgery (opioids being the exclusive analgesics used in the included studies).

- Our primary outcome was pain intensity assessed by means of subjective pain scales in studies not assessing or using PCA and in the absence of opioid rescue medication.

We assessed our primary outcomes in a non-stratified study population and by surgery type.

\section{Secondary outcomes}

We extracted, assessed, and analysed the following secondary outcomes.

- Time from end of surgery to first request for analgesia or first trigger of PCA

- Assessment of postoperative hyperalgesia in the units used in the original studies (e.g. hyperalgesia area around the surgical wound in square centimetres)

- Major and minor adverse events, as judged by the authors of the study, such as hallucinations, nightmares, dizziness, blurred vision, sedation, nausea and vomiting 


\section{Search methods for identification of studies}

\section{Electronic searches}

We searched the following databases on 11 July 2018 for all relevant randomised controlled trials (RCTs) without language restrictions:

- Cochrane Central Register of Controlled Trials (CENTRAL; 2018, issue 7) via CRSO to week 28;

- MEDLINE (via Ovid) 1946 to July week 28 2018;

- Embase (via Ovid) 1974 to July week 282018.

We used medical subject headings (MeSH) or equivalent and text word terms. We tailored the searches to the individual databases. The search strategies for CENTRAL, MEDLINE and Embase are shown in Appendix 1; Appendix 2 and Appendix 3.

\section{Searching other resources}

We searched the metaRegister of controlled trials (mRCT) (www.controlled-trials.com/mrct), ClinicalTrials.gov (www.clinicaltrials.gov) and the World Health Organization (WHO) International Clinical Trials Registry Platform (ICTRP) (apps.who.int/trialsearch/), for trials that were completed but not published, and to identify any ongoing studies. In addition, we screened the reference lists of reviews and retrieved articles for additional studies and performed citation searches on key articles. We contacted study authors via email where necessary for additional information (e.g. for obtaining results as mean and standard deviation (SD) if data were presented as medians in the original publication).

\section{Data collection and analysis}

\section{Selection of studies}

Two review authors (ECVB and ET), independently determined eligibility by reading the abstract of each study identified by the search. We eliminated studies that clearly did not satisfy the inclusion criteria and obtained full copies of the remaining studies. Two review authors (ECVB and ET), independently read and selected relevant studies and, in the event of disagreement, a third author adjudicated (VK). We did not anonymise the studies in any way before we assessed studies for inclusion. We have included a PRISMA flow chart (Moher 2009), as recommended in Chapter 6 of the Cochrane Handbook for Systematic Reviews of Interventions (Lefebvre 2011).

\section{Data extraction and management}

Two review authors (ECVB and ET), independently extracted data using a standard form and verified for agreement before entry into Review Manager 5 (RevMan 5 (Review Manager 2014)). We collated multiple reports of the same study, so that each study rather than each report was the unit of interest in the review. We collected characteristics of the included studies in sufficient detail to populate a table of 'Characteristics of included studies' in the full review. The results are summarised and interpreted in the 'Effects of interventions' section.

\section{Assessment of risk of bias in included studies}

Two review authors (ECVB and ET), independently assessed risk of bias for each study, using the criteria outlined in the Cochrane Handbook for Systematic Reviews of Interventions (Higgins 2017), and adapted from those used by the Cochrane Pregnancy and
Childbirth Group. We resolved any disagreements by discussion. We completed a 'Risk of bias' table for each included study using the 'Risk of bias' tool in RevMan 5 (Review Manager 2014). See Characteristics of included studies.

We assessed the following for each study.

- Random sequence generation (checking for possible selection bias). We assessed the method used to generate the allocation sequence as: low risk of bias (any truly random process, e.g. random number table; computer random number generator); unclear risk of bias (method used to generate sequence not clearly stated). We excluded studies using a non-random process (e.g. odd or even date of birth; hospital or clinic record number).

- Allocation concealment (checking for possible selection bias). The method used to conceal allocation to interventions prior to assignment determines whether intervention allocation could have been foreseen in advance of, or during recruitment, or changed after assignment. We assessed the methods as: low risk of bias (e.g. telephone or central randomisation; consecutively numbered, sealed, opaque envelopes); unclear risk of bias (method not clearly stated). We excluded studies that did not conceal allocation (e.g. open list or randomisation based on an individual's ID-number).

- Blinding of participants and personnel (checking for possible performance bias). We assessed the methods used to blind study participants and personnel from knowledge of which intervention a participant received. We assessed the methods as: low risk of bias (study states that it was blinded and describes the method used to achieve blinding, e.g. matched in appearance); unclear risk of bias (study states that it was blinded but does not provide an adequate description of how it was achieved). We considered studies that were not double-blind to have high risk.

- Blinding of outcome assessment (checking for possible detection bias). We assessed the methods used to blind outcome assessors from knowledge of which intervention a participant received. We assessed the methods as: low risk of bias (study states a clear statement that outcome assessors were unaware of treatment allocation, and ideally describes how this was achieved); unclear risk of bias (study states that outcome assessors were blind to treatment allocation but lacks a clear statement on how it was achieved). We excluded studies where outcome assessment was not blinded.

- Incomplete outcome data (checking for possible attrition bias due to the amount, nature and handling of incomplete outcome data). We assessed the methods used to deal with incomplete data as: low risk ( $10 \%$ or fewer of participants did not complete the study or used 'baseline observation carried forward' analysis, or both); unclear risk of bias (used 'last observation carried forward' analysis, number of participants that were excluded from the study were not reported); high risk of bias (used 'completer' analysis or inconsistency between article text and tables).

- Selective reporting (checking for reporting bias). We recorded reporting bias, such as failing to report a planned outcome. We assessed whether primary and secondary outcome measures were pre-specified and whether these were consistent with those reported. We assessed the methods as: low risk of bias (all predefined outcomes were reported); unclear risk of bias (insufficient information of some outcomes, e.g. only $\mathrm{P}$ values 
were reported); high risk of bias (predefined outcomes were not reported or outcomes that were not predefined were reported.

- Size of study (checking for possible biases confounded by small size). We assessed studies as being at low risk of bias (200 participants or more per treatment arm); unclear risk of bias (50 to 199 participants per treatment arm); high risk of bias (fewer than 50 participants per treatment arm).

\section{Measures of treatment effect}

For continuous data with consistent methods of measurement (e.g. pain intensity assessed with a visual analogue scale (VAS) or another subjective, validated pain scale), we calculated mean differences (MDs). We calculated risk ratios (RR) for dichotomous outcomes that were sufficiently homogeneous to be combined (e.g. number of participants experiencing CNS adverse events or number of participants suffering from postoperative nausea and or vomiting, or both). We used random-effects models for both continuous and dichotomous outcomes. We used numbers needed to treat for an additional beneficial outcome (NNTB) and harmful outcome (NNTH), and pooled percentages as absolute measures of benefit or harm. We used $95 \%$ confidence intervals $(\mathrm{Cl})$ to express the uncertainty in each result.

\section{Unit of analysis issues}

We originally intended that the unit of analysis was the individual participant. We changed this to study-level data because patientlevel data were only available for two studies (Joseph 2012; Lo 2008).

\section{Dealing with missing data}

We approached the corresponding authors of the included studies for missing information or data. We derived standard deviations from confidence interval data when only confidence intervals were presented. We obtained the standard deviation for each group by dividing the length of the confidence interval by 3.92, and then multiplying by the square root of the sample size: $\mathrm{SD}=\sqrt{\mathrm{N}} \times$ (upper limit-lower limit) / 3.92. We obtained standard deviation from the standard error of a mean if only standard errors were presented, by multiplying by the square root of the sample size: $S D=S E x \sqrt{ } N$ (Higgins 2011a). We extracted means and standard deviations from graphs manually, when no numerical data were presented. Where possible and appropriate, we used intention-to-treat analyses to include all participants randomised to the study groups.

\section{Assessment of heterogeneity}

Two review authors (ECVB and ET), independently assessed the clinical homogeneity of the studies. In case of discrepancy, we consulted a third review author (VK). We used the $I^{2}$ statistic (Higgins 2003), as described in the Cochrane Handbook for Systematic Reviews of Interventions, and addressed the sources of heterogeneity as appropriate (Deeks 2017).

\section{Assessment of reporting biases}

We recorded reporting bias, such as failing to report a planned outcome.

\section{Data synthesis}

We extracted both dichotomous and continuous data from the studies. We undertook a meta-analysis if we judged participants, interventions, comparisons and outcomes to be sufficiently similar to ensure an answer that was clinically appropriate using a randomeffects model. If the data permitted, we calculated RRs, NNTBs or NNTHs with $95 \%$ Cls. We calculated MDs for continuous data. We used RevMan 5 software for the analysis (Review Manager 2014).

When there were studies with multiple treatment arms, we excluded any arms that involved an intervention not defined by the inclusion criteria for this review. We combined data involving different ketamine regimens when there were studies with several intervention groups relevant to meta-analyses, as recommended in the Cochrane Handbook for Systematic Reviews of Interventions (Higgins 2011a; table 7.7.a).

We combined intervention groups in studies investigating two intervention groups where ketamine was administered, as recommended in the Cochrane Handbook for Systematic Reviews of Interventions (Higgins 2011a; table 7.7.a). We did not double-count control group participants in studies with multiple ketamine groups following guidance in Chapter 16.5.4. of the Cochrane Handbook for Systematic Reviews of Interventions (Higgins 2011b).

The main analysis compared intravenous ketamine with placebo, or compared intravenous ketamine plus a basic analgesic regimen with the same basic analgesic regimen alone. The control was either placebo, or basic analgesic regimen without ketamine. This analysis was not stratified by type of surgery or any other factor, and is referred to as a non-stratified analysis. Subgroup and sensitivity analyses investigated factors such as type of surgery, study size, and pain intensity in control groups.

\section{Quality of the evidence}

Three review authors (RAM, ECVB and VKK), independently rated the quality of the evidence for each outcome using the GRADE system (GRADE 2004), and the guidelines provided in Chapter 12.2 of the Cochrane Handbook for Systematic Reviews of Interventions (Schünemann 2017).

The GRADE approach uses five considerations (study limitations, consistency of effect, imprecision, indirectness and publication bias), to assess the quality of the body of evidence for each outcome. The GRADE system uses the following criteria for assigning grade of evidence:

- high: we are very confident that the true effect lies close to that of the estimate of the effect;

- moderate: we are moderately confident in the effect estimate; the true effect is likely to be close to the estimate of effect, but there is a possibility that it is substantially different;

- low: our confidence in the effect estimate is limited; the true effect may be substantially different from the estimate of the effect;

- very low: we have very little confidence in the effect estimate; the true effect is likely to be substantially different from the estimate of effect.

We decreased the GRADE rating by one $(-1)$ or two $(-2)$ if we identified:

- serious $(-1)$ or very serious $(-2)$ limitation to study quality;

- important inconsistency $(-1)$;

- some $(-1)$ or major (-2) uncertainty about directness;

- imprecise or sparse data $(-1)$; 
- high probability of reporting bias $(-1)$.

Factors that would decrease the quality level of a body of evidence were:

- limitations in the design and implementation of available studies suggesting high likelihood of bias;

- indirectness of evidence (indirect population, intervention, control, or outcomes);

- unexplained heterogeneity or inconsistency of results (including problems with subgroup analyses);

- high probability of publication bias;

- imprecision beyond that expected from small studies.

We paid particular attention to inconsistency, where point estimates varied widely across studies, or $\mathrm{Cls}$ of studies showed minimal or no overlap (Guyatt 2011). Small studies have been shown to overestimate treatment effects, probably because the conduct of small studies is more likely to be less rigorous, allowing critical criteria to be compromised (Dechartres 2013; Nüesch 2010), while large studies often have smaller treatment effects (Dechartres 2014). We considered the consistency of results in sensitivity analyses according to study size and, where relevant, pain intensity with control when making GRADE assessments. These are circumstances in which the overall rating for a particular outcome needs to be adjusted, as recommended by GRADE guidelines (Guyatt 2013a). In circumstances where there were no data reported for an outcome, we would have reported the level of evidence as very low-quality (Guyatt 2013b).

We had planned to use GRADEpro GDT software to rank the quality of the evidence but decided not to use this tool because it does not consider study size, and because of the importance of interpreting pain levels as a key primary outcome. In order to deal with issues around size and sensitivity analyses for small-study effects with beneficial effects we created a simple grid to aid in making consistent judgements about GRADE. This is displayed in the table below. We did not use this for making GRADE decisions about adverse event data.

$\begin{array}{lll}\begin{array}{lll}\text { Amount of data } \\ \text { (number of participants) }\end{array} & \begin{array}{l}\text { "Test for small-study ef- } \\ \text { fects }\end{array} & \text { Action } \\ & \text { (large vs small studies)" } & \end{array}$

\begin{tabular}{llll}
\hline $\begin{array}{l}\geq 1500 \text { participants, many stud- } \\
\text { ies, events common }\end{array}$ & Small-study effects absent & Do not downgrade & $\begin{array}{l}\text { Large amount of data, no obvious } \\
\text { size bias, randomness not at issue }\end{array}$ \\
\hline $\begin{array}{l}\geq 1500 \text { participants, many stud- } \\
\text { ies, events common }\end{array}$ & $\begin{array}{l}\text { Small-study effects present, } \\
\text { or not possible to test }\end{array}$ & $\begin{array}{l}\text { Downgrade once, empha- } \\
\text { sise sensitivity analysis re- } \\
\text { sult }\end{array}$ & $\begin{array}{l}\text { Large amount of data, obvious size } \\
\text { bias, randomness not at issue }\end{array}$ \\
\hline $\begin{array}{l}400-1499 \text { participants, many } \\
\text { studies, events common }\end{array}$ & Small-study effects absent & $\begin{array}{l}\text { Downgrade once, limited } \\
\text { ability of sensitivity analy- } \\
\text { sis to determine small- } \\
\text { study effects }\end{array}$ & $\begin{array}{l}\text { Possible size bias and randomness } \\
\text { effects may be present }\end{array}$ \\
\hline $\begin{array}{l}400-1499 \text { participants, many } \\
\text { studies, events common }\end{array}$ & $\begin{array}{l}\text { Small-study effects present, } \\
\text { or not possible to test }\end{array}$ & $\begin{array}{l}\text { Downgrade twice, empha- } \\
\text { sise sensitivity analysis re- } \\
\text { sult }\end{array}$ & $\begin{array}{l}\text { Obvious size bias, and randomness } \\
\text { effects may be present }\end{array}$ \\
\hline $\begin{array}{l}\text { sult pon participants, few studies, } \\
\text { events common }\end{array}$ & $\begin{array}{l}\text { Not possible to test for } \\
\text { small-study effects }\end{array}$ & Downgrade three times & $\begin{array}{l}\text { Effects of random chance large, pos- } \\
\text { sibility of small size bias is high }\end{array}$ \\
\hline
\end{tabular}

\section{'Summary of findings' table}

We included a 'Summary of findings' table to present the main findings in a transparent and simple tabular format. In particular, we included key information concerning the quality of evidence, the magnitude of effect of the interventions examined, and the sum of available data on the following outcomes:

- total consumption of opioids in milligrams of morphine equivalents for 24 and 48 hours after surgery;

- pain intensity at rest and on movement at 24 and 48 hours after surgery;

- time from end of surgery to first request for analgesia;

- postoperative hyperalgesia;

- CNS adverse events;
- postoperative nausea and vomiting.

\section{Subgroup analysis and investigation of heterogeneity}

We analysed the following predefined subgroups separately:

- studies in which ketamine had been used preoperatively or intraoperatively, or both, as well as studies where ketamine had been administered postoperatively;

- studies in which nitrous oxide had been used as a component of general anaesthesia;

- studies with benzodiazepine premedication (CNS adverse events only). 
A minimum of two studies and 200 participants had to be available in any subgroup analysis, which we restricted to the primary outcomes.

\section{Sensitivity analysis}

We performed sensitivity analysis, if we identified any issues suitable for sensitivity analysis during the review process, and reported the findings as a summary table and discussed them in the review. We decided to perform sensitivity analyses for the primary outcomes. These involved both the size of studies (30 or more and 50 or more participants in treatment arms), and the amount of pain experienced, as both of these factors could influence results with data sets such as those in this review.

\section{Study size}

There is now increasing recognition that results based on a small number of small, underpowered studies may give an incorrect or highly imprecise answer to a clinical question. Studies in neuropathic pain have historically been relatively small, and analysis of smaller trials in Cochrane Reviews has been criticised (AlBalawi 2013; Roberts 2015). An analysis on the impact of study size in Cochrane Reviews has highlighted this issue, and pointed out that if two adequately powered studies are available, then omitting all underpowered studies makes little or no difference to the result (Turner 2013). The standard Cochrane 'Risk of bias' assessment does not include size, unless added by the review authors. Some items, like inconsistency or heterogeneity may be a consequence of small size (IntHout 2015; Turner 2013), but in any event, simulation studies demonstrate that the chances of heterogeneity tests accurately detecting true homogeneity or heterogeneity with a small number of small studies is almost random (Gavaghan 2000; Sterne 2000). Alternative approaches not available in RevMan 5 may offer a way forward in some circumstances (Kulinskaya 2015). There are potentially large effects of random chance when studies are small (Flather 1997; Moore 1998; Pogue 1997; Pogue 1998). A simulation exercise suggests that, in most circumstances, a minimum data requirement is 250 to 500 events, such as a participant achieving adequate pain relief (Thorlund 2011). For most pain studies where event rates are $20 \%$ to $60 \%$, this means about 500 to 1500 participants.

Because it became clear that the studies for the review were predominantly small, with treatment group sizes of 50 participants or fewer, we decided that it was appropriate to perform a sensitivity analysis for the primary outcomes for the non-stratified results. We did this, where data allowed, for studies larger than the median group size, and for those with at least 50 participants in a treatment group. The intention was to examine the robustness of the result in such larger studies as were available.

\section{Pain intensity}

We also considered it possible that some studies would have low pain scores. Analgesic effects are difficult to measure in the absence of pain (McQuay 2012), and because of this we considered a separate sensitivity analysis for studies with at least moderate pain in the control arm, defined as $40 / 100 \mathrm{~mm}$ or more on a VAS (Collins 1997). Low pain intensity is regarded highly by people following operation (Mhuircheartaigh 2009), and generally (Moore 2013).

\section{RE S U L T S}

\section{Description of studies}

\section{Results of the search}

Searches of databases yielded 2222 possible hits, and we identified one further record through searching other sources. We screened 1438 studies for eligibility following duplicate removal. We discarded 1098 records based on the information given in the abstract, for example, a study investigated a paediatric population or it did not concern intravenous administration, or was presented at conferences but not published as a full journal article.

We examined 340 papers and discarded 148 because, on further examination, they did not meet our inclusion criteria. For example, the study intervention did not compare ketamine to placebo or ketamine plus basic analgesic versus basic analgesic alone (e.g. there was direct comparison between ketamine versus paracetamol), or the study investigated outcomes we were not interested in (e.g. catheter-related bladder discomfort).

We evaluated 192 full-text articles for eligibility. Three studies provided data only in units not applicable to meta-analysis or only abstracts were available (Lee 2018; Lou 2017; Moon 2018). We added these studies into 'Characteristics of studies awaiting classification', as the studies were small (fewer than 50 participants per treatment arm), and results showed that there were insufficient data to change the results and conclusion. Twenty of the studies were in Czech, Chinese, French, Korean, Russian, Spanish, or Turkish, and we assessed these with the help of native speakers or colleagues with language skills comparable to a native speaker. After excluding a further 59 (Characteristics of excluded studies), we finally included a total of 130 studies (Characteristics of included studies; Figure 1). 
Figure 1. Study flow diagram

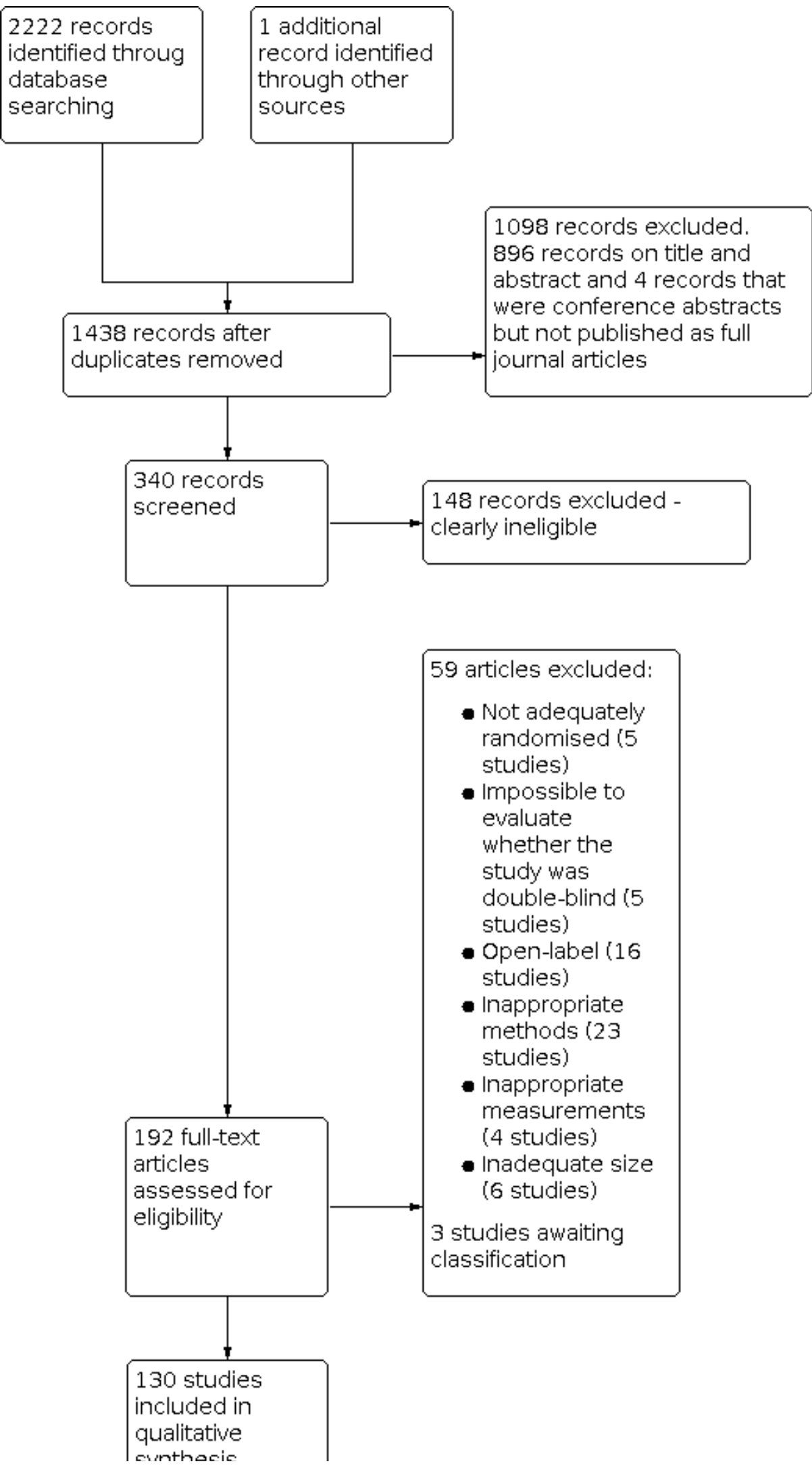


Figure 1. (Continued)

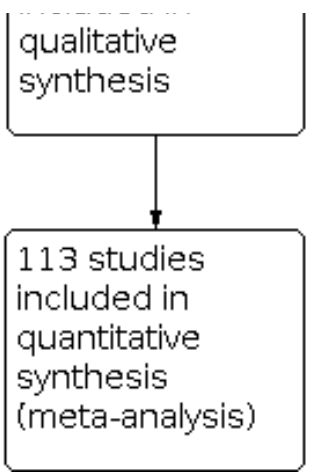

\section{Included studies}

We included 130 studies with 8341 participants. Ketamine was given to 4588 participants and 3753 received placebo or a basic analgesic alone. Ten studies investigated S-ketamine (Argiriadou 2004; Argiriadou 2011; Bornemann-Cimenti 2016; Jaksch 2002; Lahtinen 2004; Mendola 2012; Miziara 2016; Nielsen 2017; Snijdelaar 2004; Spreng 2010), one study investigated R-ketamine (Mathisen 1999), and the remaining 119 studies used racemic ketamine. Details of the included studies are in the Characteristics of included studies. Three studies comprised two treatment arms with corresponding control groups making it logical to analyse these separately for the review (Martinez 2014; Nesek-Adam 2012; Yamauchi 2008). Ayoglu 2005 reported pain intensity as VAS scores and cumulative postoperative morphine consumption up to 20 hours postoperatively. From a clinical point of view, we rounded this to 24 hours and we were able to include it in the meta-analyses.

We contacted the authors of 20 studies in order to obtain data expressed as means +/-SD. Eight authors kindly provided the necessary data. Additionally, the author of the previous Cochrane Review on this topic (and co-author of this review (RFB)) supplied data from three studies in her previous meta-analysis (Bell 2006).

Types of surgery included ear, nose or throat surgery, wisdom tooth extraction, thoracotomy, lumbar fusion surgery, microdiscectomy, hip joint replacement surgery, knee joint replacement surgery, anterior cruciate ligament repair of the knee, knee arthroscopy, mastectomy, haemorrhoidectomy, abdominal surgery (laparotomy and lumbotomy), radical prostatectomy, thyroid surgery, elective caesarean section and laparoscopic surgery.

Twenty-four studies administered ketamine as an intravenous bolus before incision. Eighty-four studies gave intraoperative intravenous ketamine during surgery as repeated boluses or as a continuous infusion; the infusion could stop at the end of surgery or last up to 72 hours after surgery. Sixteen studies investigated postoperative ketamine, in which ketamine was given solely in the postoperative period as a continuous infusion or via PCA. Six other studies used intravenous ketamine at more than one time, typically both before incision and around the time of wound closure (Dahl 2000; Gilabert Morell 2002; Karaman 2006; Kwok 2004; Lebrun 2006; Menigaux 2000).

Twenty-four studies did not provide results of the primary outcomes in units applicable to meta-analysis and thus contributed only to results concerning the secondary outcomes, adverse events, time to first analgesic request and hyperalgesia (Abdolahi
2013; Aqil 2011; Argiriadou 2004; Ataskhoyi 2013; Burstal 2001; Dal 2005; Dar 2012; Deng 2009; Du 2011; Galinski 2007; Hayes 2004; Kapfer 2005; Kim 2016; Köse 2012; Mebazaa MS 2008; Miziara 2016; Ong 2001; Ozhan 2013; Pacreu 2012; Pirim 2006; Siddiqui 2015; Singh 2013; Suzuki 1999; Yazigi 2012). Three other studies provided only qualitative data (Aida 2000; Colombani 2008; Lenzmeier 2008). Additionally, Aida 2000 and Lenzmeier 2008 did not report adverse events. Colombani 2008 expressed the occurrence of adverse events as a percentage of participants having any adverse event. Consequently, 113 studies provided data included in the metaanalyses.

Of the 130 studies, 23 stated that support was departmental or a grant, six declared there was no funding, three had at least some support from industry, and 98 made no mention of funding or support.

\section{Ketamine doses used}

\section{Racemic ketamine}

We found 35 studies that used a bolus dose of racemic ketamine less than $0.25 \mathrm{mg} / \mathrm{kg}$ (including Kapfer 2005, who administered a single $10 \mathrm{mg}$ bolus of racemic ketamine postoperatively if opioid analgesia had not produced adequate analgesia, and Ilkjaer 1998, who administered a pre-incisional intravenous racemic ketamine bolus of $10 \mathrm{mg}$ and $10 \mathrm{mg} /$ hour after surgery for 48 hours). We found 15 studies that administered a bolus dose of racemic ketamine $0.3 \mathrm{mg} / \mathrm{kg}$ intravenously. We found that in 21 studies, ketamine bolus dose was 0.5 to $1 \mathrm{mg} / \mathrm{kg}$ intravenously. We found a further six studies that used racemic ketamine as a single bolus dose more than $1 \mathrm{mg} / \mathrm{kg}$ intravenously.

The other 42 studies used ketamine infusions. If administered as a continuous infusion, most studies used a rate of 2 to $5 \mu \mathrm{g} / \mathrm{kg} /$ $\mathrm{min}$. The lowest infusion rates were $0.7 \mu \mathrm{g} / \mathrm{kg} / \mathrm{min}$ (Yamauchi 2008, cervical and lumbar spine surgery), and $0.8 \mu \mathrm{g} / \mathrm{kg} / \mathrm{min}$ (Aida 2000, gastrectomy and Sen 2009, total abdominal hysterectomy). Dualé 2009 administered 16 mg/kg/min during thoracotomy. Pirim 2006 started racemic ketamine infusion as high as $167 \mu \mathrm{g} / \mathrm{kg} / \mathrm{min}$ for five minutes and decreased it gradually to $42 \mu \mathrm{g} / \mathrm{kg} / \mathrm{min}$ which continued up to 24 hours after total abdominal hysterectomy.

\section{S-ketamine}

Of the 10 studies using S-ketamine, we found eight studies used a pre-incisional IV bolus and a continuous infusion. The bolus dose varied between $0.075 \mathrm{mg} / \mathrm{kg}$ and $0.5 \mathrm{mg} / \mathrm{kg}$. The infusion rates for S-ketamine in these studies were $0.25 \mu \mathrm{g} / \mathrm{kg} /$ 
min (group 1 of Bornemann-Cimenti 2016, abdominal surgery), and $1.25 \mu \mathrm{g} / \mathrm{kg} / \mathrm{min}$ (Lahtinen 2004, thoracotomy), $2 \mu \mathrm{g} / \mathrm{kg} /$ min (Jaksch 2002, anterior cruciate ligament repair; Snijdelaar 2004, radical prostatectomy), $4.2 \mu \mathrm{g} / \mathrm{kg} / \mathrm{min}$ (Nielsen 2017, lumbar fusion surgery), $5 \mu \mathrm{g} / \mathrm{kg} / \mathrm{min}$ (Miziara 2016, laparoscopic cholecystectomy; Spreng 2010, ambulatory haemorrhoidectomy), and $6.7 \mu \mathrm{g} / \mathrm{kg} / \mathrm{min}$ (Argiriadou 2011, thoracotomy). Argiriadou 2004 used a pre-incisional S-ketamine dose $0.5 \mathrm{mg} / \mathrm{kg}$ IV with additional S-ketamine boluses $0.2 \mathrm{mg} / \mathrm{kg}$ at 20-minute intervals during major abdominal surgery until wound closure. We found two studies that administered S-ketamine only as a continuous infusion $(2 \mu \mathrm{g} /$ $\mathrm{kg} / \mathrm{min}$ and $1.7 \mu \mathrm{g} / \mathrm{kg} / \mathrm{min}$, respectively; group 2 of BornemannCimenti 2016, abdominal surgery; Mendola 2012, thoracotomy).

\section{R-ketamine}

Finally, we found one study that administered R-ketamine as a single bolus $1 \mathrm{mg} / \mathrm{kg} \mathrm{IV}$, either pre-incisionally or at wound closure (Mathisen 1999, laparoscopic cholecystectomy).

\section{Excluded studies}

We excluded 59 studies for the following reasons:

- not adequately randomised (5 studies);

- description of methodology was deficient, for example, making it impossible to evaluate whether the study was double-blind ( 5 studies);

- open-label (16 studies);
- inappropriate methods (23 studies);

- inappropriate measurements (4 studies);

- inadequate size (6 studies).

See Characteristics of excluded studies.

\section{Studies awaiting classification}

We identified three recently published studies (Lee 2018; Lou 2017; Moon 2018), providing data only in units not applicable to meta-analysis or available only as abstracts. We put these studies into Characteristics of studies awaiting classification tables as the studies were small (fewer than 50 participants per treatment arm), and there were insufficient data to change the results and conclusion.

\section{Risk of bias in included studies}

Two review authors, ECVB and ET, independently assessed the risk of bias of the included studies with regard to the randomisation process, allocation concealment, blinding of participants and personnel, blinding of outcome assessment, attrition bias, reporting bias, size of the study, and other potential sources of bias. A third review author (VK) resolved any discrepancy that arose in the assessment process. We have included a detailed description of risk of bias in the 'Risk of bias' tables (Characteristics of included studies). See Figure 2 for 'Risk of bias' graph and Figure 3 for 'Risk of bias' summary.

\section{Figure 2. 'Risk of bias' graph: review authors' judgements about each risk of bias item presented as percentages across all included studies}

Random sequence generation (selection bias)

Allocation concealment (selection bias)

Blinding of participants and personnel (performance bias): All outcomes

Blinding of outcome assessment (detection bias): All outcomes Incomplete outcome data (attrition bias): All outcomes Selective reporting (reporting bias)

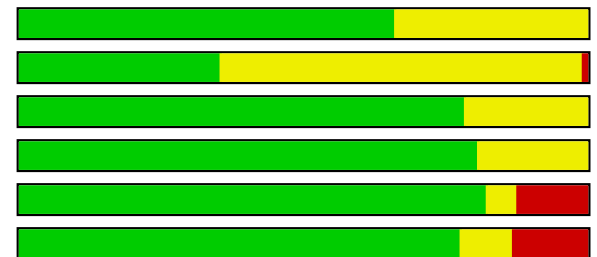

Size

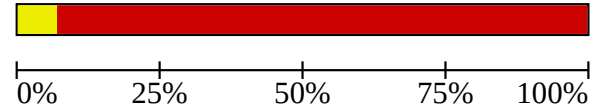

Low risk of bias

Unclear risk of bias

High risk of bias 
Figure 3. 'Risk of bias' summary: review authors' judgements about each risk of bias item for each included study

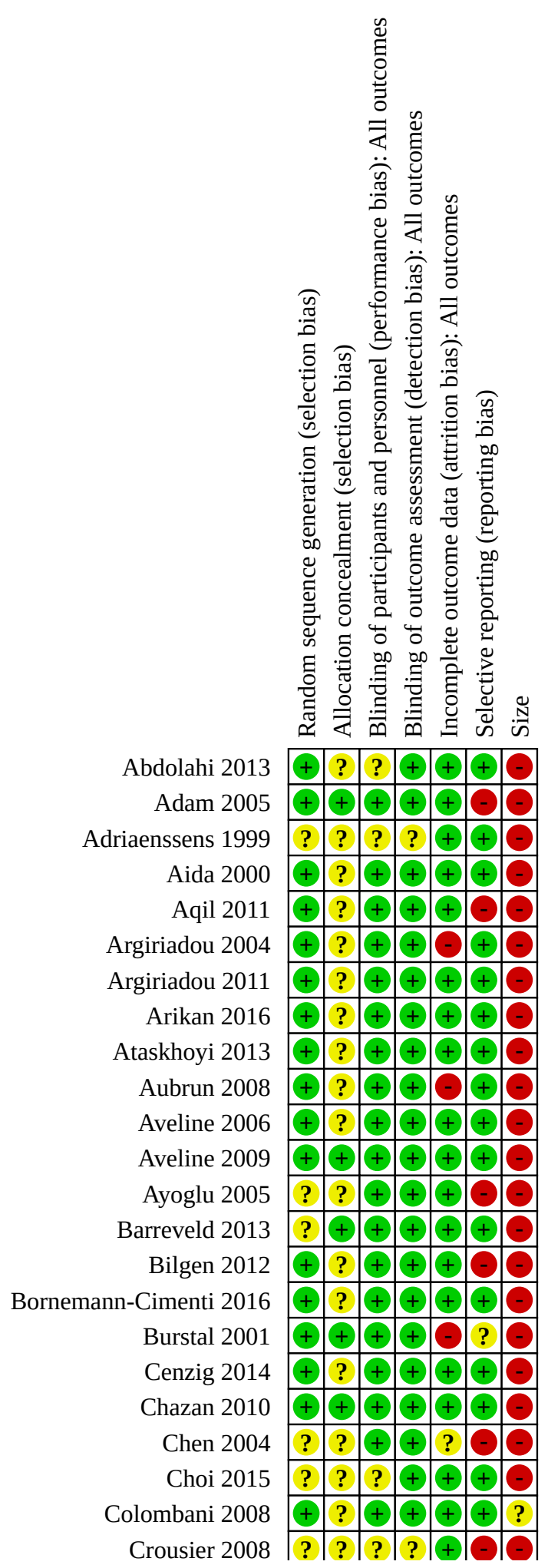


Figure 3. (Continued)

Colombani 2008

Crousier 2008

D'Alonzo 2011

Dahi-Taleghani 2014

Dahl 2000

Dal 2005

Dar 2012

De Kock 2001

Deng 2009

Du 2011

Dualé 2009

Dullenkopf 2009

Fiorelli 2015

Galinski 2007

Ganne 2005

Garcia-Navia 2016

Garg 2016

Gilabert Morell 2002

Grady 2012

Guignard 2002

Guillou 2003

Hadi 2010

Hadi 2013

Haliloglu 2015

Hasanein 2011

Hayes 2004

Helmy 2015

Hercock 1999

Hu 2014

Ilkjaer 1998

Jaksch 2002

Javery 1996

Jendoubi 2017

Joly 2005

Joseph 2012

Kafali 2004

Kakinohana 2004

Kamal 2008

Kapfer 2005

Karaman 2006

Kararmaz 2003

Karcioglu 2013

Katz 2004

Kim 2013

Kim 2016

Köse 2012

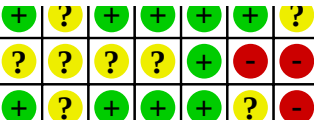

\begin{tabular}{lllllllll}
\hline & $?$ & $?$ & $?$ & + & + & ? \\
\hline
\end{tabular}

\begin{tabular}{llllllll}
\hline & $?$ & $?$ & $?$ & + & + & - \\
\hline
\end{tabular}

\begin{tabular}{l|l|l|l|lll}
\hline & $?$ & + & + & + & + & - \\
\hline
\end{tabular}

? $++\odot \odot \odot$

$+?+\odot+\odot-$

$\begin{array}{llllllll}\text { ? } & \text { ? } & + & + & \text { ? } & + & \text { ? }\end{array}$

\begin{tabular}{llllllll}
\hline+ & $?$ & + & + & + & + & - \\
\hline
\end{tabular}

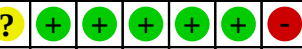

$+\odot+\odot+\oplus$

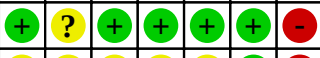

\begin{tabular}{ll|llllll} 
& $?$ & $?$ & $?$ & $?$ & $?$ & + & - \\
\hline
\end{tabular}
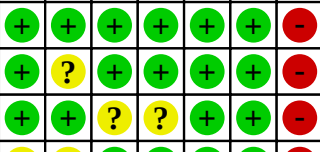

? $?+\oplus+\oplus_{-}$
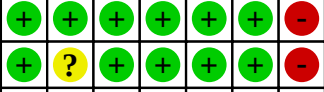

$\begin{array}{lllllll} & ? & ? & + & + & + & \text { ? }\end{array}$

\begin{tabular}{l|l|l|l|l|l|l|}
\hline$?$ & $?$ & $?$ & + & $?$ & + & - \\
\hline
\end{tabular}

? $\quad$ ? $+\odot+\odot$ ?

+ ? $++\odot+\odot$

\begin{tabular}{lllllll}
\hline$?$ & - & $?$ & + & + & - & - \\
\hline
\end{tabular}

+ ? $+\oplus+\oplus$

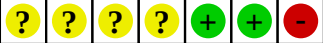

\begin{tabular}{llllllll}
\hline$?$ & + & + & + & + & + & - \\
\hline
\end{tabular}

\begin{tabular}{llllllll}
\hline$?$ & $?$ & $?$ & $?$ & + & + & - \\
\hline
\end{tabular}

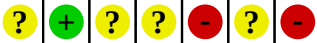

? $+\odot$ ? $+4+$

\begin{tabular}{ll|l|l|lll}
\hline$?$ & $?$ & $?$ & $?$ & + & + & - \\
\hline
\end{tabular}

\begin{tabular}{llllllll}
\hline$?$ & $?$ & + & + & + & + & - \\
\hline
\end{tabular}

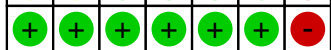

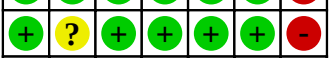

+ ?

\begin{tabular}{llllllll}
\hline$?$ & $?$ & + & + & + & + & - \\
\hline
\end{tabular}

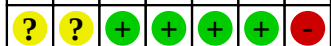

\begin{tabular}{lllllll}
\hline+ & + & + & + & + & + & - \\
\hline
\end{tabular}

\begin{tabular}{lllllll}
\hline & ? & + & + & + & + & - \\
\hline
\end{tabular}

\begin{tabular}{lllllll}
\hline+ & ? & + & + & + & + & - \\
\hline
\end{tabular}

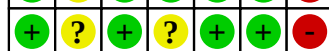

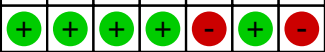

\begin{tabular}{lllllll}
\hline$?$ & $?$ & + & - & + & - \\
\hline
\end{tabular}

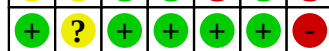

\begin{tabular}{lllllll}
\hline$?$ & + & + & + & + & + & - \\
\hline
\end{tabular}

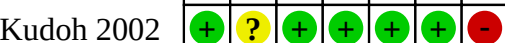


Figure 3. (Continued)

\begin{tabular}{|c|c|c|c|c|c|c|c|}
\hline Köse 2012 & $?$ & + & + & +1 & + & + & \\
\hline Kudoh 2002 & + & $?$ & + & + & + & + & \\
\hline Kwok 2004 & + & + & + & + & + & + & \\
\hline Kwon 2009 & $?$ & $?$ & $?$ & $?$ & + & + & \\
\hline Lahtinen 2004 & + & + & + & + & - & + & \\
\hline Lak 2010 & + & $?$ & $?$ & $?$ & 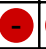 & - & \\
\hline Leal 2013 & + & $?$ & + & + & + & - & \\
\hline Leal 2015 & + & + & + & + & + & - & \\
\hline Lebrun 2006 & + & $?$ & + & + & - & + & \\
\hline Lee 2008 & $?$ & $?$ & $?$ & + & + & $?$ & \\
\hline Lehmann 2001 & + & $?$ & + & $?$ & ? & + & \\
\hline Lenzmeier 2008 & $?$ & $?$ & $?$ & $?$ & + & + & \\
\hline Lin 2016 & ? & $?$ & ? & $?$ & + & C & \\
\hline Lo 2008 & + & + & + & + & + & $?$ & \\
\hline Loftus 2010 & + & + & + & + & + & + & \\
\hline Mahran 2015 & + & + & + & + & + & + & \\
\hline Martinez 2014 & + & + & + & + & E & + & \\
\hline Mathisen 1999 & $?$ & + & + & + & - & + & \\
\hline McKay 2007 & + & + & + & + & + & + & \\
\hline Mebazaa MS 2008 & + & $?$ & + & + & + & + & \\
\hline Mendola 2012 & + & + & + & + & + & + & \\
\hline Menigaux 2000 & + & $?$ & + & + & + & + & \\
\hline Menigaux 2001 & + & $?$ & + & + & + & + & \\
\hline Michelet 2007 & + & $?$ & + & + & + & + & \\
\hline Miziara 2016 & + & + & + & + & + & + & \\
\hline Murdoch 2002 & $?$ & $?$ & + & + & + & $?$ & \\
\hline Nesek-Adam 2012 & + & + & + & + & + & + & \\
\hline Nielsen 2017 & + & + & + & + & + & + & 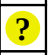 \\
\hline Ögün 2001 & $?$ & $?$ & $?$ & $?$ & + & + & \\
\hline Ong 2001 & + & $?$ & + & + & + & + & \\
\hline Ozhan 2013 & + & $?$ & + & + & + & + & \\
\hline Pacreu 2012 & + & $?$ & + & + & + & + & \\
\hline Papaziogas 2001 & \begin{tabular}{|l|}
$?$ \\
\end{tabular} & $?$ & $?$ & $?$ & + & + & \\
\hline Parikh 2011 & + & $?$ & + & + & + & + & \\
\hline Patel 2016 & + & $?$ & + & + & + & + & \\
\hline Pirim 2006 & $?$ & + & $?$ & + & + & + & \\
\hline Remérand 2009 & + & + & + & + & + & + & $?$ \\
\hline Reza 2010 & + & + & + & + & + & + & \\
\hline Roytblat 1993 & $?$ & $?$ & + & + & + & + & \\
\hline Safavi 2011 & \begin{tabular}{|l|}
$?$ \\
\end{tabular} & + & + & + & + & + & \\
\hline Sahin 2004 & \begin{tabular}{|l|}
$?$ \\
\end{tabular} & $?$ & + & + & + & $?$ & \\
\hline Sen 2009 & + & + & + & + & + & + & \\
\hline Siddiqui 2015 & $?$ & $?$ & $?$ & $?$ & - & - & \\
\hline Singh 2013 & + & + & + & + & $?$ & $?$ & \\
\hline Snijdelaar 2004 & + & + & + & + & E & + & \\
\hline Song 2013 & + & + & + & + & + & + & \\
\hline Song 2014 & 1 & ? & ? & & & + & \\
\hline
\end{tabular}


Figure 3. (Continued)

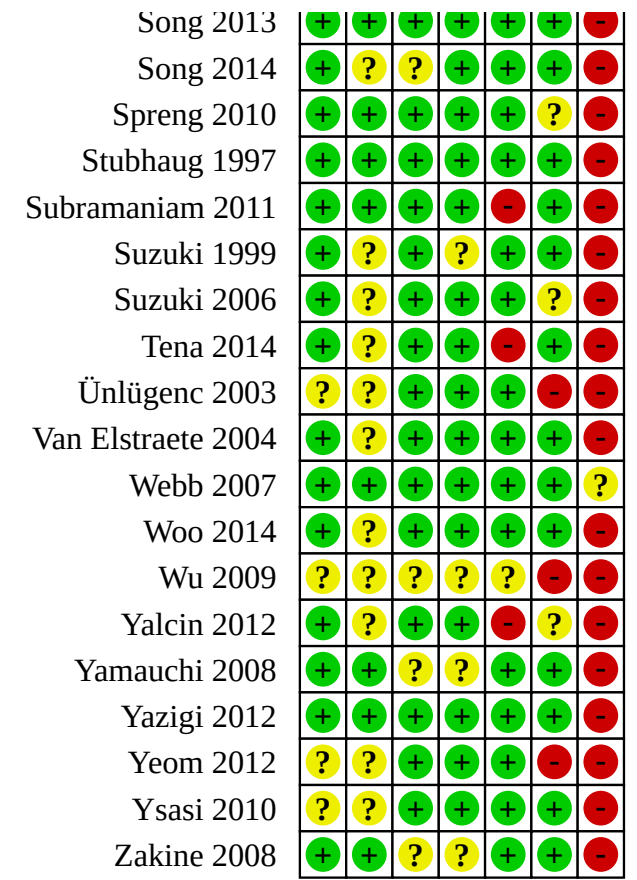

\section{Allocation}

\section{Random sequence generation}

We judged random sequence generation as adequate and the risk of bias low in 86 of the included studies. For example, we considered a computer-generated list of random numbers, shuffling envelopes or cards to be adequate sequence generation. We judged the remaining 44 studies to be at unclear risk of bias for this domain.

\section{Allocation concealment}

We regarded allocation concealment methods appropriate and the risk of bias low if the group allocation was concealed by opaque, sealed envelopes or if there was central randomisation by a third party (e.g. by a hospital pharmacy). Forty-six studies fulfilled this criteria and were at low risk of bias for this domain. Eighty-three studies did not give a detailed description of the group allocation. In this case, we judged the risk of bias concerning allocation concealment as unclear. Although we planned to exclude high risk of allocation concealment, we included Hasanein 2011 despite being judged as high risk of bias. In this study the attending anaesthesiologist was aware of the treatment allocation but study participants, and remaining personnel in the operating room and those recording data were unaware of treatment allocation. We assessed this as high risk of bias but included the study because attending anaesthesiologists did not take part in the further steps of the study.

\section{Blinding}

\section{Blinding of participants and personnel (performance bias)}

Of the 130 studies, 102 provided blinding methods in detail, allowing them to be classified as having low risk of bias concerning blinding of both participants, personnel and outcome assessment.
Twenty-eight of 130 included studies were described as doubleblind but did not describe the method used to achieve blinding of participants and personnel. We classified these as having unclear risk of bias. We classified Hasanein 2011 as unclear risk, as the attending anaesthetist was aware of allocation, though participants and outcome assessors were blinded.

\section{Blinding of outcome assessment (detection bias)}

The majority of studies $(n=105)$, reported the blinding of outcome assessment in detail and we classified them as having low risk of bias. Twenty-five of 130 included studies did not provide explicit information about how they achieved blinding of outcome assessment. We classified these studies as having unclear risk of bias. In Hasanein 2011, the attending anaesthetist was aware of treatment allocation, but as study participants and remaining personnel in the operating room and those recording data were unaware of the allocation, we regarded this as being sufficiently blinded. We therefore classified Hasanein 2011 as having unclear risk of bias.

\section{Incomplete outcome data}

In most studies ( $\mathrm{n}=107$ ), $10 \%$ of participants or fewer failed to complete the study. We judged these to have low risk of attrition bias. Seven studies lacked adequate reporting of excluded participants and we judged their attrition bias as unclear. In fifteen studies, more than $10 \%$ of participants were excluded from the study or failed to complete. We judged their attrition bias as high. Where the exclusion rate exceeded $10 \%$, the typical exclusion rate of participants was $11 \%$ to $13 \%$. Burstal 2001, Lak 2010, Mathisen 1999, Subramaniam 2011 and Tena 2014 excluded 16\%, 20\%, 17\%, $21 \%$ and $17 \%$ of study participants, respectively. Additionally, in one study (Siddiqui 2015), there was an inconsistency between the text and the table of results and we classified the study as having high risk of bias. 


\section{Selective reporting}

Seventeen out of 130 studies (Adam 2005; Aqil 2011; Ayoglu 2005; Bilgen 2012; Chen 2004; Crousier 2008; Dar 2012; Hasanein 2011; Kafali 2004; Lak 2010; Leal 2013; Leal 2015; Lin 2016; Siddiqui 2015; Ünlügenc 2003; Wu 2009; Yeom 2012), either did not report outcomes predefined in the methods or published results of outcomes that were not predefined. We judged these studies as having a high risk of bias concerning selective reporting. Twelve other studies (Burstal 2001; D'Alonzo 2011; Ilkjaer 1998; Hadi 2013; Lee 2008; Lo 2008; Murdoch 2002; Sahin 2004; Singh 2013; Spreng 2010; Suzuki 2006; Yalcin 2012), provided only P values or an imprecise description of adverse events, and we judged their risk of bias for selective reporting as being unclear. In one study (Burstal 2001), the surgeon decided about the cessation of the PCA, which potentially affected this study's results concerning opioid consumption. We judged this to have unclear risk of bias concerning selective reporting. D'Alonzo 2011 reported that the anaesthetic procedure was left to the discretion of the anaesthetist and an epidural catheter was inserted when needed to control pain in a number of participants ( 16 in the ketamine group and 19 in the control group), so the judgement for reporting bias was unclear. Suzuki 2006 reported that the epidural infusion of morphine and ropivacaine was temporarily suspended in three participants in the ketamine group and five participants in the control group due to hypotension and we classified this study's reporting bias to be unclear. We judged the remaining studies as low risk of bias.

\section{Other potential sources of bias}

We noted that 19 studies did not report power analysis. Three trials were clearly underpowered (Crousier 2008; Lo 2008; Subramaniam 2011), and two studies (Du 2011; Köse 2012), did not base the power analysis.

\section{Size of study}

The study population was fewer than 50 participants per treatment arm in 121 studies and we judged their risk of other bias as high. Nine studies (Colombani 2008; Dahi-Taleghani 2014; Deng 2009; Guillou 2003; Loftus 2010; Mebazaa MS 2008; Nielsen 2017; Remérand 2009; Webb 2007) randomised more than 50 participants per study group and we classified their risk of other bias as unclear. We did not judge any of the studies as low risk of bias concerning study size.

The small size of studies did result in several analyses displaying high $\mathrm{I}^{2}$ statistic values, above $90 \%$. Such a situation is likely to arise due to random chance effects with small studies (Gavaghan 2000; Moore 1998; Sterne 2000).

\section{Effects of interventions}

See: Summary of findings 1 Perioperative intravenous ketamine compared to placebo for acute postoperative pain in adults

We did not stratify the main analysis by type of surgery or any other factor, such as dose or timing of ketamine administration, and therefore we ran a non-stratified analysis. We conducted analyses that compared intravenous ketamine with placebo, or compared intravenous ketamine plus a basic analgesic regimen with the same basic analgesic regimen alone. The control was either placebo, or basic analgesic regimen without ketamine.
We performed sensitivity analyses in our primary analyses due to small sample sizes of studies (most had fewer than 50 participants in each treatment group), or due to considerable variation in pain levels with control. We also conducted subgroup analyses for our primary outcomes according to timing of ketamine administration, and co-administration of nitrous oxide. For CNS adverse events, we conducted a subgroup analysis relating to use of benzodiazepine premedication. These subgroup analyses follow the non-stratified analyses for the primary and secondary outcomes. We did not perform any analysis according to ketamine dose, as total doses were broadly similar and so did not allow for any sensible subgroup analysis.

\section{Primary outcomes (non-stratified study population) \\ Postoperative opioid consumption}

We converted to morphine equivalents using conversion equations found in the literature, if the opioid administered for postoperative analgesia was different from morphine (e.g. fentanyl, hydromorphone, oxycodone, ketobemidone, meperidine, nalbuphine, or piritramide). We used the following conversion ratios: 10:1 for IV meperidine:IV morphine (Woodhouse 1996; Pereira 2001), 1:1 for IV nalbuphine:IV morphine (Zeng 2015), 1:100 for IV fentanyl:IV morphine (Patanwala 2007), 1:5 for IV hydromorphone:IV morphine (Patanwala 2007), 2:3 for IV oxycodone:IV morphine (Anderson 2001; Silvasti 1998), 1:1 for IV ketobemidone:IV morphine (Lundeberg 2012), and 2:3 for IV piritramide:IV morphine (Kay 1971; Kumar 1999). In choosing this outcome, we recognised that we would be using opioid consumption generally reported as a mean or a median. However, neither of these is truly satisfactory, since the distribution of postoperative opioid consumption is highly skewed (Moore 2011). We have used this outcome because it is commonly reported in individual studies, and used in pooled analyses. The distribution is so skewed that mean, median, and mode are all very different to one another, though the median value appears to be more conservative in reporting lower consumption. We therefore report median and mean values where these are available.

\section{4-hour opioid consumption in a non-stratified study population}

Sixty-five studies with 4004 participants provided data for 24hour opioid consumption postoperatively (Adriaenssens 1999; Argiriadou 2011; Aubrun 2008; Aveline 2006; Aveline 2009; Ayoglu 2005; Barreveld 2013; Bilgen 2012; Cenzig 2014; Crousier 2008; Dahi-Taleghani 2014; Dahl 2000; Dualé 2009; Dullenkopf 2009; Fiorelli 2015; Ganne 2005; Garcia-Navia 2016; Garg 2016; Gilabert Morell 2002; Guignard 2002; Guillou 2003; Hadi 2010; Hadi 2013; Haliloglu 2015; Hasanein 2011; Helmy 2015; Hercock 1999; Ilkjaer 1998; Jaksch 2002; Javery 1996; Jendoubi 2017; Kafali 2004; Kamal 2008; Karaman 2006; Katz 2004; Kwon 2009; Leal 2013; Leal 2015; Lehmann 2001; Lin 2016; Loftus 2010; Mahran 2015; Menigaux 2000; Michelet 2007; Murdoch 2002; Nielsen 2017; Ögün 2001; Parikh 2011; Remérand 2009; Reza 2010; Roytblat 1993; Safavi 2011; Sahin 2004; Sen 2009; Snijdelaar 2004; Song 2013; Song 2014; Stubhaug 1997; Subramaniam 2011; Ünlügenc 2003; Webb 2007; Woo 2014; Yalcin 2012; Ysasi 2010; Zakine 2008). Ketamine was given to 2128 participants and control to 1876 . Most studies (56 of 65), had fewer than 50 participants in one treatment group; the median ketamine treatment group size was 29 participants. The median opioid consumption in control arms was $31 \mathrm{mg}$ morphine equivalents (mean $42 \mathrm{mg}$ ). 
Participants treated with ketamine consumed $7.6 \mathrm{mg}$ less morphine equivalent opioid in the first 24 hours after surgery $(95 \% \mathrm{Cl}-8.9$ to -6.4; Analysis 1.1).

\section{Sensitivity analyses}

We performed a sensitivity analysis using only those studies with a treatment group size of 30 participants or more. We included 2546 participants (64\% of the total). In these larger studies, participants receiving ketamine consumed $7 \mathrm{mg}$ less morphine equivalent opioid in the first 24 hours after surgery $(95 \% \mathrm{Cl}-9.3$ to -5.5$)$. Using only the nine studies with ketamine treatment group size of 50 participants or more (1072 participants, $27 \%$ ), the participants who received ketamine consumed $5 \mathrm{mg}$ less morphine equivalent opioid in the first 24 hours after surgery $(95 \% \mathrm{Cl}-9.9$ to -0.4$)$.

We assessed the quality of evidence for this outcome as moderate, downgraded once because the magnitude of effect fell with larger studies (small study effect) (Summary of findings 1 ).

Results by surgery type are shown in Summary table A.

\section{8-hour opioid consumption in a non-stratified study population}

Thirty-seven studies with 2449 participants assessed opioid consumption during the first 48 hours postoperatively (Adam 2005; Adriaenssens 1999; Argiriadou 2011; Arikan 2016; Aubrun 2008; Aveline 2009; Bilgen 2012; Bornemann-Cimenti 2016; Choi 2015; Dahl 2000; Fiorelli 2015; Ganne 2005; Garg 2016; Gilabert Morell 2002; Guillou 2003; Jaksch 2002; Kafali 2004; Kamal 2008; Kararmaz 2003; Katz 2004; Kim 2013; Kwon 2009; Lahtinen 2004; Lak 2010; Loftus 2010; Martinez 2014; Menigaux 2000; Michelet 2007; Papaziogas 2001; Remérand 2009; Snijdelaar 2004; Song 2013; Subramaniam 2011; Webb 2007; Woo 2014; Yalcin 2012; Zakine 2008). Of these, 1342 participants received ketamine while

\begin{tabular}{|c|c|c|c|}
\hline Surgery & Studies & Participants & $\begin{array}{l}\text { Morphine equivalents (mg) } \\
\text { MD }(95 \% \mathrm{Cl})\end{array}$ \\
\hline
\end{tabular}

\section{Opioid consumption at 0-24 hours}

\begin{tabular}{llll}
\hline All studies & 65 & 4004 & $-7.6(-8.9$ to -6.4$)$ \\
\hline Thoracotomy & 4 & 421 & $-5.8(-10.3$ to -1.4$)$ \\
\hline Major orthopaedic & 10 & 797 & $-19.7(-28.6$ to -10.2$)$ \\
\hline Major abdominal & 16 & 1029 & $-10.3(-13.8$ to -6.8$)$ \\
\hline Total abdominal hysterectomy & 9 & 511 & $-5.2(-10.8$ to 0.4$)$ \\
\hline Laparoscopic procedures & 4 & 199 & $-2.7(-6.2$ to 0.8$)$ \\
\hline
\end{tabular}

\section{Opioid consumption at $0-48$ hours}

\begin{tabular}{llcl}
\hline All studies & 37 & 2449 & $-12.6(-15.1$ to -10.2$)$ \\
\hline Thoracotomy & 3 & 191 & $-12.5(-18.3$ to -6.7$)$ \\
\hline Major orthopaedic & 9 & 557 & $-18.7(-27.5$ to -9.9$)$ \\
\hline
\end{tabular}

Perioperative intravenous ketamine for acute postoperative pain in adults (Review)
1107 participants received control treatment. Most studies (30 of 37), had fewer than 50 participants in one treatment group; the median ketamine treatment group size was 30 participants. The median opioid consumption in control arms was $59 \mathrm{mg}$ morphine equivalents (mean $67 \mathrm{mg}$ ).

Participants receiving ketamine consumed $12.6 \mathrm{mg}$ of morphine equivalent less opioid $(95 \% \mathrm{Cl}-15.1$ to -10.2$)$, in the first 48 hours after surgery (Analysis 1.2).

\section{Sensitivity analyses}

We performed a sensitivity analysis using only those studies with a treatment group size of 30 or more ( $n=1718$ participants; $70 \%$ of the total). In these larger studies, we found that participants consumed $13 \mathrm{mg}$ of morphine equivalent less opioid in the first 48 hours after surgery $(95 \% \mathrm{Cl}-19$ to -7.8$)$, after ketamine administration. Using only the seven studies with ketamine treatment group size of 50 participants or more (759 participants, 30\%), the participants consumed $6 \mathrm{mg}$ of morphine equivalent less opioid in the first 48 hours after surgery $(95 \% \mathrm{Cl}-11$ to -0.3$)$, after ketamine treatment.

We assessed the quality of evidence for this outcome as moderate, downgraded once because the magnitude of effect fell with larger studies (small study effect) (Summary of findings 1 ).

Summary table A shows results for the 24-hour and 48-hour opioid consumption data, both for all studies and according to different types of surgery. Analyses for the different types of surgery are in Appendix 4 . The analyses by surgery type were not subject to any sensitivity analysis by study size or pain intensity level. In general, results by surgery type were similar to that for all surgery though in some cases there was no evidence of a difference.

Summary table A: postoperative opioid consumption 0 to 24 hours and 0 to 48 hours (data for all studies and by type of surgery) 


\begin{tabular}{lccc} 
Major abdominal & 10 & 704 & $-14.3(-21.2$ to -7.5$)$ \\
\hline Total abdominal hysterectomy & 5 & 378 & $-15.3(-33.2$ to 2.6) \\
\hline Laparoscopic procedures & 2 & 85 & $-4.5(-12.2$ to 3.3) \\
\hline
\end{tabular}

\section{Postoperative pain intensity}

Pain intensity had to be assessed using a validated measure of pain at rest and during movement (0 to $100 \mathrm{VAS}$ ), or other validated scale; $0=$ no pain). We converted to a VAS of 0 to 100 by multiplying each reported pain score by 10 or 25 , as appropriate in studies where pain intensity was assessed using a VAS of 0 to 10 , a numerical rating scale (NRS) of 0 to 10 , or a verbal rating scale (VRS; a 5-point scale from no pain to unbearable pain or equivalent wording).

\section{Pain intensity at rest at $\mathbf{2 4}$ hours in a non-stratified study population}

We found that 82 studies with 5004 participants assessed pain intensity at 24 hours (Adam 2005; Adriaenssens 1999; Argiriadou 2011; Arikan 2016; Aubrun 2008; Aveline 2006; Aveline 2009; Ayoglu 2005; Bornemann-Cimenti 2016; Cenzig 2014; Chen 2004; Choi 2015; D'Alonzo 2011; Dahi-Taleghani 2014; Dahl 2000; De Kock 2001; Dualé 2009; Fiorelli 2015; Ganne 2005; Grady 2012; Guillou 2003; Hadi 2013; Haliloglu 2015; Hercock 1999; Hu 2014; Jaksch 2002; Javery 1996; Jendoubi 2017; Joly 2005; Joseph 2012; Kafali 2004; Kakinohana 2004; Kamal 2008; Karcioglu 2013; Katz 2004; Kim 2013; Kudoh 2002; Kwok 2004; Kwon 2009; Lahtinen 2004; Lak 2010; Leal 2013; Leal 2015; Lebrun 2006; Lee 2008; Lehmann 2001; Lin 2016; Lo 2008; Loftus 2010; Mahran 2015; Mathisen 1999; Mendola 2012; Menigaux 2000; Menigaux 2001; Michelet 2007; Nesek-Adam 2012; Nielsen 2017; Ögün 2001; Papaziogas 2001; Parikh 2011; Patel 2016; Remérand 2009; Reza 2010; Safavi 2011; Sen 2009; Snijdelaar 2004; Song 2013; Spreng 2010; Subramaniam 2011; Suzuki 2006; Tena 2014; Ünlügenc 2003; Van Elstraete 2004; Webb 2007; Woo 2014; Wu 2009; Yalcin 2012; Yamauchi 2008; Yazigi 2012; Yeom 2012; Ysasi 2010; Zakine 2008). Of these, 2465 participants received ketamine and 2539 served as controls. Most studies (72 of 82) had fewer than 50 participants in one treatment group; the median ketamine treatment group size was 30 participants. The median pain score in control arms was $25 / 100 \mathrm{~mm}$ (mean 26/100 mm), and 68/82 studies had pain scores below $40 / 100 \mathrm{~mm}$, indicating that pain was only mild in those studies.

Pain scores measured with VAS ( 0 to $100 \mathrm{~mm}$ ), were $5 \mathrm{~mm}$ lower after ketamine treatment $(95 \% \mathrm{Cl}-6.6$ to -3.6$)$, compared to participants receiving control treatment (Analysis 1.3).

\section{Sensitivity analyses}

We performed a sensitivity analysis using only those studies with a treatment group size of 30 or more. We included 3369 participants ( $67 \%$ of the total), in the analysis. In these larger studies, in participants treated with ketamine, pain scores were $4 \mathrm{~mm}$ lower $(95 \% \mathrm{Cl}-6.1$ to -2.3$)$.

Using only the 10 studies with ketamine treatment group size of 50 participants or more (1176 participants, 24\%), in participants treated with ketamine pain scores were $5 \mathrm{~mm}$ lower $(95 \% \mathrm{Cl}-8.7$ to $-0.6)$.
Pain scores for the control arms of the 82 studies varied between 4 $\mathrm{mm}$ and $66 \mathrm{~mm} / 100 \mathrm{~mm}$. Using only the 14 studies with ketamine control group pain scores of $40 / 100 \mathrm{~mm}$ or more (860 participants, $17 \%$ of the total), pain scores were $17 \mathrm{~mm}$ lower $(95 \% \mathrm{Cl}-25$ to -9.0$)$, in participants treated with ketamine compared with control.

We assessed the quality of evidence for this outcome as high. We did not downgrade for small study effect because the magnitude of effect was not smaller in the larger studies (Summary of findings 1 ). We also had confidence because the effect of ketamine was larger in studies with higher initial pain intensity.

Results by surgery type are shown in Summary table B.

\section{Pain intensity during movement at 24 hours in a non-stratified study population}

We found 29 studies with 1806 participants that provided data for pain intensity at 24 hours during movement (Argiriadou 2011; Aveline 2009; Bornemann-Cimenti 2016; De Kock 2001; Guillou 2003; Hercock 1999; Jendoubi 2017; Joly 2005; Joseph 2012; Kakinohana 2004; Kamal 2008; Katz 2004; Kim 2013; Lahtinen 2004; Mahran 2015; Menigaux 2000; Nielsen 2017; Sen 2009; Snijdelaar 2004; Song 2013; Spreng 2010; Subramaniam 2011; Suzuki 2006; Tena 2014; Van Elstraete 2004; Webb 2007; Wu 2009; Yamauchi 2008; Yazigi 2012).

The stimulus for pain intensity during movement varied in the studies according to type of surgical procedure. Nine studies did not define the movement stimulus and used wording such as "during movement" or "at mobilization". Eight studies assessed pain during coughing, forced coughing or peak flow expiration. Two studies assessed pain during knee flexion. Two studies assessed pain on movement when the study participant rolled from supine to a side-lying position and performed two maximal inspirations. Two studies defined movement as rolling, sitting or coughing. The remaining six studies assessed pain on movement respectively when the participant tried to change position, lifted a leg when lying supine, defecated, moved a shoulder, moved to a sitting position or swallowed.

Ketamine was given to 964 participants and control to 842. Most studies (26 out of 29 ) had fewer than 50 participants in each study group; the median ketamine treatment group size was 30 participants. The median pain score in control arms was $43 / 100 \mathrm{~mm}$ (mean 42/100 mm), and 10 of 29 studies had pain scores below $40 / 100 \mathrm{~mm}$, indicating that pain was only mild in those studies.

Pain scores measured with VAS ( 0 to $100 \mathrm{~mm}$ ), were $6 \mathrm{~mm}$ lower after ketamine treatment $(95 \% \mathrm{Cl}-10.7$ to -0.5$)$, compared to participants receiving control treatment (Analysis 1.4).

\section{Sensitivity analyses}

We performed a sensitivity analysis using only those studies with a treatment group size of 30 or more. We included 1210 participants 
(67\% of the total), in the analysis. In these larger studies, in participants treated with ketamine, pain scores were $4 \mathrm{~mm}$ lower (95\% $\mathrm{Cl}-9.9$ to -2.7$)$.

Using only the three studies with ketamine treatment group size of 50 participants or more (395 participants, 22\%), in participants treated with ketamine, pain scores were $1 \mathrm{~mm}$ lower $(95 \% \mathrm{Cl}-16$ to 18).

Pain scores for the control arms of the 29 studies varied between $12 \mathrm{~mm}$ and $69 \mathrm{~mm} / 100 \mathrm{~mm}$. Using the 19 studies with ketamine control group pain scores of $40 / 100 \mathrm{~mm}$ or more (1300 participants, $72 \%)$, in participants treated with ketamine, pain scores were $7 \mathrm{~mm}$ lower $(95 \% \mathrm{Cl}-14$ to -0.1$)$.

We assessed the quality of evidence for this outcome as moderate, downgraded once because the magnitude of effect fell with larger studies (small study effect; Summary of findings 1 ).

Results by surgery type are shown in Summary table B.

\section{Pain intensity at rest at $\mathbf{4 8}$ hours in a non-stratified study population}

We found 49 studies with 2962 participants that provided data for pain intensity at rest at 48 hours after surgery (Adam 2005; Adriaenssens 1999; Argiriadou 2011; Arikan 2016; Aveline 2006; Aveline 2009; Bornemann-Cimenti 2016; Chazan 2010; Chen 2004; Dahl 2000; De Kock 2001; Fiorelli 2015; Ganne 2005; Grady 2012; Guillou 2003; Hu 2014; Jaksch 2002; Jendoubi 2017; Joly 2005; Joseph 2012; Kafali 2004; Kakinohana 2004; Kamal 2008; Katz 2004; Kim 2013; Kudoh 2002; Kwon 2009; Lahtinen 2004; Lak 2010; Lebrun 2006; Lo 2008; Loftus 2010; Mendola 2012; Menigaux 2000; Menigaux 2001; Michelet 2007; Papaziogas 2001; Remérand 2009; Snijdelaar 2004; Song 2013; Subramaniam 2011; Suzuki 2006; Webb 2007; Woo 2014; Wu 2009; Yamauchi 2008; Yazigi 2012; Yeom 2012; Zakine 2008). Ketamine was given to 1591 participants and control to 1371 . Most studies (43 out of 49), had fewer than 50 participants in each study group; the median ketamine treatment group size was 30 participants. The median pain score in control arms was $21 / 100$ $\mathrm{mm}$ (mean $23 / 100 \mathrm{~mm}$ ), and 43 of 49 studies had pain scores below $40 / 100 \mathrm{~mm}$, indicating that pain was only mild in those studies.

We found pain scores measured with VAS ( 0 to $100 \mathrm{~mm}$ ), were $5 \mathrm{~mm}$ lower after ketamine treatment $(95 \% \mathrm{Cl}-6.7$ to -3.4$)$ compared to participants receiving control treatment (Analysis 1.5).

\section{Sensitivity analyses}

We performed a sensitivity analysis using only those studies with a treatment group size of 30 or more. We included 1972 participants ( $67 \%$ of the total), in the analysis. In these larger studies, in participants treated with ketamine, pain scores were $4 \mathrm{~mm}$ lower $(95 \% \mathrm{Cl}-5.9$ to -1.9$)$.

Using only the seven studies with ketamine treatment group size of 50 participants or more (752 participants, $25 \%$ ), in participants treated with ketamine, pain scores were $4 \mathrm{~mm}$ lower $(95 \% \mathrm{Cl}-11$ to 2.4).

Pain scores for the control arms of the 29 studies varied between 2 $\mathrm{mm}$ and $53 \mathrm{~mm} / 100 \mathrm{~mm}$. Using only the six studies with ketamine control group pain scores of $40 / 100 \mathrm{~mm}$ or more (359 participants, $12 \%)$, in participants treated with ketamine, pain scores were 10 $\mathrm{mm}$ lower $(95 \% \mathrm{Cl}-19$ to -1.1$)$.
We assessed the quality of evidence for this outcome as high. We did not downgrade for small study effect because the magnitude of effect was not smaller in the larger studies (Summary of findings 1 ). We also had confidence because the effect of ketamine was larger in studies with higher initial pain intensity.

Results by surgery type are shown in Summary table B.

\section{Pain intensity during movement at $\mathbf{4 8}$ hours in a non-stratified study population}

Twenty-three studies with 1353 participants provided data for pain intensity during movement at 48 hours (Argiriadou 2011; Aveline 2009; Bornemann-Cimenti 2016; De Kock 2001; Guillou 2003; Jaksch 2002; Jendoubi 2017; Joly 2005; Joseph 2012; Kakinohana 2004; Kamal 2008; Katz 2004; Kim 2013; Lahtinen 2004; Menigaux 2000; Snijdelaar 2004; Song 2013; Subramaniam 2011; Suzuki 2006; Webb 2007; Wu 2009; Yamauchi 2008; Yazigi 2012).

Studies addressed pain on movement during cough or peak flow expiration ( 6 studies), on knee flexion (3 studies), lifting leg at supine position (1 study), rolling, sitting or coughing (2 studies), rolling from supine to side-lying position and performing two maximal inspirations ( 2 studies), on changing position (1 study), or did not specify movement stimulus in detail and used wording such as "during movement" or "at mobilization" (8 studies).

Ketamine was given to 739 participants and control to 614 . Most studies (21 out of 23), had fewer than 50 participants in each study group; the median ketamine treatment group size was 27 participants. The median pain score in control arms was $37 / 100 \mathrm{~mm}$ (mean $37 / 100 \mathrm{~mm}$ ), and 15 of 23 studies had pain scores below $40 / 100 \mathrm{~mm}$, indicating that pain was only mild in those studies.

Pain scores measured with VAS (0 to $100 \mathrm{~mm}$ ), were $6 \mathrm{~mm}$ lower after ketamine treatment $(95 \% \mathrm{Cl}-10.2$ to -1.3$)$ compared to participants receiving control treatment (Analysis 1.6).

\section{Sensitivity analyses}

We performed a sensitivity analysis using only those studies with a treatment group size of 30 or more. We included 853 participants (63\% of the total), in the analysis. In these larger studies, in participants treated with ketamine, pain scores were $5 \mathrm{~mm}$ lower (95\% $\mathrm{Cl}-11$ to 0.8$)$.

Using only the two studies with ketamine treatment group size of 50 participants or more (250 participants, $18 \%$ ), we found participants treated with ketamine pain scores were $2 \mathrm{~mm}$ higher $(95 \% \mathrm{Cl}-14$ to 17$)$.

Pain scores for the control arms of the 23 studies varied between $5 \mathrm{~mm}$ and $70 \mathrm{~mm} / 100 \mathrm{~mm}$. We found eight studies with ketamine control group pain scores of $40 / 100 \mathrm{~mm}$ or more (379 participants, $28 \%$ ), in participants treated with ketamine, pain scores were 10 $\mathrm{mm}$ lower $(95 \% \mathrm{Cl}-14$ to -6.1$)$.

We assessed the quality of evidence for this outcome as low, downgraded once because the magnitude of effect fell with larger studies (small study effect), and once because there were fewer than 1500 participants in the analysis (Summary of findings 1 ).

Summary table B shows results for the 24 -hour and 48 -hour pain intensity data at rest and during movement, both for all studies and according to different types of surgery. The detailed results for 
these are in Appendix 5. The analyses by surgery type were not subject to any sensitivity analysis by study size or pain intensity level. In general, results by surgery type were similar to that for all surgery though in some cases there was no evidence of a difference. (data for all studies (non-stratified) and stratified by type of surgery)

\begin{tabular}{|c|c|c|c|}
\hline Surgery & Studies & Participants & $\begin{array}{l}\text { VAS } 0-100(\mathrm{~mm}) \\
\text { MD }(95 \% \mathrm{Cl})\end{array}$ \\
\hline
\end{tabular}

\section{Pain at rest at 24 hours}

\begin{tabular}{llll}
\hline All studies & 82 & 5004 & $-5(-6.6$ to -3.6$)$ \\
\hline Thoracotomy & 13 & 782 & $-4(-8.8$ to 1.0$)$ \\
\hline Major orthopaedic & 11 & 843 & $-6(-9.9$ to -3.0$)$ \\
\hline Major abdominal & 18 & 1178 & $-7(-11$ to -4.2$)$ \\
\hline Total abdominal hysterectomy & 8 & 493 & $-3(-4.6$ to -0.5$)$ \\
\hline Laparoscopic procedures & 9 & 484 & $-2(-6.7$ to 2.0
\end{tabular}

\section{Pain during movement at 24 hours}

\begin{tabular}{llll}
\hline All studies & 29 & 1806 & $-6(-11$ to -0.5$)$ \\
\hline Thoracotomy & 5 & 315 & $-7(-20$ to 5.5$)$ \\
\hline Major orthopaedic & 4 & 279 & $-7(-12$ to -0.8$)$ \\
\hline Major abdominal & 9 & 666 & $-3(-11$ to 5.7) \\
\hline Total abdominal hysterectomy & no data & \\
\hline Laparoscopic procedures & no data & \\
\hline
\end{tabular}

\section{Pain at rest at 48 hours}

\begin{tabular}{llll}
\hline All studies & 49 & 2962 & $-5(-6.7$ to -3.4$)$ \\
\hline Thoracotomy & 9 & 530 & $-7(-10$ to -3.4$)$ \\
\hline Major orthopaedic & 7 & 453 & $-1(-4.1$ to 1.3$)$ \\
\hline Major abdominal & 13 & 891 & $-6(-8.9$ to -3.1$)$ \\
\hline Total abdominal hysterectomy & no data & \\
\hline Laparoscopic procedures & no data
\end{tabular}

\section{Pain during movement at 48 hours}

\begin{tabular}{llll}
\hline All studies & 23 & 1353 & $-6(-10$ to -1.3$)$ \\
\hline Thoracotomy & 5 & 298 & $-11(-15$ to -6.0$)$ \\
\hline Major orthopaedic & 4 & 157 & $-7(-13$ to -1.6$)$ \\
\hline
\end{tabular}




\section{Secondary outcomes (non-stratified study population) Time to first analgesic request}

We found 31 studies with 1678 participants that provided data on how ketamine affects the time to first analgesic request (Adam 2005; Aqil 2011; Ataskhoyi 2013; Aveline 2009; Cenzig 2014; Choi 2015; Dal 2005; Dar 2012; Gilabert Morell 2002; Hadi 2010; Hadi 2013; Helmy 2015; Jaksch 2002; Kafali 2004; Kakinohana 2004; Karaman 2006; Kararmaz 2003; Köse 2012; Lahtinen 2004; Leal 2013; Lin 2016; Menigaux 2000; Nesek-Adam 2012; Ong 2001; Papaziogas 2001; Parikh 2011; Roytblat 1993; Safavi 2011; Sahin 2004; Song 2014; Ysasi 2010). These studies included trials where ketamine was administered pre- or intraoperatively but not after surgery. The median time to first request with control was 18 minutes (mean 39 minutes).

We found that 933 participants who received ketamine requested analgesia a mean of 54 minutes later $(95 \% \mathrm{Cl} 37$ to 71$)$, than 745 participants in the control group (Analysis 1.7). A single study reported an extraordinary increase of over 1000 minutes (Parikh 2011). Omitting this still provided evidence of a difference with an increase of 22 minutes ( $95 \% \mathrm{Cl} 15$ to 29 ).

We assessed the quality of evidence for this outcome as moderate. We downgraded the quality of evidence once because we could not test for small-study effects despite there being more than 1500 participants in the analysis (Summary of findings 1 ).

\section{Studies assessing postoperative hyperalgesia}

We found seven studies with 333 participants (Bornemann-Cimenti 2016 Burstal 2001; De Kock 2001; Joly 2005; Leal 2015; Song 2014; Stubhaug 1997), providing data for hyperalgesia assessed at 24 hours postoperatively. They expressed results as area of hyperalgesia. We were able to derive the area as square centimetres in two studies (Joly 2005; Leal 2015). In three studies mean values for affected area were smaller than the SD for treatment, control, or both groups (158 participants, $47 \%$ of total; Bornemann-Cimenti 2016; Burstal 2001; De Kock 2001). Only two of 14 treatment groups involved 30 participants or more.

Overall 188 participants received ketamine and 145 received control treatment. The area of hyperalgesia for those receiving control treatment was $15 \mathrm{~cm}^{2}$. Ketamine treatment reduced the area of postoperative hyperalgesia by $7 \mathrm{~cm}^{2}(95 \% \mathrm{Cl}-11.9$ to -2.2 ; Analysis 1.9).

We assessed the quality of evidence for this outcome as very low. We downgraded the quality of evidence three times to very low because there were fewer than 400 participants in the analysis (Summary of findings 1 ).

Bornemann-Cimenti 2016 assessed hyperalgesia after major abdominal surgery. In this study, ketamine administration lasted up to 48 hours after surgery along with a PCA device administering piritramide. Burstal 2001 administered ketamine via PCA after abdominal hysterectomy. Participants also received analgesia via epidural catheter. De Kock 2001 and Joly 2005 investigated ketamine during major abdominal surgery. Participants received ketamine as a pre-incisional bolus followed by an infusion that lasted up to 48 hours after surgery. Leal 2015 and Song 2014 administered ketamine intraoperatively during laparoscopic procedures along with remifentanil infusion. Remifentanil is known for its hyperalgesic effect. Stubhaug 1997 administered ketamine as a pre-incisional bolus followed by infusion that lasted up to 48 hours after nephrectomy. Participants also received intercostal nerve blockades.

\section{Adverse events with ketamine}

Because reports did not categorise adverse events as major or minor, we pooled all adverse event reports together, and report hallucination, dizziness, confusion, drowsiness, sedation, nightmares and visual disturbances separately from postoperative nausea and vomiting.

\section{Central nervous system (CNS) adverse events}

For this analysis, we pooled all CNS adverse events (hallucination, dizziness, confusion, drowsiness, sedation, nightmares and visual disturbances). One hundred and five studies with 6538 participants provided dichotomous data on CNS adverse events. Twelve studies (742 participants) did not report on CNS adverse events (Aida 2000; Choi 2015; Dahl 2000; Dahi-Taleghani 2014; Grady 2012; Helmy 2015; Kakinohana 2004; Ong 2001; Patel 2016; Song 2014; Ünlügenc 2003; Yalcin 2012).

We found 53 studies (2832 participants), that reported that no CNS adverse events occurred in either study group (Adam 2005; Adriaenssens 1999; Argiriadou 2004; Argiriadou 2011; Ataskhoyi 2013; Aveline 2009; Chen 2004; D'Alonzo 2011; Dal 2005; De Kock 2001; Du 2011; Fiorelli 2015; Ganne 2005; Garcia-Navia 2016; Gilabert Morell 2002; Guignard 2002; Hadi 2010; Hadi 2013; Haliloglu 2015; Hasanein 2011; Hercock 1999; Jaksch 2002; Jendoubi 2017; Kafali 2004; Karaman 2006; Karcioglu 2013; Kim 2013; Köse 2012; Kwok 2004; Kwon 2009; Lebrun 2006; Lehmann 2001; Lin 2016; Mahran 2015; Mathisen 1999; Mendola 2012; Menigaux 2000; Menigaux 2001; Michelet 2007; Pacreu 2012; Papaziogas 2001; Parikh 2011; Pirim 2006; Roytblat 1993; Sahin 2004; Snijdelaar 2004; Spreng 2010; Suzuki 1999; Van Elstraete 2004; Woo 2014; Yeom 2012; Ysasi 2010; Zakine 2008).

We found 52 studies (3706 participants), that reported CNS adverse events (Aqil 2011; Arikan 2016; Aubrun 2008; Aveline 2006; Ayoglu 2005; Barreveld 2013; Bilgen 2012; Burstal 2001; Cenzig 2014; Chazan 2010; Deng 2009; Dualé 2009; Dullenkopf 2009; Galinski 2007; Garg 2016; Guillou 2003; Hayes 2004; Hu 2014; Ilkjaer 1998; Joly 2005; Joseph 2012; Kamal 2008; Kapfer 2005; Kararmaz 2003; Katz 2004; Kim 2016; Kudoh 2002; Lahtinen 2004; Lak 2010; Leal 2013; Leal 2015; Lo 2008; Loftus 2010; Martinez 2014; McKay 2007; 
Mebazaa MS 2008; Miziara 2016; Nesek-Adam 2012; Nielsen 2017; Remérand 2009; Reza 2010; Safavi 2011; Sen 2009; Siddiqui 2015; Singh 2013; Song 2013; Subramaniam 2011; Tena 2014; Webb 2007; Wu 2009; Yalcin 2012; Yazigi 2012).

Combining both of these groups, we found that studies had observed CNS adverse events in 187 of 3614 (5\%), participants receiving ketamine compared to 122 of 2924 (4\%), participants receiving control treatment. The RR was $1.17(95 \% \mathrm{Cl} 0.95$ to 1.43 ; Analysis 1.8).

We assessed the quality of evidence for this outcome as high due to consistency across a large body of data. Studies did not note any CNS adverse events in 53 studies with $43 \%$ of participants, and the other studies had low rates of CNS adverse events (Summary of findings 1). In the studies that reported at least one CNS adverse event, there was also no evidence of a difference between ketamine and placebo, with RR 1.17 (95\% Cl 0.95 to 1.43; Analysis 1.10).

In addition, four studies reported CNS adverse events as continuous data, using a specific score (Bornemann-Cimenti 2016; Stubhaug 1997; Suzuki 2006; Yamauchi 2008). BornemannCimenti 2016 assessed postoperative delirium using the Intensive Care Delirium Screening Checklist (ICDSC). The ICDSC score was increased in the low-dose ketamine study group (ketamine 0.25 $\mathrm{mg} / \mathrm{kg}$ bolus and $0.125 \mathrm{mg} / \mathrm{kg} / \mathrm{h}$ infusion for 48 hours), compared with the minimal-dose ketamine group (a $0.015 \mathrm{mg} / \mathrm{kg} / \mathrm{h}$ infusion), and the placebo group. In the remaining three studies, there was no difference in the degree of CNS adverse events between treatment and control groups.

Six studies reported the number of participants withdrawn from the study because of CNS adverse events (Burstal 2001; Joseph 2012; Lahtinen 2004; Song 2013; Subramaniam 2011; Webb 2007). For all studies reporting the outcome of CNS adverse event withdrawal, 12 of $5884(0.2 \%)$, participants having ketamine were withdrawn, and 3 of $3447(0.09 \%)$, participants with control.

\section{Postoperative nausea and vomiting}

We combined the data concerning nausea and vomiting, or both. Four studies reported that postoperative nausea and vomiting did not occur in either study group (Fiorelli 2015; Galinski 2007; Helmy 2015; Menigaux 2001).

Ninety-five studies with 5965 participants provided dichotomous data on nausea and vomiting, or nausea, or vomiting. In 91 studies postoperative nausea and vomiting occurred in both study groups (Abdolahi 2013; Adriaenssens 1999; Aqil 2011; Argiriadou 2004; Arikan 2016; Ataskhoyi 2013; Aubrun 2008; Aveline 2006; Aveline 2009; Ayoglu 2005; Bilgen 2012; Cenzig 2014; Chazan 2010; Crousier 2008; Dahi-Taleghani 2014; Dal 2005; Dar 2012; Deng 2009; Dualé 2009; Garcia-Navia 2016; Garg 2016; Gilabert Morell 2002; Grady 2012; Guignard 2002; Guillou 2003; Hadi 2013; Haliloglu 2015; Hasanein 2011; Hu 2014; Jaksch 2002; Jendoubi 2017; Joly 2005; Joseph 2012; Kafali 2004; Kakinohana 2004; Kamal 2008; Kapfer 2005; Karaman 2006; Kararmaz 2003; Kim 2013; Kim 2016; Köse 2012; Kwok 2004; Kwon 2009; Lahtinen 2004; Lak 2010; Leal 2013; Leal 2015; Lehmann 2001; Lin 2016; Lo 2008; Loftus 2010; Mahran 2015; Martinez 2014; McKay 2007; Mebazaa MS 2008; Mendola 2012; Menigaux 2000; Michelet 2007; Miziara 2016; Nesek-Adam 2012; Nielsen 2017; Ögün 2001; Ong 2001; Ozhan 2013; Pacreu 2012; Papaziogas 2001; Parikh 2011; Pirim 2006; Remérand 2009; Reza 2010; Roytblat 1993; Safavi 2011; Sen 2009; Siddiqui 2015; Singh
2013; Snijdelaar 2004; Song 2013; Spreng 2010; Stubhaug 1997; Subramaniam 2011; Suzuki 1999; Tena 2014; Ünlügenc 2003; Van Elstraete 2004; Woo 2014; Wu 2009; Yazigi 2012; Yeom 2012; Ysasi 2010; Zakine 2008).

In the 95 studies 761 of 3263 (23\%), participants who received ketamine and 731 of 2702 (27\%), participants who received control treatment suffered from postoperative nausea and vomiting. Ketamine treatment reduced the incidence of postoperative nausea and vomiting (RR $0.88,95 \% \mathrm{Cl} 0.81$ to 0.96 ; Analysis 1.11). We calculated that the NNTB to prevent one episode of postoperative nausea and vomiting with perioperative intravenous ketamine administration was 24 (95\% Cl 16 to 54).

We assessed the quality of evidence for this outcome as high due to consistency across a large body of data (Summary of findings 1 ).

\section{Subgroup analyses of primary and secondary outcomes}

We carried out a number of subgroup analyses to investigate factors that may have influenced the overall results. We used analyses that compared intravenous ketamine with placebo, or compared intravenous ketamine plus a basic analgesic regimen with the same basic analgesic regimen alone using a non-stratified study population.

\section{Subgroup analysis of pre-incisional and postoperative ketamine}

\section{4-hour opioid consumption}

We found 19 studies that administered ketamine as a pre-incisional bolus at the beginning of surgery and reported 24-hour opioid consumption (Argiriadou 2011; Aveline 2006; Bilgen 2012; Cenzig 2014; Dahl 2000; Dullenkopf 2009; Fiorelli 2015; Garcia-Navia 2016; Gilabert Morell 2002; Helmy 2015; Kafali 2004; Karaman 2006; Kwon 2009; Lehmann 2001; Menigaux 2000; Reza 2010; Roytblat 1993; Sahin 2004; Song 2013). The studies included 573 participants who received ketamine and 472 participants who received control treatment. Pre-incisionally administered ketamine reduced 24hour opioid consumption by $5.5 \mathrm{mg}$ morphine equivalents compared with control $(95 \% \mathrm{Cl}-8.0$ to -3.1 ; Analysis 2.1). We assessed the quality of evidence for this outcome as moderate. We downgraded the quality of evidence once from high to moderate because studies were small and because we could not test for smallstudy effects ( 18 of the 19 studies included in this analysis had fewer than 50 participants in each treatment group).

We found nine studies that administered ketamine in the postoperative period and reported 24-hour opioid consumption (Adriaenssens 1999; Barreveld 2013; Dahi-Taleghani 2014; Garg 2016; Guillou 2003; Javery 1996; Kamal 2008; Michelet 2007; Ünlügenc 2003). We found 293 participants who received ketamine and 301 participants who received control treatment. Ketamine treatment reduced opioid consumption at 24 hours by $9 \mathrm{mg}$ morphine equivalents compared with control $(95 \% \mathrm{Cl}-13.8$ to -3.5 ; Analysis 2.1). We assessed the quality of evidence for this outcome as moderate. We downgraded the quality of evidence once from high to moderate because studies were small and because we could not test for small-study effects (all studies included in this analysis had fewer than 50 participants in each treatment group).

The test for difference (Analysis 2.1), showed no evidence of a difference $(P=0.28)$. 


\section{8-hour opioid consumption}

We found nine studies that administered ketamine as a preincisional bolus and reported opioid consumption at 48 hours after surgery (Bilgen 2012; Dahl 2000; Fiorelli 2015; Gilabert Morell 2002; Kafali 2004; Kwon 2009; Menigaux 2000; Papaziogas 2001; Song 2013). We found that 305 participants received ketamine and 229 participants received control treatment. Pre-incisionally administered ketamine reduced opioid consumption at 48 hours by $3.9 \mathrm{mg}$ morphine equivalents, compared with control $(95 \% \mathrm{Cl}$ -7.0 to -0.7 ; Analysis 2.2). We assessed the quality of evidence for this outcome as moderate. We downgraded the quality of evidence once from high to moderate because studies were small and because we could not test for small-study effects (all studies included in this analysis had fewer than 50 participants in each treatment group).

We found seven studies that administered ketamine in the postoperative period and reported 48-hour opioid consumption (Adriaenssens 1999; Arikan 2016; Garg 2016; Guillou 2003; Kamal 2008; Lak 2010; Michelet 2007). We found 207 participants who received ketamine and 218 participants who received control treatment. Ketamine treatment reduced opioid consumption at 48 hours by $21 \mathrm{mg}$ morphine equivalents compared with control (95\% Cl -27.4 to -14.2 ; Analysis 2.2). We assessed the quality of evidence for this outcome as moderate. We downgraded the quality of evidence once from high to moderate because studies were small and because we could not test for small-study effects (all studies included in this analysis had fewer than 50 participants in each treatment group).

The test for difference (Analysis 2.2) showed evidence of a difference between pre-incisional and postoperative ketamine $(\mathrm{P}=$ $0.000001)$.

\section{Pain intensity at $\mathbf{2 4}$ hours}

We found 20 studies that administered ketamine as a pre-incisional bolus and reported pain intensity at 24 hours after surgery (Aveline 2006; Cenzig 2014; D'Alonzo 2011; Dahl 2000; Fiorelli 2015; Kafali 2004; Kwok 2004; Kwon 2009; Lebrun 2006; Lee 2008; Lehmann 2001; Mathisen 1999; Menigaux 2000; Menigaux 2001; Nesek-Adam 2012; Papaziogas 2001; Patel 2016; Reza 2010; Roytblat 1993; Safavi 2011). Five hundred and sixty-four participants received ketamine and 511 participants received control treatment. Pain intensity was $7 \mathrm{~mm}$ lower ( $95 \% \mathrm{Cl}-10.1$ to -3.2$)$, among participants who received ketamine compared to controls (Analysis 2.3). We assessed the quality of evidence for this outcome as low. We downgraded the quality of evidence twice from high to low, once because there were fewer than 1500 participants in the analysis and once because we could not test for small-study effects (19 of the 20 studies included in this analysis had fewer than 50 participants in each treatment group).

We found nine studies that administered ketamine postoperatively and reported pain intensity at 24 hours after surgery (Adriaenssens 1999; Dahi-Taleghani 2014; Guillou 2003; Javery 1996; Kamal 2008; Lak 2010; Lo 2008; Michelet 2007; Ünlügenc 2003). We found that 282 participants received ketamine and 289 participants received control treatment. Pain intensity was $8 \mathrm{~mm}$ lower among participants who received ketamine compared with control $(95 \%$ $\mathrm{Cl}-12.6$ to -4.1 ; Analysis 2.3). We assessed the quality of evidence for this outcome as low. We downgraded the quality of evidence twice from high to low, once because there were fewer than 1500 participants in the analysis and once because we could not test for small-study effects (eight of the nine studies included in this analysis had fewer than 50 participants in each treatment group).

The test for difference (Analysis 2.3) showed no evidence of a difference $(P=0.55)$.

\section{Pain intensity at $\mathbf{4 8}$ hours}

We found nine studies that administered ketamine as a preincisional bolus and reported pain intensity at 48 hours after surgery (Aveline 2006; Dahl 2000; Fiorelli 2015; Kafali 2004; Kwon 2009; Lebrun 2006; Menigaux 2000; Menigaux 2001; Papaziogas 2001). We found 282 participants received ketamine and 227 participants received control treatment. Ketamine treatment lowered reduced pain intensity by $4 \mathrm{~mm}(95 \% \mathrm{Cl}-7.5$ to -1.2$)$, compared with controls (Analysis 2.4). We assessed the quality of evidence for this outcome as low. We downgraded the quality of evidence twice from high to low, once because there were fewer than 1500 participants in the analysis and once because we could not test for small-study effects (eight of the nine studies included in this analysis had fewer than 50 participants in each treatment group).

We found six studies that administered ketamine postoperatively and reported pain intensity at 48 hours after surgery (Adriaenssens 1999; Guillou 2003; Kamal 2008; Lak 2010; Lo 2008; Michelet 2007). We found 160 participants who received ketamine and 171 participants who received control treatment. Pain intensity measured as VAS was $8 \mathrm{~mm}$ lower among participants who received ketamine compared with control $(95 \% \mathrm{Cl}-15.8$ to -0.3 ; Analysis 2.4). We assessed the quality of evidence for this outcome as very low. We downgraded the quality of evidence three times from high to very low because there were fewer than 400 participants in the analysis.

The test for difference (Analysis 2.4) showed no evidence of a difference $(P=0.39)$.

\section{Time to first request for analgesia after pre-incisional ketamine administration}

We found 13 studies where ketamine was administered preincisionally that reported time to first request for analgesia (Aqil 2011; Ataskhoyi 2013; Cenzig 2014; Gilabert Morell 2002; Helmy 2015; Kafali 2004; Menigaux 2000; Nesek-Adam 2012; Ong 2001; Papaziogas 2001; Roytblat 1993; Safavi 2011; Sahin 2004). We found 352 participants who received ketamine and 291 participants who received control treatment. Ketamine administration delayed time to first request for analgesia by a mean of 38 minutes $(95 \% \mathrm{Cl} 20.9$ to 54.5; Analysis 2.5). We assessed the quality of evidence for this outcome as low. We downgraded the quality of evidence twice from high to low, once because there were fewer than 1500 participants in the analysis and once because we could not test for small-study effects ( 12 of the 13 studies included in this analysis had fewer than 50 participants in each treatment group).

\section{We found no studies for postoperative ketamine.}

\section{Effect of co-administration of ketamine and nitrous oxide}

We conducted separate analyses of studies where ketamine was administered together with nitrous oxide. Nitrous oxide did not seem to change the effect of ketamine. 


\section{4-hour opioid consumption}

Thirty-three studies administered ketamine where nitrous oxide was used as a component of general anaesthesia (Adriaenssens 1999; Aveline 2006; Aveline 2009; Bilgen 2012; Cenzig 2014; Crousier 2008; Dahi-Taleghani 2014; Dahl 2000; Dullenkopf 2009; Garg 2016; Gilabert Morell 2002; Grady 2012; Guillou 2003; Hadi 2010; Hadi 2013; Haliloglu 2015; Hercock 1999; Karaman 2006; Katz 2004; Leal 2013; Lehmann 2001; Menigaux 2000; Murdoch 2002; Ögün 2001; Parikh 2011; Remérand 2009; Reza 2010; Roytblat 1993; Safavi 2011; Sahin 2004; Sen 2009; Ünlügenc 2003; Zakine 2008). There were 1247 participants who received ketamine and 929 participants who received control treatment. Ketamine administration reduced postoperative opioid consumption at 24 hours by $7 \mathrm{mg}$ morphine equivalents compared with control $(95 \% \mathrm{Cl}-9.8$ to -4.8 ; Analysis 3.1).

We assessed the quality of evidence for this outcome as low. We downgraded the quality of evidence twice from high to low, once because there were fewer than 1500 participants in the analysis and once because we could not test for small-study effects ( 27 of the 33 studies included in this analysis had fewer than 50 participants in each treatment group).

\section{8-hour opioid consumption}

Fifteen studies administered ketamine where nitrous oxide was used as a component of general anaesthesia (Adam 2005; Adriaenssens 1999; Arikan 2016; Aveline 2009; Bilgen 2012; Dahl 2000; Garg 2016; Guillou 2003; Katz 2004; Kim 2013; Lak 2010; Menigaux 2000; Remérand 2009; Snijdelaar 2004; Zakine 2008). There were 657 participants who received ketamine and 453 participants who received control treatment. Ketamine administration reduced postoperative opioid consumption by 15 mg morphine equivalents compared with control $(95 \% \mathrm{Cl}-21.1$ to -8.4; Analysis 3.2).

We assessed the quality of evidence for this outcome as low. We downgraded the quality of evidence twice from high to low, once because there were fewer than 1500 participants in the analysis and once because we could not test for small-study effects ( 13 of the 15 studies included in this analysis had fewer than 50 participants in each treatment group).

\section{Pain intensity at rest at 24 hours}

Thirty-two studies administered ketamine where nitrous oxide was used as a component of general anaesthesia (Adam 2005; Adriaenssens 1999; Arikan 2016; Aubrun 2008; Aveline 2006; Aveline 2009; Cenzig 2014; Dahl 2000; Guillou 2003; Hadi 2010; Haliloglu 2015; Hercock 1999; Katz 2004; Kim 2013; Kwok 2004; Lak 2010; Lee 2008; Lehmann 2001; Menigaux 2000; Menigaux 2001; Ögün 2001; Parikh 2011; Remérand 2009; Reza 2010; Safavi 2011; Sen 2009; Snijdelaar 2004; Suzuki 2006; Ünlügenc 2003; Yamauchi 2008; Yeom 2012; Zakine 2008). There were 1145 participants who received ketamine and 908 participants who received control treatment. Pain intensity measured as VAS was $8 \mathrm{~mm}$ lower among participants who had received ketamine compared with control $(95 \% \mathrm{Cl}-10.8$ to -5.4; Analysis 3.3).

We assessed the quality of evidence for this outcome as moderate. We downgraded the quality of evidence once from high to moderate because we could not test for small-study effects despite there being more than 1500 participants in the analysis (26 of the
31 studies included in this analysis had fewer than 50 participants in each treatment group).

\section{Pain intensity during movement at $\mathbf{2 4}$ hours}

Ten studies administered ketamine where nitrous oxide was used as a component of general anaesthesia (Aveline 2009; Guillou 2003; Hercock 1999; Katz 2004; Kim 2013; Menigaux 2000; Sen 2009; Snijdelaar 2004; Suzuki 2006; Yamauchi 2008). There were 354 participants who received ketamine and 259 participants who received control treatment. Pain intensity measured as VAS at 24 hours during movement was $7 \mathrm{~mm}$ lower $(95 \% \mathrm{Cl}-19.0$ to 6.0$)$, after ketamine administration compared with control (Analysis 3.4).

We assessed the quality of evidence for this outcome as low. We downgraded the quality of evidence twice from high to low, once because there were fewer than 1500 participants in the analysis and once because we could not test for small-study effects (nine of the 10 studies included in this analysis had fewer than 50 participants in each treatment group).

\section{Pain intensity at rest at 48 hours}

Eighteen studies administered ketamine where nitrous oxide was used as a component of general anaesthesia (Adam 2005; Adriaenssens 1999; Arikan 2016; Aveline 2006; Aveline 2009; Dahl 2000; Guillou 2003; Katz 2004; Kim 2013; Lak 2010; Menigaux 2000; Menigaux 2001; Remérand 2009; Snijdelaar 2004; Suzuki 2006; Yamauchi 2008; Yeom 2012; Zakine 2008). There were 689 participants who received ketamine and 513 participants who received control treatment. Pain intensity measured as VAS at rest at 48 hours was $6 \mathrm{~mm}$ lower after ketamine administration compared with control ( $95 \% \mathrm{Cl}-9.9$ to -2.8 ; Analysis 3.5).

We assessed the quality of evidence for this outcome as low. We downgraded the quality of evidence twice from high to low, once because there were fewer than 1500 participants in the analysis and once because we could not test for small-study effects (14 of the 18 studies included in this analysis had fewer than 50 participants in each treatment group).

\section{Pain intensity during movement at $\mathbf{4 8}$ hours}

Eight studies administered ketamine where nitrous oxide was used as a component of general anaesthesia (Aveline 2009; Guillou 2003; Katz 2004; Kim 2013; Menigaux 2000; Snijdelaar 2004; Suzuki 2006; Yamauchi 2008). There were 310 participants who received ketamine and 213 participants who received control treatment. Pain intensity measured as VAS at 48 hours during movement was $5 \mathrm{~mm}$ lower after ketamine administration compared with control (95\% $\mathrm{Cl}-13.1$ to 4.1 ; Analysis 3.6).

We assessed the quality of evidence for this outcome as low. We downgraded the quality of evidence twice from high to low, once because there were fewer than 1500 participants in the analysis and once because we could not test for small-study effects (fewer than 50 participants in each treatment group).

\section{Effect of co-administration of benzodiazepine premedication CNS adverse events}

We found 65 studies (3943 participants), that used benzodiazepine premedication before ketamine administration. 
Thirty-four studies (1739 participants), did not find any CNS adverse events (Adam 2005; Adriaenssens 1999; Argiriadou 2004; Argiriadou 2011; Ataskhoyi 2013; Aveline 2009; De Kock 2001; Fiorelli 2015; Ganne 2005; Garcia-Navia 2016; Gilabert Morell 2002; Guignard 2002; Hadi 2010; Hadi 2013; Hasanein 2011; Jaksch 2002; Jendoubi 2017; Kwon 2009; Lebrun 2006; Lehmann 2001; Mahran 2015; Mathisen 1999; Mendola 2012; Menigaux 2000; Menigaux 2001; Michelet 2007; Pacreu 2012; Papaziogas 2001; Pirim 2006; Roytblat 1993; Snijdelaar 2004; Suzuki 1999; Van Elstraete 2004; Zakine 2008).

Thirty-one studies (2204 participants), reported CNS adverse events after benzodiazepine premedication (Aqil 2011; Arikan 2016; Aubrun 2008; Aveline 2006; Chazan 2010; Dualé 2009; Dullenkopf 2009; Galinski 2007; Garg 2016; Guillou 2003; Hayes 2004; Hu 2014; Ilkjaer 1998; Joly 2005; Joseph 2012; Kamal 2008; Kararmaz 2003; Katz 2004; Kim 2016; Leal 2013; Loftus 2010; Martinez 2014; Mebazaa MS 2008; Remérand 2009; Sen 2009; Siddiqui 2015; Singh 2013; Subramaniam 2011; Tena 2014; Yalcin 2012; Yazigi 2012).

These trials reported CNS adverse events in 123 of 2179 (5.6\%), participants treated with ketamine and 91 of 1764 (5.2\%), participants receiving control. The RR was 1.1 (95\% Cl 0.9 to 1.4 ; Analysis 4.1). This was very similar to the result overall.

We assessed the quality of evidence for this outcome as high.

\section{DISCUSSION}

\section{Summary of main results}

The aim of this review was to evaluate the efficacy and safety of perioperative intravenous ketamine in adult patients when used for the treatment or prevention of acute pain following general anaesthesia.

We included 130 studies (8341 participants) in the review. Of these, 4588 participants received ketamine and 3753 received placebo or a basic analgesic alone.

The mean age for participants who received ketamine was 48 years, and 49 years for those who received control treatment. The distribution between men and women was equal. Types of surgery included ear, nose or throat surgery, wisdom tooth extraction, thoracotomy, lumbar fusion surgery, microdiscectomy, hip joint replacement surgery, knee joint replacement surgery, anterior cruciate ligament repair of the knee, knee arthroscopy, mastectomy, haemorrhoidectomy, abdominal surgery (laparotomy and lumbotomy), thyroid surgery, elective caesarean section and laparoscopic surgery.

Perioperative intravenous ketamine was compared with placebo in a large number of randomised studies and participants. Perioperative intravenous ketamine administration resulted in 19\% reduction in postoperative opioid consumption both at 24 hours and 48 hours. Pain scores decreased by $19 \%$ at rest and by $22 \%$ during movement at 24 hours after surgery. At 48 hours, pain score reductions were $14 \%$ at rest and $16 \%$ on movement.

These results were, within the bounds of available data, consistent when analysed by subgroups of operation type or timing of administration, and sensitivity to study size and initial pain intensity.

The doses of ketamine used in the studies were broadly similar, precluding any sensible assessment of the effects of ketamine dose. Most studies using racemic ketamine administered $0.25 \mathrm{mg} / \mathrm{kg}$ or less. Pre-incisional doses of S-ketamine were typically $0.5 \mathrm{mg} / \mathrm{kg}$. Infusion rates were similar for racemic and S-ketamine.

Perioperative intravenous ketamine reduced postoperative opioid consumption over 24 hours by almost $8 \mathrm{mg}$ morphine equivalents (19\% from $42 \mathrm{mg}$ consumed by participants given placebo, moderate-quality evidence). Over 48 hours, opioid consumption was almost $13 \mathrm{mg}$ lower (19\% from $67 \mathrm{mg}$ with placebo, moderatequality evidence).

Perioperative intravenous ketamine also reduced pain at rest at 24 hours (5/100 mm lower, 19\% lower from 26/100 mm with placebo, high-quality evidence), and 48 hours $(5 / 100 \mathrm{~mm}, 22 \%$ lower from $23 / 100 \mathrm{~mm}$, high-quality evidence). Pain during movement was also reduced at 24 hours $(6 / 100 \mathrm{~mm}, 14 \%$ lower from $42 / 100$ $\mathrm{mm}$, moderate-quality evidence), and 48 hours (6/100 mm, 15\% lower from $37 \mathrm{~mm}$, low-quality evidence). A clinically important difference in pain is generally regarded as being around a $30 \%$ pain reduction (Farrar 2000, though that is determined in people with moderate or severe pain. Here, the mean pain scores are below $30 / 100 \mathrm{~mm}$ at 24 hours and $40 / 100 \mathrm{~mm}$ or below at 48 hours, which is at the limits of the inclusion criterion of $40 / 100 \mathrm{~mm}$ for moderate pain for many clinical trials. In arthritis, for instance, mean pain changes of $7 \mathrm{~mm}$ to $15 \mathrm{~mm}$ are seen for our most effective analgesics (Moore 2010). People in pain regard mild pain (typically below $30 / 100 \mathrm{~mm}$ ) as an acceptable outcome when their pain is moderate or severe (Moore 2013). The goal of perioperative interventions is to avoid postoperative pain, and that generally means using a range of concomitant interventions to prevent it. People with postoperative pain scores below about $30 / 100$ consider their experience is 'very good or excellent' (Mhuircheartaigh 2009). In that circumstance, it can be argued that ketamine effects are probably clinically relevant.

There was some evidence that ketamine was more efficacious in sensitivity analyses when pain scores were higher than 40/100 mm with control, that is, when pain was moderate or severe. Clinically, it is evident that if a certain patient group has little pain overall, it is not desirable to use an additional analgesic, such as ketamine, to the treatment regime. Summary table $\mathrm{C}$ demonstrates the difficulty in being able to make any definitive statement on the effect of higher pain scores. Numerically there was a larger effect on pain scores at rest and on movement at 24 and 48 hours. Where the high-pain-score trials were only a small part of the total, the size was large, but where these predominated, it was small (as for pain during movement at 24 hours). In the former case, the amounts of data are small, so that confidence intervals are wide. So the effects of ketamine may be larger at higher pain scores, but we cannot be sure.

\section{Summary table C: effect of ketamine on VAS - all studies versus} high pain score 


\begin{tabular}{llll}
\hline Analysis & Studies & Participants & $\begin{array}{l}\text { Participants (\% } \\
\text { of total) }\end{array}$ \\
\hline
\end{tabular}

\section{Pain at rest at 24 hours}

\begin{tabular}{lcccc}
\hline All studies & 82 & 5004 & 100 & $-5(-6.6$ to -3.6$)$ \\
\hline Pain in control $\geq 40 / 100 \mathrm{~mm}$ & 14 & 860 & 17 & $-17(-25$ to -9$)$ \\
\hline
\end{tabular}

Pain during movement at 24 hours

\begin{tabular}{lcccc}
\hline All studies & 29 & 1806 & 100 & $-6(-11$ to -0.5$)$ \\
\hline Pain in control $\geq 40 / 100 \mathrm{~mm}$ & 19 & 1300 & 72 & $-7(-14$ to -0.1$)$ \\
\hline
\end{tabular}

\section{Pain at rest at $\mathbf{4 8}$ hours}

\begin{tabular}{llccc}
\hline All studies & 49 & 2962 & 100 & $-5(-6.7$ to -3.4$)$ \\
\hline Pain in control $\geq 40 / 100 \mathrm{~mm}$ & 6 & 259 & 12 & $-10(-19$ to -1.1 \\
\hline
\end{tabular}

Pain during movement at 48 hours

\begin{tabular}{lllll}
\hline All studies & 23 & 1353 & 100 & $-6(-10$ to -1.3$)$ \\
\hline Pain in control $\geq 40 / 100 \mathrm{~mm}$ & 8 & 379 & 28 & $-10(-14$ to -6.1$)$ \\
\hline
\end{tabular}

It has been suggested that concurrent administration of another NMDA-antagonist, nitrous oxide, could reduce the analgesic effect of ketamine when used as a component of general anaesthesia. We found no evidence of a reduced effect with ketamine and nitrous oxide as a component of general anaesthesia, with results very similar to those of the overall analysis.

Ketamine increased the time for the first postoperative analgesic request, by a mean of 54 minutes from 39 minutes with placebo. Despite a single study reporting an extraordinary increase of over 1000 minutes, its omission still provided evidence of a difference with an increase of 22 minutes.

Only seven studies with 333 participants investigated ketamine's effect on postoperative hyperalgesia, even though Bell 2006 pointed out the need for further research on this topic more than 10 years ago. The methods used for evaluating hyperalgesia in individual studies varied from asking the participants about an abnormal sensation on the wound to objective assessment of mechanical allodynia or hyperalgesia with von Frey filaments and mapping of these hyperalgesia areas around the surgical wound. Additionally, the time period for the assessment of hyperalgesia and presentation of the results were heterogeneous, thus limiting the eligibility of the data for quantitative analysis. In this review, ketamine was found to reduce postoperative hyperalgesia, though we recognise that the number of studies contributing to this outcome was small. The study methods were heterogeneous, contributing to the low-quality of evidence, and we were unable to draw any conclusions.
The occurrence of CNS adverse events was not significantly different in participants receiving ketamine (high-quality evidence). The included studies observed CNS adverse events in 187 participants (5\%), receiving ketamine compared to 122 participants (4\%), receiving control treatment. Results were no different in studies using benzodiazepine premedication (high-quality evidence). We were unable to include a large (672 participants), recent, study of the effects of ketamine on postoperative delirium because anaesthetic techniques were not standardised (Avidan 2017). Delirium was the primary outcome, and the study showed no difference between ketamine given in $0.5 \mathrm{mg} / \mathrm{kg}$ and $1.0 \mathrm{mg} / \mathrm{kg}$ bolus doses and placebo ( $19 \%$ for ketamine and placebo).

We found ketamine treatment reduced postoperative nausea and vomiting from $27 \%$ with placebo to $23 \%$ with ketamine; the NNTB to prevent one episode of postoperative nausea and vomiting with perioperative intravenous ketamine administration was 24 (95\% $\mathrm{Cl} 16$ to 54; high-quality evidence). However, the effect size was smaller than previously reported (Bell 2006; Laskowski 2011).

\section{Overall completeness and applicability of evidence}

Based on large number of studies and consistency of response when results were subjected to subgroup and sensitivity analyses, we conclude that there is a general applicability of the evidence regarding intravenous perioperative ketamine. There are some limitations, discussed below, but the main clinical point will be how to use the evidence as part of multimodal anaesthetic techniques to improve postoperative outcomes and patient experience.

A positive bias in favour of a therapy might be found where there are small numbers of small studies (Dechartes 2013; Dechartres 
2014; Fanelli 2017; Nguyen 2017; Nüesch 2010), even by the random play of chance (Brok 2009; Moore 1998; Thorlund 2011). Overemphasising results of underpowered studies or analyses has been criticised (AlBalawi 2013; Roberts 2015; Turner 2013). This review included a large number of small studies, which was probably the source of a high degree of heterogeneity in many analyses (Gavaghan 2000; Sterne 2000).

Small size was the major source of potential bias that might limit both the completeness and the applicability of any results. In the event, examining results in studies of group size larger than the median (30 participants per treatment arm), and performing analyses after eliminating studies at high risk of small size bias (fewer than 50 participants per treatment arm), generally supported the overall results, though data were occasionally limited. This provided confidence in the overall results.

Pain intensity varied between studies from very low pain scores with placebo equivalent to no or only mild pain, to scores indicating moderate or severe pain. The measurement of analgesic effect is accepted to be possible only when pain is present (McQuay 2012). We therefore examined results according to pain scores with placebo, and obtained similar or better analgesic effects with ketamine in studies where pain with placebo was moderate or severe. This also provided confidence in the overall results.

We recognise that the mean analgesic consumption as a measure for assessing analgesic efficacy of an intervention has been criticised because the distribution of analgesic consumption is not Gaussian but highly skewed, where a small number of participants consume over $50 \%$ of the analgesics in a study (Moore 2011). But the mean analgesic consumption as an outcome measure for analgesic efficacy is commonly used and reported in clinical studies, and is the only metric available. Opioids are associated with a large number of adverse events when used during surgery (Macintyre 2010).

We also recognise that pooling data from all operation types might weaken the overall applicability of the evidence. A series of subgroup analyses therefore explored the effects of ketamine by operation type (Appendix 4; Appendix 5). These results generally supported the overall results, although they were limited by small numbers in some cases. We were not able to conduct sensitivity analyses of each operation type due to the small number of participants.

We derived the available evidence on adverse events from a large population with an adequate number of CNS and emetic events, which allowed us to draw conclusions (Moore 1998). There was inadequate evidence to be conclusive about hyperalgesia, though there was an indication that ketamine may reduce postoperative hyperalgesia.

\section{Quality of the evidence}

We judged the risk of bias to be generally low, with few exceptions that failed to present all specified outcomes or presented results that had not been predefined, evoking suspicions of selective reporting. Although there was a large number of studies, many were small in size, with group sizes below 50 participants and thus at potential for high risk of bias. We demonstrated through sensitivity analysis that no size-related bias was apparent.
Our overall judgement of outcome quality was moderate. There were many studies and participants in many analyses; where we were able to demonstrate an absence of any small-study effect, we did not downgrade the evidence, but if that was not possible because of an insufficiency of larger studies, we downgraded because of potential small-study bias.

For adverse events, we typically judged this outcome to be high quality because of a consistent effect found over a large body of data.

\section{Potential biases in the review process}

We are not aware of any biases during the review process. The review authors (ECVB and ET), worked independently and agreed 'Risk of bias' assessments of individual studies, occasionally deferring to a third review author (VKK), when discrepancies arose. The review authors RAM, ECVB, and VKK assessed and agreed GRADE quality of the evidence.

\section{Agreements and disagreements with other studies or reviews}

Schmid 1999 stated that the intravenous administration route was effective in reducing postoperative pain intensity, supporting the findings of this review. However, we excluded from our review seven of the trials that investigated intravenous ketamine that they had included in their review because they did not meet our inclusion criteria (Clausen 1975; Edwards 1993; Jahangir 1993; Joachimmson 1986; Maurset 1989; Owen 1987; Wilder-Smith 1998).

The review by Subramaniam 2004 examined epidural and intravenous ketamine as an adjuvant analgesic to opioids. An analysis of 28 studies with intravenous ketamine administration found that adjuvant ketamine reduces postoperative pain intensity at 24 hours. Subramaniam 2004 supports the findings of this review. Additionally, the prevalence of CNS adverse events ( $9 \%$ with ketamine and 5\% among control group), and postoperative nausea and vomiting (18\% with ketamine and $27 \%$ among control participants), were comparable to our findings.

Elia and Tramér (Elia 2005), published their review in 2005 and their findings concerning the effect of pre-incisionally administered ketamine on cumulative morphine consumption at 24 hours (weighted mean difference $-16 \mathrm{mg}$ in favour of ketamine), are similar to our findings and support the findings of this review. Furthermore, the RR for nausea and vomiting in our review is equivalent to that found by Elia and Tramér (RR $0.89,95 \% \mathrm{Cl} 0.52$ to 1.51$)$.

Based on the data from 37 trials, Bell 2006 concluded that perioperative ketamine reduced pain intensity, rescue analgesic requirements and postoperative nausea and vomiting. Bell 2006 included trials with epidural, intramuscular and intravenous administration routes. The findings of Bell 2006 support the findings of this review.

Laskowski 2011 observed beneficial effects of intravenous ketamine for postoperative analgesia in procedures involving the upper abdomen and thorax (i.e. especially painful procedures). Our findings are similar to this. Ketamine also reduced the incidence of postoperative nausea and vomiting. The findings of Laskowski 2011 support the findings of this review. In contrast to Laskowski's 
findings, we did not observe a higher incidence of CNS adverse events with ketamine use.

Heesen 2014 found that intravenous ketamine during general anaesthesia did not delay the time to first request for opioid or reduce the total dose of postoperative opioid consumption. This does not support the findings of our review. However, Heesen 2014 focused on one study population (patients undergoing caesarean section), which may explain the discrepancy with our result.

\section{AUTHORS' CONCLUSIONS}

\section{Implications for practice}

\section{For people with acute postoperative pain}

Perioperative intravenous ketamine reduces postoperative opioid consumption and pain intensity, especially after thoracic surgery, major orthopaedic surgery and major abdominal surgery. In a non-stratified study population, perioperative intravenous ketamine administration reduces postoperative pain and opioid consumption to a lesser extent. Traditionally, 30\% reduction in pain intensity and opioid consumption has been considered meaningful. However, even a smaller reduction in opioid consumption may be beneficial to those who are vulnerable to opioid-induced adverse events, for example, elderly people or those susceptible to postoperative nausea and vomiting, as well as individuals with opioid dependency. In a non-stratified study population, perioperative intravenous ketamine delays time to first request for analgesia. Ketamine may also reduce postoperative hyperalgesia, though more data are needed to support this preliminary result. Perioperative intravenous ketamine does not increase the risk of central nervous system (CNS) adverse events. The risk for postoperative nausea and vomiting is reduced.

\section{For clinicians}

Perioperative intravenous ketamine is beneficial for individuals undergoing thoracic, major orthopaedic, or major abdominal surgery. It may be more effective in situations with a higher background level of pain. Ketamine reduces postoperative opioid consumption and the risk for postoperative nausea and vomiting and may therefore be beneficial for individuals who are vulnerable to opioid-induced adverse events, for example, the elderly, those susceptible to postoperative nausea and vomiting, as well as individuals with opioid tolerance or dependency. Ketamine may also reduce postoperative hyperalgesia, but more data are needed to support this finding.

\section{For policy makers}

Perioperative intravenous ketamine should be targeted to those who are likely to benefit from ketamine's analgesic and opioidsparing effect.

\section{For funders}

The amount and quality of evidence around the benefits and harms of perioperative intravenous ketamine is moderate or high. The results are buttressed by several subgroup and sensitivity analyses that support the main findings. These include type of surgery, co-administration with nitrous oxide or use of benzodiazepine premedicant, timing of use, and level of pain intensity with controls. There is no evidence that perioperative intravenous ketamine increased the risk of CNS adverse events.

\section{Implications for research}

\section{General implications}

This review of intravenous perioperative ketamine revealed no major problems with the evidence available, other than the generally small size of studies. While this pattern has not been uncommon in anaesthetic research, there is growing evidence that small study size is associated with potential major biases. These are such as to raise ethical as well as scientific considerations for future studies of similar size. This might mean reconsideration of how studies are performed in future. Perhaps multicentre, randomised, controlled study design with more than 200 participants per treatment arm, compared to randomised studies with fewer than 50 participants per treatment arm, would increase confidence in any findings.

\section{Design}

Many of the studies in this review had low pain intensity with controls. That is good, because low pain intensity is valued by people in pain, including postoperatively (Mhuircheartaigh 2009; Moore 2013). The value of pain intensity reduction is probably more highly regarded by people with moderate or severe pain than with those with moderate or no pain. For future studies, it may be more informative to explore multimodal anaesthesia with intravenous ketamine with one component in situations with higher levels of pain.

\section{Measurement (endpoints)}

Mean opioid consumption has been shown to be highly skewed, and probably meaningless (Moore 2011). Future studies might usefully concentrate on reporting the number of people with high opioid consumption. Relevant endpoints could also include patient-reported outcome measures for postoperative recovery, for example, patient satisfaction.

\section{Other}

Future studies should assess the effect of ketamine's different timing and dosing regimens on postoperative pain, opioid consumption and adverse events. Additionally, subgroups who may benefit from ketamine's analgesic and opioid-sparing effect warrant more research. These subgroups are, for example, the elderly and other individuals who are sensitive to adverse events that often accompany opioid medication. So far, the data on ketamine's effect on individuals with a history of substance abuse are limited. Additionally, determining whether specific study populations are more susceptible to ketamine-related adverse events, as well as clarifying ketamine's role in prevention of persistent postsurgical pain among patients with a high risk of chronic pain, would also be of clinical interest. Ketamine's antihyperalgesic effect also warrants more research because so far the data are sparse. Studies examining the effect of ketamine as adjuvant to specific opioids would also be of interest, since recent animal data suggest that ketamine and morphine have beneficial interactions (Lilius 2015).

\section{ACKNOWLEDGEMENTS}

This review has been supported financially in part by the Finnish state funding for university-level health research (grant TYH2014305) and by a grant from the Helsinki University Hospital Research Funds. 
Anna Erskine, Emma Fisher and Kerry Harding at Cochrane Pain, Palliative and Supportive Care (PaPaS), Oxford, UK, gave irreplaceable input in the revising process.

Joanne Abbott (PaPaS), assisted with the database searches.

As we included studies irrespective of language, we would like to express our gratitude to colleagues and professionals who helped us in the translation process: Katerina Andreeva, Jiae Choi, Maija Hukka, Jae Hung Jung, Maija Kaukonen, Kun Hyung Kim, Martin Lehecka, Heng-Lien Lo, Myonghwa Park, Seyeon Park, Kauhan Derya Sentürk and Kristian Seppänen.

Päivi Koroknay-Pál gave valuable advice with Microsoft EXCEL.
Sheena Derry read through and commented on the review. She also provided valuable assistance in the use of Review Manager 5 (Review Manager 2014), during the writing of the review.

We would like to thank Ewan McNicol, Thomas Hamilton, Daryl I Smith and Catherine Hofstetter (peer reviewers), for their valuable comments and advice during the preparation of this systematic review.

Cochrane Review Group funding acknowledgement: this project was supported by the National Institute for Health Research, via Cochrane Infrastructure funding to Cochrane Pain, Palliative and Supportive Care (PaPaS). Disclaimer: the views and opinions expressed therein are those of the review authors and do not necessarily reflect those of the Systematic Reviews Programme, NIHR, National Health Service (NHS) or the Department of Health. 


\section{R E F E R E N C E S}

\section{References to studies included in this review}

Abdolahi 2013 \{published data only\}

Abdolahi M, Soltani HA, Montazeri K, Soleymani B. Preemptive low-dose of ketamine does not effective on anesthetic consumption, perioperative analgesic requirement and postoperative pain, nausea and vomiting in painful ophthalmic surgery. Journal of Research in Medical Sciences 2013;18(7):583-7.

\section{Adam 2005 \{published data only\}}

Adam F, Chauvin M, Du Manoir B, Langlois M, Sessler DI, Fletcher D. Small dose ketamine improves postoperative analgesia and rehabilitation after total knee arthroplasty. Anesthesia and Analgesia 2005;100(2):475-80. [DOI: 10.1213/01.ANE.0000142117.82241.DC]

\section{Adriaenssens 1999 \{published data only\}}

Adriaenssens G, Vermeyen KM, Hoffmann VLH, Mertens E, Adriaensen HF. Postoperative analgesia with i.v. patientcontrolled morphine: effect of adding ketamine. British Journal of Anaesthesia 1999;83(3):393-6.

\section{Aida 2000 \{published data only\}}

Aida S, Yamakura T, Baba H, Taga K, Fukuda S, Shimoji K. Preemptive analgesia by intravenous low-dose ketamine and epidural morphine in gastrectomy. Anesthesiology 2000;92:1624-30.

\section{Aqil 2011 \{published data only\}}

Aqil M, Haq AU, Rasheed A, Hussain A, Khan M, Abdulhamid A$\mathrm{S}$. Is preemptive analgesic effect of ketamine dose dependent? Effect of increasing dose on post septorhinoplasty pain. Pakistan Journal of Medical Sciences 2011;27(3):608-12.

\section{Argiriadou 2004 \{published data only\}}

Argiriadou H, Himmelseher S, Papagiannopoulou P, Georgiou M, Kanakoudis F, Giala M, et al. Improvement of pain treatment after major abdominal surgery by intravenous $S(+)$ ketamine. Anesthesia and Analgesia 2004;98:1413-8. [DOI: 10.1213/01.ANE.0000111204.31815.2D]

\section{Argiriadou 2011 \{published data only\}}

Argiriadou H, Papagiannopoulou P, Foroulis CN, Anastasiadis K, Thomaidou E, Papakonstantinou C, et al. Intraoperative infusion of $\mathrm{S}(+)$-ketamine enhances post-thoracotomy pain control compared with perioperative parecoxib when used in conjunction with thoracic paravertebral ropivacaine infusion. Journal of Cardiothoracic and Vascular Anesthesia 2011;25(3):455-61. [DOI: 10.1053/j.jvca.2010.07.011]

\section{Arikan 2016 \{published data only\}}

Arikan M, Aslan B, Arikan O, Horasanli E, But A. Comparison of the effects of magnesium and ketamine on postoperative pain and morphine consumption. A double-blind randomized controlled study. Acta Cirurgica Brasileira 2016;31(1):67-73. [DOI: http://dx.doi.org/10.1590/S0102-865020160010000010]
Ataskhoyi 2013 \{published data only\}

Ataskhoyi S, Negargar S, Hatami-Marandi P. Effects of the addition of low-dose ketamine to propofol-fentanyl anaesthesia during diagnostic gynaecological laparoscopy. European Journal of Obstetrics and Gynecology and Reproductive Biology 2013;170:247-50.

\section{Aubrun 2008 \{published data only\}}

Aubrun F, Gaillat C, Rosenthal D, Dupuis M, Mottet P, Marchetti F, et al. Effect of a low dose ketamine regimen on pain, mood, cognitive function and memory after major gynaecological surgery: a randomized, double-blind, placebo-controlled trial. European Journal of Anaesthesiology 2008;25:97-105. [DOI: $10.1017 /$ S0265021507002566]

\section{Aveline 2006 \{published data only\}}

Aveline C, Le Hetet H, Vautier P, Gautier JF, Bonnet F. Peroperative ketamine and morphine for postoperative pain control after lumbar disk surgery. European Journal of Pain 2006;10:653-8. [DOI: 10.1016/j.ejpain.2005.10.005]

\section{Aveline 2009 \{published data only\}}

Aveline C, Gautier JF, Vautier P, Cognet F, Le Hetet H, Attali JY, et al. Postoperative analgesia and early rehabilitation after total knee replacement: a comparison of continuous low-dose intravenous ketamine versus nefopam. European Journal of Pain 2009;13:613-9. [DOI: 10.1016/j.ejpain.2008.08.003]

\section{Ayoglu 2005 \{published data only\}}

Ayoglu H, Karadeniz Ü, Kunduracilar Z, Ayoglu FN, Erdemli Ö. The analgesic effect of magnesium sulphate and ketamine in patients undergoing laparoscopic cholecystectomy. The Pain Clinic 2005;17(1):45-53.

\section{Barreveld 2013 \{published data only\}}

Barreveld AM, Correll DJ, Liu X, Max B, McGowan JA, Shovel L, et al. Ketamine decreases postoperative pain scores in patients taking opioids for chronic pain: results of a prospective, randomized, double-blind study. Pain Medicine 2013;14:925-34.

\section{Bilgen 2012 \{published data only\}}

Bilgen S, Köner Ö, Türe H, Menda F, Ficicioglu C, Aykac B. Effect of three different doses of ketamine prior to general anesthesia on postoperative pain following Caesarean delivery: a prospective randomized study. Minerva Anestesiologica 2012;78(4):442-9.

\section{Bornemann-Cimenti 2016 \{published data only\}}

Bornemann-Cimenti H, Wejbora M, Michaeli K, Edler A Sandner-Kiesling A. The effects of minimal-dose versus lowdose S-ketamine on opioid consumption, hyperalgesia, and postoperative delirium: a triple-blinded, randomized, activeand placebo-controlled clinical trial. Minerva Anestesiologica 2016;82(10):1069-76.

\section{Burstal 2001 \{published data only\}}

Burstal R, Danjoux G, Hayes C, Lantry G. PCA ketamine and morphine after abdominal hysterectomy. Anaesthesia and Intensive Care 2001;29(3):246-51. 


\section{Cenzig 2014 \{published data only\}}

Cenzig P, Gokcinar D, Topcu H, Cicek GS, Gogus N. Intraoperative low-dose ketamine infusion reduces acute postoperative pain following total knee replacement surgery: a prospective, randomized, placebo-controlled trial. Journal of the College of Physicians and Surgeons Pakistan 2014;24(5):299-303.

\section{Chazan 2010 \{published data only\}}

Chazan S, Buda I, Nesher N, Paz J, Weinbroum AA. Low-dose ketamine via intravenous patient-controlled analgesia device after various transthoracic procedures improves analgesia and patient and family satisfaction. Pain Management Nursing 2010;11(3):169-76. [DOI: 10.1016/j.pmn.2009.06.003]

Chen 2004 \{published data only\}

Chen JY, Bai L, You Yu YF, Zhou SJ. Effect of low dose ketamine during anesthesia on postoperative analgesia. Fudan University Journal of Medical Sciences 2004;31(1):81-3.

\section{Choi 2015 \{published data only\}}

Choi E, Lee H, Park HS, Lee HY, Kim YJ, Baik HJ. Effect of intraoperative infusion of ketamine on remifentanilinduced hyperalgesia. Korean Journal of Anesthesiology 2015;68(5):476-80. [DOI: 10.4097/kjae.2015.68.5.476]

\section{Colombani 2008 \{published data only\}}

Colombani S, Kabbani Y, Mathoulin-Pélissier S, Gékiere JP, Dixmérias F, Monnin D, et al. Administration of ketamine during induction and maintenance of anaesthesia in postoperative pain prevention. Clinical trial in oncology [Apport de l'administration de kétamine a l'induction et en entretien anesthésique dans la prévention de la douleur postopératoire. Essai clinique en oncologie]. Annales Francaises d'Anesthesie et de Reanimation 2008;27(3):202-7.

\section{Crousier 2008 \{published data only\}}

Crousier M, Cognat V, Khaled M, Guegniaud PY, Piriou V. Effect of ketamine on prevention of postmastectomy chronic pain. A pilot study [Effet de la kétamine dans la prévention des douleurs chronique post-mastectomies]. Annales Francaises d'Anesthesie et de Reanimation 2008;27:987-93. [DOI: 10.1016/ j.annfar.2008.10.008]

\section{D'Alonzo 2011 \{published data only\}}

D'Alonzo RC, Bennett-Guerrero E, Podgoreanu M, D'Amico TA, Harpole DH, Shaw AD. A randomized, double blind, placebo controlled clinical trial of the preoperative use of ketamine for reducing inflammation and pain after thoracic surgery. Journal of Anesthesia 2011;25:672-8. [DOI: 10.1007/s00540-011-1206-4]

\section{Dahi-Taleghani 2014 \{published data only\}}

Dahi-Taleghani M, Fazli B, Ghasemi M, Vosoughian M, Dabbagh A. Effect of intravenous patient controlled ketamine analgesia on postoperative pain in opium abusers. Anesthesiology and Pain Medicine 2014;4(1):e14129.

\section{Dahl 2000 \{published data only\}}

Dahl V, Ernoe PE, Steen T, Raeder JC, White PF. Does ketamine have preemptive effects in women undergoing abdominal hysterectomy procedures? Anesthesia and Analgesia 2000;90:1419-22.

Dal 2005 \{published data only\}

Dal D, Kose A, Honca M, Akinci SB, Basgul E, Aypar U. Efficacy of prophylactic ketamine in preventing postoperative shivering. British Journal of Anaesthesia 2005;95(2):189-92. [DOI: 10.1093/ bja/aei148]

\section{Dar 2012 \{published data only\}}

Dar AM, Qasi SM, Sidiq S. A placebo-controlled comparison of ketamine with pethidine for the prevention of postoperative shivering. Southern African Journal of Anaesthesia and Analgesia 2012;18(6):340-3. [DOI: 10.1080/22201173.2012.10872875]

\section{De Kock 2001 \{published data only\}}

De Kock M, Lavand'homme P, Waterloos H. 'Balanced analgesia' in the perioperative period: is there a place for ketamine? Pain 2001:92:373-80.

\section{Deng 2009 \{published data only\}}

Deng G, Zheng J, Wang S, Tian B, Zhang S. Remifentanil combined with low-dose ketamine for postoperative analgesia of lower limb fracture: a double-blind, controlled study. Chinese Journal of Traumatology 2009;12(4):223-7. [DOI: 10.3760/ cma.j.issn.1008-1275.2009.04.007]

\section{Du 2011 \{published data only\}}

Du J, Huang YG, Yu XR, Zhao N. Effects of preoperative ketamine on the endocrine-metabolic and inflammatory response to laparoscopic surgery. Chinese Medical Journal 2011;124(22):721-5.

\section{Dualé 2009 \{published data only\}}

Dualé C, Sibaud F, Guastella V, Vallet L, Gimbert YA, Taheri H, et al. Perioperative ketamine does not prevent chronic pain after thoracotomy. European Journal of Pain 2009;13:497-505. [DOI: 10.1016/j.ejpain.2008.06.013]

\section{Dullenkopf 2009 \{published data only\}}

Dullenkopf A, Müller R, Dillmann F, Wiedemeier P, Hegi TR, Gautschi S. An intraoperative pre-incision single dose of intravenous ketamine does not have an effect on postoperative analgesic requirements under clinical conditions. Anaesthesia and Intensive Care 2009;37(5):753-7.

\section{Fiorelli 2015 \{published data only\}}

Fiorelli A, Mazzella A, Passavanti B, Sansone P, Chiodini P, lannotti $M$ et al. Is pre-emptive administration of ketamine a significant adjunction to intravenous morphine analgesia for controlling postoperative pain? A randomized, double-blind, placebo-controlled clinical trial. Interactive Cardiovascular and Thoracic Surgery 2015;21:284-91. [DOI: 10.1093/icvts/ivv154]

\section{Galinski 2007 \{published data only\}}

Galinski SF, Pereira JA, Maestre Y, Francés S, Escolano F, Puig MM. The combination of intravenous dexamethasone and ketamine does not improve postoperative analgesia when compared to each drug individually. Pain Clinic 2007;19(5):223-9. [DOI: 10.1016/j.ajem.2006.11.016] 
Ganne 2005 \{published data only\}

Ganne O, Abisseror M, Menault P, Malhiere S, Chambost V, Charpiat $B$, et al. Low-dose ketamine failed to spare morphine after a remifentanil-based anaesthesia for ear, nose and throat surgery. European Journal of Anaesthesiology 2005;22:426-30. [DOI: 10.1017/S0265021505000724]

\section{Garcia-Navia 2016 \{published data only\}}

García-Navia JT, López JT, Egea-Guerrero JJ, Arenas AV, Gutiérrez TV. Effect of a single dose of lidocaine and ketamine on intraoperative opioids requirements in patients undergoing elective gynecological laparotomies under general anesthesia. A randomized, placebo controlled pilot study. Farmacia Hospitalaria 2016;40(1):44-51. [DOI: 10.7399/fh.2016.40.1.9339]

\section{Garg 2016 \{published data only\}}

Garg N, Panda NB, Gandhi GA, Bhagat H, Batra YK, Grover VK, et al. Comparison of small dose ketamine and dexmedetomidine infusion for postoperative analgesia in spine surgery - a prospective randomized double-blind placebo controlled study. Journal of Neurosurgical Anesthesiology 2016;28(1):27-31.

\section{Gilabert Morell 2002 \{published data only\}}

Gilabert Morell A, Sánchez Pérez C. Effect of low-dose intravenous ketamine in postoperative analgesia for hysterecomy and adnexectomy [Efecto de dosis bajas intravenosas de ketamina en la analgesia postoperatoria de histerectomía y anexectomía]. Revista Espańola de Anestesiologíga y Reanimación 2002;49(5):247-53.

\section{Grady 2012 \{published data only\}}

Grady MV, Mascha E, Sessler DI, Kurz A. The effect of perioperative intravenous lidocaine and ketamine on recovery after abdominal hysterectomy. Anesthesia and Analgesia 2012;115(5):1078-84. [DOI: 10.1213/ANE0b013e3182662e01]

\section{Guignard 2002 \{published data only\}}

Guignard B, Coste C, Costes H, Sessler DI, Lebrault C, Morris W, et al. Supplementing desflurane-remifentanil anesthesia with small-dose ketamine reduces perioperative opioid analgesic requirements. Anesthesia and Analgesia 2002;95:103-8. [DOI: 10.1213/01.ANE.0000020699.65934.0F]

\section{Guillou 2003 \{published data only\}}

Guillou N, Tanguy M, Seguin P, Branger P, Campion J, Mallédant $Y$. The effects of small-dose ketamine on morphine consumption in surgical intensive care unit patients after major abdominal surgery. Anesthesia and Analgesia 2003;97:843-7. [DOI: 10.1213/01.ANE.0000075837.67275.36]

\section{Hadi 2010 \{published data only\}}

Hadi BA, Al Ramadani R, Daas R, Naylor I, Zelkó R. Remifentanil in combination with ketamine versus remifentanil in spinal fusion surgery - a double blind study. International Journal of Clinical Pharmacology and Therapeutics 2010;48(8):542-8. [DOI: 10.5414/CPP48542]

\section{Hadi 2013 \{published data only\}}

Hadi BA, Daas R, Zelkó R. A randomized, controlled trial of a clinical pharmacist intervention in microdiscectomy surgery - low dose intravenous ketamine as an adjunct to standard therapy. Saudi Pharmaceutical Journal 2013;21:169-75. [DOI: 10.1016/j.jsps.2012.08.002]

Haliloglu 2015 \{published data only\}

Haliloglu M, Ozdemir M, Uzture N, Cenksoy PO, Bakan N. Perioperative low-dose ketamine improves postoperative analgesia following cesarean delivery with general anesthesia. The Journal of Maternal-Fetal \& Neonatal Medicine 2016;29(6):962-6. [DOI: 10.3109/14767058.2015.1027190]

\section{Hasanein 2011 \{published data only\}}

Hasanein R, El-Sayed W, Nabil N, Elsayed G. The effect of combined remifentanil and low dose ketamine infusion in patients undergoing laparoscopic gastric bypass. Egyptian Journal of Anaesthesia 2011;27:255-60. [DOI: 10.1016/ j.egja.2011.07.009]

\section{Hayes 2004 \{published data only\}}

Hayes C, Armstrong-Brown A, Burstal R. Perioperative intravenous ketamine infusion for the prevention of persistent post-amputation pain: a randomized, controlled trial. Anaesthesia and Intensive Care 2004;32(3):330-8.

\section{Helmy 2015 \{published data only\}}

Helmy N, Badawy AA, Hussein M, Reda H. Comparison of the preemptive analgesia of low dose ketamine versus magnesium sulphate on parturient undergoing cesarean section under general anesthesia. Egyptian Journal of Anaesthesia 2015;31:53-8. [DOI: 10.1016/j.egja.2014.12.006]

\section{Hercock 1999 \{published data only\}}

Hercock T, Gillham MJ, Sleigh J, Jones SF. The addition of ketamine to patient controlled morphine analgesia does not improve quality of analgesia after total abdominal hysterectomy. Acute Pain 1999;2(2):68-72.

\section{Hu 2014 \{published data only\}}

Hu J, Zhang F, Tong J, Ouyang W. Chronic postthoracotomy pain and perioperative ketamine infusion. Journal of Pain and Palliative Care Pharmacotherapy 2014;28:117-21. [DOI: 10.3109/15360288.2014.908992]

\section{Ilkjaer 1998 \{published data only\}}

Ilkjaer S, Nikolajsen L, Hansen TM, Wernberg M, Brennum J, Dahl JB. Effect of i.v. ketamine in combination with epidural bupivacaine or epidural morphine on postoperative pain and wound tenderness after renal surgery. British Journal of Anaesthesia 1998;81:707-12.

\section{Jaksch 2002 \{published data only\}}

Jaksch W, Lang S, Reichhalter R, Raab G, Dann K, Fitzal S. Perioperative small-dose $S(+)$-ketamine has no incremental beneficial effects on postoperative pain when standardpractice opioid infusions are used. Anesthesia and Analgesia 2002;94:981-6.

\section{Javery 1996 \{published data only\}}

Javery KB, Ussery TW, Steger HG, Colclough GW. Comparison of morphine and morphine with ketamine for postoperative analgesia. Canadian Journal of Anaesthesiology 1996;43(3):212-5. 


\section{Jendoubi 2017 \{published data only\}}

Jendoubi A, Naceur IB, Bouzouita A, Trifa M, Ghedira S, Chebil $S$ et al. A comparison between intravenous lidocaine and ketamine on acute and chronic pain after open nephrectomy: a prospective, double-blind, randomized, placebo-controlled study. Saudi Journal of Anaesthesia 2017;11(2):177-84. [DOI: 10.4103/1658-354X203027]

\section{Joly 2005 \{published data only\}}

Joly V, Richebe P, Guignard B, Fletcher D, Maurette P, Sessler $D I$ et al. Remifentanil-induced postoperative hyperalgesia and its prevention with small-dose ketamine. Anesthesiology 2005;103:147-55.

\section{Joseph 2012 \{published data only\}}

Joseph C, Gaillat F, Duponq R, Lieven R, Baumstarck K, Thomas $\mathrm{P}$, et al. Is there any benefit to adding intravenous ketamine to patient-controlled epidural analgesia after thoracic surgery? A randomised double-blind study. European Journal of Cardio-Thoracic Surgery 2012;42:e58-65. [DOI: 10.1093/ejcts/ ezs398]

\section{Kafali 2004 \{published data only\}}

Kafali H, Aldemir B, Kaygusuz K, Gürsoy S, Kunt N. Small-dose ketamine decreases postoperative morphine requirements. European Journal of Anaesthesiology 2004;21:914.

\section{Kakinohana 2004 \{published data only\}}

Kakinohana M, Huga Y, Sasara T, Saikawa A, Miyata Y, Tomiyama $\mathrm{H}$, et al. Addition of ketamine to propofol-fentanyl anaesthesia can reduce post-operative pain and epidural analgesic consumption in upper abdominal surgery. Acute Pain 2004;5:75-9. [DOI: 0.1016/j.acpain.2003.12.001]

\section{Kamal 2008 \{published data only\}}

Kamal HM. Ketamine as an adjuvant to morphine for patient controlled analgesia in morbidly obese patients. Journal of Medical Sciences 2008;8(4):364-70.

\section{Kapfer 2005 \{published data only\}}

Kapfer B, Alfonsi P, Guignard B, Sessler DI, Chauvin M. Nefopam and ketamine comparably enhance postoperative analgesia. Anesthesia \& Analgesia 2005;100(1):169-74. [DOI: 10.1213/01.ANE.0000138037.19757.ED]

\section{Karaman 2006 \{published data only\}}

Karaman S, Kocabas S, Zincircioglu C, Firat V. Has ketamine preemptive analgesic effect in patients undergoing abdominal hysterectomy? [Abdominal histerektomi operasyonlarinda ketaminin preemptif analjezik etkisi var mi?]. The Journal of Turkish Society of Algology 2006;18(3):36-44.

\section{Kararmaz 2003 \{published data only\}}

Kararmaz A, Kaya S, Karaman H, Turhanoglu H, Ozyilmaz MA. Intraoperative intravenous ketamine in combination with epidural analgesia: postoperative analgesia after renal surgery. Anesthesia and Analgesia 2003;97:1092-6. [DOI: 10.1213/01.ANE.0000080205.24285.36]

\section{Karcioglu 2013 \{published data only\}}

Karcioglu M, Davarci I, Tuzcu K, Bozdogan YB, Turhanoglu S, Aydogan A, et al. Addition of ketamine to propofol-alfentanil anesthesia may reduce postoperative pain in laparoscopic cholecystectomy. Surgical Laparoscopy Endoscopy \& Percutaneous Techniques 2013;23:197-202. [DOI: 10.1097/ SLE.0b013e3182827f09]

\section{Katz 2004 \{published data only\}}

Katz J, Schmid R, Snijdelaar DG, Coderre TJ, McCartney CJL, Wowk A. Pre-emptive analgesia using intravenous fentanyl plus low-dose ketamine for radical prostatectomy under general anesthesia does not produce short-term or long-term reductions in pain or analgesic use. Pain 2004;110:707-18. [DOI: 10.1016/j.pain.2004.05.011]

Kim 2013 \{published data only\}

Kim SH, Kim SI, Ok SY, Park SY, Kim MG, Lee SJ et al. Opioid sparing effect of low dose ketamine in patients with intravenous patient-controlled analgesia using fentanyl after lumbar spinal fusion surgery. Korean Journal of Anesthesiology 2013;64(6):524-8. [DOI: 10.4097/kjae.2013.64.6.524]

\section{Kim 2016 \{published data only\}}

Kim DH, Choi JY, Ryu JH. Prospective, randomized, and controlled trial on ketamine infusion during bilateral axillobreast approach (BABA) robotic or endoscopic thyroidectomy: effects on postoperative pain and recovery profiles. Medicine 2016;95(49):e5485. [DOI: 10.1097/MD.0000000000005485]

Köse 2012 \{published data only\}

Köse EA, Honca M, Akinci SB, Dal D, Aypar Ü. Efficacy of prophylactic ketamine in preventing postoperative shivering. Journal of Clinical and Analytical Medicine 2012;3(2):182-5. [DOI: 10.4328]

\section{Kudoh 2002 \{published data only\}}

Kudoh A, Takahira Y, Katagai H, Takazawa T. Small-dose ketamine improves the postoperative state of depressed patients. Anesthesia and Analgesia 2002;95:114-8. [DOI: 10.1213/01.ANE.0000020693.B7]

Kwok 2004 \{published data only\}

Kwok RFK, Lim J, Chan MTV, Gin T, Chiu WKY. Preoperative ketamine improves postoperative analgesia after gynecologic laparoscopic surgery. Anesthesia and Analgesia 2004;98:1044-9. [DOI: 10.1213/01.ANE.0000105911.66089.59]

Kwon 2009 \{published data only\}

Kwon OS, Lee HJ, Yoon JY, Kim CH, Kwon JY, Kim HK. Intraoperative low dose ketamine reduce postoperative pain after combined anesthesia with propofol and remifentanil in mastectomy patients. Korean Journal Anestesiology 2009;57(5):604-9. [DOI: 10.4097/kae.2009.57.5.604]

\section{Lahtinen 2004 \{published data only\}}

Lahtinen P, Kokki H, Hakala T, Hynynen M. S(+) -ketamine as an analgesic adjunct reduces opioid consumption after cardiac surgery. Anesthesia and Analgesia 2004;99:1295-301. [DOI: 10.1213/01.ANE.0000133913.07342.B9] 
Lak 2010 \{published data only\}

Lak M, Foroozanmehr MJ, Ramazani MA, Araghizadeh H, Zahedi-Shoolami L. Assessment of ketamine effect as adjuvant to morphine in post-operative pain reduction in donor kidney transplanted. Iranian Red Crescent Medican Journal 2010;12(1):38-44.

\section{Leal 2013 \{published data only\}}

Leal PC, Sakata RK, Salomao R, Sadatsune EJ, Issy AM. Assessment of the effect of ketamine in combination with remifentanil on postoperative pain. Brazilian Journal of Anesthesiology 2013;63(2):178-82.

\section{Leal 2015 \{published data only\}}

Leal PC, Salomao R, Brunialti MKC, Sakata RK. Evaluation of the effect of ketamine on remifentanil-induced hyperalgesia: a double-blind, randomised study. Journal of Clinical Anesthesia 2015;27:331-7. [DOI: 10.1016/j.jclinane.2015.02.002]

\section{Lebrun 2006 \{published data only\}}

Lebrun T, Van Elstraete AC, Sandefo I, Polin B, Pierre-Louis L. Lack of a pre-emptive effect of low-dose ketamine on postoperative pain following oral surgery. Canadian Journal of Anesthesiology 2006;53(2):146-52.

\section{Lee 2008 \{published data only\}}

Lee EM, Lee H, Kim CH, Lee GY. A double-blinded, randomized, placebo controlled study of the effect a small dose of ketamine has on postoperative pain on sevoflurane-remifentanil anesthesia. Korean Journal of Anesthesiology 2008;54:146-51.

\section{Lehmann 2001 \{published data only\}}

Lehmann KA, Klaschik M. Lack of pre-emptive analgesic effect of low-dose ketamine in postoperative patients. A prospective, randomised doubleblind study [Klinische untersuchung über die präemptive analgesie durch niedrig dosiertes ketamin]. Schmerz 2001;15:248-53.

\section{Lenzmeier 2008 \{published data only\}}

Lenzmeier B, Moore RL, Cordts P, Garrett N. Menstrual cycle-related variations in postoperative analgesia with the preemptive use of $\mathrm{N}$-methyl $\mathrm{D}$-aspartate antagonist ketamine. Dimensions of Critical Care Nursing 2008;27(6):271-6.

\section{Lin 2016 \{published data only\}}

Lin H, Jia D. Effect of preemptive ketamine administration on postoperative visceral pain after gynecological laparoscopic surgery. Journal of Huazhong University of Science and Technology [Medical Sciences] 2016;36(4):584-7. [DOI: 10.1007/ s11596-016-1629-0]

\section{Lo 2008 \{published data only\}}

Lo A, MacPherson N, Spiwak R. Prospective randomized trial of patient-controlled analgesia with ketamine and morphine or morphine alone after hysterectomy. Canadian Journal of Hospital Pharmacy 2008;61(5):334-9.

\section{Loftus 2010 \{published data only\}}

Loftus RW, Yeager MP, Clark JA, Brown JR, Abdu WA, Sengupta DK, et al. Intraoperative ketamine reduces perioperative opiate consumption in opiate-dependent patients with chronic back pain undergoing back surgery. Anesthesiology 2010;113:639-46.

Mahran 2015 \{published data only\}

Mahran E, Hassan ME. Comparison of pregabalin versus ketamine in postoperative pain management in breast cancer surgery. Saudi Journal of Anaesthesia 2015;9(3):253-7. [DOI: 10.4103/1658-354X.154X.154697]

Martinez 2014 \{published data only\}

Martinez V, Cymerman A, Ammar SB, Fiaud JF, Rapon C, Poindessous $F$, et al. The analgesic efficiency of combined pregabalin and ketamine for total hip arthroplasty: a randomised, double-blind, controlled study. Anaesthesia 2014;69:46-52. [DOI: 10.1111/anae.12495]

Mathisen 1999 \{published data only\}

Mathisen LC, Aasbo V, Raeder J. Lack of pre-emptive analgesic effect of (R)-ketamine in laparoscopic cholecystectomy. Acta Anaesthesiologica Scandinavica 1999;43:220-4.

McKay 2007 \{published data only\}

McKay WP, Donais P. Bowel function after bowel surgery: morphine with ketamine or placebo; a randomized controlled trial pilot study. Acta Anaesthesiologica Scandinavica 2007;51:1166-71. [DOI: 10.1111/j.1399-6576.2007.01436.x]

Mebazaa MS 2008 \{published data only\}

Mebazaa MS, Mestiri T, Kaabi B, Ben Ammar MS. Clinical benefits related to the combination of ketamine with morphine for patient controlled analgesia after major abdominal surgery [Benefices cliniques de l'association ketamine morphine en analgesie controlee par le patient apres chirurgie abdominale majeure]. La Tunisie Medicale 2008;86(5):435-40.

\section{Mendola 2012 \{published data only\}}

Mendola C, Cammarota G, Netto R, Cecci G, Pisterna A, Ferrante $D$ et al. $S(+)$-ketamine for control of perioperative pain and prevention of post thoracotomy pain syndrome: a randomized, double-blind study. Minerva Anestesiologica 2012;78:757-66.

\section{Menigaux 2000 \{published data only\}}

Menigaux C, Fletcher D, Dupont X, Guignard B, Guirimand F, Chauvin M. The benefits of intraoperative small-dose ketamine on postoperative pain after anterior cruciate ligament repair. Anesthesia \& Analgesia 2000;90:129-35.

\section{Menigaux 2001 \{published data only\}}

Menigaux C, Guignard B, Fletcher D, Sessler DI, Dupont X, Chauvin M. Intraoperative small-dose ketamine enhances analgesia after outpatient knee arthroscopy. Anesthesia and Analgesia 2001;93:606-12.

\section{Michelet 2007 \{published data only\}}

Michelet P, Guervilly C, Hélaine A, Avaro JP, Blayac D, Gaillat F, et al. Adding ketamine to morphine for patient-controlled analgesia after thoracic surgery: influence on morphine consumption, respiratory function, and nocturnal desaturation. British Journal of Anaesthesiology 2007;99(3):396-403. [DOI: 0.1093/bja/aem168] 
Miziara 2016 \{published data only\}

Miziara LE, Simoni RF, Esteves LO, Cangiani LH, Grillo-Filho GFR, Paula AGL. Efficacy of continuous $\mathrm{S}(+)$-ketamine infusion for postoperative pain control: a randomised placebo-controlled trial. Anesthesiology Research and Practice 2016;2016(Article ID 6918327):1-7. [DOI: 10.1155/2016/6918327]

Murdoch 2002 \{published data only\}

Murdoch CJ, Crooks BA, Miller CD. Effect of the addition of ketamine to morphine in patient-controlled analgesia. Anaesthesia 2002;57:484-8. [DOI: 10.1046/ j.0003-2409.2001.02409.x]

\section{Nesek-Adam 2012 \{published data only\}}

Nesek-Adam V, Grizelj-Stojcić, Mrsić V, Rasić Z, Schwartz D. Preemptive use of diclofenac in combination with ketamine in patients undergoing laparoscopic cholecystectomy: a randomized, double-blind, placebo-controlled study. Surgical Laparoscopy Endoscopy \& Percutaneous Techniques 2012;22(3):232-8.

\section{Nielsen 2017 \{published data only\}}

Nielsen RV, Fomsgaard JS, Siegel H, Martusevicius R, Nikolajsen L, Dahl JB, et al. Intraoperative ketamine reduces immediate postoperative opioid consumption after spinal fusion surgery in chronic pain patients with opioid dependency: a randomized, blinded trial. Pain 2017;158(3):463-70. [DOI: 10.1097/j.pain.0000000000000782]

\section{Ögün 2001 \{published data only\}}

Ögun CÖ, Duman A, Ökesli S. The comparison of postoperative analgesic effects of preemptive ketamine and fentanyl use in mastectomy operations. The Journal of the Turkish Society of Algology 2001;13(2):31-40.

Ong 2001 \{published data only\}

Ong EL, Osborne GA. Ketamine for co-induction of anaesthesia in oral surgery. Ambulatory Surgery 2001;9:131-5.

\section{Ozhan 2013 \{published data only\}}

Ozhan Y, Bakan N, Karaoren GY, Tomruk SG, Topac Z. Effects of subanesthetic ketamine on pain and cognitive functions on TIVA [TIVA'da subanestezik ketaminin agri ve kognitif fonksiyonlara etkisi]. Journal of Clinical and Analytical Medicine 2015;6(4):452-7. [DOI: 10.4328/JCAM.2161]

\section{Pacreu 2012 \{published data only\}}

Pacreu S, Fernández Candil J, Moltó L, Carazo J, Fernández Galinski S. The perioperative combination of methadone and ketamine reduces post-operative opioid usage compared with methadone alone. Acta Anaesthesiologica Scandinavica 2012;56:1250-6. [DOI: 10.1111/j.1399-6576.2012.02743.x]

\section{Papaziogas 2001 \{published data only\}}

Papaziogas B, Argiriadou H, Papagiannopoulou P, Pavlidis T, Georgiou M, Sfyra M, et al. Preincisional intravenous lowdose ketamine and local infiltration with ropivacaine reduces postoperative pain after laparoscopic cholecystectomy. Surgical Endoscopy 2001;15:1030-3. [DOI: 10.1007/s004640090124]

\section{Parikh 2011 \{published data only\}}

Parikh B, Maliwad J, Shah VR. Preventive analgesia: effect of small dose of ketamine on morphine requirement after renal surgery. Journal of Anaesthesiology Clinical Pharmacology 2011;27(4):485-8.

\section{Patel 2016 \{published data only\}}

Patel J, Thosani R, Kothari J, Garg P, Pandya H. Clonidine and ketamine for stable hemodynamics in off-pump coronary artery bypass. Asian Cardiovascular \& Thoracic Annals 2016;24(7):638-46. [DOI: 10.1177/0218492316663359]

Pirim 2006 \{published data only\}

Pirim A, Karaman S, Uyar M, Certug A. Addition of ketamine infusion to patient controlled analgesia with intravenous morphine after abdominal hysterectomy. The Journal of the Turkish Society of Algology 2006;18(1):52-8.

\section{Remérand 2009 \{published data only\}}

Remérand F, Le Tendre C, Baud A, Couvret C, Pourrat X, Favard L, et al. The early and delayed analgesic effects of ketamine after total hip arthroplasty: a prospective, randomized, controlled, double-blind study. Pain Medicine 2009;109(6):1963-71. [DOI: 10.1213/ANE.0b013e3181bdc8a0]

\section{Reza 2010 \{published data only\}}

Reza FM, Zahra F, Esmaeel F, Hossein A. Preemptive analgesic effect of ketamine in patients undergoing elective cesarean section. The Clinical Journal of Pain 2010;26(3):223-6.

Roytblat 1993 \{published data only\}

Roytblat L, Korotkoruthko A, Katz J, Glazer M, Greemberg L, Fisher A. Postoperative pain: the effect of low-dose ketamine in addition to general anesthesia. Regional Anesthesia and Pain Management 1993;77:1161-5.

\section{Safavi 2011 \{published data only\}}

Safavi M, Honarmand A, Nematollahy Z. Pre-incisional analgesia with intravenous or subcutaneous infiltration of ketamine reduces postoperative pain in patients after open cholecystectomy: a randomized, double-blind, placebocontrolled study. Pain Medicine 2011;12:1418-26.

\section{Sahin 2004 \{published data only\}}

Sahin A, Canbay O, Cuhadar A, Celebi N, Aypar U. Bolus ketamine does not decrease hyperalgesia after remifentanil infusion. The Pain Clinic 2004;16(4):407-11.

\section{Sen 2009 \{published data only\}}

Sen H, Sizlan A, Yanarates O, Emirkadi H, Ozkan S, Dagli G, et al. A comparison of gabapentin and ketamine in acute and chronic pain after hysterectomy. Pain Medicine 2009;109(5):1645-50. [DOI: 10.1213/ANE.0b013e3181b65ea0]

\section{Siddiqui 2015 \{published data only\}}

Siddiqui KM, Khan FA. Effect of preinduction low-dose ketamine on intraoperative and immediate postoperative analgesia requirement in day care surgery: a randomized controlled trial. Saudi Journal of Anaesthesia 2015;9(4):422-7. [DOI: 10.4103/1658-354X.159468] 
Singh 2013 \{published data only\}

Singh H, Kundra S, Singh RM, Grewal A, Kaul TK, Sood D. Preemptive analgesia with ketamine for laparoscopic cholecystectomy. Journal of Anaesthesiology Clinical Pharmacology 2013;29(4):478-84. [DOI: 10.4103/0970-9185.119141]

\section{Snijdelaar 2004 \{published data only\}}

Snijdelaar DG, Cornelisse HB, Schmid RL, Katz J. A randomised, controlled study of peri-operative low dose $\mathrm{S}(+)$-ketamine in combination with postoperative patient-controlled $\mathrm{S}(+)$-ketamine and morphine after radical prostatectomy. Anaesthesia 2004;59:222-8.

\section{Song 2013 \{published data only\}}

Song JW, Kim JK, Song Y, Yang SY, Park SJ, Kwak YL. Effect of ketamine as an adjunct to intravenous patient-controlled analgesia, in patients at high risk of postoperative nausea and vomiting undergoing lumbar spinal surgery. British Journal of Anaesthesia 2013;111(4):630-5. [DOI: 10.1093/bja/aet192]

\section{Song 2014 \{published data only\}}

Song YK, Lee C, Seo DH, Park SN, Moon SY, Park CH. Interaction between postoperative shivering and hyperalgesia caused by high-dose remifentanil. Korean Journal of Anesthesiology 2014;66(1):44-51. [DOI: 10.4097/kjae.2014.66.1.44]

\section{Spreng 2010 \{published data only\}}

Spreng UJ, Dahl V, Raeder J. Effects of perioperative S(+) ketamine infusion added to multimodal analgesia undergoing ambulatory haemorrhoidectomy. Scandinavian Journal of Pain 2010;1:100-5. [DOI: 10.1016/j.sjpain.2010.01.009]

\section{Stubhaug 1997 \{published data only\}}

Stubhaug A, Breivik H, Eide PK, Kreunen M, Foss A. Mapping of punctuate hyperalgesia around a surgical incision demonstrates that ketamine is a powerful suppressor of central sensitization to pain following surgery. Acta Anaesthesiologica Scandinavica 1997;41:1124-32. [DOI: 10.1111/j.1399-6576.1997.tb04854.x]

\section{Subramaniam 2011 \{published data only\}}

Subramaniam K, Akhouri V, Glazer PA, Rachlin J, Kunze L, Cronin M, et al. Intra- and postoperative very low dose intravenous ketamine infusion does not increase pain relief after major spine surgery in patients with preoperative narcotic analgesic intake. Pain Medicine 2011;12:1276-83.

\section{Suzuki 1999 \{published data only\}}

Suzuki M, Tsueda K, Lansing PS, Tolan MM, Fuhrman TM, Ignacio $\mathrm{Cl}$, et al. Small-dose ketamine enhances morphineinduced analgesia after outpatient surgery. Anesthesia and Analgesia 1999;89:98-103.

\section{Suzuki 2006 \{published data only\}}

Suzuki M, Haraguti S, Sugimoto K, Kikutani T, Shimada Y, Sakamoto A. Low-dose intravenous ketamine potentiates epidural analgesia after thoracotomy. Anesthesiology 2006;105:111-9.

\section{Tena 2014 \{published data only\}}

Tena B, Gomar C, Rios J. Perioperative epidural or intravenous ketamine does not improve the effectiveness of thoracic epidural analgesia for acute and chronic pain after thoracotomy. Clinical Journal of Pain 2014;30(6):490-500.

Ünlügenc 2003 \{published data only\}

Ünlügenc H, Özalevi M, Güler T, Isik G. Postoperative pain management with intravenous patient-controlled morphine: comparison of the effect of adding magnesium or ketamine. European Journal of Anaesthesiology 2003;20:416-21.

Van Elstraete 2004 \{published data only\} Van Elstraete AC, Lebrun T, Sandefi I, Polin B. Ketamine does not decrease postoperative pain after remifentanilbased anaesthesia for tonsillectomy in adults. Acta Anaesthesiologica Scandinavica 2004;48:756-60. [DOI: 10.1111/ j.1399-6576.2004.00399.x]

\section{Webb 2007 \{published data only\}}

Webb AR, Skinner BS, Leong S, Kolawole H, Crofts T, Taverner M, et al. The addition of a small-dose ketamine infusion to tramadol for postoperative analgesia: a doubleblinded, placebo-controlled, randomized trial after abdominal surgery. Pain Medicine 2007;104(4):912-7. [DOI: 10.1213/01.ane.0000256961.01813.da]

\section{Woo 2014 \{published data only\}}

Woo JH, Kim YJ, Baik HJ, Han JI, Chung RH. Does intravenous ketamine enhance analgesia after arthroscopic shoulder surgery with ultrasound guided single-injection interscalene block? a randomized, prospective, double-blind trial. Journal of Korean Medical Science 2014;29:1001-6. [DOI: 10.3346/ jkms.2014.29.7.1001]

\section{Wu 2009 \{published data only\}}

Wu Y, Li H, Xiong J, Xu Z, Ma L, Huang X, et al. Effects of patient-controlled analgesia with small dose ketamine combined with morphine and the influence thereof on plasma beta-endorphin level in patients after radical operation for esophageal carcinoma. Journal of the Chinese Medical Association 2009;89(5):314-7.

Yalcin 2012 \{published data only\}

Yalcin N, Uzun ST, Reisli R, Borazan H, Otelcioglu S. A comparison of ketamine and paracetamol for preventing remifentanil induced hyperalgesia in patients undergoing total abdominal hysterectomy. International Journal of Medical Sciences 2012;9:327-33. [DOI: 10.7150/ijms.4222]

\section{Yamauchi 2008 \{published data only\}}

Yamauchi M, Asano M, Watanabe M, Iwasaki S, Furuse S, Namiki A. Continuous low-dose ketamine improves the analgesic effects of fentanyl patient-controlled analgesia after cervical spine surgery. Anesthesia and Analgesia 2008;107(3):1041-4. [DOI: 10.1213/ane.0b013e31817f1e4a]

\section{Yazigi 2012 \{published data only\}}

Yazigi A, Abou-Zeid H, Srouji T, Madi-Jebara S, Haddad F, Jabbour K. The effects of low-dose intravenous ketamine on 
continuous intercostal analgesia following thoracotomy. Annals of Cardiac Anaesthesia 2012;15(1):32-8.

Yeom 2012 \{published data only\}

Yeom JH, Chon MS, Jeon WJ, Shim JH. Peri-operative ketamine with the ambulatory elastometric infusion pump as an adjuvant to manage acute postoperative pain after spinal fusion in adults: a prospective randomized trial. Korean Journal of Anesthesiology 2012;63(1):54-8. [DOI: 10.4097/kjae.2012.63.1.54]

Ysasi 2010 \{published data only\}

Ysasi A, Calderón E, Wendt T, Gracia T, Torres LM, Llorens R. Efficacy of low doses of ketamine in postoperative analgesia and the use of morphine after myocardial revascularisation surgery [Efecto de dosis bajas de ketamine en la analgesia postoperatoria y consumo de morfina tras cirurgía de revascularizatión miocárdica]. Revista de la Sociedad Espanola del Dolor 2010;17(4):190-5. [DOI: 10.1016/j.resed.2010.04.002]

Zakine 2008 \{published data only\}

Zakine J, Samarcq D, Lorne E, Moubarak M, Montravers P, Beloucif $S$, et al. Postoperative ketamine administration decreases morphine consumption in major abdominal surgery. A prospective, randomized, double-blind, controlled study. Pain Medicine 2008;106:1856-61. [DOI: 10.1213/ ane.0b013e3181732776]

\section{References to studies excluded from this review}

\section{Abrishamkar 2012 \{published data only\}}

Abrishamkar S, Eshraghi N, Feizi A, Talakoub R, Rafiei A, Rahmani P. Analgesic effects of ketamine infusion on postoperative pain after fusion and instrumentation of the lumbar spine: a prospective randomized clinical trial. Medical Archives 2012;66(2):107-10.

\section{Adams 2003 \{published data only\}}

Adams Ha, Meyer H, Stoppa A, Müller-Goch A, Bayer P, Hecker H. Anaesthesia for caesarean section. Comparison of two general anaesthetic regimens and spinal anaesthesia [Anästhesie zur Sectio caesarea. Ein Vergleich von zwei Verfahren der Allgemeinanästhesie sowie der Spinalanästhesie]. Anaesthesist 2003;52:23-32. [DOI: 10.1007/s00101-002-0440-4]

\section{Aghamohammadi 2012 \{published data only\}}

Aghamohammadi D, Hosseinzadeh H, Eidy M, Vizhe ZM, Fakhri MBA, Movassagi R, et al. Multimodal preincisional premedication to prevent acute pain after cholecystectomy. Journal of Cardiovascular and Thoracic Research 2012;4(3):65-8. [DOI: 10.5681/jcvtr.2012.016]

\section{Akca 2016 \{published data only\}}

Acka B, Aydogan-Eren E, Canbay Ö, Karagöz AH, Ûzümcügil F, Ankay-Yilbas A, et al. Comparison of efficacy of prophylactic ketamine and dexmedetomidine on postoperative bladder catheter-related discomfort. Saudi Medical Journal 2016;37(1):55-9. [DOI: 10.15537/smj.2016.1.14122]

\section{Avidan 2017 \{published data only\}}

Avidan MS, Maybrier HR, Abdallah AB, Jacobsohn E, Vlisides PE, Pryor KO, et al. Intraoperative ketamine for prevention of postoperative delirium or pain after major surgery in older adults: an international, multicentre, double-blind, randomised clinical trial. Lancet 2017;390:267-75. [DOI: 10.1016/S0140-6736(17)31467-8]

Behdad 2011 \{published data only\}

Behdad A, Hosseinpour M, Khorasani P. Preemptive use of ketamine on post operative pain of appendectomy. Korean Journal of Pain 2011;24(3):137-40. [DOI: 10.3344/ kjp.2011.24.3.137]

\section{Bentley 2005 \{published data only\}}

Bentley MW, Stas JM, Johnson JM, Viet BC, Garrett N. Effects of preincisional ketamine treatment on natural killer cell activity and postoperative pain management after oral maxillofacial surgery. American Association of Nurse Anesthetists 2005;73(6):427-36.

\section{Bilgin 2005 \{published data only\}}

Bilgin $\mathrm{H}$, Özcan B, Bilgin T, Kerimoglu B, Uckunkaya N, Toker A, et al. The influence of timing of systemic ketamine administration on postoperative morphine consumption. Journal of Clinical Anesthesia 2005;17:592-7. [DOI: 10.1016/ j.jclinane.2005.04.005]

\section{Clausen 1975 \{published data only\}}

Clausen L, Sinclair DM, Van Hasselt CH. Intravenous ketamine for postoperative analgesia. South African Medical Journal 1975;49(35):1437-40.

\section{Edwards 1993 \{published data only\}}

Edwards ND, Fletcher A, Cole JR, Peacock JE. Combined infusions of morphine and ketamine for postoperative pain in elderly patients. Anaesthesia 1993;48:124-7. [DOI: 10.1111/ j.1365-2044.1993.tb06849.x]

\section{Gillies 2007 \{published data only\}}

Gillies A, Lindholm D, Angliss M, Orr A. The use of ketamine as rescue analgesia in the recovery room following morphine administration -a double-blind randomised controlled trial in postoperative patients. Anaesthesia and Intensive Care 2007;35(2):199-203.

\section{Guan 2008 \{published data only\}}

Guan JQ, Gan XL, Hei ZQ, Gao WL, Cai J. Effects of ketamine on analgesia of morphine and levels of cell factors in colorectal cancer. Chinese Journal of New Drugs 2008;17(18):1615-8.

Heinke 1999 \{published data only\}

Heinke W, Grimm D. Preemptive effects caused by co-analgesia with ketamine in gynecological laparotomies? Anaesthesiologie und Reanimation 1999;24(3):60-4.

\section{Hong 2011 \{published data only\}}

Hong BH, Lee WY, Kim YH, Yoon SH, Lee WH. Effects of intraoperative low dose ketamine on remifentanil-induced hyperalgesia in gynecologic surgery with sevoflurane anesthesia. Korean Journal of Anesthesiology 2011;61(3):238-43. [DOI: 10.4097/kjae.2011.61.3.238] 
Ito 1974 \{published data only\}

Ito $\mathrm{Y}$, Ichiyanagi K. Post-operative pain relief with ketamine infusion. Anaesthesia 1974;29:222-9.

\section{Jahangir 1993 \{published data only\}}

Jahangir SM, Islam M, Aziz L. Ketamine infusion for postoperative analgesia in asthmatics: a comparison with intermittent meperidine. Anesthesia and Analgesia 1993;76:45-9.

\section{Jensen 2008 \{published data only\}}

Jensen LL, Handberg G, Helbo-Hansen HS, Skaarup I, Munk T, Lund N. No morphine sparing effect of ketamine added to morphine for patient-controlled intraveous analgesia after uterine artery embolization. Acta Anaesthesiologica Scandinavica 2008;52:479-86. [DOI: 10.1111/ j.1399-6576.2008.01602.x]

\section{Jiang 2016 \{published data only\}}

Jiang M, Wang MH, Wang XB, Liu L, Wu JL, Yang XL et al. Effect of intraoperative application of ketamine on postoperative depressed mood on patients undergoing elective orthopedic surgery. Journal of Anesthesia 2016;30:232-7. [DOI: 10.1007/ s00540-015-2096-7]

\section{Joachimmson 1986 \{published data only\}}

Joachimmsson PO, Hedstrand U, Eklund A. Low-dose ketamine infusion for analgesia during postoperative ventilator treatment. Acta Anaesthesiologica Scandinavica 1986;30(8):697-702. [DOI: 10.1111/j.1399-6576.1986.tb02505.x]

\section{Kadic 2016 \{published data only\}}

Kadic L, Van Haren FG, Wilder-Smith O, Bruhn J, Driessen JJ, De Waal Malefijt MC. The effect of pregabalin and S-ketamine in total knee arthroplasty patients: a randomized study. Journal of Anaesthesiology Clinical Pharmacology 2016;32(4):476-82. [DOI: 10.4103/0970-9185.194762]

\section{Kim 2001 \{published data only\}}

Kim CJ, Chea JS, Chung MY, Song DH, Park JJ, Lee BH. The analgesic effect of combined infusions of morphine and ketamine using an intravenous PCA after a cesarean section. Korean Journal of Anesthesiology 2001;40:509-14. [DOI: 10.4097/ kjae.2001.40.4.509]

\section{Kim 2005 \{published data only\}}

Kim YJ, Baik HJ, Kim JH. The effects of the intravenous continuous infusion of low-dose ketamine on postoperative pain after total intravenous anesthesia. Korean Journal of Anesthesiology 2005;48:163-70. [DOI: 10.4097] kjae.2005.48.2.163]

\section{Kollender 2008 \{published data only\}}

Kollender Y, Bickels J, Stocki D, Maruoani N, Chazan S, Nirkin A, et al. Subanaesthetic ketamine spares postoperative morphine and controls pain better than standard morphine does alone in orthopaedic-oncological patients. European Journal of Cancer 2008;44:954-62. [DOI: 10.1016/j.ejca.2008.02.021]
Kose 2008 \{published data only\}

Kose EA, Dal D, Akinci SB, Saricaoglu F, Aypar U. The efficacy of ketamine for the treatment of postoperative shivering. Anesthesia and Analgesia 2008;106(1):120-2. [DOI: 10.1213/01.ane.0000296458.16313.7c]

Launo 2004 \{published data only\}

Launo C, Bassi C, Spagnolo L, Badano S, Ricci C, Lizzi A, et al. Preemptive ketamine during general anesthesia for postoperative analgesia in patients undergoing laparoscopic cholecystectomy. Minerva Anestesiologica 2004;70:727-38.

Lee 2005 \{published data only\}

Lee HD, Kim HK, Lee SN, Lee SY, Lee JH, Park DH. The effect of low dose i.v. ketamine in combination with epidural morphine on postoperative pain. Korean Journal of Anesthesiology 2005;49:81-5. [DOI: 10.4097/kjae.2005.49.1.81]

\section{Lee 2006 \{published data only\}}

Lee YS, Kim WY, Cha MH, Kim JH, Kim JH, Park YC, et al. Effects of preincisional ketamine on postoperative pain after laparoscopic assisted vaginal hysterectomy. Anesthesia and Pain Medicine 2006;1:44-7.

Lee 2013 \{published data only\}

Lee W, Shin D, Cho K, Kim MH. Comparison of dexmedetomidine and ketamine for the analgesic effect using intravenous patientcontrolled analgesia after gynecological abdominal surgery. Korean Journal of Anesthesiology 2013;65(6 Suppl):S132-4. [DOI: 10.4097/kjae.2013.65.6S.S132]

\section{Lee 2014 \{published data only\}}

Lee MH, Chung MH, Han CS, Lee JH, Choi YR, Choi EM, et al. Comparison of effects of intraoperative esmolol and ketamine infusion on acute postoperative pain after remifentanil-based anesthesia in patients undergoing laparoscopic cholecystectomy. Korean Journal of Anesthesia 2014;66(3):222-9. [DOI: 10.4097/kjae.2014.66.3.222]

\section{Liang 2006 \{published data only\}}

Liang S, Chen Y, Lin C. Low-dose ketamine combined with fentanyl for intravenous postoperative analgesia in elderly patients. Journal of Southern Medical University 2006;26(11):1663-4.

\section{Lux 2009 \{published data only\}}

Lux EA, Hinrichs T, Mathejka E, Wilhelm W. Ketamine racemate and fast track anaesthesia: influence on recovery times and postoperative opioid needs [Ketaminrazemat bei "fast-track" -anästhesie. Einfluss auf aufwachzeiten und postoperativen opioidbedarf]. Anaesthesist 2009;58(10):1027-34. [DOI: 10.1007/ s00101-009-1607-z]

\section{Malek 2006 \{published data only\}}

Malek J, Kurzová A, Bendová M, Nosková P, Strunová M, Vedral T. The prospective study on the effect of a preemptive longterm postoperative administration of a low-dose ketamine on the incidence of chronic post-mastectomy pain [Efekt perioperacního podávání ketaminu na potlacení vzniku chronické bolesti po operaci prsu -prospktivní studie]. Anestesziologie a intevzivní medicína 2006;17:34-7. 
Maurset 1989 \{published data only\}

Maurset A, Skoglund LA, Hustveit O, Oye I. Comparison of ketamine and pethidine in experimental and postoperative pain. Pain 1989;36:37-41. [DOI: 10.1016/0304-3959(89)90109-7]

\section{Nayar 2009 \{published data only\}}

Nayar R, Sahajanand H. Does anesthetic induction for Cesarean section with a combination of ketamine and thiopentone confer any benefits over thiopentone or ketamine alone? A prospective randomized study. Minerva Anestesiologica 2009;75(4):185-90.

\section{Ndoye 2008 \{published data only\}}

NDoye Diop M, Khalil Y, Diatta B, Seck M, Ndiaye M, Niang B, et al. Prevention of the acute tolerance with opioids by ketamine [Prevention de la tolerance aigue au fentanyl par la ketamine a faible poids]. Dakar Medical Journal 2008;53(2):122-6.

\section{Nesher 2008 \{published data only\}}

Nesher N, Serovian I, Marouani N, Chazan S, Weinbroum AA. Ketamine spares morphine consumption after transthoracic lung and heart surgery without adverse effects. Pharmacological Research 2008;58:38-44. [DOI: 10.1016/ j.phrs.2008.06.003]

\section{Nesher 2009 \{published data only\}}

Nesher N, Eksterin MP, Paz Y, Marouani N, Chazan S, Weinbroum AA. Morphine with adjuvant ketamine vs higher dose of morphine alone for immediate postthoracotomy analgesia. Chest 2009;136(1):245-52. [DOI: 10.1378/ chest.08-0246]

\section{Nikolayev 2008 \{published data only\}}

Nikolayev AP, Nikoda VV, Svetlov VA. Multimodal approach to postoperative analgesia in patients with neuropathic pain. Anesteziologiia i Reanimatologiia 2008;5:99-103.

\section{Nitta 2013 \{published data only\}}

Nitta R, Goyagi T, Nishikawa T. Combination of oral clonidine and intravenous low-dose ketamine reduces the consumption of postoperative patient-controlled analgesia morphine after spine surgery. Acta Anaesthesiologica Taiwanica 2013;51:14-7. [DOI: 10.1016/j.aat.2013.03.003]

\section{Nourozi 2010 \{published data only\}}

Nourozi A, Talebi H, Fateh S, Mohammadzadeh A, EghtesadiAraghi P, Ahmadi Z, et al. Effect of adding ketamine to pethidine on postoperative pain in patients undergoing major abdominal operations: double blind randomized controlled trial. Pakistan Journal of Biological Sciences 2010;13(24):1214-8. [DOI: 10.3923/ pjbs.2010.1214.1218]

\section{Oliveira 2005 \{published data only\}}

Oliveira CMB, Issy AM, Sakata RK, Garcia JBS, Martins CR. Preemptive effect of IV S(+) -ketamine for hysterectomy. Acute Pain 2005;7:139-43. [DOI: 10.1016/j.acpain.2005.08.001]

\section{Owen 1987 \{published data only\}}

Owen H, Reekie RM, Clements JA, Watson R, Nimmo WS. Analgesia from morphine and ketamine. Anaesthesia 1987;42:1051-6. [DOI: 10.1111/j.1365-2044.1987.tb05167.x]

\section{Park 2004 \{published data only\}}

Park HJ, Kim ST. The effect of intravenous ketamine on the recovery from total intravenous anesthesia with propofol. Korean Journal of Anesthesiology 2004;46(5):517-23. [DOI: 10.4097/kjae.2004.46.5.517]

\section{Perrin 2009 \{published data only\}}

Perrin SB, Purcell AN. Intraoperative ketamine may influence persistent pain following knee arthroplasty under combined general and spinal anaesthesia: a pilot study. Anaesthesia and Intensive Care 2009;37(2):248-53.

\section{Reeves 2001 \{published data only\}}

Reeves M, Lindholm DE, Myles PE, Fletcher H, Hunt JO. Adding ketamine to morphine for patient-controlled analgesia after major abdominal surgery: a double-blinded, randomized, controlled trial. Anesthesia and Analgesia 2001;93:116-20. [DOI: 10.1097/00000539-200107000-00025]

\section{Sadove 1971 \{published data only\}}

Sadove MS, Shulman M, Hatano S, Fevold N. Analgesic effects of ketamine administered in subdissociative doses. Anesthesia and Analgesia 1971;50(3):452-7.

\section{Sollazzi 2008 \{published data only\}}

Sollazzi L, Modesti C, Vitale F, Sacco T, Ciocchetti P, Idra AS, et al. Preinductive use of clonidine and ketamine improves recovery and reduces postoperative pain after bariatric surgery. Surgery for Obesity and Related Diseases 2009;5:67-71. [DOI: 10.1016/ j.soard.2008.09.018]

\section{Song 2004 \{published data only\}}

Song X, Li X, Zhao H, Yang T, Wang F. Pre-emptive analgesia effects of ketamine on postoperative pain management and stress responses. Journal of Jilin University (Medicine Edition) 2004;30(4):605-7.

\section{Sveticic 2008 \{published data only\}}

Sveticic G, Farzanegan F, Zmoos P, Zmoos S, Eichenberger U, Curatolo M. Is the combination of morphine with ketamine better than morphine alone for postoperative intravenous patient-controlled analgesia. Anesthesia and Analgesia 2008;106(1):287-93. [DOI: 10.1213/01.ane.0000289637.11065.8f]

\section{Talu 2002 \{published data only\}}

Talu Gk, Özyacin S, Dereli N, Sentürk M, Yücel A. The effect of ketamine administered preoperatively through different routes on thoracotomy pain: a randomized, double blind, placebo controlled study [Torakotomi Agrisinda preoperatif farkli yollardan uygulanan ketaminin etkinligi: randomize, cift kör, plasebo kontrollü klinik calisma]. Journal of Turkish Society of Algology 2002;14(2):54-9.

\section{Thomas 2012 \{published data only\}}

Thomas M, Tennant I, Augier R, Gordon-Strachan G, Harding H. The role of pre-induction ketamine in the management of postoperative pain in patients undergoing elective gynaecological surgery at the university hospital of the West Indies. West Indian Medical Journal 2012;61(3):224-9. 
Tverskoy 1994 \{published data only\}

Tverskoy M, Ozy Y, Isakson A, Finger J, Bradley EL Jr, Kissin I. Preemptive effect of fentanyl and ketamine on postoperative pain and wound hyperalgesia. Anesthesia and Analgesia 1994;78(2):205-9.

\section{Tverskoy 1996 \{published data only\}}

Tverskoy M, Oren M, Vaskovich M, Dashakovsky I, Kissin I. Ketamine enhances local anesthetic and analgesic effects of bupivacaine by peripheral mechanism: a study in postoperative patients. Neuroscience Letters 1996;215:5-8. [DOI: 10.1016/ S0304-3940(96)12922-0]

\section{Ünlügenc 2002 \{published data only\}}

Ünlügenc H, Gündüz M, Özalevli M, Akman H. A comparative study on the analgesic effect of tramadol, tramadol plus magnesium, and tramadol plus ketamine for postoperative pain management after major abdominal surgery. Acta Anaesthesiologica Scandinavica 2002;46:1025-30.

\section{Urban 2008 \{published data only\}}

Urban MK, Deau JTY, Wukovits B, Lipnistky JY. Ketamine as an adjunct to postoperative pain management in opioid tolerant patients after spinal fusions: a prospective randomized trial. Hospital for Special Surgery Journal 2008;4(1):62-5. [DOI: 10.1007/s11420-007-9069-9]

\section{Weinbroum 2003 \{published data only\}}

Weinbroum AA. A single small dose of postoperative ketamine provides rapid and sustained improvement in morphine analgesia in the presence of morphine-resistant pain. Anesthesia and Analgesia 2003;96:879-95. [DOI: 10.1213/01.ANE.0000048088.17761.B4]

\section{Wilder-Smith 1998 \{published data only\}}

Wilder-Smith OHG, Arendt-Nielsen L, Gäumann D, Tassonyi E, Rifat KR. Sensory changes and pain after abdominal hysterectomy: a comparison of anesthetic supplementation with fentanyl versus magnesium or ketamine. Anesthesia and Analgesia 1998;86:95-101. [DOI: 10.1213/00000539-199801000-00019]

\section{Xie 2003 \{published data only\}}

Xie H, Wang X, Liu G, Wang G. Analgesic effects and pharmacokinetics of a low dose of ketamine preoperatively administered epidurally or intravenously. Clinical Journal of Pain 2003;19:317-22.

\section{Xu 2017 \{published data only\}}

Xu Y, Li Y, Huang X, Chen D, She B, Ma D. Single bolus lowdose of ketamine does not prevent postpartum depression: a randomized, double-blind, placebo-controlled, prospective trial. Archives of Gynecology and Obstetrics 2017;295:1167-74. [DOI: 10.1007/s00404-017-4334-8]

\section{References to studies awaiting assessment}

\section{Lee 2018 \{published data only\}}

Lee J, Park HP, Jeong MH, Song JD, Kim HC. Efficacy of ketamine for postoperative pain following robotic thyroidectomy: A prospective randomised study. Journal of International Medical Research 2018;46(3):1109-20.

Lou 2017 \{published data only\}

Lou QB, Nan K, Xiang FF, Zhu WS, Zhang XT, Li J. Effect of perioperative multi-day low-dose ketamine infusion on prevention of postmastectomy pain syndrome. National Medical Journal of China 2017;97(46):3636-41.

Moon 2018 \{published data only\}

Moon YE, Kim MH, Lee HM, Yoon HM, Jeon YH. Preventative effect of ketamine on postsurgical hyperalgesia induced at a body part remote from the surgical site. Minerva Anestesiologica 2018;84(4):481-7.

\section{Additional references}

\section{AlBalawi 2013}

AlBalawi Z, McAlister FA, Thorlund K, Wong M, Wetterslev J. Random error in cardiovascular meta-analyses: how common are false positive and false negative results? International Journal of Cardiology 2013;168(2):1102-7. [DOI: 10.1016/ j.ijcard.2012.11.048]

\section{Anderson 2001}

Anderson R, Saiers JH, Abram S, Schlict C. Accuracy in equianalgesic dosing: conversion dilemmas. Journal of Pain and Symptom Management 2001;21(5):397-406. [DOI: 10.1016/ S0885-3924(01)00271-8]

\section{Angst 2006}

Angst MS, Clark JD. Opioid-induced hyperalgesia. A qualitative systematic review. Anesthesiology 2006;104(3):570-87.

\section{Arendt-Nielsen 1996}

Arendt-Nielsen L, Nielsen J, Petersen-Felix S, Schnider TW, Zbinden AM. Effect of racemic mixture and the (S+) isomer of ketamine on temporal and spatial summation of pain. British Journal of Anaesthesia 1996;77:625-31. [DOI: 10.1093/ bja/77.5.625]

\section{Aroni 2009}

Aroni F, lacovidou N, Dontas I, Pourzotaki C, Xanthos T. Pharmacological aspects and potential new clinical applications of ketamine: reevaluation of an old drug. Journal of Clinical Pharmacology 2009;49:957-64. [DOI: 10.1177/0091270009337941]

\section{Beals 2003}

Beals JK, Carter LB, Jevtovic-Todorovic V. Neurotoxicity of nitrous oxide and ketamine is more severe in aged than in young rat brain. Annals of the New York Academy of Sciences 2003;993:115; discussion 123-4. [DOI: 10.1111/ j.1749-6632.2003.tb07518.x]

\section{Begon 2001}

Begon S, Pickering G, Eschalier A, Mazur A, Rayssiguier Y, Dubray $C$. Role of spinal NMDA receptors, protein kinase $C$ and nitric oxide synthase in the hyperalgesia induced by magnesium deficiency in rats. British Journal of Pharmacology 2001;134:1227-36. [DOI: 10.1038/sj.bjp.0704354] 


\section{Bell 2017}

Bell RF, Eccleston C, Kalso EA. Ketamine as an adjuvant to opioids for cancer pain. Cochrane Database of Systematic Reviews 2017, Issue 6. Art. No: CD003351. [DOI: 10.1002/14651858.CD003351.pub3]

\section{Benarroch 2013}

Benarroch EE. HCN channels: function and clinical implications. Neurology 2013;80:304-10.

\section{Bredlaw 2013}

Bredlaw AL, Thakur R, Korones DN, Dworkin RH. Ketamine for pain in adults and children with cancer; a systematic review and synthesis of the literature. Pain Medicine 2013;14(10):1505-17. [DOI: 10.1111/pme.12182]

\section{Bulutcu 2002}

Bulutcu F, Dogrul A, Oguz Güc M. The involvement of nitric oxide in the analgesic effects of ketamine. Life Sciences 2002;71:841-53. [DOI: 10.1016/S0024-3205(02)01765-4]

\section{Chen 2009}

Chen X, Shu S, Bayliss DA. HCN1 channel subunits are a molecular substrate for hypnotic actions of ketamine. Journal of Neuroscience 2009;29(3):600-9. [DOI: 10.1523/ JNEUROSCI.3481-08.2009]

\section{Collins 1997}

Collins SL, Moore RA, McQuay HJ. The visual analogue pain intensity scale: what is moderate pain in millimetres? Pain 1997;72(1-2):95-7. [DOI: 10.1016/S0304-3959(97)00005-5]

\section{Colvin 2010}

Colvin LA, Fallon MT. Opioid-induced hyperalgesia: a clinical challenge. British Journal of Anaesthesia 2010;104(2):125-7. [DOI: 10.1093/bja/aep392]

\section{Dechartres 2013}

Dechartres A, Trinquart L, Boutron I, Ravaud P. Influence of trial sample size on treatment effect estimates: metaepidemiological study. BMJ 2013;346:f2304. [DOI: 10.1136/ bmj.f2304]

\section{Dechartres 2014}

Dechartres A, Altman DG, Trinquart L, Boutron I, Ravaud P. Association between analytic strategy and estimates of treatment outcomes in meta-analyses. JAMA 2014;312(6):623-30. [DOI: 10.1001/jama.2014.8166]

\section{Deeks 2017}

Deeks JJ, Higgins JP, Altman DG (editors) on behalf of the Cochrane Statistical Methods Group. Chapter 9: Analysing data and undertaking meta-analyses. In: Higgins JPT, Churchill R, Chandler J, Cumpston MS (editors), Cochrane Handbook for Systematic Reviews of Interventions version 5.2.0 (updated June 2017), Cochrane, 2017. Available from www.training.cochrane.org/handbook.

\section{Eikermann 2012}

Eikermann M, Grosse-Sundrup M, Zaremba S, Henry ME, Bittner EA, Hoffmann U, et al. Ketamine activates breathing and abolishes the coupling between loss of consciousness and upper airway dilator muscle dysfunction. Anesthesiology 2012;116(1):35-46. [DOI: 10.1097/ALN.0b013e31823d010a]

\section{Elia 2005}

Elia N, Tramer M. Ketamine and postoperative pain - a quantitative systematic review of randomised trials. Pain 2005;113:61-70. [DOI: 10.1016/j.pain.2004.09.036]

\section{Farrar 2000}

Farrar JT, Portenoy RK, Berlin JA, Kinman JL, Strom BL. Defining the clinically important difference in pain outcome measures. Pain 2000;88(3):987-94.

\section{Fisher 2000}

Fisher K, Coderre T, Hagen NA. Targeting the N-methyl-Daspartate receptor for chronic pain management: preclinical animal studies, recent clinical experience and future research directions. Journal of Pain and Symptom Management 2000;20(5):358-73. [DOI: 10.1016/S0885-3924(00)00213-X]

\section{Flather 1997}

Flather MD, Farkouh ME, Pogue JM, Yusuf S. Strengths and limitations of meta-analysis: larger studies may be more reliable. Controlled Cinical Trials 1997;18(6):568-79. [DOI: 10.1016/S0197-2456(97)00024-X]

\section{Gavaghan 2000}

Gavaghan DJ, Moore RA, McQuay HJ. An evaluation of homogeneity tests in meta-analyses in pain using simulations of individual patient data. Pain 2000;85(3):415-24. [DOI: 10.1016/ S0304-3959(99)00302-4]

\section{Geisslinger 1993}

Geisslinger G, Hering W, Thomann P, Knoll R, Kamp HD, Brune K. Pharmacokinetics and pharmaco-dynamics of ketamine enantiomers in surgical patients using a stereoselective analytical method. British Journal of Anesthesia 1993;70(6):666-71. [DOI: 10.1093/bja/70.6.666]

\section{GRADE 2004}

GRADE Working Group. Grading quality of evidence and strength of recommendations. BMJ 2004;328:1490-4.

\section{Guyatt 2011}

Guyatt GH, Oxman AD, Kunz R, Woodcock J, Brozek J, Helfand $M$ et al. GRADE guidelines: 7 . Rating the quality of evidence--inconsistency. Journal of Cinical Epidemiology 2011;64(12):1294-302. [DOI: 10.1016/j.jclinepi.2011.03.017]

\section{Guyatt 2013a}

Guyatt G, Oxman AD, Sultan S, Brozek J, Glasziou P, AlonsoCoello $P$ et al. GRADE guidelines: 11 . Making an overall rating of confidence in effect estimates for a single outcome and for all outcomes. Journal of Clinical Epidemiology 2013;66:151-7. [DOI: 10.1016/j.jclinepi.2012.01.006]

\section{Guyatt 2013b}

Guyatt GH, Oxman AD, Santesso N, Helfand M, Vist G, Kunz R, et al. GRADE guidelines: 12. Preparing summary of findings 
tables-binary outcomes. Journal of Clinical Epidemiology 2013;66:158-72. [DOI: 10.1016/j.jclinepi.2012.01.012]

\section{Hayashi 2011}

Hayashi Y, Kawaji K, Sun L, Zhang X, Kogano K, Yokoyama T, et al. Microglial Ca-activated K-channels are possible molecular targets for the analgesic effects of s-ketamine on neuropathic pain. Journal of Neuroscience 2011;31(48):17,370-82. [DOI: 10.1523/JNEUROSCI.4152-11.2011]

\section{Heesen 2014}

Heesen M, Böhmer J, Brinck EC, Kontinen VK, Klöhr S, Rossaint R, et al. Intravenous ketamine during spinal and general anaesthesia for caesarean section: systematic review and meta-analysis. Acta Anaesthesiologica Scandinavica 2015;59(4):414-26. [DOI: 10.1111/aas.12468]

\section{Higgins 2003}

Higgins JP, Thompson SG, Deeks JJ, Altman DG. Measuring inconsistency in meta-analyses. BMJ 2003;327:557-60.

\section{Higgins 2011a}

Higgins JP, Deeks JJ (editors). Chapter 7: Selecting studies and collecting data. In: Higgins JPT, Green S (editors), Cochrane Handbook for Systematic Reviews of Interventions Version 5.1.0 (updated March 2011). The Cochrane Collaboration, 2011. Available from www.handbook.cochrane.org.

\section{Higgins 2011b}

Higgins JP, Deeks JJ, Altman DG (editors). Chapter 16: Special topics in statistics. In: Higgins JPT, Green S (editors), Cochrane Handbook for Systematic Reviews of Interventions Version 5.1.0 (updated March 2011). The Cochrane Collaboration, 2011. Available from www.handbook.cochrane.org.

\section{Higgins 2017}

Higgins JP, Altman DG, Sterne JA (editors). Chapter 8: Assessing risk of bias in included studies. In: Higgins JPT, Churchill R, Chandler J, Cumpston MS (editors), Cochrane Handbook for Systematic Reviews of Interventions version 5.2.0 (updated June 2017), Cochrane, 2017. Available from www.training.cochrane.org/handbook.

\section{Hirota 2011}

Hirota K, Lambert DG. Ketamine: new uses for an old drug? British Journal of Anaesthesia 2011;107(2):123-6. [DOI: 10.1093/ bja/aer221]

\section{IntHout 2015}

IntHout J, loannidis JP, Borm GF, Goeman JJ. Small studies are more heterogeneous than large ones: a meta-meta-analysis. Journal of Clinical Epidemiology 2015;68:860-9. [DOI: 10.1016/ j.jclinepi.2015.03.017]

\section{Jevtović-Todorović 1998}

Jevtović-Todorović V, Todorović SM, Mennerick S, Powell S, Dikranian K, Benshoff N, et al. Nitrous oxide (laughing gas) is an NMDA antagonist, neuroprotectant and neurotoxin. Nature Medicine 1998;4(4):460-3. [DOI: 10.1038/nm0498-460]

\section{Jevtovic-Todorovic 2000}

Jevtovic-Todorovic V, Benshoff N, Olney JW. Ketamine potentiates cerebrocortical damage induced by the common anaesthetic agent nitrous oxide in adult rats. British Journal of Pharmacology 2000;130(7):1692-8. [DOI: 10.1038/ sj.bjp.0703479]

\section{Katz 2009}

Katz J, Seltzer Z. Transition from acute to chronic postsurgical pain: risk factors and protective factors. Expert Review of Neurotherapeutics 2009;9(5):723-44. [DOI: 10.1586/ern.09.20]

\section{Kay 1971}

Kay B. A clinical investigation of piritramide in the treatment of postoperative pain. British Journal of Anaesthesia 1971;43:1167-71. [DOI: 10.1093/bja/43.12.1167]

\section{Kehlet 2006}

Kehlet $\mathrm{H}$, Jensen TJ, Woolf CJ. Persistent postsurgical pain: risk factors and prevention. Lancet 2006;367:1618-25.

\section{Kulinskaya 2015}

Kulinskaya E, Dollinger MB. An accurate test for homogeneity of odds ratios based on Cochran's Q-statistic. BMC Medical Research Methodology 2015;15:49. [DOI: 10.1186/ s12874-015-0034-x]

\section{Kumar 1999}

Kumar N, Rowbotham DJ. Editorial II. Piritramide. British Journal of Anaesthesia 1999;82(1):3-5.

\section{Laskowski 2011}

Laskowski K, Stirling A, McKay WP, Hyun JL. A systematic review of intravenous ketamine for postoperative analgesia. Canadian Journal of Anaesthesia 2011;58:911-23.

\section{Lee 2011}

Lee M, Silverman S, Hansen H, Patel V, Manchikanti L. A comprehensive review of opioid-induced hyperalgesia. Pain Physician 2011;14:145-61.

\section{Lefebvre 2011}

Lefebvre C, Manheimer E, Glanville J. Chapter 6: Searching for studies. In: Higgins JPT, Green S (editors). Cochrane Handbook for Systematic Reviews of Interventions Version 5.1.0 (updated March 2011). The Cochrane Collaboration, 2011. Available from www.handbook.cochrane.org.

\section{Lilius 2015}

Lilius TO, Jokinen V, Neuvonen MS, Niemi M, Kalso EA, Rauhala PV. Ketamine coadministration attenuates morphine tolerance and leads to increased brain concentrations of both drugs in the rat. British Journal of Pharmacology 2015;172(11):2799-813.

\section{Low 2012}

Low Y, Clarke CF, Huh BK. Opioid-induced hyperalgesia: a review of epidemiology, mechanisms and management. Singapore Medical Journal 2012;53(5):537-60. 


\section{Lundeberg 2012}

Lundeberg S. Pharmacokinetic and pharmacodynamic aspects on opioid administration, morphine and ketobemidone, in the pediatric population [Doctoral thesis]. Stockholm, Sweden: Department of Physiology and Pharmacology, Section of Anesthesiology and Intensive Care, Karolinska Institutet, 2012.

\section{Macintyre 2010}

Macintyre PE, Schug SA, Scott DA, Visser EJ, Walker SM, APM:SE Working Group of the Australian and New Zealand College of Anaesthetists and Faculty of Pain Medicine. Acute Pain Management: Scientific Evidence. 3rd edition. Melbourne ANZCA \& FPM, 2010. [ONLINE ISBN: 978-0-9775174-5-9]

\section{Mao 2002}

Mao J. Opioid-induced abnormal pain sensitivity: implications in clinical opioid therapy. Pain 2002;100:213-7.

\section{Marland 2013}

Marland S, Ellerton J, Andolfatto G, Strapazzon G, Thomassen O, Brandner B, et al. Ketamine: use in anesthesia. CNS Neuroscience and Therapeutics 2013;19:381-9. [DOI: 10.1111/ cns.12072]

\section{McQuay 2012}

McQuay HJ, Derry S, Eccleston C, Wiffen PJ, Moore RA. Evidence for analgesic effect in acute pain - 50 years on. Pain 2012;153(7):1364-7. [DOI: 10.1016/j.pain.2012.01.024]

\section{Mhuircheartaigh 2009}

Mhuircheartaigh RJ, Moore RA, McQuay HJ. Analysis of individual patient data from clinical trials: epidural morphine for postoperative pain. British Journal of Anaesthesia 2009;103(6):974-81. [DOI: 10.1093/bja/aep300]

\section{Mion 2013}

Mion G, Villevieille T. Ketamine pharmacology: an update (pharmacodynamics and molecular aspects, recent findings). CNS Neuroscience \& Therapeutics 2013;19:370-80. [DOI: 10.1111/ cns.12099]

\section{Moher 2009}

Moher D, Liberati A, Tetzlaff J, Altman DG, the PRISMA Group. Preferred reporting items for systematic reviews and meta-analyses: the PRISMA statement. PLoS Medicine 2009;6(7):e1000097.

\section{Moore 1998}

Moore RA, Gavaghan D, Tramer MR, Collins SL, McQuay HJ. Size is everything -large amounts of information are needed to overcome random effects in estimating direction and magnitude of treatment effects. Pain 1998;78:209-16. [DOI: 10.1016/S0304-3959(98)00140-7]

\section{Moore 2008}

Moore RA. Systematic reviews: Are we getting it right yet? In: Moore RA, Kalso E, McQuay HJ, editors(s). Systematic Reviews in Pain Research: Methodology Refined. 1 edition. Seattle: IASP Press, 2008:3-14.

\section{Moore 2010}

Moore RA, Moore OA, Derry S, Peloso PM, Gammaitoni AR, Wang H. 10. Responder analysis for pain relief and numbers needed to treat in a meta-analysis of etoricoxib osteoarthritis trials: bridging a gap between clinical trials and clinical practice. Annals of the Rheumatic Diseases 2010;69(2):374-9. [DOI: 10.1136/ard.2009.107805]

\section{Moore 2011}

Moore RA, Ní Mhuirchearthaigh RJ, Derry S, McQuay HJ. Mean analgesic consumption is inappropriate for testing analgesic efficacy in post-operative pain: analysis and alternative suggestion. European Journal of Anaesthesiology 2011;28:427-32. [DOI: 10.1097/EJA.0b013e328343c569]

\section{Moore 2013}

Moore RA, Straube S, Aldington D. Pain measures and cut-offs - 'no worse than mild pain' as a simple, universal outcome. Anaesthesia 2013;68(4):400-12. [DOI: 10.1111/anae.12148]

\section{Nüesch 2010}

Nüesch E, Trelle S, Reichenbach S, Rutjes AW, Tschannen B, Altman DG, et al. Small study effects in meta-analyses of osteoarthritis trials: meta-epidemiological study. BMJ 2010;341:c3515. [DOI: 10.1136/bmj.c3515]

\section{Patanwala 2007}

Patanwala AE, Duby J, Waters D, Erstad BL. Opioid conversion in acute care. The Annals of Pharmacotherapy 2007;41:255-67. [DOI: 10.1345/aph.1H421]

\section{Peltoniemi 2016}

Peltoniemi MA, Hagelberg NM, Olkkola KT, Saari TI. Ketamine. A review of clinical pharmacokinetics and pharmacodynamics in anesthesia and pain therapy. Clinical Pharmacokinetics 2016;55:1059-77.

\section{Pereira 2001}

Pereira J, Lawlor P, Vigano A, Dorgan M, Bruera E. Equianalgesic dose ratios for opioids: a critical review and proposals for long-term dosing. Journal of Pain and Symptom Management 2001;22(2):672-87. [DOI: 10.1016/S0885-3924(01)00294-9]

\section{Pogue 1997}

Pogue JM, Yusuf S. Cumulating evidence from randomized trials: utilizing sequential monitoring boundaries for cumulative meta-analysis. Controlled Clinical Trials 1997;18(6):580-93. [DOI: 10.1016/S0197-2456(97)00051-2]

\section{Pogue 1998}

Pogue JM, Yusuf S. Overcoming the limitations of current meta-analysis of randomised controlled trials. Lancet 1998;351(9095):47-52. [DOI: 10.1016/S0140-6736(97)08461-4]

\section{Prabhakar 2014}

Prabhakar A, Mancuso KF, Owen CP, Lissauer J, Merritt CK, Urman RD, et al. Perioperative analgesia outcomes and strategies. Best Practice \& Research Clinical Anaesthesiology 2014;28:105-15. 


\section{Price 2000}

Price DD, Mayer DJ, Mao J, Caruso F. NMDA-receptor antagonists and opioid receptor interactions as related to analgesia and tolerance. Journal of Pain and Symptom Management 2000;S1:S7-15. [DOI: 10.1016/S0885-3924(99)00121-9]

\section{Review Manager 2014 [Computer program]}

Nordic Cochrane Centre, The Cochrane Collaboration Review Manager 5 (RevMan 5). Version 5.3. Copenhagen: Nordic Cochrane Centre, The Cochrane Collaboration, 2014.

\section{Roberts 2015}

Roberts I, Ker K, Edwards P, Beecher D, Manno D, Sydenham E. The knowledge system underpinning healthcare is not fit for purpose and must change. BMJ 2015;250:h2463. [DOI: 10.1136/ bmj.h2463]

\section{Ruschweyeh 2011}

Ruschweyeh, Wilder-Smith O, Drdla R, Liu X-G, Sandkühler J. Long-term potentiation in spinal nociceptive pathways as a novel target for pain therapy. Molecular Pain 2011;7:20.

\section{Sandkühler 2012}

Sandkühler J, Gruber-Schoffnegger D. Hyperalgesia by synaptic long-term potentiation (LTP): an update. Current Opinion in Pharmacology 2012;12(1):18-27. [DOI: 10.1016/ j.coph.2011.10.018]

\section{Schmid 1999}

Schmid RL, Sandler NA, Katz J. Use and efficacy of lowdose ketamine in the management of acute postoperative pain: a review of current techniques and outcomes. Pain 1999;82(2):111-25. [DOI: 10.1016/S0304-3959(99)00044-5]

\section{Schünemann 2017}

Schünemann HJ, Oxman AD, Vist GE, Higgins JP, Deeks JJ, Glasziou P, et al, on behalf of the Cochrane Applicability and Recommendations Methods Group. Chapter 12: Interpreting results and drawing conclusions. In: Higgins JPT, Churchill R, Chandler J, Cumpston MS (editors), Cochrane Handbook for Systematic Reviews of Interventions version 5.2.0 (updated June 2017). Cochrane, 2017. Available from www.training.cochrane.org/handbook.

\section{Sigtermans 2009}

Sigtermans M, Dahan A, Mooren R, Bauer M, Kest B, Sarton E, et al. S(+)-ketamine effect on experimental pain and cardiac output a population pharmacokinetic-pharmacodynamic modeling study in healthy volunteers. Anesthesiology 2009;111:892-903. [DOI: 10.1097/ALN.0b013e3181b437b1]

\section{Silvasti 1998}

Silvasti M, Rosenberg P, Seppala T, Svartling N, Pitkanen M. Comparison of analgesic efficacy of oxycodone and morphine in postoperative intravenous patient-controlled analgesia. Acta Anaesthesiologica Scandinavica 1998;42:576-80. [DOI: 10.1111/ j.1399-6576.1998.tb05169.x]

\section{Silverman 2009}

Silverman JM. Opioid induced hyperalgesia: clinical implications for the pain practitioner. Pain Physician 2009;12:679-84.

\section{Sterne 2000}

Sterne JA, Gavaghan D, Egger M. Publication and related bias in meta-analysis: power of statistical tests and prevalence in the literature. Journal of Clinical Epidemiology 2000;53(11):1119-26. [DOI: 10.1016/S0895-4356(00)00242-0]

\section{Subramaniam 2004}

Subramaniam K, Subramaniam B, Steinbrook R. Ketamine as adjuvant analgesic to opioids: a quantitative and qualitative systematic review. Anesthesia and Analgesia 2004;99:482-95. [DOI: 10.1213/01.ANE.0000118109.12855.07]

\section{Thorlund 2011}

Thorlund K, Imberger G, Walsh M, Chu R, Gluud C, Wetterslev J, et al. The number of patients and events required to limit the risk of overestimation of intervention effects in meta-analysis-a simulation study. PLoS One 2011;6(10):e25491. [DOI: 10.1371/ journal.pone.0025491]

\section{Turner 2013}

Turner RM, Bird SM, Higgins JP. The impact of study size on meta-analyses: examination of underpowered studies in Cochrane Reviews. PLoS One 2013;8(3):e59202. [DOI: 10.1371/ journal.pone.0059202]

\section{VanDenKerkhof 2013}

VanDenKerkhof EG, Peters ML, Bruce J. Chronic pain after surgery. Time for standardization? A framework to establish core risk factor and outcome domains for epidemiological studies. Clinical Journal of Pain 2013;29(1):2-8. [DOI: 10.1097/ AJP.0b013e31824730c2]

\section{Woodhouse 1996}

Woodhouse A, Hobbes AFT, Mather LE, Gibson M. A comparison of morphine, pethidine and fentanyl in the postsurgical patientcontrolled analgesia environment. Pain 1996;64:115-21. [DOI: 10.1016/0304-3959(95)00082-8]

\section{Zeng 2015}

Zeng Z, Lu J, Shu C, Chen Y, Guo Y, Wu Q, et al. A comparison of nalbuphine with morphine for analgesic effects and safety: meta-analysis of randomized controlled trials. Scientific reports 2015;5:1-8. [DOI: 10.1038/srep10927]

\section{Zhou 2013}

Zhou C, Douglas JE, Kumar NN, Shu S, Bayliss DA, Chen XC. Forebrain $\mathrm{HCN} 1$ channels contribute to hypnotic actions of ketamine. Anesthesiology 2013;118(4):785-95. [DOI: 10.1097/ ALN.0b013e318287b7c8]

\section{References to other published versions of this review Bell 2006}

Bell FR, Dahl JD, Moore RA, Kalso EA. Perioperative ketamine for acute postoperative pain. Cochrane Database of 
Systematic Reviews 2006, Issue 1. Art. No: CD004603. [DOI:

10.1002/14651858.CD004603.pub2]

CHARACTERISTICS OF STUDIES

Characteristics of included studies [ordered by study ID]

Abdolahi 2013

\section{Study characteristics}

\begin{tabular}{|c|c|}
\hline Methods & Randomised, placebo control \\
\hline Participants & $\mathrm{N}=88$, about $41 \%$ women \\
\hline Interventions & Ketamine $0.5 \mathrm{mg} / \mathrm{kg}$ bolus IV during induction of anaesthesia \\
\hline Outcomes & $\begin{array}{l}\text { Pain intensity (VAS). Analgesic consumption. Pain outcomes reported during the recovery room stay. } \\
\text { PONV }\end{array}$ \\
\hline Surgery type & Ophthalmic surgery (retinal detachment, strabismus, keratoplasty) \\
\hline $\begin{array}{l}\text { Group numbers after end } \\
\text { of study (treatment/con- } \\
\text { trol) }\end{array}$ & $44 / 44$ \\
\hline \multirow{2}{*}{$\begin{array}{l}\text { Age of patient population } \\
\text { (treatment/control) }\end{array}$} & $35 \pm 13.4$ \\
\hline & $36.3 \pm 17.8$ \\
\hline Notes & No funding \\
\hline
\end{tabular}

\section{Risk of bias}

\begin{tabular}{|c|c|c|}
\hline Bias & Authors' judgement & Support for judgement \\
\hline $\begin{array}{l}\text { Random sequence genera- } \\
\text { tion (selection bias) }\end{array}$ & Low risk & Computer-generated random number table \\
\hline $\begin{array}{l}\text { Allocation concealment } \\
\text { (selection bias) }\end{array}$ & Unclear risk & Allocation concealment method not described \\
\hline $\begin{array}{l}\text { Blinding of participants } \\
\text { and personnel (perfor- } \\
\text { mance bias) } \\
\text { All outcomes }\end{array}$ & Unclear risk & Not described \\
\hline $\begin{array}{l}\text { Blinding of outcome as- } \\
\text { sessment (detection bias) } \\
\text { All outcomes }\end{array}$ & Low risk & $\begin{array}{l}\text { Blinded investigator not participating in patient care performed data collec- } \\
\text { tion }\end{array}$ \\
\hline $\begin{array}{l}\text { Incomplete outcome data } \\
\text { (attrition bias) } \\
\text { All outcomes }\end{array}$ & Low risk & No withdrawals \\
\hline $\begin{array}{l}\text { Selective reporting (re- } \\
\text { porting bias) }\end{array}$ & Low risk & Predefined outcomes reported \\
\hline Size & High risk & 44 participants per treatment arm \\
\hline
\end{tabular}


Adam 2005

\section{Study characteristics}

\begin{tabular}{|c|c|}
\hline Methods & Randomised, double-blind, placebo control \\
\hline Participants & $\mathrm{N}=40$, about $68 \%$ women \\
\hline Interventions & $\begin{array}{l}\text { Ketamine } 0.5 \mathrm{mg} / \mathrm{kg} \text { bolus IV just after the induction of anaesthesia followed by a continuous infusion } \\
\text { of } 3 \mu \mathrm{g} / \mathrm{kg} / \mathrm{min} \text { intraoperatively and then } 1.5 \mu \mathrm{g} / \mathrm{kg} / \mathrm{min} \text { for } 48 \mathrm{~h} \text { postoperatively }\end{array}$ \\
\hline Outcomes & $\begin{array}{l}\text { Pain intensity (VAS) before and after mobilisation. PCA morphine consumption. Main outcomes report- } \\
\text { ed hourly for } 4 \mathrm{~h} \text {, then every } 4 \mathrm{~h} \text { for } 48 \mathrm{~h} \text {. Time to first analgesic request. AEs }\end{array}$ \\
\hline Surgery type & Total knee arthroplasty \\
\hline $\begin{array}{l}\text { Group numbers after end } \\
\text { of study (treatment/con- } \\
\text { trol) }\end{array}$ & $20 / 20$ \\
\hline \multirow{2}{*}{$\begin{array}{l}\text { Age of patient population } \\
\text { (treatment/control) }\end{array}$} & $68 \pm 8$ \\
\hline & $69 \pm 6$ \\
\hline Notes & No mention of sponsorship or funding \\
\hline
\end{tabular}

\section{Risk of bias}

\begin{tabular}{|c|c|c|}
\hline Bias & Authors' judgement & Support for judgement \\
\hline $\begin{array}{l}\text { Random sequence genera- } \\
\text { tion (selection bias) }\end{array}$ & Low risk & A random number table was generated \\
\hline $\begin{array}{l}\text { Allocation concealment } \\
\text { (selection bias) }\end{array}$ & Low risk & Allocation concealed in sealed and sequentially numbered envelopes \\
\hline $\begin{array}{l}\text { Blinding of participants } \\
\text { and personnel (perfor- } \\
\text { mance bias) } \\
\text { All outcomes }\end{array}$ & Low risk & $\begin{array}{l}\text { Blinded personnel; a nurse not involved in the evaluation of the participants } \\
\text { prepared study drugs }\end{array}$ \\
\hline $\begin{array}{l}\text { Blinding of outcome as- } \\
\text { sessment (detection bias) } \\
\text { All outcomes }\end{array}$ & Low risk & $\begin{array}{l}\text { Blinded personnel; none of the other investigators involved in participant } \\
\text { management and data collection was aware of the group assignment. }\end{array}$ \\
\hline $\begin{array}{l}\text { Incomplete outcome data } \\
\text { (attrition bias) } \\
\text { All outcomes }\end{array}$ & Low risk & $5 \%$ was withdrawn \\
\hline $\begin{array}{l}\text { Selective reporting (re- } \\
\text { porting bias) }\end{array}$ & High risk & No data available of all predefined AEs \\
\hline Size & High risk & 20 participants per treatment arm \\
\hline
\end{tabular}


Adriaenssens 1999

\section{Study characteristics}

\begin{tabular}{|c|c|}
\hline Methods & Randomised, double-blind, placebo control \\
\hline Participants & $\mathrm{N}=30$, about $77 \%$ women \\
\hline Interventions & Ketamine IV infusion initially $10 \mu \mathrm{g} / \mathrm{kg} / \mathrm{min}$, gradually decreased to $2.5 \mu \mathrm{g} / \mathrm{kg} / \mathrm{min}$ for $48 \mathrm{~h}$ after surgery \\
\hline Outcomes & $\begin{array}{l}\text { Pain intensity (VAS). PCA morphine consumption. AEs. Outcomes reported at 0, 1, 2, 4, 6, 12, 24, } 36 \text { and } \\
48 \mathrm{~h} \text { after surgery }\end{array}$ \\
\hline Surgery type & Laparotomy \\
\hline $\begin{array}{l}\text { Group numbers after end } \\
\text { of study (treatment/con- } \\
\text { trol) }\end{array}$ & $15 / 15$ \\
\hline \multirow{3}{*}{$\begin{array}{l}\text { Age of patient population } \\
\text { (treatment/control) }\end{array}$} & Mean values (range) \\
\hline & $53(27-83)$ \\
\hline & $51(17-82)$ \\
\hline Notes & No mention of sponsorship or funding \\
\hline
\end{tabular}

\section{Risk of bias}

\begin{tabular}{|c|c|c|}
\hline Bias & Authors' judgement & Support for judgement \\
\hline $\begin{array}{l}\text { Random sequence genera- } \\
\text { tion (selection bias) }\end{array}$ & Unclear risk & $\begin{array}{l}\text { Randomisation process not described, only mentioned "patients were ran- } \\
\text { domly allocated" }\end{array}$ \\
\hline $\begin{array}{l}\text { Allocation concealment } \\
\text { (selection bias) }\end{array}$ & Unclear risk & Allocation concealment not described \\
\hline $\begin{array}{l}\text { Blinding of participants } \\
\text { and personnel (perfor- } \\
\text { mance bias) } \\
\text { All outcomes }\end{array}$ & Unclear risk & Not described \\
\hline $\begin{array}{l}\text { Blinding of outcome as- } \\
\text { sessment (detection bias) } \\
\text { All outcomes }\end{array}$ & Unclear risk & Said to be double-blind but blinding process not described \\
\hline $\begin{array}{l}\text { Incomplete outcome data } \\
\text { (attrition bias) } \\
\text { All outcomes }\end{array}$ & Low risk & No withdrawals \\
\hline $\begin{array}{l}\text { Selective reporting (re- } \\
\text { porting bias) }\end{array}$ & Low risk & All pre-defined outcomes reported \\
\hline Size & High risk & 15 participants per treatment arm \\
\hline
\end{tabular}


Aida 2000

\section{Study characteristics}

\begin{tabular}{ll}
\hline Methods & Randomised, double-blind, placebo control \\
\hline Participants & $\mathrm{N}=121$, of whom 60 participants in groups 2 and 4 (IV ketamine and control). $40 \%$ women \\
\hline Interventions & $\begin{array}{l}\text { 1. Ketamine } 1 \mathrm{mg} / \mathrm{kg} \mathrm{IV} \mathrm{prior} \mathrm{to} \mathrm{surgical} \mathrm{incision,} 0.5 \mathrm{mg} / \mathrm{kg} / \mathrm{h} \text { infusion IV until skin closure } \\
\text { 2. Morphine ED bolus prior to surgical incision }+ \text { infusion }+ \text { placebo IV bolus + continuous infusion until } \\
\text { skin closure }\end{array}$ \\
$\begin{array}{l}\text { 3. Placebo ED + ketamine IV bolus } 1 \mathrm{mg} / \mathrm{kg}+\text { infusion } 0.5 \mathrm{mg} / \mathrm{kg} / \mathrm{h} \\
\text { 4. Morphine ED + ketamine IV }\end{array}$
\end{tabular}

\begin{tabular}{ll}
\hline Outcomes & Pain intensity (VAS). Maximum $48 \mathrm{~h}$ pain (categorical scale). PCA morphine consumption. Outcomes re- \\
ported at $6,12,24$ and $48 \mathrm{~h}$
\end{tabular}

\begin{tabular}{ll}
\hline Surgery type & Distal or total gastrectomy \\
\hline $\begin{array}{l}\text { Group numbers after end } \\
\text { of study (treatment/con- } \\
\text { trol) }\end{array}$ & \\
\hline
\end{tabular}

\begin{tabular}{ll}
\hline $\begin{array}{l}\text { Age of patient population } \\
\text { (treatment/control) }\end{array}$ & $62 \pm 14$ \\
& $63 \pm 13$
\end{tabular}

Notes Support from institutional and/or departmental sources

\section{Risk of bias}

\begin{tabular}{|c|c|c|}
\hline Bias & Authors' judgement & Support for judgement \\
\hline $\begin{array}{l}\text { Random sequence genera- } \\
\text { tion (selection bias) }\end{array}$ & Low risk & $\begin{array}{l}\text { Quote: "According to a computer-generated table of random number assign- } \\
\text { ments, each patient was assigned to one of four groups." }\end{array}$ \\
\hline $\begin{array}{l}\text { Allocation concealment } \\
\text { (selection bias) }\end{array}$ & Unclear risk & Allocation concealment method not described \\
\hline $\begin{array}{l}\text { Blinding of participants } \\
\text { and personnel (perfor- } \\
\text { mance bias) } \\
\text { All outcomes }\end{array}$ & Low risk & $\begin{array}{l}\text { Quote: "The study supervisor prepared the drug solutions, which were sealed } \\
\text { in an envelope and transferred to the anesthesiologist blinded to the solu- } \\
\text { tions." }\end{array}$ \\
\hline $\begin{array}{l}\text { Blinding of outcome as- } \\
\text { sessment (detection bias) } \\
\text { All outcomes }\end{array}$ & Low risk & Blinded personnel \\
\hline $\begin{array}{l}\text { Incomplete outcome data } \\
\text { (attrition bias) } \\
\text { All outcomes }\end{array}$ & Low risk & No withdrawals \\
\hline $\begin{array}{l}\text { Selective reporting (re- } \\
\text { porting bias) }\end{array}$ & Low risk & Predefined outcomes were reported \\
\hline Size & High risk & 29 and 31 participants per treatment arms, respectively \\
\hline
\end{tabular}


Aqil 2011

\section{Study characteristics}

\begin{tabular}{ll}
\hline Methods & Randomised, double-blind, placebo control \\
\hline Participants & $\mathrm{N}=120$, about $58 \%$ women \\
\hline Interventions & 1. Ketamine $0.5 \mathrm{mg} / \mathrm{kg}$ bolus IV at induction \\
2. Ketamine $1 \mathrm{mg} / \mathrm{kg}$ bolus IV \\
3. Ketamine $1.5 \mathrm{mg} / \mathrm{kg}$ bolus IV \\
\hline
\end{tabular}

Outcomes

Pain intensity (VAS). Pain intensity results not reported though predefined in methods. Analgesic consumption (ketoprofen) at $24 \mathrm{~h}$. AEs

\begin{tabular}{|c|c|c|}
\hline Surgery type & \multicolumn{2}{|l|}{ Septorhinoplasty } \\
\hline $\begin{array}{l}\text { Group numbers after end } \\
\text { of study (treatment/con- } \\
\text { trol) }\end{array}$ & \multicolumn{2}{|l|}{$90 / 30$} \\
\hline Age of patient population & \multicolumn{2}{|l|}{$22.9 \pm 4.5$} \\
\hline fercentrot & \multicolumn{2}{|l|}{$22.3 \pm 3.89$} \\
\hline Notes & \multicolumn{2}{|c|}{ No mention of sponsorship or funding } \\
\hline \multicolumn{3}{|l|}{ Risk of bias } \\
\hline Bias & Authors' judgement & Support for judgement \\
\hline $\begin{array}{l}\text { Random sequence genera- } \\
\text { tion (selection bias) }\end{array}$ & Low risk & Computer-generated random numbers \\
\hline $\begin{array}{l}\text { Allocation concealment } \\
\text { (selection bias) }\end{array}$ & Unclear risk & Allocation concealment method not described \\
\hline $\begin{array}{l}\text { Blinding of participants } \\
\text { and personnel (perfor- } \\
\text { mance bias) } \\
\text { All outcomes }\end{array}$ & Low risk & Blinded personnel and identical study drug syringes \\
\hline $\begin{array}{l}\text { Blinding of outcome as- } \\
\text { sessment (detection bias) } \\
\text { All outcomes }\end{array}$ & Low risk & Blinded personnel \\
\hline $\begin{array}{l}\text { Incomplete outcome data } \\
\text { (attrition bias) } \\
\text { All outcomes }\end{array}$ & Low risk & No withdrawals \\
\hline $\begin{array}{l}\text { Selective reporting (re- } \\
\text { porting bias) }\end{array}$ & High risk & $\begin{array}{l}\text { VAS scores not reported but defined in methods. Time to first request for anal- } \\
\text { gesia reported but not predefined }\end{array}$ \\
\hline Size & High risk & 30 participants per treatment arm \\
\hline
\end{tabular}


Argiriadou 2004

\section{Study characteristics}

\begin{tabular}{ll}
\hline Methods & Randomised, double-blind, placebo control \\
\hline Participants & $\mathrm{N}=45,20 \%$ women \\
\hline Interventions & $\begin{array}{l}\text { 1. S-ketamine } 0.5 \mathrm{mg} / \mathrm{kg} \text { bolus IV prior to surgical incision } \\
\text { 2. Pre-incisional S-ketamine } 0.5 \mathrm{mg} / \mathrm{kg} \text { bolus IV + intraoperative } 0.2 \mathrm{mg} / \mathrm{kg} \text { boluses IV at 20-min intervals } \\
\text { until skin closure }\end{array}$
\end{tabular}

$\begin{array}{ll}\text { Outcomes } & \text { Pain intensity (VAS). Cumulative consumption of diclofenac and dextropropoxyphene. Outcomes } \\ \text { recorded at 3, } 6 \text { and } 24 \mathrm{~h} \text { after awakening. }\end{array}$

\begin{tabular}{ll}
\hline Surgery type & Major abdominal surgery \\
\hline $\begin{array}{l}\text { Group numbers after end } \\
\text { of study (treatment/con- } \\
\text { trol) }\end{array}$ & $30 / 15$ \\
\hline
\end{tabular}

$\begin{array}{ll}\begin{array}{l}\text { Age of patient population } \\ \text { (treatment/control) }\end{array} & 61 \pm 14 \\ & 61 \pm 10\end{array}$

Notes Supported in part by Pfizer Parke-Davis Pharmaceuticals, Freiburg- Karlsruhe, Germany.

\section{Risk of bias}

\begin{tabular}{|c|c|c|}
\hline Bias & Authors' judgement & Support for judgement \\
\hline \multirow{2}{*}{$\begin{array}{l}\text { Random sequence genera- } \\
\text { tion (selection bias) }\end{array}$} & Low risk & Computer-generated randomisation table. \\
\hline & & $\begin{array}{l}\text { Quote: "With use of a computer-generated randomization table, patients were } \\
\text { assigned to one of three groups." }\end{array}$ \\
\hline $\begin{array}{l}\text { Allocation concealment } \\
\text { (selection bias) }\end{array}$ & Unclear risk & Allocation concealment method not described \\
\hline $\begin{array}{l}\text { Blinding of participants } \\
\text { and personnel (perfor- } \\
\text { mance bias) } \\
\text { All outcomes }\end{array}$ & Low risk & $\begin{array}{l}\text { Quote: "Patients and personnel who participated in the study were unaware of } \\
\text { group assignment." }\end{array}$ \\
\hline $\begin{array}{l}\text { Blinding of outcome as- } \\
\text { sessment (detection bias) } \\
\text { All outcomes }\end{array}$ & Low risk & $\begin{array}{l}\text { Quote: "Patients and personnel who participated in the study were unaware of } \\
\text { group assignment." }\end{array}$ \\
\hline $\begin{array}{l}\text { Incomplete outcome data } \\
\text { (attrition bias) } \\
\text { All outcomes }\end{array}$ & High risk & $11 \%$ was withdrawn \\
\hline $\begin{array}{l}\text { Selective reporting (re- } \\
\text { porting bias) }\end{array}$ & Low risk & Predefined outcomes reported adequately \\
\hline Size & High risk & 30 and 15 participants per treatment arm \\
\hline
\end{tabular}


Argiriadou 2011

\section{Study characteristics}

\begin{tabular}{|c|c|c|}
\hline Methods & \multicolumn{2}{|c|}{ Randomised, double-blind, placebo control } \\
\hline Participants & \multicolumn{2}{|l|}{$\mathrm{N}=80,21 \%$ women } \\
\hline Interventions & \multicolumn{2}{|c|}{$\begin{array}{l}\text { Pre-incisional S-ketamine } 0.5 \mathrm{mg} / \mathrm{kg} \text { bolus IV + intraoperative infusion } 6.7 \mu \mathrm{g} / \mathrm{kg} / \mathrm{min} \text { until } 20 \mathrm{~min} \text { be- } \\
\text { fore the end of surgery }\end{array}$} \\
\hline Outcomes & \multicolumn{2}{|c|}{$\begin{array}{l}\text { Pain intensity (VAS) at } 4,12,24 \text { and } 48 \mathrm{~h} \text { after surgery at rest and during movement (coughing). Supple- } \\
\text { mental analgesic requirement. Pulmonary function. Return of bowel functions. Length of ICU and hos- } \\
\text { pital stay }\end{array}$} \\
\hline Surgery type & \multicolumn{2}{|c|}{ Elective open thoracotomy } \\
\hline $\begin{array}{l}\text { Group numbers after end } \\
\text { of study (treatment/con- } \\
\text { trol) }\end{array}$ & \multicolumn{2}{|l|}{$27 / 26$} \\
\hline \multirow{2}{*}{$\begin{array}{l}\text { Age of patient population } \\
\text { (treatment/control) }\end{array}$} & \multicolumn{2}{|l|}{$52 \pm 17$} \\
\hline & \multicolumn{2}{|l|}{$59 \pm 11$} \\
\hline Notes & \multicolumn{2}{|l|}{ No funding } \\
\hline \multicolumn{3}{|l|}{ Risk of bias } \\
\hline Bias & Authors' judgement & Support for judgement \\
\hline $\begin{array}{l}\text { Random sequence genera- } \\
\text { tion (selection bias) }\end{array}$ & Low risk & Computer-generated randomisation schedule \\
\hline $\begin{array}{l}\text { Allocation concealment } \\
\text { (selection bias) }\end{array}$ & Unclear risk & Allocation concealment method not described \\
\hline $\begin{array}{l}\text { Blinding of participants } \\
\text { and personnel (perfor- } \\
\text { mance bias) } \\
\text { All outcomes }\end{array}$ & Low risk & Blinded personnel \\
\hline $\begin{array}{l}\text { Blinding of outcome as- } \\
\text { sessment (detection bias) } \\
\text { All outcomes }\end{array}$ & Low risk & Blinded personnel \\
\hline $\begin{array}{l}\text { Incomplete outcome data } \\
\text { (attrition bias) } \\
\text { All outcomes }\end{array}$ & Low risk & $2 \%$ was withdrawn \\
\hline $\begin{array}{l}\text { Selective reporting (re- } \\
\text { porting bias) }\end{array}$ & Low risk & All predefined outcomes reported \\
\hline Size & High risk & 26,27 and 27 participants per treatment arm \\
\hline
\end{tabular}


Arikan 2016

\section{Study characteristics}

\begin{tabular}{ll}
\hline Methods & Randomised, double-blind, placebo control \\
\hline Participants & $\mathrm{N}=120$, of whom 80 participants in IV ketamine and control treatment arms. 100\% women \\
\hline Interventions & Postoperatively: \\
& $\begin{array}{l}\text { 1. ketamine } 0.2 \mathrm{mg} / \mathrm{kg} \text { IV bolus followed by an infusion of ketamine } 0.05 \mathrm{mg} / \mathrm{kg} / \mathrm{h} \text { for } 48 \mathrm{~h} \\
\text { 2. } 0.9 \% \text { saline bolus IV followed by an infusion of } 0.9 \% \text { saline for } 48 \mathrm{~h} \\
\text { 3. (IV bolus of magnesium } 50 \mathrm{mg} / \mathrm{kg} \text { followed by an infusion of magnesium } 10 \mathrm{mg} / \mathrm{kg} / \mathrm{h} \text { ) }\end{array}$ \\
\hline $\begin{array}{ll}\text { Outcomes } \\
\text { eratively. AEs }\end{array}$ \\
\hline $\begin{array}{l}\text { Surgery type } \\
\text { Total abdominal hysterectomy }\end{array}$ \\
$\begin{array}{l}\text { Group numbers after end } \\
\text { of study (treatment/con- } \\
\text { trol) }\end{array}$ & \begin{tabular}{l}
$40 / 40$ \\
\hline $\begin{array}{l}\text { Age of patient population } \\
\text { (treatment/control) }\end{array}$
\end{tabular} \\
\hline $\begin{array}{l}\text { Notes } \\
59.35 \pm 4.96\end{array}$ \\
\hline
\end{tabular}

\section{Risk of bias}

\begin{tabular}{lll}
\hline Bias & Authors' judgement & Support for judgement \\
\hline $\begin{array}{l}\text { Random sequence genera- } \\
\text { tion (selection bias) }\end{array}$ & Low risk & Computer-generated randomisation list \\
\hline $\begin{array}{l}\text { Allocation concealment } \\
\text { (selection bias) }\end{array}$ & Unclear risk & Allocation concealment method not described \\
\hline $\begin{array}{l}\text { Blinding of participants } \\
\text { and personnel (perfor- } \\
\text { mance bias) } \\
\text { All outcomes }\end{array}$ & Low risk & Blinded participants and personnel \\
\hline $\begin{array}{l}\text { Blinding of outcome as- } \\
\text { sessment (detection bias) } \\
\text { All outcomes }\end{array}$ & Low risk & Blinded personnel \\
\hline $\begin{array}{l}\text { Incomplete outcome data } \\
\text { (attrition bias) } \\
\text { All outcomes }\end{array}$ & Low risk & No withdrawals \\
\hline $\begin{array}{l}\text { Selective reporting (re- } \\
\text { porting bias) }\end{array}$ & Low risk & Predefined outcomes reported \\
\hline \begin{tabular}{l} 
Size \\
\hline
\end{tabular} & High risk & 40 participants per treatment arm \\
\hline
\end{tabular}


Ataskhoyi 2013

\section{Study characteristics}

\begin{tabular}{|c|c|}
\hline Methods & Randomised, double-blind, placebo control \\
\hline Participants & $N=60,100 \%$ women \\
\hline Interventions & Pre-incisional ketamine $0.5 \mathrm{mg} / \mathrm{kg}$ bolus IV \\
\hline Outcomes & $\begin{array}{l}\text { Pain intensity (VAS) reported at 1, 2, 3, 6, } 12 \text { and } 24 \mathrm{~h} \text { postoperatively. Time to first request for analge- } \\
\text { sia. Analgesic consumption reported at } 24 \mathrm{~h} \text {. AEs }\end{array}$ \\
\hline Surgery type & Diagnostic gynaecological laparoscopy \\
\hline $\begin{array}{l}\text { Group numbers after end } \\
\text { of study (treatment/con- } \\
\text { trol) }\end{array}$ & $30 / 30$ \\
\hline \multirow{2}{*}{$\begin{array}{l}\text { Age of patient population } \\
\text { (treatment/control) }\end{array}$} & $32.7 \pm 3.4$ \\
\hline & $34.3 \pm 5.4$ \\
\hline Notes & No mention of sponsorship or funding \\
\hline
\end{tabular}

\section{Risk of bias}

\begin{tabular}{|c|c|c|}
\hline Bias & Authors' judgement & Support for judgement \\
\hline $\begin{array}{l}\text { Random sequence genera- } \\
\text { tion (selection bias) }\end{array}$ & Low risk & Computer-generated randomisation \\
\hline $\begin{array}{l}\text { Allocation concealment } \\
\text { (selection bias) }\end{array}$ & Unclear risk & Allocation concealment method not described \\
\hline $\begin{array}{l}\text { Blinding of participants } \\
\text { and personnel (perfor- } \\
\text { mance bias) } \\
\text { All outcomes }\end{array}$ & Low risk & Blinded personnel \\
\hline $\begin{array}{l}\text { Blinding of outcome as- } \\
\text { sessment (detection bias) } \\
\text { All outcomes }\end{array}$ & Low risk & Blinded outcome assessors \\
\hline $\begin{array}{l}\text { Incomplete outcome data } \\
\text { (attrition bias) } \\
\text { All outcomes }\end{array}$ & Low risk & No withdrawals \\
\hline $\begin{array}{l}\text { Selective reporting (re- } \\
\text { porting bias) }\end{array}$ & Low risk & Predefined outcomes reported \\
\hline Size & High risk & 30 participants per treatment arm \\
\hline
\end{tabular}

\section{Study characteristics}


Aubrun 2008 (Continued)

\begin{tabular}{ll} 
Methods & Randomised, double-blind, placebo control \\
\hline Participants & $\mathrm{N}=90,100 \%$ women \\
\hline Interventions & Pre-incisional ketamine $0.15 \mathrm{mg} / \mathrm{kg}$ bolus IV + IV PCA ketamine $0.5 \mathrm{mg} / \mathrm{bolus}$ \\
\hline Outcomes & Pain intensity (VAS). Analgesic consumption. AEs. Outcomes reported every $6 \mathrm{~h}$ up to $48 \mathrm{~h}$ \\
\hline $\begin{array}{l}\text { Surgery type } \\
\begin{array}{l}\text { Group numbers after end } \\
\text { of study (treatment/con- } \\
\text { trol) }\end{array}\end{array}$ & Major gynaecological operation \\
\hline
\end{tabular}

\begin{tabular}{ll}
\hline $\begin{array}{l}\text { Age of patient population } \\
\text { (treatment/control) }\end{array}$ & $50 \pm 10$ \\
& $49 \pm 12$ \\
\hline Notes & Support provided by departmental sources \\
\hline
\end{tabular}

\section{Risk of bias}

\begin{tabular}{lll}
\hline Bias & Authors' judgement & Support for judgement \\
\hline $\begin{array}{l}\text { Random sequence genera- } \\
\text { tion (selection bias) }\end{array}$ & Low risk & Random number table \\
\hline $\begin{array}{l}\text { Allocation concealment } \\
\text { (selection bias) }\end{array}$ & Unclear risk & Allocation concealment method not described \\
\hline $\begin{array}{l}\text { Blinding of participants } \\
\text { and personnel (perfor- } \\
\text { mance bias) }\end{array}$ & Low risk & Blinded personnel \\
$\begin{array}{l}\text { All outcomes } \\
\text { Blinding of outcome as- } \\
\text { sessment (detection bias) }\end{array}$ & Low risk & \\
$\begin{array}{l}\text { All outcomes } \\
\text { Incomplete outcome data }\end{array}$ & High risk & Blinded personnel \\
$\begin{array}{l}\text { (attrition bias) } \\
\text { All outcomes }\end{array}$ & & $12 \%$ was withdrawn \\
\hline $\begin{array}{l}\text { Selective reporting (re- } \\
\text { porting bias) }\end{array}$ & Low risk & \\
\hline \begin{tabular}{l} 
Size \\
\hline
\end{tabular} & High risk & Predefined outcomes reported \\
\hline
\end{tabular}

Aveline 2006

\section{Study characteristics}

\begin{tabular}{ll}
\hline Methods & Randomised, double-blind, ketamine vs ketamine + morphine vs morphine \\
\hline Participants & $\mathrm{N}=69$, of whom 45 participants in IV ketamine and control arms. About 50\% women \\
\hline
\end{tabular}


Aveline 2006 (Continued)

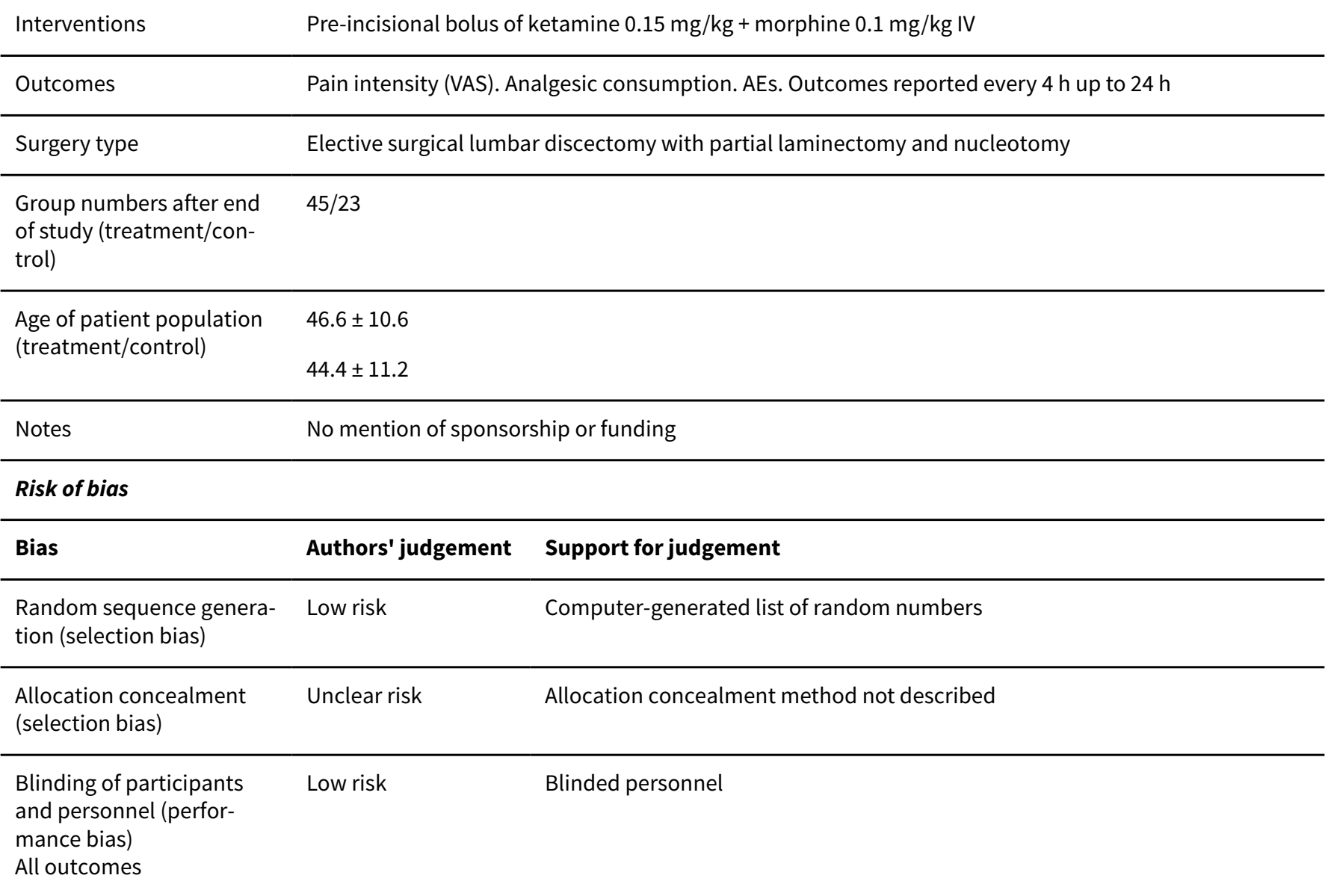

\begin{tabular}{lll}
\hline $\begin{array}{l}\text { Blinding of outcome as- } \\
\text { sessment (detection bias) } \\
\text { All outcomes }\end{array}$ & Low risk & Blinded personnel \\
\hline $\begin{array}{l}\text { Incomplete outcome data } \\
\text { (attrition bias) } \\
\text { All outcomes }\end{array}$ & Low risk & $2 \%$ was withdrawn \\
\hline $\begin{array}{l}\text { Selective reporting (re- } \\
\text { porting bias) }\end{array}$ & Low risk & Predefined outcomes reported \\
\hline Size & High risk & 22,23 and 23 participants per treatment arm \\
\hline
\end{tabular}

Aveline 2009

\section{Study characteristics}

\begin{tabular}{ll}
\hline Methods & Randomised, double-blind, placebo control \\
\hline Participants & $\mathrm{N}=75$, of whom 49 participants in IV ketamine and control arms. About $61 \%$ women \\
\hline Interventions & $\begin{array}{l}\text { Pre-incisional ketamine } 0.2 \mathrm{mg} / \mathrm{kg} \text { bolus IV + continuous IV infusion } 120 \mathrm{\mu g} / \mathrm{kg} / \mathrm{h} \mathrm{until} \mathrm{the} \mathrm{end} \mathrm{of} \\
\text { surgery, then } 60 \mu \mathrm{g} / \mathrm{kg} / \mathrm{h} \text { until the second postoperative day }\end{array}$ \\
\hline
\end{tabular}


Aveline 2009 (Continued)

Outcomes

Pain intensity (VAS). Analgesic consumption. Time to first morphine demand, AEs. Pain outcomes reported at 2, 6, 1224 and $48 \mathrm{~h}$

\begin{tabular}{|c|c|c|}
\hline Surgery type & \multicolumn{2}{|c|}{ Elective unilateral total knee replacement } \\
\hline $\begin{array}{l}\text { Group numbers after end } \\
\text { of study (treatment/con- } \\
\text { trol) }\end{array}$ & \multicolumn{2}{|l|}{$25 / 24$} \\
\hline \multirow{2}{*}{$\begin{array}{l}\text { Age of patient population } \\
\text { (treatment/control) }\end{array}$} & \multicolumn{2}{|l|}{$71 \pm 9$} \\
\hline & \multicolumn{2}{|l|}{$70 \pm 7$} \\
\hline Notes & \multicolumn{2}{|c|}{ No mention of sponsorship or funding } \\
\hline \multicolumn{3}{|l|}{ Risk of bias } \\
\hline Bias & Authors' judgement & Support for judgement \\
\hline $\begin{array}{l}\text { Random sequence genera- } \\
\text { tion (selection bias) }\end{array}$ & Low risk & Computer-generated list of random numbers \\
\hline $\begin{array}{l}\text { Allocation concealment } \\
\text { (selection bias) }\end{array}$ & Low risk & Sequence allocation concealed by opaque, sealed envelopes \\
\hline $\begin{array}{l}\text { Blinding of participants } \\
\text { and personnel (perfor- } \\
\text { mance bias) } \\
\text { All outcomes }\end{array}$ & Low risk & Blinded personnel \\
\hline $\begin{array}{l}\text { Blinding of outcome as- } \\
\text { sessment (detection bias) } \\
\text { All outcomes }\end{array}$ & Low risk & Blinded personnel \\
\hline $\begin{array}{l}\text { Incomplete outcome data } \\
\text { (attrition bias) } \\
\text { All outcomes }\end{array}$ & Low risk & $4 \%$ was withdrawn \\
\hline $\begin{array}{l}\text { Selective reporting (re- } \\
\text { porting bias) }\end{array}$ & Low risk & Predefined outcomes reported \\
\hline Size & High risk & 25,24 and 25 participants per treatment arm \\
\hline
\end{tabular}

\section{Ayoglu 2005}

\section{Study characteristics}

\begin{tabular}{ll}
\hline Methods & Randomised, double-blind, placebo control \\
\hline Participants & $\mathrm{N}=60$, of whom 40 participants in IV ketamine and control treatment arms. $65 \%$ women \\
\hline Interventions & Pre-incisional ketamine $0.5 \mathrm{mg} / \mathrm{kg}$ bolus IV + infusion $0.15 \mathrm{mg} / \mathrm{kg} / \mathrm{h}$ for the next $4 \mathrm{~h}$ \\
\hline Outcomes & $\begin{array}{l}\text { Pain intensity (VRS, NRS). PCA morphine requirement. Pain outcomes reported hourly up to } 4 \mathrm{~h} \text { postop- } \\
\text { eratively, then at } 8 \text { and } 20 \mathrm{~h} \text { postoperatively. AEs }\end{array}$ \\
\hline
\end{tabular}


Ayoglu 2005 (Continued)

\begin{tabular}{ll} 
Surgery type & Laparoscopic cholecystectomy \\
\hline $\begin{array}{l}\text { Group numbers after end } \\
\text { of study (treatment/con- } \\
\text { trol) }\end{array}$ & $20 / 20$ \\
\hline $\begin{array}{l}\text { Age of patient population } \\
\text { (treatment/control) }\end{array}$ & $52.9 \pm 9$ \\
& $49.1 \pm 3.7$ \\
\hline Notes & Third group received a bolus and infusion of magnesium sulphate. No mention of sponsorship or fund- \\
& ing
\end{tabular}

\section{Risk of bias}

\begin{tabular}{|c|c|c|}
\hline Bias & Authors' judgement & Support for judgement \\
\hline $\begin{array}{l}\text { Random sequence genera- } \\
\text { tion (selection bias) }\end{array}$ & Unclear risk & Randomisation done by using coloured balls \\
\hline $\begin{array}{l}\text { Allocation concealment } \\
\text { (selection bias) }\end{array}$ & Unclear risk & Allocation concealment method not described \\
\hline $\begin{array}{l}\text { Blinding of participants } \\
\text { and personnel (perfor- } \\
\text { mance bias) } \\
\text { All outcomes }\end{array}$ & Low risk & Blinded personnel \\
\hline $\begin{array}{l}\text { Blinding of outcome as- } \\
\text { sessment (detection bias) } \\
\text { All outcomes }\end{array}$ & Low risk & Blinded personnel \\
\hline $\begin{array}{l}\text { Incomplete outcome data } \\
\text { (attrition bias) } \\
\text { All outcomes }\end{array}$ & Low risk & No withdrawals \\
\hline $\begin{array}{l}\text { Selective reporting (re- } \\
\text { porting bias) }\end{array}$ & High risk & Unpleasant dreams not predefined in 'methods' but reported in 'results' \\
\hline Size & High risk & 20 participants per treatment arm. Lacks power analysis \\
\hline
\end{tabular}

Barreveld 2013

\section{Study characteristics}

\begin{tabular}{ll}
\hline Methods & Randomised, double-blind, placebo control \\
\hline Participants & $\mathrm{N}=59,56 \%$ women \\
\hline Interventions & Postoperative ketamine $0.2 \mathrm{mg} / \mathrm{kg} / \mathrm{h}$ infusion IV for $24 \mathrm{~h}$ \\
\hline Outcomes & Pain intensity (NRS for categorical pain states). Analgesic consumption at 24 h. AEs \\
\hline Surgery type & Nononcologic surgery leading to hospitalisation \\
\hline
\end{tabular}


Barreveld 2013 (Continued)

Group numbers after end of study (treatment/control)
$29 / 30$

$48.5 \pm 11.9$

$55 \pm 11.2$

\section{Notes}

Intraoperative anaesthetic management was at the discretion of the attending anaesthetist (not standardised). No mention of sponsorship or funding

\section{Risk of bias}

\begin{tabular}{lll}
\hline Bias & Authors' judgement & Support for judgement \\
\hline $\begin{array}{l}\text { Random sequence genera- } \\
\text { tion (selection bias) }\end{array}$ & Unclear risk & Randomisation process not described in detail \\
\hline $\begin{array}{l}\text { Allocation concealment } \\
\text { (selection bias) }\end{array}$ & Low risk & $\begin{array}{l}\text { Central allocation by Investigational Drug Service (IDS (a third party)). IDS al- } \\
\text { so prepared study solutions that were identical in appearance and labelled as } \\
\text { "ketamine/placebo" }\end{array}$ \\
\hline $\begin{array}{l}\text { Blinding of participants } \\
\text { and personnel (perfor- } \\
\text { mance bias) } \\
\text { All outcomes }\end{array}$ & Low risk & Blinded personnel \\
\hline
\end{tabular}

\begin{tabular}{|c|c|c|}
\hline $\begin{array}{l}\text { Blinding of outcome as- } \\
\text { sessment (detection bias) } \\
\text { All outcomes }\end{array}$ & Low risk & Blinded personnel assessed outcomes \\
\hline $\begin{array}{l}\text { Incomplete outcome data } \\
\text { (attrition bias) } \\
\text { All outcomes }\end{array}$ & Low risk & $8 \%$ was withdrawn \\
\hline $\begin{array}{l}\text { Selective reporting (re- } \\
\text { porting bias) }\end{array}$ & Low risk & Predefined outcomes reported \\
\hline Size & High risk & 29 and 30 participants per treatment arm \\
\hline
\end{tabular}

\section{Bilgen 2012}

\section{Study characteristics}

\begin{tabular}{ll}
\hline Methods & Randomised, double-blind, placebo control \\
\hline Participants & $\mathrm{N}=140,100 \%$ women \\
\hline Interventions & Before induction of anaesthesia \\
& $\begin{array}{l}\text { 1. Ketamine } 0.25 \mathrm{mg} / \mathrm{kg} \\
\text { 2. Ketamine } 0.5 \mathrm{mg} / \mathrm{kg}\end{array}$ \\
3. Ketamine $1 \mathrm{mg} / \mathrm{kg}$ bolus IV
\end{tabular}


Bilgen 2012 (Continued) Pain intensity (NRS). Analgesic consumption. Outcomes reported at 2, 6, 12, 18, 24, 48 h and 2 weeks, 1
and 6 months and 1 year postoperatively

\begin{tabular}{|c|c|c|}
\hline Surgery type & \multicolumn{2}{|l|}{ Caesarean section } \\
\hline $\begin{array}{l}\text { Group numbers after end } \\
\text { of study (treatment/con- } \\
\text { trol) }\end{array}$ & \multicolumn{2}{|l|}{$105 / 35$} \\
\hline \multirow{2}{*}{$\begin{array}{l}\text { Age of patient population } \\
\text { (treatment/control) }\end{array}$} & \multicolumn{2}{|l|}{$31 \pm 4$} \\
\hline & \multicolumn{2}{|l|}{$32 \pm 4$} \\
\hline Notes & \multicolumn{2}{|c|}{ No mention of sponsorship or funding } \\
\hline \multicolumn{3}{|l|}{ Risk of bias } \\
\hline Bias & Authors' judgement & Support for judgement \\
\hline $\begin{array}{l}\text { Random sequence genera- } \\
\text { tion (selection bias) }\end{array}$ & Low risk & Computer-generated random number table \\
\hline $\begin{array}{l}\text { Allocation concealment } \\
\text { (selection bias) }\end{array}$ & Unclear risk & Allocation method not described \\
\hline $\begin{array}{l}\text { Blinding of participants } \\
\text { and personnel (perfor- } \\
\text { mance bias) } \\
\text { All outcomes }\end{array}$ & Low risk & Blinded personnel \\
\hline $\begin{array}{l}\text { Blinding of outcome as- } \\
\text { sessment (detection bias) } \\
\text { All outcomes }\end{array}$ & Low risk & Blinded personnel \\
\hline $\begin{array}{l}\text { Incomplete outcome data } \\
\text { (attrition bias) } \\
\text { All outcomes }\end{array}$ & Low risk & No withdrawals \\
\hline $\begin{array}{l}\text { Selective reporting (re- } \\
\text { porting bias) }\end{array}$ & High risk & Outcomes that are not defined in "methods" are reported \\
\hline Size & High risk & 35 participants per treatment arm \\
\hline
\end{tabular}

Bornemann-Cimenti 2016

\section{Study characteristics}

\begin{tabular}{ll}
\hline Methods & Randomised, triple-blind, placebo control \\
\hline Participants & $\mathrm{N}=60$, about $52 \%$ women \\
\hline Interventions & After induction of anaesthesia \\
& $\begin{array}{l}\text { 1. } 0.25 \mathrm{mg} / \mathrm{kg} \text { IV bolus of S-ketamine followed by a } 0.125 \mathrm{mg} / \mathrm{kg} / \mathrm{h} \text { infusion for } 48 \mathrm{~h} \\
\text { 2. } 0.9 \% \mathrm{IV} \text { saline bolus followed by a } 0.015 \mathrm{mg} / \mathrm{kg} / \mathrm{h} \text { infusion of S-ketamine for } 48 \mathrm{~h}\end{array}$ \\
\hline
\end{tabular}


Bornemann-Cimenti 2016 (Continued)

3. $0.9 \%$ saline bolus IV followed by a $0.9 \%$ saline infusion for $48 \mathrm{~h}$

Outcomes Postoperative opioid consumption, pain intensity (NRS), hyperalgesia at the incision site, delirium scores. Pain outcomes reported over time every $4 \mathrm{~h}$ up to $48 \mathrm{~h}$. Hyperalgesia and ICDSC reported at $48 \mathrm{~h}$

\begin{tabular}{ll}
\hline Surgery type & Major abdominal surgery \\
\hline $\begin{array}{l}\text { Group numbers after end } \\
\text { of study (treatment/con- }\end{array}$ & $37 / 19$ \\
trol) &
\end{tabular}

Age of patient population $\quad 60.2 \pm 9$
(treatment/control)

$61 \pm 12.4$

Notes Institutional funding

\section{Risk of bias}

\begin{tabular}{|c|c|c|}
\hline Bias & Authors' judgement & Support for judgement \\
\hline $\begin{array}{l}\text { Random sequence genera- } \\
\text { tion (selection bias) }\end{array}$ & Low risk & Computer-generated randomisation list \\
\hline $\begin{array}{l}\text { Allocation concealment } \\
\text { (selection bias) }\end{array}$ & Unclear risk & $\begin{array}{l}\text { Allocation concealment method not described in detail. Only mentioned that } \\
\text { an anaesthetist with no further involvement in the study prepared and la- } \\
\text { belled study drug syringes with "study medication" and the randomisation } \\
\text { number of the participant }\end{array}$ \\
\hline $\begin{array}{l}\text { Blinding of participants } \\
\text { and personnel (perfor- } \\
\text { mance bias) } \\
\text { All outcomes }\end{array}$ & Low risk & $\begin{array}{l}\text { An independent anaesthesiologist prepared study drugs. Participants and } \\
\text { nursing staff were blinded }\end{array}$ \\
\hline $\begin{array}{l}\text { Blinding of outcome as- } \\
\text { sessment (detection bias) } \\
\text { All outcomes }\end{array}$ & Low risk & Blinded personnel made outcome assessment \\
\hline $\begin{array}{l}\text { Incomplete outcome data } \\
\text { (attrition bias) } \\
\text { All outcomes }\end{array}$ & Low risk & $7 \%$ was withdrawn \\
\hline $\begin{array}{l}\text { Selective reporting (re- } \\
\text { porting bias) }\end{array}$ & Low risk & Predefined outcomes reported \\
\hline Size & High risk & 20 participants per treatment arm \\
\hline
\end{tabular}

Burstal 2001

\section{Study characteristics}

\begin{tabular}{ll}
\hline Methods & Randomised, double-blind. Ketamine + morphine vs morphine \\
\hline Participants & $\mathrm{N}=70,100 \%$ women \\
\hline Interventions & Postoperative PCA ketamine $2 \mathrm{mg} / \mathrm{bolus} \mathrm{IV}$ \\
\hline \hline
\end{tabular}

Perioperative intravenous ketamine for acute postoperative pain in adults (Review) 
Burstal 2001 (Continued)

Outcomes

Pain intensity (VAS) and PCA morphine consumption reported at 24 and $48 \mathrm{~h}$. Area of allodynia (von Frey) reported at $48 \mathrm{~h}$

\begin{tabular}{|c|c|c|}
\hline Surgery type & \multicolumn{2}{|c|}{ Total abdominal hysterectomy } \\
\hline Group numbers after end & \multirow{2}{*}{\multicolumn{2}{|c|}{$\begin{array}{l}37 / 33 \\
\text { Allodynia subset: } 25 / 18\end{array}$}} \\
\hline trol) & & \\
\hline \multirow{4}{*}{$\begin{array}{l}\text { Age of patient population } \\
\text { (treatment/control) }\end{array}$} & \multicolumn{2}{|l|}{ Median values, IQR } \\
\hline & \multicolumn{2}{|l|}{$43(10)$} \\
\hline & \multicolumn{2}{|l|}{$45(7)$} \\
\hline & \multicolumn{2}{|c|}{ Allodynia subset: 45 (10), 44 (7) } \\
\hline Notes & \multicolumn{2}{|c|}{ No mention of sponsorship or funding } \\
\hline \multicolumn{3}{|l|}{ Risk of bias } \\
\hline Bias & Authors' judgement & Support for judgement \\
\hline $\begin{array}{l}\text { Random sequence genera- } \\
\text { tion (selection bias) }\end{array}$ & Low risk & Computer-generated groups \\
\hline $\begin{array}{l}\text { Allocation concealment } \\
\text { (selection bias) }\end{array}$ & Low risk & Quote: "A sealed envelope system" \\
\hline $\begin{array}{l}\text { Blinding of participants } \\
\text { and personnel (perfor- } \\
\text { mance bias) } \\
\text { All outcomes }\end{array}$ & Low risk & Blinded personnel \\
\hline $\begin{array}{l}\text { Blinding of outcome as- } \\
\text { sessment (detection bias) } \\
\text { All outcomes }\end{array}$ & Low risk & Blinded personnel \\
\hline $\begin{array}{l}\text { Incomplete outcome data } \\
\text { (attrition bias) } \\
\text { All outcomes }\end{array}$ & High risk & $16 \%$ was withdrawn \\
\hline $\begin{array}{l}\text { Selective reporting (re- } \\
\text { porting bias) }\end{array}$ & Unclear risk & $\begin{array}{l}\text { Predefined outcomes reported but surgeon or participant decided on PCA ces- } \\
\text { sation based on how they felt }\end{array}$ \\
\hline Size & High risk & 37 and 33 participants per treatment arm \\
\hline
\end{tabular}

\section{Cenzig 2014}

\section{Study characteristics}

\begin{tabular}{ll}
\hline Methods & Randomised, double-blind, placebo control \\
\hline Participants & $\mathrm{N}=60$, about $73 \%$ women \\
\hline Interventions & Intraoperative ketamine infusion $6 \mu \mathrm{g} / \mathrm{kg} / \mathrm{min}$ after orotracheal intubation until wound closure \\
\hline \hline
\end{tabular}


Cenzig 2014 (Continued)

Outcomes Pain intensity (VAS) and analgesic consumption, reported at 1, 3, 6, 12 and $24 \mathrm{~h}$. Time to first analgesic request. AEs during the first $24 \mathrm{~h}$

\begin{tabular}{|c|c|c|}
\hline Surgery type & \multicolumn{2}{|c|}{ Total knee replacement surgery } \\
\hline $\begin{array}{l}\text { Group numbers after end } \\
\text { of study (treatment/con- } \\
\text { trol) }\end{array}$ & \multicolumn{2}{|l|}{$30 / 30$} \\
\hline \multirow{2}{*}{$\begin{array}{l}\text { Age of patient population } \\
\text { (treatment/control) }\end{array}$} & \multicolumn{2}{|l|}{$58.2 \pm 9.58$} \\
\hline & \multicolumn{2}{|l|}{$58.8 \pm 11.5$} \\
\hline Notes & \multicolumn{2}{|c|}{ No mention of sponsorship or funding } \\
\hline \multicolumn{3}{|l|}{ Risk of bias } \\
\hline Bias & Authors' judgement & Support for judgement \\
\hline $\begin{array}{l}\text { Random sequence genera- } \\
\text { tion (selection bias) }\end{array}$ & Low risk & Computer-generated random number table \\
\hline $\begin{array}{l}\text { Allocation concealment } \\
\text { (selection bias) }\end{array}$ & Unclear risk & Allocation concealment method not described in detail \\
\hline $\begin{array}{l}\text { Blinding of participants } \\
\text { and personnel (perfor- } \\
\text { mance bias) } \\
\text { All outcomes }\end{array}$ & Low risk & Blinded personnel \\
\hline $\begin{array}{l}\text { Blinding of outcome as- } \\
\text { sessment (detection bias) } \\
\text { All outcomes }\end{array}$ & Low risk & Nurses unaware of the study protocol performed assessments \\
\hline $\begin{array}{l}\text { Incomplete outcome data } \\
\text { (attrition bias) } \\
\text { All outcomes }\end{array}$ & Low risk & No withdrawals \\
\hline $\begin{array}{l}\text { Selective reporting (re- } \\
\text { porting bias) }\end{array}$ & Low risk & Predefined outcomes reported adequately \\
\hline Size & High risk & 30 participants per treatment arm \\
\hline
\end{tabular}

Chazan 2010

\section{Study characteristics}

\begin{tabular}{ll}
\hline Methods & Randomised, double-blind \\
\hline Participants & $\mathrm{N}=46$, about $35 \%$ women \\
\hline Interventions & Postoperative ketamine $5 \mathrm{mg}+$ morphine $1 \mathrm{mg} / \mathrm{bolus} \mathrm{IV} \mathrm{via} \mathrm{PCA} \mathrm{vs} \mathrm{morphine} \mathrm{alone}$ \\
\hline Outcomes & Pain intensity (VAS) reported at 24 and $48 \mathrm{~h}$. Analgesic consumption reported at $72 \mathrm{~h}$. AEs. \\
\hline
\end{tabular}


Chazan 2010 (Continued)

\begin{tabular}{|c|c|c|}
\hline Surgery type & \multicolumn{2}{|c|}{ Minimally invasive direct coronary artery bypass, off-pump coronary artery bypass, thoracotomy } \\
\hline $\begin{array}{l}\text { Group numbers after end } \\
\text { of study (treatment/con- } \\
\text { trol) }\end{array}$ & \multicolumn{2}{|l|}{$24 / 22$} \\
\hline \multirow{2}{*}{$\begin{array}{l}\text { Age of patient population } \\
\text { (treatment/control) }\end{array}$} & \multicolumn{2}{|l|}{$60 \pm 16$} \\
\hline & \multicolumn{2}{|l|}{$57 \pm 18$} \\
\hline Notes & \multicolumn{2}{|c|}{ No mention of sponsorship or funding } \\
\hline \multicolumn{3}{|l|}{ Risk of bias } \\
\hline Bias & Authors' judgement & Support for judgement \\
\hline $\begin{array}{l}\text { Random sequence genera- } \\
\text { tion (selection bias) }\end{array}$ & Low risk & Computer-generated randomisation sequence \\
\hline $\begin{array}{l}\text { Allocation concealment } \\
\text { (selection bias) }\end{array}$ & Low risk & $\begin{array}{l}\text { Quote: "The allocation sequence of the patients was generated and concealed } \\
\text { at the computer" }\end{array}$ \\
\hline $\begin{array}{l}\text { Blinding of participants } \\
\text { and personnel (perfor- } \\
\text { mance bias) } \\
\text { All outcomes }\end{array}$ & Low risk & Blinded personnel and participants \\
\hline
\end{tabular}

\begin{tabular}{|c|c|c|}
\hline $\begin{array}{l}\text { Blinding of outcome as- } \\
\text { sessment (detection bias) } \\
\text { All outcomes }\end{array}$ & Low risk & Blinded personnel \\
\hline $\begin{array}{l}\text { Incomplete outcome data } \\
\text { (attrition bias) } \\
\text { All outcomes }\end{array}$ & Low risk & No withdrawals \\
\hline $\begin{array}{l}\text { Selective reporting (re- } \\
\text { porting bias) }\end{array}$ & Low risk & Predefined outcomes reported \\
\hline Size & High risk & 22 and 24 participants per treatment arm \\
\hline
\end{tabular}

\section{Chen 2004}

\section{Study characteristics}

\begin{tabular}{ll}
\hline Methods & Randomised, double-blind, placebo control \\
\hline Participants & $\mathrm{N}=40$. Demographic data of study participants not presented \\
\hline Interventions & $\begin{array}{l}\text { Pre-incisional ketamine } 0.3 \mathrm{mg} / \mathrm{kg} \text { bolus IV followed by an infusion } 3 \mu \mathrm{g} / \mathrm{kg} / \mathrm{min} \text { until } 15 \mathrm{mins} \text { prior to } \\
\text { completion of the operation }\end{array}$ \\
\hline Outcomes & $\begin{array}{l}\text { Pain intensity (VAS). Analgesic consumption. Pain outcomes reported at 3, 6, 12, 24 and 48 h postopera- } \\
\text { tively. Psychotomimetic AEs }\end{array}$ \\
\hline Surgery type & Upper abdominal surgery \\
\hline
\end{tabular}


Chen 2004 (Continued)

Group numbers after end

$20 / 20$

of study (treatment/con-

trol)

\begin{tabular}{ll}
$\begin{array}{l}\text { Age of patient population } \\
\text { (treatment/control) }\end{array}$ & Not mentioned \\
\hline Notes & Article in Chinese. No mention of sponsorship or funding
\end{tabular}

\section{Risk of bias}

\begin{tabular}{|c|c|c|}
\hline Bias & Authors' judgement & Support for judgement \\
\hline $\begin{array}{l}\text { Random sequence genera- } \\
\text { tion (selection bias) }\end{array}$ & Unclear risk & Only mentioned "randomised" \\
\hline $\begin{array}{l}\text { Allocation concealment } \\
\text { (selection bias) }\end{array}$ & Unclear risk & Allocation concealment method not described \\
\hline $\begin{array}{l}\text { Blinding of participants } \\
\text { and personnel (perfor- } \\
\text { mance bias) } \\
\text { All outcomes }\end{array}$ & Low risk & Blinded personnel \\
\hline $\begin{array}{l}\text { Blinding of outcome as- } \\
\text { sessment (detection bias) } \\
\text { All outcomes }\end{array}$ & Low risk & Blinded personnel \\
\hline $\begin{array}{l}\text { Incomplete outcome data } \\
\text { (attrition bias) } \\
\text { All outcomes }\end{array}$ & Unclear risk & Withdrawals not reported \\
\hline $\begin{array}{l}\text { Selective reporting (re- } \\
\text { porting bias) }\end{array}$ & High risk & Not all predefined outcomes reported \\
\hline Size & High risk & 20 participants per treatment arm \\
\hline
\end{tabular}

Choi 2015

\section{Study characteristics}

\begin{tabular}{ll}
\hline Methods & Randomised, double-blind \\
\hline Participants & $\mathrm{N}=75,100 \%$ women \\
\hline Interventions & Pre-incisional ketamine $0.5 \mathrm{mg} / \mathrm{kg}$ bolus IV followed by an infusion $5 \mu \mathrm{g} / \mathrm{kg} / \mathrm{min}$ \\
\hline Outcomes & $\begin{array}{l}\text { Analgesic consumption reported at 48 h postoperatively. Pain intensity (NRS) reported at 0, 1, } 6 \text { and } 24 \\
\text { h postoperatively. Time to first analgesic demand. Hyperalgesia (sensory threshold) }\end{array}$ \\
\hline $\begin{array}{l}\text { Surgery type } \\
\begin{array}{l}\text { Group numbers after end } \\
\text { of study (treatment/con- } \\
\text { trol) }\end{array}\end{array}$ & \begin{tabular}{l}
$25 / 25$ \\
\hline
\end{tabular}
\end{tabular}


Choi 2015 (Continued)

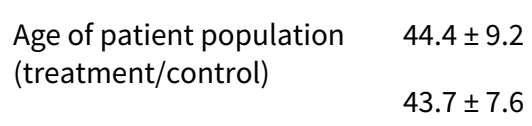

Notes No mention of sponsorship or funding

\section{Risk of bias}

\begin{tabular}{|c|c|c|}
\hline Bias & Authors' judgement & Support for judgement \\
\hline $\begin{array}{l}\text { Random sequence genera- } \\
\text { tion (selection bias) }\end{array}$ & Unclear risk & Only mentioned "randomised" \\
\hline $\begin{array}{l}\text { Allocation concealment } \\
\text { (selection bias) }\end{array}$ & Unclear risk & Allocation concealment method not described \\
\hline $\begin{array}{l}\text { Blinding of participants } \\
\text { and personnel (perfor- } \\
\text { mance bias) } \\
\text { All outcomes }\end{array}$ & Unclear risk & Not described \\
\hline $\begin{array}{l}\text { Blinding of outcome as- } \\
\text { sessment (detection bias) } \\
\text { All outcomes }\end{array}$ & Low risk & Blinded investigator \\
\hline $\begin{array}{l}\text { Incomplete outcome data } \\
\text { (attrition bias) } \\
\text { All outcomes }\end{array}$ & Low risk & No withdrawals \\
\hline $\begin{array}{l}\text { Selective reporting (re- } \\
\text { porting bias) }\end{array}$ & Low risk & Predefined outcomes reported \\
\hline Size & High risk & 25 participants per treatment arm \\
\hline
\end{tabular}

\section{Colombani 2008}

\section{Study characteristics}

\begin{tabular}{ll}
\hline Methods & Randomised, double-blind, placebo control \\
\hline Participants & $\mathrm{N}=208,100 \%$ women \\
\hline Interventions & $\begin{array}{l}\text { Pre-incisional ketamine } 0.15 \mathrm{mg} / \mathrm{kg} \text { bolus IV followed by an infusion } 2 \mu \mathrm{g} / \mathrm{kg} / \mathrm{min} \text { until the end of } \\
\text { surgery }\end{array}$ \\
\hline Outcomes & $\begin{array}{l}\text { Analgesic consumption reported at 48 h postoperatively. Pain intensity (VAS), reported as participant } \\
\text { proportion with VAS score }>4\end{array}$ \\
\hline $\begin{array}{l}\text { Surgery type } \\
\text { Breast surgery (partial resection of breast with axillary lymph node evacuation or mastectomy with or } \\
\text { without axillary lymph node evacuation) }\end{array}$ \\
$\begin{array}{l}\text { Group numbers after end } \\
\text { of study (treatment/con- } \\
\text { trol) }\end{array}$ & \begin{tabular}{l} 
106/102 \\
\hline
\end{tabular}
\end{tabular}


Colombani 2008 (Continued)

Age of patient population (treatment/control)

\section{$57.6 \pm 12.5$}

$59 \pm 11.4$

\section{Notes}

Article in French. Only participant proportion (\%) with VAS score > 4 reported. No mention of sponsorship or funding

\section{Risk of bias}

\begin{tabular}{|c|c|c|}
\hline Bias & Authors' judgement & Support for judgement \\
\hline $\begin{array}{l}\text { Random sequence genera- } \\
\text { tion (selection bias) }\end{array}$ & Low risk & Randomisation list generated at the department of biostatistics \\
\hline $\begin{array}{l}\text { Allocation concealment } \\
\text { (selection bias) }\end{array}$ & Unclear risk & Allocation concealment method not described in detail \\
\hline $\begin{array}{l}\text { Blinding of participants } \\
\text { and personnel (perfor- } \\
\text { mance bias) } \\
\text { All outcomes }\end{array}$ & Low risk & Blinded participants and personnel \\
\hline $\begin{array}{l}\text { Blinding of outcome as- } \\
\text { sessment (detection bias) } \\
\text { All outcomes }\end{array}$ & Low risk & Blinded participants and personnel \\
\hline $\begin{array}{l}\text { Incomplete outcome data } \\
\text { (attrition bias) } \\
\text { All outcomes }\end{array}$ & Low risk & $5 \%$ was withdrawn \\
\hline $\begin{array}{l}\text { Selective reporting (re- } \\
\text { porting bias) }\end{array}$ & Low risk & Predefined outcomes reported \\
\hline Size & Unclear risk & 106 and 102 participants per treatment arm \\
\hline
\end{tabular}

Crousier 2008

\section{Study characteristics}

\begin{tabular}{ll}
\hline Methods & Randomised, double-blind, placebo control \\
\hline Participants & $\mathrm{N}=36,100 \%$ women \\
\hline Interventions & Pre-incisional ketamine $0.5 \mathrm{mg} / \mathrm{kg}$ bolus IV followed by an infusion 0.25 mg/kg/h until wound closure \\
\hline Outcomes & $\begin{array}{l}\text { Pain intensity (VAS) and analgesic consumption reported at 24 h. AEs. Hyperalgesia on 5th postopera- } \\
\text { tive day and after 3 months }\end{array}$ \\
\hline $\begin{array}{l}\text { Surgery type } \\
\begin{array}{l}\text { Group numbers after end } \\
\text { of study (treatment/con- } \\
\text { trol) }\end{array}\end{array}$ & \begin{tabular}{l} 
Mastectomy \\
\hline
\end{tabular}
\end{tabular}

Age of patient population
(treatment/control)


Crousier 2008 (Continued)

$$
49 \pm 12
$$

Notes No mention of sponsorship or funding

\section{Risk of bias}

\begin{tabular}{|c|c|c|}
\hline Bias & Authors' judgement & Support for judgement \\
\hline $\begin{array}{l}\text { Random sequence genera- } \\
\text { tion (selection bias) }\end{array}$ & Unclear risk & Randomisation process not described \\
\hline $\begin{array}{l}\text { Allocation concealment } \\
\text { (selection bias) }\end{array}$ & Unclear risk & Allocation concealment method not described \\
\hline $\begin{array}{l}\text { Blinding of participants } \\
\text { and personnel (perfor- } \\
\text { mance bias) } \\
\text { All outcomes }\end{array}$ & Unclear risk & Only mentioned double-blind \\
\hline $\begin{array}{l}\text { Blinding of outcome as- } \\
\text { sessment (detection bias) } \\
\text { All outcomes }\end{array}$ & Unclear risk & Only mentioned double-blind \\
\hline $\begin{array}{l}\text { Incomplete outcome data } \\
\text { (attrition bias) } \\
\text { All outcomes }\end{array}$ & Low risk & $3 \%$ was withdrawn \\
\hline $\begin{array}{l}\text { Selective reporting (re- } \\
\text { porting bias) }\end{array}$ & High risk & $\begin{array}{l}\text { Pain score measures at certain time points predefined in methods but only av- } \\
\text { erage pain scores in the postoperative period reported }\end{array}$ \\
\hline Size & High risk & 18 participants per treatment arm \\
\hline
\end{tabular}

\section{D'Alonzo 2011}

\section{Study characteristics}

\begin{tabular}{ll}
\hline Methods & Randomised, double-blind, placebo control \\
\hline Participants & $\mathrm{N}=40$, about 43\% women \\
\hline Interventions & Ketamine $0.5 \mathrm{mg} / \mathrm{kg}$ bolus IV prior to chest wall incision \\
\hline Outcomes & Pain intensity (NRS). Reported at baseline, 4 and 24 h postoperatively \\
\hline $\begin{array}{l}\text { Surgery type } \\
\text { Group numbers after end } \\
\text { of study (treatment/con- } \\
\text { trol) }\end{array}$ & $20 / 20$ \\
\hline $\begin{array}{l}\text { Age of patient population } \\
\text { (treatment/control) }\end{array}$ & $61 \pm 12$ \\
\hline $\begin{array}{l}\text { Notes } \\
\text { Video assisted thoracoscopic surgery or thoracotomy }\end{array}$ \\
\hline
\end{tabular}


D'Alonzo 2011 (Continued)

Risk of bias

\begin{tabular}{|c|c|c|}
\hline Bias & Authors' judgement & Support for judgement \\
\hline $\begin{array}{l}\text { Random sequence genera- } \\
\text { tion (selection bias) }\end{array}$ & Low risk & Computer-generated randomisation list \\
\hline $\begin{array}{l}\text { Allocation concealment } \\
\text { (selection bias) }\end{array}$ & Unclear risk & Allocation concealment method not described in detail \\
\hline $\begin{array}{l}\text { Blinding of participants } \\
\text { and personnel (perfor- } \\
\text { mance bias) } \\
\text { All outcomes }\end{array}$ & Low risk & Blinded personnel \\
\hline $\begin{array}{l}\text { Blinding of outcome as- } \\
\text { sessment (detection bias) } \\
\text { All outcomes }\end{array}$ & Low risk & Blinded personnel \\
\hline $\begin{array}{l}\text { Incomplete outcome data } \\
\text { (attrition bias) } \\
\text { All outcomes }\end{array}$ & Low risk & No withdrawals \\
\hline $\begin{array}{l}\text { Selective reporting (re- } \\
\text { porting bias) }\end{array}$ & Unclear risk & $\begin{array}{l}\text { Predefined outcomes reported but the anaesthetic procedure was left to the } \\
\text { discretion of the anaesthesiologist; general anaesthesia was supplemented by } \\
\text { an epidural catheter placement as needed to control pain (16 participants in } \\
\text { the treatment group, } 19 \text { participants in the control group) }\end{array}$ \\
\hline Size & High risk & 20 participants per treatment arm \\
\hline
\end{tabular}

Dahi-Taleghani 2014

\section{Study characteristics}

\begin{tabular}{ll}
\hline Methods & Randomised, double-blind, placebo control \\
\hline Participants & $\mathrm{N}=140,100 \%$ men \\
\hline Interventions & Postoperative ketamine $2 \mathrm{mg}+$ morphine $2 \mathrm{mg}$ via PCA \\
\hline Outcomes & $\begin{array}{l}\text { Pain intensity (VAS) reported at 1, } 6 \text { and } 24 \mathrm{~h} \text { postoperatively. Analgesic consumption reported at 24 h } \\
\text { postoperatively. AEs }\end{array}$ \\
\hline $\begin{array}{l}\text { Surgery type } \\
\begin{array}{l}\text { Group numbers after end } \\
\text { of study (treatment/con- } \\
\text { trol) }\end{array}\end{array}$ & \begin{tabular}{l}
$70 / 70$ \\
\hline
\end{tabular}
\end{tabular}

\section{Age of patient population (treatment/control)}

$39.1 \pm 7.2$

$38.3 \pm 7.5$

Notes

The study was supported financially in part by a research grant from the Anesthesiology Research Center, Shadid Beheshti University of Medical Sciences, Tehran, Iran 
Dahi-Taleghani 2014 (Continued)

Risk of bias

\begin{tabular}{lll}
\hline Bias & Authors' judgement & Support for judgement \\
\hline $\begin{array}{l}\text { Random sequence genera- } \\
\text { tion (selection bias) }\end{array}$ & Low risk & Computer-generated random number table \\
\hline $\begin{array}{l}\text { Allocation concealment } \\
\text { (selection bias) }\end{array}$ & Unclear risk & Allocation concealment method not described \\
\hline $\begin{array}{l}\text { Blinding of participants } \\
\text { and personnel (perfor- } \\
\text { mance bias) }\end{array}$ & Unclear risk & Only mentioned double-blind \\
All outcomes & \\
\hline $\begin{array}{l}\text { Blinding of outcome as- } \\
\text { sessment (detection bias) }\end{array}$ & Unclear risk & Only mentioned double-blind \\
All outcomes & & \\
\hline $\begin{array}{l}\text { Incomplete outcome data } \\
\text { (attrition bias) }\end{array}$ & Low risk & No withdrawals \\
All outcomes & & \\
\hline $\begin{array}{l}\text { Selective reporting (re- } \\
\text { porting bias) }\end{array}$ & Low risk & All predefined outcomes reported \\
\hline \begin{tabular}{l} 
Size \\
\hline
\end{tabular} & Unclear risk & 70 participants per treatment arm \\
\hline
\end{tabular}

\section{Dahl 2000}

\section{Study characteristics}

\begin{tabular}{ll}
\hline Methods & Randomised, double-blind, placebo control \\
\hline Participants & $\mathrm{N}=89,100 \%$ women \\
\hline Interventions & $\begin{array}{l}\text { 1. Ketamine bolus 0.4 mg/kg IV prior to skin incision } \\
\text { 2. Ketamine bolus 0.4 mg/kg IV at skin closure }\end{array}$ \\
\hline Outcomes & $\begin{array}{l}\text { Pain intensity (VAS, VRS) reported every } 1 \text { h up to } 6 \text { h postoperatively, then 6-24 and 24-96 h. Analgesic } \\
\text { consumption at 0-6, 6-24 and 24-48 h postoperatively. Level of activity. AEs }\end{array}$ \\
\hline $\begin{array}{l}\text { Surgery type } \\
\text { Group numbers after end } \\
\text { of study (treatment/con- } \\
\text { trol) }\end{array}$ & $60 / 29$ \\
\hline $\begin{array}{l}\text { Age of patient population } \\
\text { (treatment/control) }\end{array}$ & $50.1 \pm 5.3$ \\
\hline $\begin{array}{l}\text { Notes } \\
48 \pm 7\end{array}$ \\
\hline
\end{tabular}

\section{Risk of bias}


Dahl 2000 (Continued)

\begin{tabular}{|c|c|c|}
\hline Bias & Authors' judgement & Support for judgement \\
\hline $\begin{array}{l}\text { Random sequence genera- } \\
\text { tion (selection bias) }\end{array}$ & Low risk & Computer-generated table of random numbers \\
\hline $\begin{array}{l}\text { Allocation concealment } \\
\text { (selection bias) }\end{array}$ & Unclear risk & Allocation concealment method not described in detail \\
\hline $\begin{array}{l}\text { Blinding of participants } \\
\text { and personnel (perfor- } \\
\text { mance bias) } \\
\text { All outcomes }\end{array}$ & Unclear risk & Blinding process not described \\
\hline $\begin{array}{l}\text { Blinding of outcome as- } \\
\text { sessment (detection bias) } \\
\text { All outcomes }\end{array}$ & Unclear risk & Blinding process not described \\
\hline $\begin{array}{l}\text { Incomplete outcome data } \\
\text { (attrition bias) } \\
\text { All outcomes }\end{array}$ & Low risk & $10 \%$ was withdrawn \\
\hline $\begin{array}{l}\text { Selective reporting (re- } \\
\text { porting bias) }\end{array}$ & Low risk & Predefined outcomes reported \\
\hline Size & High risk & $\begin{array}{l}33 \text { and } 27 \text { participants per treatment arm and } 29 \text { participants in the control } \\
\text { group }\end{array}$ \\
\hline
\end{tabular}

\section{Dal 2005}

\section{Study characteristics}

\begin{tabular}{ll}
\hline Methods & Randomised, double-blind, placebo control \\
\hline Participants & $\mathrm{N}=90$, of whom 60 participants in IV ketamine and control treatment arms. 78\% women \\
\hline Interventions & Ketamine $0.5 \mathrm{mg} / \mathrm{kg}$ bolus IV 20 mins before the end of surgery \\
\hline Outcomes & $\begin{array}{l}\text { Postoperative shivering. Pain intensity (VAS) reported on arrival in the recovery room and at 1st and } \\
\text { 2nd h postoperatively. Time to first analgesic requirement. AEs }\end{array}$ \\
\hline $\begin{array}{l}\text { Surgery type } \\
\text { Various procedures. General anaesthesia for an anticipated duration of 60-180 mins excluding proce- } \\
\text { dures that might require administration of blood or blood products and urological endoscopic opera- } \\
\text { tions }\end{array}$ \\
\hline $\begin{array}{l}\text { Group numbers after end } \\
\text { of study (treatment/con- } \\
\text { trol) }\end{array}$ \\
$\begin{array}{l}\text { Age of patient population } \\
\text { (treatment/control) }\end{array}$ & $\begin{array}{l}\text { 45 (21-66) } \\
\text { 418-65) }\end{array}$ \\
\hline \begin{tabular}{l} 
Notes \\
\hline
\end{tabular}
\end{tabular}

\section{Risk of bias}


Dal 2005 (Continued)

\begin{tabular}{|c|c|c|}
\hline Bias & Authors' judgement & Support for judgement \\
\hline $\begin{array}{l}\text { Random sequence genera- } \\
\text { tion (selection bias) }\end{array}$ & Unclear risk & Randomisation not described \\
\hline $\begin{array}{l}\text { Allocation concealment } \\
\text { (selection bias) }\end{array}$ & Unclear risk & Allocation concealment method not described \\
\hline $\begin{array}{l}\text { Blinding of participants } \\
\text { and personnel (perfor- } \\
\text { mance bias) } \\
\text { All outcomes }\end{array}$ & Low risk & Blinded personnel \\
\hline $\begin{array}{l}\text { Blinding of outcome as- } \\
\text { sessment (detection bias) } \\
\text { All outcomes }\end{array}$ & Low risk & Blinded personnel \\
\hline $\begin{array}{l}\text { Incomplete outcome data } \\
\text { (attrition bias) } \\
\text { All outcomes }\end{array}$ & Low risk & No withdrawals \\
\hline $\begin{array}{l}\text { Selective reporting (re- } \\
\text { porting bias) }\end{array}$ & Low risk & Predefined outcomes reported adequately \\
\hline Size & High risk & 30 participants per treatment arm \\
\hline
\end{tabular}

Dar 2012

\section{Study characteristics}

\begin{tabular}{ll}
\hline Methods & Randomised, double-blind, placebo control \\
\hline Participants & $\mathrm{N}=90$, of whom 60 participants in IV ketamine and control treatment arms. 30\% women \\
\hline Interventions & Ketamine $0.5 \mathrm{mg} / \mathrm{kg}$ bolus IV 20 mins before the end of surgery. Various surgical procedures \\
\hline Outcomes & Postoperative shivering, pain intensity (VAS), AEs, vital parameters. Pain intensity not reported \\
\hline Surgery type & $\begin{array}{l}\text { Various procedures under general anaesthesia with an anticipated duration of 60-180 mins excluding } \\
\text { urological endoscopic operations }\end{array}$ \\
\hline $\begin{array}{l}\text { Group numbers after end } \\
\text { of study (treatment/con- } \\
\text { trol) }\end{array}$ & $30 / 30$ \\
\hline
\end{tabular}

Age of patient population
(treatment/control)
$36.63 \pm 1.2$

$38.7 \pm 1.7$

Notes No mention of sponsorship or funding

\section{Risk of bias}

Bias Authors' judgement Support for judgement


Dar 2012 (Continued)
Random sequence genera- Unclear risk
Randomisation process not described in detail tion (selection bias)

\begin{tabular}{ll}
\hline $\begin{array}{l}\text { Allocation concealment } \\
\text { (selection bias) }\end{array}$ & $\begin{array}{l}\text { Quote: "Study drugs were prepared, diluted to a volume of } 5 \text { ml and present- } \\
\text { ed as coded syringes by an anaesthetist who was not involved in the manage- } \\
\text { ment of the patients" }\end{array}$ \\
\hline
\end{tabular}

Blinding of participants $\quad$ Low risk
and personnel (perfor-
mance bias)

\begin{tabular}{|c|c|c|}
\hline $\begin{array}{l}\text { Blinding of outcome as- } \\
\text { sessment (detection bias) } \\
\text { All outcomes }\end{array}$ & Low risk & A blinded anaesthetist made assessments \\
\hline $\begin{array}{l}\text { Incomplete outcome data } \\
\text { (attrition bias) } \\
\text { All outcomes }\end{array}$ & Low risk & No withdrawals \\
\hline $\begin{array}{l}\text { Selective reporting (re- } \\
\text { porting bias) }\end{array}$ & High risk & $\begin{array}{l}\text { Predefined VAS scores are not reported. Mean time to rescue analgesia is re- } \\
\text { ported even though not predefined in methods. }\end{array}$ \\
\hline Size & High risk & 30 participants per treatment arm \\
\hline
\end{tabular}

De Kock 2001

\section{Study characteristics}

\begin{tabular}{ll}
\hline Methods & Randomised, double-blind, placebo control \\
\hline Participants & $\mathrm{N}=100$, of whom 60 participants in IV ketamine or control treatment arms. About $48 \%$ women \\
\hline Interventions & $\begin{array}{l}\text { 1. } \pm 30 \mathrm{~min} \text { before skin incision: ketamine bolus } 0.25 \mathrm{mg} / \mathrm{kg} \mathrm{IV}+\mathrm{infusion} 0.125 \mathrm{mg} / \mathrm{kg} / \mathrm{h} \mathrm{until} \mathrm{end} \mathrm{of} \\
\text { surgery }\end{array}$ \\
$\begin{array}{l}\text { 2. } \pm 30 \mathrm{~min} \text { before skin incision: ketamine bolus } 0.5 \mathrm{mg} / \mathrm{kg} \mathrm{IV} \mathrm{+} \mathrm{infusion} 0.25 \mathrm{mg} / \mathrm{kg} / \mathrm{h} \mathrm{IV} \mathrm{until} \mathrm{skin} \mathrm{closure} \\
\text { 3. Ketamine } 0.25 \mathrm{mg} / \mathrm{kg} \text { bolus } \mathrm{ED}+\text { infusion } 0.125 \mathrm{mg} / \mathrm{kg} / \mathrm{h} \text { ED until end of surgery } \\
\text { 4. Ketamine } 0.5 \mathrm{mg} / \mathrm{kg} \text { bolus ED + infusion } 0.25 \mathrm{mg} / \mathrm{kg} / \mathrm{h} \mathrm{ED} \mathrm{until} \mathrm{end} \mathrm{of} \mathrm{surgery}\end{array}$
\end{tabular}

\section{Outcomes}

Pain intensity (VAS) reported at $15 \mathrm{~min}, 2,6,12,24,36$ and $48 \mathrm{~h}$ postoperatively. Area of hyperalgesia reported at 24, 48 and $72 \mathrm{~h}$ postoperatively. Postoperative residual pain at 2 weeks, 1 and 6 months, 1 year. Cumulative number of met and unmet PCA morphine demands. AEs

\begin{tabular}{ll}
\hline Surgery type & Rectal adenocarcinoma surgery \\
\hline $\begin{array}{l}\text { Group numbers after end } \\
\text { of study (treatment/con- } \\
\text { trol) }\end{array}$ & $40 / 20$ \\
\hline $\begin{array}{l}\text { Age of patient population } \\
\text { (treatment/control) }\end{array}$ & $67 \pm 8.4$ \\
\hline Notes & $67 \pm 9$ \\
\hline
\end{tabular}


De Kock 2001 (Continued)

Risk of bias

\begin{tabular}{|c|c|c|}
\hline Bias & Authors' judgement & Support for judgement \\
\hline $\begin{array}{l}\text { Random sequence genera- } \\
\text { tion (selection bias) }\end{array}$ & Low risk & A computer-generated table of random numbers \\
\hline $\begin{array}{l}\text { Allocation concealment } \\
\text { (selection bias) }\end{array}$ & Unclear risk & Allocation concealment method not described in detail \\
\hline $\begin{array}{l}\text { Blinding of participants } \\
\text { and personnel (perfor- } \\
\text { mance bias) } \\
\text { All outcomes }\end{array}$ & Low risk & Blinded personnel \\
\hline $\begin{array}{l}\text { Blinding of outcome as- } \\
\text { sessment (detection bias) } \\
\text { All outcomes }\end{array}$ & Low risk & Blinded personnel \\
\hline $\begin{array}{l}\text { Incomplete outcome data } \\
\text { (attrition bias) } \\
\text { All outcomes }\end{array}$ & Low risk & $\begin{array}{l}\text { No withdrawals in the immediate postoperative period. } 8 \% \text { of participants } \\
\text { died during the one-year follow up }\end{array}$ \\
\hline $\begin{array}{l}\text { Selective reporting (re- } \\
\text { porting bias) }\end{array}$ & Low risk & Predefined outcomes reported \\
\hline Size & High risk & 20 participants per treatment arm \\
\hline
\end{tabular}

Deng 2009

\section{Study characteristics}

\begin{tabular}{|c|c|}
\hline Methods & Randomised, double-blind, placebo control \\
\hline Participants & $\mathrm{N}=200$, about $43 \%$ women \\
\hline \multirow[t]{3}{*}{ Interventions } & $\begin{array}{l}\text { 1. Pre-incisional ketamine } 0.5 \mathrm{mg} / \mathrm{kg} \text { bolus IV }+0.1 \mathrm{mg} / \mathrm{kg} / \mathrm{h} \text { infusion IV during surgery and for } 24 \mathrm{~h} \text { post- } \\
\text { operatively }\end{array}$ \\
\hline & $\begin{array}{l}\text { 2. Pre-incisional ketamine } 0.5 \mathrm{mg} / \mathrm{kg} \text { bolus IV }+0.05 \mathrm{mg} / \mathrm{kg} / \mathrm{h} \text { infusion IV during surgery and for } 24 \mathrm{~h} \\
\text { postoperatively }\end{array}$ \\
\hline & $\begin{array}{l}\text { 3. Pre-incisional ketamine } 0.5 \mathrm{mg} / \mathrm{kg} \text { bolus IV }+0.01 \mathrm{mg} / \mathrm{kg} / \mathrm{h} \text { infusion IV during surgery and for } 24 \mathrm{~h} \\
\text { postoperatively }\end{array}$ \\
\hline Outcomes & $\begin{array}{l}\text { Pain intensity (VAS) reported at } 24 \mathrm{~h} \text { postoperatively. PCA remifentanil consumption, reported at 0-12 } \\
\mathrm{h}, 12-24 \mathrm{~h} \text { and } 0-24 \mathrm{~h} \text { postoperatively. AEs. Participant satisfaction with analgesia }\end{array}$ \\
\hline Surgery type & Lower limb fracture operation \\
\hline $\begin{array}{l}\text { Group numbers after end } \\
\text { of study (treatment/con- } \\
\text { trol) }\end{array}$ & $150 / 50$ \\
\hline
\end{tabular}

$49.6 \pm 5.6$

$50.1 \pm 6.3$ 
Deng 2009 (Continued)
Notes
No mention of sponsorship or funding

\section{Risk of bias}

\begin{tabular}{lll}
\hline Bias & Authors' judgement & Support for judgement \\
\hline $\begin{array}{l}\text { Random sequence genera- } \\
\text { tion (selection bias) }\end{array}$ & Unclear risk & Randomisation method not described \\
\hline $\begin{array}{l}\text { Allocation concealment } \\
\text { (selection bias) }\end{array}$ & Unclear risk & Allocation concealment method not described in detail \\
\hline $\begin{array}{l}\text { Blinding of participants } \\
\text { and personnel (perfor- } \\
\text { mance bias) }\end{array}$ & Low risk & Blinded participants and personnel \\
All outcomes & \\
\hline $\begin{array}{l}\text { Blinding of outcome as- } \\
\text { sessment (detection bias) }\end{array}$ & Low risk & Blinded personnel \\
All outcomes & & \\
\hline $\begin{array}{l}\text { Incomplete outcome data } \\
\text { (attrition bias) } \\
\text { All outcomes }\end{array}$ & Unclear risk & Withdrawals not reported \\
\hline $\begin{array}{l}\text { Selective reporting (re- } \\
\text { porting bias) }\end{array}$ & Low risk & Predefined outcomes addressed \\
\hline \begin{tabular}{l} 
Size \\
\hline
\end{tabular} & Unclear risk & 50 participants per treatment arm \\
\hline
\end{tabular}

Du 2011

\section{Study characteristics}

\begin{tabular}{ll}
\hline Methods & Randomised, double-blind, placebo control \\
\hline Participants & Preinductional ketamine $0.25 \mathrm{mg} / \mathrm{kg}$ bolus IV \\
\hline Interventions & $\begin{array}{l}\text { Pain intensity (VAS) and analgesic consumption reported at } 15 \text { min intervals during the first postopera- } \\
\text { tive hour. AEs }\end{array}$ \\
\hline $\begin{array}{l}\text { Outcomes } \\
\text { Surgery type }\end{array}$ & Gynaecological laparoscopic surgery \\
\hline $\begin{array}{l}\text { Group numbers after end } \\
\text { of study (treatment/con- } \\
\text { trol) }\end{array}$ & 20/20. Power analysis calculated based on the primary outcome (serum glucose level) \\
\hline
\end{tabular}

Age of patient population
(treatment/control)

$39.1 \pm 11.5$

\section{$35.4 \pm 7.9$}

Main outcome was endocrine metabolic and inflammatory responses to preoperative low-dose ketamine. No mention of sponsorship or funding 
Du 2011 (Continued)

\section{Risk of bias}

\begin{tabular}{|c|c|c|}
\hline Bias & Authors' judgement & Support for judgement \\
\hline $\begin{array}{l}\text { Random sequence genera- } \\
\text { tion (selection bias) }\end{array}$ & Low risk & Computer-generated table of random numbers \\
\hline $\begin{array}{l}\text { Allocation concealment } \\
\text { (selection bias) }\end{array}$ & Unclear risk & Allocation concealment method not described in detail \\
\hline $\begin{array}{l}\text { Blinding of participants } \\
\text { and personnel (perfor- } \\
\text { mance bias) } \\
\text { All outcomes }\end{array}$ & Low risk & Blinded personnel \\
\hline $\begin{array}{l}\text { Blinding of outcome as- } \\
\text { sessment (detection bias) } \\
\text { All outcomes }\end{array}$ & Low risk & Blinded outcome assessors \\
\hline $\begin{array}{l}\text { Incomplete outcome data } \\
\text { (attrition bias) } \\
\text { All outcomes }\end{array}$ & Low risk & No withdrawals \\
\hline $\begin{array}{l}\text { Selective reporting (re- } \\
\text { porting bias) }\end{array}$ & Low risk & Predefined outcomes reported \\
\hline Size & High risk & 20 participants per treatment arm \\
\hline
\end{tabular}

Dualé 2009

\section{Study characteristics}

\begin{tabular}{|c|c|}
\hline Methods & Randomised, double-blind, placebo control \\
\hline Participants & $\mathrm{N}=86$, about $30 \%$ women \\
\hline Interventions & Ketamine $1 \mathrm{mg} / \mathrm{kg}$ bolus IV at induction $+1 \mathrm{mg} / \mathrm{kg} / \mathrm{h}$ infusion IV \\
\hline Outcomes & $\begin{array}{l}\text { Pain intensity (VAS) reported on arrival in the PACU and } 1,2,4,8,12,16,24,62,40 \text { and } 48 \text { h postopera- } \\
\text { tively. Analgesic consumption reported at } 24 \text { and } 48 \mathrm{~h} \text { postoperatively. AEs. NPSI scores reported at } 6 \\
\text { weeks and } 4 \text { months postoperatively }\end{array}$ \\
\hline Surgery type & Thoracotomy \\
\hline $\begin{array}{l}\text { Group numbers after end } \\
\text { of study (treatment/con- } \\
\text { trol) }\end{array}$ & $42 / 44$ \\
\hline \multirow{2}{*}{$\begin{array}{l}\text { Age of patient population } \\
\text { (treatment/control) }\end{array}$} & $61.9 \pm 8.3$ \\
\hline & $58.5 \pm 8.5$ \\
\hline Notes & No financial arrangements that may represent a conflict of interest \\
\hline
\end{tabular}

\section{Risk of bias}


Dualé 2009 (Continued)

\begin{tabular}{lll} 
Bias & Authors' judgement & Support for judgement \\
\hline $\begin{array}{l}\text { Random sequence genera- } \\
\text { tion (selection bias) }\end{array}$ & Unclear risk & Randomisation process not described in detail \\
\hline $\begin{array}{l}\text { Allocation concealment } \\
\text { (selection bias) }\end{array}$ & Low risk & $\begin{array}{l}\text { Quote: "An inclusion number was allocated randomly and kept in a sealed en- } \\
\text { velope." }\end{array}$ \\
\hline $\begin{array}{l}\text { Blinding of participants } \\
\text { and personnel (perfor- } \\
\text { mance bias) }\end{array}$ & Low risk & Blinded personnel \\
All outcomes & \\
\hline $\begin{array}{l}\text { Blinding of outcome as- } \\
\text { sessment (detection bias) } \\
\text { All outcomes }\end{array}$ & Low risk & Blinded personnel \\
\hline $\begin{array}{l}\text { Incomplete outcome data } \\
\text { (attrition bias) } \\
\text { All outcomes }\end{array}$ & Low risk & 5\% was withdrawn \\
\hline $\begin{array}{l}\text { Selective reporting (re- } \\
\text { porting bias) }\end{array}$ & Low risk & Predefined outcomes reported adequately \\
\hline \begin{tabular}{l} 
Size \\
\hline
\end{tabular} & High risk & 42 and 44 participants per treatment arm \\
\hline
\end{tabular}

Dullenkopf 2009

\section{Study characteristics}

\begin{tabular}{|c|c|}
\hline Methods & Randomised, double-blind, placebo control \\
\hline Participants & $N=120 ;$ about $59 \%$ women \\
\hline Interventions & $\begin{array}{l}\text { 1. Pre-incisional ketamine } 0.15 \mathrm{mg} / \mathrm{kg} \text { bolus IV } \\
\text { 2. Pre-incisional ketamine } 0.5 \mathrm{mg} / \mathrm{kg} \text { bolus IV }\end{array}$ \\
\hline Outcomes & $\begin{array}{l}\text { Pain intensity (VAS) at arrival in PACU and at } 3 \text { months postoperatively. Analgesic consumption report- } \\
\text { ed at } 24 \mathrm{~h} \text { postoperatively. AEs }\end{array}$ \\
\hline Surgery type & $\begin{array}{l}\text { General surgical or orthopaedic operation anticipated to last } 30 \text { to } 90 \text { mins and assumed hospital stay } \\
\text { of } 48 \mathrm{~h}\end{array}$ \\
\hline $\begin{array}{l}\text { Group numbers after end } \\
\text { of study (treatment/con- } \\
\text { trol) }\end{array}$ & $77 / 33$ \\
\hline $\begin{array}{l}\text { Age of patient population } \\
\text { (treatment/control) }\end{array}$ & $\begin{array}{l}52.6 \pm 18 \\
52.3 \pm 17.9\end{array}$ \\
\hline Notes & Basis for group size unclear (power analysis not presented). No mention of sponsorship or funding \\
\hline Risk of bias & \\
\hline
\end{tabular}


Dullenkopf 2009 (Continued)

\begin{tabular}{|c|c|c|}
\hline Bias & Authors' judgement & Support for judgement \\
\hline $\begin{array}{l}\text { Random sequence genera- } \\
\text { tion (selection bias) }\end{array}$ & Low risk & Computer-generated block randomisation \\
\hline $\begin{array}{l}\text { Allocation concealment } \\
\text { (selection bias) }\end{array}$ & Low risk & $\begin{array}{l}\text { Study solutions prepared and blinded by a hospital pharmacist (a third party). } \\
\text { Syringes containing study drugs identical in appearance }\end{array}$ \\
\hline $\begin{array}{l}\text { Blinding of participants } \\
\text { and personnel (perfor- } \\
\text { mance bias) } \\
\text { All outcomes }\end{array}$ & Low risk & Blinded personnel \\
\hline $\begin{array}{l}\text { Blinding of outcome as- } \\
\text { sessment (detection bias) } \\
\text { All outcomes }\end{array}$ & Low risk & Blinded personnel \\
\hline $\begin{array}{l}\text { Incomplete outcome data } \\
\text { (attrition bias) } \\
\text { All outcomes }\end{array}$ & Low risk & $8 \%$ was withdrawn \\
\hline $\begin{array}{l}\text { Selective reporting (re- } \\
\text { porting bias) }\end{array}$ & Low risk & Predefined outcomes reported \\
\hline Size & High risk & 36,41 and 33 participants per treatment arm \\
\hline
\end{tabular}

Fiorelli 2015

\section{Study characteristics}

\begin{tabular}{ll}
\hline Methods & Randomised, double-blind, placebo control \\
\hline Participants & $\mathrm{N}=75,27 \%$ women \\
\hline Interventions & Ketamine $1 \mathrm{mg} / \mathrm{kg}$ bolus IV before thoracotomy \\
\hline Outcomes & $\begin{array}{l}\text { Pain intensity (VAS) and morphine consumption reported at 6, 12, 24, 36 and 48 h postoperatively. AEs. } \\
\text { Inflammatory response }\end{array}$ \\
\hline $\begin{array}{l}\text { Surgery type } \\
\begin{array}{l}\text { Group numbers after end } \\
\text { of study (treatment/con- } \\
\text { trol) }\end{array}\end{array}$ & Thoracotomy \\
\hline
\end{tabular}

Age of patient population
(treatment/control)

$59.5 \pm 15.3$

$58.6 \pm 17.4$
No mention of sponsorship or funding

\section{Risk of bias}

Bias Authors' judgement Support for judgement


Fiorelli 2015 (Continued)

\begin{tabular}{lll}
$\begin{array}{l}\text { Random sequence genera- } \\
\text { tion (selection bias) }\end{array}$ & Low risk & Computer-generated randomisation list \\
\hline $\begin{array}{l}\text { Allocation concealment } \\
\text { (selection bias) }\end{array}$ & Unclear risk & Allocation concealment method not described in detail \\
\hline $\begin{array}{l}\text { Blinding of participants } \\
\text { and personnel (perfor- } \\
\begin{array}{l}\text { mance bias) } \\
\text { All outcomes }\end{array}\end{array}$ & Low risk & Blinded personnel \\
\hline
\end{tabular}

\begin{tabular}{lll}
\hline $\begin{array}{l}\text { Blinding of outcome as- } \\
\text { sessment (detection bias) } \\
\text { All outcomes }\end{array}$ & Low risk & Blinded personnel \\
\hline $\begin{array}{l}\text { Incomplete outcome data } \\
\text { (attrition bias) } \\
\text { All outcomes }\end{array}$ & Low risk & $7 \%$ was withdrawn \\
\hline $\begin{array}{l}\text { Selective reporting (re- } \\
\text { porting bias) }\end{array}$ & Low risk & All predefined outcomes reported \\
\hline Size & High risk & 38 and 37 participants per treatment arm \\
\hline
\end{tabular}

\section{Galinski 2007}

\section{Study characteristics}

\begin{tabular}{|c|c|c|}
\hline Methods & \multicolumn{2}{|c|}{ Randomised, double-blind. Ketamine + dexamethasone vs dexamethasone } \\
\hline Participants & \multicolumn{2}{|c|}{$\mathrm{N}=65$, about $26 \%$ women } \\
\hline Interventions & \multicolumn{2}{|c|}{ Pre-incisional ketamine $0.5 \mathrm{mg} / \mathrm{kg}$ bolus IV } \\
\hline Outcomes & \multicolumn{2}{|c|}{ Pain intensity (VAS) and analgesic consumption, reported at 15 and 30 min postoperatively. AEs } \\
\hline Surgery type & \multicolumn{2}{|l|}{ Inguinal hernia repair } \\
\hline $\begin{array}{l}\text { Group numbers after end } \\
\text { of study (treatment/con- } \\
\text { trol) }\end{array}$ & \multicolumn{2}{|l|}{$20 / 20$} \\
\hline $\begin{array}{l}\text { Age of patient population } \\
\text { (treatment/control) }\end{array}$ & \multicolumn{2}{|l|}{$47.55 \pm 17.46$} \\
\hline Notes & \multicolumn{2}{|c|}{ Support provided by institutional and/or departmental sources } \\
\hline \multicolumn{3}{|l|}{ Risk of bias } \\
\hline Bias & Authors' judgement & Support for judgement \\
\hline $\begin{array}{l}\text { Random sequence genera- } \\
\text { tion (selection bias) }\end{array}$ & Unclear risk & Randomisation process not described in detail \\
\hline
\end{tabular}


Galinski 2007 (Continued)

\begin{tabular}{lll}
$\begin{array}{l}\text { Allocation concealment } \\
\text { (selection bias) }\end{array}$ & Unclear risk & Allocation concealment method not described in detail \\
\hline $\begin{array}{l}\text { Blinding of participants } \\
\text { and personnel (perfor- } \\
\text { mance bias) } \\
\text { All outcomes }\end{array}$ & Unclear risk & Not described in detail, said to be double-blind \\
\hline $\begin{array}{l}\text { Blinding of outcome as- } \\
\text { sessment (detection bias) } \\
\text { All outcomes }\end{array}$ & Unclear risk & \\
\hline $\begin{array}{l}\text { Incomplete outcome data } \\
\text { (attrition bias) } \\
\text { All outcomes }\end{array}$ & Unclear risk & Withdrawals not reported \\
\hline $\begin{array}{l}\text { Selective reporting (re- } \\
\text { porting bias) }\end{array}$ & Low risk & Predefined outcomes reported \\
\hline \begin{tabular}{l} 
Size \\
\hline
\end{tabular} & High risk & 20 participants per treatment arm \\
\hline
\end{tabular}

\section{Ganne 2005}

\section{Study characteristics}

\begin{tabular}{|c|c|c|}
\hline Methods & \multicolumn{2}{|c|}{ Randomised, double-blind, placebo control } \\
\hline Participants & \multicolumn{2}{|c|}{$\mathrm{N}=61$, about $7 \%$ women } \\
\hline Interventions & \multicolumn{2}{|c|}{$\begin{array}{l}\text { Ketamine } 0.15 \mathrm{mg} / \mathrm{kg} \text { bolus IV before induction of anaesthesia }+2 \mu \mathrm{g} / \mathrm{kg} / \mathrm{min} \text { infusion IV during anaes- } \\
\text { thesia }\end{array}$} \\
\hline Outcomes & \multicolumn{2}{|c|}{$\begin{array}{l}\text { Pain intensity (VAS). Analgesic consumption. AEs. Pain outcomes reported every } 8 \mathrm{~h} \text { up to } 48 \mathrm{~h} \text { postop- } \\
\text { eratively }\end{array}$} \\
\hline Surgery type & \multicolumn{2}{|c|}{ Ear, throat and nose surgery } \\
\hline $\begin{array}{l}\text { Group numbers after end } \\
\text { of study (treatment/con- } \\
\text { trol) }\end{array}$ & \multicolumn{2}{|l|}{$30 / 31$} \\
\hline \multirow{2}{*}{$\begin{array}{l}\text { Age of patient population } \\
\text { (treatment/control) }\end{array}$} & \multicolumn{2}{|l|}{$56.9 \pm 9.5$} \\
\hline & \multicolumn{2}{|l|}{$59.3 \pm 8.9$} \\
\hline Notes & \multicolumn{2}{|c|}{ No mention of sponsorship or funding } \\
\hline \multicolumn{3}{|l|}{ Risk of bias } \\
\hline Bias & Authors' judgement & Support for judgement \\
\hline $\begin{array}{l}\text { Random sequence genera- } \\
\text { tion (selection bias) }\end{array}$ & Low risk & Computer-generated random number table \\
\hline
\end{tabular}


Ganne 2005 (Continued)

\begin{tabular}{|c|c|c|}
\hline $\begin{array}{l}\text { Allocation concealment } \\
\text { (selection bias) }\end{array}$ & Low risk & $\begin{array}{l}\text { Allocation concealment in sealed envelopes. Syringes containing study drugs } \\
\text { were identical in appearance. }\end{array}$ \\
\hline $\begin{array}{l}\text { Blinding of participants } \\
\text { and personnel (perfor- } \\
\text { mance bias) } \\
\text { All outcomes }\end{array}$ & Low risk & Blinded personnel \\
\hline $\begin{array}{l}\text { Blinding of outcome as- } \\
\text { sessment (detection bias) } \\
\text { All outcomes }\end{array}$ & Low risk & Blinded personnel \\
\hline $\begin{array}{l}\text { Incomplete outcome data } \\
\text { (attrition bias) } \\
\text { All outcomes }\end{array}$ & Low risk & $2 \%$ was withdrawn \\
\hline $\begin{array}{l}\text { Selective reporting (re- } \\
\text { porting bias) }\end{array}$ & Low risk & Predefined outcomes reported \\
\hline Size & High risk & 30 and 31 participants per treatment arm \\
\hline
\end{tabular}

Garcia-Navia 2016

\section{Study characteristics}

\begin{tabular}{|c|c|}
\hline Methods & Randomised, double-blind, placebo control \\
\hline Participants & $N=33,100 \%$ women \\
\hline Interventions & Ketamine $0.5 \mathrm{mg} / \mathrm{kg}$ bolus IV prior to incision \\
\hline Outcomes & $\begin{array}{l}\text { Opioid consumption during surgery, emergence time, pain intensity (VAS) on admission to PACU and at } \\
2,4,8 \text { and } 24 \mathrm{~h} \text { postoperatively. Analgesic consumption at } 24 \mathrm{~h} \text { postoperatively. AEs }\end{array}$ \\
\hline Surgery type & Gynecological laparotomy excluding oncologic surgery \\
\hline $\begin{array}{l}\text { Group numbers after end } \\
\text { of study (treatment/con- } \\
\text { trol) }\end{array}$ & $11 / 11$ \\
\hline \multirow{2}{*}{$\begin{array}{l}\text { Age of patient population } \\
\text { (treatment/control) }\end{array}$} & $43.1 \pm 7.2$ \\
\hline & $45.2 \pm 4.2$ \\
\hline Notes & No mention of sponsorship or funding \\
\hline
\end{tabular}

\section{Risk of bias}

\begin{tabular}{lll}
\hline Bias & Authors' judgement & Support for judgement \\
\hline $\begin{array}{l}\text { Random sequence genera- } \\
\text { tion (selection bias) }\end{array}$ & Low risk & A computer-generated list of random numbers \\
\hline $\begin{array}{l}\text { Allocation concealment } \\
\text { (selection bias) }\end{array}$ & Unclear risk & $\begin{array}{l}\text { Allocation concealment method not described. Only mentioned that an inde- } \\
\text { pendent nurse not involved in the study prepared study solutions }\end{array}$ \\
\hline
\end{tabular}


Garcia-Navia 2016 (Continued)

\begin{tabular}{|c|c|c|}
\hline $\begin{array}{l}\text { Blinding of participants } \\
\text { and personnel (perfor- } \\
\text { mance bias) } \\
\text { All outcomes }\end{array}$ & Low risk & Blinded participants and personnel \\
\hline $\begin{array}{l}\text { Blinding of outcome as- } \\
\text { sessment (detection bias) } \\
\text { All outcomes }\end{array}$ & Low risk & Blinded personnel assessed outcomes \\
\hline $\begin{array}{l}\text { Incomplete outcome data } \\
\text { (attrition bias) } \\
\text { All outcomes }\end{array}$ & Low risk & No withdrawals \\
\hline $\begin{array}{l}\text { Selective reporting (re- } \\
\text { porting bias) }\end{array}$ & Low risk & Predefined outcomes reported \\
\hline Size & High risk & 11 participants per treatment arm \\
\hline
\end{tabular}

\section{Garg 2016}

\section{Study characteristics}

\begin{tabular}{ll}
\hline Methods & Randomised, double-blind, placebo control \\
\hline Participants & $\mathrm{N}=66$, about $63 \%$ women \\
\hline Interventions & Postoperative administration of \\
& $\begin{array}{l}\text { 1. ketamine bolus } 0.25 \mathrm{mg} / \mathrm{kg} \text { IV followed by a continuous infusion at a rate of } 0.25 \mathrm{mg} / \mathrm{kg} / \mathrm{h} \mathrm{for} 24 \mathrm{~h} \text { or } \\
\text { 2. } 0.9 \% \text { saline bolus IV followed by a } 0.9 \% \text { saline infusion (or an IV bolus of dexmedetomidine } 0.5 \mu \mathrm{mg} / \mathrm{kg} \\
\text { followed by an infusion at a rate of } 0.3 \mu \mathrm{gg} / \mathrm{kg} / \mathrm{h}\end{array}$
\end{tabular}

\section{Outcomes}

Pain intensity (NRS) at 0, 2, 6, 12, 18, 24 and $48 \mathrm{~h}$ postoperatively. Pain-free period. Analgesic consumption at 12,24 and $48 \mathrm{~h}$ postoperatively. AEs

\begin{tabular}{ll}
\hline Surgery type & Spine surgery \\
\hline $\begin{array}{l}\text { Group numbers after end } \\
\text { of study (treatment/con- }\end{array}$ & $22 / 22$ \\
trol) & \\
\hline
\end{tabular}

\begin{tabular}{ll}
$\begin{array}{l}\text { Age of patient population } \\
\text { (treatment/control) }\end{array}$ & $36.45 \pm 13.39$ \\
& $36.32 \pm 14.32$ \\
\hline Notes & $\begin{array}{l}\text { Surgery types include laminectomy and excision, pedicle screw fixation, decompression and stabilisa- } \\
\text { tion, detethering and excision of tumour). No funding }\end{array}$ \\
\hline
\end{tabular}

\section{Risk of bias}

\begin{tabular}{lll}
\hline Bias & Authors' judgement & Support for judgement \\
\hline $\begin{array}{l}\text { Random sequence genera- } \\
\text { tion (selection bias) }\end{array}$ & Low risk & A computer-generated randomisation list \\
\hline
\end{tabular}


Garg 2016 (Continued)

\begin{tabular}{|c|c|c|}
\hline $\begin{array}{l}\text { Allocation concealment } \\
\text { (selection bias) }\end{array}$ & Low risk & $\begin{array}{l}\text { Allocation concealment achieved by sequentially numbered, opaque en- } \\
\text { velopes }\end{array}$ \\
\hline
\end{tabular}
(selection bias)

Unclear risk

Not described in detail. Only mentioned "double-blind"

Blinding of participants and personnel (perfor-

mance bias)

All outcomes

Blinding of outcome as-
sessment (detection bias) $\quad$ Unclear risk Not described in detail. Only mentioned double-blind

bias)

All outcomes

Incomplete outcome data Low risk $\quad$ No withdrawals
(attrition bias)

All outcomes

\begin{tabular}{lll}
\hline $\begin{array}{l}\text { Selective reporting (re- } \\
\text { porting bias) }\end{array}$ & Low risk & Predefined outcomes reported \\
\hline Size & High risk & 22 participants per treatment arm \\
\hline
\end{tabular}

\section{Gilabert Morell 2002}

\section{Study characteristics}

\begin{tabular}{|c|c|c|}
\hline Methods & \multicolumn{2}{|c|}{ Randomised, double-blind, placebo control } \\
\hline Participants & \multicolumn{2}{|l|}{$N=69,100 \%$ women } \\
\hline Interventions & \multicolumn{2}{|c|}{ 1. Ketamine $0.15 \mathrm{mg} / \mathrm{kg}$ bolus IV at induction } \\
\hline & \multicolumn{2}{|c|}{ 2. Ketamine $0.15 \mathrm{mg} / \mathrm{kg}$ bolus IV at wound closure } \\
\hline Outcomes & \multicolumn{2}{|c|}{$\begin{array}{l}\text { Pain intensity (VAS) at rest at } 1,6,24 \text { and } 48 \text { h postoperatively and during movement on } 1 \text { st and } 5 \text { th day } \\
\text { postoperatively. Time to first request of PCA. Morphine consumption at } 6,24 \text { and } 48 \text { h postoperatively. } \\
\text { AEs }\end{array}$} \\
\hline Surgery type & \multicolumn{2}{|c|}{ Hystectomy and adenectomy } \\
\hline $\begin{array}{l}\text { Group numbers after end } \\
\text { of study (treatment/con- } \\
\text { trol) }\end{array}$ & \multicolumn{2}{|l|}{$44 / 22$} \\
\hline \multirow{2}{*}{$\begin{array}{l}\text { Age of patient population } \\
\text { (treatment/control) }\end{array}$} & \multicolumn{2}{|l|}{$47.8 \pm 8.3$} \\
\hline & \multicolumn{2}{|l|}{$48 \pm 7$} \\
\hline Notes & \multicolumn{2}{|c|}{ No mention of sponsorship or funding } \\
\hline \multicolumn{3}{|l|}{ Risk of bias } \\
\hline Bias & Authors' judgement & Support for judgement \\
\hline $\begin{array}{l}\text { Random sequence genera- } \\
\text { tion (selection bias) }\end{array}$ & Unclear risk & Randomisation not described \\
\hline
\end{tabular}


Gilabert Morell 2002 (Continued)

\begin{tabular}{lll}
$\begin{array}{l}\text { Allocation concealment } \\
\text { (selection bias) }\end{array}$ & Unclear risk & Allocation concealment method not described \\
\hline $\begin{array}{l}\text { Blinding of participants } \\
\text { and personnel (perfor- } \\
\text { mance bias) } \\
\text { All outcomes }\end{array}$ & Low risk & Blinded personnel \\
\hline $\begin{array}{l}\text { Blinding of outcome as- } \\
\text { sessment (detection bias) } \\
\text { All outcomes }\end{array}$ & Low risk \\
\hline $\begin{array}{l}\text { Incomplete outcome data } \\
\text { (attrition bias) } \\
\text { All outcomes }\end{array}$ & Low risk & $4 \%$ was withdrawn \\
\hline $\begin{array}{l}\text { Selective reporting (re- } \\
\text { porting bias) }\end{array}$ & Low risk & All predefined outcomes reported \\
\hline Size & High risk & 23 participants per treatment arm \\
\hline
\end{tabular}

Grady 2012

\section{Study characteristics}

\begin{tabular}{|c|c|c|}
\hline Methods & \multicolumn{2}{|c|}{$\begin{array}{l}\text { Randomised, double-blind, placebo control. Factorial design. No direct comparison between ketamine } \\
\text { vs non-ketamine }\end{array}$} \\
\hline Participants & \multicolumn{2}{|l|}{$N=64,100 \%$ women } \\
\hline Interventions & \multicolumn{2}{|c|}{$\begin{array}{l}\text { Pre-incisional ketamine } 0.5 \mathrm{mg} / \mathrm{kg} \text { bolus IV + a continuous infusion } 0.12 \mathrm{mg} / \mathrm{kg} / \mathrm{h} \text { until } 15 \mathrm{mins} \text { before } \\
\text { completion of the operation }\end{array}$} \\
\hline Outcomes & \multicolumn{2}{|c|}{$\begin{array}{l}\text { Pain intensity (VRS) reported on PACU admit and discharge and on 1st and } 2 \text { nd postoperative days. } \\
\text { Analgesic consumption reported in PACU and on } 1 \text { st and } 2 \text { nd postoperative days. AEs }\end{array}$} \\
\hline Surgery type & \multicolumn{2}{|c|}{ Abdominal hysterectomy } \\
\hline $\begin{array}{l}\text { Group numbers after end } \\
\text { of study (treatment/con- } \\
\text { trol) }\end{array}$ & \multicolumn{2}{|l|}{$30 / 32$} \\
\hline $\begin{array}{l}\text { Age of patient population } \\
\text { (treatment/control) }\end{array}$ & \multicolumn{2}{|l|}{$46 \pm 8$} \\
\hline Notes & \multicolumn{2}{|l|}{ Internal funding } \\
\hline \multicolumn{3}{|l|}{ Risk of bias } \\
\hline Bias & Authors' judgement & Support for judgement \\
\hline $\begin{array}{l}\text { Random sequence genera- } \\
\text { tion (selection bias) }\end{array}$ & Low risk & Randomisation based on a computer-generated list \\
\hline
\end{tabular}


Grady 2012 (Continued)

\begin{tabular}{|c|c|c|}
\hline $\begin{array}{l}\text { Allocation concealment } \\
\text { (selection bias) }\end{array}$ & Low risk & Allocation maintained in sequentially numbered, sealed, opaque envelopes \\
\hline $\begin{array}{l}\text { Blinding of participants } \\
\text { and personnel (perfor- } \\
\text { mance bias) } \\
\text { All outcomes }\end{array}$ & Low risk & Blinded personnel \\
\hline $\begin{array}{l}\text { Blinding of outcome as- } \\
\text { sessment (detection bias) } \\
\text { All outcomes }\end{array}$ & Low risk & Blinded personnel \\
\hline $\begin{array}{l}\text { Incomplete outcome data } \\
\text { (attrition bias) } \\
\text { All outcomes }\end{array}$ & Low risk & $3 \%$ was withdrawn \\
\hline $\begin{array}{l}\text { Selective reporting (re- } \\
\text { porting bias) }\end{array}$ & Low risk & Predefined outcomes reported \\
\hline Size & High risk & 30 and 32 participants per treatment arm. \\
\hline
\end{tabular}

\section{Guignard 2002}

\section{Study characteristics}

\begin{tabular}{|c|c|c|}
\hline Methods & \multicolumn{2}{|c|}{ Randomised, double-blind, placebo control } \\
\hline Participants & \multicolumn{2}{|l|}{$N=50,50 \%$ women } \\
\hline Interventions & \multicolumn{2}{|c|}{ Ketamine bolus $0.15 \mathrm{mg} / \mathrm{kg}+$ infusion $2 \mu \mathrm{g} / \mathrm{kg} / \mathrm{min}$ IV from prior to skin incision until skin closure } \\
\hline Outcomes & \multicolumn{2}{|c|}{$\begin{array}{l}\text { Pain intensity (VAS and 4-point VRS; data not shown). Total morphine consumption (PCA and nurse-ad- } \\
\text { ministered) at 0-4, 5-24 and 0-24 h postoperatively. Time to first request for morphine. AEs }\end{array}$} \\
\hline Surgery type & \multicolumn{2}{|l|}{ Abdominal surgery } \\
\hline $\begin{array}{l}\text { Group numbers after end } \\
\text { of study (treatment/con- } \\
\text { trol) }\end{array}$ & \multicolumn{2}{|l|}{$25 / 25$} \\
\hline $\begin{array}{l}\text { Age of patient population } \\
\text { (treatment/control) }\end{array}$ & \multicolumn{2}{|l|}{$64 \pm 10$} \\
\hline Notes & \multicolumn{2}{|c|}{$\begin{array}{l}\text { Supported, in part, by NIH Grant GM } 58273 \text { (Bethesda, MD), the Joseph Drown Foundation (Los Angeles, } \\
\text { CA), and the Commonwealth of Kentucky Research Challenge Trust Fund (Louisville, KY). }\end{array}$} \\
\hline \multicolumn{3}{|l|}{ Risk of bias } \\
\hline Bias & Authors' judgement & Support for judgement \\
\hline $\begin{array}{l}\text { Random sequence genera- } \\
\text { tion (selection bias) }\end{array}$ & Low risk & Computer-generated random-number table \\
\hline
\end{tabular}


Guignard 2002 (Continued)

Allocation concealment Unclear risk Allocation concealment method not described in detail. Only mentioned that a (selection bias)

\section{hospital pharmacist prepared study drugs}

Blinding of participants Low risk Blinded personnel

and personnel (perfor-

mance bias)

All outcomes

Blinding of outcome as-
sessment (detection bias) $\quad$ Low risk $\quad$ Blinded person collecting data

(detection bias)

All outcomes

Incomplete outcome data Low risk No withdrawals
(attrition bias)

All outcomes

\begin{tabular}{lll}
\hline $\begin{array}{l}\text { Selective reporting (re- } \\
\text { porting bias) }\end{array}$ & Low risk & Predefined outcomes reported \\
\hline Size & High risk & 25 participants per treatment arm \\
\hline
\end{tabular}

Guillou 2003

\section{Study characteristics}

\begin{tabular}{|c|c|c|}
\hline Methods & \multicolumn{2}{|c|}{ Randomised, double-blind, placebo control } \\
\hline Participants & \multicolumn{2}{|c|}{$N=101$, about $54 \%$ women } \\
\hline Interventions & \multicolumn{2}{|c|}{$\begin{array}{l}\text { Ketamine } 0.5 \mathrm{mg} / \mathrm{kg} \text { bolus IV after surgery + infusion } 2 \mu \mathrm{g} / \mathrm{kg} / \mathrm{min} \text { for } 24 \mathrm{~h} \text { and } 1 \mu \mathrm{g} / \mathrm{kg} / \mathrm{min} \text { from 24-48 } \\
\mathrm{h}\end{array}$} \\
\hline Outcomes & \multicolumn{2}{|c|}{$\begin{array}{l}\text { Pain intensity (VAS) at rest and during movement. Cumulative dose of PCA morphine. Outcomes report- } \\
\text { ed every } 4 \mathrm{~h} \text { up to } 48 \mathrm{~h} \text { postoperatively }\end{array}$} \\
\hline Surgery type & \multicolumn{2}{|c|}{ Major abdominal surgery } \\
\hline $\begin{array}{l}\text { Group numbers after end } \\
\text { of study (treatment/con- } \\
\text { trol) }\end{array}$ & \multicolumn{2}{|l|}{$41 / 52$} \\
\hline \multirow{2}{*}{$\begin{array}{l}\text { Age of patient population } \\
\text { (treatment/control) }\end{array}$} & \multicolumn{2}{|l|}{$60 \pm 16$} \\
\hline & \multicolumn{2}{|l|}{$60 \pm 15$} \\
\hline Notes & \multicolumn{2}{|c|}{ No mention of sponsorship or funding } \\
\hline \multicolumn{3}{|l|}{ Risk of bias } \\
\hline Bias & Authors' judgement & Support for judgement \\
\hline $\begin{array}{l}\text { Random sequence genera- } \\
\text { tion (selection bias) }\end{array}$ & Unclear risk & Randomisation process not described \\
\hline
\end{tabular}


Guillou 2003 (Continued)

\begin{tabular}{|c|c|c|}
\hline $\begin{array}{l}\text { Allocation concealment } \\
\text { (selection bias) }\end{array}$ & Unclear risk & Allocation concealment method not described \\
\hline $\begin{array}{l}\text { Blinding of participants } \\
\text { and personnel (perfor- } \\
\text { mance bias) } \\
\text { All outcomes }\end{array}$ & Low risk & Blinded personnel \\
\hline $\begin{array}{l}\text { Blinding of outcome as- } \\
\text { sessment (detection bias) } \\
\text { All outcomes }\end{array}$ & Low risk & Blinded observer \\
\hline $\begin{array}{l}\text { Incomplete outcome data } \\
\text { (attrition bias) } \\
\text { All outcomes }\end{array}$ & Low risk & $8 \%$ was withdrawn \\
\hline $\begin{array}{l}\text { Selective reporting (re- } \\
\text { porting bias) }\end{array}$ & Low risk & Predefined outcomes reported \\
\hline Size & Unclear risk & 41 and 52 participants per treatment arm \\
\hline
\end{tabular}

Hadi 2010

\section{Study characteristics}

\begin{tabular}{|c|c|c|}
\hline Methods & \multicolumn{2}{|c|}{ Randomised, double-blind, placebo control } \\
\hline Participants & \multicolumn{2}{|c|}{$\mathrm{N}=30$. Table of demographic data not presented } \\
\hline Interventions & \multicolumn{2}{|c|}{ Ketamine $1 \mu \mathrm{g} / \mathrm{kg} / \mathrm{min}$ infusion IV until the end of surgery } \\
\hline Outcomes & \multicolumn{2}{|c|}{$\begin{array}{l}\text { Pain intensity (visual face-rating scale), reported as number of participants with different degrees of } \\
\text { pain. Time to first request for analgesia. Analgesic consumption reported at } 24 \mathrm{~h} \text { postoperatively. AEs }\end{array}$} \\
\hline Surgery type & \multicolumn{2}{|c|}{ Lumbar and thoracic spinal fusion surgery } \\
\hline $\begin{array}{l}\text { Group numbers after end } \\
\text { of study (treatment/con- } \\
\text { trol) }\end{array}$ & \multicolumn{2}{|l|}{$15 / 15$} \\
\hline \multirow{3}{*}{$\begin{array}{l}\text { Age of patient population } \\
\text { (treatment/control) }\end{array}$} & \multicolumn{2}{|l|}{ Age range } \\
\hline & \multicolumn{2}{|l|}{$53-59$} \\
\hline & \multicolumn{2}{|l|}{$49-58$} \\
\hline Notes & \multicolumn{2}{|c|}{ No mention of sponsorship or funding } \\
\hline \multicolumn{3}{|l|}{ Risk of bias } \\
\hline Bias & Authors' judgement & Support for judgement \\
\hline $\begin{array}{l}\text { Random sequence genera- } \\
\text { tion (selection bias) }\end{array}$ & Unclear risk & Randomisation not described \\
\hline
\end{tabular}


Hadi 2010 (Continued)

\begin{tabular}{|c|c|c|}
\hline $\begin{array}{l}\text { Allocation concealment } \\
\text { (selection bias) }\end{array}$ & Unclear risk & Allocation concealment method not described \\
\hline $\begin{array}{l}\text { Blinding of participants } \\
\text { and personnel (perfor- } \\
\text { mance bias) } \\
\text { All outcomes }\end{array}$ & Unclear risk & Not described, only mentioned double-blind \\
\hline $\begin{array}{l}\text { Blinding of outcome as- } \\
\text { sessment (detection bias) } \\
\text { All outcomes }\end{array}$ & Low risk & Data assessment by blinded pharmacy students \\
\hline $\begin{array}{l}\text { Incomplete outcome data } \\
\text { (attrition bias) } \\
\text { All outcomes }\end{array}$ & Unclear risk & Withdrawals not reported \\
\hline $\begin{array}{l}\text { Selective reporting (re- } \\
\text { porting bias) }\end{array}$ & Low risk & Predefined outcomes reported adequately \\
\hline Size & High risk & 15 participants per treatment arm \\
\hline
\end{tabular}

Hadi 2013

\section{Study characteristics}

\begin{tabular}{|c|c|c|}
\hline Methods & \multicolumn{2}{|c|}{ Randomised, double-blind, placebo control } \\
\hline Participants & \multicolumn{2}{|c|}{$N=45$, about $53 \%$ women } \\
\hline Interventions & \multicolumn{2}{|c|}{ 1. Ketamine $1 \mu \mathrm{g} / \mathrm{kg} / \mathrm{min}$ infusion IV intraoperatively } \\
\hline & \multicolumn{2}{|c|}{ 2. Ketamine $1 \mu \mathrm{g} / \mathrm{kg} / \mathrm{min}$ infusion IV intraoperatively and $24 \mathrm{~h}$ after surgery } \\
\hline Outcomes & \multicolumn{2}{|c|}{$\begin{array}{l}\text { Pain intensity (VAS). Analgesic consumption. Pain outcomes reported at } 6,12 \text { and } 24 \mathrm{~h} \text { postoperatively. } \\
\text { Time to first request for analgesia. AEs }\end{array}$} \\
\hline Surgery type & \multicolumn{2}{|c|}{ Lumbar microdiscectomy } \\
\hline $\begin{array}{l}\text { Group numbers after end } \\
\text { of study (treatment/con- } \\
\text { trol) }\end{array}$ & \multicolumn{2}{|l|}{$30 / 15$} \\
\hline Age of patient population & \multicolumn{2}{|l|}{$55 \pm 2.5$} \\
\hline & \multicolumn{2}{|l|}{$51 \pm 2.47$} \\
\hline Notes & \multicolumn{2}{|c|}{ No mention of sponsorship or funding } \\
\hline \multicolumn{3}{|l|}{ Risk of bias } \\
\hline Bias & Authors' judgement & Support for judgement \\
\hline $\begin{array}{l}\text { Random sequence genera- } \\
\text { tion (selection bias) }\end{array}$ & Unclear risk & Randomisation process not described \\
\hline
\end{tabular}


Hadi 2013 (Continued)

\begin{tabular}{|c|c|c|}
\hline $\begin{array}{l}\text { Allocation concealment } \\
\text { (selection bias) }\end{array}$ & Unclear risk & Allocation concealment method not described \\
\hline $\begin{array}{l}\text { Blinding of participants } \\
\text { and personnel (perfor- } \\
\text { mance bias) } \\
\text { All outcomes }\end{array}$ & Low risk & Pharmacist prepared study solutions \\
\hline $\begin{array}{l}\text { Blinding of outcome as- } \\
\text { sessment (detection bias) } \\
\text { All outcomes }\end{array}$ & Low risk & Blinded personnel collecting data \\
\hline $\begin{array}{l}\text { Incomplete outcome data } \\
\text { (attrition bias) } \\
\text { All outcomes }\end{array}$ & Low risk & No withdrawals \\
\hline $\begin{array}{l}\text { Selective reporting (re- } \\
\text { porting bias) }\end{array}$ & Unclear risk & Pain intensity scale reported differs (VAS, NRS) \\
\hline Size & High risk & 15 participants per treatment arm \\
\hline
\end{tabular}

\section{Haliloglu 2015}

\section{Study characteristics}

\begin{tabular}{|c|c|c|}
\hline Methods & \multicolumn{2}{|c|}{ Randomised, double-blind, placebo control } \\
\hline Participants & \multicolumn{2}{|l|}{$N=52,100 \%$ women } \\
\hline Interventions & \multicolumn{2}{|c|}{$\begin{array}{l}\text { Ketamine } 0.5 \mathrm{mg} / \mathrm{kg} \text { bolus IV at induction followed by an infusion } 10 \mathrm{mcg} / \mathrm{kg} / \mathrm{min} \text { until the end of } \\
\text { surgery }\end{array}$} \\
\hline Outcomes & \multicolumn{2}{|c|}{$\begin{array}{l}\text { Pain intensity (NRS) reported at } 15 \mathrm{~min} \text {, at } 2 \text { and } 6 \mathrm{~h} \text {, then every } 6 \mathrm{~h} \text { up to } 24 \mathrm{~h} \text { postoperatively. Anal- } \\
\text { gesic consumption reported at } 6 \text { - } \mathrm{h} \text { intervals up to } 24 \mathrm{~h} \text { postoperatively. Cumulative analgesic con- } \\
\text { sumption reported at } 24 \mathrm{~h} \text { postoperatively. Rescue analgesia. AEs }\end{array}$} \\
\hline Surgery type & \multicolumn{2}{|c|}{ Elective caesarean section } \\
\hline $\begin{array}{l}\text { Group numbers after end } \\
\text { of study (treatment/con- } \\
\text { trol) }\end{array}$ & \multicolumn{2}{|l|}{$26 / 26$} \\
\hline \multirow{2}{*}{$\begin{array}{l}\text { Age of patient population } \\
\text { (treatment/control) }\end{array}$} & \multicolumn{2}{|l|}{$29.1 \pm 2.2$} \\
\hline & \multicolumn{2}{|l|}{$29 \pm 2.2$} \\
\hline Notes & \multicolumn{2}{|c|}{ No mention of sponsorship or funding. The study authors report that they have no conflicts of interest } \\
\hline \multicolumn{3}{|l|}{ Risk of bias } \\
\hline Bias & Authors' judgement & Support for judgement \\
\hline $\begin{array}{l}\text { Random sequence genera- } \\
\text { tion (selection bias) }\end{array}$ & Low risk & Computer-generated random number table \\
\hline
\end{tabular}


Haliloglu 2015 (Continued)

$\begin{aligned} & \text { Allocation concealment } \\ & \text { (selection bias) }\end{aligned} \quad$ Unclear risk

Blinding of participants Low risk Blinded personnel

and personnel (perfor-

mance bias)

All outcomes

Blinding of outcome as- Low risk Blinded personnel

sessment (detection bias)

All outcomes

\begin{tabular}{lll}
\hline $\begin{array}{l}\text { Incomplete outcome data } \\
\text { (attrition bias) } \\
\text { All outcomes }\end{array}$ & Low risk & No withdrawals \\
\hline $\begin{array}{l}\text { Selective reporting (re- } \\
\text { porting bias) }\end{array}$ & Low risk & Predefined outcomes reported \\
\hline Size & High risk & 26 participants per treatment arm \\
\hline
\end{tabular}

Hasanein 2011

\section{Study characteristics}

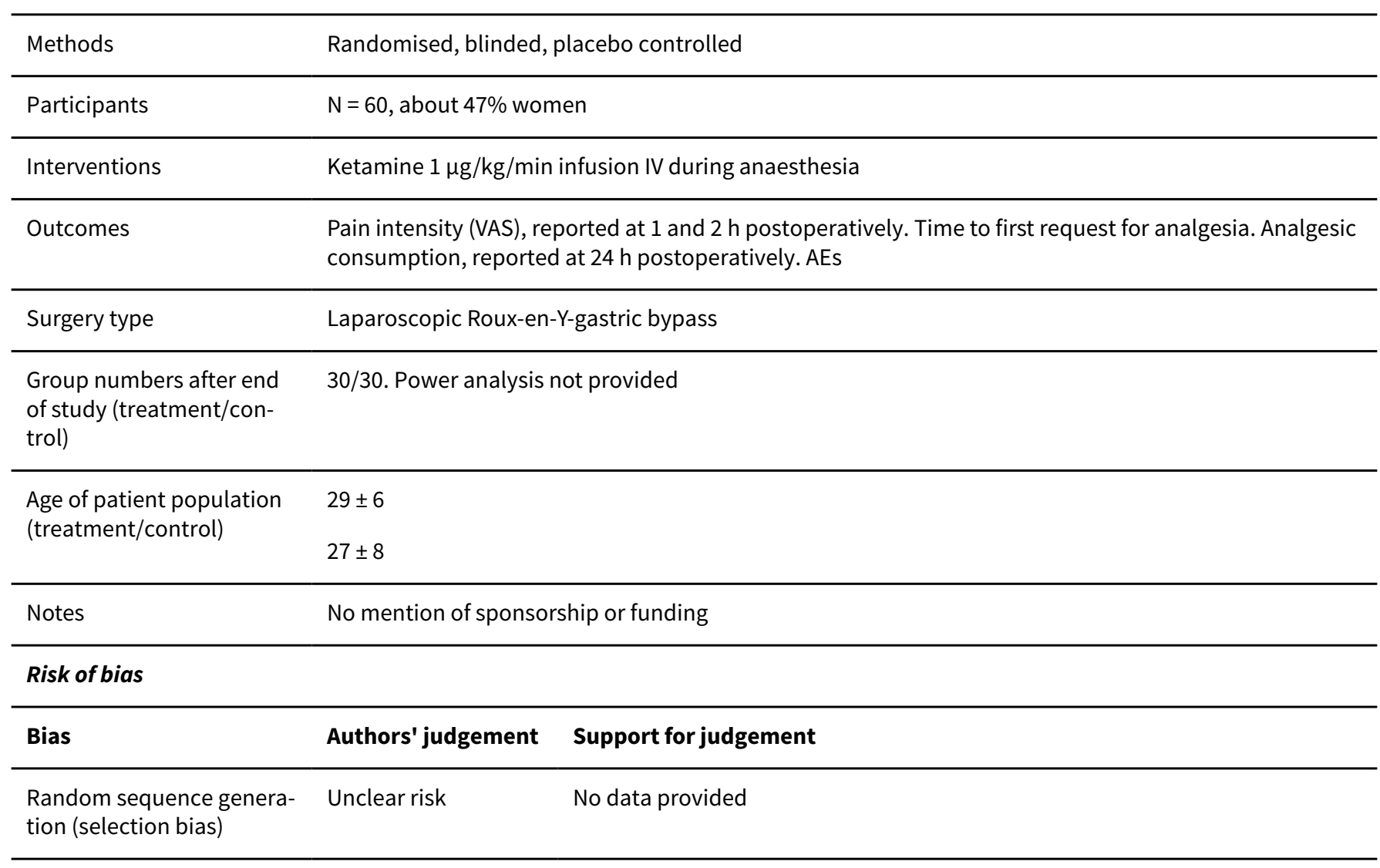


Hasanein 2011 (Continued)

Allocation concealment High risk The attending anaesthesiologist was aware of the treatment condition but (selection bias) study participants and personnel in the operating room and recording data were unaware of treatment allocation.

\begin{tabular}{|c|c|c|}
\hline $\begin{array}{l}\text { Blinding of participants } \\
\text { and personnel (perfor- } \\
\text { mance bias) }\end{array}$ & Unclear risk & $\begin{array}{l}\text { Participants and investigators recording data in the operating room were } \\
\text { blinded to the treatment but the attending anaesthesiologist was aware of the } \\
\text { treatment condition }\end{array}$ \\
\hline
\end{tabular}

All outcomes

\begin{tabular}{lll}
\hline Blinding of outcome as- & Low risk Investigators recording data were blinded \\
sessment (detection bias) &
\end{tabular}

sessment (detection bias)

All outcomes

Incomplete outcome data Low risk No withdrawals
(attrition bias)
All outcomes

\begin{tabular}{lll}
\hline $\begin{array}{l}\text { Selective reporting (re- } \\
\text { porting bias) }\end{array}$ & High risk & $\begin{array}{l}\text { Predefined outcome (PCA morphine consumption during the first } 4 \mathrm{~h} \text { after } \\
\text { surgery) is not reported }\end{array}$ \\
\hline Size & High risk & 30 participants per treatment arm \\
\hline
\end{tabular}

Hayes 2004

\section{Study characteristics}

\begin{tabular}{ll}
\hline Methods & Randomised, double-blind, placebo control \\
\hline Participants & $\mathrm{N}=45$, about $42 \%$ women \\
\hline Interventions & $\begin{array}{l}\text { Pre-induction ketamine } 0.5 \mathrm{mg} / \mathrm{kg} \text { bolus IV followed by } 0.15 \mathrm{mg} / \mathrm{kg} / \mathrm{h} \text { infusion IV for } 72 \mathrm{~h} \text { postoperative- } \\
\text { ly }\end{array}$ \\
\hline Outcomes & $\begin{array}{l}\text { Incidence of post-amputation pain (phantom and stump pain, number of participants) on 3rd and } 6 \mathrm{th} \\
\text { postoperative day and } 6 \text { months after surgery. Analgesic consumption reported at } 24 \text { and } 72 \mathrm{~h} \text { postop- } \\
\text { eratively, reported as medians. Postoperative central sensitisation on 3rd and 6th day postoperatively. } \\
\text { AEs }\end{array}$ \\
\hline
\end{tabular}

\begin{tabular}{ll}
\hline Surgery type & Above or below knee amputation \\
\hline $\begin{array}{l}\text { Group numbers after end } \\
\text { of study (treatment/con- } \\
\text { trol) }\end{array}$ & \\
\hline
\end{tabular}

\begin{tabular}{ll}
\hline $\begin{array}{l}\text { Age of patient population } \\
\text { (treatment/control) }\end{array}$ & $68.7 \pm 12.2$ \\
& $68.9 \pm 10.9$ \\
\hline Notes & No mention of sponsorship or funding \\
\hline Risk of bias & Authors' judgement Support for judgement \\
\hline Bias &
\end{tabular}


Hayes 2004 (Continued)

\begin{tabular}{|c|c|c|}
\hline $\begin{array}{l}\text { Random sequence genera- } \\
\text { tion (selection bias) }\end{array}$ & Low risk & A random-number generator \\
\hline $\begin{array}{l}\text { Allocation concealment } \\
\text { (selection bias) }\end{array}$ & Unclear risk & Allocation concealment method not described \\
\hline $\begin{array}{l}\text { Blinding of participants } \\
\text { and personnel (perfor- } \\
\text { mance bias) } \\
\text { All outcomes }\end{array}$ & Low risk & Blinded personnel \\
\hline
\end{tabular}

\begin{tabular}{lll}
\hline $\begin{array}{l}\text { Blinding of outcome as- } \\
\text { sessment (detection bias) } \\
\text { All outcomes }\end{array}$ & Low risk & Blinded personnel \\
\hline $\begin{array}{l}\text { Incomplete outcome data } \\
\text { (attrition bias) } \\
\text { All outcomes }\end{array}$ & Low risk & $7 \%$ was withdrawn in the immediate postoperative period \\
\hline $\begin{array}{l}\text { Selective reporting (re- } \\
\text { porting bias) }\end{array}$ & Low risk & Predefined outcomes reported \\
\hline Size & High risk & 20 participants per treatment arm \\
\hline
\end{tabular}

Helmy 2015

\section{Study characteristics}

\begin{tabular}{|c|c|}
\hline Methods & Randomised, double-blind, placebo control \\
\hline Participants & $\mathrm{N}=60,100 \%$ women \\
\hline Interventions & Ketamine $0.3 \mathrm{mg} / \mathrm{kg}$ bolus IV before induction of anaesthesia \\
\hline Outcomes & $\begin{array}{l}\text { Pain intensity (VAS) reported at 2, 6, } 12 \text { and } 24 \text { h postoperatively. Analgesic consumption reported at } 24 \\
\text { h postoperatively. Sedation level. PONV. Time to first analgesic request }\end{array}$ \\
\hline Surgery type & Elective caesarean section \\
\hline $\begin{array}{l}\text { Group numbers after end } \\
\text { of study (treatment/con- } \\
\text { trol) }\end{array}$ & $20 / 20$ \\
\hline \multirow{3}{*}{$\begin{array}{l}\text { Age of patient population } \\
\text { (treatment/control) }\end{array}$} & Median (range) \\
\hline & $33(24-43)$ \\
\hline & $30(22-41)$ \\
\hline Notes & The authors declare that there is no conflict of interest \\
\hline \multicolumn{2}{|l|}{ Risk of bias } \\
\hline Bias & Authors' judgement Support for judgement \\
\hline
\end{tabular}


Helmy 2015 (Continued)

Random sequence genera- Unclear risk $\quad$ Randomisation not sufficiently described
tion (selection bias)

tion (selection bias)

Quote: "using a sealed envelope method, parturients were randomly assigned into 3 groups"

\begin{tabular}{|c|c|c|}
\hline $\begin{array}{l}\text { Allocation concealment } \\
\text { (selection bias) }\end{array}$ & Unclear risk & Allocation concealment method described in detail \\
\hline $\begin{array}{l}\text { Blinding of participants } \\
\text { and personnel (perfor- } \\
\text { mance bias) } \\
\text { All outcomes }\end{array}$ & Unclear risk & Blinding not described (only said "double blind") \\
\hline $\begin{array}{l}\text { Blinding of outcome as- } \\
\text { sessment (detection bias) } \\
\text { All outcomes }\end{array}$ & Unclear risk & Blinding not described \\
\hline $\begin{array}{l}\text { Incomplete outcome data } \\
\text { (attrition bias) } \\
\text { All outcomes }\end{array}$ & Low risk & $3 \%$ was withdrawn \\
\hline $\begin{array}{l}\text { Selective reporting (re- } \\
\text { porting bias) }\end{array}$ & Low risk & Predefined outcomes reported \\
\hline Size & High risk & 19 and 20 participants per treatment arm \\
\hline
\end{tabular}

\section{Hercock 1999}

\section{Study characteristics}

\begin{tabular}{ll}
\hline Methods & $\begin{array}{l}\text { Randomised, double-blind. Intraoperative IV ketamine vs placebo/postoperative ketamine vs mor- } \\
\text { phine }\end{array}$ \\
\hline Participants & $\mathrm{N}=50,100 \%$ women \\
\hline Interventions & Ketamine $0.3 \mathrm{mg} / \mathrm{kg}$ bolus IV after induction. Postoperative IV PCA $1 \mathrm{mg} / \mathrm{bolus}$ \\
\hline Outcomes & Pain intensity. PCA morphine consumption. Outcomes reported at 24 h postoperatively \\
\hline $\begin{array}{l}\text { Surgery type } \\
\text { Group numbers after end } \\
\text { of study (treatment/con- } \\
\text { trol) }\end{array}$ & $24 / 25$ \\
\hline $\begin{array}{l}\text { Age of patient population } \\
\text { (treatment/control) }\end{array}$ & 45.7 \\
\hline $\begin{array}{l}\text { Notes } \\
\text { Risk of bias }\end{array}$ & No mention of sponsorship or funding \\
\hline \begin{tabular}{l} 
Bias \\
\hline
\end{tabular} & Authors' judgement Support for judgement \\
\hline
\end{tabular}




\section{Hercock 1999 (Continued)}

Random sequence genera- Unclear risk Randomisation process not described tion (selection bias)

\begin{tabular}{|c|c|c|}
\hline $\begin{array}{l}\text { Allocation concealment } \\
\text { (selection bias) }\end{array}$ & Low risk & $\begin{array}{l}\text { An independent anaesthetist, who subsequently had no role in the care of the } \\
\text { participant, prepared the study drugs. Allocation was concealed in sealed, se- } \\
\text { quentially numbered envelopes }\end{array}$ \\
\hline
\end{tabular}

\begin{tabular}{|c|c|c|}
\hline $\begin{array}{l}\text { Blinding of participants } \\
\text { and personnel (perfor- } \\
\text { mance bias) } \\
\text { All outcomes }\end{array}$ & Low risk & Blinded personnel \\
\hline $\begin{array}{l}\text { Blinding of outcome as- } \\
\text { sessment (detection bias) } \\
\text { All outcomes }\end{array}$ & Low risk & Blinded personnel \\
\hline $\begin{array}{l}\text { Incomplete outcome data } \\
\text { (attrition bias) } \\
\text { All outcomes }\end{array}$ & Low risk & $2 \%$ was withdrawn \\
\hline $\begin{array}{l}\text { Selective reporting (re- } \\
\text { porting bias) }\end{array}$ & Low risk & Predefined outcomes reported \\
\hline Size & High risk & 24 and 25 participants per treatment arm \\
\hline
\end{tabular}

Hu 2014

\section{Study characteristics}

\begin{tabular}{ll}
\hline Methods & Randomised, double-blind, placebo control \\
\hline Participants & $\mathrm{N}=78$, about $19 \%$ women \\
\hline Interventions & Pre-incisional ketamine $0.1 \mathrm{mg} / \mathrm{kg}$ bolus IV followed by an infusion $2 \mu \mathrm{g} / \mathrm{kg} / \mathrm{min}$ for $72 \mathrm{~h}$ \\
\hline Outcomes & $\begin{array}{l}\text { Pain intensity (NRS) reported on the } 1 \mathrm{st} 7 \text { postoperative days and at } 2 \text { and } 6 \text { months after surgery. Anal- } \\
\text { gesic consumption reported at } 72 \mathrm{~h} \text { postoperatively. AEs. Evaluation of chronic postoperative pain }\end{array}$ \\
\hline $\begin{array}{l}\text { Surgery type } \\
\begin{array}{l}\text { Group numbers after end } \\
\text { of study (treatment/con- } \\
\text { trol) }\end{array}\end{array}$ & $31 / 47$ \\
\hline
\end{tabular}

Age of patient population
(treatment/control)

\section{$51.39 \pm 9.85$}

$48.28 \pm 13.95$

Notes No mention of sponsorship or funding

\section{Risk of bias}

Bias Authors' judgement Support for judgement


Hu 2014 (Continued)

\begin{tabular}{lll}
$\begin{array}{l}\text { Random sequence genera- } \\
\text { tion (selection bias) }\end{array}$ & Unclear risk & Randomisation process not described \\
\hline $\begin{array}{l}\text { Allocation concealment } \\
\text { (selection bias) }\end{array}$ & Unclear risk & Allocation concealment method not described \\
\hline $\begin{array}{l}\text { Blinding of participants } \\
\text { and personnel (perfor- } \\
\text { mance bias) }\end{array}$ & Unclear risk & Only mentioned "double blind" \\
All outcomes & &
\end{tabular}

\begin{tabular}{lll}
\hline $\begin{array}{l}\text { Blinding of outcome as- } \\
\text { sessment (detection bias) } \\
\text { All outcomes }\end{array}$ & Unclear risk & Only mentioned "double blind" \\
\hline $\begin{array}{l}\text { Incomplete outcome data } \\
\text { (attrition bias) } \\
\text { All outcomes }\end{array}$ & Low risk & $4 \%$ was withdrawn \\
\hline $\begin{array}{l}\text { Selective reporting (re- } \\
\text { porting bias) }\end{array}$ & Low risk & Predefined outcomes reported \\
\hline Size & High risk & 31 and 47 participants per treatment arm \\
\hline
\end{tabular}

Ilkjaer 1998

\section{Study characteristics}

\begin{tabular}{|c|c|}
\hline Methods & Randomised, double-blind, placebo control \\
\hline Participants & $\mathrm{N}=60$, about $38 \%$ women \\
\hline Interventions & Ketamine $10 \mathrm{mg}$ bolus IV before surgical incision $+10 \mathrm{mg} / \mathrm{h}$ infusion IV for $48 \mathrm{~h}$ postoperatively \\
\hline Outcomes & $\begin{array}{l}\text { Pain intensity (VAS) at rest and during movement, reported at } 4,6,8 \mathrm{~h} \text {, then beginning at } 22 \mathrm{nd} \text { postop- } \\
\text { erative } \mathrm{h} \text { every } 2 \mathrm{~h} \text { up to } 32 \mathrm{~h} \text { postoperatively, then at } 46 \text { and } 48 \mathrm{~h} \text { postoperatively. Number of PCA mor- } \\
\text { phine doses at } 0-24 \mathrm{~h} \text { and } 24-48 \mathrm{~h} \text { postoperatively. Pressure pain detection threshold at } 0,6,22,30 \text { and } \\
46 \mathrm{~h} \text { postoperatively. Pain sensitivity. AEs }\end{array}$ \\
\hline Surgery type & Elective nephrectomy or operation on pelvic structures \\
\hline $\begin{array}{l}\text { Group numbers after end } \\
\text { of study (treatment/con- } \\
\text { trol) }\end{array}$ & $24 / 28$ \\
\hline \multirow{3}{*}{$\begin{array}{l}\text { Age of patient population } \\
\text { (treatment/control) }\end{array}$} & Median age \\
\hline & $50(43-68)$ \\
\hline & $55(50-65)$ \\
\hline Notes & $\begin{array}{l}\text { Supported by a grant from the Danish Medical Council (Reg. no. 28809), Novo Nordisk Foundation, Dan- } \\
\text { ish Foundation for the Advancement of Medical Science and Agnes and Poul Friis Foundation }\end{array}$ \\
\hline
\end{tabular}

\section{Risk of bias}


Ilkjaer 1998 (Continued)

\begin{tabular}{|c|c|c|}
\hline Bias & Authors' judgement & Support for judgement \\
\hline $\begin{array}{l}\text { Random sequence genera- } \\
\text { tion (selection bias) }\end{array}$ & Unclear risk & Randomisation process not described \\
\hline $\begin{array}{l}\text { Allocation concealment } \\
\text { (selection bias) }\end{array}$ & Low risk & $\begin{array}{l}\text { Quote: "Study drugs (ketamine } 10 \mathrm{mg} / \mathrm{ml} \text { ) and placebo (isotonic saline) were } \\
\text { prepared under sterile conditions by the hospital pharmacy in identical con- } \\
\text { tainers, marked with the name of the project, the investigator's name and con- } \\
\text { secutive patient number" }\end{array}$ \\
\hline $\begin{array}{l}\text { Blinding of participants } \\
\text { and personnel (perfor- } \\
\text { mance bias) } \\
\text { All outcomes }\end{array}$ & Unclear risk & Blinding not described \\
\hline $\begin{array}{l}\text { Blinding of outcome as- } \\
\text { sessment (detection bias) } \\
\text { All outcomes }\end{array}$ & Unclear risk & Only stated "double-blind" but blinding process not described in detail \\
\hline $\begin{array}{l}\text { Incomplete outcome data } \\
\text { (attrition bias) } \\
\text { All outcomes }\end{array}$ & High risk & $13 \%$ was withdrawn from the study and completed participants were analysed \\
\hline $\begin{array}{l}\text { Selective reporting (re- } \\
\text { porting bias) }\end{array}$ & Unclear risk & $\begin{array}{l}\text { Insufficient information (precise numbers) of some outcomes, only reported P } \\
\text { values }\end{array}$ \\
\hline Size & High risk & 24 and 28 participants per treatment arm \\
\hline
\end{tabular}

Jaksch 2002

\section{Study characteristics}

\begin{tabular}{|c|c|}
\hline Methods & Randomised, double-blind, placebo-control \\
\hline Participants & $\mathrm{N}=30,50 \%$ women \\
\hline Interventions & $\begin{array}{l}\text { S-ketamine } 0.5 \mathrm{mg} / \mathrm{kg} \text { bolus IV before incision + infusion } 2 \mu \mathrm{g} / \mathrm{kg} / \mathrm{min} \text { until } 2 \mathrm{~h} \text { after emergence from } \\
\text { anaesthesia }\end{array}$ \\
\hline Outcomes & $\begin{array}{l}\text { Pain intensity (VAS) and PCA morphine consumption reported at 1, 24, 48, } 72 \text { and } 120 \mathrm{~h} \text { postoperative- } \\
\text { ly. Time to first request for analgesia }\end{array}$ \\
\hline Surgery type & Elective arthroscopic anterior cruciate ligament repair \\
\hline $\begin{array}{l}\text { Group numbers after end } \\
\text { of study (treatment/con- } \\
\text { trol) }\end{array}$ & $15 / 15$ \\
\hline \multirow{2}{*}{$\begin{array}{l}\text { Age of patient population } \\
\text { (treatment/control) }\end{array}$} & $30 \pm 8$ \\
\hline & $33 \pm 7$ \\
\hline Notes & No mention of sponsorship or funding \\
\hline
\end{tabular}

\section{Risk of bias}


Jaksch 2002 (Continued)

\begin{tabular}{lll} 
Bias & Authors' judgement & Support for judgement \\
\hline $\begin{array}{l}\text { Random sequence genera- } \\
\text { tion (selection bias) }\end{array}$ & Unclear risk & Randomisation not described \\
\hline $\begin{array}{l}\text { Allocation concealment } \\
\text { (selection bias) }\end{array}$ & Low risk & $\begin{array}{l}\text { Quote: "Envelopes containing identification of the preparation administered } \\
\text { were available for emergencies" }\end{array}$
\end{tabular}

\begin{tabular}{|c|c|c|}
\hline $\begin{array}{l}\text { Blinding of participants } \\
\text { and personnel (perfor- } \\
\text { mance bias) } \\
\text { All outcomes }\end{array}$ & Low risk & Nurses not involved in the study prepared study solutions \\
\hline $\begin{array}{l}\text { Blinding of outcome as- } \\
\text { sessment (detection bias) } \\
\text { All outcomes }\end{array}$ & Unclear risk & Blinding not described \\
\hline $\begin{array}{l}\text { Incomplete outcome data } \\
\text { (attrition bias) } \\
\text { All outcomes }\end{array}$ & Low risk & No withdrawals \\
\hline $\begin{array}{l}\text { Selective reporting (re- } \\
\text { porting bias) }\end{array}$ & Low risk & Predefined outcomes reported \\
\hline Size & High risk & 15 participants per treatment arm \\
\hline
\end{tabular}

Javery 1996

\section{Study characteristics}

\begin{tabular}{ll}
\hline Methods & Randomised, double-blind. Ketamine + morphine vs morphine \\
\hline Participants & $\mathrm{N}=42$, about $10 \%$ women \\
\hline Interventions & Postoperative IV PCA ketamine $1 \mathrm{mg} /$ bolus \\
\hline Outcomes & Pain intensity (VAS). PCA morphine consumption. AEs. Outcomes reported at 24 h postoperatively \\
\hline Surgery type & Lumbar microdiscectomy \\
\hline $\begin{array}{l}\text { Group numbers after end } \\
\text { of study (treatment/con- } \\
\text { trol) }\end{array}$ & $22 / 20$ \\
\hline $\begin{array}{l}\text { Age of patient population } \\
\text { (treatment/control) }\end{array}$ & $37.3 \pm 9.9$ \\
\hline $\begin{array}{l}\text { Notes } \\
\text { Risk of bias }\end{array}$ & No mention of sponsorship or funding \\
\hline Bias & Authors' judgement Support for judgement \\
\hline
\end{tabular}


Javery 1996 (Continued)

Random sequence genera- Unclear risk Randomisation process not described tion (selection bias)

\begin{tabular}{lll}
\hline $\begin{array}{l}\text { Allocation concealment } \\
\text { (selection bias) }\end{array}$ & Unclear risk & Allocation concealment not described \\
\hline $\begin{array}{l}\text { Blinding of participants } \\
\text { and personnel (perfor- }\end{array}$ & Unclear risk & \\
mance bias) & & \\
All outcomes &
\end{tabular}

\begin{tabular}{|c|c|c|}
\hline $\begin{array}{l}\text { Blinding of outcome as- } \\
\text { sessment (detection bias) } \\
\text { All outcomes }\end{array}$ & Unclear risk & Blinding not described \\
\hline $\begin{array}{l}\text { Incomplete outcome data } \\
\text { (attrition bias) } \\
\text { All outcomes }\end{array}$ & Low risk & No withdrawals \\
\hline $\begin{array}{l}\text { Selective reporting (re- } \\
\text { porting bias) }\end{array}$ & Low risk & Predefined outcomes reported \\
\hline Size & High risk & 20 and 22 participants per treatment arm \\
\hline
\end{tabular}

Jendoubi 2017

\section{Study characteristics}

\begin{tabular}{|c|c|}
\hline Methods & Randomised, double-blind, placebo control \\
\hline Participants & $\mathrm{N}=60$, of whom 40 participants in IV ketamine and control treatment arms. $50 \%$ women \\
\hline Interventions & $\begin{array}{l}\text { At the induction of anaesthesia, ketamine bolus } 0.15 \mathrm{mg} / \mathrm{kg} \text { IV followed by infusion of } 0.1 \mathrm{mg} / \mathrm{kg} / \mathrm{h} \text { for } \\
24 \mathrm{~h} \text { postoperatively }\end{array}$ \\
\hline Outcomes & $\begin{array}{l}\text { Cumulative morphine consumption reported every } 6 \mathrm{~h} \text { up to } 24 \mathrm{~h} \text { postoperatively. Pain intensity (VAS) } \\
\text { at rest and during movement and coughing every } 6 \mathrm{~h} \text { up to } 24 \mathrm{~h} \text { and at } 48 \mathrm{~h} \text { postoperatively. AEs }\end{array}$ \\
\hline Surgery type & Open nephrectomy \\
\hline $\begin{array}{l}\text { Group numbers after end } \\
\text { of study (treatment/con- } \\
\text { trol) }\end{array}$ & $20 / 20$ \\
\hline \multirow{2}{*}{$\begin{array}{l}\text { Age of patient population } \\
\text { (treatment/control) }\end{array}$} & $55.8 \pm 13.5$ \\
\hline & $48.3 \pm 13.5$ \\
\hline Notes & No sponsorship or funding \\
\hline \multicolumn{2}{|l|}{ Risk of bias } \\
\hline Bias & Authors' judgement Support for judgement \\
\hline
\end{tabular}


Jendoubi 2017 (Continued)

Random sequence genera- Unclear risk Not described in detail. Only stated "randomised" tion (selection bias)

\begin{tabular}{lll}
\hline $\begin{array}{l}\text { Allocation concealment } \\
\text { (selection bias) }\end{array}$ & Unclear risk & $\begin{array}{l}\text { Allocation concealment method not described in detail. Only mentioned that a } \\
\text { nurse not participating in the study prepared study drugs }\end{array}$ \\
\hline $\begin{array}{l}\text { Blinding of participants } \\
\text { and personnel (perfor- } \\
\text { mance bias) }\end{array}$ & Low risk & Blinded participants and personnel \\
All outcomes & \\
\hline
\end{tabular}

\begin{tabular}{lll}
$\begin{array}{l}\text { Blinding of outcome as- } \\
\text { sessment (detection bias) } \\
\text { All outcomes }\end{array}$ & Low risk & Blinded personnel \\
\hline $\begin{array}{l}\text { Incomplete outcome data } \\
\text { (attrition bias) } \\
\text { All outcomes }\end{array}$ & Low risk & $5 \%$ was withdrawn \\
\hline $\begin{array}{l}\text { Selective reporting (re- } \\
\text { porting bias) }\end{array}$ & Low risk & Predefined outcomes reported \\
\hline Size & High risk & 20 participants per treatment arm \\
\hline
\end{tabular}

\section{Joly 2005}

\section{Study characteristics}

\begin{tabular}{|c|c|}
\hline Methods & Randomised, double-blind, placebo control \\
\hline Participants & $\mathrm{N}=75$, of whom 50 participants in IV ketamine and control treatment arms. $64 \%$ women \\
\hline Interventions & $\begin{array}{l}\text { Pre-incisional ketamine } 0.5 \mathrm{mg} / \mathrm{kg} \text { bolus IV }+5 \mu \mathrm{g} / \mathrm{kg} / \mathrm{min} \text { infusion IV until skin closure, then } 2 \mu \mathrm{g} / \mathrm{kg} / \\
\text { min during the initial } 48 \text { postoperative hours }\end{array}$ \\
\hline Outcomes & $\begin{array}{l}\text { Pain intensity (VAS) reported at } 24 \text { and } 48 \mathrm{~h} \text { postoperatively. Analgesic consumption reported at } 48 \mathrm{~h} \\
\text { postoperatively. AEs. Hyperalgesia on day } 1 \text { and } 2 \text { postoperatively }\end{array}$ \\
\hline Surgery type & Major abdominal surgery \\
\hline $\begin{array}{l}\text { Group numbers after end } \\
\text { of study (treatment/con- } \\
\text { trol) }\end{array}$ & $24 / 25$ \\
\hline \multirow{2}{*}{$\begin{array}{l}\text { Age of patient population } \\
\text { (treatment/control) }\end{array}$} & $59 \pm 13$ \\
\hline & $56 \pm 12$ \\
\hline Notes & No mention of sponsorship or funding \\
\hline \multicolumn{2}{|l|}{ Risk of bias } \\
\hline Bias & Authors' judgement Support for judgement \\
\hline
\end{tabular}


Joly 2005 (Continued)

Random sequence genera- Low risk $\quad$ Computer-generated random number table
tion (selection bias)

\begin{tabular}{|c|c|c|}
\hline $\begin{array}{l}\text { Allocation concealment } \\
\text { (selection bias) }\end{array}$ & Low risk & Allocation concealed in sealed, sequentially numbered envelopes \\
\hline $\begin{array}{l}\text { Blinding of participants } \\
\text { and personnel (perfor- } \\
\text { mance bias) } \\
\text { All outcomes }\end{array}$ & Low risk & Blinded personnel \\
\hline $\begin{array}{l}\text { Blinding of outcome as- } \\
\text { sessment (detection bias) } \\
\text { All outcomes }\end{array}$ & Low risk & Blinded personnel \\
\hline $\begin{array}{l}\text { Incomplete outcome data } \\
\text { (attrition bias) } \\
\text { All outcomes }\end{array}$ & Low risk & $1 \%$ was withdrawn \\
\hline $\begin{array}{l}\text { Selective reporting (re- } \\
\text { porting bias) }\end{array}$ & Low risk & Predefined outcomes reported \\
\hline Size & High risk & 25 participants per treatment arm \\
\hline
\end{tabular}

\section{Joseph 2012}

\section{Study characteristics}

\begin{tabular}{ll}
\hline Methods & Randomised, double-blind, placebo control \\
\hline Participants & $\mathrm{N}=60$, about $53 \%$ women \\
\hline Interventions & $\begin{array}{l}\text { Ketamine } 0.5 \mathrm{mg} / \mathrm{kg} \text { bolus IV at anaesthesia induction + IV infusion } 3 \mu \mathrm{g} / \mathrm{kg} / \mathrm{min} \text { during surgery, then } \\
1.5 \mathrm{~kg} / \mathrm{min} \text { for } 48 \mathrm{~h} \text { postoperatively }\end{array}$ \\
\hline Outcomes & $\begin{array}{l}\text { Pain intensity (NRS). Cumulative epidural ropivacaine consumption. Supplemental IV analgesia re- } \\
\text { quirement. AEs. Outcomes reported on admission to PACU, at 12, 24, 48 h and } 1 \text { and } 3 \text { months after } \\
\text { surgery }\end{array}$ \\
\hline $\begin{array}{l}\text { Surgery type } \\
\text { Group numbers after end } \\
\text { of study (treatment/con- } \\
\text { trol) }\end{array}$ & \begin{tabular}{l} 
Thoracotomy \\
\hline $\begin{array}{l}\text { Age of patient population } \\
\text { (treatment/control) }\end{array}$
\end{tabular} \\
$\begin{array}{ll}\text { median age (range) } \\
60 \text { (24-80) }\end{array}$ \\
\hline \begin{tabular}{l} 
Notes (31-79) \\
\hline
\end{tabular}
\end{tabular}

\section{Risk of bias}


Joseph 2012 (Continued)

\begin{tabular}{|c|c|c|}
\hline Bias & Authors' judgement & Support for judgement \\
\hline $\begin{array}{l}\text { Random sequence genera- } \\
\text { tion (selection bias) }\end{array}$ & Low risk & $\begin{array}{l}\text { Quote: "Patients were randomized to one of the two groups using a comput- } \\
\text { er-generated randomization schedule." }\end{array}$ \\
\hline $\begin{array}{l}\text { Allocation concealment } \\
\text { (selection bias) }\end{array}$ & Unclear risk & $\begin{array}{l}\text { Allocation concealment method not described in detail. Only described that a } \\
\text { hospital pharmacist dispensed study drugs that were identical in appearance }\end{array}$ \\
\hline $\begin{array}{l}\text { Blinding of participants } \\
\text { and personnel (perfor- } \\
\text { mance bias) } \\
\text { All outcomes }\end{array}$ & Low risk & Blinded personnel \\
\hline $\begin{array}{l}\text { Blinding of outcome as- } \\
\text { sessment (detection bias) } \\
\text { All outcomes }\end{array}$ & Low risk & Blinded personnel \\
\hline $\begin{array}{l}\text { Incomplete outcome data } \\
\text { (attrition bias) } \\
\text { All outcomes }\end{array}$ & Low risk & $7 \%$ was withdrawn \\
\hline $\begin{array}{l}\text { Selective reporting (re- } \\
\text { porting bias) }\end{array}$ & Low risk & Results of predefined outcomes reported \\
\hline Size & High risk & 22 and 25 participants per treatment arm \\
\hline
\end{tabular}

Kafali 2004

\section{Study characteristics}

\begin{tabular}{ll}
\hline Methods & Randomised, double-blind, placebo control \\
\hline Participants & $\mathrm{N}=60$, about $48 \%$ women \\
\hline Interventions & Pre-incisional ketamine $0.15 \mathrm{mg} / \mathrm{kg}$ bolus IV \\
\hline Outcomes & $\begin{array}{l}\text { Pain intensity (VAS) reported at } 30 \text { min, then } 2,12,24 \text { and } 48 \text { h postoperatively. Time to first analgesic } \\
\text { demand. Analgesic consumption reported at } 48 \mathrm{~h} \text { postoperatively. Adverse effects }\end{array}$
\end{tabular}

\begin{tabular}{ll}
\hline Surgery type & Lower abdominal surgery \\
\hline $\begin{array}{l}\text { Group numbers after end } \\
\text { of study (treatment/con- }\end{array}$ \\
trol)
\end{tabular}

Age of patient population
(treatment/control)

$47.2 \pm 4.2$

$45.2 \pm 3$

\section{Risk of bias}

Bias Authors' judgement Support for judgement


Kafali 2004 (Continued)

\begin{tabular}{lll}
$\begin{array}{l}\text { Random sequence genera- } \\
\text { tion (selection bias) }\end{array}$ & Low risk & A random number table \\
\hline $\begin{array}{l}\text { Allocation concealment } \\
\text { (selection bias) }\end{array}$ & Unclear risk & Allocation concealment method not described \\
\hline $\begin{array}{l}\text { Blinding of participants } \\
\text { and personnel (perfor- } \\
\text { mance bias) }\end{array}$ & Unclear risk & Blinding not described, mentioned "double blind" \\
$\begin{array}{l}\text { All outcomes } \\
\text { ond }\end{array}$ & \\
\hline
\end{tabular}

\begin{tabular}{lll}
\hline $\begin{array}{l}\text { Blinding of outcome as- } \\
\text { sessment (detection bias) } \\
\text { All outcomes }\end{array}$ & Unclear risk & Blinding not described, only mentioned "double-blind" \\
\hline $\begin{array}{l}\text { Incomplete outcome data } \\
\text { (attrition bias) } \\
\text { All outcomes }\end{array}$ & Low risk & No withdrawals \\
\hline $\begin{array}{l}\text { Selective reporting (re- } \\
\text { porting bias) }\end{array}$ & High risk & Not all predefined outcomes are reported (adverse effects: pruritus) \\
\hline Size & High risk & 30 participants per treatment arm \\
\hline
\end{tabular}

\section{Kakinohana 2004}

\section{Study characteristics}

\begin{tabular}{|c|c|}
\hline Methods & Randomised, double-blind, placebo control \\
\hline Participants & $\mathrm{N}=50,58 \%$ women \\
\hline Interventions & $\begin{array}{l}\text { Pre-incisional ketamine bolus } 1 \mathrm{mg} / \mathrm{kg} \mathrm{IV}+\text { infusion } 1 \mathrm{mg} / \mathrm{kg} / \mathrm{h} \mathrm{IV} \text {, maintained until } 2 \mathrm{mg} / \mathrm{kg} \text { adminis- } \\
\text { tered }\end{array}$ \\
\hline Outcomes & $\begin{array}{l}\text { Pain intensity (VAS) reported at } 5,24 \text { and } 48 \mathrm{~h} \text { postoperatively. Cumulative PCEA volume consumed re- } \\
\text { ported at } 5 \mathrm{~h}, 5-24 \mathrm{~h}, 24-48 \mathrm{~h} \text { and at } 48 \mathrm{~h} \text { postoperatively. AEs }\end{array}$ \\
\hline Surgery type & Elective open cholecystectomy \\
\hline $\begin{array}{l}\text { Group numbers after end } \\
\text { of study (treatment/con- } \\
\text { trol) }\end{array}$ & $25 / 25$ \\
\hline \multirow{2}{*}{$\begin{array}{l}\text { Age of patient population } \\
\text { (treatment/control) }\end{array}$} & $48.1 \pm 10.1$ \\
\hline & $49.9 \pm 12.0$ \\
\hline Notes & No mention of sponsorship or funding \\
\hline \multicolumn{2}{|l|}{ Risk of bias } \\
\hline Bias & Authors' judgement Support for judgement \\
\hline
\end{tabular}


Kakinohana 2004 (Continued)

\begin{tabular}{|c|c|c|}
\hline $\begin{array}{l}\text { Random sequence genera- } \\
\text { tion (selection bias) }\end{array}$ & Unclear risk & Randomisation process not described \\
\hline $\begin{array}{l}\text { Allocation concealment } \\
\text { (selection bias) }\end{array}$ & Unclear risk & Allocation concealment method not described in detail \\
\hline $\begin{array}{l}\text { Blinding of participants } \\
\text { and personnel (perfor- } \\
\text { mance bias) } \\
\text { All outcomes }\end{array}$ & Low risk & Blinded personnel \\
\hline
\end{tabular}

\begin{tabular}{lll}
\hline $\begin{array}{l}\text { Blinding of outcome as- } \\
\text { sessment (detection bias) } \\
\text { All outcomes }\end{array}$ & Low risk & Assessment accomplished by blinded nurses \\
\hline $\begin{array}{l}\text { Incomplete outcome data } \\
\text { (attrition bias) } \\
\text { All outcomes }\end{array}$ & Low risk & No withdrawals \\
\hline $\begin{array}{l}\text { Selective reporting (re- } \\
\text { porting bias) }\end{array}$ & Low risk & Predefined outcomes reported \\
\hline Size & High risk & 25 participants per treatment arm \\
\hline
\end{tabular}

\section{Kamal 2008}

\section{Study characteristics}

\begin{tabular}{ll}
\hline Methods & Randomised, double-blind. Ketamine + morphine vs morphine \\
\hline Participants & $\mathrm{N}=80$, about 49\% women \\
\hline Interventions & Postoperative IV PCA Ketamine $1 \mathrm{mg} / \mathrm{mL}$ \\
\hline Outcomes & $\begin{array}{l}\text { Pain intensity (VAS). Analgesic consumption. Adverse effects. Pain outcomes reported every } 8 \text { h up to } \\
48 \text { h postoperatively }\end{array}$ \\
\hline $\begin{array}{l}\text { Surgery type } \\
\text { Upper abdominal surgery }\end{array}$ \\
\hline $\begin{array}{l}\text { Group numbers after end } \\
\text { of study (treatment/con- } \\
\text { trol) }\end{array}$ & $40 / 40$ \\
\hline $\begin{array}{l}\text { Age of patient population } \\
\text { (treatment/control) }\end{array}$ & $38 \pm 14$ \\
\hline $\begin{array}{l}\text { Notes } \\
\text { no }\end{array}$ & No mention of sponsorship or funding \\
\hline
\end{tabular}

\section{Risk of bias}

\begin{tabular}{lll}
\hline Bias & Authors' judgement & Support for judgement \\
\hline $\begin{array}{l}\text { Random sequence genera- } \\
\text { tion (selection bias) }\end{array}$ & Unclear risk & Randomisation process not described \\
\hline
\end{tabular}


Kamal 2008 (Continued)

\begin{tabular}{|c|c|c|}
\hline $\begin{array}{l}\text { Allocation concealment } \\
\text { (selection bias) }\end{array}$ & Unclear risk & Allocation concealment method not described \\
\hline $\begin{array}{l}\text { Blinding of participants } \\
\text { and personnel (perfor- } \\
\text { mance bias) } \\
\text { All outcomes }\end{array}$ & Low risk & A nurse not involved in the care of participants prepared study drug syringes \\
\hline $\begin{array}{l}\text { Blinding of outcome as- } \\
\text { sessment (detection bias) } \\
\text { All outcomes }\end{array}$ & Low risk & Blinded personnel \\
\hline $\begin{array}{l}\text { Incomplete outcome data } \\
\text { (attrition bias) } \\
\text { All outcomes }\end{array}$ & Low risk & No withdrawals \\
\hline $\begin{array}{l}\text { Selective reporting (re- } \\
\text { porting bias) }\end{array}$ & Low risk & Results of predefined outcomes reported \\
\hline Size & High risk & 40 participants per treatment arm \\
\hline
\end{tabular}

Kapfer 2005

\section{Study characteristics}

\begin{tabular}{|c|c|}
\hline Methods & Randomised, double-blind, placebo control \\
\hline Participants & $\mathrm{N}=65$, of whom 43 participants in IV ketamine and control treatment arms. About $37 \%$ women \\
\hline Interventions & $\begin{array}{l}\text { Postoperative ketamine } 10 \mathrm{mg} \text { bolus over a twelve-min period. IV if preceding opioid analgesia insuffi- } \\
\text { cient }\end{array}$ \\
\hline Outcomes & $\begin{array}{l}\text { Morphine consumption. Failure of morphine titration to produce adequate analgesia. Delay between } \\
\text { the end of morphine titration and reappearance of a VRS pain score } 2 \text { or more }\end{array}$ \\
\hline Surgery type & Laparotomy, lumbotomy, orthopedic surgery (hip or knee arthroplasty) \\
\hline $\begin{array}{l}\text { Group numbers after end } \\
\text { of study (treatment/con- } \\
\text { trol) }\end{array}$ & $22 / 21$ \\
\hline \multirow{2}{*}{$\begin{array}{l}\text { Age of patient population } \\
\text { (treatment/control) }\end{array}$} & $51 \pm 13$ \\
\hline & $49 \pm 15$ \\
\hline Notes & $\begin{array}{l}\text { Different types of surgery included. Supported by NIH Grant GM } 061655 \text { (Bethesda, MD), the Gheens } \\
\text { Foundation (Louisville, KY), the Joseph Drown Foundation (Los Angeles, CA), and the Commonwealth } \\
\text { of Kentucky Research Challenge Trust Fund (Louisville, KY). }\end{array}$ \\
\hline
\end{tabular}

\section{Risk of bias}

\begin{tabular}{lll}
\hline Bias & Authors' judgement & Support for judgement \\
\hline $\begin{array}{l}\text { Random sequence genera- } \\
\text { tion (selection bias) }\end{array}$ & Low risk & Computer-generated randomisation list \\
\hline
\end{tabular}


Kapfer 2005 (Continued)
Allocation concealment
Low risk
Quote: "Randomization was based on computer-generated codes that were (selection bias) maintained in sequentially numbered, opaque envelopes until just before use."

\begin{tabular}{lll}
\hline $\begin{array}{l}\text { Blinding of participants } \\
\text { and personnel (perfor- } \\
\text { mance bias) }\end{array}$ & Low risk & Blinded personnel \\
$\begin{array}{l}\text { All outcomes } \\
\text { Blinding of outcome as- }\end{array}$ & Low risk & Blinded personnel \\
$\begin{array}{l}\text { sessment (detection bias) } \\
\text { All outcomes }\end{array}$ & \\
\hline $\begin{array}{l}\text { Incomplete outcome data } \\
\text { (attrition bias) }\end{array}$ & Low risk & 2\% was withdrawn \\
All outcomes & & \\
\hline
\end{tabular}

\begin{tabular}{lll}
\hline $\begin{array}{l}\text { Selective reporting (re- } \\
\text { porting bias) }\end{array}$ & Low risk & Predefined outcomes reported \\
\hline Size & High risk & 22 and 21 participants per treatment arm \\
\hline
\end{tabular}

Karaman 2006

\section{Study characteristics}

\begin{tabular}{|c|c|c|}
\hline Methods & \multicolumn{2}{|c|}{ Randomised, double-blind, placebo control } \\
\hline Participants & \multicolumn{2}{|l|}{$N=60,100 \%$ women } \\
\hline Interventions & \multicolumn{2}{|c|}{$\begin{array}{l}\text { 1. Pre-incisional ketamine } 0.4 \mathrm{mg} / \mathrm{kg} \text { bolus IV } \\
\text { 2. Ketamine } 0.4 \mathrm{mg} / \mathrm{kg} \text { bolus IV at wound closure }\end{array}$} \\
\hline Outcomes & \multicolumn{2}{|c|}{$\begin{array}{l}\text { Pain intensity (VAS, VRS). Analgesic consumption. Time to first analgesic request. AEs. Pain outcomes } \\
\text { reported at } 1,2,3,4,8,12 \text { and } 24 \mathrm{~h} \text { postoperatively }\end{array}$} \\
\hline Surgery type & \multicolumn{2}{|c|}{ Total abdominal hysterectomy } \\
\hline $\begin{array}{l}\text { Group numbers after end } \\
\text { of study (treatment/con- } \\
\text { trol) }\end{array}$ & \multicolumn{2}{|l|}{$40 / 20$} \\
\hline $\begin{array}{l}\text { Age of patient population } \\
\text { (treatment/control) }\end{array}$ & \multicolumn{2}{|l|}{$48.2 \pm 5.3$} \\
\hline Notes & \multicolumn{2}{|c|}{ Article in Turkish. No mention of sponsorship or funding } \\
\hline \multicolumn{3}{|l|}{ Risk of bias } \\
\hline Bias & Authors' judgement & Support for judgement \\
\hline $\begin{array}{l}\text { Random sequence genera- } \\
\text { tion (selection bias) }\end{array}$ & Low risk & Randomisation achieved by shuffling envelopes \\
\hline
\end{tabular}


Karaman 2006 (Continued)

\begin{tabular}{|c|c|c|}
\hline $\begin{array}{l}\text { Allocation concealment } \\
\text { (selection bias) }\end{array}$ & Unclear risk & Allocation concealment method not described \\
\hline $\begin{array}{l}\text { Blinding of participants } \\
\text { and personnel (perfor- } \\
\text { mance bias) } \\
\text { All outcomes }\end{array}$ & Low risk & Blinded personnel \\
\hline $\begin{array}{l}\text { Blinding of outcome as- } \\
\text { sessment (detection bias) } \\
\text { All outcomes }\end{array}$ & Low risk & Blinded personnel \\
\hline $\begin{array}{l}\text { Incomplete outcome data } \\
\text { (attrition bias) } \\
\text { All outcomes }\end{array}$ & Low risk & No withdrawals \\
\hline $\begin{array}{l}\text { Selective reporting (re- } \\
\text { porting bias) }\end{array}$ & Low risk & Predefined outcomes reported \\
\hline Size & High risk & 20 participants per treatment arm \\
\hline
\end{tabular}

\section{Kararmaz 2003}

\section{Study characteristics}

\begin{tabular}{ll}
\hline Methods & Randomised, double-blind, placebo control \\
\hline Participants & $\mathrm{N}=40,80 \%$ women \\
\hline Interventions & Ketamine $0.5 \mathrm{mg} / \mathrm{kg}$ bolus IV $+0.5 \mathrm{mg} / \mathrm{kg} / \mathrm{h}$ IV infusion until skin closure \\
\hline Outcomes & $\begin{array}{l}\text { Pain intensity (VAS) reported at 0.5, 1, 2, 4, 6, 12, 24 and } 48 \text { h postoperatively. Mean PCEA analgesic con- } \\
\text { sumption on 1st and 2nd postoperative day. Time to first analgesic request. AEs }\end{array}$ \\
\hline $\begin{array}{l}\text { Surgery type } \\
\text { Group numbers after end } \\
\text { of study (treatment/con- } \\
\text { trol) }\end{array}$ & $20 / 20$ \\
\hline $\begin{array}{l}\text { Age of patient population renal surgery } \\
\text { treatment/control) }\end{array}$ & $36.7 \pm 13.8$ \\
\hline \begin{tabular}{l} 
Notes \\
\hline
\end{tabular} & No mention of funding or sponsorship
\end{tabular}

\section{Risk of bias}

\begin{tabular}{lll}
\hline Bias & Authors' judgement & Support for judgement \\
\hline $\begin{array}{l}\text { Random sequence genera- } \\
\text { tion (selection bias) }\end{array}$ & Low risk & Computer-generated random sequence \\
\hline $\begin{array}{l}\text { Allocation concealment } \\
\text { (selection bias) }\end{array}$ & Unclear risk & Allocation concealment method not described \\
\hline
\end{tabular}




\section{Kararmaz 2003 (Continued)}

\begin{tabular}{|c|c|c|}
\hline $\begin{array}{l}\text { Blinding of participants } \\
\text { and personnel (perfor- } \\
\text { mance bias) } \\
\text { All outcomes }\end{array}$ & Low risk & Blinded personnel \\
\hline $\begin{array}{l}\text { Blinding of outcome as- } \\
\text { sessment (detection bias) } \\
\text { All outcomes }\end{array}$ & Low risk & Blinded personnel \\
\hline $\begin{array}{l}\text { Incomplete outcome data } \\
\text { (attrition bias) } \\
\text { All outcomes }\end{array}$ & Low risk & No withdrawals \\
\hline $\begin{array}{l}\text { Selective reporting (re- } \\
\text { porting bias) }\end{array}$ & Low risk & Predefined outcomes reported \\
\hline Size & High risk & 20 participants per treatment arm \\
\hline
\end{tabular}

\section{Karcioglu 2013}

\section{Study characteristics}

\begin{tabular}{|c|c|c|}
\hline Methods & \multicolumn{2}{|c|}{ Randomised, double-blind, placebo control } \\
\hline Participants & \multicolumn{2}{|c|}{$N=40$, gender of participants not presented in demographic data } \\
\hline Interventions & \multicolumn{2}{|c|}{$\begin{array}{l}\text { Ketamine } 1 \mathrm{mg} / \mathrm{kg} \text { bolus IV at anaesthesia induction }+25 \mathrm{mcg} / \mathrm{kg} / \mathrm{min} \text { IV infusion until the end of } \\
\text { surgery }\end{array}$} \\
\hline Outcomes & \multicolumn{2}{|c|}{$\begin{array}{l}\text { Pain intensity (VAS) reported at PACU discharge and at } 24 \mathrm{~h} \text { postoperatively. Analgesic consumption re- } \\
\text { ported as proportion (\%) of participants receiving analgesics. AEs }\end{array}$} \\
\hline Surgery type & \multicolumn{2}{|c|}{ Laparoscopic cholecystectomy } \\
\hline $\begin{array}{l}\text { Group numbers after end } \\
\text { of study (treatment/con- } \\
\text { trol) }\end{array}$ & \multicolumn{2}{|l|}{$17 / 20$} \\
\hline Age of patient population & \multicolumn{2}{|l|}{$38.6 \pm 11.7$} \\
\hline & \multicolumn{2}{|l|}{$43.4 \pm 12.1$} \\
\hline Notes & \multicolumn{2}{|c|}{ No mention of sponsorship or funding. The authors declare no conflicts of interest } \\
\hline \multicolumn{3}{|l|}{ Risk of bias } \\
\hline Bias & Authors' judgement & Support for judgement \\
\hline $\begin{array}{l}\text { Random sequence genera- } \\
\text { tion (selection bias) }\end{array}$ & Low risk & Computer-generated random sequence \\
\hline $\begin{array}{l}\text { Allocation concealment } \\
\text { (selection bias) }\end{array}$ & Unclear risk & Allocation concealment method not described \\
\hline
\end{tabular}


Karcioglu 2013 (Continued)
Blinding of participants
Low risk
Blinded personnel and personnel (performance bias)

All outcomes

\begin{tabular}{lll}
\hline $\begin{array}{l}\text { Blinding of outcome as- } \\
\text { sessment (detection bias) } \\
\text { All outcomes }\end{array}$ & Unclear risk & Not reported \\
\hline $\begin{array}{l}\text { Incomplete outcome data } \\
\text { (attrition bias) } \\
\text { All outcomes }\end{array}$ & Low risk & $8 \%$ was withdrawn \\
\hline $\begin{array}{l}\text { Selective reporting (re- } \\
\text { porting bias) }\end{array}$ & Low risk & $\begin{array}{l}\text { Predefined outcomes reported (three participants withdrawn from the keta- } \\
\text { mine group because of hypertension) }\end{array}$ \\
\hline Size & High risk & 17 and 20 participants per treatment arm \\
\hline
\end{tabular}

\section{Katz 2004}

\section{Study characteristics}

\begin{tabular}{|c|c|}
\hline Methods & Randomised, double-blind, placebo control \\
\hline Participants & $\mathrm{N}=168,100 \%$ men \\
\hline Interventions & $\begin{array}{l}\text { 1. Pre-incisional ketamine } 0.2 \mathrm{mg} / \mathrm{kg} \text { bolus IV }+0.0025 \mathrm{mg} / \mathrm{kg} / \mathrm{min} \text { infusion IV for } 70 \mathrm{mins} \\
\text { 2. Ketamine } 0.2 \mathrm{mg} / \mathrm{kg} \text { bolus IV } 70 \text { mins after the incision }+0.0025 \mathrm{mg} / \mathrm{kg} / \mathrm{min} \text { infusion IV up to } 80 \mathrm{mins}\end{array}$ \\
\hline Outcomes & $\begin{array}{l}\text { Pain intensity (VAS), reported at } 3,6,12,24,48 \text { and } 72 \mathrm{~h} \text { postoperatively. Analgesic consumption re- } \\
\text { ported at } 0-3,3-6,6-12,12-24,24-48 \text { and } 48-72 \mathrm{~h} \text { postoperatively. Touch and pain threshold (von Frey) }\end{array}$ \\
\hline Surgery type & Radical prostatectomy \\
\hline $\begin{array}{l}\text { Group numbers after end } \\
\text { of study (treatment/con- } \\
\text { trol) }\end{array}$ & $97 / 46$ \\
\hline
\end{tabular}

\begin{tabular}{ll}
\hline $\begin{array}{l}\text { Age of patient population } \\
\text { (treatment/control) }\end{array}$ & $62 \pm 6.8$ \\
& $61 \pm 6.7$
\end{tabular}

Notes The study was supported by Grants MT-12052 and MOP-37845 from the Canadian Institutes of Health Research (CIHR), Ontario, Canada, and a CIHR Investigator Award to the lead author.

\section{Risk of bias}

Bias Authors' judgement Support for judgement

Random sequence genera- Low risk Computer-generated randomisation list
tion (selection bias)

\begin{tabular}{ll}
$\begin{array}{l}\text { Allocation concealment } \\
\text { (selection bias) }\end{array}$ & Low risk \\
& $\begin{array}{l}\text { Quote: "An opaque envelope containing the patient number and group assign- } \\
\text { ment was prepared, sealed and numbered for each patient by the hospital } \\
\text { pharmacist" (a third party) }\end{array}$ \\
\hline
\end{tabular}


Katz 2004 (Continued)

\begin{tabular}{|c|c|c|}
\hline $\begin{array}{l}\text { Blinding of participants } \\
\text { and personnel (perfor- } \\
\text { mance bias) } \\
\text { All outcomes }\end{array}$ & Low risk & Blinded personnel \\
\hline $\begin{array}{l}\text { Blinding of outcome as- } \\
\text { sessment (detection bias) } \\
\text { All outcomes }\end{array}$ & Low risk & Blinded personnel \\
\hline $\begin{array}{l}\text { Incomplete outcome data } \\
\text { (attrition bias) } \\
\text { All outcomes }\end{array}$ & High risk & $11 \%$ was withdrawn \\
\hline $\begin{array}{l}\text { Selective reporting (re- } \\
\text { porting bias) }\end{array}$ & Low risk & Predefined outcomes reported \\
\hline Size & High risk & 47,46 and 50 participants per treatment arm \\
\hline
\end{tabular}

Kim 2013

\section{Study characteristics}

\begin{tabular}{|c|c|c|}
\hline Methods & \multicolumn{2}{|c|}{ Randomised, double-blind, placebo control } \\
\hline Participants & \multicolumn{2}{|c|}{$N=60$, about $47 \%$ women } \\
\hline Interventions & \multicolumn{2}{|c|}{$\begin{array}{l}\text { 1. Pre-incisional ketamine } 0.5 \mathrm{mg} / \mathrm{kg} \text { bolus IV }+1 \mathrm{mcg} / \mathrm{kg} / \mathrm{min} \text { intraoperative infusion IV until } 48 \mathrm{~h} \text { post- } \\
\text { operatively } \\
\text { 2. Pre-incisional ketamine } 0.5 \mathrm{mg} / \mathrm{kg} \text { bolus IV }+2 \mathrm{mcg} / \mathrm{kg} / \mathrm{min} \text { intraoperative infusion IV until } 48 \mathrm{~h} \text { post- } \\
\text { operatively }\end{array}$} \\
\hline Outcomes & \multicolumn{2}{|c|}{$\begin{array}{l}\text { Pain intensity (VAS) reported at 1, 6, } 12 \text { and } 24 \mathrm{~h} \text { postoperatively. Analgesic consumption reported at } 48 \\
\text { h postoperatively. AES }\end{array}$} \\
\hline Surgery type & \multicolumn{2}{|c|}{ Lumbar spinal fusion surgery } \\
\hline $\begin{array}{l}\text { Group numbers after end } \\
\text { of study (treatment/con- } \\
\text { trol) }\end{array}$ & \multicolumn{2}{|l|}{$35 / 17$} \\
\hline $\begin{array}{l}\text { Age of patient population } \\
\text { (treatment/control) }\end{array}$ & \multicolumn{2}{|l|}{$55.5 \pm 11.8$} \\
\hline Notes & \multicolumn{2}{|c|}{ No mention of sponsorship or funding } \\
\hline \multicolumn{3}{|l|}{ Risk of bias } \\
\hline Bias & Authors' judgement & Support for judgement \\
\hline $\begin{array}{l}\text { Random sequence genera- } \\
\text { tion (selection bias) }\end{array}$ & Unclear risk & Randomisation method not described \\
\hline $\begin{array}{l}\text { Allocation concealment } \\
\text { (selection bias) }\end{array}$ & Unclear risk & Allocation concealment method not described \\
\hline
\end{tabular}


Kim 2013 (Continued)

\begin{tabular}{|c|c|c|}
\hline $\begin{array}{l}\text { Blinding of participants } \\
\text { and personnel (perfor- } \\
\text { mance bias) } \\
\text { All outcomes }\end{array}$ & Low risk & Blinded personnel \\
\hline $\begin{array}{l}\text { Blinding of outcome as- } \\
\text { sessment (detection bias) } \\
\text { All outcomes }\end{array}$ & Low risk & Blinded personnel \\
\hline $\begin{array}{l}\text { Incomplete outcome data } \\
\text { (attrition bias) } \\
\text { All outcomes }\end{array}$ & High risk & $12 \%$ was withdrawn \\
\hline $\begin{array}{l}\text { Selective reporting (re- } \\
\text { porting bias) }\end{array}$ & Low risk & All predefined outcomes reported \\
\hline Size & High risk & 18,17 and 17 participants per treatment arm \\
\hline
\end{tabular}

Kim 2016

\section{Study characteristics}

\begin{tabular}{|c|c|c|}
\hline Methods & \multicolumn{2}{|c|}{ Randomised, double-blind, placebo control } \\
\hline Participants & \multicolumn{2}{|l|}{$N=58,95 \%$ women } \\
\hline Interventions & \multicolumn{2}{|c|}{$\begin{array}{l}\text { After the induction on anaesthesia, ketamine bolus } 1 \mathrm{mg} / \mathrm{kg} \text { IV followed by a continuous infusion } 60 \\
\mathrm{\mu g} / \mathrm{kg} / \mathrm{h} \text { until skin closure }\end{array}$} \\
\hline Outcomes & \multicolumn{2}{|c|}{$\begin{array}{l}\text { Pain intensity (NRS). Analgesic consumption. Pain outcomes reported at 1, 6, } 24 \text { and } 48 \text { h postopera- } \\
\text { tively. AEs }\end{array}$} \\
\hline Surgery type & \multicolumn{2}{|c|}{ Bilateral axillo-breast approach robotic or endoscopic thyroidectomy } \\
\hline $\begin{array}{l}\text { Group numbers after end } \\
\text { of study (treatment/con- } \\
\text { trol) }\end{array}$ & \multicolumn{2}{|l|}{$28 / 29$} \\
\hline \multirow{2}{*}{$\begin{array}{l}\text { Age of patient population } \\
\text { (treatment/control) }\end{array}$} & \multicolumn{2}{|l|}{$40 \pm 9$} \\
\hline & \multicolumn{2}{|l|}{$39 \pm 8$} \\
\hline Notes & \multicolumn{2}{|c|}{ No mention of sponsorship or funding. The study authors have no conflicts of interest. } \\
\hline \multicolumn{3}{|l|}{ Risk of bias } \\
\hline Bias & Authors' judgement & Support for judgement \\
\hline $\begin{array}{l}\text { Random sequence genera- } \\
\text { tion (selection bias) }\end{array}$ & Low risk & $\begin{array}{l}\text { A computer-generated table of random numbers (Random-Allocation Soft- } \\
\text { ware Version 1.0) }\end{array}$ \\
\hline $\begin{array}{l}\text { Allocation concealment } \\
\text { (selection bias) }\end{array}$ & Unclear risk & Allocation concealment method not described in detail \\
\hline
\end{tabular}


Kim 2016 (Continued)

\begin{tabular}{|c|c|c|}
\hline $\begin{array}{l}\text { Blinding of participants } \\
\text { and personnel (perfor- } \\
\text { mance bias) } \\
\text { All outcomes }\end{array}$ & Low risk & Blinded participants and personnel \\
\hline $\begin{array}{l}\text { Blinding of outcome as- } \\
\text { sessment (detection bias) } \\
\text { All outcomes }\end{array}$ & Low risk & Blinded personnel collected data \\
\hline $\begin{array}{l}\text { Incomplete outcome data } \\
\text { (attrition bias) } \\
\text { All outcomes }\end{array}$ & Low risk & $2 \%$ was withdrawn \\
\hline $\begin{array}{l}\text { Selective reporting (re- } \\
\text { porting bias) }\end{array}$ & Low risk & Predefined outcomes reported \\
\hline Size & High risk & 28 and 29 participants per treatment arm \\
\hline
\end{tabular}

Köse 2012

\section{Study characteristics}

\begin{tabular}{|c|c|c|}
\hline Methods & \multicolumn{2}{|c|}{ Randomised, double-blind, placebo control } \\
\hline Participants & \multicolumn{2}{|c|}{$\mathrm{N}=150$, of whom 120 participants in IV ketamine and control treatment arms. About $74 \%$ women } \\
\hline Interventions & \multicolumn{2}{|c|}{$\begin{array}{l}\text { Ketamine } 0.1 \mathrm{mg} / \mathrm{kg} \text { OR ketamine } 0.25 \mathrm{mg} / \mathrm{kg} \text { OR ketamine } 0.5 \mathrm{mg} / \mathrm{kg} \text { bolus IV } 20 \text { mins before the end } \\
\text { of surgery. Heterogeneous surgery types }\end{array}$} \\
\hline Outcomes & \multicolumn{2}{|c|}{$\begin{array}{l}\text { Pain intensity (VAS) reported at } 0,1 \text { and } 2 \mathrm{~h} \text { postoperatively. Time to first analgesic request. AEs. Post- } \\
\text { operative shivering }\end{array}$} \\
\hline Surgery type & \multicolumn{2}{|c|}{ Various operations under general anaesthesia } \\
\hline $\begin{array}{l}\text { Group numbers after end } \\
\text { of study (treatment/con- } \\
\text { trol) }\end{array}$ & \multicolumn{2}{|l|}{$90 / 30$} \\
\hline $\begin{array}{l}\text { Age of patient population } \\
\text { (treatment/control) }\end{array}$ & \multicolumn{2}{|l|}{$41.2 \pm 12$} \\
\hline Notes & \multicolumn{2}{|c|}{ Primary endpoint was postoperative shivering. No mention of sponsorship or funding } \\
\hline \multicolumn{3}{|l|}{ Risk of bias } \\
\hline Bias & Authors' judgement & Support for judgement \\
\hline $\begin{array}{l}\text { Random sequence genera- } \\
\text { tion (selection bias) }\end{array}$ & Unclear risk & Randomisation process not described \\
\hline $\begin{array}{l}\text { Allocation concealment } \\
\text { (selection bias) }\end{array}$ & Low risk & $\begin{array}{l}\text { Allocation in closed envelopes, identical study drug syringes prepared and la- } \\
\text { belled by an independent investigator not participating in the further study }\end{array}$ \\
\hline
\end{tabular}


Köse 2012 (Continued)

\begin{tabular}{|c|c|c|}
\hline $\begin{array}{l}\text { Blinding of participants } \\
\text { and personnel (perfor- } \\
\text { mance bias) } \\
\text { All outcomes }\end{array}$ & Low risk & Blinded participants and personnel \\
\hline $\begin{array}{l}\text { Blinding of outcome as- } \\
\text { sessment (detection bias) } \\
\text { All outcomes }\end{array}$ & Low risk & Blinded investigator made assessments \\
\hline $\begin{array}{l}\text { Incomplete outcome data } \\
\text { (attrition bias) } \\
\text { All outcomes }\end{array}$ & Low risk & No withdrawals \\
\hline $\begin{array}{l}\text { Selective reporting (re- } \\
\text { porting bias) }\end{array}$ & Low risk & Predefined outcomes reported \\
\hline Size & High risk & 30 participants per treatment arm \\
\hline
\end{tabular}

Kudoh 2002

\section{Study characteristics}

\begin{tabular}{ll}
\hline Methods & Randomised, double-blind, placebo control \\
\hline Participants & $\mathrm{N}=70$. Participants diagnosed as having major depression. Gender of participants not presented \\
\hline Interventions & Pre-incisional ketamine $1 \mathrm{mg} / \mathrm{kg}$ bolus IV \\
\hline Outcomes & $\begin{array}{l}\text { Pain intensity (VAS) reported every } 8 \mathrm{~h} \text { for the first } 24 \mathrm{~h} \text {, then every } 24 \mathrm{~h} \text { till 4th postoperative day. Post- } \\
\text { operative confusion }\end{array}$ \\
\hline $\begin{array}{l}\text { Surgery type } \\
\text { Oroup numbers after end } \\
\begin{array}{l}\text { of study (treatment/con- } \\
\text { trol) }\end{array}\end{array}$ & $35 / 35$ \\
\hline
\end{tabular}

Age of patient population
(treatment/control)

$48.2 \pm 7.4$

Notes No mention of sponsorship or funding

\section{Risk of bias}

\begin{tabular}{lll}
\hline Bias & Authors' judgement & Support for judgement \\
\hline $\begin{array}{l}\text { Random sequence genera- } \\
\text { tion (selection bias) }\end{array}$ & Low risk & Computer-generated random number table \\
\hline $\begin{array}{l}\text { Allocation concealment } \\
\text { (selection bias) }\end{array}$ & Unclear risk & Allocation concealment method not described \\
\hline $\begin{array}{l}\text { Blinding of participants } \\
\text { and personnel (perfor- } \\
\text { mance bias) }\end{array}$ & Low risk & Blinded participants \\
\hline
\end{tabular}


Kudoh 2002 (Continued)

All outcomes

\begin{tabular}{|c|c|c|}
\hline $\begin{array}{l}\text { Blinding of outcome as- } \\
\text { sessment (detection bias) } \\
\text { All outcomes }\end{array}$ & Low risk & Blinded personnel \\
\hline $\begin{array}{l}\text { Incomplete outcome data } \\
\text { (attrition bias) } \\
\text { All outcomes }\end{array}$ & Low risk & No withdrawals \\
\hline $\begin{array}{l}\text { Selective reporting (re- } \\
\text { porting bias) }\end{array}$ & Low risk & Predefined outcomes reported \\
\hline Size & High risk & 35 participants per treatment arm \\
\hline
\end{tabular}

Kwok 2004

\section{Study characteristics}

\begin{tabular}{|c|c|}
\hline Methods & Randomised, double-blind, placebo control \\
\hline Participants & $N=135,100 \%$ women \\
\hline Interventions & $\begin{array}{l}\text { 1. Pre-incisional bolus of ketamine } 0.15 \mathrm{mg} / \mathrm{kg} \text { IV } \\
\text { 2. At wound closure: ketamine bolus } 0.15 \mathrm{mg} / \mathrm{kg} \text { IV }\end{array}$ \\
\hline Outcomes & $\begin{array}{l}\text { Pain intensity (VAS) reported hourly up to } 7 \mathrm{~h} \text { postoperatively, then at } 24 \mathrm{~h} \text {. Analgesic consumption un- } \\
\text { til VAS }<20 \mathrm{~mm} \text {. AEs }\end{array}$ \\
\hline Surgery type & Laparoscopic gynaecological surgery \\
\hline $\begin{array}{l}\text { Group numbers after end } \\
\text { of study (treatment/con- } \\
\text { trol) }\end{array}$ & $90 / 45$ \\
\hline $\begin{array}{l}\text { Age of patient population } \\
\text { (treatment/control) }\end{array}$ & $\begin{array}{l}33 \pm 6.1 \\
34 \pm 6\end{array}$ \\
\hline Notes & No mention of sponsorship or funding \\
\hline
\end{tabular}

\section{Risk of bias}

\begin{tabular}{lll}
\hline Bias & Authors' judgement & Support for judgement \\
\hline $\begin{array}{l}\text { Random sequence genera- } \\
\text { tion (selection bias) }\end{array}$ & Low risk & Computer-generated random numbers \\
\hline $\begin{array}{l}\text { Allocation concealment } \\
\text { (selection bias) }\end{array}$ & Low risk & Allocation was concealed in opaque envelopes \\
\hline $\begin{array}{l}\text { Blinding of participants } \\
\text { and personnel (perfor- } \\
\text { mance bias) }\end{array}$ & Low risk & Blinded personnel \\
All outcomes & & \\
\hline
\end{tabular}


Kwok 2004 (Continued)

$\begin{array}{lll}\text { Blinding of outcome as- } & \text { Low risk } & \text { Blinded personnel } \\ \text { sessment (detection bias) } & \end{array}$

All outcomes

\begin{tabular}{lll}
\hline $\begin{array}{l}\text { Incomplete outcome data } \\
\text { (attrition bias) } \\
\text { All outcomes }\end{array}$ & Low risk & No withdrawals \\
\hline $\begin{array}{l}\text { Selective reporting (re- } \\
\text { porting bias) }\end{array}$ & Low risk & Predefined outcomes reported \\
\hline Size & High risk & 45 participants per treatment arm \\
\hline
\end{tabular}

Kwon 2009

\section{Study characteristics}

\begin{tabular}{ll}
\hline Methods & Randomised, double-blind, placebo control \\
\hline Participants & $\mathrm{N}=40,100 \%$ women \\
\hline Interventions & Intraoperative ketamine $0.3 \mathrm{mg} / \mathrm{kg}$ bolus IV followed by an infusion $3 \mu \mathrm{g} / \mathrm{kg} / \mathrm{min}$ \\
\hline Outcomes & $\begin{array}{l}\text { Pain intensity (VAS) reported at } 24 \text { and } 48 \mathrm{~h} \text { postoperatively. Analgesic consumption reported at 0-6 h, } \\
\text { 6-12 h, 12-24 h and } 24-48 \text { h postoperatively. AEs }\end{array}$ \\
\hline $\begin{array}{l}\text { Surgery type } \\
\text { Mroup numbers after end } \\
\text { of study (treatment/con- } \\
\text { trol) }\end{array}$ & $20 / 20$ \\
\hline $\begin{array}{l}\text { Age of patient population } \\
\text { treatment/control) }\end{array}$ & $50.5 \pm 8.8$ \\
\hline $\begin{array}{l}\text { Notes } \\
47.2 \pm 7.4\end{array}$
\end{tabular}

\section{Risk of bias}

\begin{tabular}{lll}
\hline Bias & Authors' judgement & Support for judgement \\
\hline $\begin{array}{l}\text { Random sequence genera- } \\
\text { tion (selection bias) }\end{array}$ & Unclear risk & Randomisation not described \\
\hline $\begin{array}{l}\text { Allocation concealment } \\
\text { (selection bias) }\end{array}$ & Unclear risk & Allocation concealment method not described \\
\hline $\begin{array}{l}\text { Blinding of participants } \\
\text { and personnel (perfor- } \\
\text { mance bias) }\end{array}$ & Unclear risk & Blinding not described \\
All outcomes & \\
\hline $\begin{array}{l}\text { Blinding of outcome as- } \\
\text { sessment (detection bias) }\end{array}$ & Unclear risk & Not described in detail \\
\hline
\end{tabular}


Kwon 2009 (Continued)

All outcomes

\begin{tabular}{lll}
\hline $\begin{array}{l}\text { Incomplete outcome data } \\
\text { (attrition bias) } \\
\text { All outcomes }\end{array}$ & Low risk & No withdrawals \\
\hline $\begin{array}{l}\text { Selective reporting (re- } \\
\text { porting bias) }\end{array}$ & Low risk & Prespecified outcomes reported \\
\hline Size & High risk & 20 participants per treatment arm \\
\hline
\end{tabular}

\section{Lahtinen 2004}

\section{Study characteristics}

\begin{tabular}{ll}
\hline Methods & Randomised, double-blind, placebo control \\
\hline Participants & $\mathrm{N}=102$, about $11 \%$ women \\
\hline Interventions & $\begin{array}{l}\text { S-ketamine } 75 \mu \mathrm{g} / \mathrm{kg} \text { bolus IV immediately after anaesthesia induction + intraoperative IV infusion } 1.25 \\
\mathrm{\mu g} / \mathrm{kg} / \mathrm{min} \text { for } 48 \mathrm{~h} \text { after arrival to the PACU }\end{array}$ \\
\hline Outcomes & $\begin{array}{l}\text { Pain intensity (VAS) reported after extubation, after titration of oxycodone until VAS }<30 \mathrm{~mm} \text {, on the } \\
\text { day of surgery at } 12 \mathrm{pm} \text {, on first postoperative day at } 8 \text { am, } 4 \text { pm and } 12 \text { pm, on second postoperative } \\
\text { day at } 8 \mathrm{pm} \text { and } 4 \mathrm{pm} . \text { Cumulative analgesic consumption at } 48 \mathrm{~h} \text { postoperatively. AEs }\end{array}$ \\
\hline
\end{tabular}

\begin{tabular}{ll} 
Surgery type Sternotomy \\
\hline
\end{tabular}

\section{Group numbers after end $\quad 44 / 46$}

of study (treatment/con-

trol)

\begin{tabular}{ll}
\hline $\begin{array}{l}\text { Age of patient population } \\
\text { (treatment/control) }\end{array}$ & $59 \pm 5$ \\
& $58 \pm 7$ \\
\hline Notes & No mention of sponsorship or funding
\end{tabular}

\section{Risk of bias}

\begin{tabular}{|c|c|c|}
\hline Bias & Authors' judgement & Support for judgement \\
\hline $\begin{array}{l}\text { Random sequence genera- } \\
\text { tion (selection bias) }\end{array}$ & Low risk & $\begin{array}{l}\text { Quote: "Randomization was performed with a computer program by using } \\
\text { random numbers and a balanced design." }\end{array}$ \\
\hline $\begin{array}{l}\text { Allocation concealment } \\
\text { (selection bias) }\end{array}$ & Low risk & $\begin{array}{l}\text { Mentioned: "the code remained blinded until the end of the study" and that } \\
\text { study drug syringes were identical in appearance but not described in detail } \\
\text { how the allocation was concealed }\end{array}$ \\
\hline $\begin{array}{l}\text { Blinding of participants } \\
\text { and personnel (perfor- } \\
\text { mance bias) } \\
\text { All outcomes }\end{array}$ & Low risk & Blinded personnel \\
\hline $\begin{array}{l}\text { Blinding of outcome as- } \\
\text { sessment (detection bias) }\end{array}$ & Low risk & Blinded personnel \\
\hline
\end{tabular}


Lahtinen 2004 (Continued)

All outcomes

\begin{tabular}{lll}
$\begin{array}{l}\text { Incomplete outcome data } \\
\text { (attrition bias) } \\
\text { All outcomes }\end{array}$ & High risk & $11 \%$ was withdrawn \\
\hline $\begin{array}{l}\text { Selective reporting (re- } \\
\text { porting bias) }\end{array}$ & Low risk & All predefined outcomes reported adequately \\
\hline Size & High risk & 44 and 46 participants per treatment arm \\
\hline
\end{tabular}

\section{Lak 2010}

\section{Study characteristics}

\begin{tabular}{ll}
\hline Methods & Randomised, double-blind, placebo control \\
\hline Participants & $\mathrm{N}=60,12 \%$ women \\
\hline Interventions & $\begin{array}{l}\text { Ketamine } 0.5 \mathrm{mg} / \mathrm{kg} \text { bolus IV postoperatively + IV infusion } 2 \mu \mathrm{g} / \mathrm{kg} / \mathrm{min} \text { for } 24 \mathrm{~h}, \text { then } 1 \mu \mathrm{g} / \mathrm{kg} / \mathrm{min} \text { the } \\
\text { following } 24 \mathrm{~h}\end{array}$ \\
\hline $\begin{array}{l}\text { Outcomes } \\
\text { Pain intensity (VAS and face pain scale) reported hourly during the first } 4 \mathrm{~h}, \text { then at } 8,12,24 \text { and } 48 \mathrm{~h} \\
\text { postoperatively. Analgesic consumption reported at } 48 \text { h postoperatively. AEs }\end{array}$ \\
\hline $\begin{array}{l}\text { Group numbers after end } \\
\text { of study (treatment/con- } \\
\text { trol) }\end{array}$ & $25 / 25$ \\
\hline $\begin{array}{l}\text { Age of patient population } \\
\text { (treatment/control) }\end{array}$ & $27.3 \pm 5.5$ \\
\hline \begin{tabular}{l} 
Notes \\
\hline
\end{tabular} & No mention of sponsorship or funding \\
\hline
\end{tabular}

\section{Risk of bias}

\begin{tabular}{lll}
\hline Bias & Authors' judgement & Support for judgement \\
\hline $\begin{array}{l}\text { Random sequence genera- } \\
\text { tion (selection bias) }\end{array}$ & Low risk & $\begin{array}{l}\text { Quote: "Randomization was assigned to patients of the two groups according } \\
\text { to random numbers table" }\end{array}$ \\
\hline $\begin{array}{l}\text { Allocation concealment } \\
\text { (selection bias) }\end{array}$ & Unclear risk & Allocation concealment method not described \\
\hline $\begin{array}{l}\text { Blinding of participants } \\
\text { and personnel (perfor- } \\
\text { mance bias) } \\
\text { All outcomes }\end{array}$ & Unclear risk & Described as "double blind" but not described \\
\hline
\end{tabular}

Blinding of outcome as- Unclear risk Blinding process not described sessment (detection bias)

All outcomes 
Lak 2010 (Continued)

Incomplete outcome data High risk $20 \%$ was withdrawn
(attrition bias)
All outcomes

\begin{tabular}{lll}
\hline $\begin{array}{l}\text { Selective reporting (re- } \\
\text { porting bias) }\end{array}$ & High risk & Pain outcome not reported using predefined pain scale (face pain scale) \\
\hline Size & High risk & 25 participants per treatment arm \\
\hline
\end{tabular}

Leal 2013

\section{Study characteristics}

\begin{tabular}{ll}
\hline Methods & Randomised, double-blind, placebo control \\
\hline Participants & $\mathrm{N}=40$, about $83 \%$ women \\
\hline Interventions & Ketamine $5 \mu \mathrm{g} / \mathrm{kg} / \mathrm{min}$ infusion IV until wound closure \\
\hline Outcomes & $\begin{array}{l}\text { Pain intensity (NRS) reported every } 30 \text { min up to } 4 \mathrm{~h} \text {, then every } 6 \text { h up to } 24 \mathrm{~h} \text { postoperatively. Time to } \\
\text { the first analgesic supplementation. Cumulative analgesic consumption reported at } 24 \text { h postopera- } \\
\text { tively. AEs }\end{array}$
\end{tabular}

\begin{tabular}{ll}
\hline Surgery type & Laparoscopic cholecystectomy \\
\hline $\begin{array}{l}\text { Group numbers after end } \\
\text { of study (treatment/con- } \\
\text { trol) }\end{array}$ & \\
\hline
\end{tabular}

$\begin{array}{ll}\begin{array}{l}\text { Age of patient population } \\ \text { (treatment/control) }\end{array} & 46 \pm 12.5 \\ & 45.5 \pm 16.1\end{array}$

\begin{tabular}{|c|c|c|}
\hline Notes & \multicolumn{2}{|c|}{ No mention of sponsorship or funding } \\
\hline \multicolumn{3}{|l|}{ Risk of bias } \\
\hline Bias & Authors' judgement & Support for judgement \\
\hline $\begin{array}{l}\text { Random sequence genera- } \\
\text { tion (selection bias) }\end{array}$ & Low risk & Randomisation achieved by drawing envelopes \\
\hline $\begin{array}{l}\text { Allocation concealment } \\
\text { (selection bias) }\end{array}$ & Unclear risk & Allocation concealment method not described \\
\hline $\begin{array}{l}\text { Blinding of participants } \\
\text { and personnel (perfor- } \\
\text { mance bias) } \\
\text { All outcomes }\end{array}$ & Low risk & Blinded personnel \\
\hline $\begin{array}{l}\text { Blinding of outcome as- } \\
\text { sessment (detection bias) } \\
\text { All outcomes }\end{array}$ & Low risk & Blinded personnel \\
\hline $\begin{array}{l}\text { Incomplete outcome data } \\
\text { (attrition bias) }\end{array}$ & Low risk & No withdrawals \\
\hline
\end{tabular}


Leal 2013 (Continued)

All outcomes

\begin{tabular}{lll}
\hline $\begin{array}{l}\text { Selective reporting (re- } \\
\text { porting bias) }\end{array}$ & High risk & $\begin{array}{l}\text { AEs are defined and reported in 'results' section, but not predefined in 'meth- } \\
\text { ods' section }\end{array}$ \\
\hline Size & High risk & 20 participants per treatment arm \\
\hline
\end{tabular}

Leal 2015

\section{Study characteristics}

\begin{tabular}{ll}
\hline Methods & Randomised, double-blind, placebo control \\
\hline Participants & $\mathrm{N}=56$, about $84 \%$ women \\
\hline Interventions & Ketamine $5 \mu \mathrm{g} / \mathrm{kg} / \mathrm{min}$ infusion IV until skin closure \\
\hline Outcomes & $\begin{array}{l}\text { Pain intensity (NRS) reported in } 30-\text { min intervals up to } 4 \mathrm{~h} \text {, then in } 6 \text { - } \mathrm{h} \text { intervals up to } 24 \mathrm{~h} \text { postopera- } \\
\text { tively. Analgesic consumption reported at } 24 \mathrm{~h} \text { postoperatively. Time to first morphine supplementa- } \\
\text { tion. Extent of hyperalgesia reported at } 24 \mathrm{~h} \text { after surgery. AEs }\end{array}$ \\
\hline
\end{tabular}

\begin{tabular}{ll}
\hline Surgery type & Laparoscopic cholecystectomy \\
\hline $\begin{array}{l}\text { Group numbers after end } \\
\text { of study (treatment/con- } \\
\text { trol) }\end{array}$ & $28 / 28$ \\
\hline $\begin{array}{l}\text { Age of patient population } \\
\text { treatment/control) }\end{array}$ & $45.8 \pm 13.1$ \\
& $43.4 \pm 15.9$ \\
\hline Notes & $\begin{array}{l}\text { Funded by grant 2009/5335-4, São Paulo Research Foundation and Coordenacão de Aperfeicoamento } \\
\text { de Pessoal De Nivel Superior }\end{array}$ \\
\hline
\end{tabular}

\section{Risk of bias}

\begin{tabular}{|c|c|c|}
\hline Bias & Authors' judgement & Support for judgement \\
\hline $\begin{array}{l}\text { Random sequence genera- } \\
\text { tion (selection bias) }\end{array}$ & Low risk & Randomisation achieved by a computer program "Randomizer" \\
\hline $\begin{array}{l}\text { Allocation concealment } \\
\text { (selection bias) }\end{array}$ & Low risk & Opaque, sealed envelopes contained allocation information \\
\hline $\begin{array}{l}\text { Blinding of participants } \\
\text { and personnel (perfor- } \\
\text { mance bias) } \\
\text { All outcomes }\end{array}$ & Low risk & Blinded personnel \\
\hline $\begin{array}{l}\text { Blinding of outcome as- } \\
\text { sessment (detection bias) } \\
\text { All outcomes }\end{array}$ & Low risk & Binded personnel \\
\hline $\begin{array}{l}\text { Incomplete outcome data } \\
\text { (attrition bias) } \\
\text { All outcomes }\end{array}$ & Low risk & $7 \%$ was withdrawn \\
\hline
\end{tabular}


Leal 2015 (Continued)

\begin{tabular}{lll}
$\begin{array}{l}\text { Selective reporting (re- } \\
\text { porting bias) }\end{array}$ & High risk & Adverse effects not defined in 'methods' section but are reported \\
\hline Size & High risk & 28 participants per treatment arm
\end{tabular}

\section{Lebrun 2006}

\section{Study characteristics}

\begin{tabular}{|c|c|c|}
\hline Methods & \multicolumn{2}{|c|}{ Randomised, double-blind, placebo control } \\
\hline Participants & \multicolumn{2}{|c|}{$\mathrm{N}=84$, about $42 \%$ women } \\
\hline Interventions & \multicolumn{2}{|c|}{$\begin{array}{l}\text { 1. Ketamine } 0.3 \mathrm{mg} / \mathrm{kg} \text { bolus IV at anaesthesia induction } \\
\text { 2. Ketamine } 0.3 \mathrm{mg} / \mathrm{kg} \text { bolus IV at the end of surgery }\end{array}$} \\
\hline Outcomes & \multicolumn{2}{|c|}{$\begin{array}{l}\text { Pain intensity (VAS) reported at } 0.5,1,2,4,24 \text { and } 48 \mathrm{~h} \text { postoperatively. Time to first request for analge- } \\
\text { sia. AEs. Analgesic consumption in PACU }\end{array}$} \\
\hline Surgery type & \multicolumn{2}{|c|}{ Third molar surgical removal } \\
\hline $\begin{array}{l}\text { Group numbers after end } \\
\text { of study (treatment/con- } \\
\text { trol) }\end{array}$ & \multicolumn{2}{|l|}{$54 / 30$} \\
\hline $\begin{array}{l}\text { Age of patient population } \\
\text { (treatment/control) }\end{array}$ & \multicolumn{2}{|l|}{$19 \pm 6.7$} \\
\hline Notes & \multicolumn{2}{|c|}{ No mention of sponsorship or funding } \\
\hline \multicolumn{3}{|l|}{ Risk of bias } \\
\hline Bias & Authors' judgement & Support for judgement \\
\hline $\begin{array}{l}\text { Random sequence genera- } \\
\text { tion (selection bias) }\end{array}$ & Low risk & Computer-generated random numbers \\
\hline $\begin{array}{l}\text { Allocation concealment } \\
\text { (selection bias) }\end{array}$ & Unclear risk & $\begin{array}{l}\text { Allocation concealment not described in detail. Only mentioned that a nurse } \\
\text { not involved in the study prepared study drugs }\end{array}$ \\
\hline $\begin{array}{l}\text { Blinding of participants } \\
\text { and personnel (perfor- } \\
\text { mance bias) } \\
\text { All outcomes }\end{array}$ & Low risk & Blinded personnel and participants \\
\hline $\begin{array}{l}\text { Blinding of outcome as- } \\
\text { sessment (detection bias) } \\
\text { All outcomes }\end{array}$ & Low risk & Blinded personnel \\
\hline $\begin{array}{l}\text { Incomplete outcome data } \\
\text { (attrition bias) } \\
\text { All outcomes }\end{array}$ & High risk & $13 \%$ was withdrawn \\
\hline
\end{tabular}


Lebrun 2006 (Continued)

\begin{tabular}{lll}
$\begin{array}{l}\text { Selective reporting (re- } \\
\text { porting bias) }\end{array}$ & Low risk & Predefined outcomes reported \\
\hline Size & High risk & 31,23 and 30 participants per treatment arm
\end{tabular}

Lee 2008

\section{Study characteristics}

\begin{tabular}{ll}
\hline Methods & Randomised, double-blind, placebo control \\
\hline Participants & $\mathrm{N}=32$, about $55 \%$ women \\
\hline Interventions & Pre-incisional ketamine $0.15 \mathrm{mg} / \mathrm{kg}$ bolus IV \\
\hline Outcomes & $\begin{array}{l}\text { Pain intensity (NRS) reported on arrival to PACU and at } 5,10,15 \text { and } 30 \text { min, then at } 5 \text { and } 24 \mathrm{~h} \text { postop- } \\
\text { eratively. Analgesic consumption (either ketorolac or tramadol) reported as additional count per day }\end{array}$ \\
\hline $\begin{array}{l}\text { Surgery type } \\
\begin{array}{l}\text { Group numbers after end } \\
\text { of study (treatment/con- } \\
\text { trol) }\end{array}\end{array}$ & \begin{tabular}{l} 
Laparoscopic cholecystectomy \\
\hline
\end{tabular}
\end{tabular}

$\begin{array}{ll}\begin{array}{l}\text { Age of patient population } \\ \text { (treatment/control) }\end{array} & 40.7 \pm 7.8 \\ & 43.8 \pm 10.8\end{array}$

$43.8 \pm 10.8$

Notes Article in Korean. No mention of sponsorship or funding

\section{Risk of bias}

\begin{tabular}{lll}
\hline Bias & Authors' judgement & Support for judgement \\
\hline $\begin{array}{l}\text { Random sequence genera- } \\
\text { tion (selection bias) }\end{array}$ & Unclear risk & Study authors do not describe randomisation \\
\hline $\begin{array}{l}\text { Allocation concealment } \\
\text { (selection bias) }\end{array}$ & Unclear risk & Allocation concealment method not described \\
\hline $\begin{array}{l}\text { Blinding of participants } \\
\text { and personnel (perfor- } \\
\text { mance bias) } \\
\text { All outcomes }\end{array}$ & Unclear risk & Blinding not described, only stated "double blind" in the title \\
\hline
\end{tabular}

\begin{tabular}{|c|c|c|}
\hline $\begin{array}{l}\text { Blinding of outcome as- } \\
\text { sessment (detection bias) } \\
\text { All outcomes }\end{array}$ & Low risk & A blinded investigator assessed postoperative pain scores \\
\hline $\begin{array}{l}\text { Incomplete outcome data } \\
\text { (attrition bias) } \\
\text { All outcomes }\end{array}$ & Low risk & $3 \%$ was withdrawn \\
\hline $\begin{array}{l}\text { Selective reporting (re- } \\
\text { porting bias) }\end{array}$ & Unclear risk & The study protocol was not available \\
\hline
\end{tabular}


Lee 2008 (Continued)
Size
High risk
16 and 15 participants per treatment arm

Lehmann 2001

\section{Study characteristics}

\begin{tabular}{ll}
\hline Methods & Randomised, double-blind, placebo control \\
\hline Participants & $\mathrm{N}=80$, about $58 \%$ women \\
\hline Interventions & Pre-incisional bolus of ketamine $0.15 \mathrm{mg} / \mathrm{kg} \mathrm{IV}$ \\
\hline Outcomes & $\begin{array}{l}\text { Pain intensity (VAS, VRS). PCA piritramide consumption. Rescue medication. AEs. Pain outcomes re- } \\
\text { ported hourly up to } 6 \mathrm{~h} \text { postoperatively, then at } 12 \text { and } 24 \mathrm{~h} \text { after surgery }\end{array}$ \\
\hline $\begin{array}{l}\text { Surgery type } \\
\text { Lroup numbers after end } \\
\text { of study (treatment/con- } \\
\text { trol) }\end{array}$ & \begin{tabular}{l}
$40 / 40$ \\
\hline $\begin{array}{l}\text { Age of patient population } \\
\text { (treatment/control) }\end{array}$
\end{tabular} \\
$\begin{array}{l}46 \pm 12 \\
43 \pm 10\end{array}$ \\
\hline $\begin{array}{l}\text { Notes } \\
\text { In the English abstract, the operation is laparoscopic surgery, in the original article (in German), it is } \\
\text { said to be laparotomy. We contacted the study author and he clarified that the procedure was laparo- } \\
\text { tomy or proctologic surgery. No mention of sponsorship or funding }\end{array}$ \\
\hline
\end{tabular}

\section{Risk of bias}

\begin{tabular}{lll}
\hline Bias & Authors' judgement & Support for judgement \\
\hline $\begin{array}{l}\text { Random sequence genera- } \\
\text { tion (selection bias) }\end{array}$ & Low risk & Computer-generated randomisation list \\
\hline $\begin{array}{l}\text { Allocation concealment } \\
\text { (selection bias) }\end{array}$ & Unclear risk & Allocation concealment method not described in detail \\
\hline $\begin{array}{l}\text { Blinding of participants } \\
\text { and personnel (perfor- } \\
\text { mance bias) }\end{array}$ & Low risk & A person not involved in patient care prepared the study solutions \\
$\begin{array}{l}\text { All outcomes } \\
\text { Blinding of outcome as- } \\
\text { sessment (detection bias) } \\
\text { All outcomes }\end{array}$ & Unclear risk & Not described \\
\hline $\begin{array}{l}\text { Incomplete outcome data } \\
\text { (attrition bias) } \\
\text { All outcomes }\end{array}$ & Unclear risk & Withdrawals not reported \\
\hline $\begin{array}{l}\text { Selective reporting (re- } \\
\text { porting bias) }\end{array}$ & Low risk & Predefined outcomes reported. Results as percentage \\
\hline
\end{tabular}


Lehmann 2001 (Continued)
Size
High risk
40 participants per treatment arm

Lenzmeier 2008

\section{Study characteristics}

\begin{tabular}{|c|c|c|}
\hline Methods & \multicolumn{2}{|c|}{ Randomised, double-blind, placebo control } \\
\hline Participants & \multicolumn{2}{|l|}{$\mathrm{N}=22,100 \%$ women } \\
\hline Interventions & \multicolumn{2}{|c|}{ Ketamine $0.5 \mathrm{mg} / \mathrm{kg}$ bolus IV at anaesthesia induction } \\
\hline Outcomes & \multicolumn{2}{|c|}{$\begin{array}{l}\text { Pain intensity (VAS) reported upon admission to and at discharge from PACU. Opioid consumption dur- } \\
\text { ing PACU stay }\end{array}$} \\
\hline Surgery type & \multicolumn{2}{|c|}{ Laparoscopic abdominal procedures } \\
\hline $\begin{array}{l}\text { Group numbers after end } \\
\text { of study (treatment/con- } \\
\text { trol) }\end{array}$ & \multicolumn{2}{|l|}{$11 / 11$} \\
\hline \multirow{2}{*}{$\begin{array}{l}\text { Age of patient population } \\
\text { (treatment/control) }\end{array}$} & \multicolumn{2}{|l|}{$29.7 \pm 8.5$} \\
\hline & \multicolumn{2}{|l|}{$31.6 \pm 6.7$} \\
\hline Notes & \multicolumn{2}{|c|}{ A pilot study, findings reported as descriptive statistics. No mention of sponsorship or funding } \\
\hline \multicolumn{3}{|l|}{ Risk of bias } \\
\hline Bias & Authors' judgement & Support for judgement \\
\hline $\begin{array}{l}\text { Random sequence genera- } \\
\text { tion (selection bias) }\end{array}$ & Unclear risk & Sequence generation not described \\
\hline $\begin{array}{l}\text { Allocation concealment } \\
\text { (selection bias) }\end{array}$ & Unclear risk & Allocation concealment method not described \\
\hline $\begin{array}{l}\text { Blinding of participants } \\
\text { and personnel (perfor- } \\
\text { mance bias) } \\
\text { All outcomes }\end{array}$ & Unclear risk & Mentioned "double-blind" but blinding process not described \\
\hline
\end{tabular}

\begin{tabular}{lll}
\hline $\begin{array}{l}\text { Blinding of outcome as- } \\
\text { sessment (detection bias) } \\
\text { All outcomes }\end{array}$ & Unclear risk & Blinding not described \\
\hline $\begin{array}{l}\text { Incomplete outcome data } \\
\text { (attrition bias) } \\
\text { All outcomes }\end{array}$ & Low risk & No withdrawals \\
\hline $\begin{array}{l}\text { Selective reporting (re- } \\
\text { porting bias) }\end{array}$ & Low risk & Predefined outcomes reported \\
\hline Size & High risk & 11 participants per treatment arm. \\
\hline
\end{tabular}


Lin 2016

\section{Study characteristics}

\begin{tabular}{|c|c|c|}
\hline Methods & \multicolumn{2}{|c|}{ Randomised, double-blind, placebo control } \\
\hline Participants & \multicolumn{2}{|l|}{$90,100 \%$ women } \\
\hline Interventions & \multicolumn{2}{|c|}{ Pre-incisional ketamine $0.3 \mathrm{mg} / \mathrm{kg}$ bolus IV } \\
\hline Outcomes & \multicolumn{2}{|c|}{$\begin{array}{l}\text { Pain intensity (VAS) reported at 2, 6, } 12 \text { and } 24 \text { h postoperatively. Mean analgesic dose per participant. } \\
\text { Time to first analgesic request. AEs }\end{array}$} \\
\hline Surgery type & \multicolumn{2}{|c|}{ Gynecological laparoscopic surgery } \\
\hline $\begin{array}{l}\text { Group numbers after end } \\
\text { of study (treatment/con- } \\
\text { trol) }\end{array}$ & \multicolumn{2}{|l|}{$30 / 29$} \\
\hline $\begin{array}{l}\text { Age of patient population } \\
\text { (treatment/control) }\end{array}$ & \multicolumn{2}{|l|}{$\begin{array}{l}39.6 \pm 9.8 \\
43.4 \pm 10.1\end{array}$} \\
\hline Notes & \multicolumn{2}{|c|}{ No mention of sponsorship or funding } \\
\hline \multicolumn{3}{|l|}{ Risk of bias } \\
\hline Bias & Authors' judgement & Support for judgement \\
\hline $\begin{array}{l}\text { Random sequence genera- } \\
\text { tion (selection bias) }\end{array}$ & Unclear risk & Randomisation method not described \\
\hline $\begin{array}{l}\text { Allocation concealment } \\
\text { (selection bias) }\end{array}$ & Unclear risk & Allocation concealment method not described in detail \\
\hline $\begin{array}{l}\text { Blinding of participants } \\
\text { and personnel (perfor- } \\
\text { mance bias) } \\
\text { All outcomes }\end{array}$ & Unclear risk & Only mentioned double-blind with no further description \\
\hline $\begin{array}{l}\text { Blinding of outcome as- } \\
\text { sessment (detection bias) } \\
\text { All outcomes }\end{array}$ & Unclear risk & Not described in detail \\
\hline $\begin{array}{l}\text { Incomplete outcome data } \\
\text { (attrition bias) } \\
\text { All outcomes }\end{array}$ & Low risk & $2 \%$ was withdrawn \\
\hline $\begin{array}{l}\text { Selective reporting (re- } \\
\text { porting bias) }\end{array}$ & High risk & $\begin{array}{l}\text { Results of time to first request for analgesia are reported but this outcome was } \\
\text { not predefined in methods }\end{array}$ \\
\hline Size & High risk & 29 and 30 participants per treatment arm \\
\hline
\end{tabular}


Lo 2008

\section{Study characteristics}

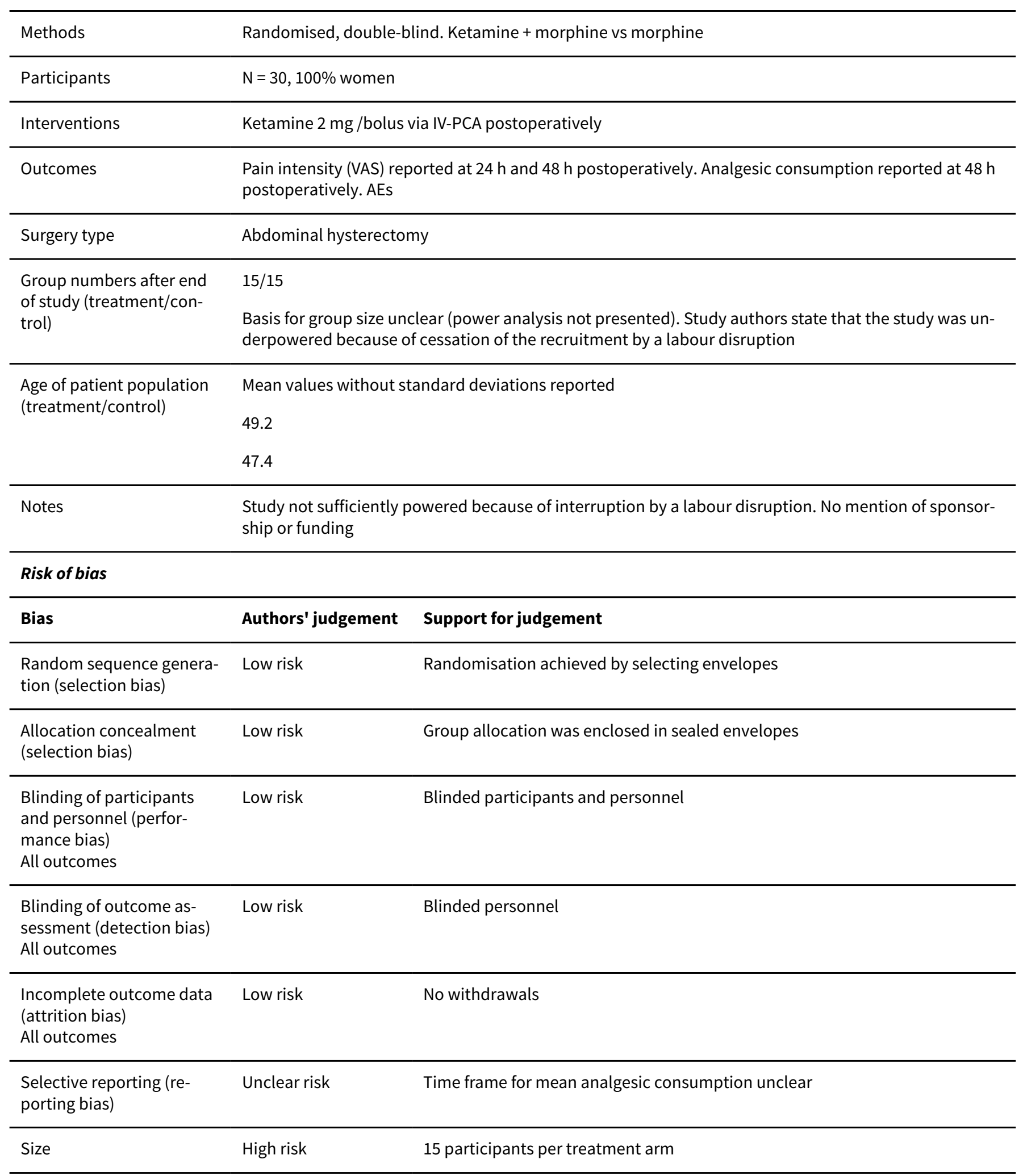


Loftus 2010

\section{Study characteristics}

\begin{tabular}{ll}
\hline Methods & Randomised, double-blind, placebo control \\
\hline Participants & $\mathrm{N}=101$, about $40 \%$ women \\
\hline Interventions & Ketamine $0.5 \mathrm{mg} / \mathrm{kg}$ bolus IV on anaesthesia induction + infusion $10 \mu \mathrm{gg} / \mathrm{kg} / \mathrm{min}$ IV until wound closure \\
\hline Outcomes & Pain intensity (VAS). Analgesic consumption. AEs. \\
\hline $\begin{array}{l}\text { Surgery type } \\
\begin{array}{l}\text { Group numbers after end } \\
\text { of study (treatment/con- } \\
\text { trol) }\end{array}\end{array}$ & Major lumbar spine surgery \\
\hline
\end{tabular}

$51.7 \pm 14.2$

$51.4 \pm 14.4$

Age of patient population (treatment/control)

No mention of sponsorship or funding

\section{Risk of bias}

\begin{tabular}{|c|c|c|}
\hline Bias & Authors' judgement & Support for judgement \\
\hline $\begin{array}{l}\text { Random sequence genera- } \\
\text { tion (selection bias) }\end{array}$ & Low risk & Computer-generated block randomisation scheme \\
\hline $\begin{array}{l}\text { Allocation concealment } \\
\text { (selection bias) }\end{array}$ & Low risk & $\begin{array}{l}\text { The study infusions were prepared by the investigational pharmacy (a third } \\
\text { party) preoperatively and labelled as "study drug/placebo" }\end{array}$ \\
\hline $\begin{array}{l}\text { Blinding of participants } \\
\text { and personnel (perfor- } \\
\text { mance bias) } \\
\text { All outcomes }\end{array}$ & Low risk & Blinded personnel \\
\hline $\begin{array}{l}\text { Blinding of outcome as- } \\
\text { sessment (detection bias) } \\
\text { All outcomes }\end{array}$ & Low risk & Blinded personnel \\
\hline $\begin{array}{l}\text { Incomplete outcome data } \\
\text { (attrition bias) } \\
\text { All outcomes }\end{array}$ & Low risk & $\begin{array}{l}\text { Less than } 10 \% \text { of the data pertaining to the primary outcome were missing in } \\
\text { the final analysis. Missing data were due to unanticipated early participant dis- } \\
\text { charge with equal numbers in both treatment groups. No participants enrolled } \\
\text { in the study were excluded from the primary analysis }\end{array}$ \\
\hline $\begin{array}{l}\text { Selective reporting (re- } \\
\text { porting bias) }\end{array}$ & Low risk & Predefined outcomes reported adequately \\
\hline Size & Unclear risk & 50 and 52 participants per treatment arm \\
\hline
\end{tabular}


Mahran 2015

\section{Study characteristics}

\begin{tabular}{|c|c|c|}
\hline Methods & \multicolumn{2}{|c|}{ Randomised, double-blind, placebo control } \\
\hline Participants & \multicolumn{2}{|c|}{$\mathrm{N}=90$, of whom 60 participants in IV ketamine and control treatment arms. $100 \%$ women } \\
\hline Interventions & \multicolumn{2}{|c|}{$\begin{array}{l}\text { Ketamine } 0.5 \mathrm{mg} / \mathrm{kg} \text { bolus IV before anaesthesia induction + intraoperative IV infusion } 0.25 \mathrm{mg} / \mathrm{kg} / \mathrm{h} \text { till } \\
\text { the end of skin closure }\end{array}$} \\
\hline Outcomes & \multicolumn{2}{|c|}{$\begin{array}{l}\text { Pain intensity (VAS) reported at } 30 \mathrm{~min}, 2,4,6,12 \text { and } 24 \text { h postoperatively. Cumulative analgesic con- } \\
\text { sumption at } 24 \mathrm{~h} \text {. AEs }\end{array}$} \\
\hline Surgery type & \multicolumn{2}{|c|}{ Breast cancer surgery (radical mastectomy) } \\
\hline $\begin{array}{l}\text { Group numbers after end } \\
\text { of study (treatment/con- } \\
\text { trol) }\end{array}$ & \multicolumn{2}{|l|}{$30 / 30$} \\
\hline $\begin{array}{l}\text { Age of patient population } \\
\text { (treatment/control) }\end{array}$ & \multicolumn{2}{|l|}{$53.1 \pm 6.2$} \\
\hline Notes & \multicolumn{2}{|c|}{ No mention of sponsorship or funding } \\
\hline \multicolumn{3}{|l|}{ Risk of bias } \\
\hline Bias & Authors' judgement & Support for judgement \\
\hline $\begin{array}{l}\text { Random sequence genera- } \\
\text { tion (selection bias) }\end{array}$ & Low risk & Computer-generated random number assignment \\
\hline $\begin{array}{l}\text { Allocation concealment } \\
\text { (selection bias) }\end{array}$ & Low risk & Allocation concealed in sequentially numbered, opaque envelopes \\
\hline $\begin{array}{l}\text { Blinding of participants } \\
\text { and personnel (perfor- } \\
\text { mance bias) } \\
\text { All outcomes }\end{array}$ & Low risk & Blinded personnel \\
\hline $\begin{array}{l}\text { Blinding of outcome as- } \\
\text { sessment (detection bias) } \\
\text { All outcomes }\end{array}$ & Low risk & Blinded personnel \\
\hline $\begin{array}{l}\text { Incomplete outcome data } \\
\text { (attrition bias) } \\
\text { All outcomes }\end{array}$ & Low risk & No withdrawals \\
\hline $\begin{array}{l}\text { Selective reporting (re- } \\
\text { porting bias) }\end{array}$ & Low risk & All predefined dropouts reported \\
\hline Size & High risk & 30 participants per treatment arm \\
\hline
\end{tabular}

\section{Study characteristics}


Martinez 2014 (Continued)

\begin{tabular}{|c|c|}
\hline Methods & Randomised, double-blind. Ketamine vs placebo. Ketamine vs pregabalin \\
\hline Participants & $\mathrm{N}=142$. Total hip arthroplasty under general anaesthesia. About $48 \%$ women \\
\hline Interventions & Ketamine $0.5 \mathrm{mg} / \mathrm{kg}$ bolus IV at induction $+3 \mu \mathrm{g} / \mathrm{kg} / \mathrm{min}$ IV infusion until skin closure \\
\hline Outcomes & $\begin{array}{l}\text { Pain intensity (NRS) reported in the recovery room, at } 24 \text { and } 48 \mathrm{~h} \text {. Analgesic consumption at } 48 \mathrm{~h} \text {. Pres- } \\
\text { sure pain threshold measured on the first and second postoperative days. AEs }\end{array}$ \\
\hline Surgery type & Total hip arthroplasty \\
\hline \multirow{4}{*}{$\begin{array}{l}\text { Group numbers after end } \\
\text { of study (treatment/con- } \\
\text { trol) }\end{array}$} & Ketamine: 34 \\
\hline & Placebo: 38 \\
\hline & Ketamine + pregabalin 35 \\
\hline & Pregabalin: 35 \\
\hline
\end{tabular}

\begin{tabular}{ll}
\hline $\begin{array}{l}\text { Age of patient population } \\
\text { (treatment/control) }\end{array}$ & $60 \pm 17$ \\
& $64 \pm 11$ \\
& $64 \pm 9$ \\
& $59 \pm 12$
\end{tabular}

Notes Divided into 2 for analysis: ketamine vs placebo and ketamine + pregabalin vs pregabalin. No external funding

\section{Risk of bias}

\begin{tabular}{|c|c|c|}
\hline Bias & Authors' judgement & Support for judgement \\
\hline $\begin{array}{l}\text { Random sequence genera- } \\
\text { tion (selection bias) }\end{array}$ & Low risk & Computer-generated random numbers \\
\hline $\begin{array}{l}\text { Allocation concealment } \\
\text { (selection bias) }\end{array}$ & Low risk & Allocation concealed in opaque envelopes \\
\hline $\begin{array}{l}\text { Blinding of participants } \\
\text { and personnel (perfor- } \\
\text { mance bias) } \\
\text { All outcomes }\end{array}$ & Low risk & Study solutions were prepared by an independent person. \\
\hline $\begin{array}{l}\text { Blinding of outcome as- } \\
\text { sessment (detection bias) } \\
\text { All outcomes }\end{array}$ & Low risk & Blinded personnel \\
\hline $\begin{array}{l}\text { Incomplete outcome data } \\
\text { (attrition bias) } \\
\text { All outcomes }\end{array}$ & High risk & $13 \%$ was withdrawn \\
\hline $\begin{array}{l}\text { Selective reporting (re- } \\
\text { porting bias) }\end{array}$ & Low risk & All predefined outcomes reported adequately \\
\hline Size & High risk & $34,38,35$ and 35 participants per treatment arm. \\
\hline
\end{tabular}


Mathisen 1999

\section{Study characteristics}

\begin{tabular}{ll}
\hline Methods & Randomised, double-blind, placebo control \\
\hline Participants & $\mathrm{N}=60$, about $82 \%$ women \\
\hline Interventions & $\begin{array}{l}\text { 1. Pre-incisional bolus of R-ketamine } 1 \mathrm{mg} / \mathrm{kg} \mathrm{IV} \\
\text { 2. IV-bolus of R-ketamine } 1 \mathrm{mg} / \mathrm{kg} \text { at wound closure }\end{array}$ \\
\hline Outcomes & $\begin{array}{l}\text { Pain intensity (VAS, VRS) reported at } 1,2,3,4 \text { and } 24 \mathrm{~h} \text { and } 7 \text { days after surgery. PCA opioid consump- } \\
\text { tion reported at } 1,2,3 \text { and } 4 \text { h postoperatively. Analgesics after discharge. AEs }\end{array}$ \\
\hline $\begin{array}{l}\text { Surgery type } \\
\text { Elective laparoscopic cholecystectomy } \\
\text { of study (treatment/con- } \\
\text { trol) }\end{array}$ & $32 / 18$ \\
\hline $\begin{array}{l}\text { Age of patient population } \\
\text { (treatment/control) }\end{array}$ & $48 \pm 15.8$ \\
\hline \begin{tabular}{l} 
Notes \\
\hline
\end{tabular} & $50 \pm 13$ \\
\hline
\end{tabular}

\section{Risk of bias}

\section{Bias Authors' judgement Support for judgement}

Random sequence genera- Unclear risk Randomisation not described

tion (selection bias)

$\begin{array}{ll}\begin{array}{l}\text { Allocation concealment } \\ \text { (selection bias) }\end{array} & \text { Contral allocation } \\ & \begin{array}{l}\text { Quote: "The hospital pharmacy prepared the drugs in identical ampules } \\ \text { marked with patient number and injection number in a randomized, dou- } \\ \text { ble-blind manner." }\end{array}\end{array}$

ble-blind manner."

$\begin{array}{lll}\text { Blinding of participants } \quad \text { Low risk } & \text { Blinded personne } \\ \text { and personnel (perfor- } & \end{array}$

and personnel (perfor-

mance bias)

All outcomes

\begin{tabular}{lll}
\hline $\begin{array}{l}\text { Blinding of outcome as- } \\
\text { sessment (detection bias) } \\
\text { All outcomes }\end{array}$ & Low risk & Blinded personnel \\
\hline $\begin{array}{l}\text { Incomplete outcome data } \\
\begin{array}{l}\text { (attrition bias) } \\
\text { All outcomes }\end{array}\end{array}$ & High risk & $17 \%$ was withdrawn \\
\hline $\begin{array}{l}\text { Selective reporting (re- } \\
\text { porting bias) }\end{array}$ & Low risk & Predefined outcomes reported \\
\hline Size & High risk & 20 participants per treatment arm \\
\hline
\end{tabular}


McKay 2007

\section{Study characteristics}

\begin{tabular}{|c|c|c|}
\hline Methods & \multicolumn{2}{|c|}{ Randomised, double-blind, placebo control } \\
\hline Participants & \multicolumn{2}{|c|}{$N=41$, about $46 \%$ women } \\
\hline Interventions & \multicolumn{2}{|c|}{ Pre-incisional ketamine $1.5 \mathrm{mg} / \mathrm{kg}$ bolus IV followed by ketamine infusion $2.5 \mu \mathrm{g} / \mathrm{kg} / \mathrm{min}$} \\
\hline Outcomes & \multicolumn{2}{|c|}{$\begin{array}{l}\text { Pain intensity (VAS; reported as AUC: units }(\mathrm{cm}) \mathrm{x} \text { half days). AEs. Return of bowel function. Time to am- } \\
\text { bulation. Length of hospital stay. Analgesic consumption reported as total opioid use for each study } \\
\text { participant, time frame unclear }\end{array}$} \\
\hline Surgery type & \multicolumn{2}{|c|}{ Laparotomy (bowel resection) } \\
\hline $\begin{array}{l}\text { Group numbers after end } \\
\text { of study (treatment/con- } \\
\text { trol) }\end{array}$ & \multicolumn{2}{|l|}{$19 / 22$} \\
\hline $\begin{array}{l}\text { Age of patient population } \\
\text { (treatment/control) }\end{array}$ & \multicolumn{2}{|l|}{$49.5 \pm 16.3$} \\
\hline Notes & \multicolumn{2}{|c|}{$\begin{array}{l}\text { Primary outcome was return of bowel function. The study was funded in part by a grant from the Royal } \\
\text { University Hospital Foundation, Saskatoon, Canada }\end{array}$} \\
\hline \multicolumn{3}{|l|}{ Risk of bias } \\
\hline Bias & Authors' judgement & Support for judgement \\
\hline $\begin{array}{l}\text { Random sequence genera- } \\
\text { tion (selection bias) }\end{array}$ & Low risk & Computer-generated random number table \\
\hline $\begin{array}{l}\text { Allocation concealment } \\
\text { (selection bias) }\end{array}$ & Low risk & $\begin{array}{l}\text { Central allocation. Hospital pharmacy (a third party) randomised participants } \\
\text { and prepared study drug solutions }\end{array}$ \\
\hline $\begin{array}{l}\text { Blinding of participants } \\
\text { and personnel (perfor- } \\
\text { mance bias) } \\
\text { All outcomes }\end{array}$ & Low risk & Blinded personnel \\
\hline $\begin{array}{l}\text { Blinding of outcome as- } \\
\text { sessment (detection bias) } \\
\text { All outcomes }\end{array}$ & Low risk & Blinded personnel \\
\hline $\begin{array}{l}\text { Incomplete outcome data } \\
\text { (attrition bias) } \\
\text { All outcomes }\end{array}$ & Low risk & No withdrawals \\
\hline $\begin{array}{l}\text { Selective reporting (re- } \\
\text { porting bias) }\end{array}$ & Low risk & Predefined outcomes reported \\
\hline Size & High risk & 19 and 22 participants per treatment arm \\
\hline
\end{tabular}


Mebazaa MS 2008

\section{Study characteristics}

\begin{tabular}{ll}
\hline Methods & Randomised, double-blind. Ketamine + morphine vs morphine \\
\hline Participants & $\mathrm{N}=138$, about 49\% women \\
\hline Interventions & Ketamine $1 \mathrm{mg} /$ bolus IV via PCA \\
\hline Outcomes & $\begin{array}{l}\text { Pain intensity (VAS). Analgesic consumption. AEs. Outcomes reported every 4 h up to 48 h postopera- } \\
\text { tively }\end{array}$ \\
\hline $\begin{array}{l}\text { Surgery type } \\
\text { Lroup numbers after end } \\
\text { of study (treatment/con- } \\
\text { trol) }\end{array}$ & $67 / 67$ \\
\hline $\begin{array}{l}\text { Age of patient population } \\
\text { (treatment/control) }\end{array}$ & $46 \pm 13$ \\
\hline $\begin{array}{l}\text { Notes } \\
46 \pm 14\end{array}$
\end{tabular}

\section{Risk of bias}

\begin{tabular}{|c|c|c|}
\hline Bias & Authors' judgement & Support for judgement \\
\hline $\begin{array}{l}\text { Random sequence genera- } \\
\text { tion (selection bias) }\end{array}$ & Low risk & $\begin{array}{l}\text { Randomisation was stratified according to the type of surgery (i.e. visceral or } \\
\text { gynaecological) and performed according to a table of random numbers per } \\
\text { blocks of six }\end{array}$ \\
\hline $\begin{array}{l}\text { Allocation concealment } \\
\text { (selection bias) }\end{array}$ & Unclear risk & Allocation concealment method not described \\
\hline $\begin{array}{l}\text { Blinding of participants } \\
\text { and personnel (perfor- } \\
\text { mance bias) } \\
\text { All outcomes }\end{array}$ & Low risk & Blinded personnel \\
\hline $\begin{array}{l}\text { Blinding of outcome as- } \\
\text { sessment (detection bias) } \\
\text { All outcomes }\end{array}$ & Low risk & Blinded personnel \\
\hline $\begin{array}{l}\text { Incomplete outcome data } \\
\text { (attrition bias) } \\
\text { All outcomes }\end{array}$ & Low risk & $3 \%$ was withdrawn \\
\hline $\begin{array}{l}\text { Selective reporting (re- } \\
\text { porting bias) }\end{array}$ & Low risk & Predefined outcomes reported \\
\hline Size & Unclear risk & 67 participants per treatment arm \\
\hline
\end{tabular}

\section{Study characteristics}


Mendola 2012 (Continued)

\begin{tabular}{|c|c|}
\hline Methods & Randomised, double-blind, placebo control \\
\hline Participants & $\mathrm{N}=66$, about $32 \%$ women \\
\hline Interventions & S-ketamine $0.1 \mathrm{mg} / \mathrm{kg} / \mathrm{h}$ infusion IV during surgery and for $60 \mathrm{~h}$ postoperatively \\
\hline Outcomes & $\begin{array}{l}\text { Pain intensity (NRS) on 1st, } 2 \text { nd and } 3 \text { rd day postoperatively and then monthly up to } 6 \text { months. Anal- } \\
\text { gesic consumption reported on 1st, } 2 \text { nd and 3rd days postoperatively. AEs. NPSI at } 1,3 \text { and } 6 \text { months } \\
\text { postoperatively }\end{array}$ \\
\hline Surgery type & Thoracotomy \\
\hline $\begin{array}{l}\text { Group numbers after end } \\
\text { of study (treatment/con- } \\
\text { trol) }\end{array}$ & $32 / 30$ \\
\hline
\end{tabular}

$\begin{array}{ll}\begin{array}{l}\text { Age of patient population } \\ \text { (treatment/control) }\end{array} & 62 \pm 10.4 \\ & 65.7 \pm 10.9\end{array}$

Notes No mention of sponsorship or funding

\section{Risk of bias}

\begin{tabular}{|c|c|c|}
\hline Bias & Authors' judgement & Support for judgement \\
\hline $\begin{array}{l}\text { Random sequence genera- } \\
\text { tion (selection bias) }\end{array}$ & Low risk & Randomisation achieved by the statistic laboratory of the institution \\
\hline $\begin{array}{l}\text { Allocation concealment } \\
\text { (selection bias) }\end{array}$ & Low risk & $\begin{array}{l}\text { Central allocation. Hospital pharmacy (a third party) prepared the study infu- } \\
\text { sions that were coded }\end{array}$ \\
\hline $\begin{array}{l}\text { Blinding of participants } \\
\text { and personnel (perfor- } \\
\text { mance bias) } \\
\text { All outcomes }\end{array}$ & Low risk & Blinded personnel \\
\hline $\begin{array}{l}\text { Blinding of outcome as- } \\
\text { sessment (detection bias) } \\
\text { All outcomes }\end{array}$ & Low risk & Blinded personnel \\
\hline $\begin{array}{l}\text { Incomplete outcome data } \\
\text { (attrition bias) } \\
\text { All outcomes }\end{array}$ & Low risk & $6 \%$ was withdrawn \\
\hline $\begin{array}{l}\text { Selective reporting (re- } \\
\text { porting bias) }\end{array}$ & Low risk & Predefined outcomes addressed \\
\hline Size & High risk & 32 and 30 participants per treatment arm \\
\hline
\end{tabular}

Menigaux 2000

\section{Study characteristics}


Menigaux 2000 (Continued)

\begin{tabular}{ll} 
Participants & $\mathrm{N}=45$, about $33 \%$ women \\
\hline Interventions & Ketamine $0.15 \mathrm{mg} / \mathrm{kg}$ bolus IV before surgical incision or at wound closure \\
\hline Outcomes & $\begin{array}{l}\text { Pain intensity (VAS, VRS) reported at } 1,2,3 \mathrm{~h} \text {, then every } 4 \mathrm{~h} \text { up to } 48 \mathrm{~h} \text { after surgery. PCA morphine con- } \\
\text { sumption. Incremental doses reported hourly up to } 3 \mathrm{~h} \text { postoperatively, then every } 4 \mathrm{~h} \text { up to } 48 \mathrm{~h} \text {. Cu- } \\
\text { mulative morphine consumption reported at } 24 \text { and } 48 \mathrm{~h} \text { after surgery. AEs. Time to first analgesic re- } \\
\text { quest }\end{array}$
\end{tabular}

\begin{tabular}{ll}
\hline Surgery type & Elective arthroscopic anterior cruciate ligament repair \\
\hline $\begin{array}{l}\text { Group numbers after end } \\
\text { of study (treatment/con- } \\
\text { trol) }\end{array}$ & $30 / 15$ \\
\hline $\begin{array}{l}\text { Age of patient population } \\
\text { (treatment/control) }\end{array}$ & $26 \pm 6$ \\
\hline Notes & $28 \pm 7$ \\
\hline
\end{tabular}

\section{Risk of bias}

\begin{tabular}{|c|c|c|}
\hline Bias & Authors' judgement & Support for judgement \\
\hline $\begin{array}{l}\text { Random sequence genera- } \\
\text { tion (selection bias) }\end{array}$ & Low risk & Random number table \\
\hline $\begin{array}{l}\text { Allocation concealment } \\
\text { (selection bias) }\end{array}$ & Unclear risk & Allocation concealment method not described in detail \\
\hline $\begin{array}{l}\text { Blinding of participants } \\
\text { and personnel (perfor- } \\
\text { mance bias) } \\
\text { All outcomes }\end{array}$ & Low risk & Blinded personnel \\
\hline $\begin{array}{l}\text { Blinding of outcome as- } \\
\text { sessment (detection bias) } \\
\text { All outcomes }\end{array}$ & Low risk & Blinded personnel at data collection \\
\hline $\begin{array}{l}\text { Incomplete outcome data } \\
\text { (attrition bias) } \\
\text { All outcomes }\end{array}$ & Low risk & No withdrawals \\
\hline $\begin{array}{l}\text { Selective reporting (re- } \\
\text { porting bias) }\end{array}$ & Low risk & Predefined outcomes clearly reported \\
\hline Size & High risk & 15 participants per treatment arm \\
\hline
\end{tabular}

Menigaux 2001

\section{Study characteristics}

Methods Randomised, double-blind, placebo control


Menigaux 2001 (Continued)

\begin{tabular}{ll} 
Participants & $\mathrm{N}=50,34 \%$ women \\
\hline Interventions & Pre-incisional bolus of ketamine $0.15 \mathrm{mg} / \mathrm{kg} \mathrm{IV}$ \\
\hline Outcomes & $\begin{array}{l}\text { Pain intensity (VAS, VRS) reported every } 15 \text { mins for } 1 \mathrm{~h}, \text { then every } 2 \mathrm{~h} \text { up to } 6 \mathrm{~h} \text { after surgery and on } \\
\text { 1st, 2nd and 3rd day after surgery. Rescue medication (mean number of analgesic tablets per partici- } \\
\text { pant required during } 3 \text { days). AEs }\end{array}$
\end{tabular}

\begin{tabular}{|c|c|c|}
\hline Surgery type & \multicolumn{2}{|c|}{ Outpatient knee arthroscopy } \\
\hline $\begin{array}{l}\text { Group numbers after end } \\
\text { of study (treatment/con- } \\
\text { trol) }\end{array}$ & \multicolumn{2}{|l|}{$25 / 25$} \\
\hline \multirow{2}{*}{$\begin{array}{l}\text { Age of patient population } \\
\text { (treatment/control) }\end{array}$} & \multicolumn{2}{|l|}{$37 \pm 9$} \\
\hline & \multicolumn{2}{|l|}{$36 \pm 12$} \\
\hline Notes & \multicolumn{2}{|c|}{$\begin{array}{l}\text { Supported by NIH Grant GM 58273, the Joseph Drown Foundation, and the Commonwealth of Ken- } \\
\text { tucky Research Challenge Trust Fund }\end{array}$} \\
\hline \multicolumn{3}{|l|}{ Risk of bias } \\
\hline Bias & Authors' judgement & Support for judgement \\
\hline $\begin{array}{l}\text { Random sequence genera- } \\
\text { tion (selection bias) }\end{array}$ & Low risk & A computer-generated random number table \\
\hline $\begin{array}{l}\text { Allocation concealment } \\
\text { (selection bias) }\end{array}$ & Unclear risk & Allocation concealment method not described in detail \\
\hline $\begin{array}{l}\text { Blinding of participants } \\
\text { and personnel (perfor- } \\
\text { mance bias) } \\
\text { All outcomes }\end{array}$ & Low risk & Blinded personnel \\
\hline $\begin{array}{l}\text { Blinding of outcome as- } \\
\text { sessment (detection bias) } \\
\text { All outcomes }\end{array}$ & Low risk & Blinded personnel \\
\hline $\begin{array}{l}\text { Incomplete outcome data } \\
\text { (attrition bias) } \\
\text { All outcomes }\end{array}$ & Low risk & No withdrawals \\
\hline $\begin{array}{l}\text { Selective reporting (re- } \\
\text { porting bias) }\end{array}$ & Low risk & Predefined outcomes reported \\
\hline Size & High risk & 25 participants per treatment arm \\
\hline
\end{tabular}

Michelet 2007

\section{Study characteristics}

Methods

Randomised, double-blind. Ketamine + morphine vs morphine 


\begin{tabular}{ll}
$\begin{array}{l}\text { Michelet } 2007 \text { (Continued) } \\
\text { Participants }\end{array}$ & $\mathrm{N}=50,28 \%$ women \\
\hline Interventions & $\begin{array}{l}\text { Pain intensity (VAS) and cumulative analgesic consumption, reported at baseline, at 12, 24, 36, 48 and } \\
60 \text { h postoperatively. AEs }\end{array}$ \\
\hline Outcomes & Thoracotomy \\
\hline $\begin{array}{l}\text { Surgery type } \\
\text { Group numbers after end } \\
\text { of study (treatment/con- } \\
\text { trol) }\end{array}$ & \begin{tabular}{l}
$24 / 24$ \\
\hline $\begin{array}{l}\text { Age of patient population } \\
\text { treatment/control) }\end{array}$
\end{tabular} \\
$\begin{array}{ll}\text { Mean (range) } \\
64 \text { (42-77) }\end{array}$ \\
\hline \begin{tabular}{l}
63 (42-76) \\
\hline Notes
\end{tabular} & No financial support \\
\hline
\end{tabular}

\section{Risk of bias}

\begin{tabular}{lll}
\hline Bias & Authors' judgement & Support for judgement \\
\hline $\begin{array}{l}\text { Random sequence genera- } \\
\text { tion (selection bias) }\end{array}$ & Low risk & Table of random numbers \\
\hline $\begin{array}{l}\text { Allocation concealment } \\
\text { (selection bias) }\end{array}$ & Unclear risk & $\begin{array}{l}\text { Allocation concealment method not described in detail. Only mentioned that a } \\
\text { hospital pharmacist prepared study drugs }\end{array}$ \\
\hline $\begin{array}{l}\text { Blinding of participants } \\
\text { and personnel (perfor- } \\
\text { mance bias) }\end{array}$ & Low risk & Blinded personnel \\
All outcomes & \\
\hline $\begin{array}{l}\text { Blinding of outcome as- } \\
\text { sessment (detection bias) } \\
\text { All outcomes }\end{array}$ & Low risk & Blinded personnel \\
\hline $\begin{array}{l}\text { Incomplete outcome data } \\
\text { (attrition bias) }\end{array}$ & Low risk & 4\% was withdrawn \\
\hline $\begin{array}{l}\text { Sll outcomes } \\
\text { pelective reporting (re- }\end{array}$ & Low risk & Results of predefined outcomes reported \\
\hline \begin{tabular}{l} 
Size \\
\hline
\end{tabular} & High risk & 24 participants per treatment arm \\
\hline
\end{tabular}

Miziara 2016

\section{Study characteristics}

Methods Randomised, double-blind, placebo control


Miziara 2016 (Continued)

\begin{tabular}{ll} 
Participants & $\mathrm{N}=48$, patient demographic data table not presented \\
\hline Interventions & S-ketamine infusion $5 \mu \mathrm{g} / \mathrm{kg} / \mathrm{min}$ beginning 5 mins before surgery and lasting till the end of surgery \\
\hline Outcomes & $\begin{array}{l}\text { Pain intensity (VNS 0-10) reported during PACU stay, at } 4 \text { and } 12 \mathrm{~h} \text { postoperatively. Analgesic consump- } \\
\text { tion reported during PACU stay, at 4-12 } \mathrm{h} \text { and as cumulative analgesic consumption at } 12 \mathrm{~h} \text { postopera- } \\
\text { tively. AEs }\end{array}$
\end{tabular}

\begin{tabular}{ll}
\hline Surgery type & Laparoscopic cholecystectomy \\
\hline $\begin{array}{l}\text { Group numbers after end } \\
\text { of study (treatment/con- }\end{array}$ & \\
trol) & \\
\hline
\end{tabular}

Age of patient population No data available

(treatment/control)

Notes No mention of sponsorship or funding. Study authors state that they have no conflicts interest

\section{Risk of bias}

\begin{tabular}{|c|c|c|}
\hline Bias & Authors' judgement & Support for judgement \\
\hline $\begin{array}{l}\text { Random sequence genera- } \\
\text { tion (selection bias) }\end{array}$ & Low risk & Randomisation achieved using a randomisation software \\
\hline $\begin{array}{l}\text { Allocation concealment } \\
\text { (selection bias) }\end{array}$ & Low risk & $\begin{array}{l}\text { Allocation sequence was concealed in sequentially numbered, sealed en- } \\
\text { velopes }\end{array}$ \\
\hline $\begin{array}{l}\text { Blinding of participants } \\
\text { and personnel (perfor- } \\
\text { mance bias) } \\
\text { All outcomes }\end{array}$ & Low risk & Blinded participants and personnel \\
\hline $\begin{array}{l}\text { Blinding of outcome as- } \\
\text { sessment (detection bias) } \\
\text { All outcomes }\end{array}$ & Low risk & Blinded personnel \\
\hline $\begin{array}{l}\text { Incomplete outcome data } \\
\text { (attrition bias) } \\
\text { All outcomes }\end{array}$ & Low risk & $7 \%$ was withdrawn \\
\hline $\begin{array}{l}\text { Selective reporting (re- } \\
\text { porting bias) }\end{array}$ & Low risk & Predefined outcomes reported \\
\hline Size & High risk & 21 participants per treatment arm \\
\hline
\end{tabular}

Murdoch 2002

\section{Study characteristics}

\begin{tabular}{ll}
\hline Methods & Randomised, double-blind. Ketamine + morphine vs morphine \\
\hline Participants & $\mathrm{N}=42,100 \%$ women \\
\hline
\end{tabular}


Murdoch 2002 (Continued)

\begin{tabular}{ll} 
Interventions & Postoperative IV PCA ketamine $0.75 \mathrm{mg} / \mathrm{bolus}+$ morphine \\
\hline Outcomes & $\begin{array}{l}\text { Pain intensity (VRS), reported every } 4 \mathrm{~h} \text { up to } 16 \mathrm{~h} \text { postoperatively, then at } 24 \mathrm{~h} . \text { PCA morphine con } \\
\text { sumption reported at } 24 \mathrm{~h} . \mathrm{AEs}\end{array}$
\end{tabular}

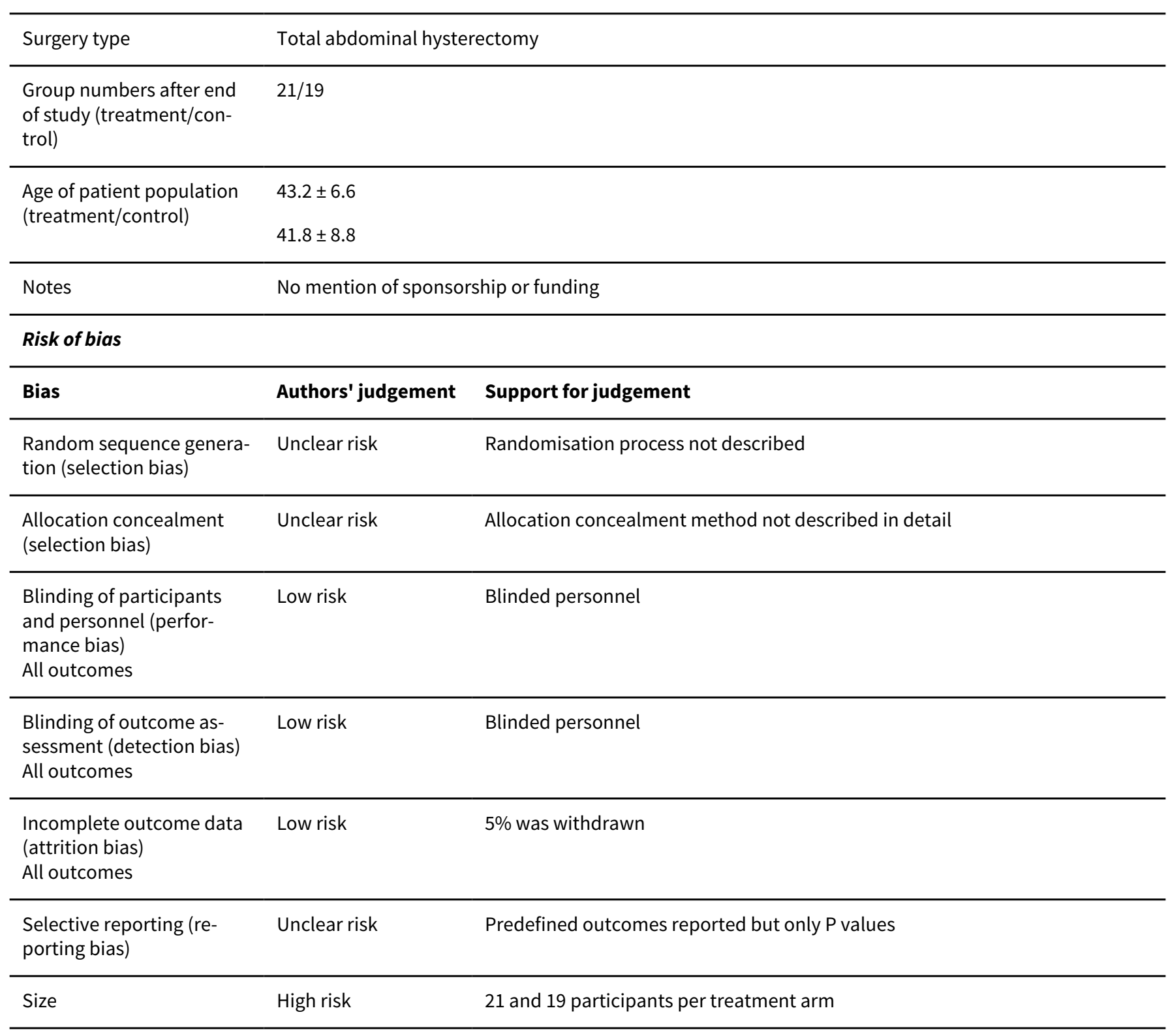

Nesek-Adam 2012

\section{Study characteristics}

\begin{tabular}{ll}
\hline Methods & Randomised, double-blind \\
\hline Participants & $\mathrm{N}=80$, about $73 \%$ women \\
\hline Interventions & 1. Pre-incisional ketamine $0.15 \mathrm{mg} / \mathrm{kg}$ bolus IV vs placebo
\end{tabular}


Nesek-Adam 2012 (Continued)

2. Pre-incisional ketamine $0.15 \mathrm{mg} / \mathrm{kg}$ + diclofenac $1 \mathrm{mg} / \mathrm{kg}$ bolus IV vs pre-incisional diclofenac $1 \mathrm{mg} /$ $\mathrm{kg}$ IV

\begin{tabular}{ll}
\hline Outcomes & $\begin{array}{l}\text { Pain intensity (VAS), reported every } 2 \mathrm{~h} \text { up to } 6 \mathrm{~h} \text { postoperatively, then at } 12 \text { and } 24 \mathrm{~h} . \text { Time to first anal- } \\
\text { gesic request. AEs }\end{array}$ \\
\hline Surgery type & Laparoscopic cholecystectomy \\
\hline $\begin{array}{l}\text { Group numbers after end } \\
\text { of study (treatment/con- } \\
\text { trol) }\end{array}$ & $20 / 20 / 20 / 20$ \\
\hline $\begin{array}{l}\text { Age of patient population } \\
\text { (treatment/control) }\end{array}$ & $\begin{array}{l}\text { Ketamine group } 50.7 \pm 11.9 \\
\text { Placebo group 53.6 } \pm 9.6\end{array}$ \\
& Diclofenac group 45.7 \pm 15.3 \\
& Ketamine + diclofenac group $52.7 \pm 15.5$ \\
\hline Notes & $\begin{array}{l}\text { streatment arms. Ketamine vs placebo and ketamine }+ \text { diclofenac vs diclofenac. No mention of spon- } \\
\text { sorship or funding }\end{array}$
\end{tabular}

\section{Risk of bias}

\begin{tabular}{lll}
\hline Bias & Authors' judgement & Support for judgement \\
\hline $\begin{array}{l}\text { Random sequence genera- } \\
\text { tion (selection bias) }\end{array}$ & Low risk & A computer-generated random number table \\
\hline $\begin{array}{l}\text { Allocation concealment } \\
\text { (selection bias) }\end{array}$ & Low risk & $\begin{array}{l}\text { Quote: "An envelope containing group assignment was prepared, sealed, and } \\
\text { numbered for each patient." }\end{array}$ \\
\hline $\begin{array}{l}\text { Blinding of participants } \\
\text { and personnel (perfor- } \\
\text { mance bias) }\end{array}$ & Low risk & Blinded personnel \\
All outcomes & & \\
\hline $\begin{array}{l}\text { Blinding of outcome as- } \\
\text { sessment (detection bias) } \\
\text { All outcomes }\end{array}$ & Low risk & Blinded personnel \\
\hline $\begin{array}{l}\text { Incomplete outcome data } \\
\text { (attrition bias) }\end{array}$ & Low risk & No withdrawals \\
\hline $\begin{array}{l}\text { All outcomes } \\
\text { Selective reporting (re- } \\
\text { porting bias) }\end{array}$ & Low risk & Predefined outcomes reported \\
\hline \begin{tabular}{l} 
Size \\
\hline
\end{tabular} & 20 participants per treatment arm \\
\hline
\end{tabular}

\section{Nielsen 2017}

\section{Study characteristics}

Methods Randomised, double-blind, placebo control


Nielsen 2017 (Continued)

\begin{tabular}{ll} 
Participants & $\mathrm{N}=150,65 \%$ women \\
\hline Interventions & Intraoperative S-ketamine bolus $0.5 \mathrm{mg} / \mathrm{kg}$ and infusion $0.25 \mathrm{mg} / \mathrm{kg} / \mathrm{h}$ \\
\hline Outcomes & $\begin{array}{l}\text { Analgesic consumption, reported at the first hour in the PACU and then at 24 h. Postoperative pain in- } \\
\text { tensity (VAS), reported every } 2 \mathrm{~h} \text { up to } 24 \mathrm{~h} \text { postoperatively at rest and during mobilisation. AEs (PONV } \\
\text { reported every } 6 \mathrm{~h} \text { up to } 24 \mathrm{~h} \text { postoperatively, CNS AEs reported as number of participants experienc- } \\
\text { ing CNS adverse event during 0-24 h). Persistent pain } 6 \text { months postoperatively }\end{array}$ \\
\hline $\begin{array}{l}\text { Surgery type } \\
\text { Lumbar fusion surgery }\end{array}$ \\
$\begin{array}{l}\text { Group numbers after end } \\
\text { of study (treatment/con- } \\
\text { trol) }\end{array}$ \\
$\begin{array}{l}75 / 75 \\
\begin{array}{l}\text { Age of patient population } \\
\text { (treatment/control) }\end{array}\end{array}$ \\
$\begin{array}{l}57 \pm 14 \\
55 \pm 13\end{array}$ \\
\hline $\begin{array}{l}\text { Notes } \\
\text { hagen University Hospital }\end{array}$
\end{tabular}

\section{Risk of bias}

\begin{tabular}{|c|c|c|}
\hline Bias & Authors' judgement & Support for judgement \\
\hline $\begin{array}{l}\text { Random sequence genera- } \\
\text { tion (selection bias) }\end{array}$ & Low risk & A computer-generated randomisation list \\
\hline $\begin{array}{l}\text { Allocation concealment } \\
\text { (selection bias) }\end{array}$ & Low risk & $\begin{array}{l}\text { Central allocation. Hospital pharmacy (a third party) prepared and pre-packed } \\
\text { identical ampoules in consecutively numbered boxes according to the com- } \\
\text { puter-generated randomisation list. Information about each participant's } \\
\text { treatment was concealed in consecutively numbered, sealed, opaque en- } \\
\text { velopes }\end{array}$ \\
\hline $\begin{array}{l}\text { Blinding of participants } \\
\text { and personnel (perfor- } \\
\text { mance bias) } \\
\text { All outcomes }\end{array}$ & Low risk & Blinded participants and personnel \\
\hline $\begin{array}{l}\text { Blinding of outcome as- } \\
\text { sessment (detection bias) } \\
\text { All outcomes }\end{array}$ & Low risk & Blinded personnel \\
\hline $\begin{array}{l}\text { Incomplete outcome data } \\
\text { (attrition bias) } \\
\text { All outcomes }\end{array}$ & Low risk & $3 \%$ was withdrawn in the immediate postoperative period \\
\hline $\begin{array}{l}\text { Selective reporting (re- } \\
\text { porting bias) }\end{array}$ & Low risk & Predefined outcomes reported \\
\hline Size & Unclear risk & 75 participants per treatment arm \\
\hline
\end{tabular}


Ögün 2001

\section{Study characteristics}

\begin{tabular}{ll}
\hline Methods & Randomised, double-blind, placebo control \\
\hline Participants & $\mathrm{N}=47,100 \%$ women \\
\hline Interventions & Ketamine $1 \mathrm{mg} / \mathrm{kg}$ bolus IV pre-incisionally and after excision of tissue specimen \\
\hline Outcomes & $\begin{array}{l}\text { Pain intensity (VAS, VRS) reported at 0, 1, 2, 4, 6, 12 and 24 h postoperatively. Analgesic consumption } \\
\text { reported at 24 h postoperatively. AEs }\end{array}$ \\
\hline $\begin{array}{l}\text { Surgery type } \\
\begin{array}{l}\text { Group numbers after end } \\
\text { of study (treatment/con- } \\
\text { trol) }\end{array}\end{array}$ & Mastectomy \\
\hline
\end{tabular}

\begin{tabular}{ll}
\hline $\begin{array}{l}\text { Age of patient population } \\
\text { (treatment/control) }\end{array}$ & $47.2 \pm 12.7$ \\
& $47.8 \pm 14.2$ \\
\hline Notes & Article in Turkish. No mention of sponsorship or funding
\end{tabular}

\section{Risk of bias}

\begin{tabular}{|c|c|c|}
\hline Bias & Authors' judgement & Support for judgement \\
\hline $\begin{array}{l}\text { Random sequence genera- } \\
\text { tion (selection bias) }\end{array}$ & Unclear risk & Randomisation process not described \\
\hline $\begin{array}{l}\text { Allocation concealment } \\
\text { (selection bias) }\end{array}$ & Unclear risk & Allocation concealment process not described \\
\hline $\begin{array}{l}\text { Blinding of participants } \\
\text { and personnel (perfor- } \\
\text { mance bias) } \\
\text { All outcomes }\end{array}$ & Unclear risk & Only mentioned double-blind but lacks description of how it was achieved \\
\hline $\begin{array}{l}\text { Blinding of outcome as- } \\
\text { sessment (detection bias) } \\
\text { All outcomes }\end{array}$ & Unclear risk & Only mentioned double-blind but lacks description of how it was achieved \\
\hline $\begin{array}{l}\text { Incomplete outcome data } \\
\text { (attrition bias) } \\
\text { All outcomes }\end{array}$ & Low risk & No withdrawals \\
\hline $\begin{array}{l}\text { Selective reporting (re- } \\
\text { porting bias) }\end{array}$ & Low risk & Predefined outcomes reported \\
\hline Size & High risk & 15 and 16 participants per treatment arm \\
\hline
\end{tabular}

\section{Study characteristics}


Ong 2001 (Continued)

\begin{tabular}{ll} 
Methods & Randomised, double-blind, placebo control \\
\hline Participants & $\mathrm{N}=40$, gender of participants not specified \\
\hline Interventions & Ketamine $0.3 \mathrm{mg} / \mathrm{kg}$ bolus IV prior to induction \\
\hline Outcomes & $\begin{array}{l}\text { Pain intensity (VAS) reported on arrival to PACU, at } 1 \mathrm{~h} \text { postoperatively and on discharge. Analgesic re- } \\
\text { quirement during PACU stay. AEs }\end{array}$
\end{tabular}

\begin{tabular}{|c|c|c|}
\hline Surgery type & \multicolumn{2}{|c|}{ Extraction of wisdom teeth } \\
\hline $\begin{array}{l}\text { Group numbers after end } \\
\text { of study (treatment/con- } \\
\text { trol) }\end{array}$ & \multicolumn{2}{|l|}{$20 / 20$} \\
\hline \multirow{2}{*}{$\begin{array}{l}\text { Age of patient population } \\
\text { (treatment/control) }\end{array}$} & \multicolumn{2}{|l|}{$24.1 \pm 15.3$} \\
\hline & \multicolumn{2}{|l|}{$24.1 \pm 6.6$} \\
\hline Notes & \multicolumn{2}{|c|}{ No mention of sponsorship or funding } \\
\hline \multicolumn{3}{|l|}{ Risk of bias } \\
\hline Bias & Authors' judgement & Support for judgement \\
\hline $\begin{array}{l}\text { Random sequence genera- } \\
\text { tion (selection bias) }\end{array}$ & Low risk & Randomisation by drawing cards from an envelope \\
\hline $\begin{array}{l}\text { Allocation concealment } \\
\text { (selection bias) }\end{array}$ & Unclear risk & Allocation concealment method not described \\
\hline $\begin{array}{l}\text { Blinding of participants } \\
\text { and personnel (perfor- } \\
\text { mance bias) } \\
\text { All outcomes }\end{array}$ & Low risk & Blinded personnel \\
\hline $\begin{array}{l}\text { Blinding of outcome as- } \\
\text { sessment (detection bias) } \\
\text { All outcomes }\end{array}$ & Low risk & Blinded personnel \\
\hline $\begin{array}{l}\text { Incomplete outcome data } \\
\text { (attrition bias) } \\
\text { All outcomes }\end{array}$ & Low risk & No withdrawals \\
\hline $\begin{array}{l}\text { Selective reporting (re- } \\
\text { porting bias) }\end{array}$ & Low risk & Predefined outcomes reported \\
\hline Size & High risk & 20 participants per treatment arm \\
\hline
\end{tabular}

\section{Ozhan 2013}

\section{Study characteristics}

Methods Randomised, double-blind, placebo control


Ozhan 2013 (Continued)

\begin{tabular}{ll} 
Participants & $\mathrm{N}=60$, about $82 \%$ women \\
\hline Interventions & Pre-incisional ketamine $0.25 \mathrm{mg} / \mathrm{kg}$ bolus IV \\
\hline Outcomes & $\begin{array}{l}\text { Analgesic consumption at } 24 \text { h postoperatively. Preoperative and postoperative cognitive function (Mi- } \\
\text { ni-Mental Test). AEs. Recovery time }\end{array}$ \\
\hline $\begin{array}{l}\text { Surgery type } \\
\text { Lroup numbers after end } \\
\text { of study (treatment/con- } \\
\text { trol) }\end{array}$ & $30 / 30$ \\
\hline $\begin{array}{l}\text { Age of patient population } \\
\text { (treatment/control) }\end{array}$ & $46 \pm 12.3$ \\
\hline $\begin{array}{l}\text { Notes } \\
47 \pm 11.5\end{array}$ & $\begin{array}{l}\text { No mention of sponsorship or funding. The study authors declare that they have no competing inter- } \\
\text { ests }\end{array}$
\end{tabular}

\section{Risk of bias}

\begin{tabular}{|c|c|c|}
\hline Bias & Authors' judgement & Support for judgement \\
\hline $\begin{array}{l}\text { Random sequence genera- } \\
\text { tion (selection bias) }\end{array}$ & Low risk & Computer-generated list of random numbers \\
\hline $\begin{array}{l}\text { Allocation concealment } \\
\text { (selection bias) }\end{array}$ & Unclear risk & Allocation concealment method not described in detail \\
\hline $\begin{array}{l}\text { Blinding of participants } \\
\text { and personnel (perfor- } \\
\text { mance bias) } \\
\text { All outcomes }\end{array}$ & Low risk & Blinded participants and personnel \\
\hline $\begin{array}{l}\text { Blinding of outcome as- } \\
\text { sessment (detection bias) } \\
\text { All outcomes }\end{array}$ & Low risk & Blinded personnel \\
\hline $\begin{array}{l}\text { Incomplete outcome data } \\
\text { (attrition bias) } \\
\text { All outcomes }\end{array}$ & Low risk & No withdrawals \\
\hline $\begin{array}{l}\text { Selective reporting (re- } \\
\text { porting bias) }\end{array}$ & Low risk & Predefined outcomes reported \\
\hline Size & High risk & 30 participants per treatment arm \\
\hline
\end{tabular}

\section{Pacreu 2012}

\section{Study characteristics}

$\begin{array}{ll}\text { Methods } & \text { Randomised, double-blind. Intraoperative ketamine + postoperative IV-PCA containing ketamine + } \\ \text { methadone VS intraoperative placebo + postoperative IV-PCA with methadone alone }\end{array}$


Pacreu 2012 (Continued)

\begin{tabular}{|c|c|}
\hline Participants & $\mathrm{N}=22,70 \%$ women \\
\hline Interventions & $\begin{array}{l}\text { Pre-incisional ketamine } 0.5 \mathrm{mg} / \mathrm{kg} \text { bolus IV + intraoperative ketamine } 2.5 \mu \mathrm{g} / \mathrm{kg} / \mathrm{min} \text { infusion IV + post- } \\
\text { operative IV-PCA } 0.5 \mathrm{mg} / \text { bolus }\end{array}$ \\
\hline Outcomes & $\begin{array}{l}\text { Pain intensity (NRS). Analgesic consumption. AEs } \\
\text { Main outcomes at } 24 \text { and } 48 \mathrm{~h}\end{array}$ \\
\hline Surgery type & Lumbar arthrodesis \\
\hline $\begin{array}{l}\text { Group numbers after end } \\
\text { of study (treatment/con- } \\
\text { trol) }\end{array}$ & $10 / 10$ \\
\hline Notes & Institutional support \\
\hline
\end{tabular}

\section{Risk of bias}

\begin{tabular}{|c|c|c|}
\hline Bias & Authors' judgement & Support for judgement \\
\hline $\begin{array}{l}\text { Random sequence genera- } \\
\text { tion (selection bias) }\end{array}$ & Low risk & $\begin{array}{l}\text { A computer-generated randomisation list was performed by the Department } \\
\text { of Biostatististics }\end{array}$ \\
\hline $\begin{array}{l}\text { Allocation concealment } \\
\text { (selection bias) }\end{array}$ & Unclear risk & Allocation concealment method not described in detail \\
\hline $\begin{array}{l}\text { Blinding of participants } \\
\text { and personnel (perfor- } \\
\text { mance bias) } \\
\text { All outcomes }\end{array}$ & Low risk & Blinded personnel \\
\hline $\begin{array}{l}\text { Blinding of outcome as- } \\
\text { sessment (detection bias) } \\
\text { All outcomes }\end{array}$ & Low risk & Blinded personnel \\
\hline $\begin{array}{l}\text { Incomplete outcome data } \\
\text { (attrition bias) } \\
\text { All outcomes }\end{array}$ & Low risk & $9 \%$ was withdrawn \\
\hline $\begin{array}{l}\text { Selective reporting (re- } \\
\text { porting bias) }\end{array}$ & Low risk & Predefined outcomes reported \\
\hline Size & High risk & 10 participants per treatment arm \\
\hline
\end{tabular}

\section{Papaziogas 2001}

\section{Study characteristics}

Methods Randomised, double-blind. Ketamine IV + ropivacaine SC vs ropivacaine SC


Papaziogas 2001 (Continued)
Participants
$\mathrm{N}=55$, of whom 35 participants in IV ketamine and control treatment arms

\begin{tabular}{|c|c|}
\hline Interventions & Pre-incisional bolus of ketamine $1 \mathrm{mg} / \mathrm{kg}$ IV \\
\hline Outcomes & $\begin{array}{l}\text { Pain intensity (VAS, VRS) reported at baseline, at 3, 6, 12, } 24 \text { and } 48 \mathrm{~h} \text { postoperatively. Analgesic con- } \\
\text { sumption reported at } 48 \mathrm{~h} \text { postoperatively. Rescue analgesia. Time to first request for analgesia. AEs }\end{array}$ \\
\hline
\end{tabular}

Surgery type Elective laparoscopic cholecystectomy

Group numbers after end

$18 / 17$

of study (treatment/con-

trol)

\begin{tabular}{lc}
\hline $\begin{array}{l}\text { Age of patient population } \\
\text { (treatment/control) }\end{array}$ & $41.3 \pm 13.6$ \\
& $47.9 \pm 16.7$
\end{tabular}

Notes Third group received saline IV and saline SC. No mention of sponsorship or funding

\section{Risk of bias}

\begin{tabular}{|c|c|c|}
\hline Bias & Authors' judgement & Support for judgement \\
\hline $\begin{array}{l}\text { Random sequence genera- } \\
\text { tion (selection bias) }\end{array}$ & Unclear risk & Randomisation not described \\
\hline $\begin{array}{l}\text { Allocation concealment } \\
\text { (selection bias) }\end{array}$ & Unclear risk & Allocation concealment method not described \\
\hline $\begin{array}{l}\text { Blinding of participants } \\
\text { and personnel (perfor- } \\
\text { mance bias) } \\
\text { All outcomes }\end{array}$ & Unclear risk & Blinding process not described \\
\hline $\begin{array}{l}\text { Blinding of outcome as- } \\
\text { sessment (detection bias) } \\
\text { All outcomes }\end{array}$ & Unclear risk & Blinding process not described \\
\hline $\begin{array}{l}\text { Incomplete outcome data } \\
\text { (attrition bias) } \\
\text { All outcomes }\end{array}$ & Low risk & $4 \%$ was withdrawn \\
\hline $\begin{array}{l}\text { Selective reporting (re- } \\
\text { porting bias) }\end{array}$ & Low risk & Predefined outcomes reported \\
\hline Size & High risk & 18,17 and 18 participants per treatment arm \\
\hline
\end{tabular}

Parikh 2011

\section{Study characteristics}

\begin{tabular}{ll}
\hline Methods & Randomised, double-blind, placebo control \\
\hline Participants & $\mathrm{N}=60$, about 38\% women \\
\hline
\end{tabular}


Parikh 2011 (Continued) $\begin{array}{ll}\text { Interventions } & \begin{array}{l}\text { Ketamine } 0.15 \mathrm{mg} / \mathrm{kg} \text { bolus IV } 30 \mathrm{~min} \text { before start of surgery }+2 \mu \mathrm{mg} / \mathrm{kg} / \mathrm{min} \text { infusion IV till start of skin } \\ \text { closure }\end{array}\end{array}$

\begin{tabular}{ll}
\hline Outcomes & $\begin{array}{l}\text { Pain intensity (VAS), reported at 15, 30 and } 60 \text { min after surgery, then every } 4 \mathrm{~h} \text { up to } 16 \mathrm{~h} \text { and finally at } \\
\text { 24 h postoperatively. Time to first analgesic request. Analgesic consumption reported at 24 h postoper- } \\
\text { atively. AEs }\end{array}$ \\
\hline Surgery type & Open renal surgery by flank incision \\
\hline $\begin{array}{l}\text { Group numbers after end } \\
\text { of study (treatment/con- } \\
\text { trol) }\end{array}$ & $30 / 30$ \\
\hline
\end{tabular}

Age of patient population

$39.2 \pm 12.2$

(treatment/control)

$42.2 \pm 10.5$

Notes No financial support

\section{Risk of bias}

\begin{tabular}{|c|c|c|}
\hline Bias & Authors' judgement & Support for judgement \\
\hline $\begin{array}{l}\text { Random sequence genera- } \\
\text { tion (selection bias) }\end{array}$ & Low risk & Randomisation by shuffling envelopes \\
\hline $\begin{array}{l}\text { Allocation concealment } \\
\text { (selection bias) }\end{array}$ & Unclear risk & Allocation concealment method not described \\
\hline $\begin{array}{l}\text { Blinding of participants } \\
\text { and personnel (perfor- } \\
\text { mance bias) } \\
\text { All outcomes }\end{array}$ & Low risk & Blinded personnel \\
\hline $\begin{array}{l}\text { Blinding of outcome as- } \\
\text { sessment (detection bias) } \\
\text { All outcomes }\end{array}$ & Low risk & Blinded observers \\
\hline $\begin{array}{l}\text { Incomplete outcome data } \\
\text { (attrition bias) } \\
\text { All outcomes }\end{array}$ & Low risk & No withdrawals \\
\hline $\begin{array}{l}\text { Selective reporting (re- } \\
\text { porting bias) }\end{array}$ & Low risk & Predefined outcomes reported \\
\hline Size & High risk & 30 participants per treatment arm \\
\hline
\end{tabular}

\section{Patel 2016}

\section{Study characteristics}

\begin{tabular}{ll}
\hline Methods & Randomised, double-blind. Ketamine + clonidine vs clonidine \\
\hline Participants & $\mathrm{N}=75$, of whom 50 participants in IV ketamine and control treatment arms. $10 \%$ women \\
\hline
\end{tabular}


Patel 2016 (Continued)

\begin{tabular}{ll} 
Interventions & Ketamine $1 \mathrm{mg} / \mathrm{kg}$ bolus IV during induction of anaesthesia \\
\hline Outcomes & $\begin{array}{l}\text { Pain intensity (VAS) reported at 4, 8, } 12 \text { and } 24 \text { h postoperatively. Analgesic consumption reported as } \\
\text { number of doses at } 24 \mathrm{~h} \text {. Intraoperative haemodynamic parameters }\end{array}$
\end{tabular}

\begin{tabular}{|c|c|c|}
\hline Surgery type & \multicolumn{2}{|c|}{ Off-pump coronary artery bypass } \\
\hline $\begin{array}{l}\text { Group numbers after end } \\
\text { of study (treatment/con- } \\
\text { trol) }\end{array}$ & \multicolumn{2}{|l|}{$25 / 25$} \\
\hline $\begin{array}{l}\text { Age of patient population } \\
\text { (treatment/control) }\end{array}$ & \multicolumn{2}{|l|}{$\begin{array}{l}60.91 \pm 6.17 \\
58.52 \pm 8.29\end{array}$} \\
\hline Notes & \multicolumn{2}{|l|}{ No funding } \\
\hline \multicolumn{3}{|l|}{ Risk of bias } \\
\hline Bias & Authors' judgement & Support for judgement \\
\hline $\begin{array}{l}\text { Random sequence genera- } \\
\text { tion (selection bias) }\end{array}$ & Low risk & A computer-generated randomisation list \\
\hline $\begin{array}{l}\text { Allocation concealment } \\
\text { (selection bias) }\end{array}$ & Unclear risk & Allocation concealment method not described in detail \\
\hline $\begin{array}{l}\text { Blinding of participants } \\
\text { and personnel (perfor- } \\
\text { mance bias) } \\
\text { All outcomes }\end{array}$ & Low risk & Blinded participants and personnel \\
\hline $\begin{array}{l}\text { Blinding of outcome as- } \\
\text { sessment (detection bias) } \\
\text { All outcomes }\end{array}$ & Low risk & Blinded personnel \\
\hline $\begin{array}{l}\text { Incomplete outcome data } \\
\text { (attrition bias) } \\
\text { All outcomes }\end{array}$ & Low risk & No withdrawals \\
\hline $\begin{array}{l}\text { Selective reporting (re- } \\
\text { porting bias) }\end{array}$ & Low risk & Predefined outcomes reported \\
\hline Size & High risk & 25 participants per treatment arm \\
\hline
\end{tabular}

Pirim 2006

\section{Study characteristics}

\begin{tabular}{ll}
\hline Methods & Randomised, double-blind, placebo control \\
\hline Participants & $\mathrm{N}=45,100 \%$ women \\
\hline Interventions & $\begin{array}{l}\text { Postoperative ketamine infusion initially } 10 \mathrm{mg} / \mathrm{kg} / \mathrm{min} \text { IV and gradually lowered to } 2.5 \mathrm{mg} / \mathrm{kg} / \mathrm{min} \text { for } \\
24 \text { h postoperatively }\end{array}$ \\
\hline
\end{tabular}


Pirim 2006 (Continued)

Outcomes

Pain intensity (VAS, VRS). Analgesic consumption. Pain outcomes reported at 1, 2, 4, 6, 12 and $24 \mathrm{~h}$ postoperatively. AEs reported at 24 and $48 \mathrm{~h}$ postoperatively

\begin{tabular}{|c|c|c|}
\hline Surgery type & \multicolumn{2}{|c|}{ Total abdominal hysterectomy } \\
\hline $\begin{array}{l}\text { Group numbers after end } \\
\text { of study (treatment/con- } \\
\text { trol) }\end{array}$ & \multicolumn{2}{|l|}{$23 / 22$} \\
\hline \multirow{2}{*}{$\begin{array}{l}\text { Age of patient population } \\
\text { (treatment/control) }\end{array}$} & \multicolumn{2}{|l|}{$46.7 \pm 6$} \\
\hline & \multicolumn{2}{|l|}{$45.9 \pm 5.5$} \\
\hline Notes & \multicolumn{2}{|c|}{ No mention of sponsorship or funding } \\
\hline \multicolumn{3}{|l|}{ Risk of bias } \\
\hline Bias & Authors' judgement & Support for judgement \\
\hline $\begin{array}{l}\text { Random sequence genera- } \\
\text { tion (selection bias) }\end{array}$ & Unclear risk & Randomisation process not described in detail \\
\hline $\begin{array}{l}\text { Allocation concealment } \\
\text { (selection bias) }\end{array}$ & Low risk & Allocation concealed in envelopes \\
\hline $\begin{array}{l}\text { Blinding of participants } \\
\text { and personnel (perfor- } \\
\text { mance bias) } \\
\text { All outcomes }\end{array}$ & Unclear risk & Not described \\
\hline $\begin{array}{l}\text { Blinding of outcome as- } \\
\text { sessment (detection bias) } \\
\text { All outcomes }\end{array}$ & Low risk & Assessments made by a researcher who was unaware of the study groups \\
\hline $\begin{array}{l}\text { Incomplete outcome data } \\
\text { (attrition bias) } \\
\text { All outcomes }\end{array}$ & Low risk & No withdrawals \\
\hline $\begin{array}{l}\text { Selective reporting (re- } \\
\text { porting bias) }\end{array}$ & Low risk & Predefined outcomes reported \\
\hline Size & High risk & 22 and 23 participants per treatment arm \\
\hline
\end{tabular}

Remérand 2009

\section{Study characteristics}

\begin{tabular}{ll}
\hline Methods & Randomised, double-blind, placebo control \\
\hline Participants & $\mathrm{N}=154,49 \%$ women \\
\hline Interventions & Pre-incisional ketamine $0.5 \mathrm{mg} / \mathrm{kg}$ bolus $\mathrm{IV}+2 \mu \mathrm{g} / \mathrm{kg} / \mathrm{min}$ infusion IV for $24 \mathrm{~h}$ \\
\hline Outcomes & Pain intensity (NRS) reported on $1 \mathrm{st}$ and 2 nd day postoperatively. Analgesic consumption reported at \\
& PACU, at 24 and $48 \mathrm{~h}$ postoperatively and on days 4 and 7 postoperatively. AEs \\
\hline
\end{tabular}


Remérand 2009 (Continued)

$\begin{array}{ll}\text { Surgery type } & \text { Total hip arthroplasty } \\ \begin{array}{l}\text { Group numbers after end } \\ \text { of study (treatment/con- } \\ \text { trol) }\end{array}\end{array}$

$\begin{array}{ll}\begin{array}{l}\text { Age of patient population } \\ \text { (treatment/control) }\end{array} & 64 \pm 13 \\ & 65 \pm 14\end{array}$

$65+14$

Notes Supported by institutional and/or departmental sources

\section{Risk of bias}

\begin{tabular}{lll}
\hline Bias & Authors' judgement & Support for judgement \\
\hline $\begin{array}{l}\text { Random sequence genera- } \\
\text { tion (selection bias) }\end{array}$ & Low risk & Computer-generated randomisation process \\
\hline $\begin{array}{l}\text { Allocation concealment } \\
\text { (selection bias) }\end{array}$ & Low risk & $\begin{array}{l}\text { Quote: "Before the study began, as part of a computer-generated randomiza- } \\
\text { tion process, } 160 \text { identical white envelopes were prepared, numbered, and } \\
\text { sealed by a person external to our clinical unit. Each envelope contained de- } \\
\text { tailed instructions of the preparation of } 2 \text { syringes (ketamine or saline)." }\end{array}$ \\
\hline
\end{tabular}

\begin{tabular}{lll}
\hline $\begin{array}{l}\text { Blinding of participants } \\
\text { and personnel (perfor- } \\
\text { mance bias) } \\
\text { All outcomes }\end{array}$ & Low risk & \\
\hline $\begin{array}{l}\text { Blinding of outcome as- } \\
\text { sessment (detection bias) } \\
\text { All outcomes }\end{array}$ & Low risk & Blinded personnel \\
\hline $\begin{array}{l}\text { Incomplete outcome data } \\
\text { (attrition bias) }\end{array}$ & Low risk & \\
All outcomes & & $\begin{array}{l}\text { No withdrawals in the immediate postoperative period. At } 6 \text { months, } 8 \% \text { was } \\
\text { withdrawn }\end{array}$ \\
\hline
\end{tabular}

\begin{tabular}{lll}
$\begin{array}{l}\text { Selective reporting (re- } \\
\text { porting bias) }\end{array}$ & Low risk & Predefined outcomes reported adequately \\
\hline Size & Unclear risk & 79 and 74 participants per treatment arm \\
\hline
\end{tabular}

Reza 2010

\section{Study characteristics}

\begin{tabular}{ll}
\hline Methods & Randomised, double-blind, placebo control \\
\hline Participants & $\mathrm{N}=60,100 \%$ women \\
\hline Interventions & Ketamine $0.5 \mathrm{mg} / \mathrm{kg}$ bolus IV before anaesthesia induction \\
\hline Outcomes & Pain intensity (VAS), reported at 2, 6,12 and 24 h postoperatively. Analgesic consumption, reported at \\
& $0-2 \mathrm{~h}$ and 2-24 h postoperatively. AEs \\
\hline
\end{tabular}


Reza 2010 (Continued)

\begin{tabular}{ll} 
Surgery type & Elective caesarean section \\
\hline $\begin{array}{l}\text { Group numbers after end } \\
\text { of study (treatment/con- } \\
\text { trol) }\end{array}$ & $30 / 30$
\end{tabular}

$\begin{array}{ll}\begin{array}{l}\text { Age of patient population } \\ \text { (treatment/control) }\end{array} & 27 \pm 5.1 \\ & 27 \pm 4.5\end{array}$

Notes No mention of sponsorship or funding

\begin{tabular}{|c|c|c|}
\hline \multicolumn{3}{|l|}{ Risk of bias } \\
\hline Bias & Authors' judgement & Support for judgement \\
\hline $\begin{array}{l}\text { Random sequence genera- } \\
\text { tion (selection bias) }\end{array}$ & Low risk & Computer-generated random numbers \\
\hline $\begin{array}{l}\text { Allocation concealment } \\
\text { (selection bias) }\end{array}$ & Low risk & Allocation was concealed in opaque envelopes \\
\hline $\begin{array}{l}\text { Blinding of participants } \\
\text { and personnel (perfor- } \\
\text { mance bias) } \\
\text { All outcomes }\end{array}$ & Low risk & Blinded participants and personnel \\
\hline $\begin{array}{l}\text { Blinding of outcome as- } \\
\text { sessment (detection bias) } \\
\text { All outcomes }\end{array}$ & Low risk & Blinded personnel \\
\hline $\begin{array}{l}\text { Incomplete outcome data } \\
\text { (attrition bias) } \\
\text { All outcomes }\end{array}$ & Low risk & No withdrawals \\
\hline $\begin{array}{l}\text { Selective reporting (re- } \\
\text { porting bias) }\end{array}$ & Low risk & Predefined outcomes adequately addressed \\
\hline Size & High risk & 30 participants per treatment arm \\
\hline
\end{tabular}

\section{Roytblat 1993}

\section{Study characteristics}

\begin{tabular}{ll}
\hline Methods & Randomised, double-blind, placebo control \\
\hline Participants & $\mathrm{N}=22,100 \%$ women \\
\hline Interventions & Pre-incisional bolus of ketamine $0.15 \mathrm{mg} / \mathrm{kg} \mathrm{IV}$ \\
\hline Outcomes & $\begin{array}{l}\text { Pain intensity (VAS, VRS). PCA morphine consumption. Time to first request for analgesia. AEs. Pain out- } \\
\text { comes recorded at } 1,2,3,4,5,6,12 \text { and } 24 \text { h after surgery }\end{array}$ \\
\hline Surgery type & Elective open cholecystectomy \\
\hline
\end{tabular}


Roytblat 1993 (Continued)

Group numbers after end

$11 / 11$ of study (treatment/control)

$\begin{array}{ll}\begin{array}{l}\text { Age of patient population } \\ \text { (treatment/control) }\end{array} & 55.1 \pm 10.7 \\ & 54.8 \pm 14.8\end{array}$

Notes No mention of sponsorship or funding

\section{Risk of bias}

Bias Authors' judgement Support for judgement

Random sequence genera- Unclear risk $\quad$ Randomisation process not described
tion (selection bias)

tion (selection bias)

Allocation concealment $\quad$ Unclear risk $\quad$ Allocation concealment method not described in detail
(selection bias)

\begin{tabular}{ll}
\hline $\begin{array}{l}\text { Blinding of participants } \\
\text { and personnel (perfor- }\end{array}$ & Low risk
\end{tabular}

mance bias)

All outcomes

\begin{tabular}{|c|c|c|}
\hline $\begin{array}{l}\text { Blinding of outcome as- } \\
\text { sessment (detection bias) } \\
\text { All outcomes }\end{array}$ & Low risk & Personnel involved in data collection were blinded \\
\hline $\begin{array}{l}\text { Incomplete outcome data } \\
\text { (attrition bias) } \\
\text { All outcomes }\end{array}$ & Low risk & No withdrawals \\
\hline $\begin{array}{l}\text { Selective reporting (re- } \\
\text { porting bias) }\end{array}$ & Low risk & Predefined outcomes reported \\
\hline Size & High risk & 11 participants per treatment arm \\
\hline
\end{tabular}

Safavi 2011

\section{Study characteristics}

\begin{tabular}{ll}
\hline Methods & Randomised, double-blind, placebo control \\
\hline Participants & $\mathrm{N}=120$, of whom 60 participants in IV ketamine and control treatment arms. About $48 \%$ women \\
\hline Interventions & Pre-incisional ketamine $1 \mathrm{mg} / \mathrm{kg}$ bolus IV \\
\hline Outcomes & $\begin{array}{l}\text { Pain intensity (VAS), reported at } 15 \text { and } 30 \text { min, then at } 1,2,3,4,8,12 \text { and } 24 \mathrm{~h} \text { postoperatively. Anal- } \\
\text { gesic consumption reported at } 24 \mathrm{~h} \text { postoperatively. Time to first request for rescue analgesia. AEs }\end{array}$ \\
\hline Surgery type & Open cholecystectomy \\
\hline
\end{tabular}


Safavi 2011 (Continued)

Group numbers after end of study (treatment/control)

\begin{tabular}{ll}
$\begin{array}{l}\text { Age of patient population } \\
\text { (treatment/control) }\end{array}$ & $47.7 \pm 11.8$ \\
& $54.1 \pm 11.3$ \\
\hline Notes & $\begin{array}{l}\text { Four study groups in the study: } 1 \text { group where participants received ketamine IV, } 2 \text { groups where ket- } \\
\text { amine was given SC in different doses and } 1 \text { control group where the participants received saline. No } \\
\text { mention of sponsorship or funding }\end{array}$
\end{tabular}

\section{Risk of bias}

\begin{tabular}{|c|c|c|}
\hline Bias & Authors' judgement & Support for judgement \\
\hline $\begin{array}{l}\text { Random sequence genera- } \\
\text { tion (selection bias) }\end{array}$ & Unclear risk & Randomisation process not described \\
\hline $\begin{array}{l}\text { Allocation concealment } \\
\text { (selection bias) }\end{array}$ & Low risk & $\begin{array}{l}\text { A research nurse prepared envelopes that were sealed, numbered and stored } \\
\text { in a box }\end{array}$ \\
\hline $\begin{array}{l}\text { Blinding of participants } \\
\text { and personnel (perfor- } \\
\text { mance bias) } \\
\text { All outcomes }\end{array}$ & Low risk & Blinded personnel \\
\hline $\begin{array}{l}\text { Blinding of outcome as- } \\
\text { sessment (detection bias) } \\
\text { All outcomes }\end{array}$ & Low risk & Blinded personnel \\
\hline $\begin{array}{l}\text { Incomplete outcome data } \\
\text { (attrition bias) } \\
\text { All outcomes }\end{array}$ & Low risk & No withdrawals \\
\hline $\begin{array}{l}\text { Selective reporting (re- } \\
\text { porting bias) }\end{array}$ & Low risk & Predefined outcome adequately addressed \\
\hline Size & High risk & 30 participants per treatment arm \\
\hline
\end{tabular}

\section{Sahin 2004}

\section{Study characteristics}

\begin{tabular}{ll}
\hline Methods & Randomised, double-blind, placebo control \\
\hline Participants & $\mathrm{N}=47$, of whom 33 participants in IV ketamine and control treatment arms. About $52 \%$ women \\
\hline Interventions & Pre-incisional ketamine $0.5 \mathrm{mg} / \mathrm{kg}$ bolus IV \\
\hline Outcomes & $\begin{array}{l}\text { Pain intensity (VAS), reported in } 15-m i n \text { intervals during the 1st h. Cumulative analgesic consumption } \\
\text { reported at } 24 \text { h postoperatively. Time to first analgesic request. AEs }\end{array}$ \\
\hline Surgery type & Lumbar discectomy \\
\hline
\end{tabular}


Sahin 2004 (Continued)

$$
\begin{aligned}
& \text { Group numbers after end } \\
& \text { of study (treatment/con- } \\
& \text { trol) }
\end{aligned}
$$

\begin{tabular}{lc}
\hline $\begin{array}{l}\text { Age of patient population } \\
\text { (treatment/control) }\end{array}$ & $46.5 \pm 7.3$ \\
& $48.3 \pm 11.2$
\end{tabular}

Notes

3 study groups. The treatment group received ketamine bolus IV + remifentanil intraoperatively. The 2nd group where participants received saline bolus IV + remifentanil intraoperatively served as a control group. The 3rd group (14 participants) received saline bolus IV + saline infusion intraoperatively

\begin{tabular}{|c|c|c|}
\hline Bias & Authors' judgement & Support for judgement \\
\hline $\begin{array}{l}\text { Random sequence genera- } \\
\text { tion (selection bias) }\end{array}$ & Unclear risk & Randomisation process not described \\
\hline $\begin{array}{l}\text { Allocation concealment } \\
\text { (selection bias) }\end{array}$ & Unclear risk & Allocation concealment method not described \\
\hline $\begin{array}{l}\text { Blinding of participants } \\
\text { and personnel (perfor- } \\
\text { mance bias) } \\
\text { All outcomes }\end{array}$ & Low risk & Blinded personnel \\
\hline $\begin{array}{l}\text { Blinding of outcome as- } \\
\text { sessment (detection bias) } \\
\text { All outcomes }\end{array}$ & Low risk & Blinded personnel \\
\hline $\begin{array}{l}\text { Incomplete outcome data } \\
\text { (attrition bias) } \\
\text { All outcomes }\end{array}$ & Low risk & No withdrawals \\
\hline $\begin{array}{l}\text { Selective reporting (re- } \\
\text { porting bias) }\end{array}$ & Unclear risk & $\begin{array}{l}\text { Predefined outcomes reported but misses exact numbers of PONV, only men- } \\
\text { tioned that the incidences of nausea, vomiting were similar among groups }\end{array}$ \\
\hline Size & High risk & 17 and 16 participants per treatment arm \\
\hline
\end{tabular}
and was excluded from the analysis. No mention of sponsorship or funding

\section{Risk of bias}

Sen 2009

\section{Study characteristics}

\begin{tabular}{ll}
\hline Methods & Randomised, double-blind, placebo control \\
\hline Participants & $\mathrm{N}=60,100 \%$ women \\
\hline Interventions & Pre-incisional ketamine $0.3 \mathrm{mg} / \mathrm{kg}$ bolus IV $+0.05 \mathrm{mg} / \mathrm{kg} / \mathrm{h}$ infusion IV until the end of surgery \\
\hline Outcomes & $\begin{array}{l}\text { Pain intensity (VRS). Analgesic consumption. AEs. Main outcomes reported every } 4 \mathrm{~h} \text { up to } 24 \mathrm{~h} \text { postop- } \\
\text { eratively }\end{array}$ \\
\hline Surgery type & Abdominal hysterectomy \\
\hline
\end{tabular}


Sen 2009 (Continued)

$$
\begin{aligned}
& \text { Group numbers after end } \\
& \text { of study (treatment/con- } \\
& \text { trol) }
\end{aligned}
$$

$20 / 20$

$\begin{array}{lr}\begin{array}{l}\text { Age of patient population } \\ \text { (treatment/control) }\end{array} & 46 \pm 6 \\ & 46 \pm 7\end{array}$

Notes

\begin{tabular}{|c|c|c|}
\hline \multicolumn{3}{|l|}{ Risk of bias } \\
\hline Bias & Authors' judgement & Support for judgement \\
\hline $\begin{array}{l}\text { Random sequence genera- } \\
\text { tion (selection bias) }\end{array}$ & Low risk & Computer-generated table of random numbers \\
\hline $\begin{array}{l}\text { Allocation concealment } \\
\text { (selection bias) }\end{array}$ & Low risk & $\begin{array}{l}\text { Central allocation: hospital pharmacy (a third party) prepared study drugs that } \\
\text { were labelled identically }\end{array}$ \\
\hline $\begin{array}{l}\text { Blinding of participants } \\
\text { and personnel (perfor- } \\
\text { mance bias) } \\
\text { All outcomes }\end{array}$ & Low risk & Blinded personnel \\
\hline $\begin{array}{l}\text { Blinding of outcome as- } \\
\text { sessment (detection bias) } \\
\text { All outcomes }\end{array}$ & Low risk & Blinded personnel \\
\hline $\begin{array}{l}\text { Incomplete outcome data } \\
\text { (attrition bias) } \\
\text { All outcomes }\end{array}$ & Low risk & No withdrawals \\
\hline $\begin{array}{l}\text { Selective reporting (re- } \\
\text { porting bias) }\end{array}$ & Low risk & Predefined outcomes reported adequately \\
\hline Size & High risk & 20 participants per treatment arm \\
\hline
\end{tabular}
The third group in this study was treated with oral gabapentin. Supported by institutional and departmental sources at GATA Haydarpasa Eğitim Hastanesi.

\section{Siddiqui 2015}

\section{Study characteristics}

\begin{tabular}{ll}
\hline Methods & Randomised, double-blind, placebo control \\
\hline Participants & $\mathrm{N}=60$, gender of participants not specified \\
\hline Interventions & Ketamine $0.3 \mathrm{mg} / \mathrm{kg}$ bolus IV following induction \\
\hline Outcomes & Pain intensity (VAS) reported at 30 min postoperatively as number of participants with VAS-score 1-3, \\
& $4-5,6-8$ and 9-10, respectively. Analgesic consumption during recovery room stay. AEs \\
\hline Surgery type & Elective day care surgery (procedures not defined) \\
\hline
\end{tabular}


Siddiqui 2015 (Continued)

Group numbers after end of study (treatment/control)

$\begin{array}{ll}\begin{array}{ll}\text { Age of patient population } \\ \text { (treatment/control) }\end{array} & 36.1 \pm 10.6 \\ & 36.1 \pm 9\end{array}$

Notes No mention of sponsorship or funding

\section{Risk of bias}

Bias Authors' judgement Support for judgement

Random sequence genera- Unclear risk $\quad$ Randomisation process not described in detail
tion (selection bias)

tion (selection bias)

Allocation concealment $\quad$ Unclear risk Allocation concealment method not described in detail
(selection bias)

Blinding of participants $\quad$ Unclear risk
and personnel (perfor-
mance bias)

\begin{tabular}{|c|c|c|}
\hline $\begin{array}{l}\text { Blinding of outcome as- } \\
\text { sessment (detection bias) } \\
\text { All outcomes }\end{array}$ & Unclear risk & Blinding method not described in detail \\
\hline $\begin{array}{l}\text { Incomplete outcome data } \\
\text { (attrition bias) } \\
\text { All outcomes }\end{array}$ & High risk & Discrepancy between text, "there were no dropouts" and a results table \\
\hline $\begin{array}{l}\text { Selective reporting (re- } \\
\text { porting bias) }\end{array}$ & High risk & All predefined outcomes are not reported (rescue analgesia) \\
\hline Size & High risk & 29 participants per treatment arm \\
\hline
\end{tabular}

Singh 2013

\section{Study characteristics}

\begin{tabular}{ll}
\hline Methods & Randomised, double-blind, placebo control \\
\hline Participants & $\mathrm{N}=80$. Only mentioned that adult patients of either gender were randomised \\
\hline Interventions & $\begin{array}{l}\text { 1. Pre-incisional ketamine } 1 \mathrm{mg} / \mathrm{kg} \text { bolus IV } \\
\text { 2. Pre-incisional ketamine } 0.75 \mathrm{mg} / \mathrm{kg} \text { bolus IV } \\
\text { 3. Pre-incisional ketamine } 0.5 \mathrm{mg} / \mathrm{kg} \text { bolus IV }\end{array}$ \\
\hline Outcomes & $\begin{array}{l}\text { Pain intensity (VAS), reported every } 30 \text { min for first } 2 \mathrm{~h} \text {, every } 1 \mathrm{~h} \text { for the next } 4 \mathrm{~h} \text {, and then at } 12 \mathrm{~h} \text { and } \\
\text { number of analgesic doses given to participants in different groups. AEs }\end{array}$ \\
\hline Surgery type & Laparoscopic cholecystectomy \\
\hline \hline
\end{tabular}


Singh 2013 (Continued)

Group numbers after end $\quad 20 / 20 / 20 / 20$
of study (treatment/con-
trol)

\begin{tabular}{ll}
$\begin{array}{l}\text { Age of patient population } \\
\text { (treatment/control) }\end{array}$ & NA \\
\hline Notes & $\begin{array}{l}\text { 3 groups with } 3 \text { different ketamine doses given pre-incisionally IV vs placebo. No mention of sponsor- } \\
\text { ship or funding }\end{array}$ \\
\hline
\end{tabular}

\section{Risk of bias}

\begin{tabular}{|c|c|c|}
\hline Bias & Authors' judgement & Support for judgement \\
\hline $\begin{array}{l}\text { Random sequence genera- } \\
\text { tion (selection bias) }\end{array}$ & Low risk & Computer-generated random numbers \\
\hline $\begin{array}{l}\text { Allocation concealment } \\
\text { (selection bias) }\end{array}$ & Low risk & Allocation was concealed in sealed, opaque envelopes \\
\hline $\begin{array}{l}\text { Blinding of participants } \\
\text { and personnel (perfor- } \\
\text { mance bias) } \\
\text { All outcomes }\end{array}$ & Low risk & Blinded personnel \\
\hline $\begin{array}{l}\text { Blinding of outcome as- } \\
\text { sessment (detection bias) } \\
\text { All outcomes }\end{array}$ & Low risk & Blinded personnel \\
\hline $\begin{array}{l}\text { Incomplete outcome data } \\
\text { (attrition bias) } \\
\text { All outcomes }\end{array}$ & Unclear risk & Withdrawals not reported \\
\hline $\begin{array}{l}\text { Selective reporting (re- } \\
\text { porting bias) }\end{array}$ & Unclear risk & No numerical results but percentages reported. Withdrawals not reported \\
\hline Size & High risk & 20 participants per treatment arm \\
\hline
\end{tabular}

\section{Snijdelaar 2004}

\section{Study characteristics}

Methods Randomised, double-blind. Intraoperative S-ketamine vs placebo/postoperative S-ketamine + mor-
phine vs morphine and placebo

\begin{tabular}{ll}
\hline Participants & $\mathrm{N}=28$. Radical retropubic prostatectomy. $100 \% \mathrm{men}$ \\
\hline Interventions & $\begin{array}{l}\text { Intraoperative S-ketamine } 0.1 \mathrm{mg} / \mathrm{kg} \text { bolus IV followed by a continuous infusion of } 2 \mu \mathrm{mg} / \mathrm{kg} / \mathrm{min} \mathrm{IV} \text { until } \\
\text { skin closure + postoperative IV PCA S-ketamine } 0.5 \mathrm{mg} / \mathrm{bolus}\end{array}$ \\
\hline Outcomes & $\begin{array}{l}\text { Pain intensity (VAS) reported hourly up to } 4 \mathrm{~h} \text {, then at } 8 \text { and } 12 \mathrm{~h} \text {, then every } 6 \mathrm{~h} \text { up to } 48 \mathrm{~h} \text { postopera- } \\
\text { tively. PCA morphine consumption, reported hourly during the first } 4 \mathrm{~h} \text {, then every } 6 \mathrm{~h} \text { up to } 48 \mathrm{~h} \text { post- } \\
\text { operatively. Hyperalgesia (pain perception threshold, pressure algometry). AEs }\end{array}$ \\
\hline Surgery type & Radical retropubic prostatectomy \\
\hline Pens
\end{tabular}


Snijdelaar 2004 (Continued)

Group numbers after end of study (treatment/control)
$13 / 12$

$60.1 \pm 4.7$

$61.7 \pm 4.7$

Age of patient population (treatment/control)

Dr D G Snijdelaar is supported by an Independent Investigator Grant from Parke-Davis (now Pfizer). Dr J. Katz is supported by a Canada Research Chair in Health Psychology at York University from the Canadian Institutes of Health Research.

\section{Risk of bias}

\begin{tabular}{|c|c|c|}
\hline Bias & Authors' judgement & Support for judgement \\
\hline $\begin{array}{l}\text { Random sequence genera- } \\
\text { tion (selection bias) }\end{array}$ & Low risk & Randomisation by using the random function of Microsoft EXCEL 97 \\
\hline $\begin{array}{l}\text { Allocation concealment } \\
\text { (selection bias) }\end{array}$ & Low risk & $\begin{array}{l}\text { Central allocation. Study syringes prepared and dispensed by the hospital } \\
\text { pharmacy (a third party). Coded syringes that were prepared in a blinded fash- } \\
\text { ion for each participant and retained by the pharmacy }\end{array}$ \\
\hline $\begin{array}{l}\text { Blinding of participants } \\
\text { and personnel (perfor- } \\
\text { mance bias) } \\
\text { All outcomes }\end{array}$ & Low risk & Blinded personnel prepared study drugs \\
\hline $\begin{array}{l}\text { Blinding of outcome as- } \\
\text { sessment (detection bias) } \\
\text { All outcomes }\end{array}$ & Low risk & Blinded personnel collected data \\
\hline $\begin{array}{l}\text { Incomplete outcome data } \\
\text { (attrition bias) } \\
\text { All outcomes }\end{array}$ & High risk & $11 \%$ was withdrawn \\
\hline $\begin{array}{l}\text { Selective reporting (re- } \\
\text { porting bias) }\end{array}$ & Low risk & Predefined outcomes reported \\
\hline Size & High risk & 13 and 12 participants per treatment arm \\
\hline
\end{tabular}

Song 2013

\section{Study characteristics}

\begin{tabular}{ll}
\hline Methods & Randomised, double-blind, placebo control \\
\hline Participants & $\mathrm{N}=50,100 \%$ women \\
\hline Interventions & $\begin{array}{l}\text { Ketamine } 0.3 \mathrm{mg} / \mathrm{kg} \text { bolus IV at induction + postoperative IV-PCA with ketamine } 3 \mathrm{mg} / \mathrm{kg}, \text { background } \\
\text { infusion } 2 \mathrm{~mL} / \mathrm{h}+2 \mathrm{~mL} / \text { bolus on-demand }\end{array}$ \\
\hline Outcomes & $\begin{array}{l}\text { Pain intensity (VAS) reported at } 0-6 \mathrm{~h}, 6-12 \mathrm{~h}, 12-24 \mathrm{~h}, 24-36 \mathrm{~h} \text { and } 36-48 \text { h postoperatively. Cumulative } \\
\text { volume of IV-PCA consumed reported at } 6,12,24,36 \text { and } 48 \text { h postoperatively. AEs }\end{array}$ \\
\hline
\end{tabular}


Song 2013 (Continued)

\begin{tabular}{ll} 
Surgery type & Lumbar spinal fusion \\
\hline $\begin{array}{l}\text { Group numbers after end } \\
\text { of study (treatment/con- } \\
\text { trol) }\end{array}$ & $24 / 25$
\end{tabular}

\begin{tabular}{ll}
$\begin{array}{ll}\text { Age of patient population } \\
\text { (treatment/control) }\end{array}$ & Mean (range) \\
& $57(30-65)$ \\
& $58(34-65)$ \\
\hline Notes & Financial support provided from departmental sources
\end{tabular}

\section{Risk of bias}

\begin{tabular}{|c|c|c|}
\hline Bias & Authors' judgement & Support for judgement \\
\hline $\begin{array}{l}\text { Random sequence genera- } \\
\text { tion (selection bias) }\end{array}$ & Low risk & Random number table \\
\hline $\begin{array}{l}\text { Allocation concealment } \\
\text { (selection bias) }\end{array}$ & Low risk & Sequentially numbered, opaque, sealed envelopes \\
\hline $\begin{array}{l}\text { Blinding of participants } \\
\text { and personnel (perfor- } \\
\text { mance bias) } \\
\text { All outcomes }\end{array}$ & Low risk & Blinded personnel \\
\hline $\begin{array}{l}\text { Blinding of outcome as- } \\
\text { sessment (detection bias) } \\
\text { All outcomes }\end{array}$ & Low risk & Blinded personnel \\
\hline $\begin{array}{l}\text { Incomplete outcome data } \\
\text { (attrition bias) } \\
\text { All outcomes }\end{array}$ & Low risk & $2 \%$ was withdrawn \\
\hline $\begin{array}{l}\text { Selective reporting (re- } \\
\text { porting bias) }\end{array}$ & Low risk & Results of predefined outcomes reported \\
\hline Size & High risk & 24 and 25 participants per treatment arm \\
\hline
\end{tabular}

\section{Song 2014}

\section{Study characteristics}

\begin{tabular}{ll}
\hline Methods & Randomised, double-blind \\
\hline Participants & $\mathrm{N}=75,100 \%$ women \\
\hline Interventions & Pre-incisional ketamine $0.25 \mathrm{mg} / \mathrm{kg}$ bolus IV followed by an infusion of $5 \mu \mathrm{kg} / \mathrm{min}$ until skin closure \\
\hline Outcomes & $\begin{array}{l}\text { Pain intensity (VAS), reported at } 1 \mathrm{~h} \text { postoperatively. Cumulative analgesic consumption reported at } 24 \\
\text { h postoperatively. Time to first request for analgesia. Hyperalgesia reported at } 24 \mathrm{~h} \text { postoperatively }\end{array}$ \\
\hline
\end{tabular}


Song 2014 (Continued)

\begin{tabular}{ll} 
Surgery type & Laparoscopic gynaecologic surgery \\
\hline $\begin{array}{l}\text { Group numbers after end } \\
\text { of study (treatment/con- } \\
\text { trol) }\end{array}$ & $25 / 25$
\end{tabular}

$\begin{array}{ll}\begin{array}{l}\text { Age of patient population } \\ \text { (treatment/control) }\end{array} & 48.9 \pm 6.8 \\ & 49.3 \pm 5.7\end{array}$

Notes The study was supported by Wonkwang University

\section{Risk of bias}

\begin{tabular}{lll}
\hline Bias & Authors' judgement & Support for judgement \\
\hline $\begin{array}{l}\text { Random sequence genera- } \\
\text { tion (selection bias) }\end{array}$ & Low risk & Randomisation according to computer-generated random number table \\
\hline $\begin{array}{l}\text { Allocation concealment } \\
\text { (selection bias) }\end{array}$ & Unclear risk & Allocation concealment method not described \\
\hline $\begin{array}{l}\text { Blinding of participants } \\
\text { and personnel (perfor- } \\
\text { mance bias) } \\
\text { All outcomes }\end{array}$ & Unclear risk & $\begin{array}{l}\text { Participants unaware of group assignments. Not described whether anaesthe- } \\
\text { siologists in the operating room were blinded, thus unclear risk of bias }\end{array}$ \\
\hline $\begin{array}{l}\text { Blinding of outcome as- } \\
\text { sessment (detection bias) } \\
\text { All outcomes }\end{array}$ & Low risk & Blinded anaesthesiologist assessed pain \\
\hline $\begin{array}{l}\text { Incomplete outcome data } \\
\text { (attrition bias) } \\
\text { All outcomes }\end{array}$ & Low risk & No withdrawals \\
\hline $\begin{array}{l}\text { Selective reporting (re- } \\
\text { porting bias) }\end{array}$ & Low risk & Pigh risk \\
\hline \begin{tabular}{l} 
Size \\
\hline
\end{tabular} & 25 participants per treatment arm \\
\hline
\end{tabular}

Spreng 2010

\section{Study characteristics}

\begin{tabular}{ll}
\hline Methods & Randomised, double-blind, placebo control \\
\hline Participants & $\mathrm{N}=83,58 \%$ women of those included \\
\hline Interventions & $\begin{array}{l}\text { Pre-incisional S-ketamine } 0.35 \mathrm{mg} / \mathrm{kg} \text { bolus IV }+5 \mu \mathrm{g} / \mathrm{kg} / \mathrm{min} \text { infusion IV until } 2 \text { mins after the end of } \\
\text { surgery }\end{array}$ \\
\hline Outcomes & Pain intensity (VAS). Emergence time. AEs \\
& Main measurements were 1 day, 7 days, and 3 months \\
\hline
\end{tabular}


Spreng 2010 (Continued)

\begin{tabular}{ll} 
Surgery type & Ambulatory haemorrhoidectomy \\
\hline $\begin{array}{l}\text { Group numbers after end } \\
\text { of study (treatment/con- } \\
\text { trol) }\end{array}$ & $39 / 38$
\end{tabular}

$\begin{array}{ll}\begin{array}{ll}\text { Age of patient population } \\ \text { (treatment/control) }\end{array} & 46.4 \pm 4.7 \\ & 61.7 \pm 4.7\end{array}$

Notes Institutional funding, with no conflicts of interest

\section{Risk of bias}

\begin{tabular}{|c|c|c|}
\hline Bias & Authors' judgement & Support for judgement \\
\hline $\begin{array}{l}\text { Random sequence genera- } \\
\text { tion (selection bias) }\end{array}$ & Low risk & Permuted block randomisation \\
\hline $\begin{array}{l}\text { Allocation concealment } \\
\text { (selection bias) }\end{array}$ & Low risk & $\begin{array}{l}\text { Central allocation by the hospital pharmacy. } \\
\text { Quote: "Permuted block randomization, blinding and packing of the study } \\
\text { medication were performed by the hospital pharmacy. The randomization } \\
\text { codes were provided in sealed envelopes which only were opened in case of } \\
\text { emergency or after completion of the study protocol of all study participants." }\end{array}$ \\
\hline $\begin{array}{l}\text { Blinding of participants } \\
\text { and personnel (perfor- } \\
\text { mance bias) } \\
\text { All outcomes }\end{array}$ & Low risk & Blinded personnel \\
\hline $\begin{array}{l}\text { Blinding of outcome as- } \\
\text { sessment (detection bias) } \\
\text { All outcomes }\end{array}$ & Low risk & Blinded personnel \\
\hline $\begin{array}{l}\text { Incomplete outcome data } \\
\text { (attrition bias) } \\
\text { All outcomes }\end{array}$ & Low risk & $8 \%$ was withdrawn \\
\hline $\begin{array}{l}\text { Selective reporting (re- } \\
\text { porting bias) }\end{array}$ & Unclear risk & $\begin{array}{l}\text { Study authors state that treatment group had more AEs. However, the differ- } \\
\text { ence was not statistically significant }\end{array}$ \\
\hline Size & High risk & 39 and 38 participants per treatment arm \\
\hline
\end{tabular}

Stubhaug 1997

\section{Study characteristics}

\begin{tabular}{ll}
\hline Methods & Randomised, double-blind, placebo control \\
\hline Participants & $\mathrm{N}=20,50 \%$ women \\
\hline Interventions & $\begin{array}{l}\text { Ketamine } 0.5 \mathrm{mg} / \mathrm{kg} \text { bolus IV }+ \text { infusion } 2 \mu \mathrm{gg} / \mathrm{kg} / \mathrm{min} \mathrm{IV} \mathrm{for} 24 \mathrm{~h} \text {, thereafter } 1 \mathrm{mcg} / \mathrm{kg} / \mathrm{min} \text { and main- } \\
\text { tained for } 48 \mathrm{~h}\end{array}$ \\
\hline
\end{tabular}


Stubhaug 1997 (Continued)

Outcomes

Pain intensity (VAS) reported hourly during the first $4 \mathrm{~h}$. Cumulative PCA morphine consumption reported at 0-24 h, 24-48 $\mathrm{h}$ and 48-72h postoperatively. Area of punctate hyperalgesia reported on days 1,3 and 7 postoperatively. Pressure pain threshold. AEs

\begin{tabular}{|c|c|c|}
\hline Surgery type & \multicolumn{2}{|c|}{ Nephrectomy (live kidney donors) } \\
\hline $\begin{array}{l}\text { Group numbers after end } \\
\text { of study (treatment/con- } \\
\text { trol) }\end{array}$ & \multicolumn{2}{|l|}{$10 / 10$} \\
\hline Age of patient population & \multicolumn{2}{|l|}{ Median (range) } \\
\hline 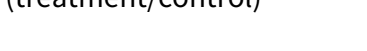 & \multicolumn{2}{|l|}{$44(32-53)$} \\
\hline & \multicolumn{2}{|l|}{$42(25-66)$} \\
\hline Notes & \multicolumn{2}{|c|}{ Baxter Norway provided Baxter Ambulatory PCA Pumps with printer. No mention of additional funding } \\
\hline \multicolumn{3}{|l|}{ Risk of bias } \\
\hline Bias & Authors' judgement & Support for judgement \\
\hline $\begin{array}{l}\text { Random sequence genera- } \\
\text { tion (selection bias) }\end{array}$ & Low risk & Random numbers (Moses Oakford algorithm) \\
\hline $\begin{array}{l}\text { Allocation concealment } \\
\text { (selection bias) }\end{array}$ & Low risk & $\begin{array}{l}\text { Quote: "Treatment allocation remained concealed to patients and investiga- } \\
\text { tors during the whole study. Study drug for each patient was prepared by the } \\
\text { hospital pharmacy in identical containers, marked with consecutive patient } \\
\text { numbers only and delivered by a portable pump via a separate i.v. line." }\end{array}$ \\
\hline $\begin{array}{l}\text { Blinding of participants } \\
\text { and personnel (perfor- } \\
\text { mance bias) } \\
\text { All outcomes }\end{array}$ & Low risk & Blinded personnel \\
\hline $\begin{array}{l}\text { Blinding of outcome as- } \\
\text { sessment (detection bias) } \\
\text { All outcomes }\end{array}$ & Low risk & Blinded personnel \\
\hline $\begin{array}{l}\text { Incomplete outcome data } \\
\text { (attrition bias) } \\
\text { All outcomes }\end{array}$ & Low risk & No withdrawals \\
\hline $\begin{array}{l}\text { Selective reporting (re- } \\
\text { porting bias) }\end{array}$ & Low risk & Predefined outcomes reported \\
\hline Size & High risk & 10 participants per treatment arm \\
\hline
\end{tabular}

Subramaniam 2011

\section{Study characteristics}

\begin{tabular}{ll}
\hline Methods & Randomised, double-blind, placebo control \\
\hline Participants & $N=38,50 \%$ women \\
\hline
\end{tabular}


Subramaniam 2011 (Continued)

\begin{tabular}{|c|c|c|}
\hline Interventions & \multicolumn{2}{|c|}{$\begin{array}{l}\text { Ketamine } 0.1 \mathrm{mg} / \mathrm{kg} \text { bolus IV at anaesthesia induction }+2 \mu \mathrm{g} / \mathrm{kg} / \mathrm{min} \text { intraoperative infusion IV and } 24 \mathrm{~h} \\
\text { postoperatively }\end{array}$} \\
\hline Outcomes & \multicolumn{2}{|c|}{$\begin{array}{l}\text { Pain intensity (VAS) and analgesic consumption reported at 1, 2, 4, 8, 12, 18, 24, } 36 \text { and } 48 \text { h postopera- } \\
\text { tively. AEs }\end{array}$} \\
\hline Surgery type & \multicolumn{2}{|c|}{ Lumbar or thoracolumbar laminectomy and fusion } \\
\hline $\begin{array}{l}\text { Group numbers after end } \\
\text { of study (treatment/con- } \\
\text { trol) }\end{array}$ & \multicolumn{2}{|l|}{$15 / 15$} \\
\hline $\begin{array}{l}\text { Age of patient population } \\
\text { (treatment/control) }\end{array}$ & \multicolumn{2}{|l|}{$57.2 \pm 12.2$} \\
\hline Notes & \multicolumn{2}{|c|}{$\begin{array}{l}\text { The study was underpowered. According to power analysis, } 26 \text { participants per study group should } \\
\text { have been recruited in order to obtain sufficient power. No mention of sponsorship or funding }\end{array}$} \\
\hline \multicolumn{3}{|l|}{ Risk of bias } \\
\hline Bias & Authors' judgement & Support for judgement \\
\hline $\begin{array}{l}\text { Random sequence genera- } \\
\text { tion (selection bias) }\end{array}$ & Low risk & Computer-generated random numbers \\
\hline $\begin{array}{l}\text { Allocation concealment } \\
\text { (selection bias) }\end{array}$ & Low risk & $\begin{array}{l}\text { Central allocation. Randomisation and allocation concealment by the hospital } \\
\text { pharmacy (a third party) }\end{array}$ \\
\hline $\begin{array}{l}\text { Blinding of participants } \\
\text { and personnel (perfor- } \\
\text { mance bias) } \\
\text { All outcomes }\end{array}$ & Low risk & Blinded personnel \\
\hline
\end{tabular}

\begin{tabular}{lll}
\hline $\begin{array}{l}\text { Blinding of outcome as- } \\
\text { sessment (detection bias) } \\
\text { All outcomes }\end{array}$ & Low risk & Blinded personnel \\
\hline $\begin{array}{l}\text { Incomplete outcome data } \\
\text { (attrition bias) } \\
\text { All outcomes }\end{array}$ & High risk & $21 \%$ was withdrawn \\
\hline $\begin{array}{l}\text { Selective reporting (re- } \\
\text { porting bias) }\end{array}$ & Low risk & Predefined outcomes adequately reported \\
\hline Size & High risk & 15 participants per treatment arm \\
\hline
\end{tabular}

\section{Suzuki 1999}

\section{Study characteristics}

\begin{tabular}{ll}
\hline Methods & Randomised, double-blind, placebo control \\
\hline Participants & $\mathrm{N}=140$, about $38 \%$ women \\
\hline Interventions & IV-bolus of ketamine at wound closure: \\
\hline
\end{tabular}

Perioperative intravenous ketamine for acute postoperative pain in adults (Review) 
Suzuki 1999 (Continued)
1. $50 \mu \mathrm{g} / \mathrm{kg}$
2. $75 \mu \mathrm{g} / \mathrm{kg}$
3. $100 \mu \mathrm{g} / \mathrm{kg}$

\begin{tabular}{ll}
\hline Outcomes & $\begin{array}{l}\text { Pain intensity (VAS) reported at 15-min intervals for the first hour. Rescue medication, reported as } \\
\text { mean amount needed during the phase } 1 \text { recovery. AEs }\end{array}$ \\
\hline Surgery type & $\begin{array}{l}\text { Elective outpatient surgery (inguinal hernia repair, excision of skin lesions, breast or lymph node biop- } \\
\text { sy) }\end{array}$ \\
\hline $\begin{array}{l}\text { Group numbers after end } \\
\text { of study (treatment/con- } \\
\text { trol) }\end{array}$ & $105 / 35$ \\
\hline $\begin{array}{l}\text { Age of patient population } \\
\text { (treatment/control) }\end{array}$ & $36 \pm 11$ \\
& $30 \pm 12$ \\
\hline Notes & $\begin{array}{l}\text { 3 different doses of ketamine IV vs placebo. } 35 \text { participants in each study group. No mention of spon- } \\
\text { sorship or funding }\end{array}$
\end{tabular}

\section{Risk of bias}

\begin{tabular}{|c|c|c|}
\hline Bias & Authors' judgement & Support for judgement \\
\hline $\begin{array}{l}\text { Random sequence genera- } \\
\text { tion (selection bias) }\end{array}$ & Low risk & Computer-generated randomisation schedule \\
\hline $\begin{array}{l}\text { Allocation concealment } \\
\text { (selection bias) }\end{array}$ & Unclear risk & Allocation concealment method not described \\
\hline $\begin{array}{l}\text { Blinding of participants } \\
\text { and personnel (perfor- } \\
\text { mance bias) } \\
\text { All outcomes }\end{array}$ & Low risk & A person not involved in the study prepared study drugs \\
\hline $\begin{array}{l}\text { Blinding of outcome as- } \\
\text { sessment (detection bias) } \\
\text { All outcomes }\end{array}$ & Unclear risk & Blinding not described \\
\hline $\begin{array}{l}\text { Incomplete outcome data } \\
\text { (attrition bias) } \\
\text { All outcomes }\end{array}$ & Low risk & No withdrawals \\
\hline $\begin{array}{l}\text { Selective reporting (re- } \\
\text { porting bias) }\end{array}$ & Low risk & Predefined outcomes reported \\
\hline Size & High risk & 35 participants per treatment arm. Heterogeneous procedures \\
\hline
\end{tabular}

\section{Study characteristics}

Methods Randomised, double-blind, placebo control


Suzuki 2006 (Continued)

\begin{tabular}{ll} 
Participants & $\mathrm{N}=50$, about $41 \%$ women \\
\hline Interventions & Ketamine $5 \mu \mathrm{g} / \mathrm{kg} / \mathrm{min}$ IV infusion after tracheal intubation lasting $72 \mathrm{~h}$ after surgery \\
\hline Outcomes & $\begin{array}{l}\text { Pain intensity (VAS) reported at } 6,12,24 \text { and } 48 \mathrm{~h} \text { postoperatively. Cumulative analgesic consump- } \\
\text { tion reported at } 0-6 \mathrm{~h}, 6-12 \mathrm{~h}, 12-24 \mathrm{~h} \text { and } 24-48 \mathrm{~h} \text { after surgery. Abnormal sensation of pain around the } \\
\text { wound on postoperative day } 7 . \text { AEs. }\end{array}$
\end{tabular}

\begin{tabular}{ll}
\hline Surgery type & Thoracotomy \\
\hline $\begin{array}{l}\text { Group numbers after end } \\
\text { of study (treatment/con- } \\
\text { trol) }\end{array}$ & \\
\hline
\end{tabular}

\begin{tabular}{ll}
\hline $\begin{array}{l}\text { Age of patient population } \\
\text { (treatment/control) }\end{array}$ & $66 \pm 14$ \\
& $66 \pm 9$
\end{tabular}

Notes No mention of sponsorship or funding

\section{Risk of bias}

\begin{tabular}{|c|c|c|}
\hline Bias & Authors' judgement & Support for judgement \\
\hline $\begin{array}{l}\text { Random sequence genera- } \\
\text { tion (selection bias) }\end{array}$ & Low risk & $\begin{array}{l}\text { Quote: "Patients were assigned to one of two groups using a computer-gener- } \\
\text { ated randomization schedule" }\end{array}$ \\
\hline $\begin{array}{l}\text { Allocation concealment } \\
\text { (selection bias) }\end{array}$ & Unclear risk & $\begin{array}{l}\text { Allocation concealment method not described in detail. Only stated that the } \\
\text { study drugs were prepared and placed in the infusion pump by an investigator } \\
\text { who did not participate in the administration of anaesthesia or the evaluation } \\
\text { of postoperative pain. }\end{array}$ \\
\hline $\begin{array}{l}\text { Blinding of participants } \\
\text { and personnel (perfor- } \\
\text { mance bias) } \\
\text { All outcomes }\end{array}$ & Low risk & Blinded personnel \\
\hline $\begin{array}{l}\text { Blinding of outcome as- } \\
\text { sessment (detection bias) } \\
\text { All outcomes }\end{array}$ & Low risk & Blinded personnel \\
\hline $\begin{array}{l}\text { Incomplete outcome data } \\
\text { (attrition bias) } \\
\text { All outcomes }\end{array}$ & Low risk & $2 \%$ was withdrawn \\
\hline $\begin{array}{l}\text { Selective reporting (re- } \\
\text { porting bias) }\end{array}$ & Unclear risk & $\begin{array}{l}\text { Predefined outcomes reported. Epidural infusion was suspended in some par- } \\
\text { ticipants (ketamine 3, control 5) due to hypotension. }\end{array}$ \\
\hline Size & High risk & 24 and 25 participants per treatment arm \\
\hline
\end{tabular}

Tena 2014

\section{Study characteristics}

Methods Randomised, double-blind, placebo control


Tena 2014 (Continued)

\begin{tabular}{ll} 
Participants & $\mathrm{N}=125$, of whom 68 participants in IV ketamine and control treatment arms. About $28 \%$ women \\
\hline Interventions & Pre-incisional ketamine $0.5 \mathrm{mg} / \mathrm{kg}$ bolus IV + postoperatively $0.25 \mathrm{mg} / \mathrm{kg} / \mathrm{h}$ infusion IV for $48 \mathrm{~h}$ \\
\hline Outcomes & $\begin{array}{l}\text { Pain intensity (VAS) reported at } 2,4,24 \text { and } 72 \mathrm{~h} \text { and } 3 \text { days, } 3 \text { months and } 6 \text { months postoperatively. } \\
\text { Hyperalgesia (von Frey filaments, electronic von Frey, electrical toothbrush) reported at } 72 \mathrm{~h}, 7 \text { days, } 3 \\
\text { months and } 6 \text { months postoperatively. AEs }\end{array}$
\end{tabular}

\begin{tabular}{ll}
\hline Surgery type & Thoracotomy \\
\hline $\begin{array}{l}\text { Group numbers after end } \\
\text { of study (treatment/con- }\end{array}$ & $33 / 35$ \\
trol)
\end{tabular}

\section{$66.5 \pm 9.9$}

The 3rd study group was not included in analysis because participants received ketamine epidurally. No mention of sponsorship or funding and the authors declare no conflict of interest

\section{Risk of bias}

\begin{tabular}{|c|c|c|}
\hline Bias & Authors' judgement & Support for judgement \\
\hline $\begin{array}{l}\text { Random sequence genera- } \\
\text { tion (selection bias) }\end{array}$ & Low risk & Computer-generated randomisation \\
\hline $\begin{array}{l}\text { Allocation concealment } \\
\text { (selection bias) }\end{array}$ & Unclear risk & Allocation concealment method not described \\
\hline $\begin{array}{l}\text { Blinding of participants } \\
\text { and personnel (perfor- } \\
\text { mance bias) } \\
\text { All outcomes }\end{array}$ & Low risk & Blinded personnel \\
\hline $\begin{array}{l}\text { Blinding of outcome as- } \\
\text { sessment (detection bias) } \\
\text { All outcomes }\end{array}$ & Low risk & Blinded personnel \\
\hline $\begin{array}{l}\text { Incomplete outcome data } \\
\text { (attrition bias) } \\
\text { All outcomes }\end{array}$ & High risk & $17 \%$ was withdrawn \\
\hline $\begin{array}{l}\text { Selective reporting (re- } \\
\text { porting bias) }\end{array}$ & Low risk & Predefined outcomes reported \\
\hline Size & High risk & 33 and 35 participants per treatment arm \\
\hline
\end{tabular}

Ünlügenc 2003

\section{Study characteristics}

Methods

Randomised, double-blind. Ketamine + morphine vs morphine 
Ünlügenc 2003 (Continued)

\begin{tabular}{ll} 
Participants & $\mathrm{N}=90$, of whom 60 participants in IV ketamine and control treatment arms. About 43\% women \\
\hline Interventions & Postoperative IV PCA ketamine $0.0125 \mathrm{mg} / \mathrm{kg} / \mathrm{bolus}+$ morphine \\
\hline Outcomes & $\begin{array}{l}\text { Pain intensity (VRS). PCA morphine consumption. Pain outcomes reported at } 15 \text { and } 30 \text { min, then at } 1, \\
2,6,12 \text { and } 24 \text { h postoperatively. AEs }\end{array}$ \\
\hline $\begin{array}{l}\text { Surgery type } \\
\text { Group numbers after end } \\
\text { of study (treatment/con- } \\
\text { trol) }\end{array}$ & $30 / 28$ \\
\hline $\begin{array}{l}\text { Age of patient population } \\
\text { (treatment/control) }\end{array}$ & $\begin{array}{l}52 \pm 4 \\
51 \pm 1.1\end{array}$ \\
\hline $\begin{array}{l}\text { Notes } \\
\text { study groups in this study. We excluded the 3rd group from the analysis because the participants al- } \\
\text { so received magnesium sulphate and the } 2 \text { other study groups did not. No mention of sponsorship or } \\
\text { funding }\end{array}$ \\
\hline
\end{tabular}

\section{Risk of bias}

\begin{tabular}{lll}
\hline Bias & Authors' judgement & Support for judgement \\
\hline $\begin{array}{l}\text { Random sequence genera- } \\
\text { tion (selection bias) }\end{array}$ & Unclear risk & Randomisation not described \\
\hline $\begin{array}{l}\text { Allocation concealment } \\
\text { (selection bias) }\end{array}$ & Unclear risk & Allocation concealment method not described \\
\hline $\begin{array}{l}\text { Blinding of participants } \\
\text { and personnel (perfor- } \\
\text { mance bias) }\end{array}$ & Low risk & Blinded personnel \\
All outcomes & \\
\hline $\begin{array}{l}\text { Blinding of outcome as- } \\
\text { sessment (detection bias) } \\
\text { All outcomes }\end{array}$ & Low risk & Blinded personnel \\
\hline $\begin{array}{l}\text { Incomplete outcome data } \\
\text { (attrition bias) } \\
\text { All outcomes }\end{array}$ & Low risk & $3 \%$ was withdrawn \\
\hline $\begin{array}{l}\text { Selective reporting (re- } \\
\text { porting bias) }\end{array}$ & High risk & All predefined AEs are not reported \\
\hline \begin{tabular}{l} 
Size \\
\hline
\end{tabular} & High risk & 30 and 29 participants per treatment arm \\
\hline
\end{tabular}

Van Elstraete 2004

\section{Study characteristics}

Methods Randomised, double-blind, placebo control


Van Elstraete 2004 (Continued)

\begin{tabular}{|c|c|}
\hline Participants & $\mathrm{N}=40,50 \%$ women \\
\hline Interventions & $\begin{array}{l}\text { Ketamine } 0.5 \mathrm{mg} / \mathrm{kg} \text { bolus IV after anaesthesia induction }+2 \mu \mathrm{g} / \mathrm{kg} / \mathrm{min} \text { infusion IV till the end of } \\
\text { surgery }\end{array}$ \\
\hline Outcomes & $\begin{array}{l}\text { Pain intensity (VAS). Analgesic consumption. Time to first request for analgesia. AEs } \\
\text { Main outcomes at } 2,4,8,12 \text {, and } 24 \text { h postoperatively }\end{array}$ \\
\hline Surgery type & Elective tonsillectomy \\
\hline $\begin{array}{l}\text { Group numbers after end } \\
\text { of study (treatment/con- } \\
\text { trol) }\end{array}$ & $20 / 20$ \\
\hline Notes & No mention of sponsorship or funding \\
\hline
\end{tabular}

\section{Risk of bias}

\begin{tabular}{|c|c|c|}
\hline Bias & Authors' judgement & Support for judgement \\
\hline $\begin{array}{l}\text { Random sequence genera- } \\
\text { tion (selection bias) }\end{array}$ & Low risk & $\begin{array}{l}\text { Quote: "Patients were randomly assigned to one of two groups using a table of } \\
\text { computer-generated random numbers." }\end{array}$ \\
\hline $\begin{array}{l}\text { Allocation concealment } \\
\text { (selection bias) }\end{array}$ & Unclear risk & $\begin{array}{l}\text { Allocation concealment method not described in detail. Only stated that a re- } \\
\text { search nurse not involved in the perioperative care of the participant prepared } \\
\text { and labelled } 2 \text { syringes per randomisation list }\end{array}$ \\
\hline $\begin{array}{l}\text { Blinding of participants } \\
\text { and personnel (perfor- } \\
\text { mance bias) } \\
\text { All outcomes }\end{array}$ & Low risk & $\begin{array}{l}\text { Quote: "The anaesthetist who was in charge of the patient during surgery was } \\
\text { unaware of the study group assignment, as were those involved in data collec- } \\
\text { tion." }\end{array}$ \\
\hline $\begin{array}{l}\text { Blinding of outcome as- } \\
\text { sessment (detection bias) } \\
\text { All outcomes }\end{array}$ & Low risk & $\begin{array}{l}\text { Quote: "The anaesthetist who was in charge of the patient during surgery was } \\
\text { unaware of the study group assignment, as were those involved in data collec- } \\
\text { tion." }\end{array}$ \\
\hline $\begin{array}{l}\text { Incomplete outcome data } \\
\text { (attrition bias) } \\
\text { All outcomes }\end{array}$ & Low risk & No withdrawals \\
\hline $\begin{array}{l}\text { Selective reporting (re- } \\
\text { porting bias) }\end{array}$ & Low risk & Predefined outcomes reported \\
\hline Size & High risk & 20 participants per treatment arm \\
\hline
\end{tabular}

Webb 2007

\section{Study characteristics}

Methods Randomised, double-blind, placebo control


Webb 2007 (Continued)

\begin{tabular}{|c|c|}
\hline Participants & $N=120$, about $38 \%$ women \\
\hline Interventions & $\begin{array}{l}\text { Ketamine } 0.3 \mathrm{mg} / \mathrm{kg} \text { bolus IV at anaesthesia induction + intraoperative infusion } 0.1 \mathrm{mg} / \mathrm{kg} / \mathrm{h} \text { for } 48 \mathrm{~h} \text { af- } \\
\text { ter surgery }\end{array}$ \\
\hline Outcomes & $\begin{array}{l}\text { Pain intensity (VRS) and analgesic consumption reported every } 4 \mathrm{~h} \text { up to } 48 \mathrm{~h} \text { postoperatively. AEs. } \\
\text { Subjective analgesic efficacy }\end{array}$ \\
\hline Surgery type & Laparotomy \\
\hline $\begin{array}{l}\text { Group numbers after end } \\
\text { of study (treatment/con- } \\
\text { trol) }\end{array}$ & $52 / 58$ \\
\hline \multirow{2}{*}{$\begin{array}{l}\text { Age of patient population } \\
\text { (treatment/control) }\end{array}$} & $63 \pm 15$ \\
\hline & $61 \pm 15$ \\
\hline Notes & No mention of sponsorship or funding \\
\hline
\end{tabular}

\section{Risk of bias}

\begin{tabular}{|c|c|c|}
\hline Bias & Authors' judgement & Support for judgement \\
\hline $\begin{array}{l}\text { Random sequence genera- } \\
\text { tion (selection bias) }\end{array}$ & Low risk & Random number tables \\
\hline $\begin{array}{l}\text { Allocation concealment } \\
\text { (selection bias) }\end{array}$ & Low risk & $\begin{array}{l}\text { Quote: "Allocation to treatment group was determined in advance according } \\
\text { to tables of random numbers and concealed from patients and hospital staff, } \\
\text { using sealed opaque envelopes." }\end{array}$ \\
\hline $\begin{array}{l}\text { Blinding of participants } \\
\text { and personnel (perfor- } \\
\text { mance bias) } \\
\text { All outcomes }\end{array}$ & Low risk & Blinded participants and personnel \\
\hline $\begin{array}{l}\text { Blinding of outcome as- } \\
\text { sessment (detection bias) } \\
\text { All outcomes }\end{array}$ & Low risk & Blinded personnel \\
\hline $\begin{array}{l}\text { Incomplete outcome data } \\
\text { (attrition bias) } \\
\text { All outcomes }\end{array}$ & Low risk & $8 \%$ withdrawn \\
\hline $\begin{array}{l}\text { Selective reporting (re- } \\
\text { porting bias) }\end{array}$ & Low risk & Predefined outcomes reported \\
\hline Size & Unclear risk & 56 and 64 participants per treatment arm \\
\hline
\end{tabular}

Woo 2014

\section{Study characteristics}

Methods Randomised, double-blind, placebo control


Woo 2014 (Continued)

\begin{tabular}{ll} 
Participants & $\mathrm{N}=40$, about 33\% women \\
\hline Interventions & $\begin{array}{l}\text { Pre-incisional ketamine } 0.3 \mathrm{mg} / \mathrm{kg} \text { bolus IV + continuous infusion } 0.15 \mathrm{mg} / \mathrm{kg} / \mathrm{min} \text { until } 5 \mathrm{mins} \text { before } \\
\text { the end of surgery }\end{array}$ \\
\hline Outcomes & $\begin{array}{l}\text { Pain intensity (NRS). Analgesic consumption. Pain outcomes reported at } 1,6,12,24,36 \text { and } 48 \mathrm{~h} \text { post- } \\
\text { operatively. AEs }\end{array}$ \\
\hline $\begin{array}{l}\text { Surgery type } \\
\text { Arthroscopic shoulder surgery }\end{array}$ \\
$\begin{array}{l}\text { Group numbers after end } \\
\text { of study (treatment/con- } \\
\text { trol) }\end{array}$ & $20 / 20$ \\
\hline
\end{tabular}

\begin{tabular}{ll}
\hline $\begin{array}{l}\text { Age of patient population } \\
\text { (treatment/control) }\end{array}$ & $42.9 \pm 19$ \\
& $50 \pm 14.1$
\end{tabular}

\begin{tabular}{|c|c|c|}
\hline Notes & No mention of sponso & hip or funding. The study authors declare no conflicts of interest \\
\hline \multicolumn{3}{|l|}{ Risk of bias } \\
\hline Bias & Authors' judgement & Support for judgement \\
\hline $\begin{array}{l}\text { Random sequence genera- } \\
\text { tion (selection bias) }\end{array}$ & Low risk & Randomisation by a computer-generated random number table \\
\hline $\begin{array}{l}\text { Allocation concealment } \\
\text { (selection bias) }\end{array}$ & Unclear risk & $\begin{array}{l}\text { Only said that an anaesthetist blinded to group assignments prepared study } \\
\text { drugs but no further description of allocation concealment }\end{array}$ \\
\hline $\begin{array}{l}\text { Blinding of participants } \\
\text { and personnel (perfor- } \\
\text { mance bias) } \\
\text { All outcomes }\end{array}$ & Low risk & Blinded personnel \\
\hline $\begin{array}{l}\text { Blinding of outcome as- } \\
\text { sessment (detection bias) } \\
\text { All outcomes }\end{array}$ & Low risk & Blinded personnel \\
\hline $\begin{array}{l}\text { Incomplete outcome data } \\
\text { (attrition bias) } \\
\text { All outcomes }\end{array}$ & Low risk & No withdrawals \\
\hline $\begin{array}{l}\text { Selective reporting (re- } \\
\text { porting bias) }\end{array}$ & Low risk & All predefined outcomes reported \\
\hline Size & High risk & 20 participants per treatment arm \\
\hline
\end{tabular}

Wu 2009

\section{Study characteristics}

\begin{tabular}{ll}
\hline Methods & Randomised, double-blind \\
\hline Participants & $N=30$, about $27 \%$ women \\
\hline
\end{tabular}


Wu 2009 (Continued)

Interventions Perioperative ketamine infusion $0.08 \mathrm{mg} / \mathrm{kg} / \mathrm{h}$ IV with morphine for $50 \mathrm{~h}$ vs morphine alone

Outcomes Pain intensity (VAS) reported at 4, 8, 20 and $24 \mathrm{~h}$ postoperatively. Analgesic consumption at 0-8 h, 8-24
$\mathrm{h}, 24-48 \mathrm{~h}$ after surgery (median values). Cumulative analgesic consumption at $48 \mathrm{~h}$ postoperatively. AEs

\begin{tabular}{ll}
\hline Surgery type & Elective radical operation for oesophageal carcinoma \\
\hline $\begin{array}{l}\text { Group numbers after end } \\
\text { of study (treatment/con- } \\
\text { trol) }\end{array}$ & $15 / 15$ \\
\hline $\begin{array}{l}\text { Age of patient population } \\
\text { (treatment/control) }\end{array}$ & $56 \pm 11$ \\
\hline Notes & $58 \pm 10$ \\
\hline
\end{tabular}

\section{Risk of bias}

\begin{tabular}{|c|c|c|}
\hline Bias & Authors' judgement & Support for judgement \\
\hline $\begin{array}{l}\text { Random sequence genera- } \\
\text { tion (selection bias) }\end{array}$ & Unclear risk & Randomisation not described \\
\hline $\begin{array}{l}\text { Allocation concealment } \\
\text { (selection bias) }\end{array}$ & Unclear risk & Allocation concealment method not described \\
\hline $\begin{array}{l}\text { Blinding of participants } \\
\text { and personnel (perfor- } \\
\text { mance bias) } \\
\text { All outcomes }\end{array}$ & Unclear risk & Mentioned "double blind" but blinding not described in detail \\
\hline $\begin{array}{l}\text { Blinding of outcome as- } \\
\text { sessment (detection bias) } \\
\text { All outcomes }\end{array}$ & Unclear risk & Mentioned "double blind" but blinding not described in detail \\
\hline $\begin{array}{l}\text { Incomplete outcome data } \\
\text { (attrition bias) } \\
\text { All outcomes }\end{array}$ & Unclear risk & No withdrawals \\
\hline $\begin{array}{l}\text { Selective reporting (re- } \\
\text { porting bias) }\end{array}$ & High risk & Predefined observer's assessment of awareness/sedation scores not provided \\
\hline Size & High risk & 15 participants per treatment arm \\
\hline
\end{tabular}

Yalcin 2012

\section{Study characteristics}

\begin{tabular}{ll}
\hline Methods & Randomised, double-blind, placebo control \\
\hline Participants & $\mathrm{N}=90,100 \%$ women \\
\hline Interventions & Ketamine $0.5 \mathrm{mg} / \mathrm{kg}$ bolus IV before anaesthesia induction $+5 \mathrm{\mu g} / \mathrm{kg} / \mathrm{min}$ infusion IV until skin closure \\
\hline
\end{tabular}


Yalcin 2012 (Continued)

Outcomes

Pain intensity (VAS). Analgesic consumption. AEs

Main outcomes at 2, 4, 6, 12 and 24 hrs postoperatively

\begin{tabular}{l} 
Surgery type \\
\hline Group numbers after end \\
of study (treatment/con- \\
trol)
\end{tabular}

Total abdominal hysterectomy

\begin{tabular}{ll}
$\begin{array}{ll}\text { Age of patient population } \\
\text { (treatment/control) }\end{array}$ & $48.3 \pm 5.7$ \\
& $48.1 \pm 6$ \\
\hline Notes & The 3rd group that received paracetamol as study drug was excluded from analysis \\
& No financial or competing interests
\end{tabular}

\section{Risk of bias}

\begin{tabular}{lll}
\hline Bias & Authors' judgement & Support for judgement \\
\hline $\begin{array}{l}\text { Random sequence genera- } \\
\text { tion (selection bias) }\end{array}$ & Low risk & A computer-generated random number system \\
\hline $\begin{array}{l}\text { Allocation concealment } \\
\text { (selection bias) }\end{array}$ & Unclear risk & Allocation concealment method described \\
\hline $\begin{array}{l}\text { Blinding of participants } \\
\begin{array}{l}\text { and personnel (perfor- } \\
\text { mance bias) }\end{array}\end{array}$ & Low risk \\
$\begin{array}{ll}\text { All outcomes } \\
\text { Blinding of outcome as- } \\
\text { sessment (detection bias) } \\
\text { All outcomes }\end{array}$ & Low risk & Blinded personnel \\
\hline $\begin{array}{l}\text { Incomplete outcome data } \\
\text { (attrition bias) } \\
\text { All outcomes }\end{array}$ & High risk & Blinded observer \\
\hline $\begin{array}{l}\text { Selective reporting (re- } \\
\text { porting bias) }\end{array}$ & Unclear risk & $12 \%$ was withdrawn \\
\hline \begin{tabular}{l} 
Size \\
\hline
\end{tabular} & High risk & Exact numbers of AEs not reported \\
\hline
\end{tabular}

Yamauchi 2008

\section{Study characteristics}

\begin{tabular}{ll}
\hline Methods & Randomised, double-blind, placebo control \\
\hline Participants & $\mathrm{N}=202$, about $30 \%$ women \\
\hline Interventions & 1. Ketamine $1 \mathrm{mg} / \mathrm{kg}$ bolus IV at skin incision $+42 \mu \mathrm{g} / \mathrm{kg} / \mathrm{h}$ infusion IV for $24 \mathrm{~h}$
\end{tabular}

Interventions

1. Ketamine $1 \mathrm{mg} / \mathrm{kg}$ bolus IV at skin incision $+42 \mu \mathrm{g} / \mathrm{kg} / \mathrm{h}$ infusion IV for $24 \mathrm{~h}$ 
Yamauchi 2008 (Continued)

2. Ketamine $1 \mathrm{mg} / \mathrm{kg}$ bolus IV at skin incision $+83 \mu \mathrm{g} / \mathrm{kg} / \mathrm{h}$ infusion IV for $24 \mathrm{~h}$

$\begin{array}{ll}\text { Outcomes } & \text { Pain intensity (VAS) at rest and during movement at 1, 6, 12, 24, 36, 48 h and 3, } 6 \text { and } 10 \text { days after } \\ \text { surgery. Analgesic consumption. AEs }\end{array}$

\begin{tabular}{ll}
\hline Surgery type & Posterior cervical spine and lumbar spine surgery \\
\hline $\begin{array}{l}\text { Group numbers after end } \\
\text { of study (treatment/con- } \\
\text { trol) }\end{array}$ & \\
\hline
\end{tabular}

\section{Age of patient population (treatment/control)}

$60.2 \pm 16.9$

$57 \pm 17.3$
The study consists of cervical and lumbar surgery participants with 2 different interventions and corresponding control groups. These treatment arms (4 treatment arms and corresponding control groups) were analysed separately. No mention of sponsorship or funding

\section{Risk of bias}

\begin{tabular}{|c|c|c|}
\hline Bias & Authors' judgement & Support for judgement \\
\hline $\begin{array}{l}\text { Random sequence genera- } \\
\text { tion (selection bias) }\end{array}$ & Low risk & Randomisation by shuffling envelopes \\
\hline $\begin{array}{l}\text { Allocation concealment } \\
\text { (selection bias) }\end{array}$ & Low risk & Allocation concealment in envelopes \\
\hline $\begin{array}{l}\text { Blinding of participants } \\
\text { and personnel (perfor- } \\
\text { mance bias) } \\
\text { All outcomes }\end{array}$ & Unclear risk & Mentioned "double-blind" but not described \\
\hline $\begin{array}{l}\text { Blinding of outcome as- } \\
\text { sessment (detection bias) } \\
\text { All outcomes }\end{array}$ & Unclear risk & Blinding not described \\
\hline $\begin{array}{l}\text { Incomplete outcome data } \\
\text { (attrition bias) } \\
\text { All outcomes }\end{array}$ & Low risk & $1 \%$ was withdrawn \\
\hline $\begin{array}{l}\text { Selective reporting (re- } \\
\text { porting bias) }\end{array}$ & Low risk & Predefined outcomes reported \\
\hline Size & High risk & $\begin{array}{l}\text { 22, } 23 \text { and } 23 \text { participants in the cervical surgery groups. } 42,46 \text { and } 44 \text { partici- } \\
\text { pants in the lumbar surgery groups }\end{array}$ \\
\hline
\end{tabular}

\section{Yazigi 2012}

\section{Study characteristics}

\begin{tabular}{ll}
\hline Methods & Randomised, double-blind, placebo control \\
\hline Participants & $\mathrm{N}=60$, about $52 \%$ women \\
\hline
\end{tabular}


Yazigi 2012 (Continued)

Interventions

Outcomes
Pre-incisional ketamine $0.1 \mathrm{mg} / \mathrm{kg}$ bolus IV $+0.05 \mathrm{mg} / \mathrm{kg} / \mathrm{h}$ infusion IV during surgery and for $72 \mathrm{~h}$ postoperatively

Pain intensity (VAS) reported every $6 \mathrm{~h}$ for 3 days postoperatively. Cumulative analgesic requirement reported at $72 \mathrm{~h}$. AEs

\begin{tabular}{|c|c|c|}
\hline Surgery type & Thoracotomy & \\
\hline $\begin{array}{l}\text { Group numbers after end } \\
\text { of study (treatment/con- } \\
\text { trol) }\end{array}$ & $30 / 30$ & \\
\hline \multirow{2}{*}{$\begin{array}{l}\text { Age of patient population } \\
\text { (treatment/control) }\end{array}$} & $57.3 \pm 11.9$ & \\
\hline & $56.9 \pm 12.5$ & \\
\hline Notes & \multicolumn{2}{|c|}{ No financial support or funding and no conflict of interest } \\
\hline \multicolumn{3}{|l|}{ Risk of bias } \\
\hline Bias & Authors' judgement & Support for judgement \\
\hline $\begin{array}{l}\text { Random sequence genera- } \\
\text { tion (selection bias) }\end{array}$ & Low risk & Random number table \\
\hline $\begin{array}{l}\text { Allocation concealment } \\
\text { (selection bias) }\end{array}$ & Low risk & $\begin{array}{l}\text { Central allocation by the hospital pharmacy (a third party). Study drugs were } \\
\text { prepared in identical containers and marked with the name of the study and a } \\
\text { consecutive number }\end{array}$ \\
\hline $\begin{array}{l}\text { Blinding of participants } \\
\text { and personnel (perfor- } \\
\text { mance bias) } \\
\text { All outcomes }\end{array}$ & Low risk & Blinded personnel \\
\hline $\begin{array}{l}\text { Blinding of outcome as- } \\
\text { sessment (detection bias) } \\
\text { All outcomes }\end{array}$ & Low risk & Blinded personnel \\
\hline $\begin{array}{l}\text { Incomplete outcome data } \\
\text { (attrition bias) } \\
\text { All outcomes }\end{array}$ & Low risk & No withdrawals \\
\hline $\begin{array}{l}\text { Selective reporting (re- } \\
\text { porting bias) }\end{array}$ & Low risk & Predefined outcomes reported \\
\hline Size & High risk & 30 participants per treatment arm \\
\hline
\end{tabular}

\section{Yeom 2012}

\section{Study characteristics}

\begin{tabular}{ll}
\hline Methods & Randomised, double-blind. Ketamine + fentanyl vs fentanyl \\
\hline Participants & $\mathrm{N}=40,70 \%$ women \\
\hline
\end{tabular}


Yeom 2012 (Continued)

\begin{tabular}{|c|c|c|}
\hline Interventions & \multicolumn{2}{|c|}{ Ketamine $0.2 \mathrm{mg} / \mathrm{kg}$ bolus IV $+30 \mu \mathrm{g} / \mathrm{mL} / \mathrm{kg}$ infusion IV intraoperatively } \\
\hline Outcomes & \multicolumn{2}{|c|}{$\begin{array}{l}\text { Pain intensity (NRS). AEs. Ketamine and fentanyl infusion rates. Outcomes recorded at 1, } 24 \text { and } 48 \text { h af- } \\
\text { ter surgery }\end{array}$} \\
\hline Surgery type & \multicolumn{2}{|l|}{ Lumbar spinal fusion } \\
\hline $\begin{array}{l}\text { Group numbers after end } \\
\text { of study (treatment/con- } \\
\text { trol) }\end{array}$ & \multicolumn{2}{|l|}{$20 / 20$} \\
\hline $\begin{array}{l}\text { Age of patient population } \\
\text { (treatment/control) }\end{array}$ & \multicolumn{2}{|l|}{$61 \pm 10$} \\
\hline Notes & \multicolumn{2}{|c|}{ No mention of sponsorship or funding } \\
\hline \multicolumn{3}{|l|}{ Risk of bias } \\
\hline Bias & Authors' judgement & Support for judgement \\
\hline $\begin{array}{l}\text { Random sequence genera- } \\
\text { tion (selection bias) }\end{array}$ & Unclear risk & Not described \\
\hline $\begin{array}{l}\text { Allocation concealment } \\
\text { (selection bias) }\end{array}$ & Unclear risk & Allocation concealment method not described in detail. \\
\hline $\begin{array}{l}\text { Blinding of participants } \\
\text { and personnel (perfor- } \\
\text { mance bias) } \\
\text { All outcomes }\end{array}$ & Low risk & Blinded personnel prepared study drugs \\
\hline $\begin{array}{l}\text { Blinding of outcome as- } \\
\text { sessment (detection bias) } \\
\text { All outcomes }\end{array}$ & Low risk & Blinded personnel assessed outcomes \\
\hline $\begin{array}{l}\text { Incomplete outcome data } \\
\text { (attrition bias) } \\
\text { All outcomes }\end{array}$ & Low risk & No withdrawals \\
\hline $\begin{array}{l}\text { Selective reporting (re- } \\
\text { porting bias) }\end{array}$ & High risk & Not all AEs are reported that were predefined in methods \\
\hline Size & High risk & 20 participants per treatment arm \\
\hline
\end{tabular}

Ysasi 2010

\section{Study characteristics}

\begin{tabular}{ll}
\hline Methods & Randomised, double-blind, placebo control \\
\hline Participants & $\mathrm{N}=60$, about $30 \%$ women \\
\hline Interventions & Ketamine infusion $8 \mu \mathrm{g} / \mathrm{kg} / \mathrm{min}$ IV during surgery \\
\hline
\end{tabular}


Ysasi 2010 (Continued)

Outcomes Pain intensity (VAS, SVS) reported at 15 and 30 mins, then 1, 2, 4, 6, 8, 12 and $24 \mathrm{~h}$ postoperatively. Analgesic consumption reported at $24 \mathrm{~h}$ postoperatively. AEs

\begin{tabular}{|c|c|c|}
\hline Surgery type & \multicolumn{2}{|c|}{ Myocardial revascularisation } \\
\hline $\begin{array}{l}\text { Group numbers after end } \\
\text { of study (treatment/con- } \\
\text { trol) }\end{array}$ & \multicolumn{2}{|l|}{$30 / 30$} \\
\hline \multirow{2}{*}{$\begin{array}{l}\text { Age of patient population } \\
\text { (treatment/control) }\end{array}$} & \multicolumn{2}{|l|}{$62.2 \pm 10$} \\
\hline & \multicolumn{2}{|l|}{$63.3 \pm 9.6$} \\
\hline Notes & \multicolumn{2}{|c|}{ Article in Spanish. No mention of sponsorship or funding } \\
\hline \multicolumn{3}{|l|}{ Risk of bias } \\
\hline Bias & Authors' judgement & Support for judgement \\
\hline $\begin{array}{l}\text { Random sequence genera- } \\
\text { tion (selection bias) }\end{array}$ & Unclear risk & Randomisation process not described \\
\hline $\begin{array}{l}\text { Allocation concealment } \\
\text { (selection bias) }\end{array}$ & Unclear risk & Allocation concealment method not described \\
\hline $\begin{array}{l}\text { Blinding of participants } \\
\text { and personnel (perfor- } \\
\text { mance bias) } \\
\text { All outcomes }\end{array}$ & Low risk & Blinded participants and personnel \\
\hline $\begin{array}{l}\text { Blinding of outcome as- } \\
\text { sessment (detection bias) } \\
\text { All outcomes }\end{array}$ & Low risk & Blinded personnel \\
\hline $\begin{array}{l}\text { Incomplete outcome data } \\
\text { (attrition bias) } \\
\text { All outcomes }\end{array}$ & Low risk & No withdrawals \\
\hline $\begin{array}{l}\text { Selective reporting (re- } \\
\text { porting bias) }\end{array}$ & Low risk & Predefined outcomes reported \\
\hline Size & High risk & 30 participants per treatment arm \\
\hline
\end{tabular}

\section{Zakine 2008}

\section{Study characteristics}

\begin{tabular}{ll}
\hline Methods & Randomised, double-blind, placebo control \\
\hline Participants & $\mathrm{N}=81,23 \%$ women \\
\hline Interventions & $\begin{array}{l}\text { 1. Pre-incisional ketamine } 0.5 \mathrm{mg} / \mathrm{kg} \text { bolus IV }+2 \mu \mathrm{g} / \mathrm{kg} / \mathrm{min} \text { infusion IV during surgery } \\
\text { 2. Pre-incisional ketamine } 0.5 \mathrm{mg} / \mathrm{kg} \text { bolus IV }+2 \mu \mathrm{gg} / \mathrm{kg} / \mathrm{min} \text { infusion IV during surgery and for } 48 \mathrm{~h} \text { post- } \\
\text { operatively }\end{array}$
\end{tabular}


Zakine 2008 (Continued)

Heterogeneous procedures

\begin{tabular}{ll}
\hline Outcomes & $\begin{array}{l}\text { Pain intensity (VAS). Analgesic consumption. AEs. Pain outcomes recorded at 4, 24 and 48 h after } \\
\text { surgery }\end{array}$ \\
\hline Surgery type & Major abdominal, urologic or vascular surgery \\
\hline $\begin{array}{l}\text { Group numbers after end } \\
\text { of study (treatment/con- } \\
\text { trol) }\end{array}$ & $50 / 27$ \\
\hline
\end{tabular}

Age of patient population median (interquartile range)

(treatment/control)
$63(12)$
$62(14)$

Notes Various procedures. Supported by a grant from the French Ministry of Health

\section{Risk of bias}

\begin{tabular}{lll}
\hline Bias & Authors' judgement & Support for judgement \\
\hline $\begin{array}{l}\text { Random sequence genera- } \\
\text { tion (selection bias) }\end{array}$ & Low risk & Computer-generated randomisation \\
\hline $\begin{array}{l}\text { Allocation concealment } \\
\text { (selection bias) }\end{array}$ & Low risk & $\begin{array}{l}\text { Quote: "Patients were randomized by means of computer-generated opaque } \\
\text { envelopes containing the patient number and group assignment." }\end{array}$ \\
\hline $\begin{array}{l}\text { Blinding of participants } \\
\text { and personnel (perfor- } \\
\text { mance bias) }\end{array}$ & Unclear risk & Mentioned "double-blind" but blinding not described in detail \\
$\begin{array}{l}\text { All outcomes } \\
\text { Blinding of outcome as- } \\
\text { sessment (detection bias) } \\
\text { All outcomes }\end{array}$ & Unclear risk & Mentioned "double-blind" but blinding not described in detail \\
\hline $\begin{array}{l}\text { Incomplete outcome data } \\
\text { (attrition bias) } \\
\text { All outcomes }\end{array}$ & Low risk & $5 \%$ was withdrawn \\
\hline $\begin{array}{l}\text { Selective reporting (re- } \\
\text { porting bias) }\end{array}$ & Low risk & 27,23 and 27 participants per treatment arm \\
\hline \begin{tabular}{l} 
Size \\
\hline
\end{tabular} & High risk & Predefined outcomes adequately reported \\
\hline
\end{tabular}

AEs: adverse events;ED: epidural, ICDSC: intensive care delirium screening checklist, h: hour(s), ICU: intensive care unit, IV: intravenous, mcg: micrograms, mg: milligrams, min: minutes, kg: kilograms, N: number of participants, NPRS: numeric pain rating scale, NPSI: neuropathic pain symptom inventory, NRS: numerical rating scale, PACU: post-anaesthesia care unit, PCA: patient-controlled analgesia, PCEA: patient-controlled epidural analgesia, PONV: post-operative nausea and vomiting; PTPS: post-thoracotomy pain syndrome; SC: subcutaneous; VAS: visual analogue scale, VNS: verbal numeric scale; VRS: verbal rating scale

Characteristics of excluded studies [ordered by study ID] 


\begin{tabular}{|c|c|}
\hline Study & Reason for exclusion \\
\hline Abrishamkar 2012 & Open-label study \\
\hline Adams 2003 & Open-label study \\
\hline Aghamohammadi 2012 & Inappropriate method - study compared 3 different active treatment regimens, with no placebo \\
\hline Akca 2016 & $\begin{array}{l}\text { Inappropriate method - pain measured but not reported, except as a binary outcome at short post- } \\
\text { operative time scales }\end{array}$ \\
\hline Avidan 2017 & Inappropriate method - different anaesthesia techniques \\
\hline Behdad 2011 & Open-label study \\
\hline Bentley 2005 & Inappropriate method - number of participants in each study group was not reported \\
\hline Bilgin 2005 & Inappropriate method - all participants treated with ketamine \\
\hline Clausen 1975 & Inappropriate pain scale (measurement) \\
\hline Edwards 1993 & Number of participants who completed the study fewer than 10 \\
\hline Gillies 2007 & Inappropriate method - mixed population of adults and children \\
\hline Guan 2008 & Number of participants who completed the study fewer than 10 \\
\hline Heinke 1999 & $\begin{array}{l}\text { Inappropriate method - the primary outcome was PCA consumption of piritramide, but fixed maxi- } \\
\text { mum dose of piritramide limited the utility of the study to detect differences }\end{array}$ \\
\hline Hong 2011 & Open-label study \\
\hline Ito 1974 & Open-label study \\
\hline Jahangir 1993 & $\begin{array}{l}\text { Inappropriate method - time with study drug infusion varied, and so groups may not have been } \\
\text { comparable in ketamine dose }\end{array}$ \\
\hline Jensen 2008 & Inappropriate method - no general anaesthesia \\
\hline Jiang 2016 & Pain scores were not reported in any detail (measurement) \\
\hline Joachimmson 1986 & $\begin{array}{l}\text { Inappropriate pain scale. Participants on ventilator and probably not able to communicate easily } \\
\text { (measurement) }\end{array}$ \\
\hline Kadic 2016 & $\begin{array}{l}\text { Inappropriate method - participants in the same study group received ketamine and pregabalin so } \\
\text { effects of ketamine could not be identified }\end{array}$ \\
\hline Kim 2001 & Inappropriate description of methods - not described and may not be blinded \\
\hline Kim 2005 & Open-label study \\
\hline Kollender 2008 & Open-label study \\
\hline Kose 2008 & No pain or analgesic consumption outcome reported (measurement) \\
\hline Launo 2004 & Inappropriate method - ketamine was compared to tramadol with no placebo \\
\hline
\end{tabular}




\begin{tabular}{|c|c|}
\hline Study & Reason for exclusion \\
\hline Lee 2005 & Open-label study \\
\hline Lee 2006 & Inappropriate description of methods - not described and may not be blinded \\
\hline Lee 2013 & Open-label study \\
\hline Lee 2014 & Open-label study \\
\hline Liang 2006 & Inappropriate description of methods - not described and may not be blinded \\
\hline Lux 2009 & Inappropriate randomisation - participants divided sequentially into 2 groups \\
\hline Malek 2006 & Inappropriate method - outcome is chronic pain \\
\hline Maurset 1989 & Number of participants who completed the study fewer than 10 \\
\hline Nayar 2009 & Open-label study \\
\hline Ndoye 2008 & Not an RCT \\
\hline Nesher 2008 & $\begin{array}{l}\text { Inappropriate randomisation. Participants assigned to } 1 \text { of } 2 \text { groups according to their national ID- } \\
\text { number }\end{array}$ \\
\hline Nesher 2009 & Inappropriate randomisation. Randomisation according to the national ID-number \\
\hline Nikolayev 2008 & Open-label study \\
\hline Nitta 2013 & Open-label study \\
\hline Nourozi 2010 & Inappropriate method - mixed population of adults and children \\
\hline Oliveira 2005 & Inappropriate method - all participants treated with ketamine \\
\hline Owen 1987 & Inappropriate method - not placebo-controlled \\
\hline Park 2004 & Methods not described and may not be blinded \\
\hline Perrin 2009 & Number of participants in group who completed the study fewer than 10 \\
\hline Reeves 2001 & $\begin{array}{l}\text { Inappropriate method - different PCA settings for different participants, so non-standardised treat- } \\
\text { ment regimens }\end{array}$ \\
\hline Sadove 1971 & Inappropriate method - not IV ketamine administration \\
\hline Sollazzi 2008 & Open-label study \\
\hline Song 2004 & Methods not described and may not be blinded \\
\hline Sveticic 2008 & $\begin{array}{l}\text { Inappropriate method - different anaesthesia techniques (general anaesthesia, regional anaesthe- } \\
\text { sia or combined) }\end{array}$ \\
\hline Talu 2002 & Inappropriate method - not IV \\
\hline Thomas 2012 & Open-label study \\
\hline
\end{tabular}




\begin{tabular}{ll}
\hline Study & Reason for exclusion \\
\hline Tverskoy 1994 & Number of participants in group who completed the study fewer than 10 \\
\hline Tverskoy 1996 & Number of participants in group who completed the study fewer than 10 \\
\hline Ünlügenc 2002 & Inappropriate method - different PCA settings for different participants \\
\hline Urban 2008 & Open-label study \\
\hline Weinbroum 2003 & $\begin{array}{l}\text { Inappropriate randomisation - participants allocated into } 1 \text { of the 2 treatment protocols on alter- } \\
\text { nate days }\end{array}$ \\
\hline Wilder-Smith 1998 & $\begin{array}{l}\text { Inappropriate method - study compared 3 different treatment regimens without a placebo com- } \\
\text { parator group }\end{array}$ \\
\hline Xie 2003 & Inappropriate method - placebo administered epidurally, so no comparison group for IV ketamine \\
\hline Xu 2017 & Inappropriate method - no general anaesthesia \\
\hline
\end{tabular}

ID-number: identification number, IV: intravenous; PCA: patient-controlled analgesia; RCT: randomised controlled trial

Characteristics of studies awaiting classification [ordered by study ID]

Lee 2018

\begin{tabular}{ll}
\hline Methods & A prospective, randomised, double-blind, placebo-controlled study \\
\hline Participants & $\mathrm{N}=64$, robotic thyroidectomy \\
\hline Interventions & $\begin{array}{l}\text { Pre-incisional ketamine } 0.15 \mathrm{mg} / \mathrm{kg} \mathrm{IV} \mathrm{followed} \mathrm{by} \mathrm{a} \mathrm{continuous} \mathrm{infusion} 2 \mathrm{mcg} / \mathrm{kg} / \mathrm{min} \text { until the } \\
\text { end of procedure }\end{array}$ \\
\hline Outcomes & $\begin{array}{l}\text { Primary endpoint: pain intensity (VAS) at } 6 \text { h postoperatively. Secondary outcomes: pain intensity } \\
\text { (VAS) at } 0,1,24 \text { and } 48 \text { h and at } 3 \text { months postoperatively at rest and while coughing. Incidence of } \\
\text { hypoesthesia, time administration of the first analgesic, number of participants requiring addition- } \\
\text { al analgesics, complications related to opioids or ketamine }\end{array}$ \\
\hline Notes & $\begin{array}{l}\text { Results for pain outcomes are provided as median (IQR) thus cannot be used in meta-analysis. AEs } \\
\text { are reported as N (\%) }\end{array}$ \\
\hline
\end{tabular}

Lou 2017

\begin{tabular}{ll}
\hline Methods & Prospective, randomised study \\
\hline Participants & $\mathrm{N}=66$. Mastectomy \\
\hline Interventions & Ketamine $0.5 \mathrm{mg} / \mathrm{kg}$ infused in $1 \mathrm{~h}$ daily for 7 days vs saline $(\mathrm{NaCl} 0.9 \%)$ \\
\hline Outcomes & $\begin{array}{l}\text { Postoperative pain (VAS) during PACU, } 4 \mathrm{~h}, 24 \mathrm{~h} \text { and } 2-5 \text { days after surgery. Analgesic requirement } \\
\text { at same time points. Hospital Anxiety and Depression Scale (HADS) } 5 \text { days after surgery. Incidence } \\
\text { of postmastectomy pain syndrome, pain site and HADS at } 3 \text { and } 6 \text { months after surgery }\end{array}$ \\
\hline Notes & Original article is in Chinese. Only abstract available \\
\hline
\end{tabular}


Moon 2018

\begin{tabular}{ll}
\hline Methods & Not known. \\
\hline Participants & $\mathrm{N}=46$. Laparoscopic hysterectomy \\
\hline Interventions & $\begin{array}{l}\text { Pre-incisional ketamine } 1 \mathrm{mg} / \mathrm{kg} \text { IV followed by a continuous infusion } 0.5 \mathrm{mg} / \mathrm{kg} / \mathrm{h} \text { vs saline ( } \mathrm{NaCl} \\
0.9 \%)\end{array}$ \\
\hline Outcomes & Primary outcome: mechanical pain threshold evaluating hyperalgesia. \\
& $\begin{array}{l}\text { Secondary outcomes: postoperative pain (VAS). Analgesic and antiemetic consumption. Incidence } \\
\text { of dizzess }\end{array}$ \\
\hline
\end{tabular}

AEs: adverse events; IQR: interquartile range,h: hour/s, IV: intravenous, kg: kilograms, mcg: micrograms, $\mathbf{m g : ~ m i l l i g r a m s , ~} \mathbf{m i n}: \mathbf{m i n u t e s ,}$ $\mathbf{N}$ : number of participants, PACU: post-anaesthesia care unit; VAS: visual analogue scale

\section{DATA AND ANALYSES}

\section{Comparison 1. Perioperative ketamine versus control in a non-stratified study population}

\begin{tabular}{|c|c|c|c|c|}
\hline Outcome or subgroup title & No. of studies & $\begin{array}{l}\text { No. of partici- } \\
\text { pants }\end{array}$ & Statistical method & Effect size \\
\hline $\begin{array}{l}1.1 \text { Opioid consumption at } 24 \\
\text { hours }\end{array}$ & 65 & 4004 & $\begin{array}{l}\text { Mean Difference (IV, Random, } \\
95 \% \mathrm{CI} \text { ) }\end{array}$ & $-7.63[-8.88,-6.39]$ \\
\hline $\begin{array}{l}1.2 \text { Opioid consumption at } 48 \\
\text { hours }\end{array}$ & 37 & 2449 & $\begin{array}{l}\text { Mean Difference (IV, Random, } \\
95 \% \mathrm{CI} \text { ) }\end{array}$ & $\begin{array}{l}-12.62[-15.06 \\
-10.18]\end{array}$ \\
\hline $\begin{array}{l}1.3 \text { Pain intensity at rest at } 24 \\
\text { hours }\end{array}$ & 82 & 5004 & $\begin{array}{l}\text { Mean Difference (IV, Random, } \\
95 \% \mathrm{CI} \text { ) }\end{array}$ & $-5.09[-6.55,-3.64]$ \\
\hline $\begin{array}{l}\text { 1.4 Pain intensity during move- } \\
\text { ment at } 24 \text { hours }\end{array}$ & 29 & 1806 & $\begin{array}{l}\text { Mean Difference (IV, Random, } \\
95 \% \mathrm{CI} \text { ) }\end{array}$ & $-5.60[-10.72,-0.48]$ \\
\hline $\begin{array}{l}1.5 \text { Pain intensity at rest at } 48 \\
\text { hours }\end{array}$ & 49 & 2962 & $\begin{array}{l}\text { Mean Difference (IV, Random, } \\
95 \% \mathrm{CI} \text { ) }\end{array}$ & $-5.03[-6.65,-3.40]$ \\
\hline $\begin{array}{l}\text { 1.6 Pain intensity during move- } \\
\text { ment at } 48 \text { hours }\end{array}$ & 23 & 1353 & $\begin{array}{l}\text { Mean Difference (IV, Random, } \\
95 \% \mathrm{CI} \text { ) }\end{array}$ & $-5.72[-10.15,-1.29]$ \\
\hline $\begin{array}{l}\text { 1.7 Time to first request for analge- } \\
\text { sia/trigger of PCA }\end{array}$ & 31 & 1678 & $\begin{array}{l}\text { Mean Difference (IV, Random, } \\
95 \% \mathrm{CI})\end{array}$ & $53.89[37.00,70.78]$ \\
\hline 1.8 CNS adverse events - all studies & 105 & 6538 & $\begin{array}{l}\text { Risk Ratio (M-H, Random, 95\% } \\
\text { Cl) }\end{array}$ & $1.17[0.95,1.43]$ \\
\hline 1.9 Hyperalgesia & 7 & 333 & $\begin{array}{l}\text { Mean Difference (IV, Random, } \\
95 \% \mathrm{Cl} \text { ) }\end{array}$ & $-7.08[-11.92,-2.23]$ \\
\hline
\end{tabular}




\begin{tabular}{lllll}
\hline Outcome or subgroup title & No. of studies & $\begin{array}{l}\text { No. of partici- } \\
\text { pants }\end{array}$ & Statistical method & Effect size \\
\hline $\begin{array}{l}\text { 1.10 CNS adverse events - studies } \\
\text { with events }\end{array}$ & 52 & 3706 & $\begin{array}{l}\text { Risk Ratio (M-H, Random, 95\% } \\
\text { Cl) }\end{array}$ & $1.17[0.95,1.43]$ \\
\hline $\begin{array}{l}\text { 1.11 Postoperative nausea and } \\
\text { vomiting - all studies }\end{array}$ & 95 & 5965 & $\begin{array}{l}\text { Risk Ratio (M-H, Random, 95\% } \\
\text { Cl) }\end{array}$ & $0.88[0.81,0.96]$ \\
\hline
\end{tabular}


Analysis 1.1. Comparison 1: Perioperative ketamine versus control in a non-stratified study population, Outcome 1: Opioid consumption at 24 hours

\begin{tabular}{|c|c|c|c|c|c|c|c|c|c|c|}
\hline \multirow{2}{*}{$\begin{array}{l}\text { Study or Subgroup } \\
\text { Adriaenssens } 1999\end{array}$} & \multicolumn{3}{|c|}{ Ketamine } & \multicolumn{3}{|c|}{ Control } & Weight & $\begin{array}{c}\text { Mean Difference } \\
\text { IV, Random, 95\% CI }\end{array}$ & \multicolumn{2}{|c|}{$\begin{array}{c}\text { Mean Difference } \\
\text { IV, Random, 95\% CI }\end{array}$} \\
\hline & 19.4 & 10.7 & 15 & 30.7 & 15.9 & 15 & $1.0 \%$ & $-11.30[-21.00,-1.60]$ & & \\
\hline Argiriadou 2011 & 12.2 & 2.8 & 27 & 21 & 5 & 26 & $2.3 \%$ & $-8.80[-10.99,-6.61]$ & $\rightarrow$ & \\
\hline Aubrun 2008 & 24.8 & 19.2 & 45 & 17.8 & 16.4 & 45 & $1.3 \%$ & $7.00[-0.38,14.38]$ & & \\
\hline Aveline 2006 & 21.8 & 9.2 & 45 & 33.9 & 5.4 & 23 & $2.1 \%$ & $-12.10[-15.58,-8.62]$ & - & \\
\hline Aveline 2009 & 39.2 & 6.5 & 25 & 56.8 & 5.9 & 24 & $2.1 \%$ & $-17.60[-21.07,-14.13]$ & & \\
\hline Ayoglu 2005 & 19.7 & 1.9 & 20 & 23.5 & 2.3 & 20 & $2.4 \%$ & $-3.80[-5.11,-2.49]$ & - & \\
\hline Barreveld 2013 & 218 & 147 & 29 & 231 & 168 & 30 & $0.0 \%$ & $-13.00[-93.48,67.48]$ & $\longleftarrow$ & \\
\hline Bilgen 2012 & 44 & 19 & 35 & 28 & 14 & 16 & $1.0 \%$ & $16.00[6.69,25.31]$ & & \\
\hline Bilgen 2012 & 42.5 & 15.1 & 70 & 28 & 14 & 18 & $1.3 \%$ & $14.50[7.13,21.87]$ & & \\
\hline Cenzig 2014 & 47 & 15.3 & 30 & 85.2 & 8.01 & 30 & $1.5 \%$ & $-38.20[-44.38,-32.02]$ & 4 & \\
\hline Crousier 2008 & 13 & 6.6 & 12 & 16.17 & 26 & 18 & $0.7 \%$ & $-3.17[-15.75,9.41]$ & & \\
\hline Dahi-Taleghani 2014 & 7 & 2 & 70 & 12 & 3 & 70 & $2.5 \%$ & $-5.00[-5.84,-4.16]$ & $=$ & \\
\hline Dahl 2000 & 19.2 & 6.3 & 29 & 20.4 & 8 & 60 & $2.2 \%$ & $-1.20[-4.26,1.86]$ & & \\
\hline Dualé 2009 & 39 & 23 & 39 & 48 & 28 & 41 & $0.8 \%$ & $-9.00[-20.21,2.21]$ & & \\
\hline Dullenkopf 2009 & 8.8 & 9 & 77 & 10.3 & 6.8 & 33 & $2.2 \%$ & $-1.50[-4.57,1.57]$ & - & \\
\hline Fiorelli 2015 & 18 & 0.4 & 38 & 22.5 & 0.3 & 37 & $2.5 \%$ & $-4.50[-4.66,-4.34]$ & - & \\
\hline Ganne 2005 & 33.3 & 14.9 & 30 & 31.9 & 15.3 & 31 & $1.3 \%$ & $1.40[-6.18,8.98]$ & & \\
\hline Garcia-Navia 2016 & 30.95 & 7.88 & 11 & 27.54 & 11.75 & 11 & $1.2 \%$ & $3.41[-4.95,11.77]$ & & \\
\hline Garg 2016 & 2.45 & 2.1 & 22 & 15.64 & 9.3 & 22 & $2.0 \%$ & $-13.19[-17.17,-9.21]$ & & \\
\hline Gilabert Morell 2002 & 11.4 & 6.7 & 44 & 15.63 & 9.31 & 22 & $1.9 \%$ & $-4.23[-8.60,0.14]$ & & \\
\hline Guignard 2002 & 42.7 & 16.3 & 25 & 64.9 & 27 & 25 & $0.7 \%$ & $-22.20[-34.56,-9.84]$ & $\longleftarrow$ & \\
\hline Guillou 2003 & 36 & 20 & 41 & 48 & 20 & 52 & $1.2 \%$ & $-12.00[-20.19,-3.81]$ & & \\
\hline Hadi 2010 & 35.2 & 8.9 & 30 & 60 & 2.6 & 15 & $2.1 \%$ & $-24.80[-28.25,-21.35]$ & 4 & \\
\hline Hadi 2013 & 35.2 & 8.9 & 30 & 60 & 2.6 & 15 & $2.1 \%$ & $-24.80[-28.25,-21.35]$ & $\leftarrow$ & \\
\hline Haliloglu 2015 & 25 & 3.7 & 26 & 36.4 & 3.6 & 26 & $2.3 \%$ & $-11.40[-13.38,-9.42]$ & 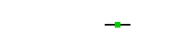 & \\
\hline Hasanein 2011 & 40 & 6.1 & 30 & 47.4 & 8 & 30 & $2.1 \%$ & $-7.40[-11.00,-3.80]$ & 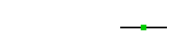 & \\
\hline Helmy 2015 & 8.2 & 3.3 & 19 & 14 & 3.8 & 20 & $2.3 \%$ & $-5.80[-8.03,-3.57]$ & - & \\
\hline Hercock 1999 & 34.5 & 15.2 & 24 & 33 & 16 & 25 & $1.1 \%$ & $1.50[-7.24,10.24]$ & & \\
\hline Ilkjaer 1998 & 28 & 21 & 30 & 36 & 23 & 30 & $0.8 \%$ & $-8.00[-19.14,3.14]$ & & \\
\hline Jaksch 2002 & 40.2 & 17 & 15 & 44.1 & 45 & 15 & $0.2 \%$ & $-3.90[-28.24,20.44]$ & $\leftarrow$ & \\
\hline Javery 1996 & 25.82 & 16.4 & 22 & 51.1 & 20.8 & 20 & $0.8 \%$ & $-25.28[-36.68,-13.88]$ & 4 & \\
\hline Jendoubi 2017 & 10.7 & 2.3 & 20 & 15.9 & 1.7 & 20 & $2.4 \%$ & $-5.20[-6.45,-3.95]$ & - & \\
\hline Kafali 2004 & 44.2 & 4.1 & 30 & 53.9 & 4 & 30 & $2.3 \%$ & $-9.70[-11.75,-7.65]$ & - & \\
\hline Kamal 2008 & 33.3 & 7.4 & 40 & 46.9 & 7.4 & 40 & $2.1 \%$ & $-13.60[-16.84,-10.36]$ & & \\
\hline Karaman 2006 & 13.8 & 4.1 & 40 & 14.6 & 4.1 & 20 & $2.3 \%$ & $-0.80[-3.00,1.40]$ & & \\
\hline Katz 2004 & 51.9 & 33.3 & 97 & 52.3 & 32.8 & 46 & $0.8 \%$ & $-0.40[-11.97,11.17]$ & & \\
\hline Kwon 2009 & 13.3 & 4.5 & 20 & 16.9 & 11.5 & 20 & $1.7 \%$ & $-3.60[-9.01,1.81]$ & & \\
\hline Leal 2013 & 29 & 18.4 & 20 & 25.1 & 13.3 & 20 & $1.0 \%$ & $3.90[-6.05,13.85]$ & & \\
\hline Leal 2015 & 27.4 & 18.3 & 28 & 27.7 & 12.9 & 28 & $1.2 \%$ & $-0.30[-8.59,7.99]$ & & \\
\hline Lehmann 2001 & 17.5 & 11.3 & 40 & 20.7 & 14.3 & 40 & $1.6 \%$ & $-3.20[-8.85,2.45]$ & & \\
\hline Lin 2016 & 5.42 & 1.64 & 29 & 5.25 & 1.45 & 29 & $2.5 \%$ & $0.17[-0.63,0.97]$ & & \\
\hline Loftus 2010 & 142 & 82 & 52 & 202 & 176 & 50 & $0.1 \%$ & $-60.00[-113.63,-6.37]$ & $\leftarrow$ & \\
\hline Mahran 2015 & 23.1 & 6.91 & 30 & 34.43 & 10.14 & 30 & $1.9 \%$ & $-11.33[-15.72,-6.94]$ & & \\
\hline Menigaux 2000 & 26.2 & 17.9 & 30 & 49.7 & 24.2 & 15 & $0.6 \%$ & $-23.50[-37.32,-9.68]$ & $\vdash$ & \\
\hline Michelet 2007 & 25.4 & 12.8 & 24 & 30.4 & 10.2 & 24 & $1.5 \%$ & $-5.00[-11.55,1.55]$ & & \\
\hline Murdoch 2002 & 67.6 & 25.1 & 21 & 66.4 & 17.7 & 21 & $0.7 \%$ & $1.20[-11.94,14.34]$ & & \\
\hline Nielsen 2017 & 79 & 47 & 74 & 121 & 53 & 73 & $0.5 \%$ & $-42.00[-58.20,-25.80]$ & 4 & \\
\hline Ögün 2001 & 10.6 & 4.6 & 16 & 16.3 & 3.6 & 15 & $2.2 \%$ & $-5.70[-8.60,-2.80]$ & & \\
\hline Parikh 2011 & 5.8 & 1.48 & 30 & 18.1 & 1.8 & 30 & $2.5 \%$ & $-12.30[-13.13,-11.47]$ & $=$ & \\
\hline Remérand 2009 & 14 & 13 & 79 & 19 & 12 & 75 & $2.0 \%$ & $-5.00[-8.95,-1.05]$ & & \\
\hline Reza 2010 & 8 & 4.7 & 30 & 11.2 & 6.5 & 30 & $2.2 \%$ & $-3.20[-6.07,-0.33]$ & & \\
\hline Roytblat 1993 & 29.5 & 7.5 & 11 & 48.7 & 13 & 11 & $1.1 \%$ & $-19.20[-28.07,-10.33]$ & $\leftarrow$ & \\
\hline Safavi 2011 & 2.33 & 0.6 & 30 & 15.1 & 5.3 & 30 & $2.3 \%$ & $-12.77[-14.68,-10.86]$ & - & \\
\hline Sahin 2004 & 20.28 & 11.81 & 17 & 17.26 & 11.87 & 16 & $1.2 \%$ & $3.02[-5.06,11.10]$ & & \\
\hline Sen 2009 & 28 & 8 & 20 & 48 & 17 & 20 & $1.2 \%$ & $-20.00[-28.23,-11.77]$ & $\longleftarrow$ & \\
\hline Snijdelaar 2004 & 32.15 & 18.59 & 13 & 50.42 & 24.7 & 12 & $0.4 \%$ & $-18.27[-35.52,-1.02]$ & $\leftarrow$ & \\
\hline Song 2013 & 39.9 & 14.7 & 24 & 50.5 & 23.2 & 25 & $0.9 \%$ & $-10.60[-21.43,0.23]$ & & \\
\hline Song 2014 & 23.23 & 0.76 & 25 & 24.04 & 0.92 & 25 & $2.5 \%$ & $-0.81[-1.28,-0.34]$ & & \\
\hline Stubhaug 1997 & 64.5 & 22.6 & 10 & 68 & 30 & 10 & $0.3 \%$ & $-3.50[-26.78,19.78]$ & & \\
\hline Subramaniam 2011 & 103.6 & 87.8 & 15 & 96.8 & 67.85 & 15 & $0.0 \%$ & $6.80[-49.35,62.95]$ & & \\
\hline$\ddot{~}$ & 165 & 17 & $2 n$ & 10 & 16 & 30 & ว $50 /$ & J Eחז $255 \quad 1651$ & & \\
\hline
\end{tabular}




\section{Analysis 1.1. (Continued)}

\begin{tabular}{|c|c|c|c|c|c|c|}
\hline suuvnaug ıyy/ & 04.5 & $\angle<.0$ & 10 & ов & su & 10 \\
\hline Subramaniam 2011 & 103.6 & 87.8 & 15 & 96.8 & 67.85 & 15 \\
\hline Ünlügenc 2003 & 46.5 & 1.7 & 30 & 49 & 1.6 & 28 \\
\hline Webb 2007 & 25.3 & 23.3 & 52 & 43.3 & 31.9 & 59 \\
\hline Woo 2014 & 14.1 & 3 & 20 & 17.55 & 3.3 & 20 \\
\hline Yalcin 2012 & 35.34 & 13.71 & 26 & 73.03 & 22.41 & 27 \\
\hline Ysasi 2010 & 23.1 & 7.3 & 30 & 24.8 & 7.7 & 30 \\
\hline Zakine 2008 & 22.3 & 15.1 & 50 & 25.3 & 12.6 & 27 \\
\hline
\end{tabular}

Heterogeneity: $\mathrm{Tau}^{2}=16.24 ; \mathrm{Chi}^{2}=1634.83, \mathrm{df}=65(\mathrm{P}<0.00001) ; \mathrm{I}^{2}=96 \%$

Test for overall effect: $\mathrm{Z}=12.02(\mathrm{P}<0.00001)$

Test for subgroup differences: Not applicable

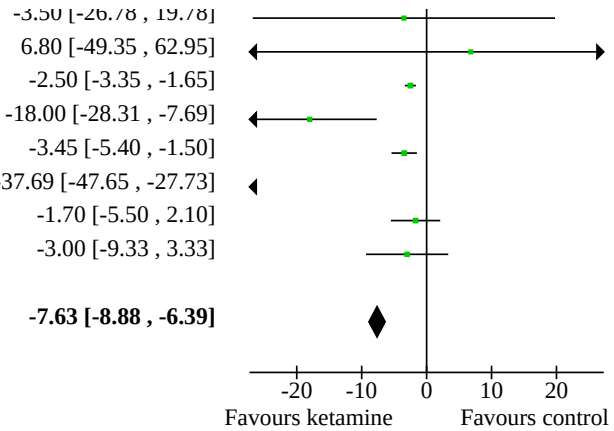

Favours ketamine $\quad$ Favours control Analysis 1.2. Comparison 1: Perioperative ketamine versus control in a non-
stratified study population, Outcome 2: Opioid consumption at 48 hours

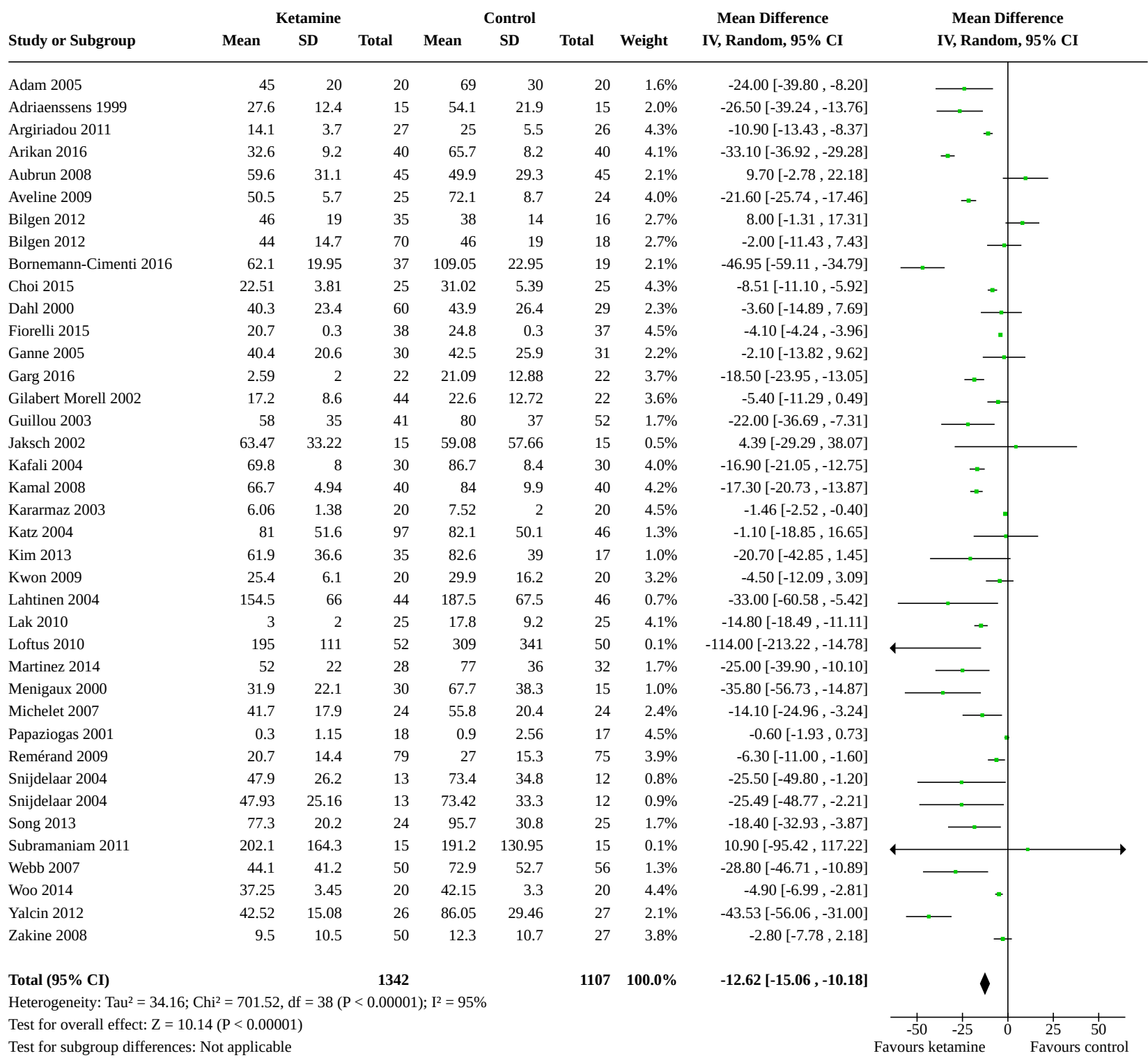


Analysis 1.3. Comparison 1: Perioperative ketamine versus control in a non-stratified study population, Outcome 3: Pain intensity at rest at 24 hours

\begin{tabular}{|c|c|c|c|c|c|c|c|c|c|c|}
\hline \multirow{2}{*}{ Study or Subgroup } & \multicolumn{3}{|c|}{ Ketamine } & \multicolumn{3}{|c|}{ Control } & \multicolumn{2}{|r|}{ Mean Difference } & \multirow{2}{*}{\multicolumn{2}{|c|}{$\begin{array}{c}\text { Mean Difference } \\
\text { IV, Random, 95\% CI }\end{array}$}} \\
\hline & Mean & SD & Total & Mean & SD & Total & Weight & IV, Random, 95\% CI & & \\
\hline Adam 2005 & 28.1 & 9.4 & 20 & 32.5 & 9.4 & 20 & $1.3 \%$ & $-4.40[-10.23,1.43]$ & & \\
\hline Adriaenssens 1999 & 25 & 18 & 15 & 36 & 24 & 15 & $0.6 \%$ & $-11.00[-26.18,4.18]$ & & \\
\hline Argiriadou 2011 & 22.3 & 3.4 & 27 & 39 & 10.2 & 26 & $1.5 \%$ & $-16.70[-20.83,-12.57]$ & - & \\
\hline Arikan 2016 & 27 & 5 & 40 & 31 & 10 & 40 & $1.5 \%$ & $-4.00[-7.46,-0.54]$ & $\rightarrow$ & \\
\hline Aubrun 2008 & 15.2 & 11.8 & 45 & 19.3 & 18.5 & 45 & $1.3 \%$ & $-4.10[-10.51,2.31]$ & & \\
\hline Aveline 2006 & 32.4 & 7.8 & 45 & 37 & 7 & 23 & $1.5 \%$ & $-4.60[-8.26,-0.94]$ & - & \\
\hline Aveline 2009 & 24 & 6 & 25 & 37 & 8 & 24 & $1.5 \%$ & $-13.00[-16.97,-9.03]$ & $\rightarrow$ & \\
\hline Ayoglu 2005 & 3.2 & 1.6 & 20 & 4.8 & 1.6 & 20 & $1.7 \%$ & $-1.60[-2.59,-0.61]$ & & \\
\hline Bornemann-Cimenti 2016 & 17.5 & 7.2 & 37 & 20 & 9 & 19 & $1.4 \%$ & $-2.50[-7.16,2.16]$ & - & \\
\hline Cenzig 2014 & 2 & 4.8 & 30 & 6.3 & 6.1 & 30 & $1.6 \%$ & $-4.30[-7.08,-1.52]$ & $\ldots$ & \\
\hline Chen 2004 & 29.4 & 21.4 & 20 & 28.9 & 20.7 & 20 & $0.7 \%$ & $0.50[-12.55,13.55]$ & & \\
\hline Choi 2015 & 10 & 5 & 25 & 20 & 15 & 25 & $1.3 \%$ & $-10.00[-16.20,-3.80]$ & $\longrightarrow$ & \\
\hline D'Alonzo 2011 & 26 & 22 & 20 & 28 & 21 & 20 & $0.7 \%$ & $-2.00[-15.33,11.33]$ & & \\
\hline Dahi-Taleghani 2014 & 10 & 5 & 70 & 17 & 8 & 70 & $1.6 \%$ & $-7.00[-9.21,-4.79]$ & - & \\
\hline Dahl 2000 & 54.5 & 22 & 60 & 58 & 19 & 29 & $1.0 \%$ & $-3.50[-12.38,5.38]$ & & \\
\hline De Kock 2001 & 18.8 & 9.8 & 40 & 18 & 12.3 & 20 & $1.3 \%$ & $0.80[-5.39,6.99]$ & & - \\
\hline Dualé 2009 & 30 & 27.7 & 39 & 32.5 & 50 & 41 & $0.5 \%$ & $-2.50[-20.10,15.10]$ & & \\
\hline Fiorelli 2015 & 41 & 5 & 38 & 48 & 6 & 37 & $1.6 \%$ & $-7.00[-9.50,-4.50]$ & - & \\
\hline Ganne 2005 & 16.3 & 14.9 & 30 & 14.2 & 10.8 & 31 & $1.3 \%$ & $2.10[-4.45,8.65]$ & & \\
\hline Grady 2012 & 37 & 22 & 30 & 36 & 17 & 32 & $1.0 \%$ & $1.00[-8.83,10.83]$ & & \\
\hline Guillou 2003 & 21.8 & 21.8 & 41 & 24.6 & 18.9 & 52 & $1.1 \%$ & $-2.80[-11.22,5.62]$ & - & - \\
\hline Hadi 2013 & 40 & 7 & 30 & 56 & 5.1 & 15 & $1.5 \%$ & $-16.00[-19.60,-12.40]$ & $\rightarrow$ & \\
\hline Haliloglu 2015 & 4.2 & 5 & 26 & 4.6 & 5.1 & 26 & $1.6 \%$ & $-0.40[-3.15,2.35]$ & & \\
\hline Hercock 1999 & 14 & 12.5 & 24 & 12.8 & 14 & 25 & $1.2 \%$ & $1.20[-6.22,8.62]$ & - & \\
\hline Hu 2014 & 26 & 12 & 31 & 29 & 12 & 47 & $1.4 \%$ & $-3.00[-8.44,2.44]$ & - & \\
\hline Jaksch 2002 & 10 & 12 & 15 & 14 & 13 & 15 & $1.0 \%$ & $-4.00[-12.95,4.95]$ & - & - \\
\hline Javery 1996 & 23 & 16.7 & 22 & 45 & 15.4 & 20 & $1.0 \%$ & $-22.00[-31.71,-12.29]$ & - & \\
\hline Jendoubi 2017 & 22 & 6.3 & 20 & 31.3 & 9.4 & 20 & $1.4 \%$ & $-9.30[-14.26,-4.34]$ & - & \\
\hline Joly 2005 & 40.7 & 19.5 & 24 & 27.7 & 16.3 & 25 & $0.9 \%$ & $13.00[2.92,23.08]$ & & \\
\hline Joseph 2012 & 25 & 29 & 22 & 17.2 & 17.8 & 25 & $0.7 \%$ & $7.80[-6.18,21.78]$ & - & \\
\hline Kafali 2004 & 11.3 & 2.7 & 30 & 17.1 & 2.2 & 30 & $1.7 \%$ & $-5.80[-7.05,-4.55]$ & - & \\
\hline Kakinohana 2004 & 6 & 7 & 25 & 15 & 12 & 25 & $1.4 \%$ & $-9.00[-14.45,-3.55]$ & - & \\
\hline Kamal 2008 & 26 & 2.5 & 40 & 28.5 & 4 & 40 & $1.7 \%$ & $-2.50[-3.96,-1.04]$ & 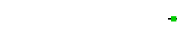 & \\
\hline Karcioglu 2013 & 13.5 & 11.2 & 17 & 48 & 19 & 20 & $0.9 \%$ & $-34.50[-44.38,-24.62]$ & $\longleftarrow$ & \\
\hline Katz 2004 & 19.4 & 2.8 & 97 & 16.9 & 1.9 & 46 & $1.7 \%$ & $2.50[1.72,3.28]$ & & $=$ \\
\hline Kim 2013 & 36.1 & 16 & 35 & 46 & 23 & 17 & $0.8 \%$ & $-9.90[-22.05,2.25]$ & & \\
\hline Kudoh 2002 & 13 & 9 & 35 & 25 & 7 & 35 & $1.5 \%$ & $-12.00[-15.78,-8.22]$ & - & \\
\hline Kwok 2004 & 12.4 & 12.5 & 90 & 15.6 & 8.5 & 45 & $1.5 \%$ & $-3.20[-6.78,0.38]$ & - & \\
\hline Kwon 2009 & 2 & 7 & 20 & 14 & 8 & 20 & $1.4 \%$ & $-12.00[-16.66,-7.34]$ & - & \\
\hline Lahtinen 2004 & 28.5 & 17.7 & 44 & 25.4 & 20 & 46 & $1.1 \%$ & $3.10[-4.69,10.89]$ & & \\
\hline Lak 2010 & 32.8 & 14 & 25 & 65.6 & 13.6 & 25 & $1.2 \%$ & $-32.80[-40.45,-25.15]$ & - & \\
\hline Leal 2013 & 15 & 13 & 20 & 5 & 7 & 20 & $1.3 \%$ & $10.00[3.53,16.47]$ & & 一 \\
\hline Leal 2015 & 14 & 15 & 28 & 8 & 10 & 28 & $1.2 \%$ & $6.00[-0.68,12.68]$ & & \\
\hline Lebrun 2006 & 28.2 & 6.9 & 54 & 35.4 & 4.2 & 30 & $1.6 \%$ & $-7.20[-9.58,-4.82]$ & - & \\
\hline Lee 2008 & 34 & 9 & 15 & 36 & 13 & 16 & $1.1 \%$ & $-2.00[-9.83,5.83]$ & - & \\
\hline Lehmann 2001 & 11 & 11 & 40 & 11 & 11 & 40 & $1.4 \%$ & $0.00[-4.82,4.82]$ & & \\
\hline Lin 2016 & 24 & 8 & 30 & 25 & 8 & 29 & $1.5 \%$ & $-1.00[-5.08,3.08]$ & & - \\
\hline Lo 2008 & 41 & 20.2 & 15 & 32 & 17.4 & 15 & $0.7 \%$ & $9.00[-4.49,22.49]$ & & \\
\hline Loftus 2010 & 47 & 27 & 52 & 48 & 24 & 50 & $0.9 \%$ & $-1.00[-10.90,8.90]$ & & \\
\hline Mahran 2015 & 29.3 & 10.2 & 30 & 30 & 11.4 & 30 & $1.4 \%$ & $-0.70[-6.17,4.77]$ & & - \\
\hline Mathisen 1999 & 20 & 14 & 32 & 24 & 18 & 18 & $1.0 \%$ & $-4.00[-13.63,5.63]$ & & \\
\hline Mendola 2012 & 9.72 & 12.2 & 32 & 7.99 & 9.4 & 30 & $1.4 \%$ & $1.73[-3.67,7.13]$ & & \\
\hline Menigaux 2000 & 24.7 & 11.7 & 30 & 42.6 & 19.8 & 15 & $0.9 \%$ & $-17.90[-28.76,-7.04]$ & - & \\
\hline Menigaux 2001 & 15.2 & 12.2 & 25 & 26.3 & 10.1 & 25 & $1.3 \%$ & $-11.10[-17.31,-4.89]$ & $=-$ & \\
\hline Michelet 2007 & 30 & 14 & 24 & 40 & 20 & 24 & $1.0 \%$ & $-10.00[-19.77,-0.23]$ & & \\
\hline Nesek-Adam 2012 & 23.3 & 11.3 & 20 & 18.4 & 11.3 & 20 & $1.2 \%$ & $4.90[-2.10,11.90]$ & & \\
\hline Nielsen 2017 & 46 & 19 & 73 & 48 & 20 & 72 & $1.3 \%$ & $-2.00[-8.35,4.35]$ & & \\
\hline Ögün 2001 & 17 & 20 & 16 & 36 & 29 & 15 & $0.5 \%$ & $-19.00[-36.65,-1.35]$ & & \\
\hline Papaziogas 2001 & 0 & 0 & 18 & 5 & 13 & 17 & & Not estimable & & \\
\hline Parikh 2011 & 21.4 & 2.4 & 30 & 21.4 & 9.5 & 30 & $1.5 \%$ & $0.00[-3.51,3.51]$ & & - \\
\hline Patel 2016 & 21 & 3 & 25 & 26.4 & 6 & 25 & $1.6 \%$ & $-5.40[-8.03,-2.77]$ & $\rightarrow$ & \\
\hline Remérand 2009 & 14 & 14 & 79 & 15 & 12 & 75 & $1.5 \%$ & $-1.00[-5.11,3.11]$ & & \\
\hline Reza 2010 & 35 & 4 & 30 & 30 & 4 & 30 & $1.6 \%$ & $5.00[2.98$. 7.02$]$ & & 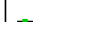 \\
\hline
\end{tabular}




\section{Analysis 1.3. (Continued)}

\begin{tabular}{|c|c|c|c|c|}
\hline I ULL $\angle$ VIV & 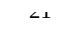 & $u$ & $<u$ & $<\mathrm{cut}$ \\
\hline Remérand 2009 & 14 & 14 & 79 & 15 \\
\hline Reza 2010 & 35 & 4 & 30 & 30 \\
\hline Safavi 2011 & 0.7 & 1.3 & 30 & 59 \\
\hline Sen 2009 & 10 & 13 & 20 & 13 \\
\hline Snijdelaar 2004 & 12 & 10 & 13 & 20 \\
\hline Song 2013 & 25 & 18 & 24 & 23 \\
\hline Spreng 2010 & 14 & 14 & 43 & 9 \\
\hline Subramaniam 2011 & 47 & 28 & 15 & 53 \\
\hline Suzuki 2006 & 9 & 4 & 24 & 25 \\
\hline Tena 2014 & 19.4 & 16.8 & 33 & 27.1 \\
\hline Ünlügenc 2003 & 10 & 1 & 30 & 10 \\
\hline Van Elstraete 2004 & 4.2 & 2 & 20 & 6.3 \\
\hline Webb 2007 & 15.6 & 16.1 & 50 & 23.9 \\
\hline Woo 2014 & 22 & 21.7 & 20 & 23 \\
\hline Wu 2009 & 17 & 7 & 15 & 20 \\
\hline Yalcin 2012 & 2.5 & 2.5 & 26 & 5 \\
\hline Yamauchi 2008 & 10.1 & 15.3 & 45 & 21.7 \\
\hline Yazigi 2012 & 10 & 3.2 & 30 & 4 \\
\hline Yeom 2012 & 36 & 20 & 20 & 51 \\
\hline Ysasi 2010 & 12.9 & 8.1 & 30 & 9.7 \\
\hline Zakine 2008 & 16.7 & 15.9 & 50 & 35 \\
\hline Total (95\% CI) & & & 2645 & \\
\hline \multirow{2}{*}{\multicolumn{5}{|c|}{$\begin{array}{l}\text { Heterogeneity: } \mathrm{Tau}^{2}=32.48 ; \mathrm{Chi}^{2}=1075.02, \mathrm{df}=80(\mathrm{P}<0.00001) ; \mathrm{I}^{2}=93 \% \\
\text { Test for overall effect: } \mathrm{Z}=6.87(\mathrm{P}<0.00001)\end{array}$}} \\
\hline & & & & \\
\hline \multicolumn{5}{|c|}{ Test for subgroup differences: Not applicable } \\
\hline
\end{tabular}

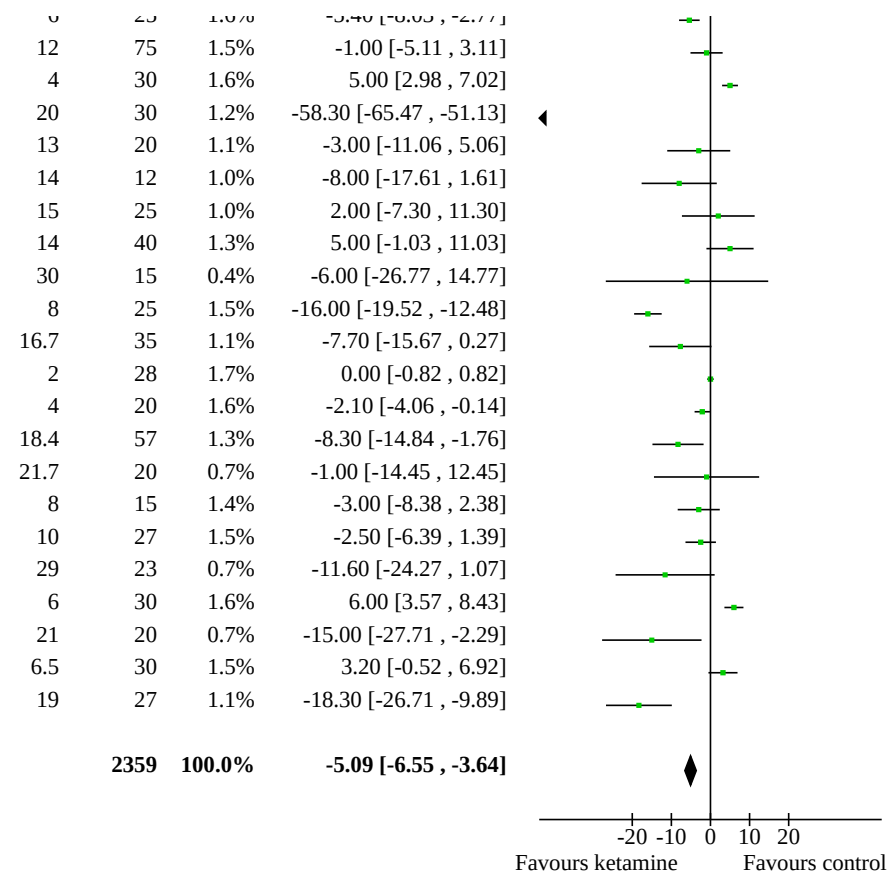


Analysis 1.4. Comparison 1: Perioperative ketamine versus control in a nonstratified study population, Outcome 4: Pain intensity during movement at 24 hours

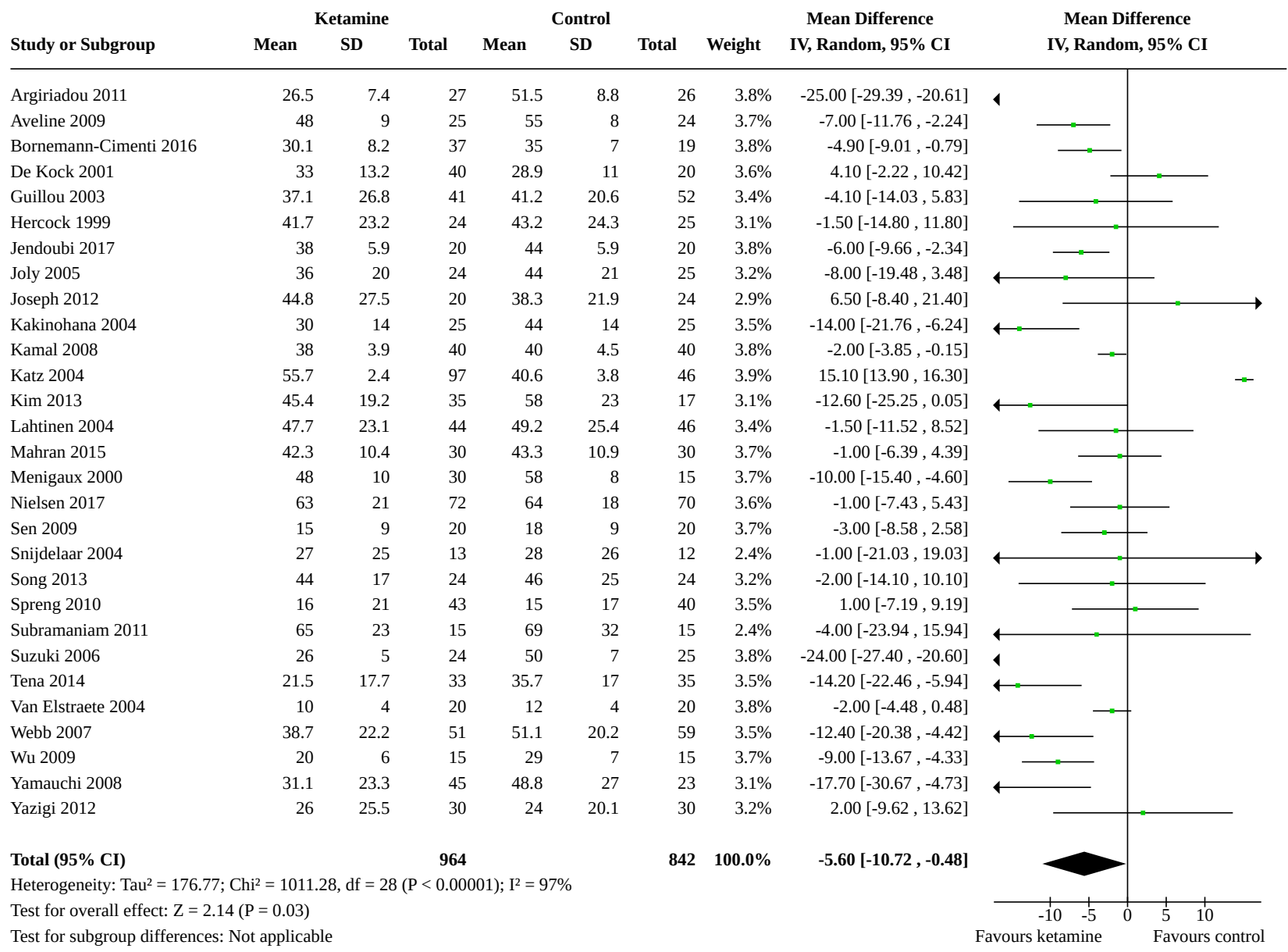


Analysis 1.5. Comparison 1: Perioperative ketamine versus control in a nonstratified study population, Outcome 5: Pain intensity at rest at 48 hours

\begin{tabular}{|c|c|c|c|c|c|c|c|c|c|c|}
\hline \multirow{2}{*}{ Study or Subgroup } & \multicolumn{3}{|c|}{ Ketamine } & \multicolumn{3}{|c|}{ Control } & \multirow{2}{*}{ Weight } & \multirow{2}{*}{$\begin{array}{c}\text { Mean Difference } \\
\text { IV, Random, 95\% CI }\end{array}$} & \multirow{2}{*}{\multicolumn{2}{|c|}{$\begin{array}{c}\text { Mean Difference } \\
\text { IV, Random, 95\% CI }\end{array}$}} \\
\hline & Mean & SD & Total & Mean & SD & Total & & & & \\
\hline Adam 2005 & 16.3 & 11.9 & 20 & 20 & 7.5 & 20 & $2.1 \%$ & $-3.70[-9.86,2.46]$ & & \\
\hline Adriaenssens 1999 & 12 & 12 & 15 & 21 & 18 & 15 & $1.3 \%$ & $-9.00[-19.95,1.95]$ & & 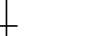 \\
\hline Argiriadou 2011 & 13.6 & 6.8 & 27 & 30.5 & 11.9 & 26 & $2.3 \%$ & $-16.90[-22.14,-11.66]$ & - & \\
\hline Arikan 2016 & 25 & 11 & 40 & 28 & 5 & 40 & $2.7 \%$ & $-3.00[-6.74,0.74]$ & & \\
\hline Aveline 2006 & 33.4 & 5.2 & 45 & 35 & 5 & 23 & $2.9 \%$ & $-1.60[-4.15,0.95]$ & & \\
\hline Aveline 2009 & 24 & 4 & 25 & 35 & 8 & 24 & $2.7 \%$ & $-11.00[-14.56,-7.44]$ & - & \\
\hline Bornemann-Cimenti 2016 & 10.5 & 6.6 & 37 & 19 & 9 & 19 & $2.5 \%$ & $-8.50[-13.07,-3.93]$ & & \\
\hline Chazan 2010 & 18 & 11 & 24 & 24 & 18 & 22 & $1.6 \%$ & $-6.00[-14.71,2.71]$ & & 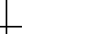 \\
\hline Chen 2004 & 23.9 & 14.4 & 20 & 26.2 & 9.1 & 20 & $1.9 \%$ & $-2.30[-9.77,5.17]$ & & - \\
\hline Dahl 2000 & 43.9 & 23.1 & 60 & 45 & 24 & 29 & $1.3 \%$ & $-1.10[-11.61,9.41]$ & & \\
\hline De Kock 2001 & 15.1 & 9 & 40 & 16.4 & 8.6 & 20 & $2.5 \%$ & $-1.30[-5.99,3.39]$ & & - \\
\hline Fiorelli 2015 & 27 & 6 & 38 & 33 & 4 & 37 & $2.9 \%$ & $-6.00[-8.30,-3.70]$ & $=$ & \\
\hline Ganne 2005 & 9.5 & 6.8 & 30 & 8.1 & 8.1 & 31 & $2.7 \%$ & $1.40[-2.35,5.15]$ & & - \\
\hline Grady 2012 & 31 & 21 & 30 & 28 & 14 & 32 & $1.6 \%$ & $3.00[-5.94,11.94]$ & & \\
\hline Guillou 2003 & 15.1 & 18 & 41 & 18.9 & 18 & 52 & $1.9 \%$ & $-3.80[-11.17,3.57]$ & & - \\
\hline Hu 2014 & 32 & 12 & 31 & 30 & 11 & 47 & $2.3 \%$ & $2.00[-3.27,7.27]$ & & \\
\hline Jaksch 2002 & 7 & 14 & 15 & 6 & 8 & 15 & $1.7 \%$ & $1.00[-7.16,9.16]$ & & \\
\hline Jendoubi 2017 & 18.8 & 6.3 & 20 & 28 & 6.3 & 20 & $2.6 \%$ & $-9.20[-13.10,-5.30]$ & - & \\
\hline Joly 2005 & 26 & 13 & 24 & 28.5 & 20.6 & 25 & $1.5 \%$ & $-2.50[-12.11,7.11]$ & & \\
\hline Joseph 2012 & 18.6 & 16.3 & 22 & 13.2 & 17.8 & 25 & $1.5 \%$ & $5.40[-4.35,15.15]$ & & - \\
\hline Kafali 2004 & 6 & 2.1 & 30 & 12.1 & 2.1 & 30 & $3.1 \%$ & $-6.10[-7.16,-5.04]$ & $=$ & \\
\hline Kakinohana 2004 & 4 & 6 & 25 & 10 & 9 & 25 & $2.6 \%$ & $-6.00[-10.24,-1.76]$ & - & \\
\hline Kamal 2008 & 19 & 1 & 40 & 22 & 2 & 40 & $3.1 \%$ & $-3.00[-3.69,-2.31]$ & $=$ & \\
\hline Katz 2004 & 14 & 2.9 & 97 & 11.3 & 1.9 & 46 & $3.1 \%$ & $2.70[1.90,3.50]$ & & $=$ \\
\hline Kim 2013 & 29 & 18.6 & 35 & 34 & 20 & 17 & $1.2 \%$ & $-5.00[-16.33,6.33]$ & & \\
\hline Kudoh 2002 & 9 & 8 & 35 & 18 & 6 & 35 & $2.7 \%$ & $-9.00[-12.31,-5.69]$ & & \\
\hline Kwon 2009 & 2 & 5 & 20 & 2 & 9 & 20 & $2.5 \%$ & $0.00[-4.51,4.51]$ & & - \\
\hline Lahtinen 2004 & 12.3 & 16.9 & 44 & 18.5 & 17.7 & 46 & $1.9 \%$ & $-6.20[-13.35,0.95]$ & & \\
\hline Lak 2010 & 24.8 & 10.4 & 25 & 46 & 9.6 & 25 & $2.3 \%$ & $-21.20[-26.75,-15.65]$ & - & \\
\hline Lebrun 2006 & 23.4 & 5.2 & 54 & 35.4 & 5.2 & 30 & $2.9 \%$ & $-12.00[-14.32,-9.68]$ & $=$ & \\
\hline Lo 2008 & 16.7 & 22.9 & 15 & 12 & 15.2 & 15 & $0.9 \%$ & $4.70[-9.21,18.61]$ & & \\
\hline Loftus 2010 & 54 & 21 & 52 & 53 & 22 & 50 & $1.7 \%$ & $1.00[-7.35,9.35]$ & & \\
\hline Mendola 2012 & 6.42 & 11.3 & 32 & 6.08 & 8.7 & 30 & $2.4 \%$ & $0.34[-4.66,5.34]$ & & - \\
\hline Menigaux 2000 & 16.7 & 12.8 & 30 & 24.3 & 12.9 & 15 & $1.8 \%$ & $-7.60[-15.57,0.37]$ & & \\
\hline Menigaux 2001 & 8.1 & 10.1 & 25 & 15.7 & 10.1 & 25 & $2.3 \%$ & $-7.60[-13.20,-2.00]$ & & \\
\hline Michelet 2007 & 29 & 16 & 24 & 42 & 21 & 24 & $1.3 \%$ & $-13.00[-23.56,-2.44]$ & & \\
\hline Papaziogas 2001 & 0 & 0 & 18 & 5 & 13 & 17 & & Not estimable & & \\
\hline Remérand 2009 & 15 & 16 & 79 & 14 & 11 & 75 & $2.5 \%$ & $1.00[-3.32,5.32]$ & & \\
\hline Snijdelaar 2004 & 8 & 8 & 13 & 9 & 8 & 12 & $2.1 \%$ & $-1.00[-7.28,5.28]$ & & - \\
\hline Song 2013 & 22 & 14 & 24 & 20 & 15 & 25 & $1.7 \%$ & $2.00[-6.12,10.12]$ & & \\
\hline Subramaniam 2011 & 43 & 22 & 15 & 48 & 26 & 15 & $0.7 \%$ & $-5.00[-22.24,12.24]$ & & \\
\hline Suzuki 2006 & 9 & 4 & 24 & 18 & 5 & 25 & $2.9 \%$ & $-9.00[-11.53,-6.47]$ & - & \\
\hline Webb 2007 & 9.7 & 10.3 & 51 & 18.4 & 16.3 & 52 & $2.3 \%$ & $-8.70[-13.96,-3.44]$ & & \\
\hline Woo 2014 & 23 & 22.9 & 20 & 22 & 21.7 & 20 & $1.0 \%$ & $1.00[-12.83,14.83]$ & & \\
\hline Wu 2009 & 15 & 7 & 15 & 21 & 7 & 15 & $2.4 \%$ & $-6.00[-11.01,-0.99]$ & - & \\
\hline Yamauchi 2008 & 7.1 & 19.9 & 45 & 15.7 & 24 & 23 & $1.2 \%$ & $-8.60[-20.00,2.80]$ & & \\
\hline Yazigi 2012 & 9 & 14 & 30 & 17 & 20 & 30 & $1.6 \%$ & $-8.00[-16.74,0.74]$ & & \\
\hline Yeom 2012 & 24 & 14 & 20 & 42 & 21 & 20 & $1.3 \%$ & $-18.00[-29.06,-6.94]$ & & \\
\hline Zakine 2008 & 12.2 & 13.5 & 50 & 25 & 17 & 27 & $1.9 \%$ & $-12.80[-20.22,-5.38]$ & & \\
\hline Total $(95 \%$ CI) & & & 1591 & & & 1371 & $100.0 \%$ & $-5.03[-6.65,-3.40]$ & 0 & \\
\hline \multicolumn{11}{|c|}{ Heterogeneity: $\mathrm{Tau}^{2}=22.21 ; \mathrm{Chi}^{2}=491.41, \mathrm{df}=47(\mathrm{P}<0.00001) ; \mathrm{I}^{2}=90 \%$} \\
\hline Test for overall effect: $\mathrm{Z}=$ & $\mathrm{P}<0.0000$ & & & & & & & & $-20-10$ & 10 \\
\hline Test for subgroup differenc & ot applicabl & & & & & & & & mine & \\
\hline
\end{tabular}


Analysis 1.6. Comparison 1: Perioperative ketamine versus control in a nonstratified study population, Outcome 6: Pain intensity during movement at 48 hours

\begin{tabular}{|c|c|c|c|c|c|c|c|c|c|c|}
\hline \multirow{2}{*}{$\begin{array}{l}\text { Study or Subgroup } \\
\text { Argiriadou } 2011\end{array}$} & \multicolumn{3}{|c|}{ Ketamine } & \multicolumn{3}{|c|}{ Control } & Weight & $\begin{array}{c}\text { Mean Difference } \\
\text { IV, Random, 95\% CI }\end{array}$ & \multicolumn{2}{|c|}{$\begin{array}{c}\text { Mean Difference } \\
\text { IV, Random, 95\% CI }\end{array}$} \\
\hline & 20.6 & 7.4 & 27 & 35.3 & 13.2 & 26 & $4.8 \%$ & $-14.70[-20.49,-8.91]$ & $\longleftarrow$ & \\
\hline Aveline 2009 & 49 & 6 & 25 & 59 & 12 & 24 & $4.8 \%$ & $-10.00[-15.35,-4.65]$ & & \\
\hline Bornemann-Cimenti 2016 & 27.5 & 6.5 & 37 & 31 & 9 & 19 & $4.9 \%$ & $-3.50[-8.06,1.06]$ & & \\
\hline De Kock 2001 & 28 & 13.4 & 40 & 28.9 & 11 & 20 & $4.7 \%$ & $-0.90[-7.26,5.46]$ & & \\
\hline Guillou 2003 & 26.8 & 26.8 & 41 & 35 & 24.7 & 51 & $4.0 \%$ & $-8.20[-18.84,2.44]$ & & \\
\hline Jaksch 2002 & 21 & 20 & 15 & 23 & 23 & 15 & $3.2 \%$ & $-2.00[-17.42,13.42]$ & & \\
\hline Jendoubi 2017 & 35.3 & 5.9 & 20 & 41.2 & 5.9 & 20 & $5.0 \%$ & $-5.90[-9.56,-2.24]$ & -1 & \\
\hline Joly 2005 & 33 & 18 & 24 & 37 & 23 & 25 & $3.8 \%$ & $-4.00[-15.54,7.54]$ & & \\
\hline Joseph 2012 & 38.2 & 18.3 & 22 & 34.2 & 29.1 & 24 & $3.4 \%$ & $4.00[-9.93,17.93]$ & & \\
\hline Kakinohana 2004 & 23 & 12 & 25 & 42 & 16 & 25 & $4.5 \%$ & $-19.00[-26.84,-11.16]$ & 4 & \\
\hline Kamal 2008 & 32 & 4.49 & 40 & 35 & 5.13 & 40 & $5.1 \%$ & $-3.00[-5.11,-0.89]$ & $\because$ & \\
\hline Katz 2004 & 47.2 & 3.1 & 97 & 37.7 & 3.8 & 46 & $5.2 \%$ & $9.50[8.24,10.76]$ & & - \\
\hline Kim 2013 & 36 & 18.3 & 35 & 45 & 19 & 17 & $3.9 \%$ & $-9.00[-19.88,1.88]$ & $\leftarrow$ & \\
\hline Lahtinen 2004 & 12.3 & 16.9 & 44 & 18.5 & 17.7 & 46 & $4.6 \%$ & $-6.20[-13.35,0.95]$ & & \\
\hline Menigaux 2000 & 28 & 18 & 30 & 36 & 10 & 15 & $4.4 \%$ & $-8.00[-16.19,0.19]$ & & \\
\hline Snijdelaar 2004 & 11 & 12 & 13 & 5 & 4 & 12 & $4.6 \%$ & $6.00[-0.90,12.90]$ & & \\
\hline Song 2013 & 42 & 20 & 24 & 40 & 21 & 25 & $3.8 \%$ & $2.00[-9.48,13.48]$ & & \\
\hline Subramaniam 2011 & 63 & 21 & 15 & 70 & 33 & 15 & $2.5 \%$ & $-7.00[-26.79,12.79]$ & $\leftarrow$ & \\
\hline Suzuki 2006 & 29 & 3.4 & 24 & 42.6 & 6.9 & 25 & $5.1 \%$ & $-13.60[-16.63,-10.57]$ & - & \\
\hline Webb 2007 & 32.9 & 16.4 & 51 & 39.4 & 17.1 & 56 & $4.7 \%$ & $-6.50[-12.85,-0.15]$ & & \\
\hline Wu 2009 & 18 & 8 & 15 & 25 & 8 & 15 & $4.8 \%$ & $-7.00[-12.73,-1.27]$ & & \\
\hline Yamauchi 2008 & 26.2 & 19.8 & 45 & 37.8 & 20 & 23 & $4.1 \%$ & $-11.60[-21.61,-1.59]$ & $\leftarrow$ & \\
\hline Yazigi 2012 & 32 & 19.5 & 30 & 44 & 19.5 & 30 & $4.1 \%$ & $-12.00[-21.87,-2.13]$ & & \\
\hline Total $(95 \%$ CI) & & & 739 & & & 614 & $100.0 \%$ & $-5.72[-10.15,-1.29]$ & & \\
\hline \multicolumn{11}{|c|}{ Heterogeneity: $\mathrm{Tau}^{2}=98.70 ; \mathrm{Chi}^{2}=409.22, \mathrm{df}=22(\mathrm{P}<0.00001) ; \mathrm{I}^{2}=95 \%$} \\
\hline \multicolumn{9}{|c|}{ Test for overall effect: $\mathrm{Z}=2.53(\mathrm{P}=0.01)$} & $-10-5$ & $\begin{array}{ll}1 & 1 \\
5 & 10\end{array}$ \\
\hline \multicolumn{9}{|c|}{ Test for subgroup differences: Not applicable } & Favours ketamine & Favours \\
\hline
\end{tabular}


Analysis 1.7. Comparison 1: Perioperative ketamine versus control in a non-stratified study population, Outcome 7: Time to first request for analgesia/trigger of PCA

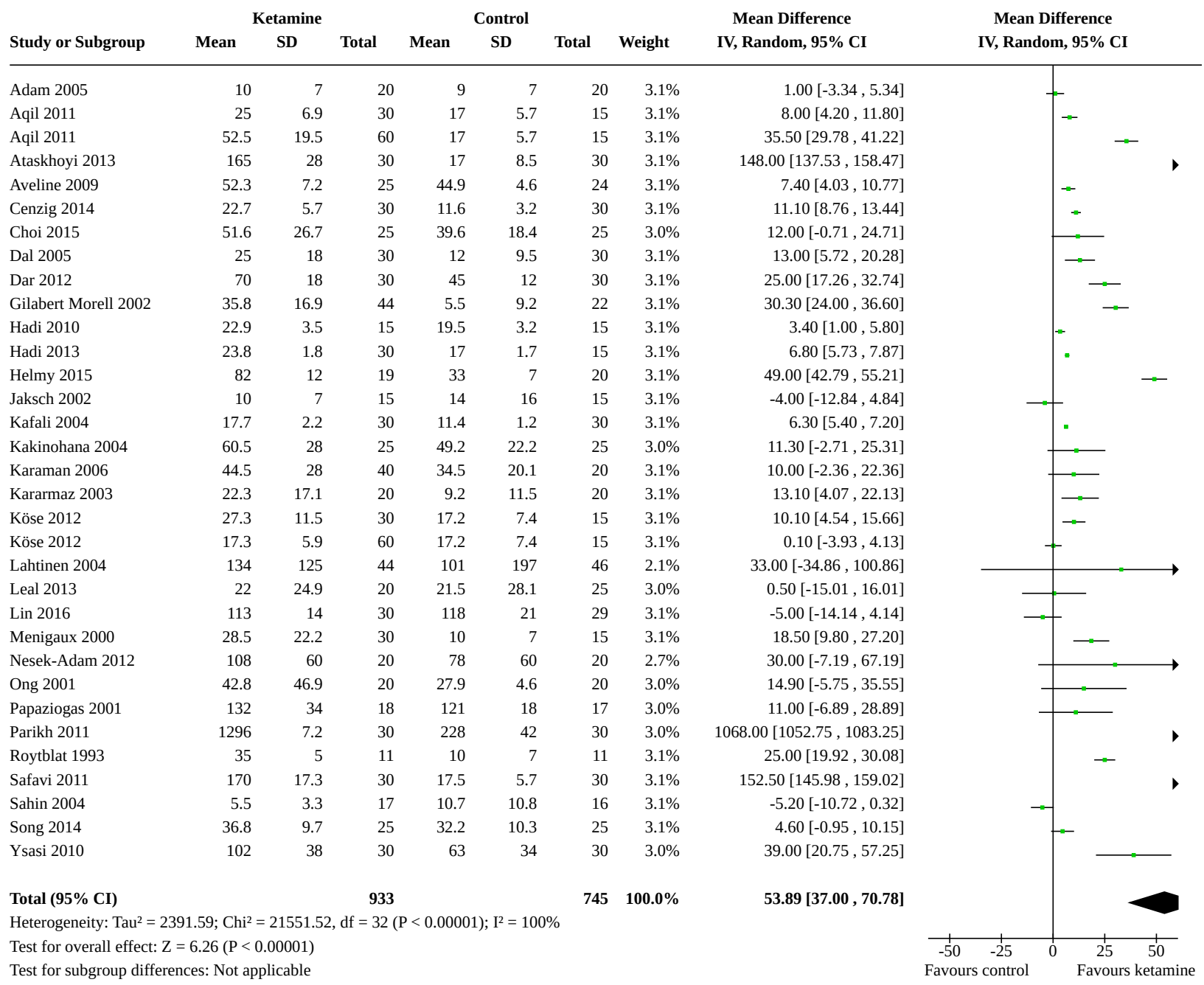


Analysis 1.8. Comparison 1: Perioperative ketamine versus control in a non-stratified study population, Outcome 8: CNS adverse events - all studies

\begin{tabular}{|c|c|c|c|c|c|c|c|}
\hline \multirow[b]{2}{*}{ Study or Subgroup } & \multicolumn{2}{|c|}{ Ketamine } & \multicolumn{2}{|c|}{ Control } & \multirow[b]{2}{*}{ Weight } & \multirow{2}{*}{$\begin{array}{c}\text { Risk Ratio } \\
\text { M-H, Random, 95\% CI }\end{array}$} & \multirow{2}{*}{$\begin{array}{c}\text { Risk Ratio } \\
\text { M-H, Random, } \mathbf{9 5 \% ~ C l}\end{array}$} \\
\hline & Events & Total & Events & Total & & & \\
\hline Adam 2005 & 0 & 20 & 0 & 20 & & Not estimable & \\
\hline Adriaenssens 1999 & 0 & 15 & 0 & 15 & & Not estimable & \\
\hline Aqil 2011 & 5 & 90 & 0 & 30 & $0.5 \%$ & $3.75[0.21,65.84]$ & \\
\hline Argiriadou 2004 & 0 & 30 & 0 & 15 & & Not estimable & \\
\hline Argiriadou 2011 & 0 & 27 & 0 & 26 & & Not estimable & \\
\hline Arikan 2016 & 1 & 40 & 0 & 40 & $0.4 \%$ & $3.00[0.13,71.51]$ & \\
\hline Ataskhoyi 2013 & 0 & 30 & 0 & 30 & & Not estimable & \\
\hline Aubrun 2008 & 5 & 45 & 3 & 45 & $2.3 \%$ & $1.67[0.42,6.56]$ & \\
\hline Aveline 2006 & 1 & 45 & 0 & 23 & $0.4 \%$ & $1.57[0.07,36.98]$ & \\
\hline Aveline 2009 & 0 & 25 & 0 & 24 & & Not estimable & \\
\hline Ayoglu 2005 & 3 & 20 & 0 & 20 & $0.5 \%$ & $7.00[0.38,127.32]$ & \\
\hline Barreveld 2013 & 1 & 32 & 0 & 32 & $0.4 \%$ & $3.00[0.13,71.00]$ & \\
\hline Bilgen 2012 & 1 & 105 & 0 & 35 & $0.4 \%$ & $1.02[0.04,24.46]$ & \\
\hline Burstal 2001 & 4 & 37 & 0 & 33 & $0.5 \%$ & $8.05[0.45,144.15]$ & - \\
\hline Cenzig 2014 & 2 & 30 & 2 & 30 & $1.2 \%$ & $1.00[0.15,6.64]$ & \\
\hline Chazan 2010 & 1 & 24 & 2 & 22 & $0.8 \%$ & $0.46[0.04,4.71]$ & \\
\hline Chen 2004 & 0 & 20 & 0 & 20 & & Not estimable & \\
\hline D'Alonzo 2011 & 0 & 20 & 0 & 20 & & Not estimable & \\
\hline Dal 2005 & 0 & 30 & 0 & 30 & & Not estimable & \\
\hline De Kock 2001 & 0 & 40 & 0 & 20 & & Not estimable & \\
\hline Deng 2009 & 7 & 150 & 3 & 50 & $2.5 \%$ & $0.78[0.21,2.89]$ & $\longrightarrow$ \\
\hline Du 2011 & 0 & 20 & 0 & 20 & & Not estimable & \\
\hline Dualé 2009 & 8 & 20 & 8 & 20 & $7.5 \%$ & $1.00[0.47,2.14]$ & $\longrightarrow$ \\
\hline Dullenkopf 2009 & 1 & 77 & 0 & 33 & $0.4 \%$ & $1.31[0.05,31.29]$ & \\
\hline Fiorelli 2015 & 0 & 38 & 0 & 37 & & Not estimable & \\
\hline Galinski 2007 & 1 & 20 & 0 & 20 & $0.4 \%$ & $3.00[0.13,69.52]$ & \\
\hline Ganne 2005 & 0 & 30 & 0 & 31 & & Not estimable & \\
\hline Garcia-Navia 2016 & 0 & 11 & 0 & 11 & & Not estimable & \\
\hline Garg 2016 & 4 & 22 & 1 & 22 & $1.0 \%$ & $4.00[0.48,33.00]$ & \\
\hline Gilabert Morell 2002 & 0 & 22 & 0 & 22 & & Not estimable & \\
\hline Guignard 2002 & 0 & 25 & 0 & 25 & & Not estimable & \\
\hline Guillou 2003 & 1 & 41 & 1 & 52 & $0.6 \%$ & $1.27[0.08,19.67]$ & \\
\hline Hadi 2010 & 0 & 15 & 0 & 15 & & Not estimable & \\
\hline Hadi 2013 & 0 & 15 & 0 & 15 & & Not estimable & \\
\hline Haliloglu 2015 & 0 & 26 & 0 & 26 & & Not estimable & \\
\hline Hasanein 2011 & 0 & 30 & 0 & 30 & & Not estimable & \\
\hline Hayes 2004 & 6 & 22 & 6 & 23 & $4.6 \%$ & $1.05[0.40,2.75]$ & \\
\hline Hercock 1999 & 0 & 24 & 0 & 25 & & Not estimable & \\
\hline Hu 2014 & 7 & 31 & 4 & 47 & $3.3 \%$ & $2.65[0.85,8.31]$ & \\
\hline Ilkjaer 1998 & 3 & 24 & 2 & 28 & $1.5 \%$ & $1.75[0.32,9.62]$ & \\
\hline Jaksch 2002 & 0 & 15 & 0 & 15 & & Not estimable & \\
\hline Jendoubi 2017 & 0 & 20 & 0 & 20 & & Not estimable & \\
\hline Joly 2005 & 1 & 24 & 0 & 25 & $0.4 \%$ & $3.12[0.13,73.04]$ & \\
\hline Joseph 2012 & 1 & 22 & 1 & 25 & $0.6 \%$ & $1.14[0.08,17.11]$ & \\
\hline Kafali 2004 & 0 & 30 & 0 & 30 & & Not estimable & \\
\hline Kamal 2008 & 1 & 40 & 1 & 40 & $0.6 \%$ & $1.00[0.06,15.44]$ & \\
\hline Kapfer 2005 & 5 & 22 & 1 & 21 & $1.0 \%$ & $4.77[0.61,37.52]$ & \\
\hline Karaman 2006 & 0 & 40 & 0 & 20 & & Not estimable & \\
\hline Kararmaz 2003 & 2 & 20 & 1 & 20 & $0.8 \%$ & $2.00[0.20,20.33]$ & \\
\hline Karcioglu 2013 & 0 & 17 & 0 & 20 & & Not estimable & \\
\hline Katz 2004 & 4 & 97 & 1 & 46 & $0.9 \%$ & $1.90[0.22,16.50]$ & \\
\hline Kim 2013 & 0 & 35 & 0 & 17 & & Not estimable & \\
\hline Kim 2016 & 8 & 28 & 11 & 29 & $7.8 \%$ & $0.75[0.36,1.59]$ & -1 \\
\hline
\end{tabular}


Analysis 1.8. (Continued)

Kim 2013

Kim 2016

Köse 2012

Kudoh 2002

Kwok 2004

Kwon 2009

Lahtinen 2004

Lak 2010

Leal 2013

Leal 2015

Lebrun 2006

Lehmann 2001

Lin 2016

Lo 2008

Loftus 2010

Mahran 2015

Martinez 2014

Mathisen 1999

McKay 2007

Mebazaa MS 2008

Mendola 2012

Menigaux 2000

Menigaux 2001

Michelet 2007

Miziara 2016

Nesek-Adam 2012

Nielsen 2017

Pacreu 2012

Papaziogas 2001

Parikh 2011

Pirim 2006

Remérand 2009

Reza 2010

Roytblat 1993

Safavi 2011

Sahin 2004

Sen 2009

Siddiqui 2015

Singh 2013

Snijdelaar 2004

Song 2013

Spreng 2010

Subramaniam 2011

Suzuki 1999

Tena 2014

Van Elstraete 2004

Webb 2007

Woo 2014

Wu 2009

Yalcin 2012

Yazigi 2012

Yeom 2012

Ysasi 2010

Zakine 2008

Total (95\% CI)

\begin{tabular}{|c|c|c|c|}
\hline 35 & 0 & 17 & \\
\hline 28 & 11 & 29 & $7.8 \%$ \\
\hline 90 & 0 & 30 & \\
\hline 35 & 8 & 35 & $4.2 \%$ \\
\hline 90 & 0 & 45 & \\
\hline 20 & 0 & 20 & \\
\hline 44 & 0 & 46 & $0.5 \%$ \\
\hline 25 & 0 & 25 & $0.4 \%$ \\
\hline 20 & 1 & 20 & $1.1 \%$ \\
\hline 28 & 2 & 28 & $1.8 \%$ \\
\hline 54 & 0 & 30 & \\
\hline 40 & 0 & 40 & \\
\hline 30 & 0 & 29 & \\
\hline 15 & 1 & 15 & $0.8 \%$ \\
\hline 52 & 1 & 50 & $0.6 \%$ \\
\hline 30 & 0 & 30 & \\
\hline 34 & 2 & 38 & $0.5 \%$ \\
\hline 40 & 0 & 20 & \\
\hline 19 & 0 & 22 & $0.5 \%$ \\
\hline 67 & 1 & 67 & $0.6 \%$ \\
\hline 32 & 0 & 30 & \\
\hline 30 & 0 & 15 & \\
\hline 25 & 0 & 25 & \\
\hline 24 & 0 & 24 & \\
\hline 21 & 0 & 21 & $0.4 \%$ \\
\hline 20 & 1 & 20 & $0.6 \%$ \\
\hline 74 & 4 & 73 & $0.9 \%$ \\
\hline 10 & 0 & 10 & \\
\hline 18 & 0 & 17 & \\
\hline 30 & 0 & 30 & \\
\hline 23 & 0 & 22 & \\
\hline 79 & 31 & 75 & $26.9 \%$ \\
\hline 30 & 0 & 30 & $0.5 \%$ \\
\hline 11 & 0 & 11 & \\
\hline 30 & 2 & 30 & $1.2 \%$ \\
\hline 17 & 0 & 16 & \\
\hline 20 & 2 & 20 & $1.5 \%$ \\
\hline 29 & 0 & 20 & $0.5 \%$ \\
\hline 60 & 0 & 20 & $0.5 \%$ \\
\hline 13 & 0 & 12 & \\
\hline 24 & 0 & 25 & $0.5 \%$ \\
\hline 43 & 0 & 40 & \\
\hline 15 & 9 & 15 & $6.4 \%$ \\
\hline 105 & 0 & 35 & \\
\hline 33 & 2 & 35 & $1.6 \%$ \\
\hline 20 & 0 & 20 & \\
\hline 56 & 5 & 64 & $3.4 \%$ \\
\hline 20 & 0 & 20 & \\
\hline 15 & 2 & 15 & $1.3 \%$ \\
\hline 26 & 0 & 27 & $0.5 \%$ \\
\hline 30 & 0 & 30 & $0.5 \%$ \\
\hline 20 & 0 & 20 & \\
\hline 30 & 0 & 30 & \\
\hline 50 & 0 & 27 & \\
\hline
\end{tabular}

3614
Not estimab
$0.75[0.36,1.59]$
Not estimab
$0.63[0.23,1.72]$
Not estimab
Not estimab
$9.40[0.52,169.65]$

$3.00[0.13,70.30]$

$7.00[0.95,51.80]$

2.50 [0.53, 11.82]

Not estimable

Not estimable

Not estimable

$2.00[0.20,19.78]$

$0.96[0.06,14.96]$

Not estimable

0.22 [0.01, 4.48]

Not estimable

$14.95[0.90,249.15]$

$1.00[0.06,15.66]$

Not estimable

Not estimable

Not estimable

Not estimable

$3.00[0.13,69.70]$

$1.00[0.07,14.90]$

0.25 [0.03, 2.15]

Not estimable

Not estimable

Not estimable

Not estimable

$0.86[0.57,1.28]$

5.00 [0.25, 99.95]

Not estimable

$1.00[0.15,6.64]$

Not estimable

$1.50[0.28,8.04]$

$3.50[0.18,69.23]$

$1.72[0.09,34.42]$

Not estimable

$7.28[0.40,133.89]$

Not estimable

0.56 [0.24, 1.27]

Not estimable

$2.12[0.42,10.82]$

Not estimable

1.37 [0.44, 4.25]

Not estimable

$1.00[0.16,6.20]$

$15.56[0.93,259.28]$

5.00 [0.25, 99.95]

Not estimable

Not estimable

Not estimable

$1.17[0.95,1.43]$

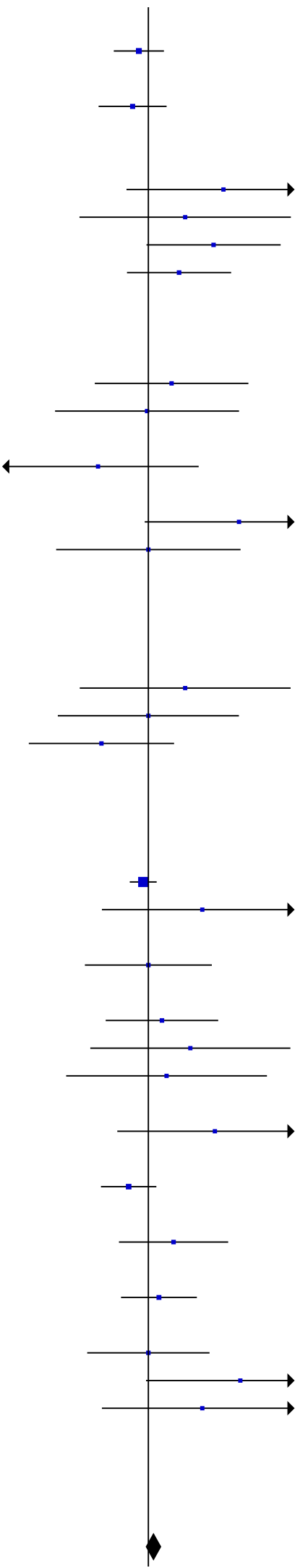




\section{Analysis 1.8. (Continued)}

Total (95\% CI)

3614

$2924 \quad 100.0 \%$

$1.17[0.95,1.43]$

Total events:

$187 \quad 122$

Heterogeneity: $\mathrm{Tau}^{2}=0.00 ; \mathrm{Chi}^{2}=44.83, \mathrm{df}=51(\mathrm{P}=0.72) ; \mathrm{I}^{2}=0 \%$

Test for overall effect: $\mathrm{Z}=1.44(\mathrm{P}=0.15)$

Test for subgroup differences: Not applicable

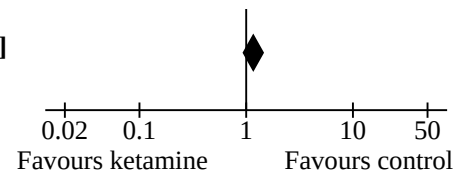

Analysis 1.9. Comparison 1: Perioperative ketamine versus control in a non-stratified study population, Outcome 9: Hyperalgesia

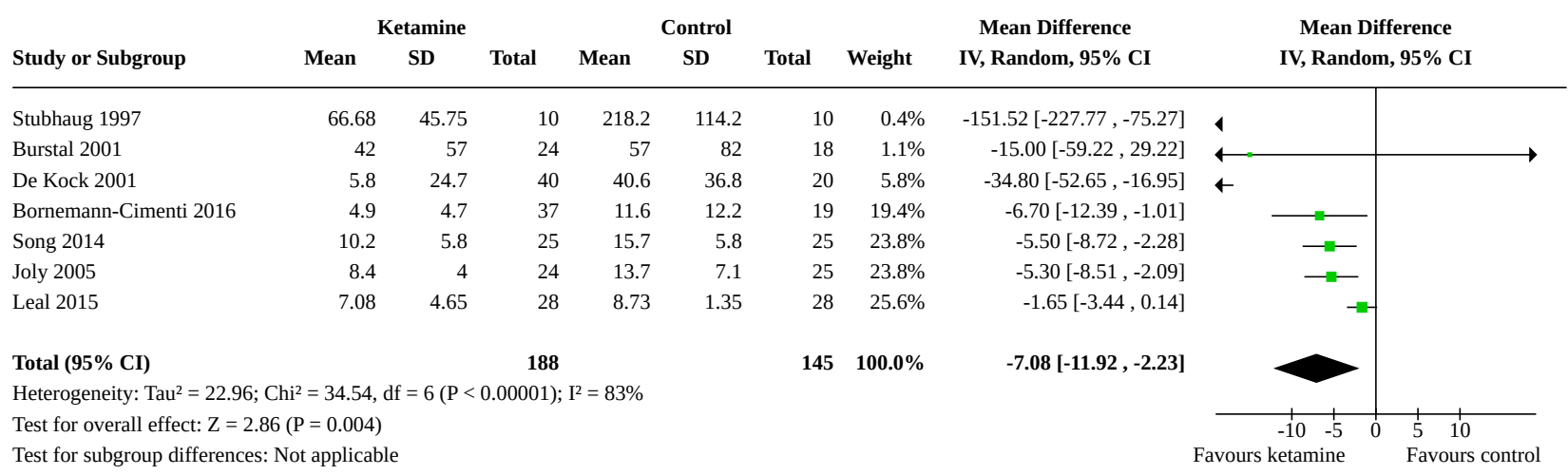


Analysis 1.10. Comparison 1: Perioperative ketamine versus control in a non-stratified study population, Outcome 10: CNS adverse events - studies with events

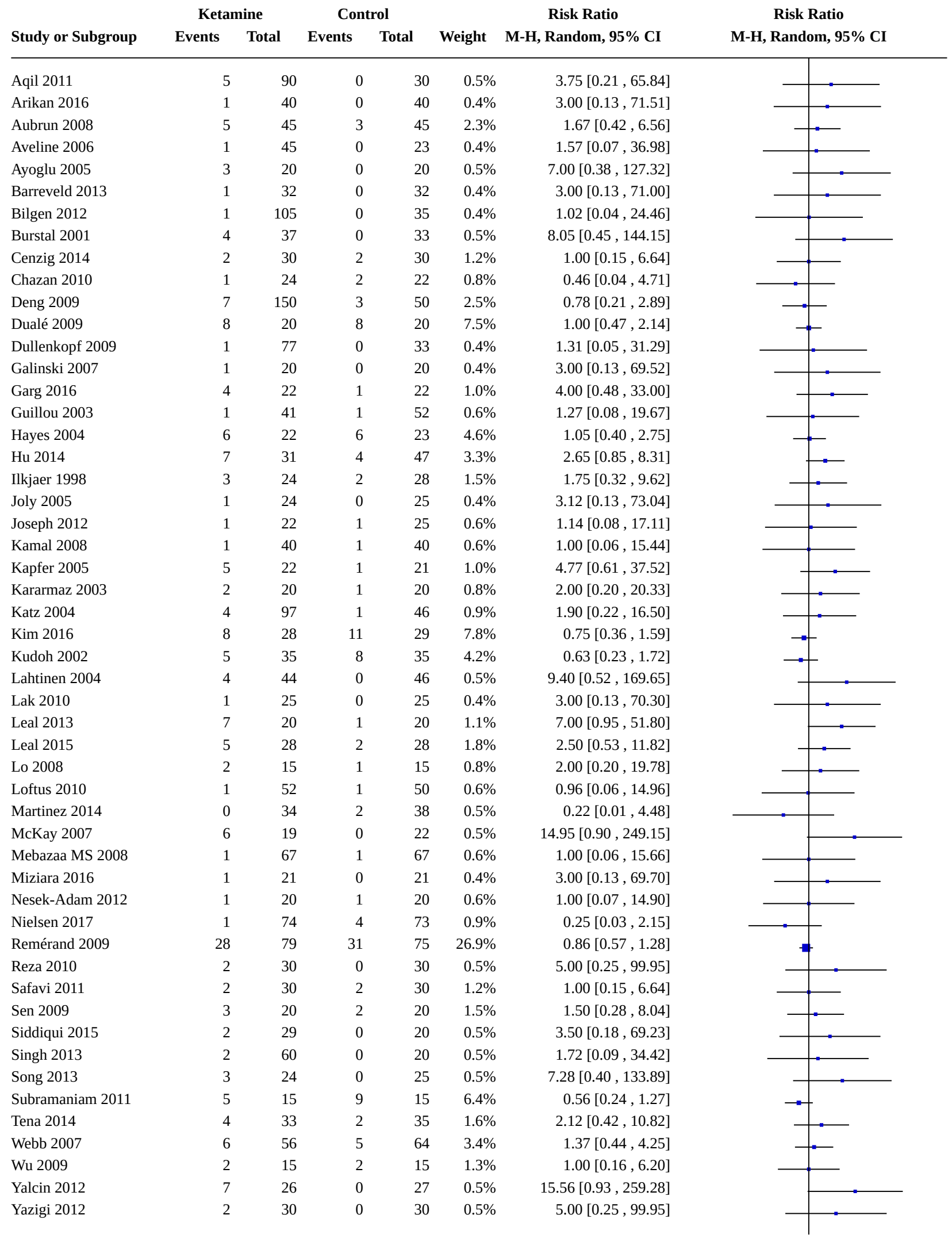




\section{Analysis 1.10. (Continued)}

Yazigi 2012 2

Total (95\% CI)

Total events:

187

2029

0

30

$0.5 \%$

Heterogeneity: $\mathrm{Tau}^{2}=0.00 ; \mathrm{Chi}^{2}=44.83, \mathrm{df}=51(\mathrm{P}=0.72) ; \mathrm{I}^{2}=0 \%$

Test for overall effect: $\mathrm{Z}=1.44(\mathrm{P}=0.15)$

Test for subgroup differences: Not applicable
$5.00[0.25,99.95]$

$1.17[0.95,1.43]$

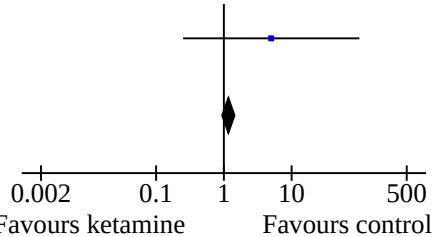


Analysis 1.11. Comparison 1: Perioperative ketamine versus control in a non-stratified study population, Outcome 11: Postoperative nausea and vomiting - all studies

\begin{tabular}{|c|c|c|c|c|c|c|c|}
\hline \multirow[b]{2}{*}{ Study or Subgroup } & \multicolumn{2}{|c|}{ Ketamine } & \multicolumn{2}{|c|}{ Control } & \multirow[b]{2}{*}{ Weight } & \multirow{2}{*}{$\begin{array}{c}\text { Risk Ratio } \\
\text { M-H, Random, 95\% CI }\end{array}$} & \multirow{2}{*}{$\begin{array}{c}\text { Risk Ratio } \\
\text { M-H, Random, 95\% CI }\end{array}$} \\
\hline & Events & Total & Events & Total & & & \\
\hline Galinski 2007 & 0 & 20 & 0 & 20 & & Not estimable & \\
\hline Helmy 2015 & 0 & 20 & 0 & 30 & & Not estimable & \\
\hline Menigaux 2001 & 0 & 25 & 0 & 25 & & Not estimable & \\
\hline Fiorelli 2015 & 0 & 38 & 0 & 37 & & Not estimable & \\
\hline Abdolahi 2013 & 0 & 44 & 1 & 44 & $0.1 \%$ & $0.33[0.01,7.97]$ & \\
\hline Snijdelaar 2004 & 0 & 13 & 1 & 12 & $0.1 \%$ & $0.31[0.01,6.94]$ & \\
\hline Woo 2014 & 2 & 20 & 0 & 20 & $0.1 \%$ & $5.00[0.26,98.00]$ & \\
\hline Pacreu 2012 & 2 & 10 & 0 & 10 & $0.1 \%$ & $5.00[0.27,92.62]$ & \\
\hline Parikh 2011 & 0 & 30 & 4 & 30 & $0.1 \%$ & $0.11[0.01,1.98]$ & - \\
\hline Dar 2012 & 1 & 30 & 1 & 30 & $0.1 \%$ & $1.00[0.07,15.26]$ & \\
\hline Kafali 2004 & 1 & 30 & 2 & 30 & $0.1 \%$ & $0.50[0.05,5.22]$ & \\
\hline Safavi 2011 & 2 & 30 & 1 & 30 & $0.1 \%$ & $2.00[0.19,20.90]$ & \\
\hline Miziara 2016 & 1 & 21 & 2 & 21 & $0.1 \%$ & $0.50[0.05,5.10]$ & \\
\hline Dal 2005 & 1 & 30 & 3 & 30 & $0.1 \%$ & $0.33[0.04,3.03]$ & \\
\hline Garg 2016 & 3 & 22 & 1 & 22 & $0.2 \%$ & $3.00[0.34,26.66]$ & \\
\hline Bilgen 2012 & 6 & 105 & 1 & 35 & $0.2 \%$ & $2.00[0.25,16.04]$ & \\
\hline Kararmaz 2003 & 1 & 20 & 6 & 20 & $0.2 \%$ & $0.17[0.02,1.26]$ & - \\
\hline Adriaenssens 1999 & 1 & 15 & 6 & 15 & $0.2 \%$ & $0.17[0.02,1.22]$ & \\
\hline Stubhaug 1997 & 1 & 10 & 5 & 10 & $0.2 \%$ & $0.20[0.03,1.42]$ & - \\
\hline Tena 2014 & 2 & 33 & 2 & 35 & $0.2 \%$ & $1.06[0.16,7.10]$ & \\
\hline Roytblat 1993 & 2 & 11 & 2 & 11 & $0.2 \%$ & $1.00[0.17,5.89]$ & \\
\hline Hasanein 2011 & 2 & 30 & 3 & 30 & $0.2 \%$ & $0.67[0.12,3.71]$ &. \\
\hline Kwon 2009 & 2 & 20 & 3 & 20 & $0.3 \%$ & $0.67[0.12,3.57]$ & \\
\hline Guillou 2003 & 2 & 41 & 4 & 52 & $0.3 \%$ & $0.63[0.12,3.29]$ & \\
\hline Spreng 2010 & 2 & 43 & 4 & 40 & $0.3 \%$ & $0.47[0.09,2.40]$ & - \\
\hline Deng 2009 & 6 & 150 & 2 & 50 & $0.3 \%$ & $1.00[0.21,4.80]$ & \\
\hline Crousier 2008 & 2 & 18 & 4 & 18 & $0.3 \%$ & $0.50[0.10,2.40]$ & \begin{tabular}{l|l}
-1 & \\
\end{tabular} \\
\hline Ataskhoyi 2013 & 5 & 30 & 2 & 30 & $0.3 \%$ & $2.50[0.53,11.89]$ & 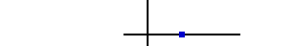 \\
\hline Lak 2010 & 3 & 25 & 3 & 25 & $0.3 \%$ & $1.00[0.22,4.49]$ & \\
\hline Kamal 2008 & 2 & 40 & 8 & 40 & $0.3 \%$ & $0.25[0.06,1.11]$ & \\
\hline Kim 2016 & 7 & 28 & 2 & 29 & $0.3 \%$ & $3.63[0.82,15.98]$ & \\
\hline Menigaux 2000 & 3 & 30 & 3 & 15 & $0.3 \%$ & $0.50[0.11,2.19]$ & - \\
\hline Yeom 2012 & 3 & 20 & 3 & 20 & $0.3 \%$ & $1.00[0.23,4.37]$ & \\
\hline Joseph 2012 & 8 & 22 & 2 & 25 & $0.3 \%$ & $4.55[1.08,19.18]$ & \\
\hline Nesek-Adam 2012 & 4 & 20 & 3 & 20 & $0.4 \%$ & $1.33[0.34,5.21]$ & \\
\hline Van Elstraete 2004 & 4 & 20 & 3 & 20 & $0.4 \%$ & $1.33[0.34,5.21]$ & \\
\hline Hu 2014 & 4 & 31 & 5 & 47 & $0.5 \%$ & $1.21[0.35,4.17]$ & \\
\hline Haliloglu 2015 & 4 & 26 & 5 & 26 & $0.5 \%$ & $0.80[0.24,2.65]$ & 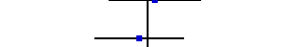 \\
\hline Guignard 2002 & 4 & 25 & 5 & 25 & $0.5 \%$ & $0.80[0.24,2.64]$ & \\
\hline Singh 2013 & 11 & 60 & 3 & 20 & $0.5 \%$ & $1.22[0.38,3.95]$ & \\
\hline Subramaniam 2011 & 3 & 15 & 7 & 15 & $0.5 \%$ & $0.43[0.14,1.35]$ & \\
\hline Wu 2009 & 3 & 15 & 7 & 15 & $0.5 \%$ & $0.43[0.14,1.35]$ & \\
\hline Papaziogas 2001 & 5 & 18 & 4 & 17 & $0.5 \%$ & $1.18[0.38,3.67]$ & \\
\hline Siddiqui 2015 & 4 & 29 & 8 & 29 & $0.6 \%$ & $0.50[0.17,1.48]$ & -1 \\
\hline Kapfer 2005 & 8 & 22 & 4 & 21 & $0.6 \%$ & $1.91[0.67,5.40]$ & \\
\hline Aveline 2009 & 4 & 25 & 9 & 24 & $0.7 \%$ & $0.43[0.15,1.20]$ & \\
\hline Martinez 2014 & 6 & 34 & 6 & 38 & $0.7 \%$ & $1.12[0.40,3.14]$ & 1 \\
\hline Mahran 2015 & 6 & 30 & 6 & 30 & $0.7 \%$ & $1.00[0.36,2.75]$ & \\
\hline Köse 2012 & 7 & 90 & 6 & 30 & $0.7 \%$ & $0.39[0.14,1.07]$ & \\
\hline Garcia-Navia 2016 & 3 & 11 & 9 & 11 & $0.7 \%$ & $0.33[0.12,0.91]$ & \\
\hline Jaksch 2002 & 7 & 15 & 4 & 15 & $0.7 \%$ & $1.75[0.64,4.75]$ & \\
\hline Arikan 2016 & 5 & 40 & 10 & 40 & $0.7 \%$ & $0.50[0.19,1.33]$ & 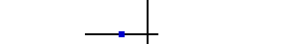 \\
\hline Argiriadou 2004 & 7 & 30 & 5 & 15 & $0.7 \%$ & $0.70[0.27,1.84]$ & $\rightarrow$ \\
\hline
\end{tabular}




\section{Analysis 1.11. (Continued)}

\begin{tabular}{|c|c|c|c|c|c|c|}
\hline Arikan 2016 & 5 & 40 & 10 & 40 & $0.7 \%$ & $0.50[0.19,1.33]$ \\
\hline Argiriadou 2004 & 7 & 30 & 5 & 15 & $0.7 \%$ & $0.70[0.27,1.84]$ \\
\hline Ünlügenc 2003 & 5 & 30 & 9 & 28 & $0.7 \%$ & $0.52[0.20,1.36]$ \\
\hline Dahi-Taleghani 2014 & 10 & 70 & 6 & 70 & $0.8 \%$ & $1.67[0.64,4.34]$ \\
\hline Michelet 2007 & 6 & 24 & 7 & 24 & $0.8 \%$ & $0.86[0.34,2.18]$ \\
\hline Reza 2010 & 6 & 30 & 8 & 30 & $0.8 \%$ & $0.75[0.30,1.90]$ \\
\hline Yazigi 2012 & 8 & 30 & 6 & 30 & $0.8 \%$ & $1.33[0.53,3.38]$ \\
\hline Ysasi 2010 & 6 & 30 & 9 & 30 & $0.9 \%$ & $0.67[0.27,1.64]$ \\
\hline Zakine 2008 & 6 & 50 & 10 & 27 & $0.9 \%$ & $0.32[0.13,0.79]$ \\
\hline Hadi 2013 & 6 & 30 & 8 & 15 & $0.9 \%$ & $0.38[0.16,0.88]$ \\
\hline Ayoglu 2005 & 6 & 20 & 8 & 20 & $0.9 \%$ & $0.75[0.32,1.77]$ \\
\hline Ong 2001 & 7 & 20 & 7 & 20 & $1.0 \%$ & $1.00[0.43,2.33]$ \\
\hline Kim 2013 & 10 & 35 & 6 & 17 & $1.0 \%$ & $0.81[0.35,1.86]$ \\
\hline Mendola 2012 & 10 & 32 & 7 & 30 & $1.0 \%$ & $1.34[0.59,3.06]$ \\
\hline Joly 2005 & 8 & 24 & 8 & 25 & $1.0 \%$ & $1.04[0.47,2.33]$ \\
\hline Sen 2009 & 7 & 20 & 8 & 20 & $1.1 \%$ & $0.88[0.39,1.95]$ \\
\hline Ozhan 2013 & 11 & 30 & 7 & 30 & $1.1 \%$ & $1.57[0.71,3.50]$ \\
\hline Cenzig 2014 & 7 & 30 & 14 & 30 & $1.2 \%$ & $0.50[0.24,1.06]$ \\
\hline Lin 2016 & 10 & 30 & 9 & 29 & $1.2 \%$ & $1.07[0.51,2.26]$ \\
\hline Ögün 2001 & 7 & 16 & 8 & 15 & $1.3 \%$ & $0.82[0.40,1.70]$ \\
\hline Lehmann 2001 & 12 & 40 & 10 & 40 & $1.3 \%$ & $1.20[0.59,2.45]$ \\
\hline Aveline 2006 & 13 & 45 & 10 & 23 & $1.5 \%$ & $0.66[0.35,1.28]$ \\
\hline Loftus 2010 & 14 & 52 & 13 & 50 & $1.6 \%$ & $1.04[0.54,1.98]$ \\
\hline Suzuki 1999 & 28 & 105 & 9 & 35 & $1.6 \%$ & $1.04[0.54,1.98]$ \\
\hline Lo 2008 & 8 & 15 & 10 & 15 & $1.8 \%$ & $0.80[0.44,1.45]$ \\
\hline Lahtinen 2004 & 18 & 44 & 13 & 46 & $1.9 \%$ & $1.45[0.81,2.59]$ \\
\hline Chazan 2010 & 10 & 24 & 15 & 22 & $2.0 \%$ & $0.61[0.35,1.06]$ \\
\hline Kakinohana 2004 & 14 & 25 & 12 & 25 & $2.1 \%$ & $1.17[0.68,1.99]$ \\
\hline Dualé 2009 & 19 & 39 & 15 & 41 & $2.3 \%$ & $1.33[0.79,2.23]$ \\
\hline Kwok 2004 & 30 & 90 & 15 & 45 & $2.4 \%$ & $1.00[0.60,1.66]$ \\
\hline Nielsen 2017 & 22 & 74 & 21 & 73 & $2.4 \%$ & $1.03[0.62,1.71]$ \\
\hline McKay 2007 & 10 & 19 & 16 & 22 & $2.4 \%$ & $0.72[0.44,1.19]$ \\
\hline Mebazaa MS 2008 & 17 & 67 & 30 & 67 & $2.5 \%$ & $0.57[0.35,0.92]$ \\
\hline Aqil 2011 & 43 & 90 & 12 & 30 & $2.5 \%$ & $1.19[0.73,1.95]$ \\
\hline Grady 2012 & 16 & 30 & 16 & 32 & $2.6 \%$ & $1.07[0.66,1.73]$ \\
\hline Karaman 2006 & 20 & 40 & 12 & 20 & $2.6 \%$ & $0.83[0.52,1.34]$ \\
\hline Song 2013 & 17 & 24 & 14 & 25 & $3.0 \%$ & $1.26[0.82,1.95]$ \\
\hline Jendoubi 2017 & 13 & 20 & 15 & 20 & $3.3 \%$ & $0.87[0.58,1.30]$ \\
\hline Pirim 2006 & 13 & 23 & 19 & 22 & $3.5 \%$ & $0.65[0.44,0.97]$ \\
\hline Gilabert Morell 2002 & 25 & 44 & 15 & 22 & $3.6 \%$ & $0.83[0.57,1.22]$ \\
\hline Remérand 2009 & 28 & 79 & 36 & 75 & $3.7 \%$ & $0.74[0.51,1.08]$ \\
\hline Aubrun 2008 & 23 & 45 & 32 & 45 & $4.3 \%$ & $0.72[0.51,1.01]$ \\
\hline Leal 2013 & 18 & 20 & 15 & 20 & $5.2 \%$ & $1.20[0.90,1.61]$ \\
\hline Leal 2015 & 22 & 28 & 21 & 28 & $5.3 \%$ & $1.05[0.79,1.40]$ \\
\hline Total $(95 \%$ CI) & & 3263 & & 2702 & $100.0 \%$ & $0.88[0.81,0.96]$ \\
\hline Total events: & 761 & & 731 & & & \\
\hline
\end{tabular}

Heterogeneity: $\mathrm{Tau}^{2}=0.01 ; \mathrm{Chi}^{2}=99.14, \mathrm{df}=90(\mathrm{P}=0.24) ; \mathrm{I}^{2}=9 \%$

Test for overall effect: $\mathrm{Z}=2.87(\mathrm{P}=0.004)$

Test for subgroup differences: Not applicable 
Comparison 2. Pre-incisional and postoperative ketamine versus control in a non-stratified patient population

\begin{tabular}{|c|c|c|c|c|}
\hline Outcome or subgroup title & No. of studies & $\begin{array}{l}\text { No. of partici- } \\
\text { pants }\end{array}$ & Statistical method & Effect size \\
\hline $\begin{array}{l}2.1 \text { Opioid consumption at } 24 \\
\text { hours }\end{array}$ & 28 & 1639 & $\begin{array}{l}\text { Mean Difference (IV, Random, } \\
95 \% \mathrm{CI})\end{array}$ & $-6.26[-8.42,-4.11]$ \\
\hline 2.1.1 Pre-incisional ketamine & 19 & 1045 & $\begin{array}{l}\text { Mean Difference (IV, Random, } \\
95 \% \mathrm{CI})\end{array}$ & $-5.54[-7.95,-3.12]$ \\
\hline 2.1.2 Postoperative ketamine & 9 & 594 & $\begin{array}{l}\text { Mean Difference (IV, Random, } \\
95 \% \mathrm{CI})\end{array}$ & $-8.66[-13.84,-3.49]$ \\
\hline $\begin{array}{l}2.2 \text { Opioid consumption at } 48 \\
\text { hours }\end{array}$ & 16 & 959 & $\begin{array}{l}\text { Mean Difference (IV, Random, } \\
95 \% \mathrm{CI})\end{array}$ & $-10.76[-14.84,-6.68]$ \\
\hline 2.2.1 Pre-incisional ketamine & 9 & 534 & $\begin{array}{l}\text { Mean Difference (IV, Random, } \\
95 \% \mathrm{CI})\end{array}$ & $-3.88[-7.04,-0.72]$ \\
\hline 2.2.2 Postoperative ketamine & 7 & 425 & $\begin{array}{l}\text { Mean Difference (IV, Random, } \\
95 \% \mathrm{CI} \text { ) }\end{array}$ & $-20.81[-27.39,-14.24]$ \\
\hline 2.3 Pain intensity at 24 hours & 29 & 1646 & $\begin{array}{l}\text { Mean Difference (IV, Random, } \\
95 \% \mathrm{CI})\end{array}$ & $-7.08[-9.56,-4.59]$ \\
\hline 2.3.1 Pre-incisional ketamine & 20 & 1075 & $\begin{array}{l}\text { Mean Difference (IV, Random, } \\
95 \% \mathrm{CI})\end{array}$ & $-6.65[-10.06,-3.24]$ \\
\hline 2.3.2 Postoperative ketamine & 9 & 571 & $\begin{array}{l}\text { Mean Difference (IV, Random, } \\
95 \% \mathrm{CI})\end{array}$ & $-8.30[-12.55,-4.05]$ \\
\hline 2.4 Pain intensity at 48 hours & 15 & 840 & $\begin{array}{l}\text { Mean Difference (IV, Random, } \\
95 \% \mathrm{CI} \text { ) }\end{array}$ & $-5.49[-7.72,-3.25]$ \\
\hline 2.4.1 Pre-incisional ketamine & 9 & 509 & $\begin{array}{l}\text { Mean Difference (IV, Random, } \\
95 \% \mathrm{CI})\end{array}$ & $-4.36[-7.53,-1.19]$ \\
\hline 2.4.2 Postoperative ketamine & 6 & 331 & $\begin{array}{l}\text { Mean Difference (IV, Random, } \\
95 \% \mathrm{Cl} \text { ) }\end{array}$ & $-8.02[-15.79,-0.26]$ \\
\hline $\begin{array}{l}2.5 \text { Time to first request for } \\
\text { analgesia/first trigger of PCA }\end{array}$ & 13 & 643 & $\begin{array}{l}\text { Mean Difference (IV, Random, } \\
95 \% \mathrm{CI} \text { ) }\end{array}$ & $37.70[20.87,54.52]$ \\
\hline
\end{tabular}


Analysis 2.1. Comparison 2: Pre-incisional and postoperative ketamine versus control in a non-stratified patient population, Outcome 1: Opioid consumption at 24 hours

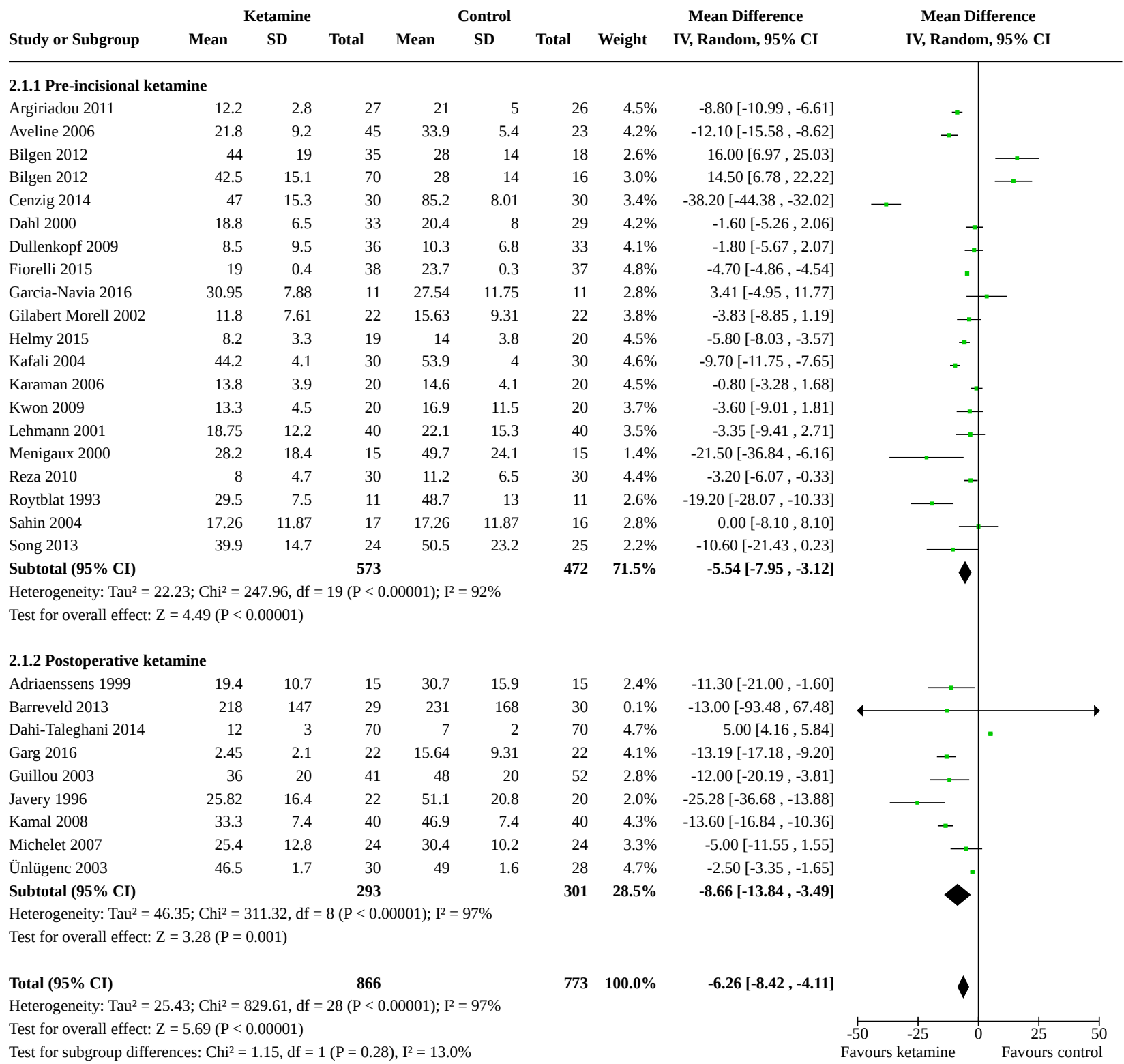


Analysis 2.2. Comparison 2: Pre-incisional and postoperative ketamine versus control in a non-stratified patient population, Outcome 2: Opioid consumption at 48 hours

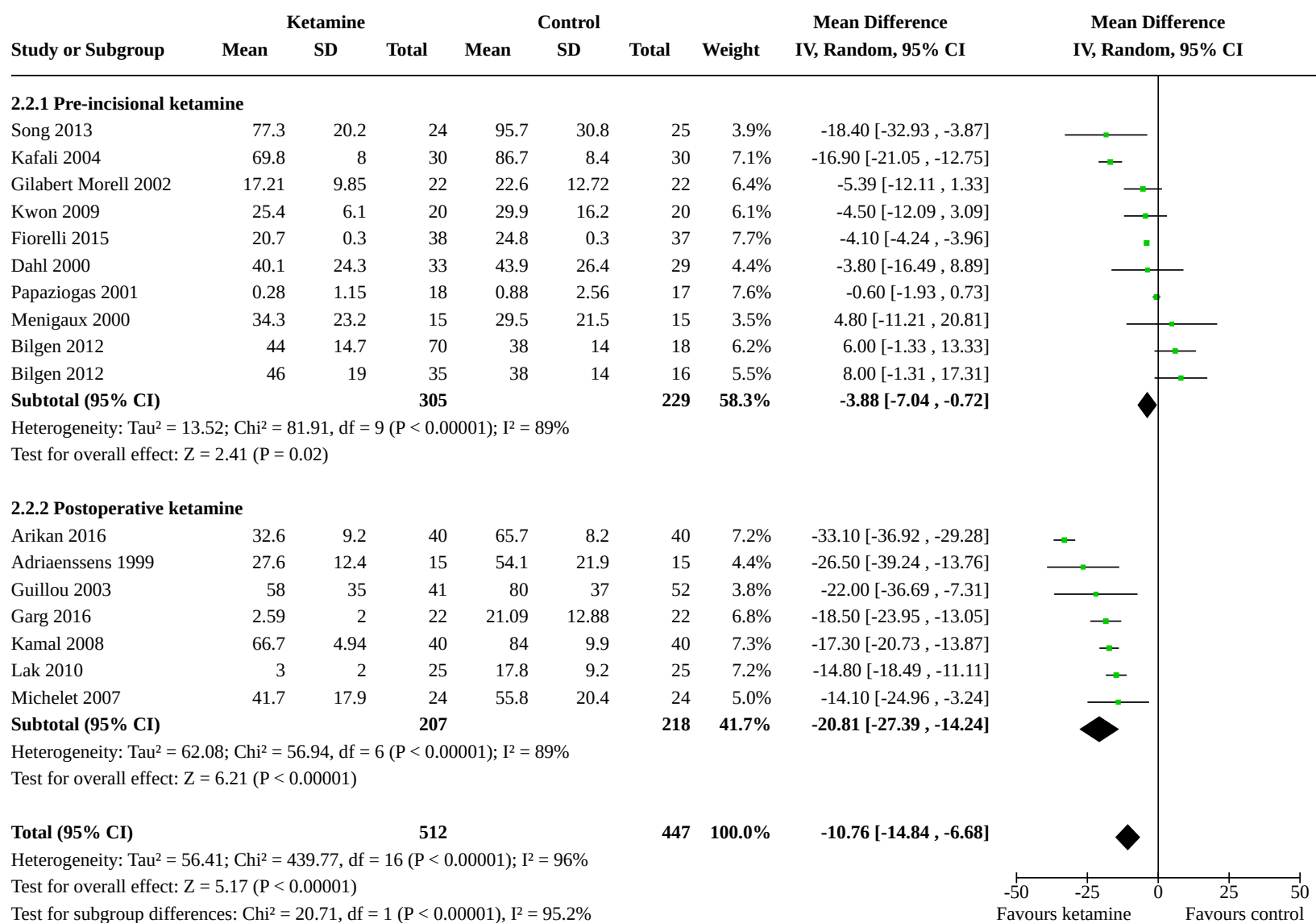


Analysis 2.3. Comparison 2: Pre-incisional and postoperative ketamine versus control in a non-stratified patient population, Outcome 3: Pain intensity at 24 hours

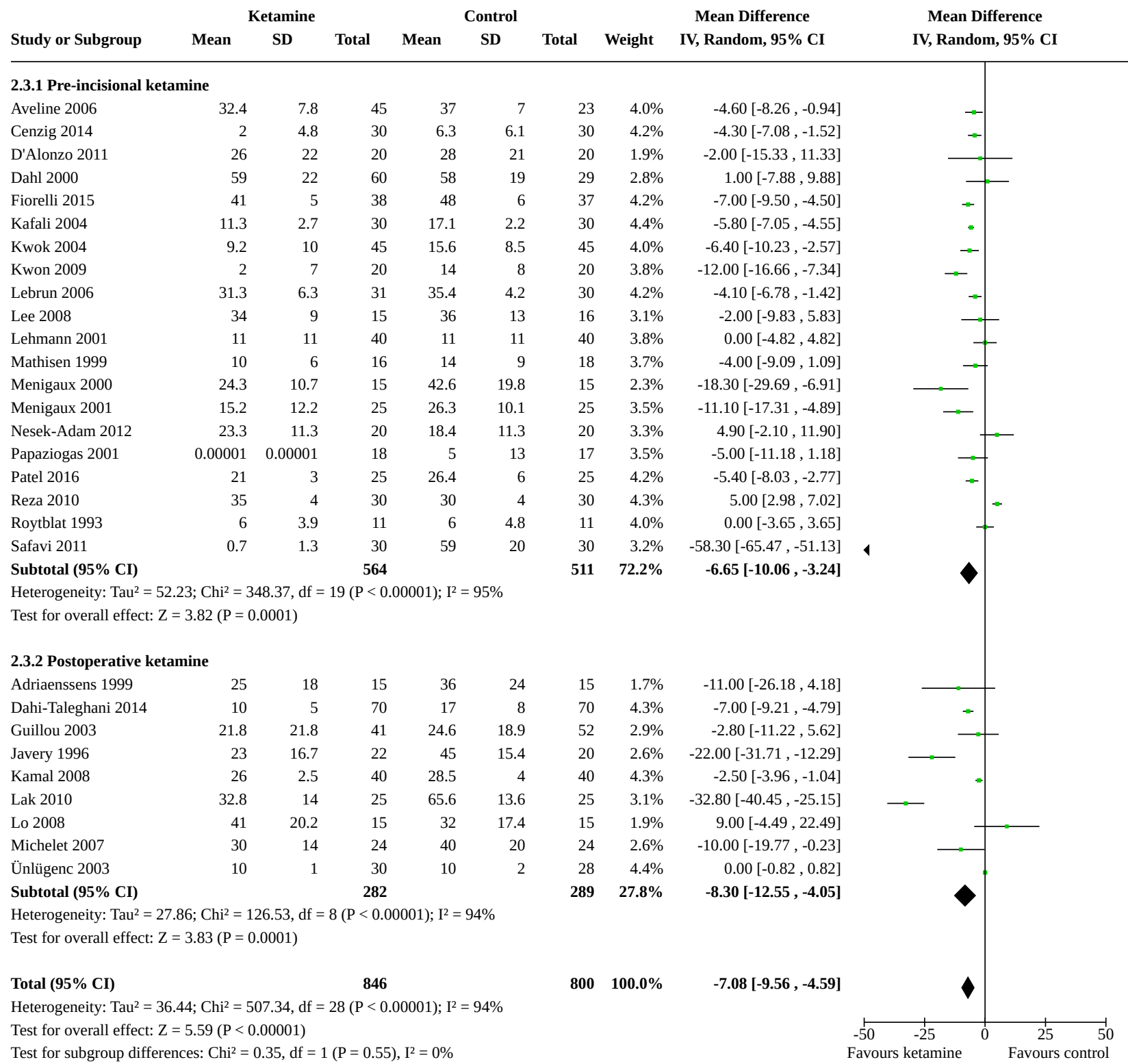


Analysis 2.4. Comparison 2: Pre-incisional and postoperative ketamine versus control in a non-stratified patient population, Outcome 4: Pain intensity at 48 hours

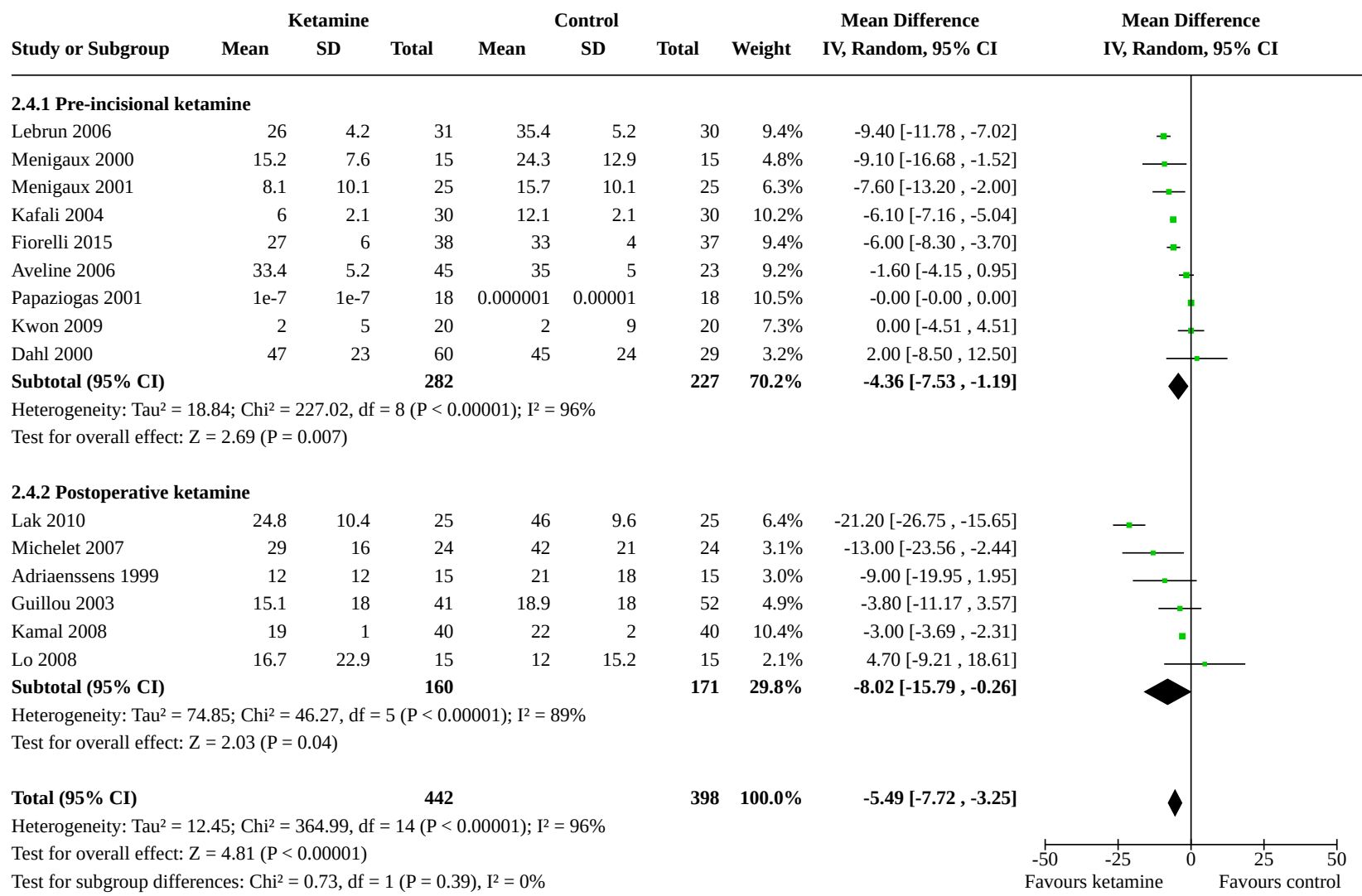

Analysis 2.5. Comparison 2: Pre-incisional and postoperative ketamine versus control in a nonstratified patient population, Outcome 5: Time to first request for analgesia/first trigger of PCA

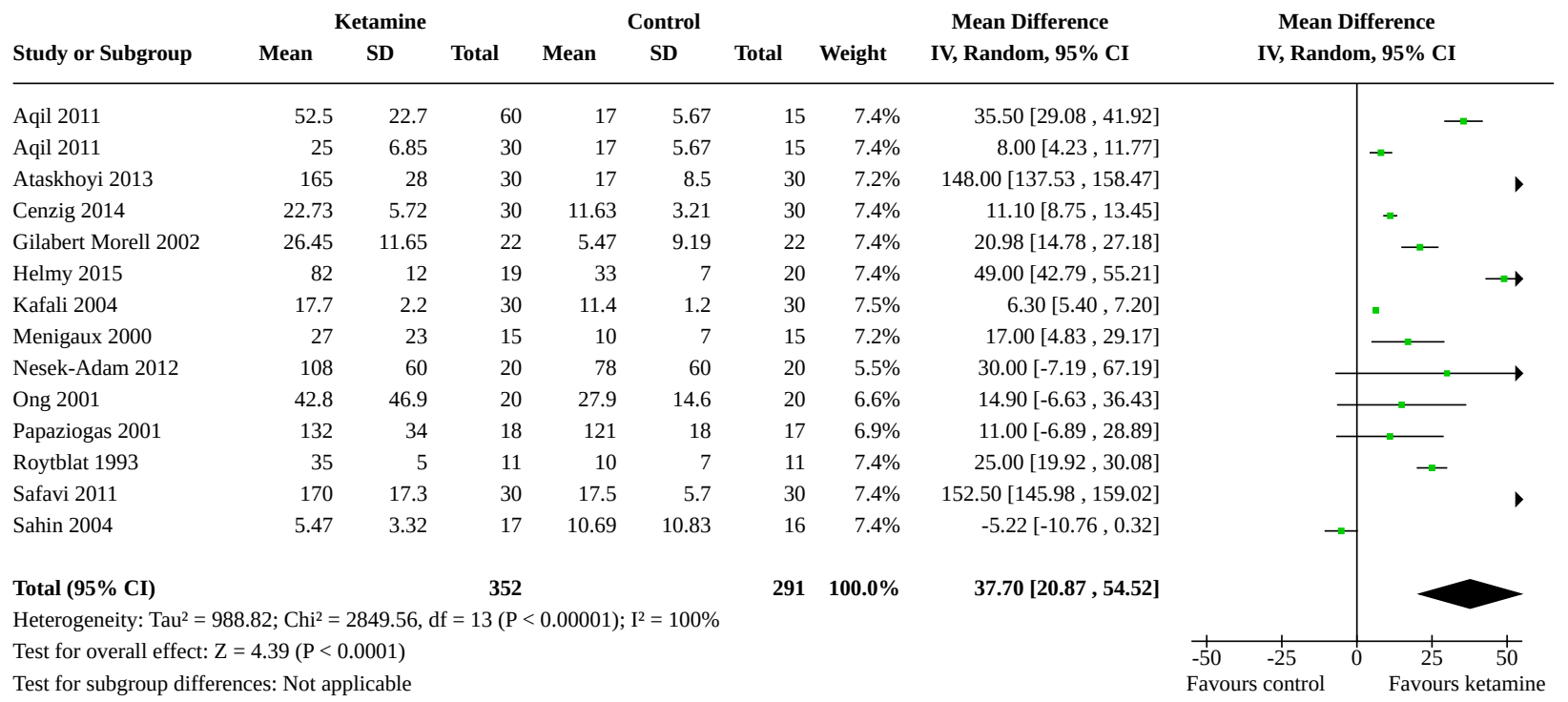


Comparison 3. Perioperative ketamine versus control co-administered with nitrous oxide in a non-stratified study population

\begin{tabular}{lllll}
\hline Outcome or subgroup title & No. of studies & $\begin{array}{l}\text { No. of partici- } \\
\text { pants }\end{array}$ & Statistical method & Effect size \\
\hline $\begin{array}{l}\text { 3.1 Opioid consumption at 24 } \\
\text { hours }\end{array}$ & 33 & 2176 & $\begin{array}{l}\text { Mean Difference (IV, Random, } \\
95 \% \text { Cl) }\end{array}$ & $-7.31[-9.78,-4.84]$ \\
\hline $\begin{array}{l}\text { 3.2 Opioid consumption at 48 } \\
\text { hours }\end{array}$ & 15 & 1110 & $\begin{array}{l}\text { Mean Difference (IV, Random, } \\
95 \% \text { CI) }\end{array}$ & $-14.78[-21.12$, \\
\hline $\begin{array}{l}\text { 3.3 Pain intensity at rest at 24 } \\
\text { hours }\end{array}$ & 32 & 2053 & $\begin{array}{l}\text { Mean Difference (IV, Random, } \\
95 \% \text { Cl) }\end{array}$ & -8.13 [-10.84, -5.42] \\
\hline $\begin{array}{l}\text { 3.4 Pain intensity during move- } \\
\text { ment at 24 hours }\end{array}$ & 10 & 613 & $\begin{array}{l}\text { Mean Difference (IV, Random, } \\
95 \% \text { CI) }\end{array}$ & $-6.50[-18.97,5.97]$ \\
\hline $\begin{array}{l}\text { 3.5 Pain intensity at rest at 48 } \\
\text { hours }\end{array}$ & 18 & 1202 & $\begin{array}{l}\text { Mean Difference (IV, Random, } \\
95 \% \text { Cl) }\end{array}$ & $-6.38[-9.91,-2.84]$ \\
\hline $\begin{array}{l}\text { 3.6 Pain intensity during move- } \\
\text { ment at 48 hours }\end{array}$ & 8 & 523 & $\begin{array}{l}\text { Mean Difference (IV, Random, } \\
95 \% \text { Cl) }\end{array}$ & $-4.47[-13.08,4.14]$ \\
\hline
\end{tabular}


Analysis 3.1. Comparison 3: Perioperative ketamine versus control co-administered with nitrous oxide in a non-stratified study population, Outcome 1: Opioid consumption at 24 hours

\begin{tabular}{|c|c|c|c|c|c|c|c|c|c|c|}
\hline \multirow{3}{*}{$\begin{array}{l}\text { Study or Subgroup } \\
\text { Adriaenssens } 1999\end{array}$} & \multicolumn{3}{|c|}{ Ketamine } & \multicolumn{3}{|c|}{ Control } & \multirow[b]{2}{*}{ Weight } & \multirow{2}{*}{$\begin{array}{c}\text { Mean Difference } \\
\text { IV, Random, 95\% CI }\end{array}$} & \multirow{2}{*}{\multicolumn{2}{|c|}{$\begin{array}{c}\text { Mean Difference } \\
\text { IV, Random, 95\% CI }\end{array}$}} \\
\hline & Mean & SD & Total & Mean & SD & Total & & & & \\
\hline & 19.4 & 10.7 & 15 & 30.7 & 15.9 & 15 & $2.3 \%$ & $-11.30[-21.00,-1.60]$ & $\leftarrow$ & \\
\hline Aveline 2006 & 21.8 & 9.2 & 45 & 33.9 & 5.4 & 23 & $3.3 \%$ & $-12.10[-15.58,-8.62]$ & & \\
\hline Aveline 2009 & 39.2 & 6.5 & 25 & 56.8 & 5.9 & 24 & $3.3 \%$ & $-17.60[-21.07,-14.13]$ & $\longleftarrow$ & \\
\hline Bilgen 2012 & 44 & 19 & 35 & 28 & 14 & 16 & $2.4 \%$ & $16.00[6.69,25.31]$ & & \\
\hline Bilgen 2012 & 42.5 & 15.1 & 70 & 28 & 14 & 18 & $2.7 \%$ & $14.50[7.13,21.87]$ & & \\
\hline Cenzig 2014 & 47 & 15.3 & 30 & 85.2 & 8.01 & 30 & $2.9 \%$ & $-38.20[-44.38,-32.02]$ & 4 & \\
\hline Crousier 2008 & 13 & 6.6 & 12 & 16.17 & 26 & 18 & $1.9 \%$ & $-3.17[-15.75,9.41]$ & & \\
\hline Dahi-Taleghani 2014 & 12 & 3 & 70 & 7 & 2 & 70 & $3.5 \%$ & $5.00[4.16,5.84]$ & & - \\
\hline Dahl 2000 & 19.2 & 6.3 & 60 & 20.4 & 8 & 29 & $3.3 \%$ & $-1.20[-4.52,2.12]$ & & - \\
\hline Dullenkopf 2009 & 8.8 & 9 & 77 & 10.3 & 6.8 & 33 & $3.4 \%$ & $-1.50[-4.57,1.57]$ & & . \\
\hline Garg 2016 & 2.45 & 2.1 & 22 & 15.64 & 9.31 & 22 & $3.3 \%$ & $-13.19[-17.18,-9.20]$ & $\longleftarrow$ & \\
\hline Gilabert Morell 2002 & 11.4 & 6.7 & 44 & 15.63 & 9.31 & 22 & $3.2 \%$ & $-4.23[-8.60,0.14]$ & & \\
\hline Grady 2012 & $1 \mathrm{e}-7$ & $1 \mathrm{e}-8$ & 30 & 0.000001 & 0.000001 & 32 & $3.6 \%$ & $-0.00[-0.00,-0.00]$ & & \\
\hline Guillou 2003 & 35.9 & 22.2 & 41 & 47.8 & 22.2 & 52 & $2.4 \%$ & $-11.90[-20.99,-2.81]$ & $\longleftarrow$ & \\
\hline Hadi 2010 & 35.2 & 8.9 & 30 & 60 & 2.6 & 15 & $3.3 \%$ & $-24.80[-28.25,-21.35]$ & 4 & \\
\hline Hadi 2013 & 35.2 & 8.9 & 30 & 60 & 2.6 & 15 & $3.3 \%$ & $-24.80[-28.25,-21.35]$ & 4 & \\
\hline Haliloglu 2015 & 25 & 3.7 & 26 & 36.4 & 3.6 & 26 & $3.5 \%$ & $-11.40[-13.38,-9.42]$ & & \\
\hline Hercock 1999 & 34.5 & 15.2 & 24 & 33 & 16 & 25 & $2.5 \%$ & $1.50[-7.24,10.24]$ & & \\
\hline Karaman 2006 & 13.8 & 4.1 & 40 & 14.6 & 4.1 & 20 & $3.5 \%$ & $-0.80[-3.00,1.40]$ & & - \\
\hline Katz 2004 & 51.9 & 33.3 & 97 & 52.3 & 32.8 & 46 & $2.0 \%$ & $-0.40[-11.97,11.17]$ & & \\
\hline Leal 2013 & 29 & 18.4 & 20 & 25.1 & 13.3 & 20 & $2.3 \%$ & $3.90[-6.05,13.85]$ & & \\
\hline Lehmann 2001 & 18.75 & 12.2 & 40 & 22.1 & 15.3 & 40 & $2.9 \%$ & $-3.35[-9.41,2.71]$ & & - \\
\hline Menigaux 2000 & 26.2 & 17.9 & 30 & 49.7 & 24.2 & 15 & $1.7 \%$ & $-23.50[-37.32,-9.68]$ & $\longleftarrow$ & \\
\hline Murdoch 2002 & 67.6 & 25.1 & 21 & 66.4 & 17.7 & 21 & $1.8 \%$ & $1.20[-11.94,14.34]$ & & \\
\hline Ögün 2001 & 10.62 & 4.6 & 16 & 16.27 & 3.58 & 15 & $3.4 \%$ & $-5.65[-8.54,-2.76]$ & - & \\
\hline Parikh 2011 & 5.8 & 1.48 & 30 & 18.1 & 1.8 & 30 & $3.5 \%$ & $-12.30[-13.13,-11.47]$ & 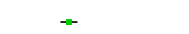 & \\
\hline Remérand 2009 & 14 & 13 & 79 & 19 & 12 & 75 & $3.3 \%$ & $-5.00[-8.95,-1.05]$ & & \\
\hline Reza 2010 & 8 & 4.7 & 30 & 11.2 & 6.5 & 30 & $3.4 \%$ & $-3.20[-6.07,-0.33]$ & & \\
\hline Roytblat 1993 & 29.5 & 4.8 & 11 & 48.7 & 6.4 & 11 & $3.1 \%$ & $-19.20[-23.93,-14.47]$ & $\leftarrow$ & \\
\hline Safavi 2011 & 1.864 & 0.43 & 30 & 12 & 4.2 & 30 & $3.5 \%$ & $-10.14[-11.65,-8.63]$ & & \\
\hline Sahin 2004 & 20.28 & 11.81 & 17 & 17.26 & 11.87 & 16 & $2.6 \%$ & $3.02[-5.06,11.10]$ & & \\
\hline Sen 2009 & 28 & 8 & 20 & 48 & 17 & 20 & $2.6 \%$ & $-20.00[-28.23,-11.77]$ & $\longleftarrow$ & \\
\hline Ünlügenc 2003 & 46.5 & 1.7 & 30 & 49 & 1.6 & 28 & $3.5 \%$ & $-2.50[-3.35,-1.65]$ & - & \\
\hline Zakine 2008 & 22.3 & 15.1 & 50 & 25.3 & 12.6 & 27 & $2.9 \%$ & $-3.00[-9.33,3.33]$ & & - \\
\hline Total (95\% CI) & & & 1247 & & & 929 & $100.0 \%$ & $-7.31[-9.78,-4.84]$ & & \\
\hline \multicolumn{11}{|c|}{ Heterogeneity: $\mathrm{Tau}^{2}=44.67 ; \mathrm{Chi}^{2}=2205.12, \mathrm{df}=33(\mathrm{P}<0.00001) ; \mathrm{I}^{2}=99 \%$} \\
\hline \multicolumn{9}{|c|}{ Test for overall effect: $Z=5.80(P<0.00001)$} & 5 & 0 \\
\hline
\end{tabular}


Analysis 3.2. Comparison 3: Perioperative ketamine versus control co-administered with nitrous oxide in a non-stratified study population, Outcome 2: Opioid consumption at 48 hours

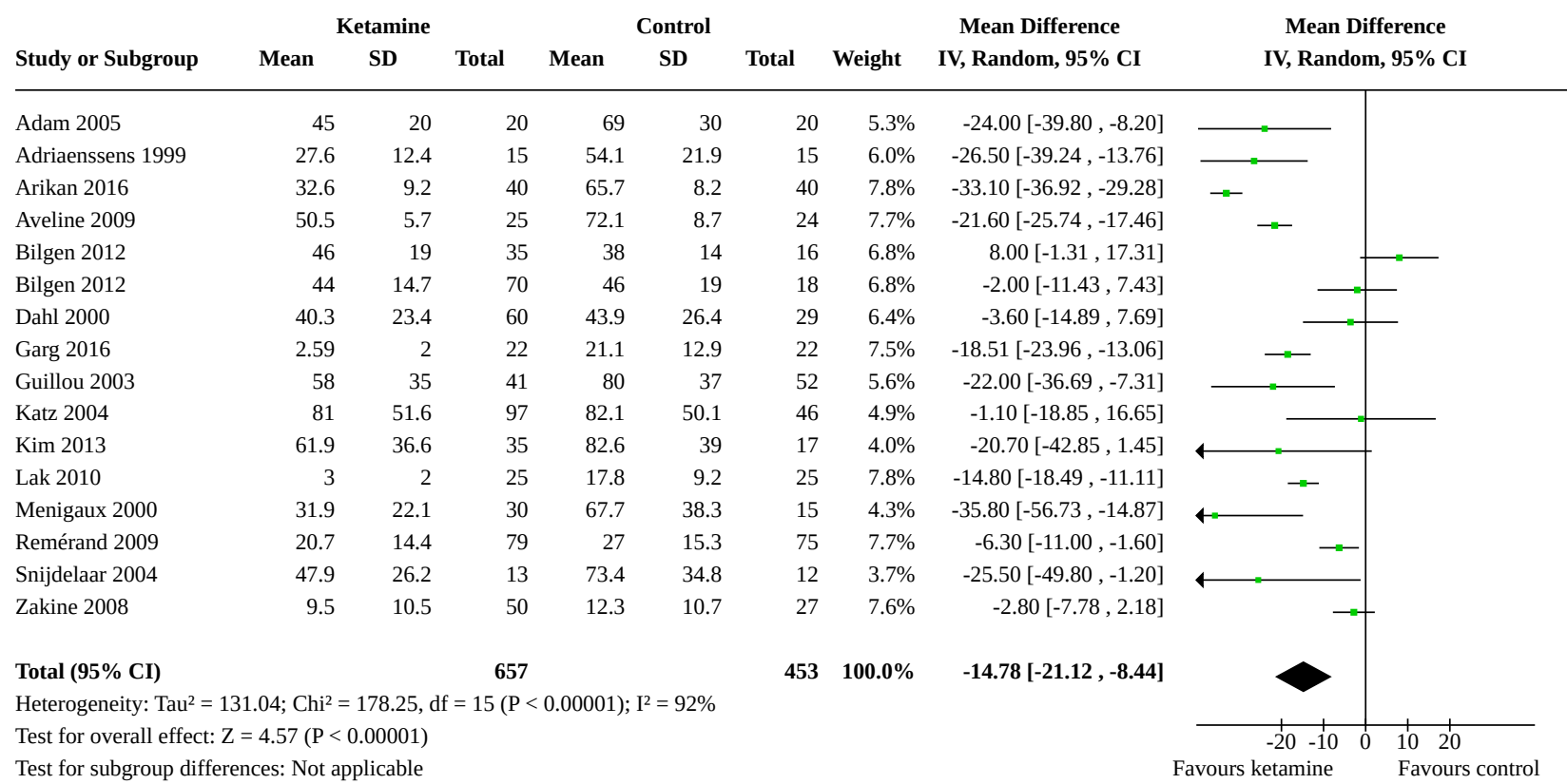


Analysis 3.3. Comparison 3: Perioperative ketamine versus control co-administered with nitrous oxide in a non-stratified study population, Outcome 3: Pain intensity at rest at 24 hours

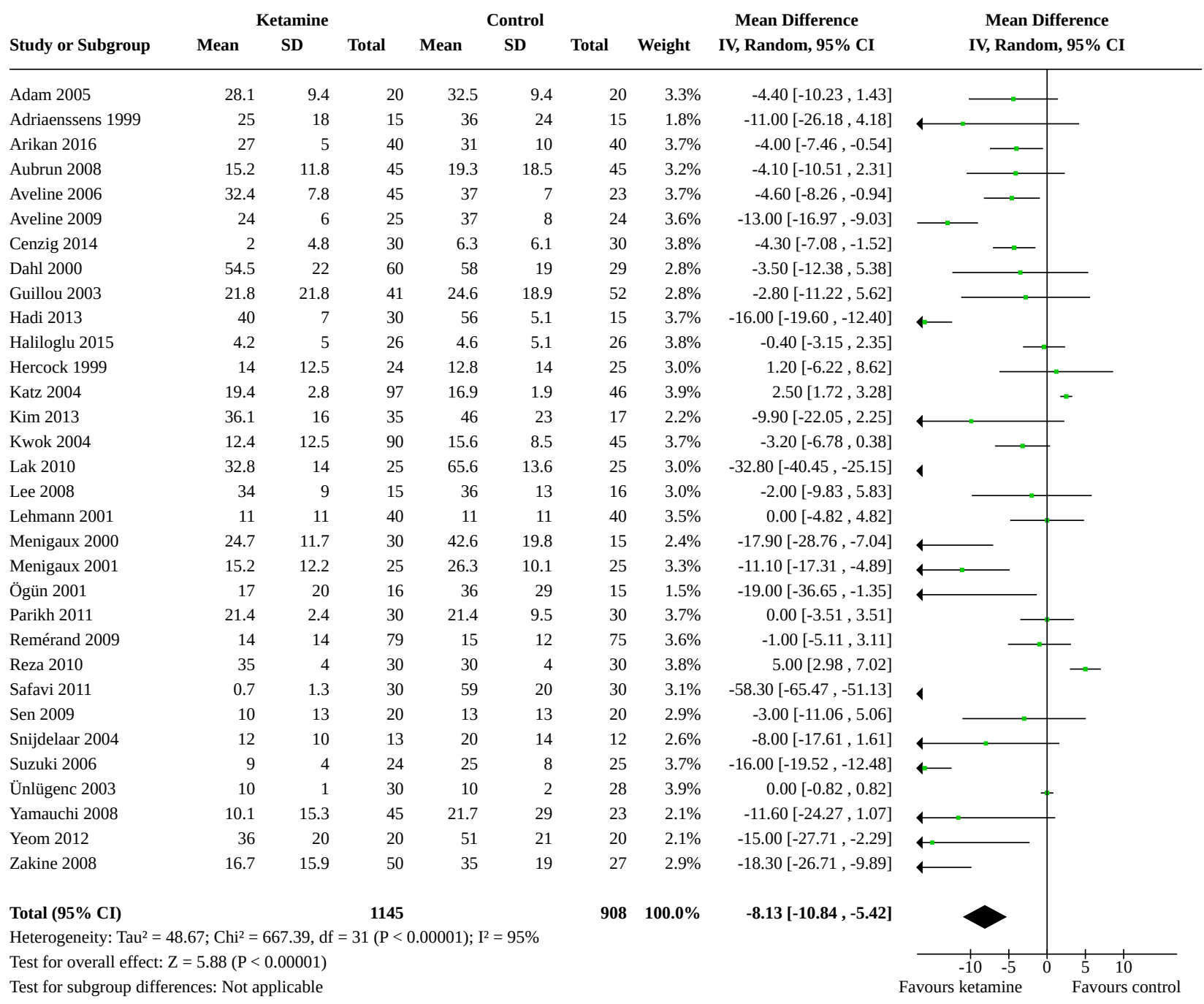


Analysis 3.4. Comparison 3: Perioperative ketamine versus control co-administered with nitrous oxide in a non-stratified study population, Outcome 4: Pain intensity during movement at 24 hours

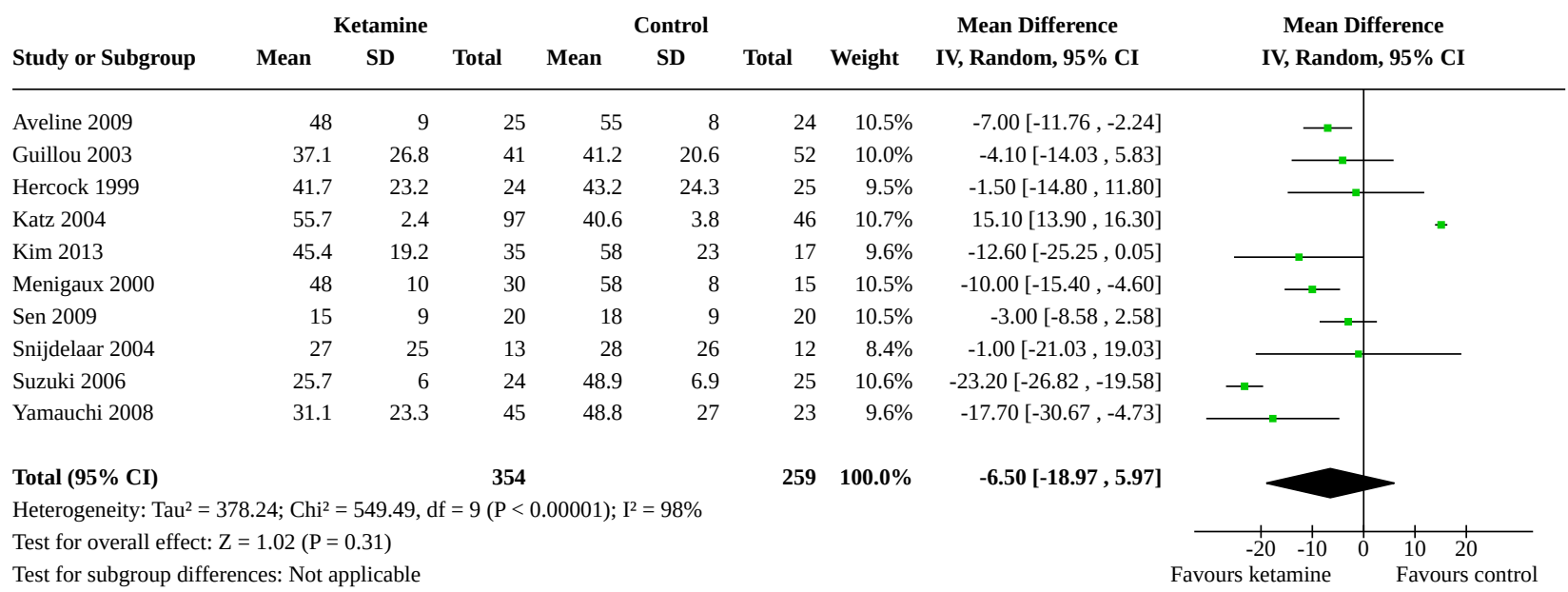

Analysis 3.5. Comparison 3: Perioperative ketamine versus control co-administered with nitrous oxide in a non-stratified study population, Outcome 5: Pain intensity at rest at 48 hours

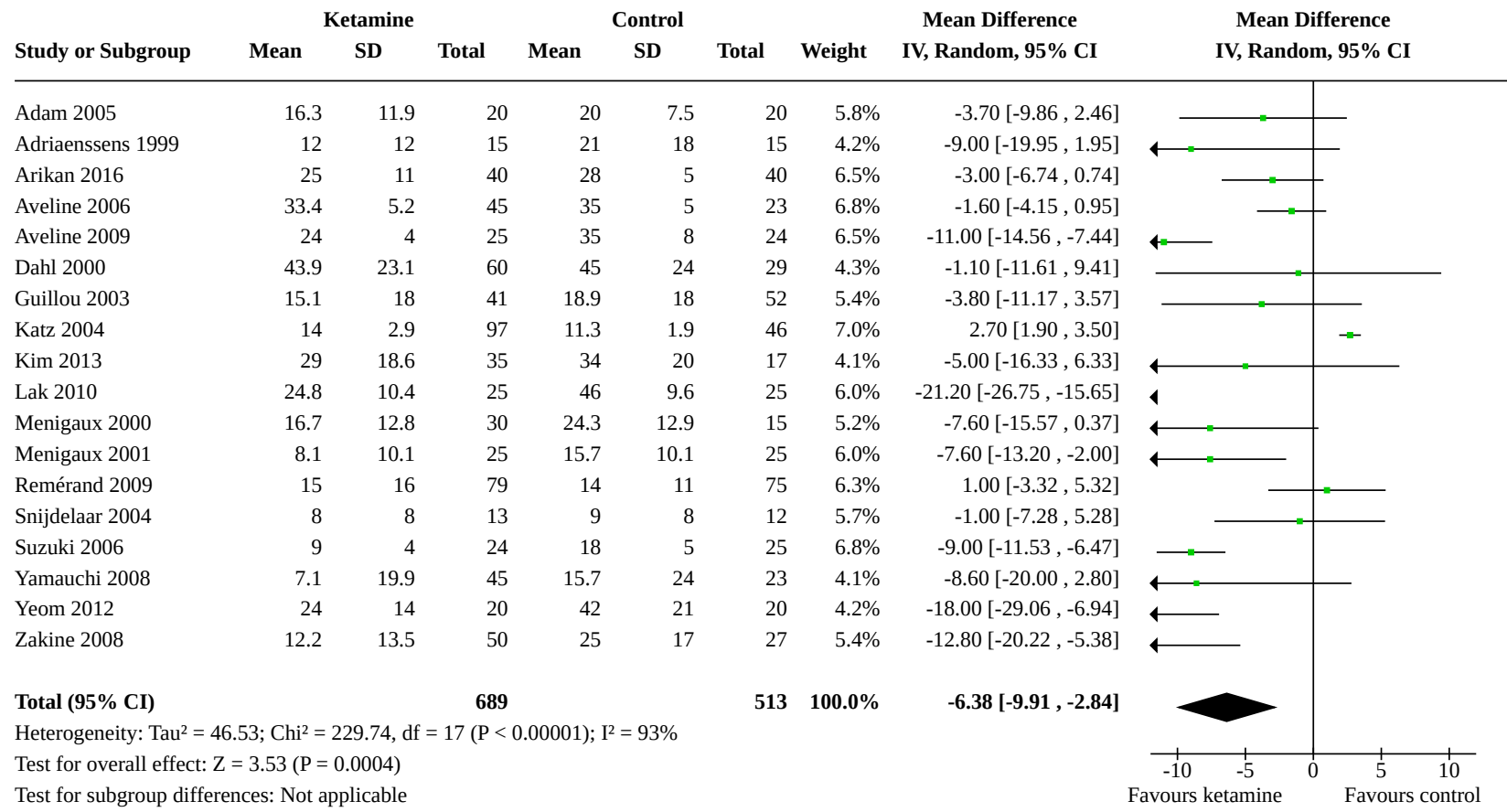


Analysis 3.6. Comparison 3: Perioperative ketamine versus control co-administered with nitrous oxide in a non-stratified study population, Outcome 6: Pain intensity during movement at 48 hours

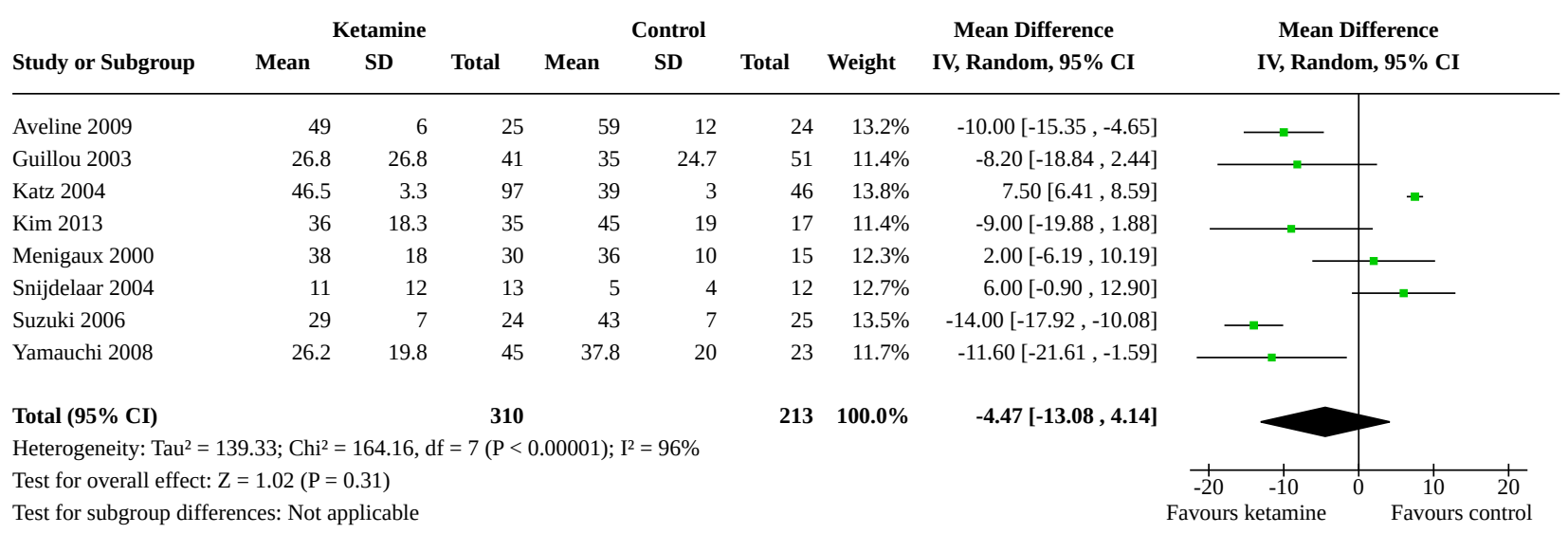

Comparison 4. CNS adverse events in studies with benzodiazepine premedication

\begin{tabular}{lllll}
\hline Outcome or subgroup title & No. of studies & $\begin{array}{l}\text { No. of partici- } \\
\text { pants }\end{array}$ & Statistical method & Effect size \\
\hline 4.1 CNS adverse events & 65 & 3943 & Risk Ratio (M-H, Random, 95\% Cl) & $1.09[0.86,1.38]$ \\
\hline
\end{tabular}


Analysis 4.1. Comparison 4: CNS adverse events in studies with benzodiazepine premedication, Outcome 1: CNS adverse events

\begin{tabular}{|c|c|c|c|c|c|c|c|}
\hline \multirow[b]{2}{*}{ Study or Subgroup } & \multicolumn{2}{|c|}{ Ketamine } & \multicolumn{2}{|c|}{ Control } & \multirow[b]{2}{*}{ Weight } & \multirow{2}{*}{$\begin{array}{c}\text { Risk Ratio } \\
\text { M-H, Random, 95\% CI }\end{array}$} & \multirow{2}{*}{$\begin{array}{c}\text { Risk Ratio } \\
\text { M-H, Random, 95\% CI }\end{array}$} \\
\hline & Events & Total & Events & Total & & & \\
\hline Adam 2005 & 0 & 20 & 0 & 20 & & Not estimable & \\
\hline Adriaenssens 1999 & 0 & 15 & 0 & 15 & & Not estimable & \\
\hline Aqil 2011 & 5 & 90 & 0 & 30 & $0.7 \%$ & $3.75[0.21,65.84]$ & \\
\hline Argiriadou 2004 & 0 & 30 & 0 & 15 & & Not estimable & \\
\hline Argiriadou 2011 & 0 & 27 & 0 & 26 & & Not estimable & \\
\hline Arikan 2016 & 1 & 40 & 0 & 40 & $0.6 \%$ & $3.00[0.13,71.51]$ & \\
\hline Ataskhoyi 2013 & 0 & 30 & 0 & 30 & & Not estimable & \\
\hline Aubrun 2008 & 5 & 45 & 3 & 45 & $3.0 \%$ & $1.67[0.42,6.56]$ & \\
\hline Aveline 2006 & 1 & 45 & 0 & 23 & $0.6 \%$ & $1.57[0.07,36.98]$ & \\
\hline Aveline 2009 & 0 & 25 & 0 & 24 & & Not estimable & \\
\hline Chazan 2010 & 1 & 24 & 2 & 22 & $1.0 \%$ & $0.46[0.04,4.71]$ & 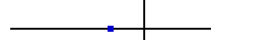 \\
\hline De Kock 2001 & 0 & 40 & 0 & 20 & & Not estimable & \\
\hline Dualé 2009 & 8 & 20 & 8 & 20 & $9.9 \%$ & $1.00[0.47,2.14]$ & - \\
\hline Dullenkopf 2009 & 1 & 77 & 0 & 33 & $0.6 \%$ & $1.31[0.05,31.29]$ & \\
\hline Fiorelli 2015 & 0 & 38 & 0 & 37 & & Not estimable & \\
\hline Galinski 2007 & 1 & 20 & 0 & 20 & $0.6 \%$ & $3.00[0.13,69.52]$ & \\
\hline Ganne 2005 & 0 & 30 & 0 & 31 & & Not estimable & \\
\hline Garcia-Navia 2016 & 0 & 11 & 0 & 11 & & Not estimable & \\
\hline Garg 2016 & 4 & 22 & 1 & 22 & $1.3 \%$ & $4.00[0.48,33.00]$ & \\
\hline Gilabert Morell 2002 & 0 & 44 & 0 & 22 & & Not estimable & \\
\hline Guignard 2002 & 0 & 25 & 0 & 25 & & Not estimable & \\
\hline Guillou 2003 & 1 & 41 & 1 & 52 & $0.8 \%$ & $1.27[0.08,19.67]$ & \\
\hline Hadi 2010 & 0 & 15 & 0 & 15 & & Not estimable & \\
\hline Hadi 2013 & 0 & 30 & 0 & 15 & & Not estimable & \\
\hline Hasanein 2011 & 0 & 30 & 0 & 30 & & Not estimable & \\
\hline Hayes 2004 & 6 & 22 & 6 & 23 & $6.1 \%$ & $1.05[0.40,2.75]$ & \\
\hline Hu 2014 & 7 & 31 & 4 & 47 & $4.4 \%$ & $2.65[0.85,8.31]$ & \\
\hline Ilkjaer 1998 & 3 & 24 & 2 & 28 & $2.0 \%$ & $1.75[0.32,9.62]$ & \\
\hline Jaksch 2002 & 0 & 15 & 0 & 15 & & Not estimable & \\
\hline Jendoubi 2017 & 0 & 20 & 0 & 20 & & Not estimable & \\
\hline Joly 2005 & 1 & 24 & 0 & 25 & $0.6 \%$ & $3.12[0.13,73.04]$ & \\
\hline Joseph 2012 & 1 & 22 & 1 & 25 & $0.8 \%$ & $1.14[0.08,17.11]$ & \\
\hline Kamal 2008 & 1 & 40 & 1 & 40 & $0.8 \%$ & $1.00[0.06,15.44]$ & \\
\hline Kararmaz 2003 & 2 & 20 & 1 & 20 & $1.1 \%$ & $2.00[0.20,20.33]$ & 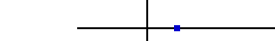 \\
\hline Katz 2004 & 4 & 97 & 1 & 46 & $1.2 \%$ & $1.90[0.22,16.50]$ & - \\
\hline Kim 2016 & 8 & 28 & 11 & 29 & $10.2 \%$ & $0.75[0.36,1.59]$ & $\rightarrow-$ \\
\hline Kwon 2009 & 0 & 20 & 0 & 20 & & Not estimable & \\
\hline Leal 2013 & 7 & 20 & 1 & 20 & $1.4 \%$ & $7.00[0.95,51.80]$ & \\
\hline Lebrun 2006 & 0 & 54 & 0 & 30 & & Not estimable & \\
\hline Lehmann 2001 & 0 & 40 & 0 & 40 & & Not estimable & \\
\hline Loftus 2010 & 1 & 52 & 1 & 50 & $0.8 \%$ & $0.96[0.06,14.96]$ & \\
\hline Mahran 2015 & 0 & 30 & 0 & 30 & & Not estimable & \\
\hline Martinez 2014 & 0 & 34 & 2 & 38 & $0.6 \%$ & $0.22[0.01,4.48]$ & \\
\hline Mathisen 1999 & 0 & 32 & 0 & 18 & & Not estimable & \\
\hline Mebazaa MS 2008 & 1 & 67 & 1 & 67 & $0.8 \%$ & $1.00[0.06,15.66]$ & \\
\hline Mendola 2012 & 0 & 32 & 0 & 30 & & Not estimable & \\
\hline Menigaux 2000 & 0 & 30 & 0 & 15 & & Not estimable & \\
\hline Menigaux 2001 & 0 & 25 & 0 & 25 & & Not estimable & \\
\hline Michelet 2007 & 0 & 24 & 0 & 24 & & Not estimable & \\
\hline Pacreu 2012 & 0 & 10 & 0 & 10 & & Not estimable & \\
\hline Papaziogas 2001 & 0 & 18 & 0 & 17 & & Not estimable & \\
\hline Pirim 2006 & 0 & 23 & 0 & 22 & & Not estimable & \\
\hline Remérand 2009 & 28 & 79 & 31 & 75 & $35.3 \%$ & $0.86[0.57,1.28]$ & \\
\hline
\end{tabular}




\section{Analysis 4.1. (Continued)}

\begin{tabular}{|c|c|c|c|c|c|}
\hline Pirim 2006 & 0 & 23 & 0 & 22 & \\
\hline Remérand 2009 & 28 & 79 & 31 & 75 & $35.3 \%$ \\
\hline Roytblat 1993 & 0 & 11 & 0 & 11 & \\
\hline Sen 2009 & 3 & 20 & 2 & 20 & $2.0 \%$ \\
\hline Siddiqui 2015 & 2 & 29 & 0 & 20 & $0.6 \%$ \\
\hline Singh 2013 & 2 & 60 & 0 & 20 & $0.6 \%$ \\
\hline Snijdelaar 2004 & 0 & 13 & 0 & 12 & \\
\hline Subramaniam 2011 & 5 & 15 & 9 & 15 & $8.3 \%$ \\
\hline Suzuki 1999 & 0 & 105 & 0 & 35 & \\
\hline Tena 2014 & 4 & 33 & 2 & 35 & $2.1 \%$ \\
\hline Van Elstraete 2004 & 0 & 20 & 0 & 20 & \\
\hline Yalcin 2012 & 7 & 26 & 0 & 27 & $0.7 \%$ \\
\hline Yazigi 2012 & 2 & 30 & 0 & 30 & $0.6 \%$ \\
\hline Zakine 2008 & 0 & 50 & 0 & 27 & \\
\hline Total $(95 \%$ CI) & & 2179 & & 1764 & $100.0 \%$ \\
\hline Total events: & 123 & & 91 & & \\
\hline \multirow{2}{*}{\multicolumn{6}{|c|}{$\begin{array}{l}\text { Heterogeneity: } \mathrm{Tau}^{2}=0.00 ; \mathrm{Chi}^{2}=24.37, \mathrm{df}=30(\mathrm{P}=0.76) ; \mathrm{I}^{2}=0 \% \\
\text { Test for overall effect: } \mathrm{Z}=0.68(\mathrm{P}=0.50)\end{array}$}} \\
\hline & & & & & \\
\hline Test for subgroup & & & & & \\
\hline
\end{tabular}

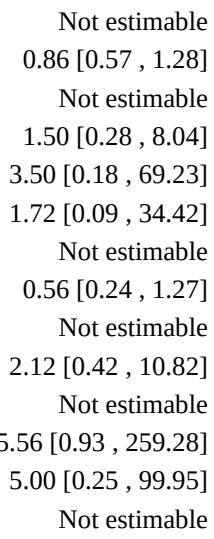

$1.09[0.86,1.38]$

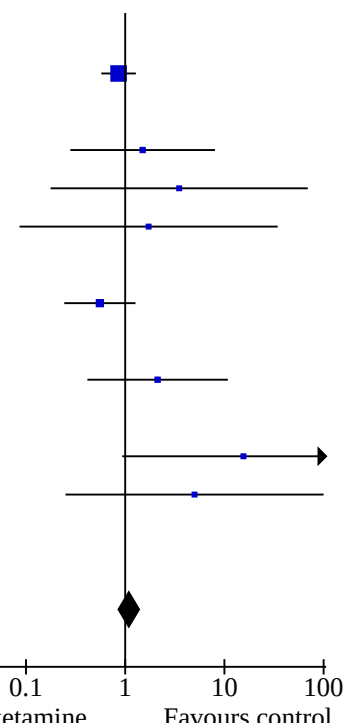

\section{Comparison 5. Perioperative ketamine versus control: thoracotomy}

\begin{tabular}{|c|c|c|c|c|}
\hline Outcome or subgroup title & No. of studies & $\begin{array}{l}\text { No. of partici- } \\
\text { pants }\end{array}$ & Statistical method & Effect size \\
\hline $\begin{array}{l}5.1 \text { Opioid consumption at } 24 \\
\text { hours }\end{array}$ & 4 & 241 & $\begin{array}{l}\text { Mean Difference (IV, Random, } \\
95 \% \mathrm{Cl} \text { ) }\end{array}$ & $-5.81[-10.28,-1.35]$ \\
\hline $\begin{array}{l}5.2 \text { Opioid consumption at } 48 \\
\text { hours }\end{array}$ & 3 & 191 & $\begin{array}{l}\text { Mean Difference (IV, Random, } \\
95 \% \mathrm{CI} \text { ) }\end{array}$ & $\begin{array}{l}-12.52[-18.34 \\
-6.71]\end{array}$ \\
\hline $\begin{array}{l}5.3 \text { Pain intensity at rest at } 24 \\
\text { hours }\end{array}$ & 13 & 782 & $\begin{array}{l}\text { Mean Difference (IV, Random, } \\
95 \% \mathrm{CI} \text { ) }\end{array}$ & $-3.90[-8.80,1.00]$ \\
\hline $\begin{array}{l}5.4 \text { Pain intensity during move- } \\
\text { ment at } 24 \text { hours }\end{array}$ & 5 & 315 & $\begin{array}{l}\text { Mean Difference (IV, Random, } \\
95 \% \mathrm{CI} \text { ) }\end{array}$ & $-7.32[-20.10,5.45]$ \\
\hline $\begin{array}{l}5.5 \text { Pain intensity at rest at } 48 \\
\text { hours }\end{array}$ & 9 & 530 & $\begin{array}{l}\text { Mean Difference (IV, Random, } \\
95 \% \mathrm{CI} \text { ) }\end{array}$ & $-6.86[-10.37,-3.35]$ \\
\hline $\begin{array}{l}\text { 5.6 Pain intensity during move- } \\
\text { ment at } 48 \text { hours }\end{array}$ & 5 & 298 & $\begin{array}{l}\text { Mean Difference (IV, Random, } \\
95 \% \mathrm{Cl})\end{array}$ & $\begin{array}{l}-10.64[-15.27 \\
-6.00]\end{array}$ \\
\hline
\end{tabular}


Analysis 5.1. Comparison 5: Perioperative ketamine versus control: thoracotomy, Outcome 1: Opioid consumption at 24 hours

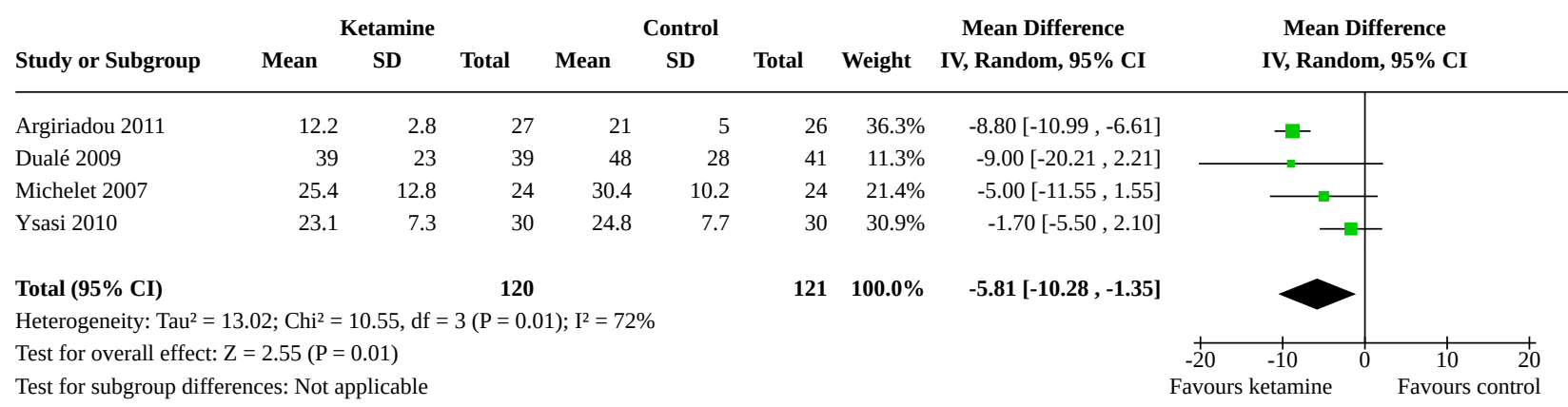

Analysis 5.2. Comparison 5: Perioperative ketamine versus control: thoracotomy, Outcome 2: Opioid consumption at 48 hours

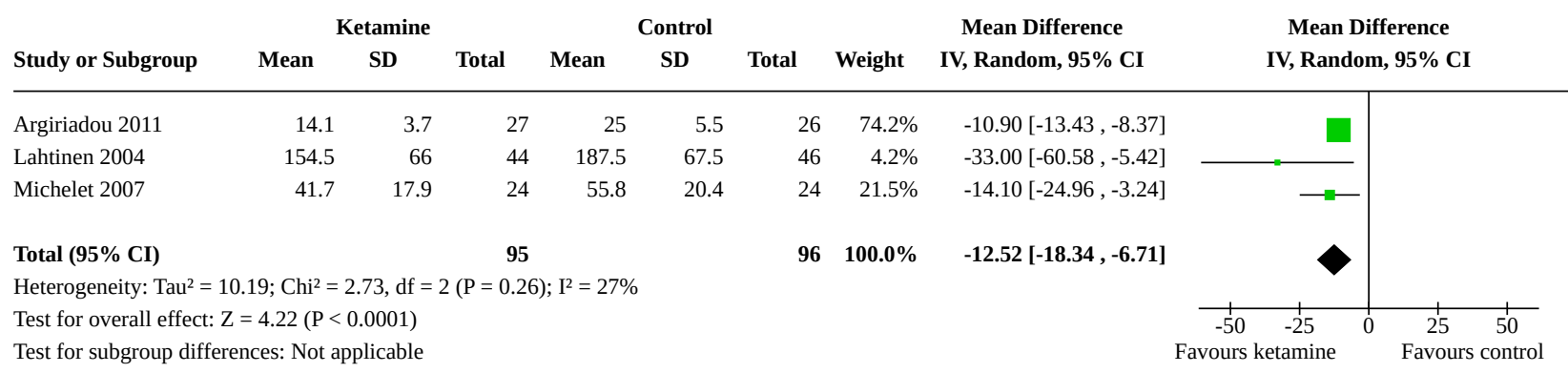

Analysis 5.3. Comparison 5: Perioperative ketamine versus control: thoracotomy, Outcome 3: Pain intensity at rest at 24 hours

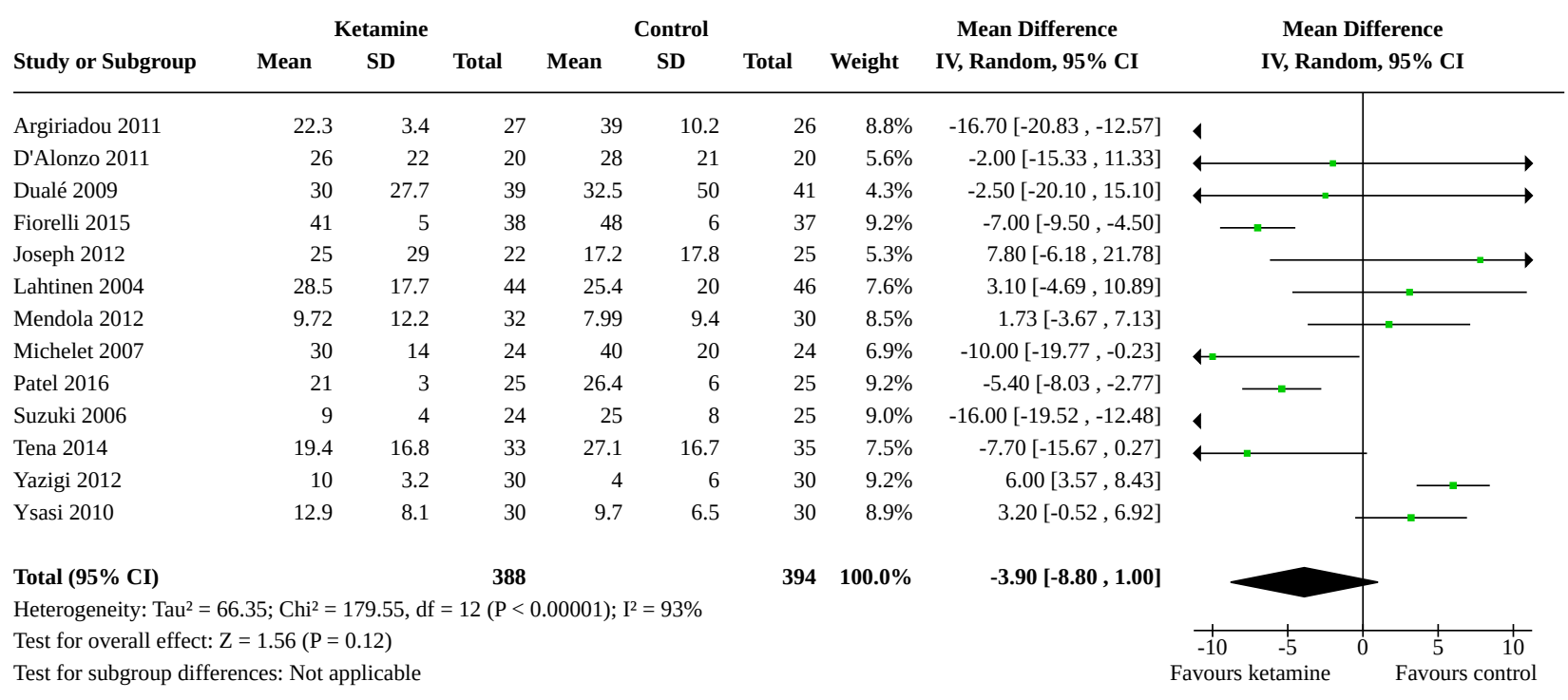


Analysis 5.4. Comparison 5: Perioperative ketamine versus control: thoracotomy, Outcome 4: Pain intensity during movement at 24 hours

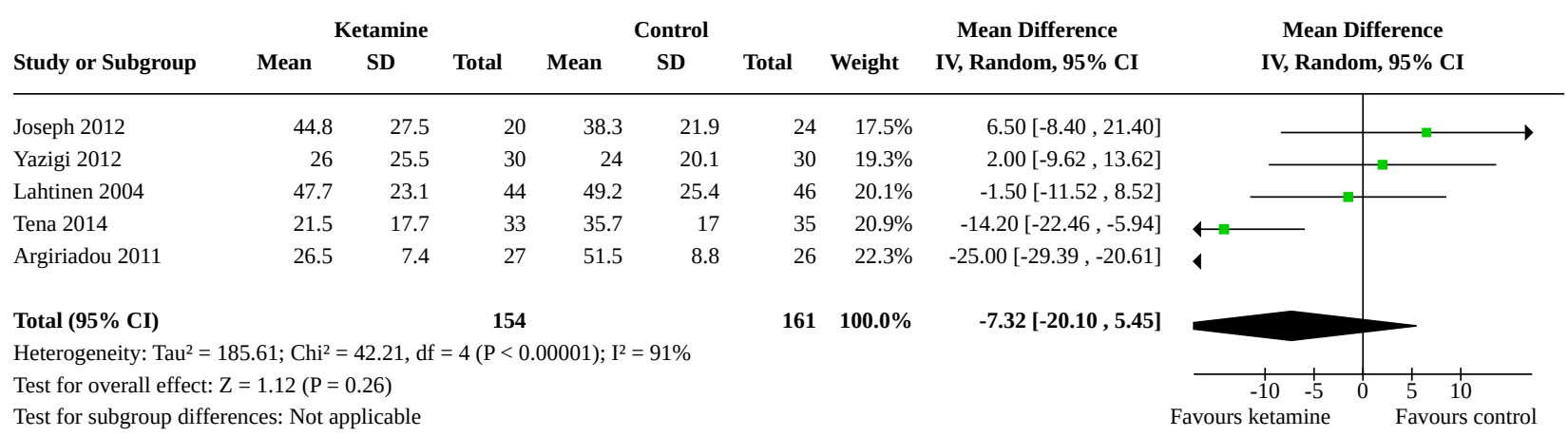

Analysis 5.5. Comparison 5: Perioperative ketamine versus control: thoracotomy, Outcome 5: Pain intensity at rest at $\mathbf{4 8}$ hours

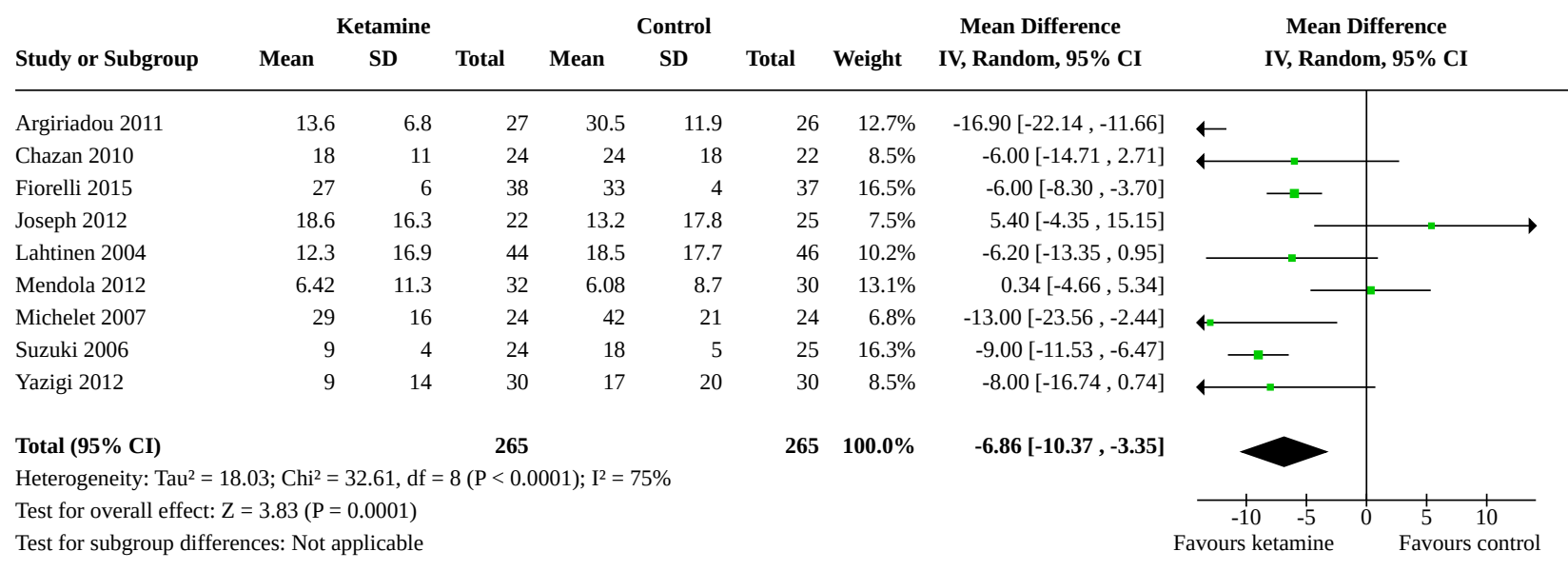

Analysis 5.6. Comparison 5: Perioperative ketamine versus control: thoracotomy, Outcome 6: Pain intensity during movement at 48 hours

\begin{tabular}{|c|c|c|c|c|c|c|c|c|c|c|}
\hline \multirow{3}{*}{$\begin{array}{l}\text { Study or Subgroup } \\
\text { Argiriadou } 2011\end{array}$} & \multicolumn{3}{|c|}{ Ketamine } & \multicolumn{3}{|c|}{ Control } & \multirow{2}{*}{ Weight } & \multirow{2}{*}{$\begin{array}{c}\text { Mean Difference } \\
\text { IV, Random, 95\% CI }\end{array}$} & \multirow{2}{*}{\multicolumn{2}{|c|}{$\begin{array}{c}\text { Mean Difference } \\
\text { IV, Random, 95\% CI }\end{array}$}} \\
\hline & Mean & SD & Total & Mean & SD & Total & & & & \\
\hline & 20.6 & 7.4 & 27 & 35.3 & 13.2 & 26 & $24.1 \%$ & $-14.70[-20.49,-8.91]$ & 4 & \\
\hline Joseph 2012 & 38.2 & 18.3 & 22 & 34.2 & 29.1 & 24 & $8.6 \%$ & $4.00[-9.93,17.93]$ & & \\
\hline Lahtinen 2004 & 12.3 & 16.9 & 44 & 18.5 & 17.7 & 46 & $20.1 \%$ & $-6.20[-13.35,0.95]$ & & \\
\hline Suzuki 2006 & 29 & 3.4 & 24 & 42.6 & 6.9 & 25 & $33.1 \%$ & $-13.60[-16.63,-10.57]$ & $\rightarrow$ & \\
\hline Yazigi 2012 & 32 & 19.5 & 30 & 44 & 19.5 & 30 & $14.0 \%$ & $-12.00[-21.87,-2.13]$ & & \\
\hline Total (95\% CI) & & & 147 & & & 151 & $100.0 \%$ & $-10.64[-15.27,-6.00]$ & & \\
\hline \multicolumn{11}{|c|}{ Heterogeneity: $\mathrm{Tau}^{2}=14.51 ; \mathrm{Chi}^{2}=9.43, \mathrm{df}=4(\mathrm{P}=0.05) ; \mathrm{I}^{2}=58 \%$} \\
\hline \multicolumn{9}{|c|}{ Test for overall effect: $\mathrm{Z}=4.50(\mathrm{P}<0.00001)$} & & $\begin{array}{ll}1 & 1 \\
5 & 10\end{array}$ \\
\hline \multicolumn{9}{|c|}{ Test for subgroup differences: Not applicable } & Favours ketan & Favours \\
\hline
\end{tabular}


Comparison 6. Perioperative ketamine versus control: major orthopaedic surgery

\begin{tabular}{|c|c|c|c|c|}
\hline Outcome or subgroup title & No. of studies & $\begin{array}{l}\text { No. of partici- } \\
\text { pants }\end{array}$ & Statistical method & Effect size \\
\hline $\begin{array}{l}6.1 \text { Opioid consumption at } 24 \\
\text { hours }\end{array}$ & 10 & 797 & $\begin{array}{l}\text { Mean Difference (IV, Random, } \\
95 \% \mathrm{CI})\end{array}$ & $\begin{array}{l}-19.68[-28.55 \\
-10.82]\end{array}$ \\
\hline $\begin{array}{l}6.2 \text { Opioid consumption at } 48 \\
\text { hours }\end{array}$ & 9 & 557 & $\begin{array}{l}\text { Mean Difference (IV, Random, } \\
95 \% \mathrm{CI})\end{array}$ & $\begin{array}{l}-18.69[-27.47 \\
-9.90]\end{array}$ \\
\hline $\begin{array}{l}6.3 \text { Pain intensity at rest at } 24 \\
\text { hours }\end{array}$ & 11 & 843 & $\begin{array}{l}\text { Mean Difference (IV, Random, } \\
95 \% \mathrm{CI})\end{array}$ & $-6.45[-9.86,-3.03]$ \\
\hline $\begin{array}{l}\text { 6.4 Pain intensity during move- } \\
\text { ment at } 24 \text { hours }\end{array}$ & 4 & 279 & $\begin{array}{l}\text { Mean Difference (IV, Random, } \\
95 \% \mathrm{CI})\end{array}$ & $-6.73[-12.64,-0.82]$ \\
\hline $\begin{array}{l}6.5 \text { Pain intensity at rest at } 48 \\
\text { hours }\end{array}$ & 7 & 453 & $\begin{array}{l}\text { Mean Difference (IV, Random, } \\
95 \% \mathrm{CI})\end{array}$ & $-1.39[-4.10,1.32]$ \\
\hline $\begin{array}{l}\text { 6.6 Pain intensity during move- } \\
\text { ment at } 48 \text { hours }\end{array}$ & 4 & 157 & $\begin{array}{l}\text { Mean Difference (IV, Random, } \\
95 \% \mathrm{CI})\end{array}$ & $-7.36[-13.12,-1.60]$ \\
\hline
\end{tabular}

\section{Analysis 6.1. Comparison 6: Perioperative ketamine versus control: major orthopaedic surgery, Outcome 1: Opioid consumption at 24 hours}

\begin{tabular}{|c|c|c|c|c|c|c|c|c|c|c|c|}
\hline \multirow{3}{*}{$\begin{array}{l}\text { Study or Subgroup } \\
\text { Cenzig } 2014\end{array}$} & \multicolumn{3}{|c|}{ Ketamine } & \multicolumn{3}{|c|}{ Control } & \multirow{2}{*}{\multicolumn{2}{|c|}{$\begin{array}{c}\text { Mean Difference } \\
\text { IV, Random, 95\% CI }\end{array}$}} & \multirow{2}{*}{\multicolumn{3}{|c|}{$\begin{array}{c}\text { Mean Difference } \\
\text { IV, Random, 95\% CI }\end{array}$}} \\
\hline & Mean & SD & Total & Mean & SD & Total & & & & & \\
\hline & 47 & 15.3 & 30 & 85.2 & 8.01 & 30 & $13.2 \%$ & $-38.20[-44.38,-32.02]$ & - & & \\
\hline Dahi-Taleghani 2014 & 7 & 2 & 70 & 12 & 3 & 70 & $14.1 \%$ & $-5.00[-5.84,-4.16]$ & - & & \\
\hline Garg 2016 & 2.45 & 2.1 & 22 & 15.64 & 9.31 & 22 & $13.7 \%$ & $-13.19[-17.18,-9.20]$ & $=$ & & \\
\hline Hadi 2010 & 35.2 & 8.9 & 30 & 60 & 2.6 & 15 & $13.8 \%$ & $-24.80[-28.25,-21.35]$ & $=$ & & \\
\hline Jaksch 2002 & 40.2 & 17 & 15 & 44.1 & 45 & 15 & $6.8 \%$ & $-3.90[-28.24,20.44]$ & & & \\
\hline Loftus 2010 & 142 & 82 & 52 & 202 & 176 & 50 & $2.3 \%$ & $-60.00[-113.63,-6.37]$ & 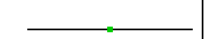 & & \\
\hline Menigaux 2000 & 26.2 & 17.9 & 30 & 49.7 & 24.2 & 15 & $10.5 \%$ & $-23.50[-37.32,-9.68]$ & -- & & \\
\hline Nielsen 2017 & 79 & 47 & 74 & 121 & 53 & 73 & $9.6 \%$ & $-42.00[-58.20,-25.80]$ & - & & \\
\hline Remérand 2009 & 14 & 13 & 79 & 19 & 12 & 75 & $13.7 \%$ & $-5.00[-8.95,-1.05]$ & $=$ & & \\
\hline Subramaniam 2011 & 103.6 & 87.8 & 15 & 96.8 & 67.85 & 15 & $2.1 \%$ & $6.80[-49.35,62.95]$ & & & \\
\hline Total (95\% CI) & & & 417 & & & 380 & $100.0 \%$ & $-19.68[-28.55,-10.82]$ & & & \\
\hline \multicolumn{12}{|c|}{ Heterogeneity: Tau$^{2}=144.84 ; \mathrm{Chi}^{2}=259.65, \mathrm{df}=9(\mathrm{P}<0.00001) ; \mathrm{I}^{2}=97 \%$} \\
\hline \multicolumn{9}{|c|}{ Test for overall effect: $\mathrm{Z}=4.35(\mathrm{P}<0.0001)$} & -100 & 50 & $\frac{1}{100}$ \\
\hline \multicolumn{9}{|c|}{ Test for subgroup differences: Not applicable } & Favours ketamine & Favour & control \\
\hline
\end{tabular}


Analysis 6.2. Comparison 6: Perioperative ketamine versus control: major orthopaedic surgery, Outcome 2: Opioid consumption at 48 hours

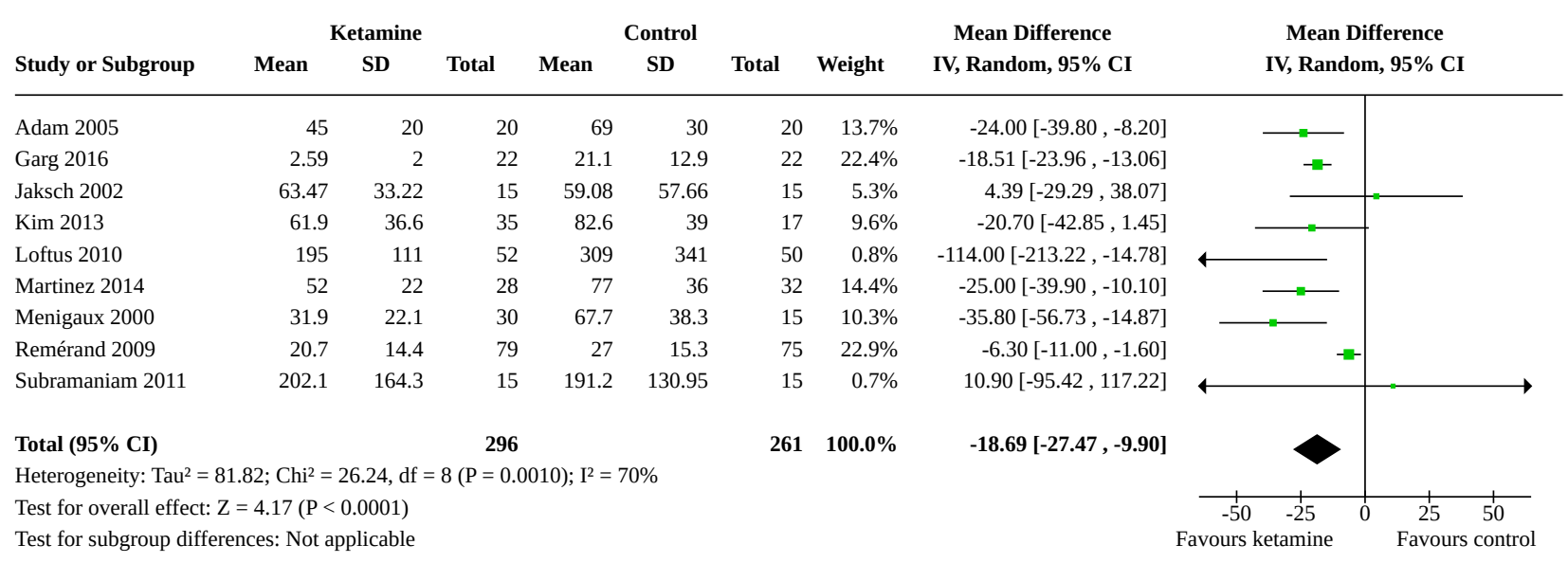

Analysis 6.3. Comparison 6: Perioperative ketamine versus control: major orthopaedic surgery, Outcome 3: Pain intensity at rest at 24 hours

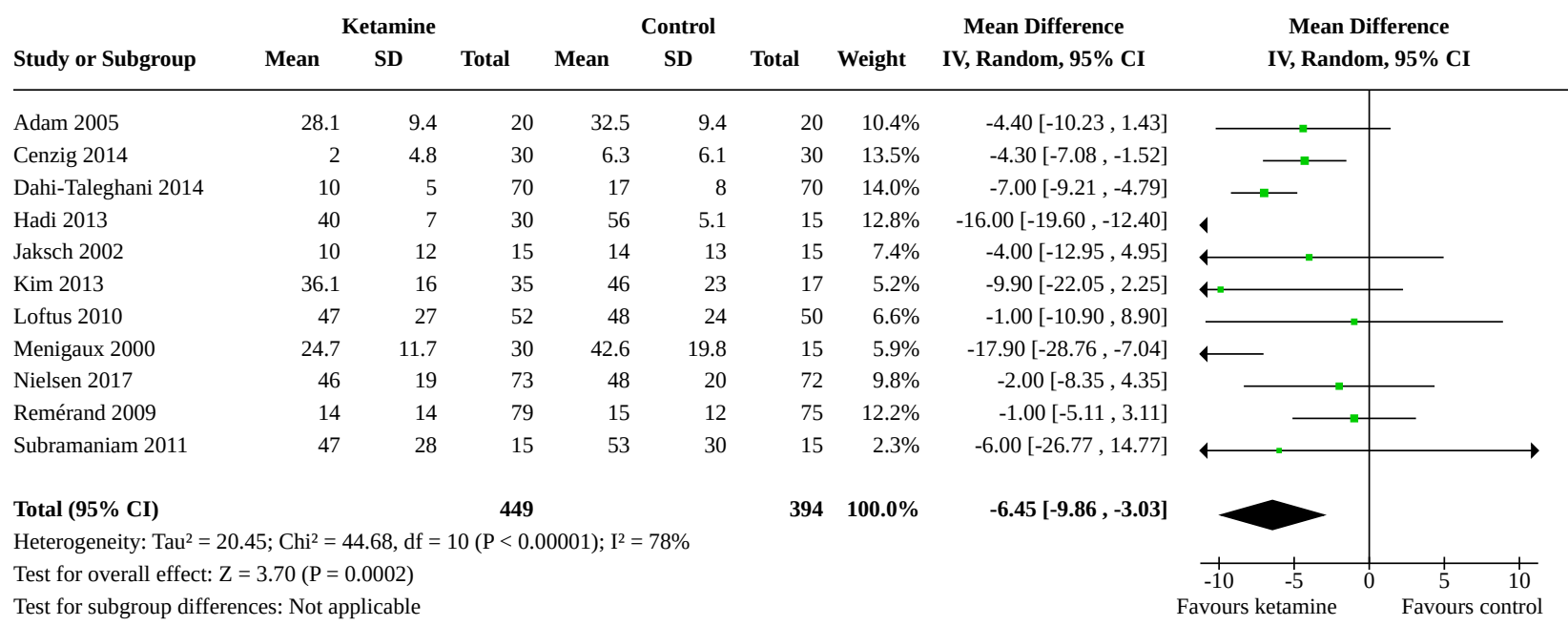

Analysis 6.4. Comparison 6: Perioperative ketamine versus control: major orthopaedic surgery, Outcome 4: Pain intensity during movement at 24 hours

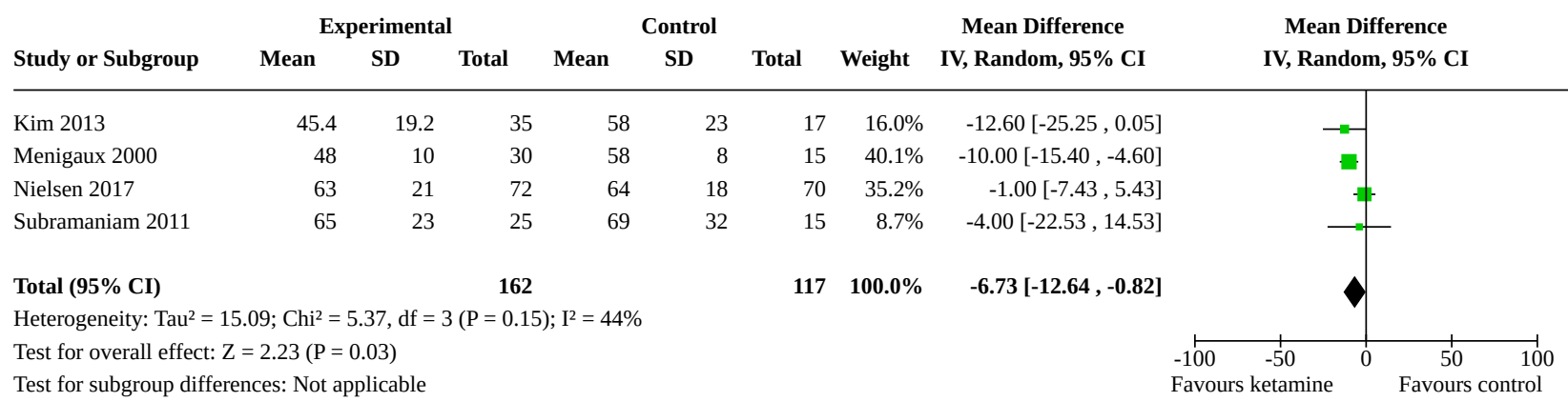


Analysis 6.5. Comparison 6: Perioperative ketamine versus control: major orthopaedic surgery, Outcome 5: Pain intensity at rest at 48 hours

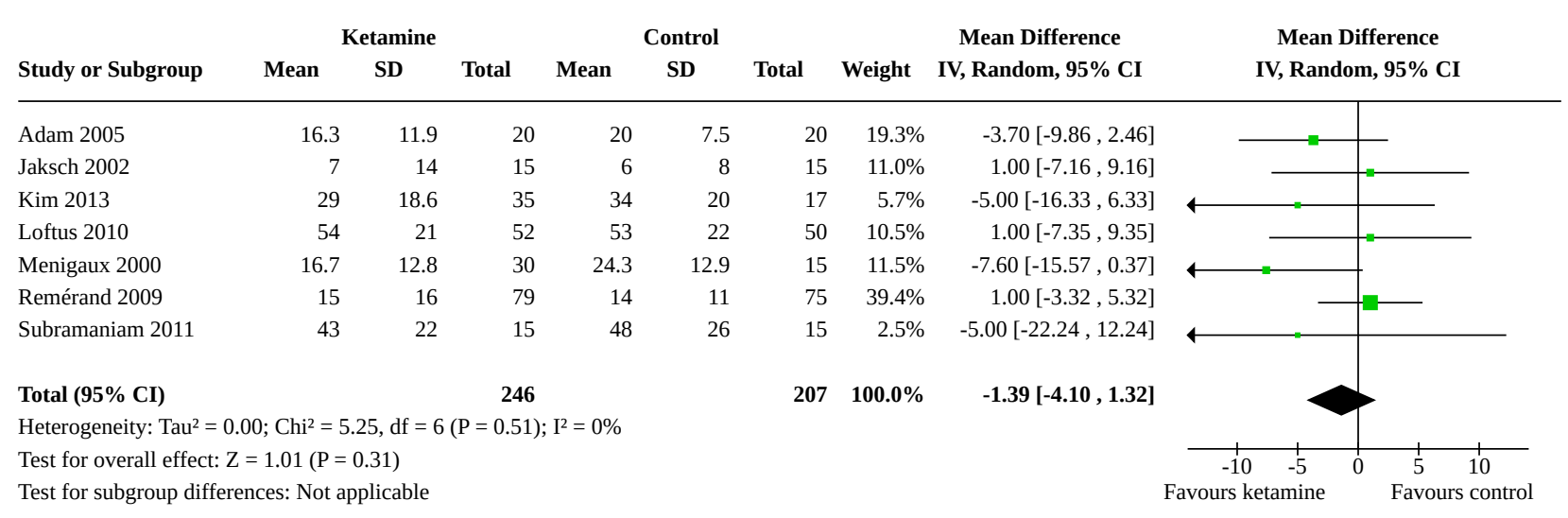

Analysis 6.6. Comparison 6: Perioperative ketamine versus control: major orthopaedic surgery, Outcome 6: Pain intensity during movement at $\mathbf{4 8}$ hours

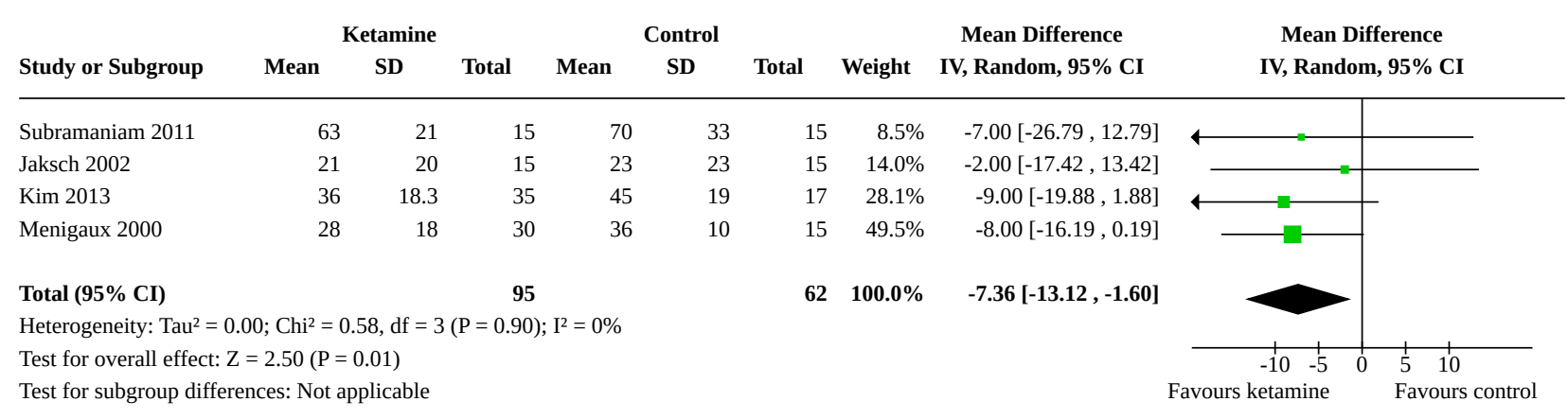

Comparison 7. Perioperative ketamine versus control: major abdominal surgery

\begin{tabular}{|c|c|c|c|c|}
\hline Outcome or subgroup title & No. of studies & $\begin{array}{l}\text { No. of partici- } \\
\text { pants }\end{array}$ & Statistical method & Effect size \\
\hline $\begin{array}{l}7.1 \text { Opioid consumption at } 24 \\
\text { hours }\end{array}$ & 16 & 1029 & $\begin{array}{l}\text { Mean Difference (IV, Random, } \\
95 \% \mathrm{CI} \text { ) }\end{array}$ & $\begin{array}{l}-10.26[-13.75 \\
-6.76]\end{array}$ \\
\hline $\begin{array}{l}7.2 \text { Opioid consumption at } 48 \\
\text { hours }\end{array}$ & 10 & 704 & $\begin{array}{l}\text { Mean Difference (IV, Random, } \\
95 \% \mathrm{CI} \text { ) }\end{array}$ & $\begin{array}{l}-14.34[-21.21 \\
-7.48]\end{array}$ \\
\hline $\begin{array}{l}7.3 \text { Pain intensity at rest at } 24 \\
\text { hours }\end{array}$ & 18 & 1178 & $\begin{array}{l}\text { Mean Difference (IV, Random, } \\
95 \% \mathrm{CI} \text { ) }\end{array}$ & $-7.42[-10.63,-4.21]$ \\
\hline $\begin{array}{l}\text { 7.4 Pain intensity during move- } \\
\text { ment at } 24 \text { hours }\end{array}$ & 9 & 666 & $\begin{array}{l}\text { Mean Difference (IV, Random, } \\
95 \% \mathrm{CI})\end{array}$ & $-2.80[-11.24,5.65]$ \\
\hline $\begin{array}{l}7.5 \text { Pain intensity at rest at } 48 \\
\text { hours }\end{array}$ & 13 & 891 & $\begin{array}{l}\text { Mean Difference (IV, Random, } \\
95 \% \mathrm{Cl})\end{array}$ & $-5.99[-8.89,-3.08]$ \\
\hline $\begin{array}{l}\text { 7.6 Pain intensity during move- } \\
\text { ment at } 48 \text { hours }\end{array}$ & 9 & 662 & $\begin{array}{l}\text { Mean Difference (IV, Random, } \\
95 \% \mathrm{Cl} \text { ) }\end{array}$ & $-2.91[-9.15,3.34]$ \\
\hline
\end{tabular}


Analysis 7.1. Comparison 7: Perioperative ketamine versus control: major abdominal surgery, Outcome 1: Opioid consumption at 24 hours

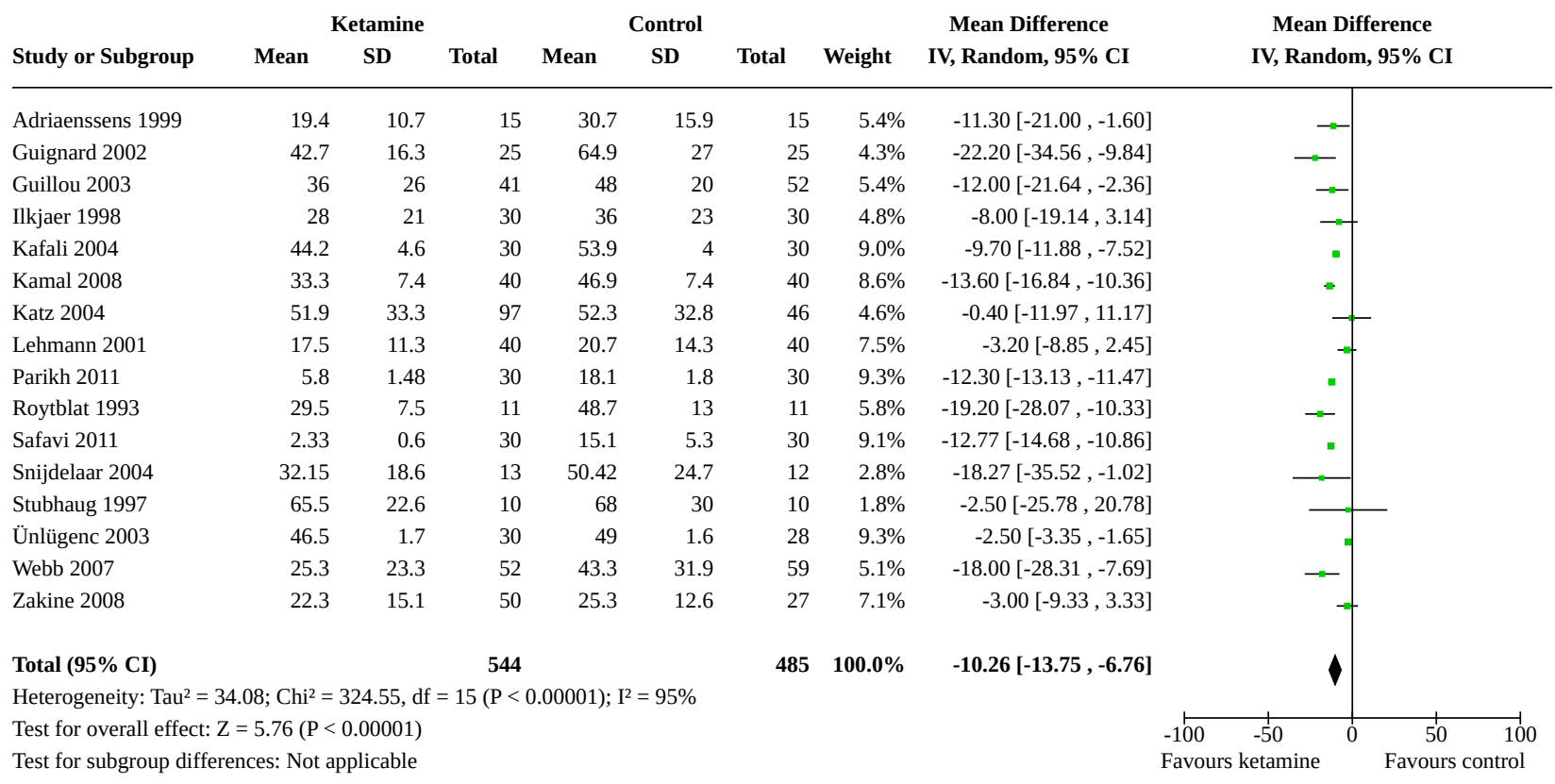

Analysis 7.2. Comparison 7: Perioperative ketamine versus control: major abdominal surgery, Outcome 2: Opioid consumption at 48 hours

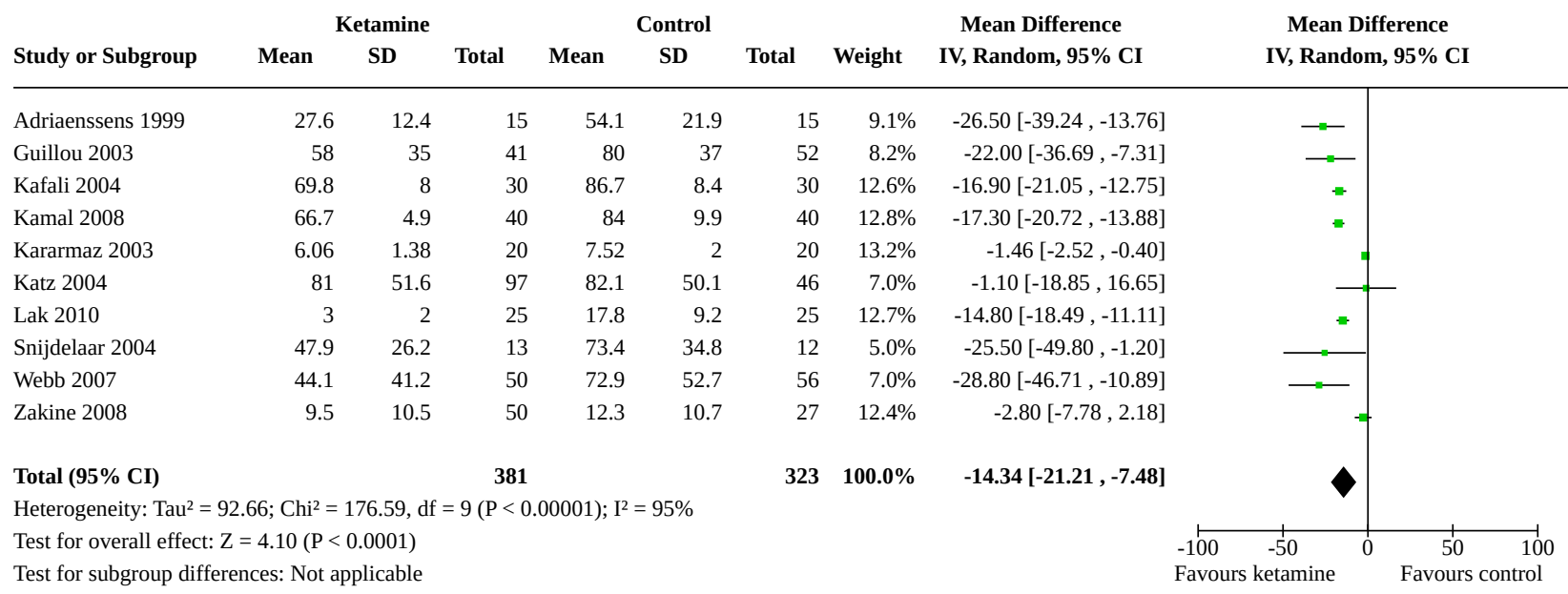


Analysis 7.3. Comparison 7: Perioperative ketamine versus control: major abdominal surgery, Outcome 3: Pain intensity at rest at 24 hours

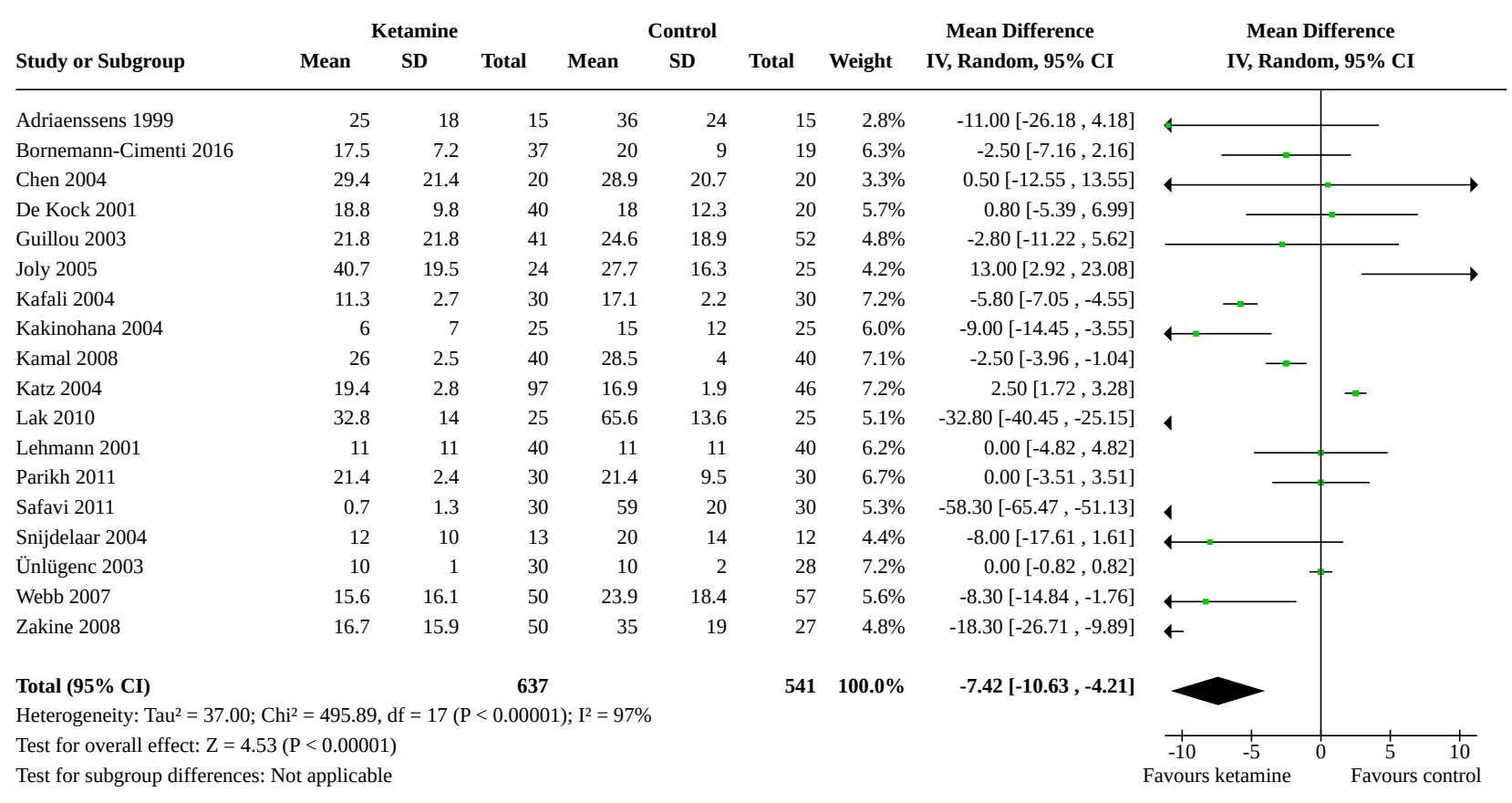

Analysis 7.4. Comparison 7: Perioperative ketamine versus control: major abdominal surgery, Outcome 4: Pain intensity during movement at 24 hours

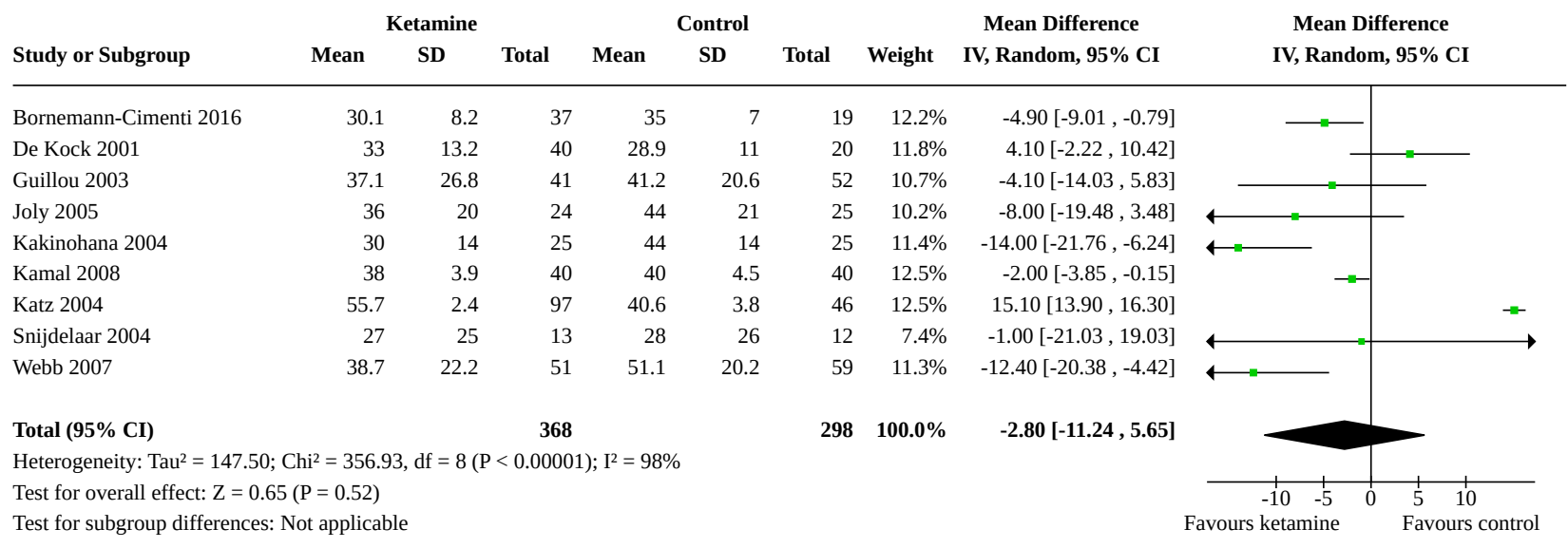


Analysis 7.5. Comparison 7: Perioperative ketamine versus control: major abdominal surgery, Outcome 5: Pain intensity at rest at 48 hours

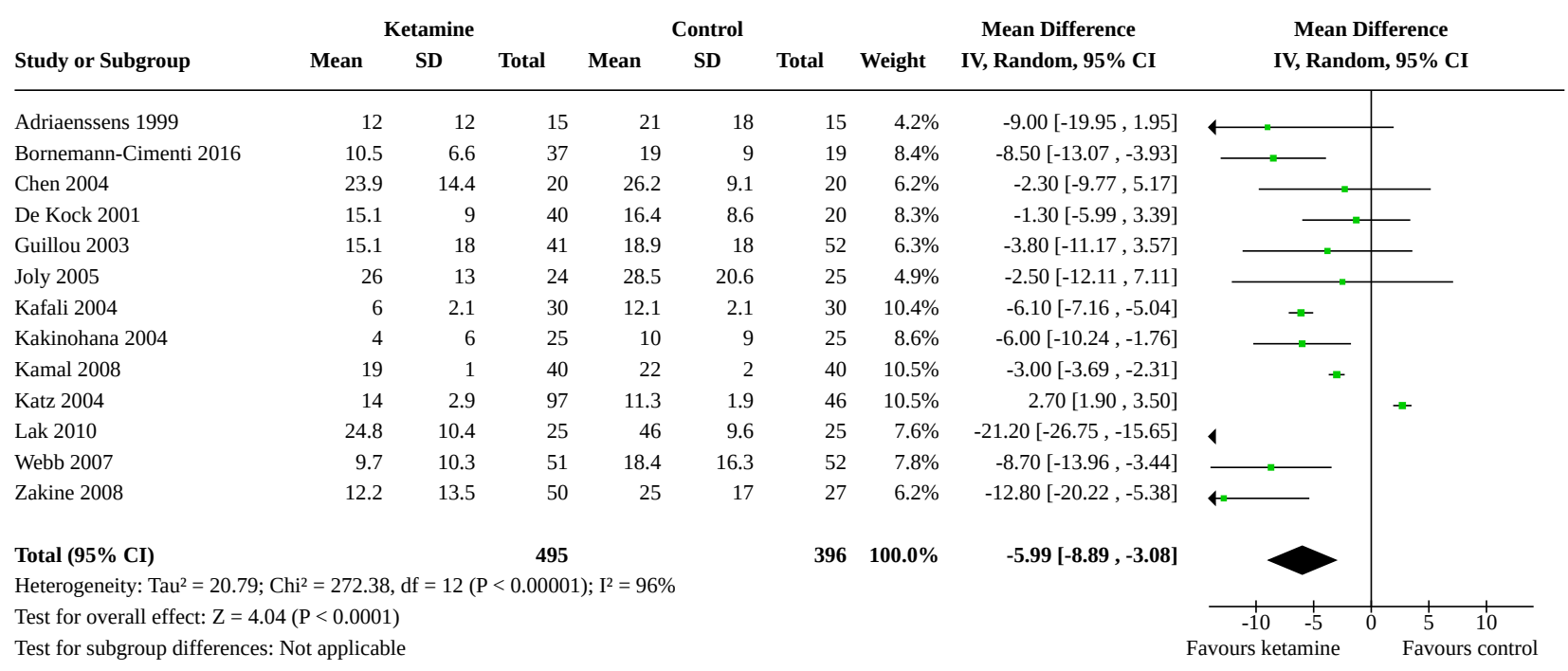

Analysis 7.6. Comparison 7: Perioperative ketamine versus control: major abdominal surgery, Outcome 6: Pain intensity during movement at 48 hours

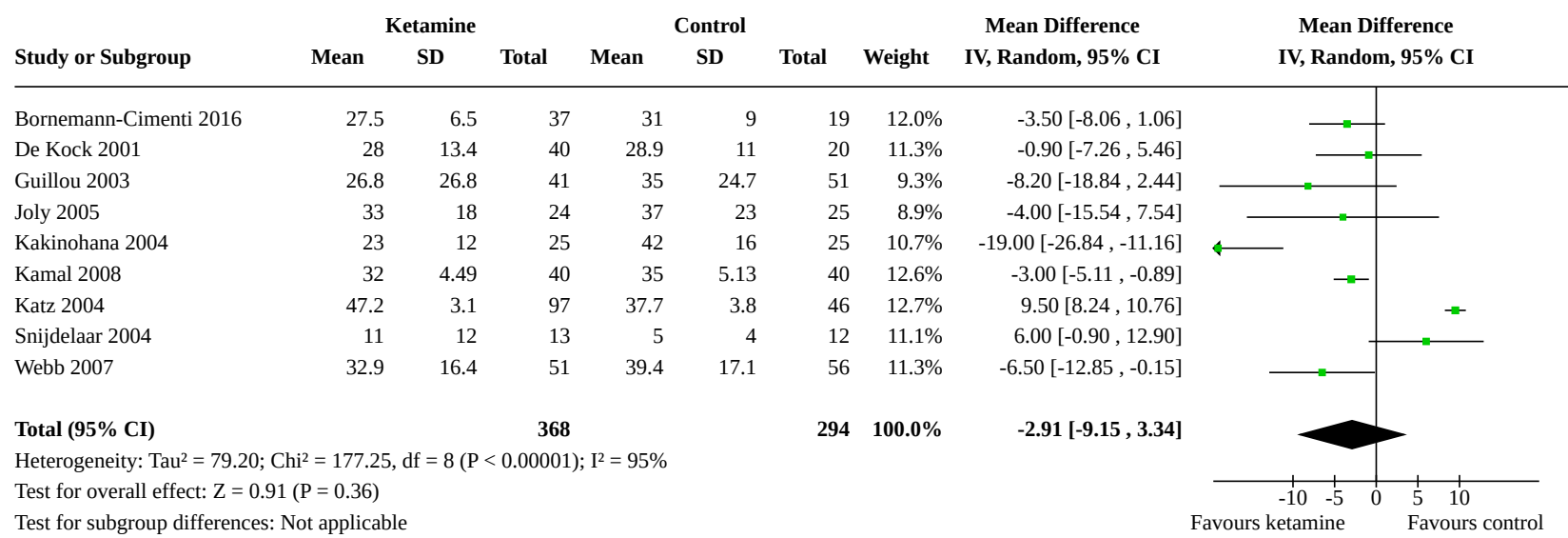

Comparison 8. Perioperative ketamine versus control: total abdominal hysterectomy

\begin{tabular}{lllll}
\hline Outcome or subgroup title & No. of studies & $\begin{array}{l}\text { No. of partici- } \\
\text { pants }\end{array}$ & Statistical method & Effect size \\
\hline $\begin{array}{l}8.1 \text { Opioid consumption at 24 } \\
\text { hours }\end{array}$ & 9 & 511 & $\begin{array}{l}\text { Mean Difference (IV, Random, } \\
95 \% \text { Cl) }\end{array}$ & $-5.18[-10.77,0.41]$ \\
\hline $\begin{array}{l}8.2 \text { Opioid consumption at 48 } \\
\text { hours }\end{array}$ & 5 & 378 & $\begin{array}{l}\text { Mean Difference (IV, Random, } \\
95 \% \text { Cl) }\end{array}$ & $-15.32[-33.20,2.56]$ \\
\hline $\begin{array}{l}8.3 \text { Pain intensity at rest at 24 } \\
\text { hours }\end{array}$ & 8 & 493 & $\begin{array}{l}\text { Mean Difference (IV, Random, } \\
95 \% \text { Cl) }\end{array}$ & $-2.58[-4.64,-0.52]$ \\
\hline
\end{tabular}


Analysis 8.1. Comparison 8: Perioperative ketamine versus control: total abdominal hysterectomy, Outcome 1: Opioid consumption at 24 hours

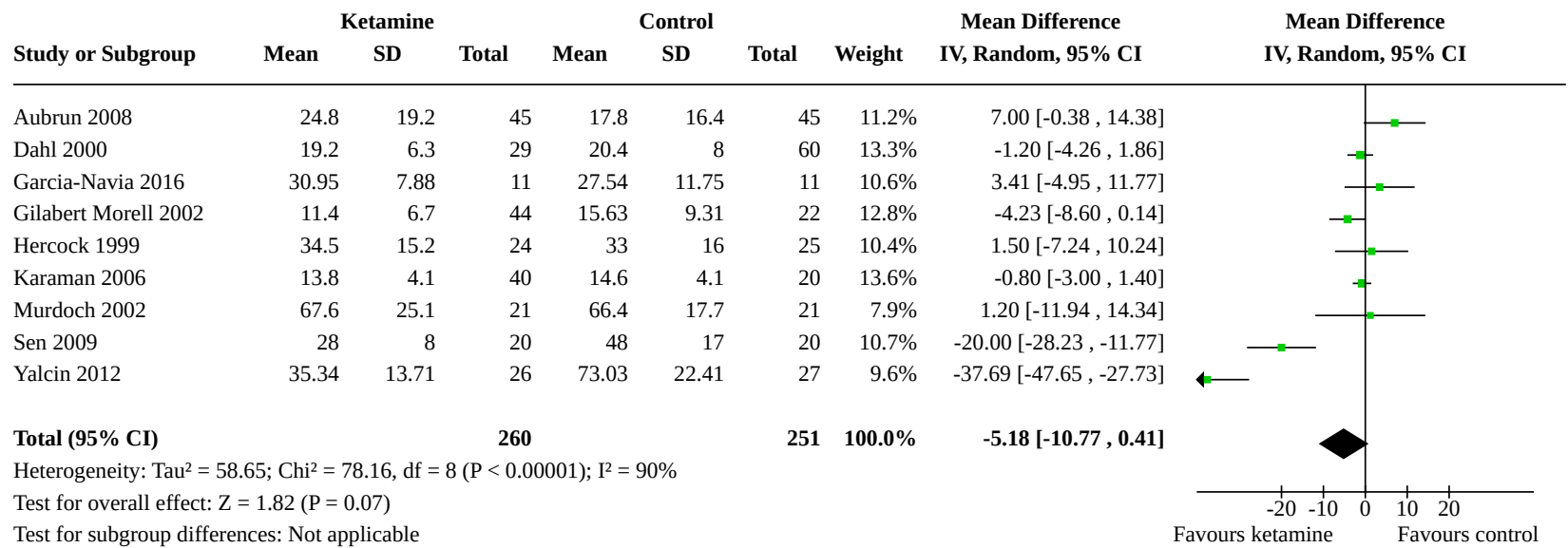

Analysis 8.2. Comparison 8: Perioperative ketamine versus control: total abdominal hysterectomy, Outcome 2: Opioid consumption at $\mathbf{4 8}$ hours

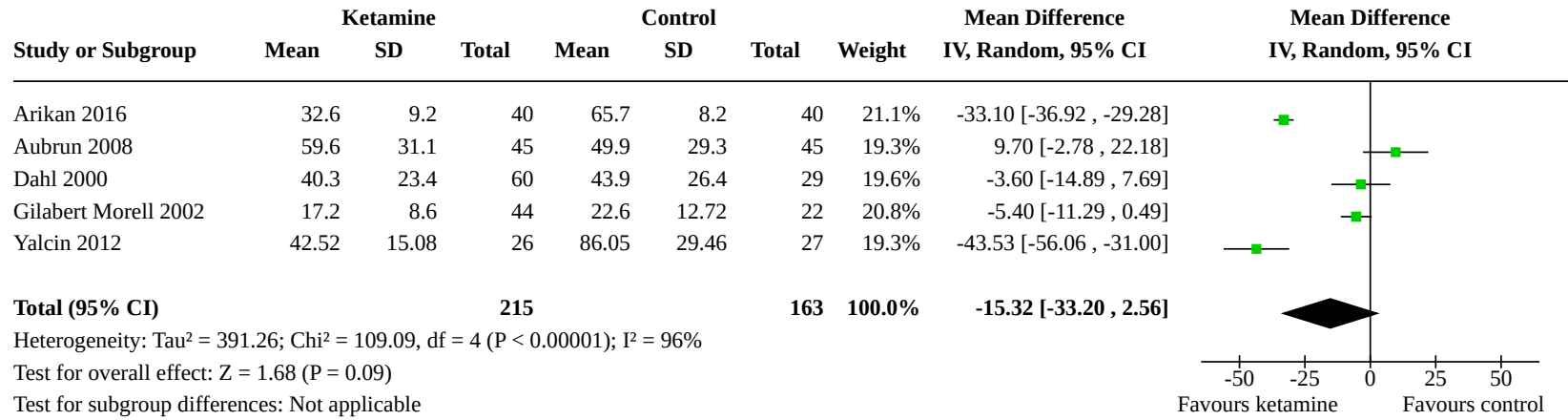

Analysis 8.3. Comparison 8: Perioperative ketamine versus control: total abdominal hysterectomy, Outcome 3: Pain intensity at rest at 24 hours

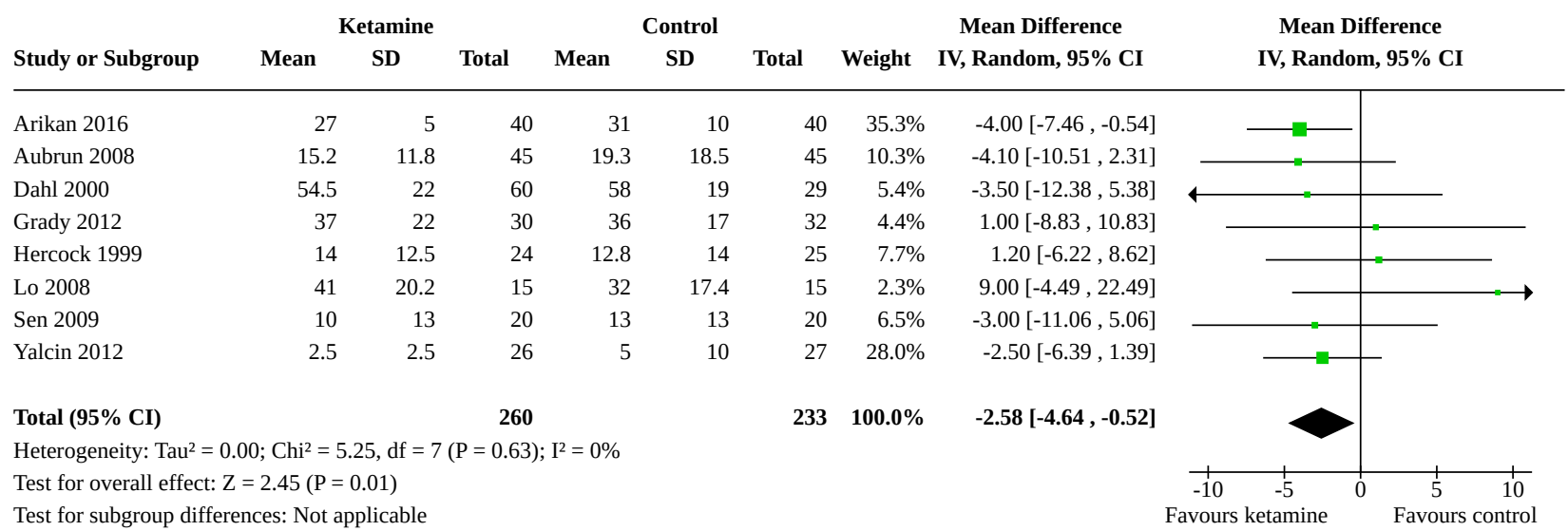


Comparison 9. Perioperative ketamine versus control: laparoscopic procedures

\begin{tabular}{lllll}
\hline Outcome or subgroup title & No. of studies & $\begin{array}{l}\text { No. of partici- } \\
\text { pants }\end{array}$ & Statistical method & Effect size \\
\hline $\begin{array}{l}\text { 9.1 Opioid consumption at 24 } \\
\text { hours }\end{array}$ & 4 & 199 & $\begin{array}{l}\text { Mean Difference (IV, Random, } \\
95 \% \text { Cl) }\end{array}$ & $-2.67[-6.19,0.84]$ \\
\hline $\begin{array}{l}\text { 9.2 Opioid consumption at 48 } \\
\text { hours }\end{array}$ & 2 & 85 & $\begin{array}{l}\text { Mean Difference (IV, Random, } \\
95 \% \text { Cl) }\end{array}$ & $-4.47[-12.21,3.27]$ \\
\hline $\begin{array}{l}\text { 9.3 Pain intensity at rest at 24 } \\
\text { hours }\end{array}$ & 9 & 484 & $\begin{array}{l}\text { Mean Difference (IV, Random, } \\
95 \% \text { Cl) }\end{array}$ & $-2.32[-6.65,2.02]$ \\
\hline
\end{tabular}

Analysis 9.1. Comparison 9: Perioperative ketamine versus control: laparoscopic procedures, Outcome 1: Opioid consumption at 24 hours

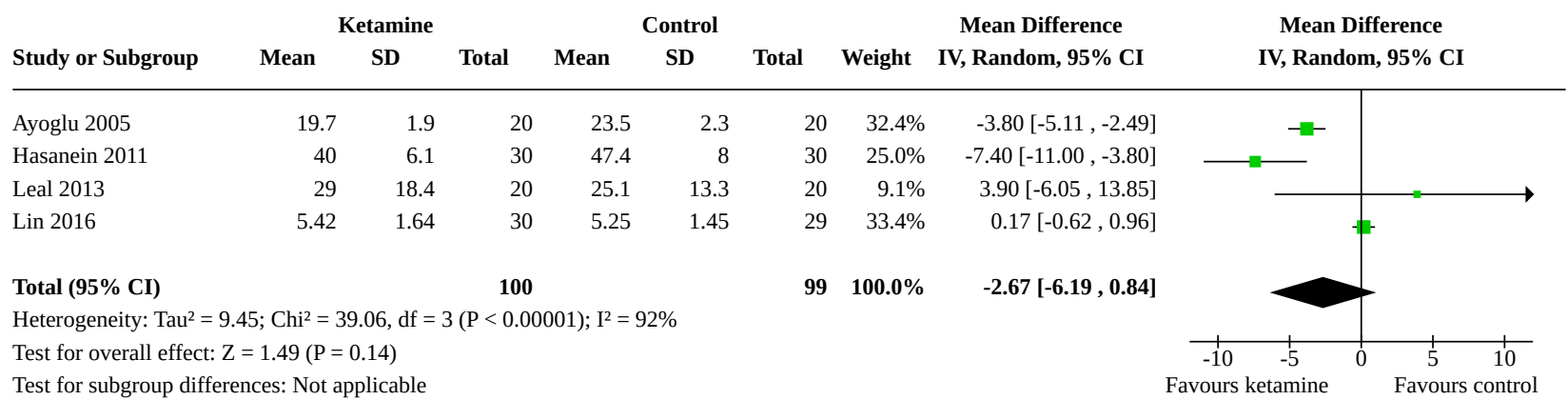

Analysis 9.2. Comparison 9: Perioperative ketamine versus control: laparoscopic procedures, Outcome 2: Opioid consumption at $\mathbf{4 8}$ hours

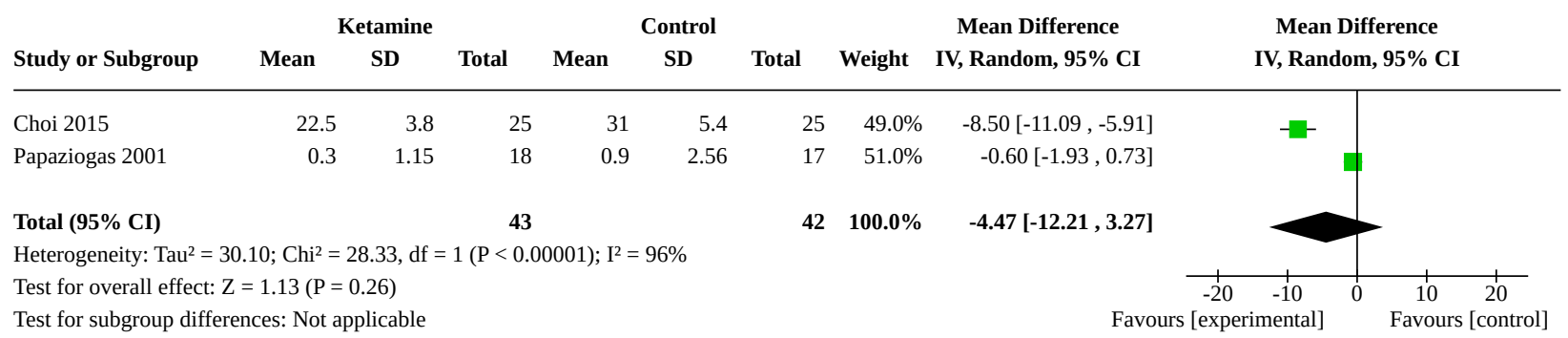


Analysis 9.3. Comparison 9: Perioperative ketamine versus control: laparoscopic procedures, Outcome 3: Pain intensity at rest at 24 hours

\begin{tabular}{|c|c|c|c|c|c|c|c|c|c|}
\hline Study or Subgroup & Mean & SD & Total & Mean & $\begin{array}{l}\text { ontrol } \\
\text { SD }\end{array}$ & Total & Weight & $\begin{array}{c}\text { Mean Difference } \\
\text { IV, Random, } 95 \% \text { CI }\end{array}$ & $\begin{array}{c}\text { Mean Difference } \\
\text { IV, Random, } 95 \% \text { C }\end{array}$ \\
\hline Ayoglu 2005 & 3.2 & 1.6 & 20 & 4.8 & 1.6 & 20 & $14.4 \%$ & $-1.60[-2.59,-0.61]$ & - \\
\hline Karcioglu 2013 & 13.5 & 11.2 & 17 & 48 & 19 & 20 & $8.3 \%$ & $-34.50[-44.38,-24.62]$ & $\leftarrow$ \\
\hline Kwok 2004 & 12.4 & 12.5 & 90 & 15.6 & 8.5 & 45 & $13.2 \%$ & $-3.20[-6.78,0.38]$ & \\
\hline Leal 2013 & 15 & 13 & 20 & 5 & 7 & 20 & $11.0 \%$ & $10.00[3.53,16.47]$ & -- \\
\hline Leal 2015 & 14 & 15 & 20 & 8 & 10 & 28 & $10.1 \%$ & $6.00[-1.55,13.55]$ & \\
\hline Lin 2016 & 24 & 8 & 30 & 25 & 8 & 29 & $12.9 \%$ & $-1.00[-5.08,3.08]$ & \\
\hline Mathisen 1999 & 20 & 14 & 32 & 24 & 18 & 18 & $8.5 \%$ & $-4.00[-13.63,5.63]$ & - \\
\hline Nesek-Adam 2012 & 23.3 & 11.3 & 20 & 18.4 & 11.3 & 20 & $10.5 \%$ & $4.90[-2.10,11.90]$ & - \\
\hline Papaziogas 2001 & 0.0001 & 0.0001 & 18 & 5 & 13 & 17 & $11.2 \%$ & $-5.00[-11.18,1.18]$ & \\
\hline Total (95\% CI) & & & 267 & & & 217 & $100.0 \%$ & $-2.32[-6.65,2.02]$ & \\
\hline \multicolumn{10}{|c|}{ Heterogeneity: $\mathrm{Tau}^{2}=33.64 ; \mathrm{Chi}^{2}=64.37, \mathrm{df}=8(\mathrm{P}<0.00001) ; \mathrm{I}^{2}=88 \%$} \\
\hline \multicolumn{9}{|c|}{ Test for overall effect: $\mathrm{Z}=1.05(\mathrm{P}=0.29)$} & 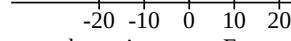 \\
\hline \multicolumn{9}{|c|}{ Test for subgroup differences: Not applicable } & ketamine \\
\hline
\end{tabular}

\section{APPENDICES}

\section{Appendix 1. CENTRAL (via CRSO)}

1. MESH DESCRIPTOR Ketamine EXPLODE ALL TREES

2. ((ketamine or ketalar or calipsol or ketanest or ketaset or calypsol or kalipsol or ci-581)):TI,AB,KY

3. \#1 OR \#2

4. MESH DESCRIPTOR Pain, Postoperative

5. ((postoperat ${ }^{\star}$ adj3 pain $\left.\left.{ }^{\star}\right)\right): T I, A B, K Y$

6. (pain* following surg*):TI,AB,KY

7. pain $^{\star}$ following treat $\left.{ }^{\star}\right): T I, A B, K Y$

8. (pain* following operation $\left.{ }^{\star}\right): T I, A B, K Y$

9. (post-operat* pain):TI,AB,KY

10. (((post adj1 surg $\left.{ }^{\star}\right)$ or postsurg ${ }^{\star}$ or post-surg $\left.)\right): T I, A B, K Y$

11. (((post adj1 operat $\left.{ }^{\star}\right)$ or postoperat ${ }^{\star}$ or post-operat $\left.\left.{ }^{\star}\right)\right): \mathrm{TI}, \mathrm{AB}, \mathrm{KY}$

12. pain $^{\star}: T I, A B, K Y$

13. \#10 OR \#11

14. \#12 AND \#13

15. (((post-operat* or postoperat* ${ }^{\star}$ or post-surg* or postsurg $\left.{ }^{\star}\right)$ adj analgesi $\left.\left.{ }^{\star}\right)\right): \mathrm{TI}, \mathrm{AB}, \mathrm{KY}$

16. (analgesi ${ }^{\star}$ following surg*):TI,AB,KY

17. (analgesi* following operat $\left.{ }^{\star}\right): T I, A B, K Y$

18. \#4 OR \#5 OR \#6 OR \#7 OR \#8 OR \#9 OR \#14 OR

19. \#15 OR \#16 OR \#17

20. \#3 AND \#18 


\section{Appendix 2. MEDLINE (via Ovid)}

1. Ketamine/

2. (ketamine or ketalar or calipsol or ketanest or ketaset or calypsol or kalipsol or ci-581).tw.

3. or/1-2

4. Pain, Postoperative/

5. (postoperat* adj3 pain $\left.{ }^{\star}\right)$.tw.

6. pain ${ }^{\star}$ following surg*.tw.

7. pain ${ }^{\star}$ following treat ${ }^{\star} . t w$.

8. pain* following operation ${ }^{\star}$.tw.

9. post-operat* pain.tw.

10. ((post adj1 surg $\left.^{\star}\right)$ or postsurg* or post-surg*).tw.

11. ((post adj1 operat $\left.{ }^{\star}\right)$ or postoperat ${ }^{\star}$ or post-operat $\left.{ }^{\star}\right)$.tw.

12. pain*.tw.

13. (10 or 11$)$ and 12

14. ((post-operat* or postoperat $^{\star}$ or post-surg ${ }^{\star}$ or postsurg* $)$ adj analgesi $\left.{ }^{\star}\right)$.tw.

15. analgesi* following surg ${ }^{\star}$. tw.

16. analgesi ${ }^{\star}$ following operat*..tw.

17.4 or 5 or 6 or 7 or 8 or 9 or 13 or 14 or 15 or 16

18. 3 and 17

19. randomized controlled trial.pt.

20. controlled clinical trial.pt.

21. randomized.ab.

22. placebo.ab.

23. drug therapy.fs.

24. randomly.ab.

25. trial.ab.

26. or/19-25

27. exp animals/ not humans.sh.

28. 26 not 27

29. 18 and 28

Appendix 3. Embase (via Ovid)

1. Ketamine/

2. (ketamine or ketalar or calipsol or ketanest or ketaset or calypsol or kalipsol or ci-581).tw.

3. or/1-2

4. Pain, Postoperative/ 
5. (postoperat* $\operatorname{adj}^{3}$ pain $\left.{ }^{\star}\right)$. tw.

6. pain ${ }^{\star}$ following surg*.tw.

7. pain ${ }^{\star}$ following treat*.tw.

8. pain* following operation ${ }^{\star}$.tw.

9. post-operat* pain.tw.

10. ((post adj1 surg $\left.^{\star}\right)$ or postsurg ${ }^{\star}$ or post-surg $\left.{ }^{\star}\right)$.tw.

11. ((post adj1 operat $\left.{ }^{\star}\right)$ or postoperat ${ }^{\star}$ or post-operat $\left.{ }^{\star}\right)$. tw.

12. pain ${ }^{\star} . t w$.

13. (10 or 11$)$ and 12

14. ((post-operat ${ }^{\star}$ or postoperat ${ }^{\star}$ or post-surg ${ }^{\star}$ or postsurg $\left.^{\star}\right)$ adj analgesi $\left.{ }^{\star}\right)$.tw.

15. analgesi* following surg*.tw.

16. analgesi ${ }^{\star}$ following operat*..tw.

17.4 or 5 or 6 or 7 or 8 or 9 or 13 or 14 or 15 or 16

18. 3 and 17

19. random\$.tw.

20. factorial\$s.tw.

21. crossover\$.tw.

22. cross over\$.tw.

23. cross-over\$.tw.

24. placebo\$.tw.

25. (doubl\$ adj blind\$).tw.

26. (singl\$ adj blind\$).tw.

27. assign\$.tw.

28. allocat\$.tw.

29. volunteer\$.tw.

30. Crossover Procedure/

31. double-blind procedure.tw.

32. Randomized Controlled Trial/

33. Single Blind Procedure/

34. or/19-33

35. (animal/ or nonhuman/) not human/

36. 34 not 35

37. 18 and 36 


\section{Appendix 4. Postoperative opioid consumption in different types of surgery}

\section{4-hour outcomes}

\section{4-hour opioid consumption after thoracotomy}

Four studies assessed opioid consumption during the first 24 hours after thoracotomy; 120 participants received ketamine and 121 participants served as controls (Argiriadou 2011; Dualé 2009; Michelet 2007; Ysasi 2010). Participants who received ketamine consumed 6 mg less opioid ( $95 \% \mathrm{Cl}-10.3$ to -1.4 ), compared to participants who received control treatment (Analysis 5.1). We assessed the quality of evidence for this outcome as very low. We downgraded the evidence three times because there were fewer than 400 participants in the analysis.

\section{4-hour opioid consumption after major orthopaedic surgery}

Ten studies provided data for 24-hour opioid consumption after major orthopaedic surgery; 417 participants received ketamine and 380 participants received control treatment (Cenzig 2014; Dahi-Taleghani 2014; Garg 2016; Hadi 2010; Jaksch 2002; Loftus 2010; Menigaux 2000; Nielsen 2017; Remérand 2009; Subramaniam 2011). Participants who had received ketamine consumed $20 \mathrm{mg}$ less opioid (95\% Cl-28.6 to -10.8), compared to controls (Analysis 6.1). We assessed the quality of evidence for this outcome as low, downgraded once because there were fewer than 1500 participants in the analysis, and once because it was not possible to test for small-study effects.

\section{4-hour opioid consumption after major abdominal surgery}

Sixteen studies gave data of pain intensity at rest at 24 hours after major abdominal surgery; 544 participants received ketamine and 485 participants received control treatment (Adriaenssens 1999; Guignard 2002; Guillou 2003; Ilkjaer 1998; Kafali 2004; Kamal 2008; Katz 2004; Lehmann 2001; Parikh 2011; Roytblat 1993; Safavi 2011; Snijdelaar 2004; Stubhaug 1997; Webb 2007; Zakine 2008; Ünlügenc 2003). Ketamine treatment reduced opioid consumption by $10 \mathrm{mg}$ of morphine equivalents $(95 \% \mathrm{Cl}-13.8$ to -6.8 ; Analysis 7.1$)$. We assessed the quality of evidence for this outcome as low, downgraded once because there were fewer than 1500 participants in the analysis, and once because it was not possible to test for small-study effects.

\section{4-hour opioid consumption after total abdominal hysterectomy}

Nine studies provided data for 24-hour opioid consumption after total abdominal hysterectomy; 260 participants received ketamine and 251 participants received control treatment (Aubrun 2008; Dahl 2000; Garcia-Navia 2016; Gilabert Morell 2002; Hercock 1999; Karaman 2006; Murdoch 2002; Sen 2009; Yalcin 2012). Ketamine administration reduced 24-hour opioid consumption after total abdominal hysterectomy by $5 \mathrm{mg}$ of morphine equivalents $(95 \% \mathrm{Cl}-10.8$ to 0.4 ; Analysis 8.1$)$. We assessed the quality of evidence for this outcome as low, downgraded once because there were fewer than 1500 participants in the analysis, and once because it was not possible to test for small-study effects.

\section{4-hour opioid consumption after laparoscopic procedures}

Four studies assessed 24-hour opioid consumption after laparoscopic procedures; 100 participants were given ketamine and 99 participants served as controls (Ayoglu 2005; Hasanein 2011; Leal 2013; Lin 2016). Ketamine treatment reduced 24-hour opioid consumption after laparoscopic procedures by $3 \mathrm{mg}$ of morphine equivalents $(95 \% \mathrm{Cl}-6.2$ to 0.8 ; Analysis 9.1$)$. We assessed the quality of evidence for this outcome as very low. We downgraded the evidence three times because there were fewer than 400 participants in the analysis.

\section{8-hour outcomes}

\section{8-hour opioid consumption after thoracotomy}

Three studies provided data of 48-hour opioid consumption after thoracotomy; 95 participants received ketamine and 96 participants served as controls (Argiriadou 2011; Lahtinen 2004; Michelet 2007). Ketamine treatment reduced 48-hour opioid consumption after thoracotomy by $13 \mathrm{mg}(95 \% \mathrm{Cl}-18.3$ to -6.7 ; Analysis 5.2). We assessed the quality of evidence for this outcome as very low. We downgraded the evidence three times because there were fewer than 400 participants in the analysis.

\section{8-hour opioid consumption after major orthopaedic surgery}

Nine studies assessed 48-hour opioid consumption after major orthopaedic surgery; 296 participants received ketamine and 261 participants received control treatment (Adam 2005; Garg 2016; Jaksch 2002; Kim 2013; Loftus 2010; Martinez 2014; Menigaux 2000; Remérand 2009; Subramaniam 2011). Participants who had received ketamine consumed $19 \mathrm{mg}$ less opioid (95\% Cl-27.5 to -9.9), compared to participants who received control treatment (Analysis 6.2). We assessed the quality of evidence for this outcome as low, downgraded once because there were fewer than 1500 participants in the analysis, and once because it was not possible to test for small-study effects.

\section{8-hour opioid consumption after major abdominal surgery}

Ten studies assessed 48-hour opioid consumption after major abdominal surgery; 381 participants received ketamine and 323 participants received control treatment (Adriaenssens 1999; Guillou 2003; Kafali 2004; Kamal 2008; Kararmaz 2003; Katz 2004; Lak 2010; Snijdelaar 2004; Webb 2007; Zakine 2008). Ketamine treatment reduced 48-hour opioid consumption by $14 \mathrm{mg}$ of morphine equivalents (95\% $\mathrm{Cl}-21.2$ to 
-7.5), after major abdominal surgery (Analysis 7.2). We assessed the quality of evidence for this outcome as low, downgraded once because there were fewer than 1500 participants in the analysis, and once because it was not possible to test for small-study effects.

\section{8-hour opioid consumption after total abdominal hysterectomy}

Five studies provided data on 48-hour opioid consumption after total abdominal hysterectomy; 215 participants received ketamine and 163 participants served as controls (Arikan 2016; Aubrun 2008; Dahl 2000; Gilabert Morell 2002; Yalcin 2012). Treatment with ketamine reduced 48 hour postoperative opioid consumption by 15 milligrams of morphine equivalents $(95 \% \mathrm{Cl}-33.2$ to 2.6 ; Analysis 8.2$)$. We assessed the quality of evidence for this outcome as very low. We downgraded the evidence three times because there were fewer than 400 participants in the analysis.

\section{8-hour opioid consumption after laparoscopic procedures}

Two studies investigated ketamine's effect on 48-hour opioid consumption after laparoscopic procedures; 43 participants received ketamine and 42 participants received control treatment (Choi 2015; Papaziogas 2001). Ketamine treatment reduced 48-hour opioid consumption by $5 \mathrm{mg}(95 \% \mathrm{Cl}-12.2$ to 3.3; Analysis 9.2). We assessed the quality of evidence for this outcome as very low. We downgraded the evidence three times because there were fewer than 400 participants in the analysis.

\section{Appendix 5. Postoperative pain in different types of surgery}

\section{Pain intensity at rest at $\mathbf{2 4}$ hours after thoracotomy}

Thirteen studies assessed pain at 24 hours at rest after thoracotomy; 388 participants received ketamine and 394 participants received control treatment (Argiriadou 2011; D'Alonzo 2011; Dualé 2009; Fiorelli 2015; Joseph 2012; Lahtinen 2004; Mendola 2012; Michelet 2007; Patel 2016; Suzuki 2006; Tena 2014; Yazigi 2012; Ysasi 2010). Visual analogue scale (VAS) scores were $4 \mathrm{~mm}$ lower (95\% Cl -8.8 to 1), among participants who received ketamine (Analysis 5.3). We assessed the quality of evidence for this outcome as low, downgraded once because there were fewer than 1500 participants in the analysis, and once because it was not possible to test for small-study effects.

\section{Pain intensity during movement at $\mathbf{2 4}$ hours after thoracotomy}

Five studies assessed postoperative pain intensity at 24 hours on movement after thoracotomy (Argiriadou 2011; Joseph 2012 Lahtinen 2004; Tena 2014; Yazigi 2012). VAS scores were $7 \mathrm{~mm}$ lower (95\% Cl -20.1 to 5.5), among 154 participants who received ketamine versus 161 participants who received control treatment (Analysis 5.4). We assessed the quality of evidence for this outcome as very low. We downgraded the evidence three times because there were fewer than 400 participants in the analysis.

\section{Pain intensity at rest at $\mathbf{4 8}$ hours after thoracotomy}

Nine studies provided data of pain intensity at rest at 48 hours after thoracotomy (Argiriadou 2011; Chazan 2010; Fiorelli 2015; Joseph 2012; Lahtinen 2004; Mendola 2012; Michelet 2007; Suzuki 2006; Yazigi 2012). VAS scores were $7 \mathrm{~mm}$ lower (95\% Cl -10.4 to -3.4), among 265 participants who received ketamine versus 265 participants who served as controls (Analysis 5.5). We assessed the quality of evidence for this outcome as low, downgraded once because there were fewer than 1500 participants in the analysis, and once because it was not possible to test for small-study effects.

\section{Pain intensity during movement at $\mathbf{4 8}$ hours after thoracotomy}

Five studies assessed pain intensity at 48 hours during movement after thoracotomy (Argiriadou 2011; Joseph 2012; Lahtinen 2004; Suzuki 2006; Yazigi 2012). VAS scores were $11 \mathrm{~mm}$ lower ( $95 \% \mathrm{Cl}-15.3$ to -6), among 147 participants who received ketamine versus 151 participants who received control treatment (Analysis 5.6). We assessed the quality of evidence for this outcome as very low. We downgraded the evidence three times because there were fewer than 400 participants in the analysis.

\section{Pain intensity at rest at $\mathbf{2 4}$ hours after major orthopaedic surgery}

Eleven studies assessed pain intensity at rest at 24 hours after major orthopaedic surgery; 449 participants received ketamine and 394 participants received control treatment (Adam 2005; Cenzig 2014; Dahi-Taleghani 2014; Hadi 2013; Jaksch 2002; Kim 2013; Loftus 2010; Menigaux 2000; Nielsen 2017; Remérand 2009; Subramaniam 2011). VAS scores were $7 \mathrm{~mm}$ lower (95\% Cl -9.9 to -3.0$)$ after ketamine treatment compared to those who received control treatment (Analysis 6.3). We assessed the quality of evidence for this outcome as low, downgraded once because there were fewer than 1500 participants in the analysis, and once because it was not possible to test for smallstudy effects.

\section{Pain intensity during movement at $\mathbf{2 4}$ hours after major orthopaedic surgery}

Four studies gave data about pain intensity at 24 hours during movement after major orthopaedic surgery; 162 participants who received ketamine had $7 \mathrm{~mm}$ lower VAS scores (95\% Cl -12.6 to -0.8), compared to 117 participants who received control treatment (Kim 2013; Menigaux 2000; Nielsen 2017; Subramaniam 2011; Analysis 6.4). We assessed the quality of evidence for this outcome as very low. We downgraded the evidence three times because there were fewer than 400 participants in the analysis. 


\section{Pain intensity at rest at $\mathbf{4 8}$ hours after major orthopaedic surgery}

Seven studies assessed pain intensity at rest at 48 hours after major orthopaedic surgery; 246 participants received ketamine and 207 participants served as controls (Adam 2005; Jaksch 2002; Kim 2013; Loftus 2010; Menigaux 2000; Remérand 2009; Subramaniam 2011). VAS scores were $1 \mathrm{~mm}$ lower (95\% Cl-4.1 to 1.3), after ketamine treatment (Analysis 6.5). We assessed the quality of evidence for this outcome as low, downgraded once because there were fewer than 1500 participants in the analysis, and once because it was not possible to test for small-study effects.

\section{Pain intensity during movement at $\mathbf{4 8}$ hours after major orthopaedic surgery}

Four studies provided data of pain intensity at 48 hours during movement after major orthopaedic surgery; 95 participants experienced 7 mm lower VAS scores $(95 \% \mathrm{Cl}-13.1$ to -1.6) after ketamine treatment compared to 62 participants who served as controls (Jaksch 2002 ; Kim 2013; Menigaux 2000; Subramaniam 2011; Analysis 6.6). We assessed the quality of evidence for this outcome as very low. We downgraded the evidence three times because there were fewer than 400 participants in the analysis.

\section{Pain intensity at rest at $\mathbf{2 4}$ hours after major abdominal surgery}

Eighteen studies gave data of pain intensity at rest at 24 hours after major abdominal surgery; 637 participants received ketamine and 541 participants received control treatment (Adriaenssens 1999; Bornemann-Cimenti 2016; Chen 2004; De Kock 2001; Guillou 2003; Joly 2005; Kafali 2004; Kakinohana 2004; Kamal 2008; Katz 2004; Lak 2010; Lehmann 2001; Parikh 2011; Safavi 2011; Snijdelaar 2004; Webb 2007; Zakine 2008; Ünlügenc 2003). VAS scores were $7 \mathrm{~mm}$ lower (95\% Cl-10.6 to -4.2), after ketamine treatment compared to controls (Analysis 7.3). We assessed the quality of evidence for this outcome as low, downgraded once because there were fewer than 1500 participants in the analysis, and once because it was not possible to test for small-study effects.

\section{Pain intensity during movement at $\mathbf{2 4}$ hours after major abdominal surgery}

Nine studies assessed pain intensity at 24 hours during movement after major abdominal surgery (Bornemann-Cimenti 2016; De Kock 2001; Guillou 2003; Joly 2005; Kakinohana 2004; Kamal 2008; Katz 2004; Snijdelaar 2004; Webb 2007). VAS scores were 3 mm lower (95\% Cl-11.2 to 5.7), among 368 participants who received ketamine compared to 298 participants who served as controls (Analysis 7.4). We assessed the quality of evidence for this outcome as low, downgraded once because there were fewer than 1500 participants in the analysis, and once because it was not possible to test for small-study effects.

\section{Pain intensity at rest at $\mathbf{4 8}$ hours after major abdominal surgery}

Thirteen studies provided data of pain intensity at rest at 48 hours after major abdominal surgery; 495 participants received ketamine and 396 participants received control treatment (Adriaenssens 1999; Bornemann-Cimenti 2016; Chen 2004; De Kock 2001; Guillou 2003; Joly 2005; Kafali 2004; Kakinohana 2004; Kamal 2008; Katz 2004; Lak 2010; Webb 2007; Zakine 2008). VAS scores were 6 mm lower (95\% Cl -8.9 to -3.1), after ketamine treatment compared to those who received control treatment (Analysis 7.5). We assessed the quality of evidence for this outcome as low, downgraded once because there were fewer than 1500 participants in the analysis, and once because it was not possible to test for small-study effects.

\section{Pain intensity during movement at $\mathbf{4 8}$ hours after major abdominal surgery}

Nine studies assessed pain intensity at 48 hours during movement after major abdominal surgery (Bornemann-Cimenti 2016; De Kock 2001; Guillou 2003; Joly 2005; Kakinohana 2004; Kamal 2008; Katz 2004; Snijdelaar 2004; Webb 2007). VAS scores were 3 mm lower (95\% $\mathrm{Cl}-9.2$ to 3.3), after ketamine treatment among 368 participants versus 294 participants who received control treatment (Analysis 7.6). We assessed the quality of evidence for this outcome as low, downgraded once because there were fewer than 1500 participants in the analysis, and once because it was not possible to test for small-study effects.

\section{Pain intensity at rest at $\mathbf{2 4}$ hours after total abdominal hysterectomy}

Eight studies provided data for pain intensity at rest at 24 hours after total abdominal hysterectomy; 260 participants received ketamine and 233 participants received control treatment (Arikan 2016; Aubrun 2008; Dahl 2000; Grady 2012; Hercock 1999; Lo 2008; Sen 2009; Yalcin 2012). Pain scores were $3 \mathrm{~mm}$ lower in VAS (95\% Cl -4.6 to -0.5), among those who received ketamine compared to controls (Analysis 8.3 ). We assessed the quality of evidence for this outcome as low, downgraded once because there were fewer than 1500 participants in the analysis, and once because it was not possible to test for small-study effects.

\section{Pain intensity during movement at 24 hours after total abdominal hysterectomy}

No data were available for analysis on pain intensity at 24 hours during movement after total abdominal hysterectomy.

\section{Pain intensity at rest at $\mathbf{4 8}$ hours after total abdominal hysterectomy}

No data were available for analysis on pain intensity at rest at 48 hours after total abdominal hysterectomy.

\section{Pain intensity during movement at 48 hours after total abdominal hysterectomy}

No data were available for analysis on pain intensity at 48 hours during movement after total abdominal hysterectomy. 


\section{Pain intensity at rest at $\mathbf{2 4}$ hours after laparoscopic procedures}

Nine studies assessed ketamine's effect on pain intensity at rest at 24 hours after laparoscopic procedures (Ayoglu 2005; Karcioglu 2013; Kwok 2004; Leal 2013; Leal 2015; Lin 2016; Mathisen 1999; Nesek-Adam 2012; Papaziogas 2001). Pain scores were 2 mm lower (95\% Cl -6.7 to 2.0), in VAS among 267 participants who received ketamine compared to 217 participants who served as controls (Analysis 9.3). We assessed the quality of evidence for this outcome as low, downgraded once because there were fewer than 1500 participants in the analysis, and once because it was not possible to test for small-study effects.

\section{Pain intensity during movement at $\mathbf{2 4}$ hours after laparoscopic procedures}

No data were available for analysis on pain intensity at 24 hours during movement after laparoscopic procedures.

\section{Pain intensity at rest at $\mathbf{4 8}$ hours after laparoscopic procedures}

No data were available for analysis on pain intensity at rest at 48 hours after laparoscopic procedures.

\section{Pain intensity during movement at $\mathbf{4 8}$ hours after laparoscopic procedures}

No data were available for analysis on pain intensity at 48 hours during movement after laparoscopic procedures.

\section{Appendix 6. GRADE: criteria for assigning grade of evidence}

The GRADE system uses the following criteria for assigning a quality level to a body of evidence (Higgins 2011).

- High: randomised trials; or double-upgraded observational studies

- Moderate: downgraded randomised trials; or upgraded observational studies

- Low: double-downgraded randomised trials; or observational studies

- Very low: triple-downgraded randomised trials; or downgraded observational studies; or case series/case reports

Factors that may decrease the quality level of a body of evidence are:

- limitations in the design and implementation of available studies suggesting high likelihood of bias;

- indirectness of evidence (indirect population, intervention, control, outcomes);

- unexplained heterogeneity or inconsistency of results (including problems with subgroup analyses);

- imprecision of results (wide confidence intervals);

- high probability of publication bias.

WHAT'S NEW

\begin{tabular}{lll}
\hline Date & Event & Description \\
\hline 21 January 2021 & Review declared as stable & See Published notes. \\
\hline
\end{tabular}

\section{H I S T O R Y}

Protocol first published: Issue 1, 2016

Review first published: Issue 12, 2018

\begin{tabular}{lll}
\hline Date & Event & Description \\
\hline 11 January 2019 & Amended & Typo corrected in author SS's declaration of interest. \\
\hline 18 September 2017 & Amended & See Published notes. \\
\hline 14 June 2017 & Amended & This protocol has been withdrawn. See Published notes. \\
\hline
\end{tabular}




\section{CONTRIBUTIONS OF AUTHORS}

ECVB and VKK developed the search strategy, ECVB wrote and VKK revised the background section, ECVB and ET were responsible for the data extraction, and ECVB, ET and VKK were responsible for the data analysis; RAM completed sensitivity analyses. VKK and RAM acted as guarantors of the review. All review authors were responsible for completing the protocol and the review, and will be responsible for updating the review in future.

\section{DECLARATIONS OF INTEREST}

ECVB: none known. ECVB is a specialist physician in anaesthesiology and intensive care medicine and she treats patients suffering from acute postoperative pain.

ET: none known. ET is a specialist physician in anaesthesiology and intensive care medicine and she treats patients suffering from acute postoperative pain.

MH: none known. MH is a specialist physician in anaesthesiology and he treats patients with acute postoperative and chronic pain.

RFB: none known. RFB is a specialist pain physician (retired).

SS's institution (University of Alberta) received fees for his contribution to an advisory board from Daiichi Sankyo, Inc. (2015). SS is a specialist occupational medicine physician and some of the patients he assesses have painful conditions.

RAM has received grant support from Grünenthal relating to individual patient-level analyses of trial data regarding tapentadol in osteoarthritis and back pain (2015), and Novartis for a network meta-analysis on acute postoperative pain using data from Cochrane Reviews. He has received honoraria for attending boards with RB on understanding pharmacokinetics of drug uptake (2015). He has received honoraria from Omega Pharma (2016), and Futura Pharma (2016), for providing advice on trial and data analysis methods.

VK: none known. VK is a specialist physician in anaesthesiology and intensive care medicine and he treats patients suffering from acute postoperative pain.

\section{SOURCES OF SUPPORT}

\section{Internal sources}

- No sources of support supplied

\section{External sources}

- State funding for university-level health research (grant TYH2014305), Finland

\section{DIFFERENCES BETWEEN PROTOCOLANDREVIEW}

- We realised that in the protocol we had inadvertently included postoperative hyperalgesia assessment as both a secondary outcome and (incorrectly) as a subgroup analysis.

- We have changed the method of testing for statistical heterogeneity to the $\mathrm{I}^{2}$ statistic.

- We have assessed the possible bias in blinding as two separate domains: blinding of participants and personnel (performance bias), and blinding of outcome assessment (checking for possible detection bias). This is also presented as two separate domains in the 'Risk of bias' table. Additionally, we present an assessment concerning selective reporting (checking for reporting bias), and considered other possible biases.

- In the review, we have specified that the opioid consumption registered is milligrams of morphine equivalents. If the opioid administered for postoperative analgesia was other than morphine we converted it to morphine equivalents using equations found in the literature. Studies refer to postoperative analgesic use, but this was exclusively use of opioids.

- We changed the unit of analysis of this review from individual, participant-level data to study-level data because participant-level data were not available except for two studies (Joseph 2012; Lo 2008).

- If confidence intervals alone were presented, we derived standard deviations from confidence interval data (Higgins 2011a).

- We observed a wide heterogeneity considering surgery types between studies to be included in this analysis. From a clinical point of view and to better serve clinical decision making, in addition to analysing a non-stratified study population, we decided to analyse the main outcomes separately for different surgery types.

- One additional subgroup analysis related to the use of benzodiazepines as premedicant. The potential for interaction was highlighted in the protocol, but not mentioned as a subgroup analysis at that time.

- We added sections, 'Subgroup analysis and investigation of heterogeneity' and 'Sensitivity analyses' to the full review. 
- We performed an additional sensitivity analysis on pain intensity with placebo, as many studies had pain scores indicating little or no pain, blunting the ability to detect an analgesic effect. Additionally, we performed sensitivity analysis according to study size (30 or more and 50 or more participants in treatment arms).

- We included assessment of the quality of evidence in this review. We did not use GRADEpro GDT 2015 to determine levels of evidence because it did not consider important issues such as small study size (which predominated in our included studies), and other factors that could have affected judgement about study quality. Because we observed a wide range of surgery types we decided to analyse the primary outcomes separately for different surgery types and present the analyses in appendices. This was to better serve clinical decision making, and to support GRADE decisions.

- We limited study size to a minimum of 10 participants completing the study in each trial arm, to be consistent with this practice in many pain and anaesthesia reviews, and because of the considerable concerns about small study size as a source of heterogeneity and bias. For these reasons we also performed a sensitivity analysis to check that the overall result was also reflected by the largest studies, least likely to suffer from problems of random chance or small study bias.

- We created a grid to help with consistent judgements about GRADE, relating to study size and presence or absence of small-study effects.

- Because reports did not categorise adverse events as major or minor, we pooled all adverse event reports together.

\section{NOTES}

\section{Assessed for updating in 2021}

In January 2021 we did not identify any potentially relevant studies likely to change the conclusions. Therefore, this review has now been stabilised following discussion with the authors and editors. The review will be reassessed for updating in two years. If appropriate, we will update the review before this date if new evidence likely to change the conclusions is published, or if standards change substantially which necessitate major revisions.

\section{IN DEX TERMS}

\section{Medical Subject Headings (MeSH)}

Acute Pain [ ${ }^{\star}$ drug therapy]; Analgesics [ ${ }^{*}$ administration \& dosage] [adverse effects]; Analgesics, Opioid [administration \& dosage]; Central Nervous System Diseases [chemically induced]; Hyperalgesia [epidemiology]; Injections, Intravenous; Ketamine [ ${ }^{\star}$ administration \& dosage] [adverse effects]; Morphine [administration \& dosage]; Pain Measurement; Pain, Postoperative [ ${ }^{*}$ drug therapy]; Postoperative Nausea and Vomiting [epidemiology] [prevention \& control]; Randomized Controlled Trials as Topic

\section{MeSH check words}

Adult; Humans 